Dittrich, Ann-Kathrin

\title{
Pädagogisches Wissen im LehrerInnenberuf. Rekonstruktive Befunde aus der schulischen Praxis
}

Bad Heilbrunn : Verlag Julius Klinkhardt 2020, 232 S. - (forschung klinkhardt)

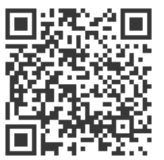

Quellenangabe/ Reference:

Dittrich, Ann-Kathrin: Pädagogisches Wissen im LehrerInnenberuf. Rekonstruktive Befunde aus der schulischen Praxis. Bad Heilbrunn : Verlag Julius Klinkhardt 2020, 232 S. - (forschung klinkhardt) URN: urn:nbn:de:0111-pedocs-204894 - DOI: 10.25656/01:20489

https://nbn-resolving.org/urn:nbn:de:0111-pedocs-204894

https://doi.org/10.25656/01:20489

in Kooperation mit / in cooperation with:

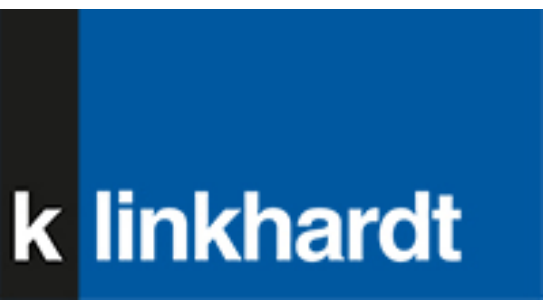

http://www.klinkhardt.de

\section{Nutzungsbedingungen}

Dieses Dokument steht unter folgender Creative Commons-Lizenz: http://creativecommons.org/licenses/by-nc-sa/4.0/deed.de - Sie dürfen das Werk bzw. den Inhalt unter folgenden Bedingungen vervielfältigen, verbreiten und öffentlich zugänglich machen sowie Abwandlungen und Bearbeitungen des Werkes bzw. Inhaltes anfertigen: Sie müssen den Namen des Autors/Rechteinhabers in der von ihm festgelegten Weise nennen. Dieses Werk bzw. der Inhalt darf nicht für kommerzielle Zwecke verwendet werden. Die neu entstandenen Werke bzw. Inhalte dürfen nur unter Verwendung von Dizenzbedingungen weitergegeben werden, die mit denen dieses Lizenzbedingungen weitergegeben werden,

Mit der Verwendung dieses Dokuments erkennen Sie die

Nutzungsbedingungen an.

\section{Terms of use}

This document is published under following Creative Commons-License: http://creativecommons.org/licenses/by-nc-sa/4.0/deed.en - You may copy, distribute and transmit, adapt or exhibit the work in the public and alter, transform or change this work as long as you attribute the work in the manner specified by the author or licensor. You are not allowed to make commercial use of the work. If you alter, transform, or change this work in any way, you may distribute the resulting work only under this or a comparable license.

By using this particular document, you accept the above-stated conditions of use.

\section{Kontakt / Contact:}

peDOcs

DIPF | Leibniz-Institut für Bildungsforschung und Bildungsinformation Informationszentrum (IZ) Bildung

E-Mail:pedocs@dipf.de

Internet: www.pedocs.de

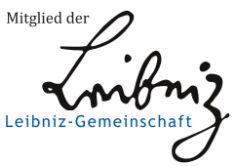




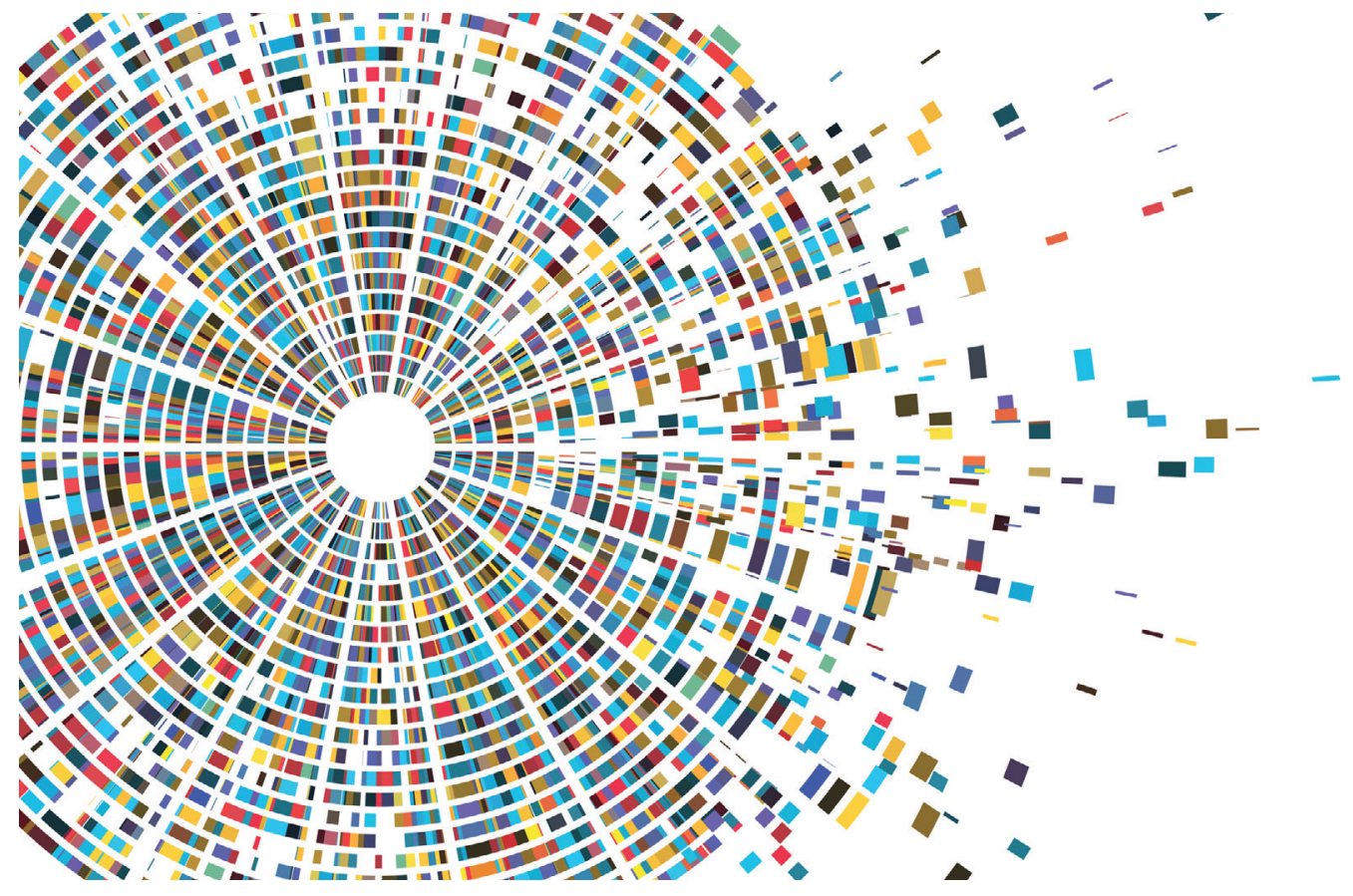

\section{Ann-Kathrin Dittrich}

\section{Pädagogisches Wissen im Lehrerlnnenberuf}

Rekonstruktive Befunde aus der schulischen Praxis 
Dittrich

Pädagogisches Wissen im LehrerInnenberuf 



\section{Ann-Kathrin Dittrich}

\section{Pädagogisches Wissen im LehrerInnenberuf}

Rekonstruktive Befunde aus der schulischen Praxis 
Die Satzeinrichtung sowie die Open Access-Publikation dieses Titels wurde mit finanzieller Unterstïtzung aus den Fördermitteln des Vizerektorats für Forschung der Leopold-Franzens-Universität Innsbruck ermöglicht.

\section{universität innsbruck}

Das vorliegende Buch basiert auf einer Dissertation mit dem Titel „Pädagogisches Wissen im LehrerInnenberuf - Konzepte und empirische Befunde“, die im Rahmen der Forschungsgruppe Teacher Education am Institut für LehrerInnenbildung und Schulforschung der Universität Innsbruck verfasst wurde.

Dieser Titel wurde in das Programm des Verlages mittels eines Peer-Review-Verfahrens aufgenommen. Für weitere Informationen siehe www.klinkhardt.de.

Bibliografische Information der Deutschen Nationalbibliothek Die Deutsche Nationalbibliothek verzeichnet diese Publikation in der Deutschen Nationalbibliografie; detaillierte bibliografische Daten sind im Internet abrufbar über http://dnb.d-nb.de.

2020.kg $\odot$ by Julius Klinkhardt.

Satz: Kay Fretwurst, Spreeau.

Bildnachweis Umschlagseite 1: @ by Tetiana Lazunova/istockphoto.

Druck und Bindung: AZ Druck und Datentechnik, Kempten.

Printed in Germany 2020.

Gedruckt auf chlorfrei gebleichtem alterungsbeständigem Papier.

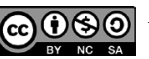

Die Publikation (mit Ausnahme aller Fotos, Grafiken und Abbildungen) ist veröffentlicht unter der Creative Commons-Lizenz: CC BY-NC-SA 4.0 International

https://creativecommons.org/licenses/by-nc-sa/4.0/ 


\section{Zusammenfassung}

Das Handeln von Lehrpersonen im Schulalltag und das damit einhergehende Professionalisierungsverständnis sind zentrale Themen in der LehrerInnenbildung (vgl. Thenorth 2006; Guerriero 2017). Das professionelle Wissen gilt mittlerweile als Schlüsselkompetenz für erfolgreiches unterrichtliches Handeln (vgl. Shulman 1987; Voss et al. 2015; European Commission 2018). Aufgrund fundamentaler Wandlungsprozesse in unserer Gesellschaft, die sich spiegelbildlich in der Schule abbilden, sind die Fragen nach dem Professionsverständnis von Lehrpersonen, vor allem das immer wichtiger werdende pädagogische Wissen (vgl. Shulmans 1987) für LehrerInnen im Zusammenhang mit Globalisierung, Multikulturalität und Technologisierung, sowie der Vermittlung der Schlüsselqualifikationen neu zu stellen. Der Fokus der vorliegenden Arbeit liegt auf der Rekonstruktion pädagogischen Wissens aus der Schulpraxis. Im Rahmen einer qualitativ-rekonstruktiven Studie wird über Interviews und Unterrichtsbeobachtungen versucht, pädagogisches Wissen von Lehrpersonen sichtbar zu machen und konzeptuelle Erkenntnisse für die LehrerInnenbildung aufzuarbeiten. Zudem werden Abweichungen und Übereinstimmungen kognitiver Muster mit ihrem pädagogischen Handeln aufgezeigt und der Transfer von pädagogischem Wissen und Handeln diskutiert. Ein weiterer Schwerpunkt liegt auf der metareflexiven Entwicklung professionellen Wissens und Handeln von Lehrpersonen, die vor dem Hintergrund handlungstheoretischer Zugänge diskutiert wird. 


\begin{abstract}
The actions of teachers in everyday school life and the associated understanding of professionalisation is a central topic in teacher education (Thenorth 2006; Guerriero 2017). Professional knowledge is therefore regarded as a key competence of successful teaching (Shulman 1987; Blömeke et al. 2008; Voss et al. 2015; European Commission 2018). As a result of fundamental processes of change in our society, set against the backdrop of globalisation, multiculturalism and technology, which are all mirrored in the school system, the professional understanding of teachers, especially the increasing importance of pedagogical knowledge, (Shulman 1987) plays a vital role in the mediation of core qualifications. The focus of this paper is the reconstruction of general pedagogical knowledge of teachers from everyday school practice. In the course of a qualitative-reconstructive study, general pedagogical knowledge of teachers is made visible with the help of interviews and lesson observations and conceptual insights gained for teacher education. Furthermore, the study presents deviations and accordances of cognitive concepts of the teachers and their acting. Another focus is on a meta-reflective development of professional knowledge and action of teachers, taking into account action-theoretical approaches.
\end{abstract}




\section{Vorwort}

Beim vorliegende Buch handelt es sich um eine adaptierte Fassung einer Dissertation, die im Rahmen einer Forschungsgruppe an der Universität Innsbruck am Institut für LehrerInnenbildung und Schulforschung im Bereich Teacher Education verfasst wurde.

Pädagogisches Wissen wird als ein zentraler professioneller Wissensbereich von Lehrpersonen verstanden, welchem aufgrund gegenwärtiger Transformationsprozesse und den damit einhergehenden neuen Anforderungen für Schule und Lehrpersonen eine wichtige Bedeutung zukommt (vgl. Shulman 1978; Bromme 1992; Voss et al. 2015; Leicht et al. 2018). Soziale, politische und ökonomische Wandlungsprozesse bedingen neben einem umfangreichen Wissen für die Umsetzung des Bildungs- und Erziehungsauftrags eine kontinuierliche Weiterentwicklung pädagogischen Wissens von Lehrpersonen (vgl. Guerriero 2017).

Die Bedeutung sowie der Diskurs über pädagogisches Wissen von Lehrpersonen hat sich durch die zweite empirische Wende ${ }^{1}$ um das Jahr 2000 verstärkt. Diese führte zu einem Trend der Messung pädagogischen Wissens mittels psychometrischer Testverfahren. Die im Rahmen der Testverfahren definierten standardisierten Konstrukte beeinflussen maßgeblich das gegenwärtige Verständnis. Stützen sich unterschiedliche Studien auf die Testung ähnlicher Konzeptualisierungen, so ist aufgrund stetiger Transformationsprozesse und der Trend zu normativen Zugänge die Aktualität und Komplexität der Konstrukte dennoch stets in Frage zu stellen (vgl. König et al. 2014; Kraler et al. 2017).

Die vorliegende Arbeit zielt auf die Rekonstruktion pädagogischen Wissens von Lehrpersonen aus der unmittelbaren Unterrichtspraxis unterschiedlicher Schulformen in Österreich ab. Die praxisnahe Forschung ermöglicht es über einen qualitativen Zugang mit Interviews und Beobachtungen, das Verständnis von Lehrpersonen und deren Herausforderungen sichtbar zu machen und den Schulalltag, ergänzend zu den existierenden normativen Studien, möglichst gegenstandsadäquat darzustellen. Wichtig ist in diesem Zusammenhang anzumerken, dass der Fokus der vorliegenden Arbeit nicht auf einer systematischen Erstellung pädagogischen Wissens liegt, sondern in der durchgeführten Untersuchung die Rekonstruktion aus der Schulpraxis im Vordergrund steht. In der vorliegenden Studie können bestehende Konzeptualisierungen pädagogischen Wissens ergänzt, weiterentwickelt und im Zusammenhang mit Transformationsprozessen diskutiert werden. Es werden zudem Übereinstimmungen und Abweichungen zwischen kognitiven Mustern pädagogischen Wissens der untersuchten Lehrpersonen und ihrem Handeln aufgezeigt sowie hinsichtlich dem Transfer von Wissen und Handeln und ihrem metareflexiven Entwicklungsprozess diskutiert.

1 Die empirische Wende der Bildungsforschung wurde vorwiegend durch unzufriedenstellenden Ergebnissen internationaler Vergleichsstudien wie PISA und TIMSS eingeleitet. Die Bildungspolitik erhofft sich durch die Kompetenzorientierung und der damit einhergehenden Output-Orientiertung zentrale Kompetenzen von Lehrpersonen über standardisierte Testverfahren zu konzeptualisieren, die Leistungen von SchülerInnen sowie das professionelle Wissen und Handeln von Lehrpersonen positiv beeinflussen. 



\section{Inhalt}

\section{Grundlagen}

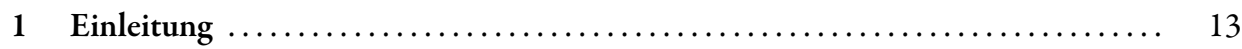

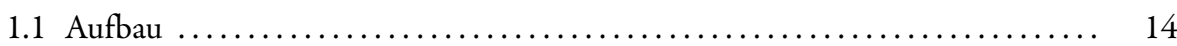

1.2 Thematische Einbettung pädagogischen Wissens ................... 15

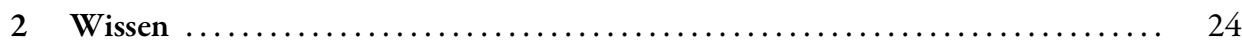

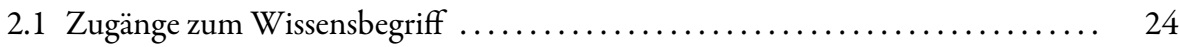

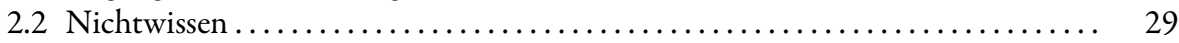

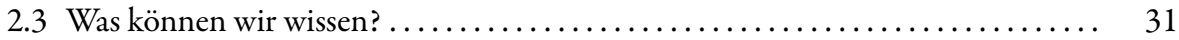

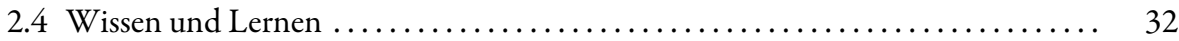

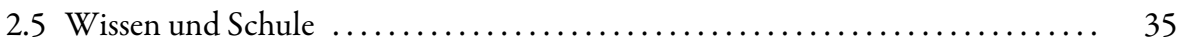

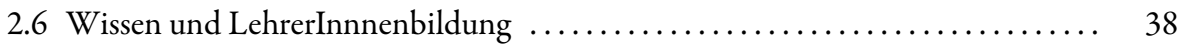

3 Konzeptualisierungen von Wissen und Professionswissen im LehrerInnenberuf ... 42

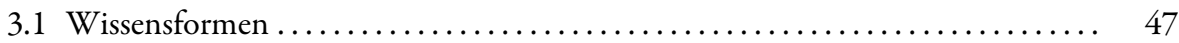

3.1.1 Deklaratives und prozedurales Wissen $\ldots \ldots \ldots \ldots \ldots \ldots \ldots \ldots \ldots \ldots \ldots$

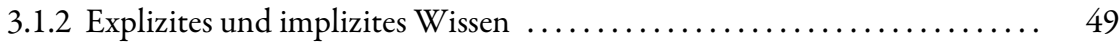

3.1.3 Handlungswirksames und träges Wissen $\ldots \ldots \ldots \ldots \ldots \ldots \ldots \ldots \ldots . \quad 50$

3.2 Wissensqualitäten ...................................... 51

3.3 Professionelles Wissen und seine Aneignung $\ldots \ldots \ldots \ldots \ldots \ldots \ldots \ldots \ldots \ldots, \quad 52$

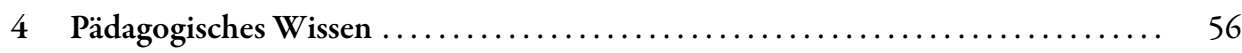

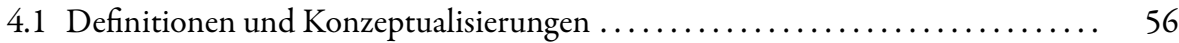

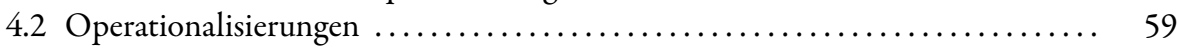

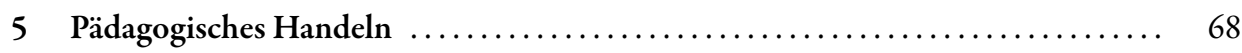

5.1 Modelle pädagogischen Handelns .............................. 68

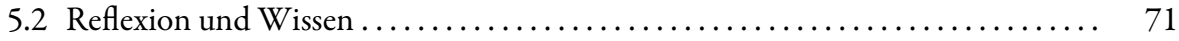

5.3 Pädagogische Interaktion im Kontext des symbolischen Interaktionismus und der sozialen Praxis ...................................... 74

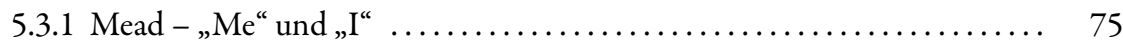

5.3.2 Giddens - Soziale Praxis .............................. 77 
II Empirischer Zugang

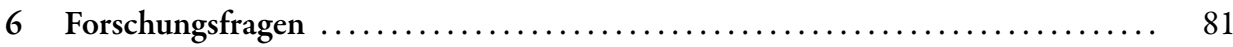

$7 \quad$ Methodologisches Vorgehen $\ldots \ldots \ldots \ldots \ldots \ldots \ldots \ldots \ldots \ldots \ldots \ldots \ldots \ldots, 83$

7.1 Qualitativ-rekonstruktiver Zugang $\ldots \ldots \ldots \ldots \ldots \ldots \ldots \ldots \ldots \ldots \ldots \ldots \ldots \ldots \ldots \ldots \ldots$

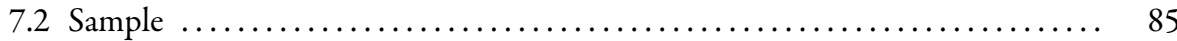

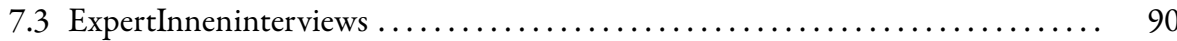

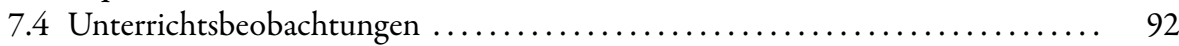

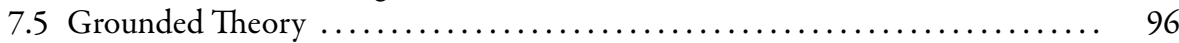

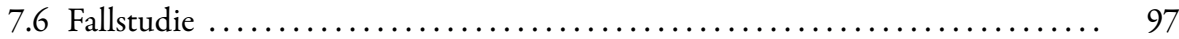

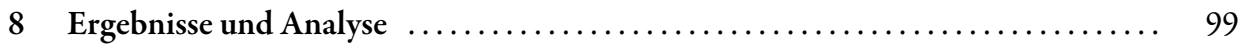

8.1 Kategorien pädagogischen Wissens aus den Interviews ................. 100

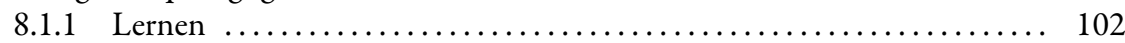

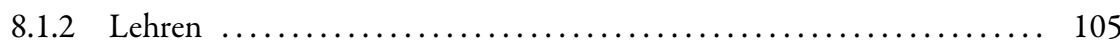

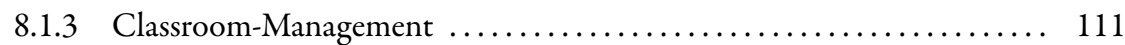

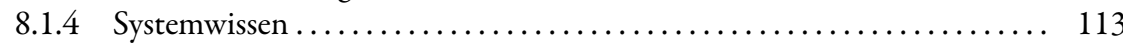

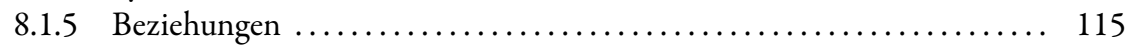

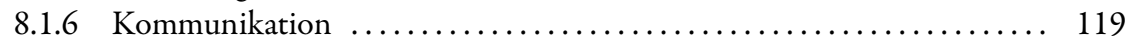

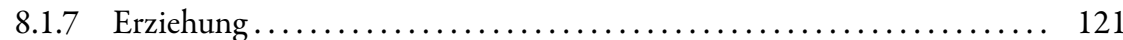

8.1 .8 Entwicklungspsychologie $\ldots \ldots \ldots \ldots \ldots \ldots \ldots \ldots \ldots \ldots \ldots \ldots, 122$

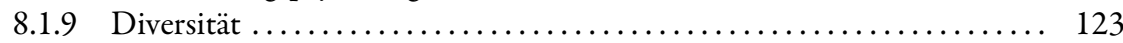

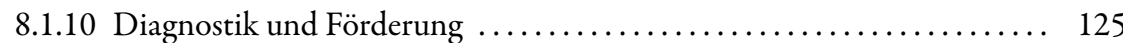

8.1 .11 Person ............................................ 126

8.2 Herausforderungen für pädagogisches Wissen $\ldots \ldots \ldots \ldots \ldots \ldots \ldots \ldots \ldots \ldots \ldots \ldots$

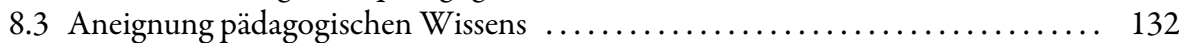

8.4 Auswirkungen von Defiziten pädagogischen Wissens ................. 134

8.5 Kategorien pädagogischen Wissens aus den Beobachtungen ............... 138

8.5.1 (Pädagogische) Interaktion . . . . . . . . . . . . . . . . . . . . . . 139

8.5 .2 Didaktik .......................................... 146

8.5.3 Kommunikation .................................... 151

8.5.4 Classroom-Management ................................. 154

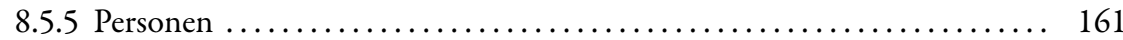

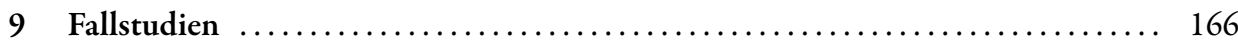

9.1 Fallstudie 1 - Lehrperson Grundschule ......................... 166

9.2 Fallstudie 2 - Lehrperson Neue Mittelschule ....................... 169

9.3 Fallstudie 3 - Lehrperson Gymnasium ......................... 174

10 Zusammengefasste Ergebnisdarstellung aus den rekonstruierten Kategorien pädagogischen Wissens ....................................... 179

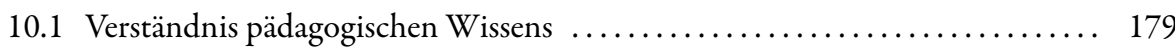

10.2 Übereinstimmungen und Unterschiede der rekonstruierten Kategorien zwischen den Erhebungsinstrumenten ....................... 184

10.3 Schulformspezifische Rekonstruktionsschwerpunkte ................ 189 
III Integration und Diskussion

11 Konzeptualisierungen pädagogischen Wissens .................... 193

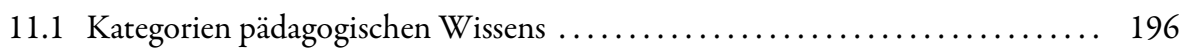

11.2 Übereinstimmungen und Unterschiede zwischen konzeptionellen Mustern pädagogischen Wissens und der Handlungsebene ................ 201

12 Befunde zur Bedeutung pädagogischen Wissens für Lehrpersonen .......... 203

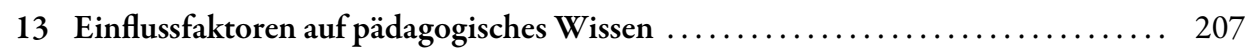

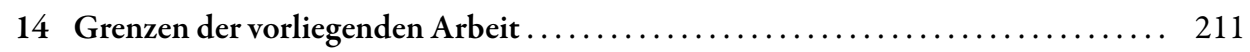

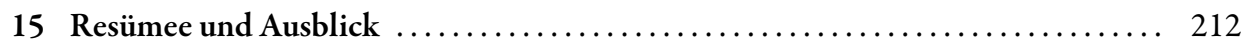

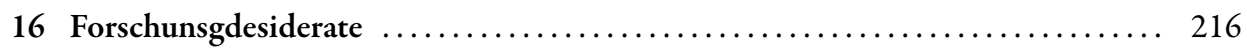

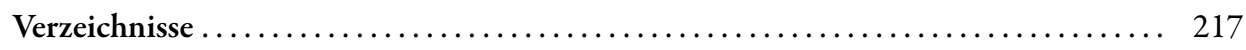

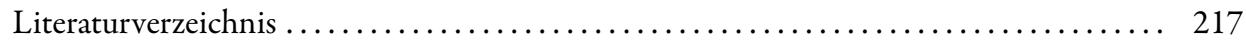

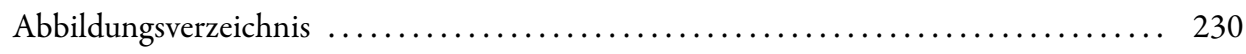

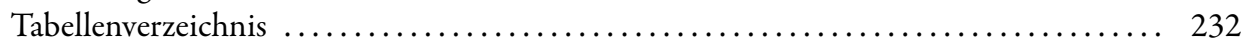





\section{Grundlagen}

\section{Einleitung}

Soziale, wirtschaftliche und politische Transformationsprozesse beeinflussen die Rolle und Aufgaben von Lehrpersonen maßgeblich (vgl. Eriksen 2001: 3ff.; Terhart 2013: 55f.). Themen wie Globalisierung, Technologisierung und Multikulturalität sowie der Trend zur Wissensgesellschaft wirken auf Lehrpersonen als Wissensvermittler sowie ihren Wissensbestand ein (vgl. Schratz \& Schrittesser 2003: 185; European Commission 2018: 5ff.).

Die Schule gilt aufgrund ihrer gesellschaftlichen Funktion als Spiegel der Gesellschaft (vgl. Kraler \& Schratz 2012: 89). Es wird erwartet, dass im Schulalltag adäquat mit Veränderungsprozessen und den damit verbundenen Herausforderungen umgegangen wird (vgl. Bauer et al. 1996: 5f.). Lehrpersonen sind für die Weitergabe bestehender Traditionen und Wissensbestände sowie für die Vermittlung neuen Wissens und von Innovationen gleichermaßen verantwortlich (vgl. Eagleton 2009: 14ff.). Sie stehen selbst im Mittelpunkt gesellschaftlichen Interesses und repräsentieren kulturelle Werte (vgl. Schratz \& Schrittesser 2003: 181). Der Wandel und die daraus entstehenden neuen Anforderungen beeinflussen das Verständnis von Schule und die Rolle von Lehrpersonen. Zentral ist die Frage nach den Inhalten, die in der Schule vermittelt werden sollen, sowie jene nach dem Wissen, welches Lehrpersonen benötigen. Sie befinden sich in einem professionellen Veränderungsprozess und müssen, wie Schratz \& Schrittesser (2003: 185) postulieren, für die Umsetzung gegenwärtiger Wissens- und Handlungsstrukturen kontinuierlich verlernen, umlernen oder neu lernen (vgl. Schratz \& Schrittesser 2003: 185). Henting (1996: 17ff.) verwies schon Mitte der Neunzigerjahre auf die Notwendigkeit, Schule und damit die LehrerInnenrolle anzupassen. Sie sind nicht nur gefordert ihren Wissensbestand weiterzuentwickeln, sondern müssen neue Perspektiven gewinnen. Lehrpersonen stehen vor der Frage, welches Wissen sie gegenwärtig brauchen, um curricular verankerte Bildungs- und Erziehungsaufgaben wahrnehmen und zusätzlichen gesellschaftlichen Anforderungen professionell gerecht werden zu können.

Wie Hatti und andere (vgl. Hatti 2003: 22; König 2010a: 40; European Commission 2015: 13) verdeutlichen, werden an Lehrpersonen große Erwartungen an die Gestaltung und (Weiter-) Entwicklung von Schule und Unterricht gestellt. Der umfangreiche Diskurs zur Rolle von Lehrpersonen und ihrem Professionswissen bestärkt diese Annahme. Von ihnen wird erwartet, dass sie aktuell relevantes Wissen aufarbeiten und ihre Wissensbestände regelmäßig professionell weiterentwickeln (vgl. Guerriero 2017: 13). Zahlreiche Studien belegen (vgl. Lipowsky 2006: 47ff.; Baumert et al. 2010: 133ff.; Lenske et al. 2015: 225), wie auch Weinert (1997:137ff.) mit dem Begriff „Humankapital“ argumentiert, die essenzielle Bedeutung des LehrerInnenwissens und demonstrieren den Zusammenhang zwischen der Unterrichtsqualität und der schuli- 
schen sowie persönlichen Entwicklung von SchülerInnen (vgl. European Commission 2015: 9; 2018). ${ }^{2}$

Fordern die Forschungsergebnisse einheitlich qualifizierte Lehrpersonen, verdeutlichen gesellschaftliche Anforderungen an die Rolle sowie historische Entwicklungen die Schwierigkeit eines einheitlichen Bilds einer professionellen Lehrperson (vgl. Enzelberger 2001: 31ff.). Der Trend zur Vereinheitlichung zentraler Merkmale von Lehrpersonen wird über vorhandene Indikatoren zur Charakterisierung des professionellen Wissens und der Kompetenzen verstärkt (vgl. European Commission 2013: 9f.). Enzelberger (2001: 31ff.) sowie Rheinberg (2001: 297) diskutieren jedoch die Problematik der Standardisierung und argumentieren, dass es den einen guten Lehrer/die eine gute Lehrerin nicht gibt.

Das derzeitige Bild von Schule definiert sich im Kontext sozialer, politischer und wirtschaftlicher Transformationsprozesse und der Bedeutung von Wissen in einer Wissensgesellschaft nicht nur über die Wissensvermittlung. Im Mittelpunkt steht die Aneignung von handlungsleitenden Fähigkeiten und Fertigkeiten. Die Schule wird zunehmend als Innovationszentrum interpretiert, in dem sich SchülerInnen selbstgesteuert Wissen und Kompetenzen aneignen und zum lebenslangen Lernen angeregt werden. Die sozialen, wirtschaftlichen und politischen Entwicklungen können nach Terhart (2013: 89f.) nur mit professionellen Lehrpersonen gefördert werden. Eine wesentliche Wissensform professioneller Lehrpersonen für die Umsetzung gegenwärtiger Anforderungen im Unterrichtsalltag ist das pädagogische Wissen.

Im vorliegenden Buch wird der Diskurs zum pädagogischen Wissen von Lehrpersonen eröffnet. Das gegenwärtige Verständnis wird maßgeblich durch die im Rahmen der Kompetenzorientierten standardisierten Testverfahren beeinflusst. Ziel der Arbeit ist die Rekonstruktion pädagogischen Wissens von Lehrpersonen aus der unmittelbaren Unterrichtspraxis. Die praxisnahe Forschung ermöglicht es, das Verständnis von Lehrpersonen und deren Herausforderungen sichtbar zu machen und den Schulalltag möglichst gegenstandsadäquat, ergänzend zu existierenden normativen Studien, darzustellen. Für eine angemessene Rekonstruktion des Unterrichtsalltags werden kognitive Muster sowie pädagogisches Handeln mit Hilfe einer qualitativen Studie rekonstruiert. Ein weiterer Fokus liegt auf einer metareflexiven Entwicklung professionsspezifischen Denkens und Handeln von Lehrpersonen mit Hilfe handlungstheoretischer Zugänge von Mead mit dem Konzept des „Me“ und „““ sowie Giddens mit seiner Theorie der sozialen Praxis. In der Arbeit werden grundlegende theoretische Zugänge und Modelle als Erklärungs- bzw. Begründungsfiguren für die Weiterentwicklung der Fragestellungen diskutiert und Zusammenhänge zwischen den rekonstruierten Ergebnissen und den theoretischen Grundlagen aufgezeigt.

\subsection{Aufbau}

Das Buch gliedert sich in drei Bereiche - einen Grundlagenteil, einen empirischen Teil sowie eine Zusammenführung und Diskussion der Ergebnisse. Der Grundlagenteil systematisiert sich über eine allgemeine Diskussion zum Wissens, Nichtwissen und der Frage was können wir überhaupt wissen. Anschließend wird das Thema Lernen und Schule mit dem Wissen in Verbindung

2 Das Angebots-Nutzungs-Modell von Helmke (2003) thematisiert als ein weiteres Modell die Bedeutung von Lehrpersonen für die Qualität des Unterrichts. Weitere Zugänge, wie zum Beispiel die Meta-Analysen von Hatti (2003) oder auch die im Rahmen der Kompetenzorientierung durchgeführte COACTIV-Studie (vgl. Voss et al. 2011) zur Messung von Professionswissen, heben hervor, dass Leistungen von SchülerInnen durch das Wissen und Handeln von Lehrpersonen maßgeblich beeinflusst werden. 
gebracht und spezifisch das professionsspezifische Wissen und ihre Formen thematisiert. Kapitel 4 eröffnet die Diskussion zum Thema pädagogisches Wissen, mit ihren Konzeptualisierungen und Operationalisierungen. Kapitel 5 knüpft mit einem Diskurs zum pädagogischen Handeln an. Das Handeln von Lehrpersonen wird in der vorliegenden Forschungsarbeit in einem eigenen Kapitel ausgeführt, da nach dem zugrundeliegenden Verständnis pädagogischen Wissens sowie der Lehr-Lern-Konfiguration das pädagogische Handeln konstitutiv mit dem Wissen verknüpft ist (vgl. Abbildung 1).

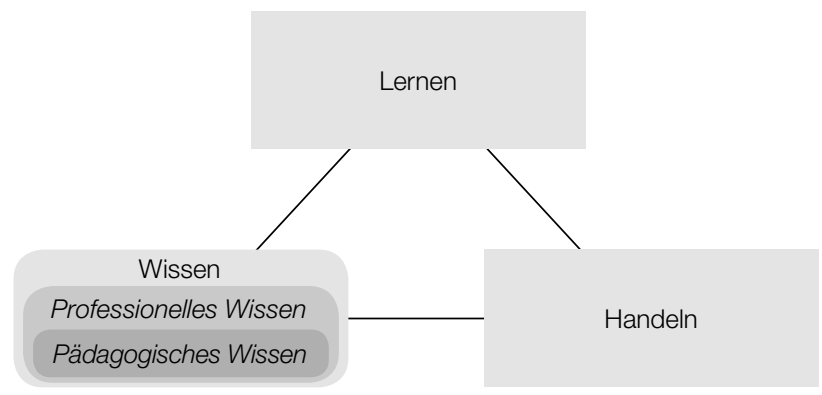

Abb. 1: Überblick zentrale Themenbereiche der Forschungsarbeit

Der Schwerpunkt des Kapitels liegt auf einer theoretischen Einbettung des Begriffs und thematisiert den Transfer von Wissen zum Handeln mithilfe von Reflexion. Auf Basis von zwei handlungstheoretischen Zugängen - Symbolischem Interaktionismus (Mead) und sozialer Praxis (Giddens) - wird pädagogisches Wissen und seine Sichtbarkeit diskutiert.

Der empirische Zugang illustriert die in der Studie untersuchten Forschungsfragen, beschreibt das angelegte Forschungsdesign und präsentiert die Untersuchungsergebnisse. Die Ergebnisdarstellung untergliedert sich in die rekonstruierten Wissensfelder aus den Erhebungsinstrumenten Interviews und Unterrichtsbeobachtungen. Zu den Wissenskategorien werden weitere Ergebnisse betreffend die Herausforderungen, die Aneignung sowie die Auswirkungen von Wissensdefiziten bei pädagogischem Wissen dargestellt. Es werden anschließend drei untersuchte Lehrpersonen mittels Fallstudien anhand individuellen Mustern illustriert. Im Anschluss erfolgt eine zusammengefasste Darstellung der rekonstruierten Kategorien sowie eine Präsentation weiterer signifikante Ergebnisse aus der Studie.

Der abschließende Teil, die Integration und Diskussion, hat die Zusammenführung der Ergebnisse mit den diskutierten theoretischen Grundlagen zum Ziel. Es werden alle rekonstruierten Ergebnisse noch einmal systematisch aufgegriffen und unter Berücksichtigung theoretischer Grundlagen hinsichtlich ihrer Bedeutung für die LehrerInnenbildung rekontextualisiert.

\subsection{Thematische Einbettung pädagogischen Wissens}

Zentral für den Erhalt von Gesellschaften ist nach Fend (2006: 36) ein Gleichgewicht des politischen, ökonomischen und des Bildungssystems. Der Austauschprozess der Funktionen und die damit verbundene Stabilität unterstützen eine innovative und moderne Gesellschaft (vgl. Fend 2006: 36). Fend entwickelt ein struktur-funktionalistisches Modell, in dem er dem Bildungssystem spezifische Funktionen zuschreibt. Es hat die Aufgabe, SchülerInnen zu handlungs- und kommunikationsfähigen Mitgliedern einer Gesellschaft zu machen (vgl. Fend 2006: 35). Die 
Frage der Funktion sowie des pädagogischen Anspruchs an Schule und das hierfür benötigte Wissen von Lehrpersonen ist im Kontext stetiger Transformationsprozesse immer wieder neu zu stellen.

Gegenwärtige Wandlungsprozesse, die in die Schule diffundieren, sind soziale, wirtschaftliche und politische Transformationen. Terhart (2013: 55f.) benennt sieben gesellschaftskulturelle Veränderungen, die sich auf formaler Bildungsebene widerspiegeln. Diese sind (1) die zunehmende geografische, soziale und kulturelle Mobilität, (2) die Gestaltung eines selbstbestimmten aktiven Lebens, (3) die zunehmende Bedeutung von umfassenden Schlüsselqualifikationen und Problemlösungsfähigkeiten, (4) soziale sowie kommunikative Kompetenzen im Berufs- und Privatleben, (5) der Umgang mit kultureller Heterogenität und Diversität, (6) die zunehmende Notwendigkeit lebenslangen Lernens sowie (7) die verminderte Bedeutung staatlicher Vorund Fürsorgeleistungen. Weitere gegenwärtig relevante Einflussfaktoren betreffen technologische Entwicklungen sowie eine Wissenskonjunktur (vgl. Thiel 2007: 153; Eriksen 2001: 3ff.). Der technologische Wandel wirkt sich durch die Implementierung unterschiedlicher Medien im Unterricht sowie eine nie zuvor gesehene Fülle an schnell zugänglichem Wissen aus. Das schnelle Abfragen von Informationen sowie die Bilderflut über unterschiedliche Medien wie Mobiltelefone oder Tablets beeinflussen das Lernen. Lehrpersonen stehen vor der pädagogischen Herausforderung, mit neuen Technologien und dem Zuwachs an Wissen und Informationen umzugehen.

Wie die Diskussion zeigt, formiert sich Schule über kulturelles Denken und Handeln und charakterisiert sich über ein Spannungsfeld zwischen Tradierung und Bewahrung und Innovation und Veränderung (vgl. Eagleton 2009: 14ff.; Kraler 2011: 43). Von Fend (2006: 48f.) benannte grundlegende gesellschaftliche Funktionen der Schule sind die Qualifikations-, Selektions-, Legitimations ${ }^{-}{ }^{3}$ sowie die Entkulturationsfunktionen, die eine Erhaltung und Reproduktion einer Gesellschaft unterstützen. Mit der Entkulturationsfunktion verdeutlicht er die Verzahnung und die Notwendigkeit stetiger Veränderungsprozesse des Bildungssystems basierend auf den von Terhart (2013), Thiel (2007) sowie Eriksen (2001) angeführten gesellschaftlichen Entwicklungen. Schon Immanuel Kant (1977: 706) verwies auf die von Fend diskutierte Wechselbeziehung von Bildung und Gesellschaft und beschrieb die Schule als Ort der „Kultivierung des Menschen".

Keck (2009: 157ff.) etwa führt diese Diskussion über das generationsbezogene Spannungsfeld Schule und Kultur fort. Betrachtet man die primäre Funktion von Schule und formaler Bildung ausgehend von Makrotheorien (vgl. Gudjons 2006: 307ff.), haben sie die Aufgabe, die „[...] Sicherstellung und Übertragung der Kultur eines Volkes an die folgende Generation" (Keck 2009: 157) sicherzustellen. Die Definition beinhaltet den Erhalt, die Reproduktion sowie die (Neu-)Konstruktion von Strukturen und ihre jeweilige fundamentale Bedeutung (vgl. Giles \& Middleton 2008: 6ff.). Die Bedeutung der Kultur im Zusammenhang mit dem Bildungs- und Erziehungsauftrag der Schule verdeutlicht die Notwendigkeit der stetigen Anpassung formaler Bildung unter Berücksichtigung sozialer, ökonomischer und politischer Transformationsprozesse. Basierend auf diesen Überlegungen muss die Frage nach adäquatem Wissen von Lehrpersonen und ihrer Funktion neu gestellt werden (vgl. Mittelstraß 2001: 33).

3 Die Qualifikationsfunktion definiert sich über die Vermittlung von Fertigkeiten und Kenntnissen, die SchülerInnen zur Teilnahme am gesellschaftlichen Leben befähigen. Die Selektionsfunktion verweist auf die Strukturübernahme der Gesellschaft in der Schule, indem SchülerInnen je nach Leistung Positionen zugeschrieben werden. Die Legitimationsfunktion kommt über die Aneignung normativer Strukturen der Gesellschaft in der Schule zum Ausdruck. 
Wissen wurde seit der Ausdifferenzierung der Gesellschaft und der Etablierung einer arbeitsteiligen Wirtschaft als besonderes Gut und häufig als Machtinstrument ${ }^{4}$, verbunden mit einer speziellen Personengruppe, verstanden. Der Umgang mit Wissen hat sich verändert und charakterisiert sich über ein facettenreiches und interdisziplinäres Verständnis (vgl. Mittelstraß 2001: 38f.). Im Kontext dieser Entwicklungen vertreten AutorInnen unterschiedliche Positionen. Spricht Rabl (2014: 11) von einer zunehmenden Bedeutung des Wissens, so konstatiert Mittelstraß (2001: 40) eine Trivialisierung des Wissensbegriffs.

Bezugnehmend auf dieses Verhältnis stehen Lehrpersonen vor der Herausforderung, relevante Informationen zu erkennen und in einen sinnvollen Wissenskontext zu bringen. Mittelstraß betont, dass Information „Wissen und der Gesellschaft Beine macht aber sie damit noch nicht das bessere Wissen ist“ (Mittelstraß 1998: 226; 2001: 41). Wird der Informationsbegriff häufig dem Wissen gleichgesetzt, zeichnet sich die Entstehung von Wissen nach Mittelstraß (1998: 226; 2001: 42) durch ihre dynamische Art und Weise aus. Die Diskussion zum Spannungsfeld Wissen im Zuge der modernen Wissensgesellschaft wird mit den von ihm generierten Ordnungskategorien Verfügungs- und Orientierungswissen diskutiert (vgl. Mittelstraß 1998: 226f.). Verfügungswissen wird als „positives Wissen, ein Wissen um Ursachen, Wirkungen und Mittel“ (Mittelstraß 1989: 19) definiert. Unter Orientierungswissen wird im Vergleich dazu ein „regulatives Wissen, ein Wissen um Ziele und Maximen“ (Mittelstraß 1989: 19) verstanden. Das Orientierungswissen, das er als Zweck- oder Zielwissen benennt, formiert sich mithilfe des Verfügungswissens. Mittelstraß verweist auf die Tatsache, dass sich durch die moderne (Wissens-)Gesellschaft eine zunehmende Differenz zwischen Verfügungswissen (Faktenwissen) und Orientierungswissen ergibt (vgl. Mittelstraß 2001: 42). Die Flut von Wissen moderner Gesellschaften führt zu einer Zunahme des Verfügungswissens und zu einem Rückgang des Orientierungswissens. In Bezug auf Lehrpersonen lässt sich die von Mittelstraß nachgezeichnete Diskussion auf die Schule übertragen. Verfügungs- und Orientierungswissen können als zwei Seiten einer Medaille beschrieben werden. Die Professionalität einer Lehrperson zeigt sich in der Integration beider Seiten. Reines Verfügungswissen, oder auch reines Orientierungswissen, existiert nach Mittelstraß nicht. Ohne Verfügungswissen ist die Lehrperson nicht handlungsfähig. Lehrpersonen als fachliche ExpertInnen mit der Aufgabe eines Erziehungsauftrags müssen für ein professionelles Agieren ein hohes Maß an inhaltlichem Wissen besitzen. Dabei ist nicht nur das Faktenwissen, sondern das Verfügungswissen zentral, welches das Handeln unmittelbar bestimmt. Das Orientierungswissen kann als das normative Wissen der adäquaten Anwendung von Verfügungswissen in einer professionellen Situation verstanden werden. Mittelstraß verweist auf die essenzielle Bedeutung des Orientierungswissens für eine dynamische, moderne, reflexive Gesellschaft.

Lehrpersonen stehen aufgrund des Verhältnisses von Wissen und Information sowie der Bedeutung von Wissen in der modernen Gesellschaft vor einer doppelten Herausforderung: Sie müssen für die Umsetzung des Bildungs- und Erziehungsauftrags wissen, welches Wissen sie

4 Die essenzielle Bedeutung von Wissen wurde von Philosophen wie Aristoteles, Francis Bacon oder Michel Foucault definiert. Bacon (1620) beschreibt in seinem Werk Wissen ist Macht die Verbindung des Wissensbegriffs mit dem Thema Macht. Wissen führe nach Bacon zu einem höheren Dasein in der Gesellschaft. Macht und Wissen wurden von Foucault (1994) als zwei zusammengehörige Konzepte in Verbindung gebracht. Er beschreibt in Überwachen und Strafen, wie durch detailliertes und tiefgründiges Wissen und Macht eine höhere Kontrolle erreicht werden kann. Aristoteles (1966) betonte im Vergleich zu den beiden erstgenannten Philosophen Wissen nicht in Zusammenhang mit Macht, verwies dennoch mit seiner Aussage „alle Menschen streben von Natur aus nach Wissen“ auf den Wissensdurst der Menschheit sowie auf die relevante gesellschaftliche Bedeutung. 
benötigen, sowie entscheiden, welches Wissen sie im gesellschaftlichen Kontext stetiger Veränderungsprozesse an SchülerInnen weitergeben.

Ein Blick in die Vergangenheit zeigt, wie sich das Wissen von Lehrpersonen seit der Entstehung der ersten Schulen in stetigem Wandel befindet (vgl. Gudjons 2000: 33ff.). Dies reicht vom Faktenwissen eines Gelehrten bis zum heutigen Verständnis der Lehrperson als ExpertIn, wobei die pädagogische Wissensdimension eine zunehmende Bedeutung annimmt. Ausgehend vom Mittelalter, mit den ersten Klosterschulen und der Entstehung von Hochschulen im 11. und 12. Jahrhundert, stützte sich die Rolle der Lehrperson auf die Idee der Vermittlung von (elementarem) Faktenwissen sowie die Vermittlung der Fertigkeiten des Lesens und Schreibens. Dieses Verständnis bedingt, dass sie über einen entsprechenden Wissenskanon (ohne pädagogische Dimensionen wie wir sie im heutigen Sinn verstehen) verfügen mussten. Im Kontext der Reformation und seit der Aufklärung hat die Bedeutung der pädagogischen Dimension, insbesondere das von Mittelstraß definierte Orientierungswissen, eine zunehmend wichtigere Rolle übernommen (vgl. Zymek 2008: 220f.; Fend 2008: 49ff.; Kintzinger 2014: 16ff.). Comenius thematisierte nach der Reformation in seinem Werk „Didactica magna“ als einer der ersten die pädagogische Dimension im Kontext einer religiösen Erziehung (vgl. Raithel et al. 2009: 93ff.). Im 18. Jahrhundert hat sich das pädagogische Wissen als wesentliche Kompetenz etabliert (vgl. Hellekamps \& Musolff 2014: 34ff.). Basierend auf seinem Autonomieverständnis entwickelte Immanuel Kant während der Aufklärung eine pädagogische Sichtweise, die das gegenwärtige Verständnis einer pädagogischen Erziehung maßgeblich beeinflusst (vgl. Giesecke 2013: 9f.). Der Prozess der pädagogischen Erziehung führt laut Kant über Disziplinierung, Kultivierung, Zivilisierung und Moralisierung. Die Bildung eines Menschen wird bei ihm in unterschiedlichen Stufen beschrieben und führt zur Freiheit eines individuellen, (selbst-)mündigen Personen (vgl. Raithel et al. 2009: 11). Pestalozzis anthropologischer Ansatz unterstützt den Gedanken einer freien Entwicklung des Menschen, welcher durch die Lehrperson im Schulalltag gefördert werden sollte (vgl. Raithel et al. 2009: 112). Diskussionen zum LehrerInnenwissen und ihr professionelles Wissen Ende der Neunzigerjahre gaben dem pädagogischen Wissen von Lehrpersonen einen neuen Stellenwert. Die Diskussion zur Sichtweise auf den Professionalisierungsstatus von Lehrpersonen verschob sich Ende des 20. Jahrhunderts von der Betrachtungsweise der Semiprofession hin zu Konzepten professionalisierter Berufe (vgl. Terhart 1998; Hellekamps \& Musolff 2014: 34ff.; Kemnitz 2014: 63). Eine der ersten Wissenstaxonomien zum Professionswissen, die pädagogisches Wissen als einen zentralen Bereich professionellen Wissens beschreibt, stammt von Shulman (1986/1987). Eine gängige Klassifizierung von Shulman (1987), die sich im Professionalisierungsdiskurs durchgesetzt hat, ist die Untergliederung professionelles Wissen in fachliches, fachdidaktisches und pädagogisches Wissen. Pädagogisches Wissen hat sich seitdem zu einer wesentlichen Form professionellen Wissens von Lehrpersonen weiterentwickelt. Die zweite empirische Wende nach den Zweitausenderjahren unterstreicht über psychometrische Testverfahren die zentrale Bedeutung pädagogischen Wissens bzw. pädagogischer Kompetenz von Lehrpersonen als wesentlicher Wissensbereich professioneller Lehrpersonen (vgl. Voss et al. 2015).

Um dem Auftrag der Schule, der Wissensvermittlung, gerecht werden zu können (vgl. Fend 2006: 48f.), benötigen Lehrpersonen aufgrund der zunehmend pluralen Gesellschaft mit ihren heterogenen Herausforderungen eine spezifische Form pädagogischen und fachlichen Wissens. Gudjons (2000: 47) beschreibt diese Anforderungen an Lehrpersonen als eine „kognitive Integration [...] curricularer und pädagogischer Wissensbestände im konkreten Lehrerhandeln“. Um das Ziel der Umsetzung dieser zu realisieren, fordert Gudjons die Integration methodischer, fachlicher sowie diagnostischer Kompetenzen mit der Unterrichtsphilosophie und dem situationsgerechten Handeln (vgl. Gudjons 2000: 47). 
Der Arbeitsbereich von Lehrpersonen umfasst nicht nur das Unterrichten. Sie nehmen professionell aktiv am Schulleben teil, müssen Erziehungs- und Entwicklungsaufgaben umsetzen sowie effizientes Lernen und Lehren garantieren (vgl. European Commission 2018: 6; 14). Terhart (2013: 55f.) sowie Fend (2008: 24ff.) betonen die Bedeutung grundlegender Werte und Normen für das Funktionieren einer Gesellschaft. Über den Aspekt gesellschaftlicher Anforderungen hinaus ist ein wesentlicher Bestandteil der Schule, gegenwarts- und zukunftsbezogenes Wissen zu vermitteln (vgl. Esslinger-Hinz \& Sliwka 2011: 23; European Commission 2018: 14). Abbildung 2 zeigt in Anlehnung an Conway et al. (2009), Terhart (2013) sowie Guerriero (2017) einen Überblick über die gegenwärtigen Anforderungen an Lehrpersonen, die sie mithilfe ihres pädagogischen Wissens erfolgreich im Schulalltag umsetzen sollten.

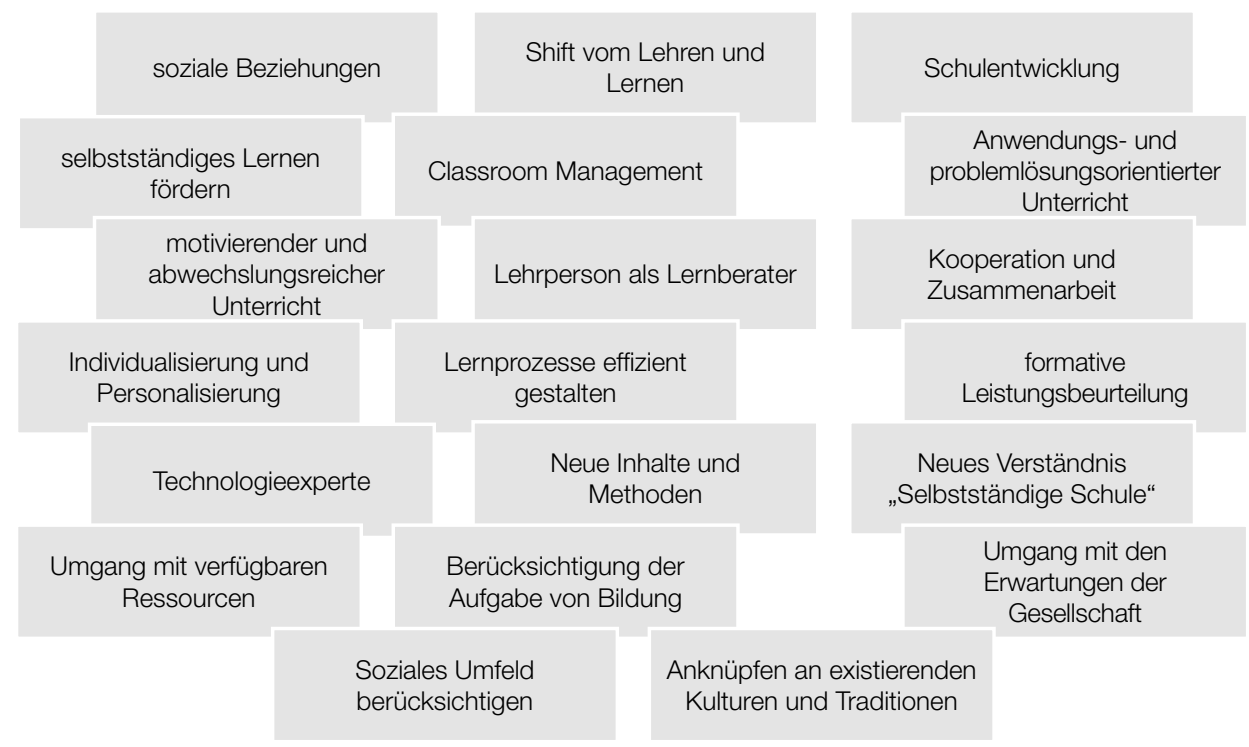

Abb. 2: Anforderungen Lehrpersonen (in Anlehnung an Conway et al. 2009; Terhart 2013: 56ff.; Guerriero 2017)

Innovative didaktische Ansätze und umfangreiches pädagogisches Wissen bieten den Lehrpersonen die Möglichkeit, sich diesen Herausforderungen zu stellen. Mit dem „shift from best practice to next practice“ fordern Kraler \& Schratz (2012: 92) nicht nur die Weiterentwicklung der Profession, sondern neue innovative Muster anzunehmen, um auf Veränderungen und Herausforderungen professionell zu reagieren. Dabei liegt der Fokus nicht nur auf „good teaching“, sondern auf „effective teaching“ (vgl. Terhart 2013: 133ff.). Diese Anforderung für effektives Unterrichten können Lehrpersonen durch die Einnahme unterschiedlicher Rollen verwirklichen. Gudjons (2000: 33ff.) beschreibt in diesem Zusammenhang die Mehrperspektivität der Rollenanteile von Lehrpersonen anhand vier unterschiedlicher LehrerInnentypen: (1) Die Lehrperson als Dompteur, der den Unterricht straff führt und die SchülerInnen zum Lernen antreibt. (2) Die Lehrperson als Entertainer, der Lernende lockt, anzieht und durch geschickte Methodik verzaubert. (3) Die Lehrperson als Neo-Romantiker, der von der natürlichen Neugier und dem unverdorbenen Wissensdurst der Kinder ausgeht. (4) Die Lehrperson als cooler Fachmann, der Wissen sachorientiert und interessant weitergibt. Die EU (2013: 13) benennt 
in Anlehnung an Paquay und Wagner (2001) sechs Bereiche einer professionellen Lehrperson, die das Verständnis Gudjons erweitern: die Lehrperson als „,reflective agent“, „knowledgeable expert", „skiful expert“, „,lassroom actor“, „social agent“ und als „lifelong learner“. Das Rollenverständnis vedeutlicht die Vielseitigkeit des LehrerInnenberufs und verweist auf die Notwendigkeit unterschiedlicher pädagogischer Wissensbereiche.

$\mathrm{Zu}$ den angeführten Anforderungsbereichen definieren das National Research Council (2012), die UNESCO (vgl. Leicht et al. 2018) sowie die OECD (2005) spezifische zentrale Fertigkeiten des 21. Jahrhunderts (vgl. Abbildung 3). Traditionelle Annahmen berufen sich auf kognitive Kompetenzen, wie kritisches Denken oder problemlösungsorientiertes Lernen, als Schlüsselfähigkeiten. Studien belegen, dass gegenwärtig vor allem intrapersonelle sowie interpersonelle Kompetenzen, die sogenannten Soft Skills, und pädagogische Wissensbereiche der Schlüssel für Bildung seien (vgl. Ontario 2016: 10; Leicht et al. 2018).

\section{Cognitive}

analysis, decision-making, critical thinking, active listening, innovation, interpretation, creativity, problem solving, adaptive learning, communication

\section{Interpersonal}

responsibility,

social influence with others, leadership, assertive communication, empathy, trust, coordination, negotiation, teamwork, cooperation, collaboration, conflict resolution, argumentation

\section{Intrapersonal}

adaptability, appreciation for diversity, self-monitoring, integrity, intellectual interest and curiosity, self-monitoring, continuous learning, initiative, productivity, metacognition, self direction, flexibility, responsibility, self-evaluation, self-monitoring

Abb. 3: Fertigkeiten des 21. Jahrhunderts (in Anlehnung an National Research Council 2012: 2)

Lehrpersonen sollen die zentralen Fertigkeiten im Unterricht integrieren und die Aneignung dieser bei SchülerInnen fördern. Die UNESCO fordert im Rahmen des SDG 4 ähnlich wie die EU qualifizierte Lehrpersonen, um zielorientierte Bildung zu garantieren, und urgiert eine Verbesserung der LehrerInnenqualität (vgl. Teacher Task Force 2018: 6ff.; European Commission 2018: 14ff.). Internationale Studien belegen den Zusammenhang zwischen der Unterrichtsqualität und SchülerInnenleistungen (vgl. Wright el al. 1997: 57ff.; Helmke 2003; Nyle et al. 2004: 237ff.; Kunter et al. 2013; Kunter et al. 2017). Das Bildungssystem versucht, mit spezifischen Formen der Professionalisierung von Lehrkräften zu reagieren.

Guerriero (2017: 13), im OECD-Kontext, sowie Terhart et al. (2014), auf den deutschen Bildungsraum bezogen, argumentieren, dass Lehrpersonen komplexe Anforderungen meistern und sich hierfür mehrdimensionale Kompetenzen aneignen müssen. Ein fundiertes Wissen, 
welches Mittelstraß mithilfe der Begriffe Orientierungs- und Verfügungswissen beschreibt (vgl. Mittelstraß 1998), ist für die Aneignung dieser Kompetenzen unabdingbar.

Seit Ende der Neunzigerjahre entwickelt sich der Fokus zunehmend dahingehend, dass komplexe Zusammenhänge über Kompetenzen formuliert werden. Weinert (2001a: 45ff.) verknüpft im Kontext des Kompetenzansatzes theoretische sowie praxisrelevante Fähigkeiten und Fertigkeiten. Professionelle Kompetenzen definieren sich über berufsbezogene Merkmale, die es ermöglichen, Situationen wahrzunehmen, zu interpretieren und angemessen auf diese zu reagieren (vgl. Shavelson 2013: 73ff.; Blömeke et al. 2015: 3ff.). Mit dem Kompetenzansatz wurden Anforderungen an Lehrpersonen neu operationalisiert und Fähigkeiten und Qualifikationen stärker in den Fokus gerückt. In Anlehnung an den Kompetenzansatz entwickelte die deutsche Kultusministerkonferenz (KMK 2004) bildungswissenschaftliche Standards, die einer inhaltlichen bildungspolitischen curricularen Festlegung zugrunde liegen und von Lehrpersonen unterschiedliche Kompetenzen fordern. Wesentlich war in diesem Zusammenhang der Perspektivenwechsel von einer Input- zu einer Outcomeorientierung sowie die Definition verschiedener Kompetenz- und Wissensbereichen von Lehrpersonen (vgl. König 2014a: 617). Brügelmann (2015) diskutiert im Rahmen der Kompetenzorientierung die Sinnhaftigkeit der Outcomeorientierung und kritisiert statische und standardisierte Verfahren, die den pädagogischen Alltag und die pädagogische Qualität nur unzureichend erfassen würden. Standardisierte Untersuchungen und ihre Verallgemeinerung bedingen Brügelman (2015) zufolge, dass die individuelle Bedeutung von Wissen verloren geht.

Unzufriedenstellende Ergebnisse internationaler Vergleichsstudien wie PISA, TIMSS oder IGLU lösten einen Trend zur Entwicklung unterschiedlicher Instrumente aus, mit denen gezielt professionelle Kompetenzen für Schule und Unterricht getestet wurden. Es wurden Testverfahren entwickelt, die professionelle Kompetenzen bzw. das Professionswissen von Lehrpersonen messen (vgl. König 2010a: 43) und zum Teil das gegenwärtige Verständnis professionellen Wissens von Lehrpersonen prägen. Ein Fokus der Operationalisierungen liegt auf der Messung pädagogischen Wissens. Die bisher existierenden Forschungsansätze bedienen sich psychometrischer Testverfahren (z.B. COACTIV, TEDS-M, LEK, ProwiN), die pädagogisches Wissen konzeptualisieren und operationalisieren (vgl. Kapitel 4) (vgl. König \& Klemenz 2005: 248). Hierzu im Konstrast bestehen bereits in den Neunzigerjahren formulierte Konzepte, die auf anthropologische Aspekte bezugnehmen und die Bedeutung pädagogischen Wissens jenseits der operationalisierten Messbarkeit betonen. Wulf (1994: 7) definierte pädagogisches Wissen und zeigt mit seinem Verständnis auf, dass dieses nicht auf standardisiert vorgegebene Konstrukte reduziert werden kann. Der Begriff zeichnet sich vielmehr über ein mehrperspektivisches Verständnis aus:

„Pädagogisches Wissen ist nicht auf die Ergebnisse pädagogischen Wissens beschränkt. Es umfasst viele Formen des Wissens. Zu ihnen gehören philosophische, wissenschaftliche, ästhetische, praktische Symbol- und Zeichensysteme, die in komplexen Beziehungen zueinanderstehen." (Wulf 1994: 7)

Für ein gegenstandsadäquates Verständnis pädagogischen Wissens ist es notwendig, das Wissen von Lehrpersonen sowie dessen Anwendung in der alltäglichen Unterrichtspraxis zu verstehen. Schön (1983) schlug in den Achtzigerjahren einen stark praxisorientierten Ansatz vor. Terhart (2012: 3ff.) setzte diesen Ansatz fort und betonte, dass die Wirkung der LehrerInnenbildung über zugrundeliegende Prozesse untersucht werden kann. Für eine methodologische Umsetzung der Praxisorientierung verweist Kemnitz (2014: 65) auf die Rekonstruktion vorhandener Strukturen, die die Wahrnehmung komplexer Strukturen ermöglicht. Wissen kann demnach, 
wie auch Schütz \& Luckmann (1988: 48) hervorheben, nur über die Lehrperson selbst bestimmt werden.

Neuere Studien zum pädagogischen Wissen stützen sich auf Verfahren, die artikulierbares Wissen abfragen (vgl. Kapitel 4.2). König (2010: 63) betont, dass weitere empirische Zugänge erforderlich sind, um pädagogisches Wissen zu konzeptualisieren. Hierzu argumentiert Lange (2008: 7ff.), dass die Definition der Wissensform nicht nur auf standardisierten Testverfahren, sondern vielmehr auf einer Rekonstruktion mithilfe des Blicks in die Unterrichtspraxis basieren sollte (vgl. Lange 2008: 7ff.). Lange (2008: 7ff.) argumentiert über eine weitere Ausdifferenzierung eine unzureichende Berücksichtigung der Transformationsprozesse und neue Anforderungsbereiche von Lehrpersonen in den existierenden Testverfahren. Dieses Verständnis unterstützt Neumann (2012: 37), der feststellt, dass Lehrpersonen im Unterrichtsalltag viel mehr wissen müssten, als über die Testverfahren erhoben werden kann. Dieses Wissen wird in Anlehnung an sein Verständnis nur über die empirische Erforschung des Unterrichtsalltags ausreichend dargestellt.

Hinsichtlich psychometrischer Testverfahren ist die Frage zu stellen, inwieweit sie gegenstandsadäquat pädagogisches Wissen abfragen können. Hamlyn (1970: 95ff.; 135ff.) zufolge definiert sich Wissen weniger über einen „state of mind“, sondern verlangt vielmehr begründete Annahmen aus der alltäglichen Praxis. Er nennt es „naiv“, Wissen zu testen, und argumentiert, dass man lediglich Leistungen im Hinblick auf spezifische Aufgaben abfrage. Bromme (1992: 10) argumentiert mit seinem Expertenansatz, dass professionelles Wissen von Lehrpersonen keine Zusammenfassung von einzelnen Bereichen ist. Das Wissen entwickelt sich über ein individuelles Verständnis mithilfe der Verknüpfung eigener Erfahrungen und der Anwendung unterschiedlicher Wissensformen (vgl. Bromme 1992: 10). Loughran (2019: 1) argumentiert, dass Lehrpersonen ihr Wissen bislang zu wenig verbalisieren und unterstützt die zentrale Bedeutung der Rekonstruktion pädagogischen Wissens aus der Unterrichtspraxis:

„Typically, teachers are not encouraged to spend time talking about teaching in ways that are theoretically robust, or to unpack their teaching in order to show others what they know, how and why. Because they are busy, doing teaching' they are not commonly afforded opportunities to ,unpack' their practice to explore and articulate the reasoning underpinning what they do." (Loughran 2019:1)

Die hier angeführte Diskussion von geforderten praxisorientierten Aspekten für die Darstellung pädagogischen Wissens von Lehrpersonen wird in der vorliegenden Studie über einen qualitativ-rekonstruktiven Zugang mit Interviews und Unterrichtsbeobachtungen realisiert. Die vorliegende Arbeit hat zum Ziel, pädagogisches Wissen unter Berücksichtigung gegenwärtigerer Transformationsprozesse und zentraler Anforderungsbereiche von Lehrpersonen im Schulalltag gegenstandsadäquat darzustellen. Schratz \& Schrittesser verweisen auf die Tatsache, dass das Ausmaß der sozialen, ökonomischen und politischen Transformationsprozesse und deren Bedeutung für die Schule bislang unzureichend erfasst wurde, was Lehrpersonen vor große Herausforderungen stellt (vgl. Schratz \& Schrittesser 2003: 185). Dabei geht es vor allem um die Darstellung der „Wirksamkeit der Wirklichkeit“. Der Wirksamkeitsbegriff wird bei Neuweg (2004: 603) diskutiert, der eine vielversprechende Möglichkeit darin sieht, „das Lehrerwissen feiner auszudifferenzieren und die Effekte einzelner Wissensbereiche und -facetten auf verschiedene Kriteriumsvariabeln zu studieren, um mehr über die Wege zu erfahren, über die Lehrerwissen seine Wirksamkeit auf das Lernen der SchülerInnen entfaltet“. Anhand vier unterschiedlicher Forschungsfragen (vgl. Kapitel 6) wird das Verständnis pädagogischen Wissens sowie die daraus entstehenden Herausforderungen vor dem Hintergrund gesellschaftlicher 
Transformationsprozesse rekonstruiert. Ein weiterer Fokus liegt auf der Übereinstimmung bzw. den Differenzen konzeptioneller Darstellungs- und Begründungsmuster und ihrem Handeln. Wird pädagogisches Wissen als Teil des Orientierungswissens (vgl. Mittelstraß 2001) konzeptualisiert, ist das Handeln von Lehrpersonen grundlegend. Bruner argumentiert die Notwendigkeit der Vernetzung von Theorie und Praxis und verdeutlicht die essenzielle Bedeutung des Wissens, wie Lehrpersonen im Klassenzimmer pädagogisch agieren und ihr Wissen anwenden:

„We need standards [...] to make our schools work well in solving the myriad tasks they face. But [...] standards alone will not work. We need a surer sense of what to teach to whom and how to go about teaching it in such a way that it will make those taught more effective, less alienated, and better human beings." (Bruner 1996: 117)

Die Frage nach dem Verhältnis von Theorie und Praxis in Bezug auf das pädagogisch professionelle Wissen und Handeln (vgl. Horvath 2009: 30) ist für die Forschungsarbeit zentral und wird in der vorliegenden Arbeit anhand unterschiedlicher Modelle diskutiert. Es wird davon ausgegangen, dass Praxiswissen mittels Theoriewissen ausdifferenziert und Theoriewissen durch Praxiswissen provoziert und über Reflexion entwickelt werden kann. Krümmel (1987: 121) bezeichnet diese Vorgänge als „Praxisfähigkeit der Theorie“, und umgekehrt als „Theoriefähigkeit der Praxis“. Ähnlich argumentiert Dann (2000: 83), dass „Handeln durch Wissen gesteuert und Wissen (...) durch Handeln bestärkt oder verändert“" wird. 


\section{Wissen}

Wissen lebt im Spannungsfeld unterschiedlicher Zugänge. Das vorliegende Kapitel hat zum Ziel, den Wissensdiskurs über einen mehrperspektivischen Zugang zu eröffnen und zentrale Aspekte im Kontext des Wissensbegriffs und im Zusammenhang mit Lernen und Schule zu diskutieren. Etymologisch stammt „Wissen“ aus der indogermanischen Form woida bzw. dem Altdeutschen wizzan ab. Übersetzt bedeutet der Wortstamm ich habe gesehen, restriktive ich weiß (vgl. Etymologisches Wörterbuch Duden 2019). Die etymologische Bedeutung illustriert, dass Wissen in Verbindung mit Beobachtungen und Erfahrungen steht. Dieses aktive Moment wird im späteren Verlauf der Forschungsarbeit hinsichtlich der formalen Bildung weiter ausgeführt. Basierend auf dieser Bedeutung haben sich in verschiedenen Wissenschaftsbereichen unterschiedliche Ansätze zum Wissensbegriff etabliert. Lexika erklären Wissen als die Gesamtheit von Kenntnissen (Fakten, Theorien, Regeln), die eine Person oder Gruppe aufweist (vgl. Baumann 2006: 29; Etymologisches Wörterbuch Duden 2019). Synonym wird der Wissensbegriff auch mit Begriffen wie „gelernt“, „erkannt“, „erfahren“ oder „im Gedächtnis haben“ gebraucht (vgl. Knoblauch 2005: 13). Im weiteren Verlauf (vgl. Kapitel 2.1) wird dieses Verständnis durch unterschiedliche Zugänge zum Wissensbegriff erweitert.

\subsection{Zugänge zum Wissensbegriff}

In der Forschung gibt es keinen einheitlichen Zugang zum Wissen. Vielmehr wird der Wissensbegriff in unterschiedlichen Feldern und interdisziplinären Zugängen definiert und begründet. Die Schwierigkeit der Abgrenzung wird über den Umstand verstärkt, dass sich die einzelnen Disziplinen selbst in ihrem Verständnis von Wissen unterscheiden. Ziel des Kapitels ist es, unterschiedliche Zugänge über einen systematischen und historiografischen Vergleich über die Philosophie/Erkenntnistheorie, Soziologie, Psychologie und Ökonomie/des Wissensmanagements aufzuzeigen und zu diskutiert (vgl. Abbildung 4).

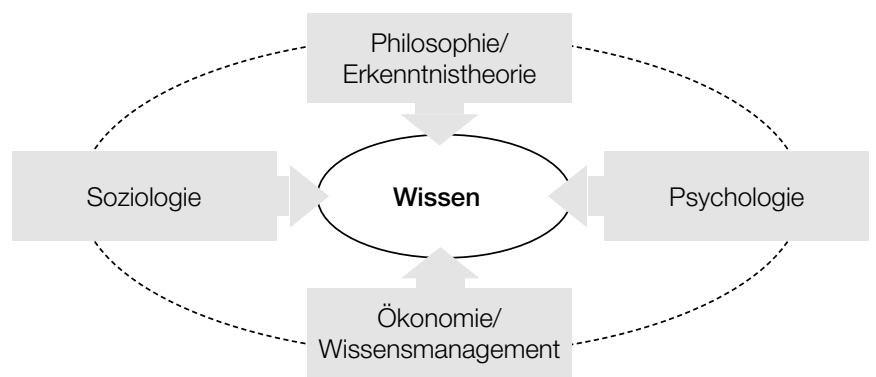

Abb. 4: Interdisziplinärer Zugang zum Wissensbegriff in der vorliegenden Arbeit

Die Komplexität des Wissensbegriffs und dessen interdisziplinäres Verständnis legen nahe, einen mehrperspektivischen Zugang zu verfolgen, insbesondere in der gegenwärtigen pluralen Gesellschaft. Von Bedeutung für das Verständnis des Wissensbegriffs sind erkenntnistheoretische sowie soziologische Zugänge, die sich mit den grundlegenden Themen der (sozialen) Erkenntnis und des Wissens beschäftigen (vgl. Knoblauch 2005: 13). Weitere wesentliche Ansätze betreffen allgemeine psychologische Zugänge über kognitive Verarbeitungsmöglichkeiten von 
Wissen. Die ökonomische Sichtweise ist relevant, da sie gegenwärtig den Begriff „Wissen“ im Kontext der Wissensgesellschaft und der Technologisierung diskutiert und die gegenwärtige Bedeutung des Terminus maßgeblich beeinflusst.

Das Verständnis des Wissensterminus stützt sich auf die Tradition der abendländischen Philosophiegeschichte des antiken Griechenland. Platon setzte mit seiner traditionellen Wissensformel $S$ weiß, dass $p$ einen Grundpfeiler und argumentiert die Bedeutung des Wissens über einen propositionalen Zugang. Er definiert Wissen als gerechtfertigte, wahre Meinung, welche sich nicht über Zufälle bilden kann, sondern rationales Denken voraussetzt (vgl. Baumann 2006: 39). Die Wissensdefinition „Wissen als wahre, gerechtfertigte Meinung" hat sich mit unterschiedlichen Akzenten ${ }^{5}$ bis heute als ein gängiges Verständnis von Wissen durchgesetzt.

Aristoteles übernahm diesen Ansatz und systematisierte den Wissensbegriff über verschiedene Stufen. Diese beziehen sich auf Wahrnehmung, Erinnerung, Erfahrung, Kunst und Weisheit und verdeutlichen gleichzeitig den Aneignungsprozess von Wissen, der von der Wahrnehmung zur Weisheit führe. In seinem Stufensystem integrierte er nicht nur das theoretische Wissen, sondern definierte es über praxisorientierte Formen. Das praktische Wissen bezog er auf einen veränderbaren Einzelfall und stellte keine allgemeingültigen, unveränderbaren Definitionen auf. Seine Klassifizierung von theoretischem und praktischem Wissen ${ }^{6}$ ist noch heute im Wissensdiskurs zentral (vgl. Gottschalk-Mazouz 2007: 24) und wird in der vorliegenden Forschungsarbeit in Verbindung mit pädagogischem Wissen (vgl. Kapitel 3/4/5) diskutiert.

Ausgehend vom Verständnis der antiken Griechen hat sich der Wissensbegriff umfassend entwickelt und korreliert zunehmend, wie auch in Bezug auf das pädagogische Wissen in Kapitel 5 diskutiert, mit gesellschaftlichen Prozessen. Im frühen Mittelalter löste sich Augustinus von Platons Gedanken zu rationaler Erkenntnis. Augustinus definierte Wissen über den Glauben und beschrieb das Erkennen der Welt über die christliche Lehre. Wissen bilde sich nach Augustinus mithilfe von Sinnesdaten und sei ohne falsche Angaben vollständig erfassbar (vgl. Schurz 1995: 40; Drews 2009: 173ff.). Die Ideenlehre an sich begründete er im Vergleich zu Platon nicht mittels der Erkenntnistheorie, vielmehr stand bei ihm die Weltanschauung und die Ontologie im Vordergrund. Die Ideen waren in seinem Verständnis in einer Welt angesiedelt, die über unserer Sinnwelt liegen und sich über den Glauben zur Wirklichkeit entwickeln (vgl. Wagenknecht 1992). Thomas von Aquin argumentierte in Anlehnung an Aristoteles, wie man über den Sinn und mithilfe des Glaubens zur Erkenntnis gelange (vgl. Schurz 1995: 41). Im Vergleich zu Augustinus stützte er sich auf eine negative Theologie: „Wir können von Gott nicht wissen, was er ist [...], sondern nur, was er nicht ist.“ (Tapp 2016: 426) Wissen ist Thomas von Aquin zufolge scientia (vgl. Tapp 2016: 427). Damit assoziierte er ein sicheres Wissen, das voraussetzte, die Ursachen von etwas Gewusstem einzusehen. Es gab bei ihm die cognitio, die er als universelles Wissen definierte. Wissen stand für Thomas von Aquin in einem Verhältnis zwischen scientia und cognitio (vgl. Tapp 2016: 428). ${ }^{7}$

5 Die Platonische Wissensformel wurde mehrfach kritisiert und weiterentwickelt. Gettier kritisiert das Wissensmodell von Platon. Er verweist auf die Bedingung, dass die gerechtfertigte Überzeugung wahr sein und diese gleichzeitig wiederum gerechtfertigt sein müsse. In Anlehnung an die Gettier-Kritik haben sich, abhängig von der Überzeugung, ob Rechtfertigung für Wissen notwendig sei, internalistische sowie externalistische Theorien entwickelt. Dazu gehört die von Gettier konzipierte kausale Konzeption des Wissens, die realibilistische Konzeption des Wissens sowie der Berücksichtigung des Kontextualisimus (vgl. Baumann 2006: 40ff.).

6 Aristoteles unterscheidet dabei drei unterschiedliche Wissensformen, die er dem theoretischen und praktischen Wissen zuordnet. Dem theoretischen Wissen ordnet er die intuitiv-subjektive Evidenz sowie die diskursiv-intersubjektive Erklärung unter. Das praktische Wissen beschreibt er als nur teilweise bis nicht sprachlichen Könnens über die Erfahrung und Urteilskraft. letzten Satz überarbeiten, der Sinn erschließt sich mir nicht

7 Thomas von Aquin beschreibt schon in seinem Werk „De magistro“ die Rolle des Wissens von Lehrpersonen. Er diskutiert in seinem Werk, welches Wissen von Lehrpersonen benötigt wird, um die Erkenntnis bzw. das selbstständige Lernen von SchülerInnen zu ermöglichen (vgl. Jüssen et al. 2006). 
Insbesondere seit der Renaissance vollzog sich eine Trennung von Naturwissenschaft und Religion. Diese Entwicklung sorgte für einen Aufschwung naturwissenschaftlicher und empiristischer Erkenntnistheorien (vgl. Schurz 1995: 41). Ein Vertreter dieser Zeit, in der experimentellen Methoden fokussiert wurden, war Bacon. Sein Modell des Wissensbegriffs verband er mit dem Gedanken der Macht:

„Wissen und Macht des Menschen treffen in demselben zusammen, weil die Unkenntnis der Ursachen die Wirkung verfehlen lässt. Die Natur lässt sich nur beherrschen, wenn man ihr gehorcht; und was in der Erkenntnis als Ursache holt, dient im Handeln als Regel.“ (Krohn 2006: 98)

Er beschrieb den Wissensterminus über das Zusammenrücken von Wissenschaft, Macht und Technik und verband dies mit dem kausalen Wissen (vgl. Krohn 2006: 98), welches im Zusammenhang mit dem ökonomischen Zugang im weiteren Verlauf näher diskutiert wird. Bacon spiegelte in seinem Verständnis eine aufklärerische Intention der neuzeitlichen Philosophie wider, indem er den religiösen Ansatz durch eine technische Begründung ersetzte (vgl. Schurz 1995: 43). Zentrale Vertreter des neuzeitlichen Rationalismus waren David Hume und Immanuel Kant. Hume stellte den Menschen mit seinem Handeln und Denken in den Mittelpunkt des Interesses. Er verfolgte den Ansatz der Beeinflussung menschlichen Wissens über die Kulturen. Seine Theorie basierte auf Beobachtungen menschlicher Natur (vgl. Schurz 1995: 49f.; Plümacher 2012: 136f.). Kant knüpfte an Humes Metaphysik an und führte dessen Gedanken weiter, indem er die Philosophie vor dem Hintergrund eines metaphysischen Zugangs interpretierte. Kant entwickelte drei fundamentale Kriterien - Raum, Zeit, Kausalität -, die er als Anschauungsformen des Denkens definierte. Denken könne nur im Kontext dieser Ordnungskategorien und über den Geist stattfinden und Wissen generieren. Dieser Prozess wird über logische Urteile über die Welt, die Vernunft, unterstützt (vgl. Schurz 1995: 51ff.; Pfister 2016: 126) und in Kapitel 2.3 bei der Frage „Was können wir wissen?“ im Detail diskutiert.

Das Verständnis der Wissensbegriffe von David Hume sowie Immanuel Kant wurden später von Edmund Husserl in seiner phänomenologischen Theorie aufgegriffen. Er verstand Wissen unter Berücksichtigung kultureller, gesellschaftlicher sowie historischer Bedingungen über den Prozess der Erfahrung. Husserl verwies bei seinem Zugang zum Wissen auf implizite Wissensformen, welche über die Praxis und Anwendung Bedeutung generierten (vgl. Plümacher 2012: 136f.; Pfister 2016: 122) und sich im Zusammenhang mit professionellem pädagogischem Wissen von Lehrpersonen als wichtig erweisen (vgl. Kapitel 3.1.2/5). Ein Fokus seiner Arbeit lag auf Fragen der Intersubjektivität. Er setzte sich mit der Frage auseinander, wie das Bewusstsein mit einem anderen Bewusstsein verbunden ist (vgl. Knoblauch 2005: 141). Dieser Ansatz wurde später von Alfred Schütz in seiner Soziologie des Wissens weitergeführt ${ }^{8}$. Schütz verbindet in seinem Zugang Sinn und Handeln zu einer untrennbaren Einheit. Sinn benennt er als ein handlungsleitendes Instrument, das Verhalten steuere und Wissen generiere (vgl. Knoblauch 2005: 142).

8 Die soziologische Wende wird anhand der Kritik von Kant argumentiert, der fragte, wie wahres Wissen möglich sei. Wissen ist nicht nur als individueller Gegenstand, sondern als soziales Ereignis konzipiert (vgl. Knoblauch 2005: 14). Die Wissenssoziologie stützt sich auf die erkennenden Menschen als „Teil eines sozialen Zusammenhangs, der selbst in den Prozess des Erkennens und den Inhalt des Erkannten bzw. Gewussten eingeht" (Knoblauch 2005: 14). Wie bei Platon, der eine Differenzierung zwischen Wissen und Meinung vornimmt und systematisiert, wird Meinung in der positivistischen Wissenssoziologie als wesentlicher Bestandteil von Wissen diskutiert und nicht nur auf gegenstandsadäquates Wissen reduziert. Das Konzept der Wahrheit wird in der Soziologie mit Geltung in Verbindung gebracht, die wiederum sozial beeinflusst ist. Der soziale Zugang etabliert sich über zwei unterschiedliche Ansätze. Einmal wird davon ausgegangen, dass Wissen, in Relation zur Gesellschaft und ihrer Wirklichkeit, mit dem Handeln in der Praxis, mit dem Habitus und dem Diskurs zusammenhänge. Der zweite Ansatz basiert auf dem Verständnis, dass Wissen als eine eigenständige Kategorie neben der Gesellschaft gelte (vgl. Knoblauch 2005: 17). 
Schütz beschäftigt sich in einem weiteren Schritt mit dem Bewusstsein im Erfahren der Welt. Handlungen und Erfahrungen generieren hierbei Beziehungssysteme. Er typisiert die Erfahrung in einem mehrstufigen, relationalen System und beschreibt, wie soziales Handeln über Erfahrung zu Bewusstsein führe. Die Zeitlichkeit sei für die Erfahrung grundlegendes Moment. Kognitives Wissen sowie leibliches Wissen könnten über die Erfahrung zur Automatisierung führen (vgl. Knoblauch 2005: 143ff.). Die wichtigsten Medien für die Vermittlung von Wissen seien Sprache und Symbole, die auf Erfahrungen hinwiesen. Die Wissensvermittlung geschehe somit über Interaktion und Zeichenhaftigkeit (vgl. Knoblauch 2005: 152ff.).

In der modernen Wissenssoziologie betont Mannheim in Anlehnung an Schütz den Zusammenhang zwischen Wissen und sozialem Sein, wobei das Wissen nicht an theoretische Grundlagen anknüpfe, sondern von Seinsfaktoren begründet sei. Diese charakterisierten sich über Gedanken und Erfahrungen in einem bestimmten Raum (Inhalt, Gehalt und Form), unter Berücksichtigung eines kollektiven Erfahrungszusammenhangs. Dabei hänge Wissen immer von denjenigen ab, die es vertreten (vgl. Knoblauch 2005: 104f.). Die wesentliche Bedeutung von Gesellschaft und subjektiven Sinnphänomenen wird bei der gesellschaftlichen Konstruktion der Wirklichkeit von Berger und Luckmann (1980: 3) in ihrem handlungsorientierten Ansatz weitergeführt. Wirklichkeit und Wissen manifestieren sich in Luckmanns Theorie über gesellschaftliches Handeln (vgl. Knoblauch 2005: 167). Mit ihrem Werk verknüpfen sie unterschiedliche Zugänge der Wissenssoziologie und begründen den Sozialkonstruktivismus. Mit dem Ansatz, dass sich Wissen über handelnde Personen definiert, verdeutlichen sie Parallelen zu Bacons Vorstellung der Idole. Wie Schütz vertreten Berger und Luckmann die Ansicht, dass sich Wissen über den Sinn einer Gesellschaft entwickelt (vgl. Knoblauch 2005: 155f.). Die gesellschaftliche Konstruktion der Wirklichkeit von Berger und Luckmann gilt als Wendepunkt, indem der Fokus verstärkt auf Kultur und Sinnphänomene ausgeweitet wurde und sich vermehrt von Mannheims Versuch der Analyse von Weltanschauungen entfernte (vgl. Knoblauch 2005: 167). Weitere Ansätze, die Wissen über Interaktionen definieren, sind während der kommunikativen Wende entstanden. Luckmann thematisiert sprachsoziologische Fragestellungen und knüpft an Ludwig Wittgestein an, der die Sprache als wichtigstes Mittel der Erkenntnis benennt. Der Ansatz von Luckmann führt den Gedanken der Sprache von Wittgestein als Form des Handelns fort. Er erweitert das Konzept, indem er Objekte in das Modell integriert, die über Zeichensysteme zum Ausdruck kommen. Die wichtigste Form des Zeichens ist die Sprache. Als ein weiterer Forschungszweig etablierte sich in den 1970er Jahren die Forschung zu kommunikativen Vorgängen. Unter Kommunikation versteht Luckmann die Informationsverarbeitung, über die Wissen weitergegeben werden kann (vgl. Knoblauch 2005: 173ff.). Bohnsack knüpft mit seiner praxeologischen Wissenssoziologie an das kommunikative Verständnis Luckmanns an und unterscheidet in seiner Theorie zwischen theoretischem kommunikativem Wissen und reflexivem handlungsleitendem Wissen. Das reflexive Wissen beschreibt er als Wissen impliziter Natur, das konstitutiv mit dem Habitus in Verbindung steht und nicht kommuniziert werden kann (vgl. Knoblauch 2005: 181f.). Habermas argumentiert in seinem Ansatz der kommunikativen Wende über die Theorie des kommunikativen Handelns, dass die Erkenntnis für die Konstruktion der Gesellschaft unverzichtbar sei: „Wissen dient zur Erzeugung und Aufrechterhaltung der Gesellschaft." (Knoblauch 2005: 183) Das Verständnis von Erkenntnis entsteht in seinem Zugang über drei von ihm entwickelte Formen, die miteinander verbunden werden: im Medium der Arbeit, der Sprache sowie der Macht. Er definiert Handeln über kommunikative Prozesse, die in einer bestimmten Lebenswelt unter Berücksichtigung unterschiedlicher Normen stattfinden. Normen gelten als handlungsleitend, die von den AkteurInnen mithilfe ihres Wissens erkannt und gedeutet werden müssen (vgl. Knoblauch 2005: 182ff.). Die Diskussion 
über die soziale Interaktion wird in Kapitel 5 anhand der handlungstheoretischen Zugänge von Mead (1934) und Giddens (1984) in Bezug auf das pädagogische professionelle Wissen von Lehrpersonen aufgegriffen und fortgeführt.

In der Psychologie hat sich insbesondere die Wissenspsychologie mit mentalen Repräsentationen beschäftigt. Es wird zwischen deklarativen (Wissen über Fakten und Begriffe) und prozeduralen Wissensformen (das handlungsleitende Wissen) unterschieden. Deklarative Wissensformen werden über kognitive Prozesse zu praxisorientiertem Handlungswissen. Das Praxiswissen wird nach wissenspsychologischem Verständnis mit dem impliziten Wissen verbunden ${ }^{9}$ (vgl. Gruber \& Stamouli 2009: 34f.). Eine vertiefte Auseinandersetzung und Diskussion mit dem deklarativen und prozeduralen Wissen findet aufgrund ihrer wesentlichen Bedeutung für das professionelle Wissen von Lehrpersonen in Kapitel 3.1 statt.

In der Ökonomie unterscheidet sich der Begriff „Wissen“ zu dem bisher angeführten Diskurs. Technologische Entwicklungen und damit einhergehende globale Verflechtungen der Wirtschaft führen zu einer Verselbstständigung des Wissensbegriffs, die sich auf Ebene des Wissens von Lehrpersonen widerspiegelt. Die technischen Entwicklungen des 16., 17. und 18. Jahrhunderts führten zu einer starken Ausdifferenzierung der Wirtschaft. Die sich etablierenden naturwissenschaftlichen Zugänge unterstützten dabei die Entwicklung der Industrialisierung. Dieser Prozess bedingte eine Ausdifferenzierung und Spezialisierung von Wissen (vgl. Bell 1968: 145ff.; Schulz-Schaeffer \& Böschen 2003: 9ff.). Alltagswissen, welches ursprünglich als Wissen galt, wurde durch wissenschaftliches Wissen ersetzt. Dies führte zu einer Zunahme von Wissen und einer starken Fokussierung auf spezifische Wissensbereiche (vgl. Schulz-Schaeffer \& Böschen 2003: 9). Die Veränderung des Wissensbegriffs impliziert neben den ökonomischen, materiellen Zugängen, dass der Terminus „Wissen“ mit Macht assoziiert wird (vgl. Knoblauch 2005: 270). Wissen, als ausdifferenziertes Spezialwissen, wird ergänzend zu den ökonomischen Steuerungsmechanismen wie Geld und Macht als ein Wachstumskriterium gegenwärtiger Gesellschaftsstrukturen und als zentrale Ressource charakterisiert (vgl. Hebestreit 2013: 37f.). „Wissensgesellschaft“, „Informationsgesellschaft“ oder „Netzwerkgesellschaft“ sind zu gängigen Ausdrücken geworden, welche in ihrem Verständnis Globalisierungsprozesse, die Verteilung von Ressourcen, Management und Technologien widerspiegeln (vgl. Knoblauch 2005: 255). Die Verteilung des Wissens wird als zentrale gesellschaftliche Determinante interpretiert, die gesellschaftliche Strukturen beeinflusst (vgl. Bell 1973).

Die soziologische Perspektive unterscheidet unter anderem zwischen einer „Wissensgesellschaft“ und einer „Informationsgesellschaft “10. In der Informationsgesellschaft liegt der Schwerpunkt bei einem wirtschaftsorientiert-technologischen Interesse. Die Wissensgesellschaft definiert sich über menschliche Fähigkeiten, wie den Erwerb, die Vermittlung sowie die Produktion von Wissen (vgl. Knoblauch 2005: 256). In Anlehnung an dieses Verständnis beschreibt Wilke (2002: 14) die Wissensgesellschaft als „eine auf Erfahrung gegründete kommunikative und konfirmierte Praxis“. Stehr (2001: 61) verfolgt den handlungsorientierten Ansatz und erklärt den Wissensbegriff als „Fähigkeit zum Handeln“. Wissen ist nicht nur an das Handeln gebunden,

9 Eine Definition der impliziten und expliziten Wissensformen werden in Kapitel 3.1 im Zusammenhang mit dem Professionswissen von Lehrpersonen angeführt und diskutiert.

10 Der Begriff Wissensgesellschaft stammt ursprünglich von Lane (1966), der die Idee verfolgte, Gesellschaft, Natur und Menschen zu befragen und ihr Wissen unter Berücksichtigung der objektiven Wahrheitssuche weiterzuentwickeln. Drucker (1969) stellt als erster Bezüge zwischen Wissensgesellschaft und Wirtschaft her. Er definiert Wissen über einen systematischen und gezielten Erwerb von Informationen zur systematischen Anwendung. Wissen wurde unter wirtschaftlicher Bedeutung zum Mittel und Zweck für Wachstum und Produktivität. 
sondern differenziert sich, wie Knoblauch (2005: 247) argumentiert, in Form und Inhalt aus. Knorr (2002) verweist auf den Begriff der Wissenskulturen, die inhaltliche Merkmale in einem bestimmten kollektiven Wissensumfeld festlegen.

Der Wissensbegriff nimmt aufgrund der Technologisierung einen neuen Stellenwert ein. Wissen im ökonomischen Kontext gilt als eine wesentliche Innovationsressource (vgl. Howaldt et al. 2004: 10f.), die Entwicklungsprozesse positiv unterstützt (vgl. Mescheder \& Sallach 2012: 9). Medien erlangen durch technologische Veränderungen eine Bedeutungsverschiebung. Sie haben McLuhan zufolge die Aufgabe, Botschaften zu verteilen, und beeinflussen maßgeblich die Gesellschaft, indem sie Informationen verbreiten, organisieren und zugänglich machen (vgl. McLuhan 1967). Medien als Informationsquelle unterstützen die Zugänglichkeit, die Quantität, die Vielfältigkeit sowie die Formen des Wissens. Dieser Prozess wird durch die zunehmende Digitalisierung und die weltweite globale Vernetzung verstärkt (vgl. Kübler 2010: 171). Internet, Medien, aber auch Bibliotheken machen Informationen zu einem grenzenlos verfügbaren Gut. Schon McLuhan (1967) argumentierte eine massive Beeinflussung der Gesellschaft über Medien. Kübler verweist auf die Notwendigkeit menschlicher Subjekte, die Informationen aufnehmen, rekonstruieren, verarbeiten und sinnvoll anwenden (vgl. Kübler 2005: 176f.; 194f.). Beck (1994: 340) unterstützt diese Position und argumentiert die Gesellschaft als Spiegel der Beschleunigungsprozesse. Er entwickelt zu den Modernisierungsprozessen wie Globalisierung ein weiteres Modell der reflexiven Moderne, die sich mit den Folgen der Modernisierung, wie Klimaveränderungen, neuen Familienstrukturen oder Mobilität, auseinandersetzt und im Zusammenhang mit dem Nichtwissen, wie im nachfolgenden Abschnitt (vgl. Abschnitt 2.2) aufgegriffen, in Beziehung steht. Rosa unterteilt soziale Beschleunigungen in Anlehnung an Becks Verständnis in technische Beschleunigungen sowie in Beschleunigungen des sozialen Wandels. Die technische Beschleunigung beinhaltet Kommunikations-, Produktions- und Transportprozesse. Diese führen zu einer Veränderung und Wahrnehmung von Raum und Zeit und beeinflussen das soziale Leben innerhalb einer Gesellschaft. Die Beschleunigung des sozialen Wandels bezieht sich auf Veränderungen und die Beschleunigung der Gesellschaft selbst, wie zum Beispiel die Einstellungen, Werte, Lebensstile, soziale Beziehungen oder auch Praxisformen und Sprache (vgl. Rosa 2013). Es entstehen neue Anforderungen an das Wissen von Lehrpersonen, die an der Fülle von Informationen erkennen müssen, welches Wissen gegenwärtig unter Berücksichtigung der Beschleunigungsprozesse von zentraler Bedeutung ist.

Die angeführte Diskussion beschreibt unterschiedliche Zugänge zum Wissensbegriff und demonstriert die Schwierigkeit, Wissen einheitlich zu definieren. Zusammenfassend verdeutlicht dieses Kapitel, wie Brülisauer (2008: 56) in seinem Buch „Was können wir wissen?“ hervorhebt, die Schwierigkeit, den Wissensbegriff einheitlich zu definieren. Der mehrperspektivische Zugang zeigt auf, dass es bisher nicht gelungen ist, allgemeingültige Bedingungen für Wissen zu definieren. Die hier kusorisch dargestellten Bedeutungen des Wissensbegriffs werfen die Frage auf, was wir letzten Endes wissen können und wie wir aus der Fülle der Informationen Wissen generieren. Geiger \& Schreyögg (2003: 7) verweisen auf Nichtwissen als Teil des Wissens: „Wenn alles Wissen ist, ist Wissen am Ende nichts?!“

\subsection{Nichtwissen}

Im Kontext der angeführten technologischen und ökonomischen Entwicklungen (vgl. Beck 1994; Rosa 2013), wie in Kapitel 2.1 beschrieben, betont Stehr (2000) die Vulnerabilität von Wissen. Diese wird von Krohn (1997: 69) als Nichtwissen definiert, welches sich durch steti- 
ge, weiterführende Veränderungsprozesse und zunehmende Flut an Informationen zunehmend vergrößert. Wehling (2001: 465) vertritt die Position Krohns und definiert Nichtwissen als „unerwartete Handlungs- und Entscheidungsfolgen jenseits kalkulierbarer und abschätzbarer Ungewissheit des Wissens" (Wehling 2001: 465).

Die „Akzent-Verschiebung vom Wissen zum Nichtwissen“, wie es Wehling (2003: 121) benennt, wurde schon in der Soziologie sowie in den Sozialwissenschaften als wichtiges Thema identifiziert (vgl. Maasen 1999: 52f.). Adorno (1959) gilt als einer der Ersten, die auf den Begriff des Nichtwissens im Zusammenhang mit dem Begriff der Unbildung hingewiesen haben. Die Analyse des Nichtwissens verdeutlicht ein heterogenes Feld unterschiedlicher Definitions- und Konstruktionszugänge.

Merton (1972: 45ff.) definiert das Nichtwissen über ein etwas noch nicht wissen. Weinstein \& Weinstein (1978: 151ff.) beschäftigen sich im Vergleich zu Merton mit dem Diskurs und fragen, warum bestimmte Elemente nicht in der Aufmerksamkeit unseres Interesses liegen. Die fehlende Aufmerksamkeit wird als Nichtwissen identifiziert.

Simmel (1992) beschäftigt sich in seinem kulturtheoretischen Modell mit dem Zusammenhang von Gesellschaft und Wissen. Er betont das Nichtwissen als grundlegende Voraussetzung für soziale Beziehungen. Wissen beschränkt sich für ihn auf bestimmte Personen und Gruppen und er charakterisiert es als Spiegel der Gesellschaft. Er unterstreicht, dass Wissen in einer Gesellschaft nicht für alle Gruppen gleich zugänglich ist. Heterogene Strukturen führen zu einer Generierung von Nichtwissen. Schütz und Luckmann (1988: 203ff.) führen in ihrem phänomenologischen Zugang den Ansatz Simmels fort und begründen das Nichtwissen mit der sozialen Verteilung des Wissens. Sie beschreiben, dass Nichtwissen für alle zugänglich ist und charakterisieren es als etwas Dynamisches, das sich jederzeit zu Wissen transformieren kann.

In Anlehnung an Schütz argumentiert Smithon (1985: 151), dass die Funktionen und Wirkungen von Wissen sozial erzeugt werden. Er verbindet das Nichtwissen mit den Begriffen „Risiko“ und „Unsicherheit“. Smithon (1989: 5) verweist auf die Schwierigkeit der Abgrenzung der Begrifflichkeiten. In Anlehnung an die Diskussion über Risiko, Unsicherheit und Nichtwissen haben Luhmann, mit der Systemtheorie, und Beck, mit seinem Ansatz der reflexiven Modernisierung, Zugänge zum Nichtwissen entwickelt. Diese Theorien beschäftigen sich mit Nichtwissen und seinen Auswirkungen auf die moderne Gesellschaft (vgl. Wehling 2001: 473). Luhmann (1997: 59) versteht unter der Gesellschaft ein von der Umwelt abgegrenztes soziales, kommunikatives System. Nichtwissen bezieht sich auf mangelnde Kommunikationsfähigkeit. Beck (1996: 313) eröffnet zum Thema Risikogesellschaft einen weiteren Diskurs. Er kritisiert die Wissensgesellschaft und die Verwissenschaftlichung im Zuge der gesellschaftsökonomischen Entwicklungen und diskutiert das dadurch entstehende Risiko. Beck (1996: 309) definiert Nichtwissen als „das gewusste, verdrängte, aufrechterhaltene, bestürmte oder anerkannte und eingestandene Nicht-Wissen“. Er verfolgt die Frage, wie mit Nichtwissen in der Gesellschaft umgegangen werden soll, damit möglichst viele Risiken vermieden werde. Er appelliert auf den Dialog sowie auf die Reflexion, die er als „Medium reflexiver Modernisierung“ beschreibt. Mithilfe des Dialogs und der Reflexion sei es möglich, Nichtwissen zu erkennen und die Flut an Informationen zu hinterfragen.

Wissen und Nichtwissen stehen in einem Spannungsfeld unterschiedlicher Zugänge und können, wie im Zusammenhang mit dem Wissensbegriff schon aufgezeigt, nicht einheitlich definiert werden. In Anlehnung an die Wissensdiskussion ergibt sich daraus die Frage, was wir überhaupt wissen können. Diese Frage wird im folgenden Abschnitt über erkenntnistheoretische Zugänge diskutiert. 


\subsection{Was können wir wissen?}

Immanuel Kant hat die Frage „Was können wir wissen?" programmatisch mit einem erkenntnistheoretischen Diskurs über die vier kantischen Fragen ${ }^{11}$ diskutiert (vgl. Brülisauer 2008: 5; Kant 1781). In der „Kritik der reinen Vernunft“ diskutiert er die Bedeutung der Vernunft und verlangt mit seinem kritischen Denkansatz, den Mut zu besitzen, sich seines eigenen Verstands zu bedienen, um aus der selbstverschuldeten Unmündigkeit auszubrechen (vgl. Kant 1781). Der Verstand nimmt in seinem Ansatz eine wesentliche Bedeutung ein. Er führt über die Erfahrung zur Erkenntnis. Kant beschäftigt sich mit der Frage, unter welcher Bedingung Erkenntnis überhaupt möglich ist. Eine wesentliche Bedingung ist das Urteilen. Er verbindet die Anschauung bzw. die Sinnlichkeit mit dem Begriff des Verstands. Die Sinnlichkeit und der Verstand sind Kant (1977) zufolge zwei grundlegende Instrumente, die nötig sind, um zur Erkenntnis zu gelangen.

Wie Erfahrung entsteht, erklärt Kant anhand eines Kategorialsystems. Als grundlegende Kategorien für die Bildung aller sinnlichen Vorstellungen für die Generierung von Wissen benennt er Raum und Zeit. Die Erkenntnis einer Person ist von deren individueller Realität und Erscheinungen im Raum-Zeit-Verhältnis abhängig. Jede Person gelangt dadurch zu unterschiedlichen Erkenntnissen, die Kant als Empfindungen beschreibt. Individuelle Empfindungen führen nicht automatisch zu Urteilen. Grundlegendes Moment ist der Verstand, der über das Denken benutzt wird. Er definiert vier Funktionen des Verstands, nämlich Quantität, Qualität, Relation und Modalität, nach denen Urteile gefällt werden. Die Kategorien bilden die Basis für eine systematische Verknüpfung des Verstands mit den Empfindungen, die Urteile binden, und zur Erkenntnis und schließlich zu Wissen führen (vgl. Kant 1977). Wissen ist in Anlehnung an das Verständnis von Kant die Erkenntnis, die durch den Verstand über Erfahrung angeeignet wird. Hume eröffnet mit seinem erkenntnistheoretischen Ansatz einen Diskurs in Anlehnung an die Frage, was wir über die Welt sagen und wissen können. Er stützt sich wie Kant auf die Erfahrung und begründet das Wissen über einen weiteren Aspekt, die Beobachtung. Er argumentiert, dass allein durch die Vernunft keine gesicherten Aussagen über die Welt getroffen werden können, und argumentiert, dass Erkenntnis über Erfahrungen und Beobachtungen entsteht. Trotz seiner Theorie stellt er die Erkenntnis in Frage und hebt in seiner „Abhandlung über die menschliche Natur" hervor, dass es keine sichere Erkenntnis der Außenwelt über rationales Denken geben kann. Sinne können getäuscht werden und Sinneswahrnehmungen entsprechen nicht der objektiven Realität der Außenwelt (vgl. Hume 1975).

Hume differenziert zwei unterschiedliche Arten der Wahrnehmung, die Eindrücke und die Vorstellungen, denen Sinneserfahrungen zugrunde liegen. Grundlegendes Moment, das diesen Prozess unterstützt, ist das Erinnern. Ausgehend von diesen Überlegungen setzt er sich mit dem Kausalitätsproblem und dem Induktionsproblem auseinander. Er argumentiert, dass Information, die über Erfahrung hinausgeht, über eine kausale Relation der Ursache-Wirkung-Beziehung vermittelt wird. Im Zusammenhang mit der Induktion verweist er kritisch auf das Lernen aus der Erfahrung. Er argumentiert, dass Lernen aus Erfahrung grundlegendes Wissen voraussetzt und die Gewohnheit als essenzieller Bereich der Erfahrung gilt. Er stützt sich wie Kant auf ein Kategorialsystem, das Raum und Zeit beinhaltet, und ergänzt es mit der Ähnlichkeit. Innerhalb von Raum, Zeit und Ähnlichkeit können sich durch kausale Beziehungen und Gewohnheiten die Wahrnehmungen zu Vorstellungen und Eindrücken entwickeln (vgl. Schurz 1995: 49ff.). Wissen ist die Erinnerung aus Erfahrungen, die durch Ähnlichkeiten entstehen.

11 Weitere Fragen, die Kant in seiner Philosophie der Aufklärung entwickelt, sind: „Was soll ich tun?“, „Was darf ich hoffen?“, „Was ist der Mensch?“ (vgl. Brülisauer 2008: 5). 
Gegenwärtige Diskurse greifen im Kern häufig auf idealistische und naturalistische Positionen zurück. Hoerster (2010) geht der Frage nach, was wir wissen können, und entwickelt die Ansätze von Kant und Hume weiter. Er skizziert in seinem Ansatz, welche Voraussetzungen erfüllt werden müssen, damit Wissen entsteht, und beruft sich auf Platon. Nach Hoerster (2010: 11ff.) können wir nur etwas wissen, wenn wir daran glauben und es dem Wahrheitsanspruch gerecht werden kann. Als weiteres Merkmal stützt er sich auf die Notwendigkeit der Rechtfertigung. Er greift auf die Argumentation von Kant zurück, dass Wissen sich über Raum und Zeit entwickeln kann, und begründet Wissen über logisches Denken sowie den Gebrauch der Sinne. Er diskutiert das Gedächtnis und das Erinnerungsvermögen als zwei weitere Möglichkeiten, Wissen zu generieren. Hoerster, als ein aktueller Vertreter, definiert in Anlehnung an Kant und Hume ein umfangreiches Verständnis von Wissen. Er charakterisiert Wissen nicht nur über die Erfahrung und das Erinnern, sondern über weitere Merkmale wie Glaube, Rechtfertigung und logisches Denken.

Gesellschaftliche Einflüsse nehmen in der Soziologie eine wesentliche Bedeutung ein. Wissen kennzeichnet sich durch eine stark soziale Funktion, indem die Gesellschaft Wissen generiert. Zudem kann sich über das Generieren von Wissen Gemeinschaft entwickeln (vgl. Knoblauch (2005: 341ff.). Knoblauch argumentiert, dass Wissen selbst sich nicht beobachten lässt, sondern nur über Kommunikation zugänglich gemacht werden kann. Kommunikation findet über Sprache und die zugrundeliegenden Interaktionen, die Handlungen, statt. Diesen Ansatz greift Habermas in seiner Theorie des kommunikativen Handelns auf. Er thematisiert die Bedeutung des kommunikativen Handelns für die soziale Interaktion einer Gesellschaft. Wissen entwickelt sich über drei miteinander in Beziehung stehende Formen: die Form der Arbeit, die Form der Sprache sowie die Form der Macht (vgl. Knoblauch 2005: 182ff.). Er diskutiert nicht nur die kommunikative Vernunft und den Wahrheitsanspruch, sondern verbindet die Erkenntnis, wie Habermas, mit normativer Gerechtigkeit (vgl. Knoblauch 2005: 182ff.). ${ }^{12}$

Gesellschaftlich ethische Einflüsse auf das Wissen werden von Heidenreich (2002: 1) hervorgehoben. Er argumentiert, dass trotz der Informationsfülle Menschen nicht alles wissen können. Die Frage nach dem Wissen enthält eine normativ ethische Implikation. Wie Kant schon bemerkte, ist Wissen keine objektive Aussage über die Welt, sondern wird von kulturellen, gesellschaftlichen und subjektiven Faktoren mitbestimmt. Wissen generiert sich in Heidenreichs Ansatz über kulturelle Bedingungen und Voraussetzungen. Diese werden durch Werte und Regeln, die in einer Gesellschaft vorherrschen, beeinflusst (vgl. Hoerster 2010: 85). Wissen ist folglich auf kulturelle Räume begrenzt.

Die Frage, was wir wissen können, kann in Anlehnung an die angeführte Diskussion unterschiedlich beantwortet werden. Wurde bisher die Frage des Wissens über einen erkenntnistheoretischen und soziologischen Diskurs diskutiert, wird im weiteren Verlauf eine Annäherung an das Wissen über einen kognitionspsychologischen und biographischen Zugang zum Lernen angestrebt.

\subsection{Wissen und Lernen}

Das vorliegende Kapitel beschäftigt sich mit Wissen und Lernen unter Berücksichtigung eines kognitionspsychologischen und biografischen Zugangs. In Anlehnung an Illeris (2007: 3) kann Lernen als Prozess beschrieben werden, der zu einer permanenten Kapazitätsänderung führt, sich jedoch von einer biologischen Reifung und Alterung unterscheidet. Das Lernen wird durch bio-

12 Die Argumentation wird in Kapitel 5 aufgegriffen und fortgeführt. Die Arbeit stützt sich auf einen handlungstheoretischen Ansatz, der Wissen über Handeln durch Kommunikation unter Berücksichtigung normativer Strukturen sichtbar machen kann. 
logische, psychologische sowie soziale Faktoren beeinflusst (vgl. Illeris 2018: 8). Lernen beinhaltet die Integration zweier unterschiedlicher Prozesse, einem externen sowie einem internen Prozess. Lerntheorien kennzeichnen sich vorwiegend darüber aus, dass sie sich nur auf einen Prozess konzentrieren. Der Behaviorismus sowie die Theorie des kognitiven Lernens fokussieren vorwiegend auf einen internen psychologischen Prozess. Illeris argumentiert in diesem Zusammenhang, dass über diese Zugänge nicht das ganze Feld Lernen abgedeckt werden kann (vgl. Illeris 2018: 9). Der externe Lernprozess vollzieht sich über die Wissensaneignung von Lernenden und seinem äußeren sozialen, kulturellen oder materiellen Umfeld. Interne psychologische Prozesse beziehen sich auf einen individuellen Aufbau und den Erwerb von Wissen. Externe und interne Prozesse stehen in enger Beziehung zueinander und beeinflussen den Wissenserwerb (vgl. Illeris 2018: 9f.).

Der kognitionspsychologische Zugang stützt sich auf das Verständnis, dass Wissen nicht übertragen werden kann. Wissen muss sich vielmehr individuell, über einen Umlernprozess und selbstorgansiertes Lernen, entwickeln (vgl. Edelmann 2000: 113ff.; Roth 2003: 20; Schmidt 2003: 46). Die Aneignung von Wissen findet über „Wahrnehmen“, „Vorstellen“, „Urteilen“ und „Sprache" statt und steht in enger Beziehung mit emotionalen und motivationalen Eigenschaften einer Person. Kognitionen sind Vorgänge, durch die eine Person Kenntnis von seiner Umwelt erlangt und durch die sich dabei Vorstellungen verändern oder entwickeln (vgl. Edelmann 2000: 113; Gropengießer 2003: 35; Illeris 2018: 7f.). Roth (2003: 24) beschreibt diesen Prozess als einen individuellen „aktiven Prozess der Bedeutungserzeugung“. Die Bildung von Kategorien bzw. Bedeutungseinheiten über sachlich-logische sowie emotionale Begriffe bilden die Grundlage kognitiver Orientierung (vgl. Edelmann 2000: 116ff.; Roth 2003: 21). Die Kategorisierung von Begriffen wird von emotionalen und motivationalen persönlichen Einstellungen und Haltungen einer Person beeinflusst (vgl. Gropengießer 2003: 25). Das limbische System ist nach Roth (2003: 22) der „eigentliche Kontrolleuer“ des Lernerfolgs. Bruner beschreibt in seiner Theorie der kognitiven Entwicklung als weiteren Einfluss auf Wissensstrukturen den kulturellen Faktor. Dieser ist von universellen, angeborenen, kognitiven Verarbeitungsfähigkeiten einer Person sowie ihrem kulturellen Milieu abhängig (vgl. Edelmann 2000: 131). Der kognitionspsychologische Zugang stützt sich neben dem Neulernen auf ein kontinuierliches Umlernen vorhandener Strukturen. Das Wissen muss vom Lernenden über die vorhandenen Bedeutungskontexte stetig selbst konstruiert werden (vgl. Edelmann 2000: 116; Roth 2003: 21). Gropengießer (2003: 36) argumentiert, dass Menschen fast immer lernen und das Lernen selbst über Reflexion gelernt werden kann. Diesen Zugang vertritt Schmidt (2003: 46), der in seinem Ansatz das Handeln, auf das reflexiv Bezug genommen werden kann, als Transfermittel des Wissens beschreibt.

Die Frage, was wir wissen können, wird in der Kognitionspsychologie über die Informationsverarbeitung beantwortet. Edelmann (2000: 165f.) sowie Roth (2003: 20) beschreiben ein Modell menschlicher Informationsverarbeitung über das Gedächtnis mittels der Phasen „Aneignung“, „Speicherung“ und „Abruf“. Die Aneignungsphase ist durch die Aufnahme und Verarbeitung der Informationen charakterisiert. Die Speicherung ist für den Wissenserwerb zentral. Sie kann über Wiederholungen, über das Verstehen der Information oder über Verbindung der Information mit Emotionen und Erinnerungen erreicht werden. Die letzte Phase, der Abruf, definiert sich über die Anwendung erlernter Wissensstrukturen und ihre Verknüpfung untereinander. Schmidt (2003: 40) beschreibt den Lernprozess nicht über die Informationsverarbeitung, sondern mithilfe eines Prozesses, der sich zwischen den Zuständen „vor dem Lernen“ und „nach dem Lernen“ abspielt. Der Lernprozess vollzieht sich über die Beschreibung der stattgefundenen Veränderungen der jeweiligen lernenden Person zwischen den Zuständen. Die Wissensaneignung findet über veränderte mentale Strukturen statt. Wenn ein Schema oder Muster erstellt 
wird, handelt es sich um kumulatives oder mechanisches Lernen. Diese Art des Lernens zeichnet sich dadurch aus, dass es sich um eine isolierte Formation ohne Kontextbezug handelt.

Für den Wissenserwerb wichtige Momente sind die Beziehung, die Verknüpfung, die Sinnhaftigkeit sowie die Erfahrung. Illeris (2018: 13) bezeichnet diesen Zustand als assimilatives Lernen. Der Wissenserwerb findet über sinnvolle Beziehungen zwischen den einzelnen kategorialen Begrifflichkeiten und ihrer Umwelt statt (vgl. Edelmann 2000: 113; 133). Göhlich und Zirfas (2007: 7) vertreten mit einer erziehungswissenschaftlichen Perspektive folgenden Ansatz: Lernen konstituiert sich über den Lernenden und dessen Beziehung zur Welt. Das Hauptziel des Lernens, so Ausubel (1974: 139), ist der „Erwerb einer klaren, stabilen und organisierten Wissensmenge“. Auswendig gelerntes Wissen kann nicht als Wissen bezeichnet werden. Vielmehr muss es mit kognitiven Strukturen verbunden sein, die in eigenen Worten ausgedrückt werden können (vgl. Edelmann 2000: 140). Von wesentlicher Bedeutung ist, wie schon Hume und Kant argumentieren (vgl. Abschnitt 2.3), die aktive Aneignung des Wissens über Erfahrung. Dieser Prozess wird Illeris (2018: 14) zufolge über den Begriff „Akkomodative“ bezeichnet. Die Erfahrung stützt sich auf individuelle persönliche Erfahrungen sowie Erfahrungen, die über Sprache und Bilder sozial vermittelt werden (vgl. Edelmann 2000: 162), und kann auf vorhandenes Wissen aufbauen (vgl. Illeris 2018: 14).

Lernen ist nicht auf spezifische Ereignisse und Inhalte begrenzt. Eine Argumentation stützt sich auf das Konzept des lebenslangen Lernens. Dieses reicht von der Kindheit bis zum Erwachsenenleben (vgl. Reetz \& Tramm 2000: 69; Preißer 2003: 87). Beck nennt Lernen den eigenen, selbstgesteuerten Lebensweg (vgl. Beck 1986: 216). Von Giddens (1997: 301) wird es als reflexives Projekt der Ichwerdung beschrieben. Die Forderung eines lebenslangen Lernprozesses wird seit den 1960er Jahren bildungspolitisch und seit den 1970er Jahren als Thema der OECD entwickelt. Die gegenwärtigen sozialen, politischen und ökonomischen Transformationsprozesse (vgl. Kapitel 1.2) bedingen ein kontinuierliches und lebenslanges Lernen, zum Zweck, Kenntnisse zu vermehren und Fähigkeiten und Kompetenzen zu verbessern (vgl. Hof \& Rosenberg 2018: 2). Lernen findet im Kontext von Raum und Zeit statt. Der zeitliche Kontext bezieht sich auf den kontinuierlichen Prozess des Wissenserwerbs im Verlauf des Lebens. Der örtliche Kontext ist über die Aneignung des Wissens an spezifischen Orten der Bildung (Schule, Universität, Beruf), oder über einen informell stattfindenden Wissenserwerb in Gesprächen mit Freuden charakterisiert (vgl. Hof \& Rosenberg 2018: 3ff.). Orientierungs- und Handlungswissen (vgl. Weinberg 2000: 119) bzw. Orientiertungs- und Verfügungswissen (vgl. Mittelstraß 1998) sind dabei Wissensformen, die angeeignet werden sollen.

Die Konstruktion von Wissen vollzieht sich über einen fortlaufenden Prozess, der über die Schule und die Ausbildung hinausreicht. Obwohl Lernen häufig mit Schule in Verbindung gebracht wird, reicht der Wissenserwerb in der Schule nicht aus, um Wissen und Handeln für die Ausübung eines spezifischen Berufs zu erwerben. Die Anforderungen des Berufslebens sind über die Aneignung spezifischen professionellen Wissens während der Ausbildung und des späteren Berufslebens selbst zu bewältigen. Diese können Jost (2003) zufolge als rekursive Wissens- und Handlungsformen beschrieben werden, die mit der sozialen Umwelt in Verbindung stehen. Über die Ausbildung kann spezifisches Wissen angeignet werden. Anknüpfungspunkte können sich je nach Ausbildungsart mit dem Wissen aus der Schulzeit ergeben. Die Ausbildung hat zum Ziel, Wissen weiterzuentwickeln, und charakterisiert sich als eine erste Phase des professionellen Wissenserwerbs. Ein höheres Kompetenzniveau führt zu einer Erweiterung und Neustrukturierung des Denkens (vgl. Keller-Schneider \& Hericks 2014: 394). Hascher (2014: 559) erkennt einen Wissenszuwachs zwischen dem Anfang der Ausbildung und dem Berufsein- 
stieg. Der Übergang von der Ausbildung zur Praxis bedeutet, das gelernte Wissen sinnvoll anzuwenden und handlungsorientiertes Wissens zu generieren und reflektieren. Keller-Schneider \& Hericks (2014: 391) argumentieren, dass erworbenes Wissen für diesen Prozess allein nicht ausreicht. Als weitere wesentliche Gelingens- und Einflussfaktoren werden persönliche Haltungen, Überzeugungen, Motivation und individuelle Fähigkeiten genannt. Der Berufseinstieg wird von Kocher (2014: 30) als „,besondere und sensible Phase der Professionalisierung“ beschrieben. Berufliche Entwicklungsaufgaben verlangen eine aktiv handelnde Person, die mit ihrer sozialen Umwelt in Beziehung steht. Zudem werden die Entwicklungsaufgaben über individuelle Ansprüche, Ressourcen und Ziele entwickelt (vgl. Buchwald \& Hobfoll 2004). Der professionsspezifische Lernprozess wird durch vertiefte Wissensstrukturen und Ressourcen verstärkt (vgl. Reh \& Schelle 2000), die zu Handlungen und neuen Erfahrungsmomenten führen. Während der Praxis befördern Fort- und Weiterbildungen sowie Lerngemeinschaften den lebenslangen, professionsspezifischen Lernprozess (vgl. Lipowsky 2014: 512/529).

Schule als zentraler Ort, an dem Kultur tradiert und Wissen weitergegeben wird, nimmt im Zusammenhang mit der Vermittlung von Wissen eine wichtige Position ein. Das folgende Kapitel (vgl. Kapitel 2.5) greift diese Thematik auf und diskutiert das Verhältnis Wissen und Schule.

\subsection{Wissen und Schule}

Wie im vorherigen Diskurs zu Wissen und Lernen aufgezeigt, lässt sich Lernen über einen Wissensaufbau beschreiben. Die Schule nimmt für den Wissenserwerb eine wichtige Stellung ein. Koch (2015: 129) bezeichnet die Schule als „Haus des Wissens“"13 und unterstreicht, wie Fend (2006: 36), die Wissensvermittlung als eine zentrale Aufgabe. Schule als Ort der Wissensvermittlung steht mit Lehr- und Lernprozessen und den vorhandenen AkteurInnen in Beziehung, die von curricularen Anforderungen geleitet werden (vgl. Abbildung 5).

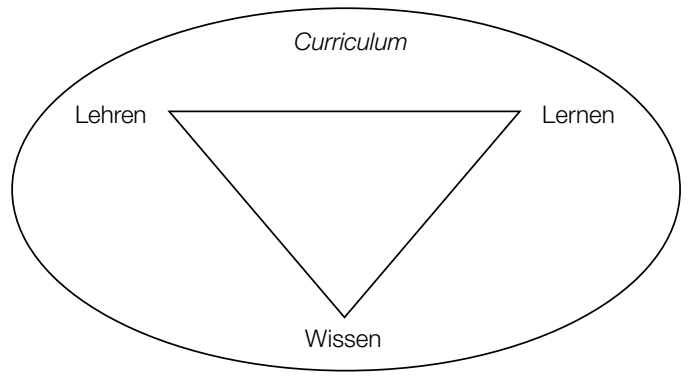

Abb. 5: Abhängigkeitsverhältnis Wissen, Lehren und Lernen

Wissen wird im schulischen Kontext mit Lernen und Lehren in Verbindung gebracht. Der Transfer des Wissens findet über das Lehren und das Lernen statt. Eine grundlegende Eigenschaft des Wissens ist, laut Koch (2015: 67), dass nur Wissen lehrbar ist. Wissen charakterisiert sich laut Koch darüber, dass es ohne inhaltliche Reduzierung mitteilbar ist. Dieses Wissen kann nur von einer Lehrperson vermittelt werden, die sich „in den Gründen auskennt [...] Wer sich

13 Der Begriff „Haus des Wissens“ stammt ursprünglich von Mittelstraß (1998), der ihn im Kontext von Wissenschaft und Universität zur Beschreibung von Forschung eingeführt hat. Koch übernimmt diesen Ausdruck und verwendet ihn, im Zusammenhang mit der Schule, korrelierend mit dem Lehren und Lernen. 
in den Gründen nicht auskennt, mag zwar Lehrer heißen, besoldet werden, aber er ist es nicht und wird, solange sein Defizit währt, keiner sein." (Koch 2015: 69)

Das Lernen in der Schule findet in Anlehnung an Koch (2015) über das Wissendwerden, über die Erkenntnis sowie über die Erfahrung statt (vgl. Abschnitt 2.3). Wissen kann in der Schule nur dann gelehrt werden, wenn die Empfänger (hier: SchülerInnen) das Lernen zulassen. Bruck argumentiert, dass „die Möglichkeit des Lehrens im Lernen [gründet]“ (Buck 1989: 1). Koch und Bruck zeigen in ihren Zugängen Parallelen zu Holzkamp (2004). Dieser beschreibt über das Modell des expansiven Lernens, wie das Lernen nicht planbar ist. Der Lernprozess ist ein von den Individuen abhängiger Prozess, der, wie in Kapitel 2.4 beschrieben, vorwiegend von der lernenden Person abhängig ist, und kann nur dann stattfinden, wenn diese motiviert ist, neues Wissen zu generieren sowie Handlungsstrategien zu entwickeln (vgl. Holzkamp 2004: 29). Dieser Prozess fasst Heinz von Förster in seiner Argumentation „Lernen ist das persönlichste auf der Welt“ zusammen (vgl. Kahl 1999).

Koch beschreibt den Weg und Zugang zum Wissen als das Wissendwerden (vgl. Koch 2015: 89). Wissendwerden bezieht sich auf das Aufzeigen von verstecktem Wissen, „das Verborgene ans Licht [...] [zu] holen und das Ungesehene sichtbar zu machen.“ (Koch 2015: 91) Er appelliert an die Selbstbelehrung der SchülerInnen. Für ihn steht nicht das Vermitteln der Lehrperson im Mittelpunkt. Vielmehr sollte die Lehrperson die SchülerInnen bei einem selbstständigen Aneignungsprozess unterstützen (vgl. Koch 2005: 92). Wissen kann Koch zufolge über das selbstständige, forschende Lernen durch die Erkenntnis entstehen. Als weiteren Zugang zum Wissen identifiziert er die Erfahrung. Er greift dabei auf das Verständnis von Kant zurück, der die Erfahrung als unseren eigentlichen Lehrer/Lehrerin bezeichnet (vgl. Koch 2005: 92).

Koch (2015: 72f.) beschreibt als ein weiterer wichtiger Bereich die Erziehung (vgl. Abbildung 6). Diese bedingt eine Steigerung von Wissen über die Welt.

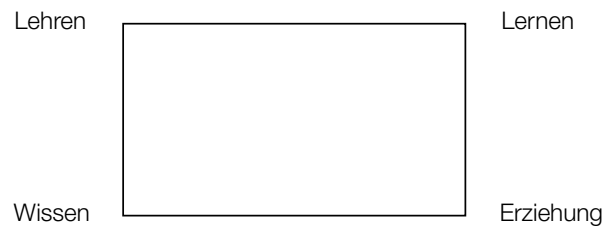

Abb. 6: Verhältnis zwischen Wissen, Lehren, Lernen und Erziehung

Er stützt sich bei seiner Argumentation auf die Bildungstheorie des erziehenden Unterrichts von Herbart ${ }^{14}$. Herbart argumentiert in der Einleitung zu „Allgemeine Pädagogik“: „Ich gestehe gleich hier keinen Begriff zu haben von Erziehung ohne Unterricht." (Herbart 1982: 22) Er entwickelt für die Beschreibung der Erziehungslehre ein dreiteiliges Konzept mit den Begrifflichkeiten „Regierung“, „Unterricht“ und „Zucht“ und verfolgt den Ansatz der Entfaltung des Individuums in der Welt über das Wissen der Welt. Nur ein erzieherischer Unterricht im Sinne einer persönlichen und praktischen Bildung kann SchülerInnen zu mündigem Handeln befähigen. Die „Regierung“ definiert er als eine Vorstufe der Erziehung. Er beschreibt sie als die Unterwerfung der Lernenden unter die Gewalt der Erziehenden (vgl. Herbart 1982: 31). „Unterricht“ und „Zucht“ nehmen in seinem Konzept zentrale Positionen ein (vgl. Geißler 1970: 89). Die

14 Herbart konzeptualisiert in seiner Bildungstheorie einen weiteren Zugang und entwickelt das Konzept der ästhetischen Bildung. 
Zucht wird einmal über die Vermittlung von Kenntnissen zum Inhalt des Unterrichts. Der Unterricht dient als Instrument der Zucht, da Herbart keinen Unterricht anerkennt, der nicht erzieht (vgl. Geißler 1970: 89). Die Hauptaufgabe des Unterrichts ist es Herbart zufolge, den Bildungsauftrag zu erfüllen und Einsicht und Erkenntnis über die Welt in ihren unterschiedlichen sozialen, geistigen und materiellen Verhältnissen zu vermitteln. Die Zucht sorgt dafür, dass der Lernende über die Bildung handeln kann (vgl. Buck 1985: 95). Erziehung und Bildung führen in Herbarts Theorie zu Wissen (vgl. Herbart 1890: 394). In Anlehnung an Koch (2015) verweisen Esslinger-Hinz \& Sliwska (2011: 22) und Gruschka (2011) auf die wesentliche Bedeutung von Erziehung. Sie argumentieren, dass Schule und Unterricht nicht nicht erziehen können.

Die Wissensvermittlung in der Schule steht in enger Beziehung mit Lehren, Lernen und Erziehung. Gesellschaftliche, ökonomische, politische und soziale Veränderungsprozesse fordern, diese Begrifflichkeiten stets neu zu hinterfragen. Hargreaves (2003) wie auch Schratz \& Schrittesser (2003) verweisen auf die Gefahr, dass gegenwärtige Transformationsprozesse oftmals im Schulleben nicht berücksichtigt werden und der Trend zu einer Stagnation besteht. Gegenwärtige Entwicklungen, die Schule beeinflussen, beziehen sich auf Prozesse der Globalisierung, auf soziale, kulturelle und geographische Mobilität, den Umgang mit Heterogenität sowie mit Technologie und den Medien. Hinzu kommen neue Anforderungen über die Zunahme von zentralen Schlüssel- und Kommunikationskompetenzen, die angemessen vermittelt werden müssen (vgl. Thiel 2007: 153; Terhart 2013: 55f.; European Commission 2013; Guerriero 2017). Die Schule als Spiegel der Gesellschaft (vgl. Kraler \& Schratz 2012: 89) fordert die adäquate Integration von Veränderungsprozessen und der damit einhergehenden Anforderungen (vgl. Abschnitt 2.1) in den Schulalltag (vgl. Bauer et al. 1996: 5f.; Hargreaves 2003). Veränderungsdynamiken bedingen, dass Lehrpersonen vor neuen Aufgaben und Anforderungen stehen und spezifisches Wissen für das Lehren, Lernen und Erziehen benötigen. Nach Bauer (2007: 12) sowie Schratz (2009: 17f.) scheitert die Schule oftmals daran, Gelingensbedingungen zu schaffen, die zeitgerechtes Lernen garantieren. Die EU (2013: 7) argumentiert, die zentrale Aufgabe von Lehrpersonen bestehe in der Weitergabe in aktuellen und zukunftsorientierten Wissens, „to help students acquire not only ,the skills that are easiest to teach and easiest to test' but more importantly, ways of thinking (creativtiy, critical thinking, problem-solving, decisionmaking and learning); ways of working (communication and collaboration; tools for working (including information and communication technologies); and skills around citizenship, life and career and personal and social responsibility for sucess in modern democracies“.

Mit dem Pragmatic Shift „from best practice to next practice“ betonen Kraler \& Schratz (2012: 92) die Notwendigkeit, Schule und Wissen neu zu denken und den Unterricht vor dem Hintergrund der im 21. Jahrhundert notwendigen Fertigkeiten der SchülerInnen auszurichten und zu öffnen. Die Öffnung der Schule generiert neue Ressourcen für Lehren, Lernen und Erziehen (vgl. Schratz 2019: 47). Wie schon Henting (2006) für eine „Entschulung“ appellierte, legt auch Schratz den Schluss nahe, dass die Öffnung der Schule und die Verbindung von Leben und Lernen das Lernen fördern kann. Sie wird als Chance gesehen, die Lernen in das Interesse des Schulalltags rückt. Dieser Perspektivenwechsel vom Lehren zum Lernen bedarf seitens der Lehrpersonen einer Weiterentwicklung ihres Wissens, damit sie diesen Prozess bestmöglich unterstützen können. Koch (2015: 109ff.) diskutiert die wesentliche Bedeutung der LehrerInnenrolle und beschreibt das Wissen über die Rolle von Lehrpersonen, die als Berater und als Instrukteur fungieren. Mithilfe der Einnahme neuer Rollen von Lehrpersonen sollen die Wissensaneignung sowie das Lernen der SchülerInnen unterstützt und passende Unterrichtssituationen hergestellt werden. Fauser et al. (2007: 10) beschreiben, wie gute Schulen unterschiedliche diagnostische Mittel einsetzen und Lernen mehrperspektivisch unter Berücksichtigung individueller Interessen der SchülerInnen be- 
trachten. Für die Umsetzung dieser Anforderungen stützt sich Schratz (2009) auf das Konzept der Lernseitigkeit. Der Fokus wird auf das Lernen der SchülerInnen gerichtet, die sich über individuelles Lernen und die dabei gemachten Erfahrungen Wissen aneignen können. Die Auseinandersetzung mit der Welt wird für diesen Prozess als wesentlich beschrieben. Lehrpersonen sind gefordert, eine förderliche Lernumgebung zu schaffen und ihr Handeln von den Lernenden aus zu denken. Die Verknüpfung von lernseitiger und lehrseitiger Orientierung wird Schratz zufolge für die Schaffung einer passenden Unterrichtssituation gefordert. Dabei stehen nicht nur Inhalt und Methodik im Vordergrund, sondern die individuellen Erfahrungen und Handlungsmomente von SchülerInnen nehmen eine zentrale Rolle ein (vgl. Schratz 2013).

Der Wissensbegriff wird seit den 2000er Jahren über Bildungsstandards und Kompetenzmodelle weiter adaptiert (vgl. Weinert 2001a: 45ff.; Bifie 2019) und beeinflusst das gegenwärtige Verständnis von Wissen in der Schule. Unzufriedenstellende Ergebnisse vergleichender internationaler Bildungsstudien wie PISA führen mit dem Ziel einer Qualitätssicherung von Schule und SchülerInnenleistungen zur Entwicklung von Bildungsstandards. Die Erwartungen an Schule, was vermittelt werden soll, haben sich verändert (vgl. Funke \& Spinath 2014: 142). Bildungsstandards definieren konkret formulierte Lernziele in Form von Könnensbeschreibungen und Kompetenzen, über die SchülerInnen verfügen müssen (vgl. Bifie 2019). Bildungsstandards zielen über standardisierte Testverfahren auf eine objektive Bewertung für die Überprüfung erreichter Kompetenzen ab. Weitere essenzielle Funktionen beziehen sich auf eine ziel- und ergebnisorientierte Unterrichtsplanung und Unterrichtsdurchführung, eine bestmögliche Förderung einzelner SchülerInnen sowie auf die Entwicklung eines kompetenz- und schülerorientierten Unterrichts (vgl. KMK 2014; Bifie 2019). Die im Rahmen der Bildungsstandards definiert Kompetenzen definiert Weinert (2001b: 27f.) als

„die bei Individuen verfügbaren oder durch sie erlernbaren kognitiven Fähigkeiten und Fertigkeiten, um bestimmte Probleme zu lösen, sowie die damit verbundenen motivationalen, volitionalen und sozialen Bereitschaften und Fähigkeiten, um die Problemlösungen in variablen Situationen erfolgreich und verantwortungsvoll nutzen zu können“.

Er verbindet über Kompetenzen Wissen mit Handeln und definiert fachliche und überfachliche Fähigkeiten und Fertigkeiten, um Probleme zu lösen und auf Situationen erfolgreich zu reagieren. Weinert (2001b: 27f.) hebt zudem die „willentliche Steuerung von Handlungen und Handlungsabsichten“ hervor und verweist auf die eigenständige Übertragung der Fähigkeiten und Fertigkeiten auf neue Situationen.

Wie in diesem Kapitel aufgezeigt, hat Schule die Weitergabe von Wissen zum Ziel, das sich aufgrund unterschiedlicher gegenwärtiger Entwicklungsprozesse verändert. Dies bedingt eine kontinuierliche Weiterentwicklung von Wissensbereichen und Fähigkeiten von Lehrpersonen, damit sie mit aktuellen und zukünftigen Anforderungen umgehen können. Im weiteren Verlauf wird der Fokus auf das Wissen im Kontext der LehrerInnenbildung gelegt.

\subsection{Wissen und LehrerInnnenbildung}

Von Lehrpersonen wird erwartet, dass sie aktuell relevantes Wissen aufarbeiten und ihre Wissensbestände regelmäßig professionell weiterentwickeln (vgl. Guerriero 2017: 13). Gegenwärtig diskutierte Entwicklungen über die Schule von heute und morgen (vgl. Kapitel 1.2/2.5) und die damit einhergehenden umfassenden Aufgaben bedingen ein umfangreiches professionelles Wissen und eine stetige professionelle Weiterentwicklung. Das Professionswissen von Lehrpersonen gilt als grundlegende Ressource für die Bewältigung beruflicher Anforderungen und das Vermitteln wich- 
tiger Wissensbereiche. Der Wissenserwerb von SchülerInnen hängt konstitutiv vom Wissen und Handeln der Lehrpersonen ab (vgl. Helmke 2003; Kunter et al. 2017). Lehrpersonen sind mithilfe ihres professionellen Wissens gefordert, das Lernen nachhaltig zu unterstützen (vgl. Schratz 2019). Gegenwärtige Transformationsprozesse führen zu einer Bedeutungsverschiebung des Wissens, indem die Vermittlung des reinen Faktenwissens an Bedeutung verliert. Im Mittelpunkt des Interesses steht das pädagogische Wissen als Teil des professionellen Wissens von Lehrpersonen. Gleichzeitig bekommt Wissen im Licht technologischer und gesellschaftlicher Entwicklungen einen veränderten Stellenwert. Lehrpersonen sind gefordert, mit der Vielzahl an Herausforderungen und Informationen sowie heterogenen Gruppen umzugehen, um ihrem Bildungs- und Erziehungsauftrag nachzukommen und einen qualitativ hochwertigen Unterricht anbieten zu können (vgl. Schratz 2019).

Die Kompetenzorientierung sowie die von der KMK (2004) definierten Standards beschreiben aufseiten der Lehrpersonen professionelle Kompetenzen, die für den LehrerInnenberuf zentral sind. Beispiele für Kompetenzmodelle werden im folgenden Kapitel (vgl. Kapitel 3) diskutiert. Angeregt durch Leistungsvergleichsstudien wurde über psychometrische Testverfahren das Wissen von Lehrpersonen operationalisiert (vgl. Bormann \& Haan 2008: 276). Studien (z.B. COACTIV, TEDS-M, ProwiN) zielen darauf ab (vgl. Kapitel 4.2), das professionelle Wissen von Lehrpersonen mittels standardisierter Testverfahren zu operationalisieren und zu konzeptualisieren (vgl. Voss et al. 2011; König et al. 2011; Lenske et al. 2015). Die Akzentverschiebung von Wissen zu Kompetenzen führte zu einem umfangreichen Kompetenzdiskurs. Bildungstheoretisch bemängelt wird ein Bedeutungsverlust des Wissens (vgl. Lenz 2005: 55). Koch (2005: 92) bemerkt, dass Wissen nicht durch Kompetenzen ersetzt werden kann, da diese auf einzelne Wissensbestände von Lehrpersonen aufbauen.

Stuckert (1980: 71) sowie Haun (2013: 209f.) diskutieren den Wissensbegriffs unter Berücksichtigung gegenwärtiger gesellschaftlicher, politischer und ökonomischer Entwicklungen. Sie betonen, wie das heutige Wissen schon morgen als überholt zu gelten scheint. Ist das Kernwissen in der Schule stabil, führen Veränderungsprozesse zu neuen Anforderungen an pädagogisches Wissen von Lehrpersonen. Helmke (2009) sowie Schratz (2019) diskutieren unterschiedliche Modelle und benennen pädagogische Wissensbereiche, die für einen gegenwarts- und zukunftsorientierten professionellen Unterricht wesentlich sind. Schratz (2019: 45) bezieht sich auf ein didaktisches Konzept der Lernseitigkeit. Er fordert insbesondere für das Lernen im 21. Jahrhundert ein lernseitiges Wissen von Lehrpersonen ein. Dieses wird über unterschiedliche generische Kompetenzen charakterisiert (vgl. Abbildung 7).

Sich mit unterschiedlichen Lebenssituationen auseinandersetzen
Individueller lebenslanger

Entwicklungsprozess und die Zukunft mitgestalten Lernprozesse mit neuen Inhalten
organisieren
Urteilskraft im Kontext gesellschaftlicher Regeln und Normen entwickeln
Heterogenität und Andersheit wahrnehmen, respektieren und damit arbeiten können

Wissen auf seinen Inhalt überprüfen und zum

Nichtwissen abgrenzen

An gesellschaftlichen Prozessen und Dialogen teilnehmen

Abb. 7: Generische Kompetenzen des lernseitigen Wissens von Lehrpersonen (in Anlehnung an Schratz 2019: 45f.) 
Die generischen Kompetenzen beziehen sich auf unterschiedliche pädagogische Wissensbereiche, die zentrale Anforderungsbereiche der Gesellschaft miteinschließen. Schratz charakterisiert die generischen Kompetenzen über den Umgang mit Heterogenität, eine professionelle Haltung von Lehrpersonen, die gesellschaftliche Kooperation und Diskursfähigkeit, das Wissen über die Gestaltung von Lernprozessen, die persönliche Weiterentwicklung der Lehrpersonen und ihr Engagement am gesellschaftlichen Leben sowie die Auseinandersetzung mit unterschiedlichen Lebenssituationen. Die von Schratz definierten Kompetenzbereiche verdeutlichen neben der lernseitigen Perspektive die Relevanz eines aktiven, handlungsfähigen und reflexiven pädagogischen Wissensrepertoires der Lehrpersonen.

Helmke (2009: 168f.) entwickelte ein weiteres Modell pädagogischer Wissensbereiche, das wesentliche „fächerübergreifende, unterrichtsrelevante Qualitätsbereiche“ diskutiert. Es illustriert anhand von zehn Merkmalen guten Unterrichts unterschiedliche Anforderungsbereiche von Lehrpersonen und verweist auf die essenzielle Bedeutung pädagogischen Wissens. Die Merkmale beziehen sich auf die Klassenführung, die Klarheit und Strukturiertheit, die Konsolidierung und Sicherung, die Aktivierung, die Motivierung, das Schaffen eines lernförderlichen Klimas, die Schülerorientierung, den Kompetenzansatz, den Umgang mit Heterogenität und die Angebotsvariation. Er charakterisiert einen guten Unterricht darüber, wie die einzelnen Merkmale in unterschiedlichen Ausprägungen situationsspezifisch und unterrichtsrelevant eingesetzt werden (vgl. Helmke 2009: 170). Schratz und Helmke verweisen in ihren Modellen auf ein mehrperspektivisches pädagogisches Wissen. Sie betonen in ihren Zugängen die Relevanz des Wissens eines schülerInnenorientierten Unterrichts. Helmke konzentriert sich stärker auf Wissensbereiche im Unterricht, während Schratz den Fokus auf die gesellschaftlichen Anforderungen und die Weiterentwicklung der Lehrperson legt. Lehrpersonen stehen in einem Spannungsfeld zwischen Tradierung und Innovation. Sie sind für die Weitergabe alter Traditionen und Wissensbestände sowie für die Implementierung neuer Wissensarten und Innovationen verantwortlich (vgl. Eagleton 2009: 14ff.). Es kommt hinzu, dass Lehrpersonen selbst als Instrument gesellschaftlichen Interesses fungieren und kulturelle Werte repräsentieren (vgl. Schratz \& Schrittesser 2003: 181). Für die kontinuierliche Weiterentwicklung der Wissensbestände, wie Schratz (2019) postuliert, ist ein professionsspezifischer Lernprozess vonnöten. Der professionelle Erwerb von Wissen findet bei den Lehrpersonen ab der Ausbildung statt (vgl. Abbildung 8).

(Lebenslanges) Lernen

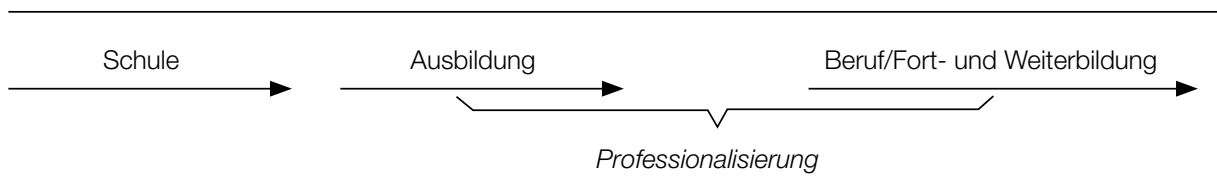

Abb. 8: Professionsspezifischer Lernprozess

Während der Ausbildung werden erste theoretische Zugänge kennengelernt sowie eigene Wissenbestände hinterfragt und weiterentwickelt. Mit der Grundausbildung während des Studiums ist der professionelle Wissenserwerb nicht abgeschlossen. Zentral für den Lernprozess sind sowohl der Berufseinstieg als auch die Berufspraxis im Sinne eines lebenslangen Lernens. Der Beziehung des Lernens zum Professionsfeld kommt eine zentrale Bedeutung zu. Die lerntheoretische Perspektive fordert eine erneute Auseinandersetzung mit den in der Ausbildung 
angelernten Wissens- und Handlungsstrukturen (vgl. Neuweg 2004). Der Übergang von der Ausbildung zur Praxis bedeutet, das gelernte Wissen sinnvoll anzuwenden und handlungsorientiertes Wissens zu generieren. Keller-Schneider \& Hericks (2014: 391) argumentieren, dass erworbenes Wissen für diesen Prozess allein nicht ausreicht. Als weitere wesentliche Gelingensund Einflussfaktoren werden persönliche Haltungen, Überzeugungen, Motivation und individuelle Fähigkeiten genannt. Der Berufseinstieg wird von Kocher (2014: 30) als „besondere und sensible Phase der Professionalisierung " beschrieben. Berufliche Entwicklungsaufgaben verlangen eine aktiv handelnde Person, die mit ihrer sozialen Umwelt in Beziehung steht. Die Entwicklungsaufgaben werden über individuelle Ansprüche, Ressourcen und Ziele entwickelt (vgl. Buchwald \& Hobfoll 2004). Der professionsspezifische Lernprozess wird mithilfe vertiefter Wissensstrukturen und Ressourcen verstärkt (vgl. Reh \& Schelle 2000), die zu Handlungen und neuen Erfahrungsmomenten führen. Die professionelle Kompetenz generiert sich aus einem Beziehungsgefüge von Professionswissen, Überzeugungen, Werthaltung, Motivation und persönlichen Fähigkeiten (vgl. Keller-Schneider \& Hericks 2014: 391). Während der Praxis befördern Fort- und Weiterbildungen sowie Lerngemeinschaften den lebenslangen, professionsspezifischen Lernprozess (vgl. Lipowsky 2014: 512/529). Der spezifische Aneignungsprozess professionellen Wissens von Lehrpersonen wird im nachfolgenden Kapitel in Abschnitt 3.2 weiter ausgeführt und im Detail beschrieben.

Ist das Professionswissen im Kontext des LehrerInnenberufs für die Umsetzung eines qualitativ hochwertigen und zeitgemäßen Unterrichts von signifikanter Bedeutung, wird dieses im Folgenden hinsichtlich ihrer Bedeutung und Formen (vgl. Kapitel 3) diskutiert. 


\section{Konzeptualisierungen von Wissen und Professionswissen im LehrerInnenberuf}

Die Bedeutung von professionellem Wissen hat sich im Kontext des Professionalisierungsdiskurses von Lehrpersonen entwickelt. Mit der empirischen Wende um das Jahr 2000 stellte sich ein neues Verständnis von professionellem Wissen von Lehrpersonen ein. Wurde der Beruf lange Zeit als Semi-Profession charaktersiert und mit unterschiedlichen Ansätzen professionellen Wissens in Verbindung gebracht ${ }^{15}$ (vgl. Shulman 1967/1968; Bromme 1992), führten die Kompetenzorientierung und die starke Fokussierung auf die Operationalisierung von Wissensfeldern von Lehrpersonen (vgl. Kapitel 2.4) zu weiteren Konzeptualisierungen spezifischen Professionswissens (vgl. Kunter et al. 2011; König et al. 2011; Voss et al. 2011). Die Wissensforschung könnte als Bindeglied zwischen der Forschung zur LehrerInnenbildung und dem aktuell gängigen Diskurs der Kompetenzforschung gesehen werden. Wissen gilt als Grundlage der definierten Kompetenzen, welches ermöglicht, Wissen mit Handeln zu verbinden und dieses zielorientiert anzuwenden (vgl. Kapitel 2.5). Über den Kompetenzansatz rückt spezifisches Wissen, mit den entsprechenden Fähigkeiten, in den Vordergrund, welches über Operationalisierungen gemessen und definiert wird. In Bezug auf das Professionswissen von Lehrpersonen, welches sich über theoretisches und handlungsorientiertes Wissen definiert, können Wissen und Kompetenz als Beziehungsgefüge professionellen Wissens beschrieben werden. ${ }^{16}$

Professionelles Wissen charakterisiert sich über theoretische und handlungsorientierte Wissensbereiche (vgl. Dann et al. 1987: 306ff.; Fried 2002: 12f.; Loughran 2019: 3). Oelkers \& Tenorth (1991: 19) betonen einen handlungsorientierten Ansatz und argumentieren, dass es nicht entscheidend ist, „ob und wie sich die Pädagogik [...] in ein metatheoretisches Schema ein $[\mathrm{zu}]$ ordnen lässt oder nicht, sondern wie das im Terrain dieser Disziplin aufzufinden und in Gebrauch befindliche Wissen beschaffen ist". Cochran-Smith \& Lyle (1999) diskutieren hierzu ein Modell, das professionelles Wissen über die Verbindung von Theorie und Handeln beschreibt und den handlungsorientierten Ansatz hervorhebt. Sie charakterisieren professionelles Wissen von Lehrpersonen über „knowledge for practice“, „knowledge in practice“ and „knowlegde of practice“. Dieser Ansatz zeigt Parallelen mit dem von Mittelstraß (1998) definierten Verfügungs- und Orientierungswissen, welcher nicht nur das Faktenwissen, sondern auch das Wissen über Ziele als wesentlichen Bereich von Lehrpersonen beinhalten.

Wie in Abschnitt 1.2 diskutiert, entwickelte Shulman (1986/1987) mit seiner Taxonomie zum LehrerInnenwissen einen Meilenstein für das gegenwärtige Verständnis professionellen

15 Der Diskurs zur Professionalität von Lehrpersonen zeigt, wie der LehrerInnenberuf in Anlehnung an den klassischen professionstheoretischen Ansatz, keine vollständige Übereinstimmung findet. Der Anforderungskomplex einer klassischen Profession, wie sie damals durch den Arztberuf formuliert wurden, kann nicht vollständig auf den LehrerInnenberuf übertragen werden (vgl. Herzmann \& König 2016: 33). Basierend auf der Professionalisierungsdiskussion haben sich unterschiedliche Ansätze wie der strukturtheoretische, biographische oder kompetenzorientierter Ansatz entwickelt, mit denen die Professionalität von Lehrpersonen begründet werden kann (vgl. Terhart 2001). Terhart (2001) hält einen strukturtheoretischen Ansatz, der das Thema „LehrerInnenberuf als Profession“ behandelt im strukturtheoretischen Diskurs am geeignetsten. Der strukturtheoretische Ansatz beschreibt das professionelle Handeln von Lehrpersonen durch Prozesse und Dynamiken und stellt die Interaktion der Lehrenden und Lernenden unter Berücksichtigung gesellschaftlicher Anforderungen, in den Mittelpunkt des Interesses (vgl. Herzmann \& König 2016: 33).

16 Die Diskussion von Wissen und Kompetenz in Bezug auf den Bildungsauftrag Schule wird in dieser Arbeit nicht weiter thematisiert. 
Wissens. ${ }^{17}$ Bromme (1992) erweitert im deutschsprachigen Raum im Kontext eines Expertenansatzes das Shulmansche Konzept. Er verdeutlicht eine Wechselbeziehung zwischen Wissen und Handeln. Für ihn ist theoretisches professionelles Wissen Voraussetzung für professionelles Handeln im Unterricht. Dieser Ansatz wird bei Shulmans Taxonomien zu wenig berücksichtigt, der sein Verständnis vorwiegend auf theoretische Wissensformen stützt. Das professionelle Wissen für die Bewältigung beruflicher Anforderungen definiert er unter Berücksichtigung theoretischer Elemente subjektiver Theorien sowie praktischer Erfahrungen:

„Die relativ schwierigen und komplexen Aufgaben des Unterrichtens erfolgreich [zu] bewältigen, [indem Lehrpersonen] über einen Bestand an professionellem Wissen verfügen, der für die Aufgabenbewältigung gebraucht wird. Das Wissen liegt der Wahrnehmung, dem Denken und dem Handeln zugrunde bzw. begleitet das Handeln. Das Wissen ist zugleich durch diese Aufgabe inhaltlich und strukturell beeinflusst." (Bromme 1992: 10)

In Anlehnung an die Klassifizierung von Shulman (1986) erweitert Bromme das Wissenskonzept anhand von fünf Wissensbereichen (vgl. Bromme 1992: 96f.). Er benennt die Wissensbereiche von Shulman und ergänzt diese um zwei weitere, die Philosophie des Schulfachs und das curriculare Wissen:

- Fachliches Wissen

- Curriculares Wissen

- Philosophie des Schulfachs

- Pädagogisches Wissen

- Fachspezifisch-pädagogisches Wissen

Bromme (1992: 96f.) definiert das Fachwissen als die zu unterrichtenden Inhalte eines spezifischen Unterrichtsfachs, das curriculare Wissen als die notwendigen, heruntergebrochenen Inhalte für ein Unterrichtsfach. Das Wissen über die Philosophie des Schulfachs definiert er anhand der normativen Aspekte einzelner Fachinhalte, die Verbindungen zwischen Theorien und sozialen Lebensbereichen generieren. Das allgemein pädagogisch-didaktische Wissen ist fachunabhängig. Diese Wissensform enthält Strukturen über den Unterrichtsablauf, Interaktionsmuster oder den Umgang mit Problemsituationen. Die sachlogische Darstellung des Inhalts über Auswahl und Gewichtung der Themenbereiche argumentiert er anhand des fachspezifisch-pädagogischen Wissens. ${ }^{18}$

Die Kompetenzorientierung verstärkt den Schwerpunkt einer handlungsorientierten Perspektive, die wie schon Oelkers \& Tenorth (1991) und Cochran-Smith \& Lyle (1999) postulieren, Wissen, Handeln und Lernen verbindet. Diese Verbindung von Lernen, Wissen und Handeln wurde auch schon von Fenstermacher (1994: 3ff.) in einen mehrdimensionalen Zusammenhang gebracht und Abhängigkeiten aufgezeigt. Die EU (2013: 8) unterstützt die positive Bedeutung des Kompetenzansatzes, indem sie dem Kompetenzbegriff für effektives Lernen und Lehren eine besondere Bedeutung beimisst. Sie definiert Kompetenzen von Lehrpersonen als „,complex combinations of knowledge, skills, understanding, values and attitutes“ (European Commission

17 Shulman spricht in seinem Aufsatz von „the teaching profession“ (vgl. Shulman 1986: 4). Übersetzt bedeutet der Begriff professionelles Wissen von Lehrpersonen. Bromme übernimmt später diesen Begriff in seinem Expertenansatz. Das Konzept professionellen Wissens von Lehrpersonen entwickelte Shulman im Rahmen einer kritischen Stellungnahme zu der damaligen öffentlichen Diskussion der Trivialisierung und Einseitigkeit des LehrerInnenberufs.

18 Die von Bromme definierten inhaltlichen Wissensbereiche finden sich auch in den von der KMK (2004) entwickelten Kompetenzbereichen von Lehrpersonen. Basierend auf den vier Kompetenzbereichen Unterrichten, Erziehung, Beurteilung und Innovieren werden zwölf Kompetenzen mit ihren Standards entwickelt, die die Anforderungsbereiche von Lehrpersonen im Detail repräsentieren. 
2013: 8) und fordert, für einen professionellen Unterricht spezifische Kompetenzbereiche für Lehrpersonen zu definieren (vgl. Kapitel 2.5 und 2.6).

Insbesondere das „fachliche Wissen“, das „fachdidaktische Wissen“ und das „pädagogische Wissen" sind Kernbereiche, die in gegenwärtigen Diskussionen über Kompetenzmodelle im deutschen Sprachraum aufgegriffen werden (vgl. Shulman 1986/1987; Bromme 1992). Ein theoretisches Rahmenmodell professioneller Kompetenz (vgl. Abbildung 9) präsentieren Kunter et al. (2011/2013).

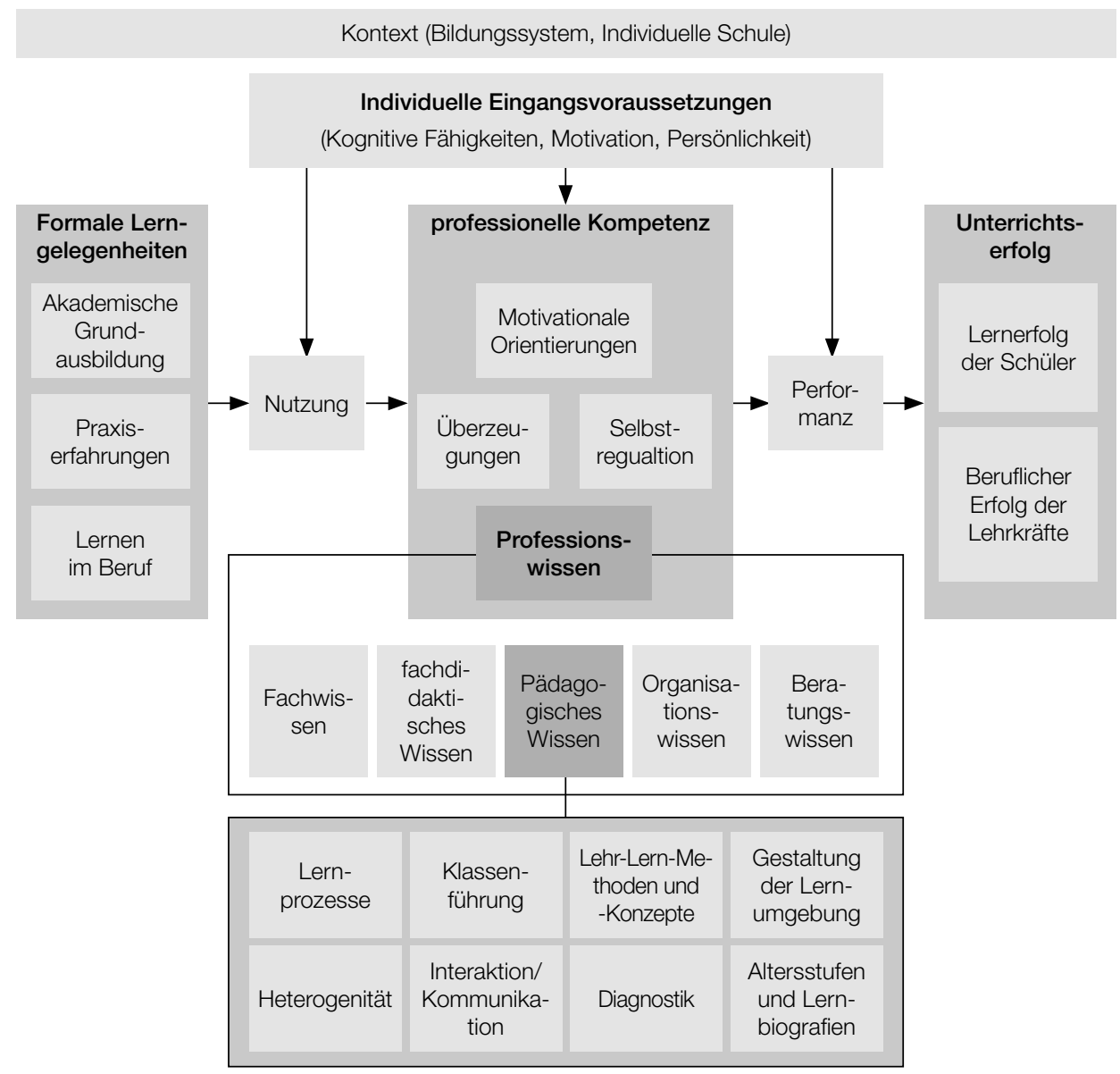

Abb. 9: Theoretisches Rahmenmodell professioneller Kompetenz (Kunter et al. 2011/2013)

Aktuelle Modelle professioneller Kompetenzen beinhalten unterschiedliche inhaltliche Anforderungsbereiche an Lehrpersonen. Bislang gibt es kein einheitliches Modell professioneller Kompetenzen. Die professionellen Kompetenzbereiche des Modells von Kunter et al. (2011/2013) beziehen sich auf affektiv-motivationale Kompetenzen, wie „Motivation“, „Überzeugung“ und „Selbstregulation“, sowie das „professionelle Wissen“. Die Kompetenzbereiche zeigen eine Übereinstimmung mit den von der European Commission (2013: 8) angeführten Definition von Kompetenzen. Die Klassifizierung der professionellen Wissensbereiche stützen sich auf die von 
Shulman und Bromme definierten Taxonomien und werden den AutorInnen durch individuell konzipierte Bereiche ergänzt. Das angeführte Kompetenzmodell (vgl. Abbildung 9) von Kunter et al. (2011/2013) führen zusätzlich das „Organisationswissen“ und „Beratungswissen“ als Formen professionellen Wissens von Lehrpersonen an. Das Kompetenzmodell spiegelt das zuvor definierte Verhältnis zwischen Lernen, Wissen und Handeln von Fenstermacher (1994) wider und verstärkt die Bedeutung eines handlungsorientierten Zugangs.

Formale Lerngelegenheiten der Lehrpersonen beeinflussen die professionellen Kompetenzen, die sich wiederum im professionellen Handeln äußern. Professionelle Kompetenzen werden über den Unterrichtserfolg ersichtlich. Dieser wird über den Lernerfolg der SchülerInnen und den beruflichen Erfolg der LehrerInnen beschrieben. Das Kompetenzmodell mit seinen Determinanten formaler Lerngelegenheiten und Konsequenzen eines erfolgreichen Unterrichts wird anhand individueller Eingangsvoraussetzungen der Lehrpersonen - „kognitive Fähigkeiten“, „Motivation“ und „Persönlichkeit“ - eingebettet. Kunter et al. (2011/2013) geben dem pädagogischen Wissen in ihrem Modell einen wesentlichen Stellenwert und definieren es über acht unterschiedliche Wissensformen: Wissen über „Lernprozesse“, „Klassenführung“, „Heterogenität“, „Altersstufen und Lernbiographie“, „Interaktion/Kommunikation“, „Lehr- und Lernmethoden“, „Diagnostik“ sowie Wissen über die „Gestaltung von der Lernumgebung“.

Die Darstellung der unterschiedlichen Charakterisierungen professionellen Wissens verdeutlicht über die unterschiedlich dargestellten Ansätze und Zugänge, wie diese heterogen definiert und konzeptualisiert werden, sich jedoch alle auf das Verständnis Shulmans mit seiner Taxonomie des fachlichen, fachdidaktischen und pädagogischen Wissens beziehen. Brommes Expertenansatz betont den handlungsbezogenen Charakter des Wissens, der gegenwärtig im Hinblick auf die Kompetenzorientierung verstärkt diskutiert wird. Kapitel 5 greift die wesentliche Bedeutung des Handelns und der Interaktion noch einmal auf und diskutieren den handlungstheoretischen Zugang im Kontext professionellen Wissens von Lehrpersonen.

$\mathrm{Zu}$ der Charakterisierung professionellen Wissens von Lehrpersonen über unterschiedliche Wissensbereiche beschäftigt sich Neuweg (2014: 583) in einem weiteren Zugang mit der Frage, in welcher Form Lehrpersonen Wissen brauchen, „damit Unterricht nicht nur gewusst wird“. Um diese Frage zu beantworten, stützt er sich auf das Verständnis der Analyse und Modellierung der LehrerInnenkognitionen. Neuweg (2014: 584f.) beschreibt die kognitiven Strukturen anhand dreier unterschiedlicher Zugänge (vgl. Abbildung 10) und klassifiziert sie an den von Fenstermacher (1994) beschriebenen Dimensionen „Lernen“, „Wissen“ und „Handeln“.

LERNEN

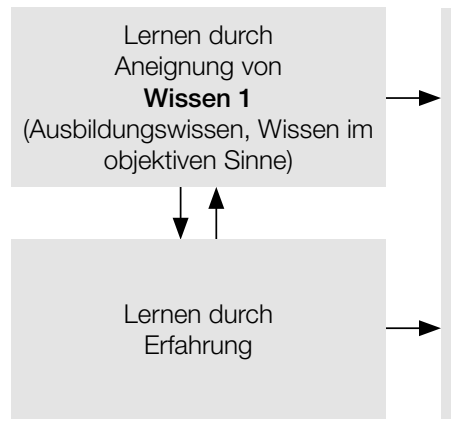

Abb. 10: Konzept des LehrerInnenwissens (Neuweg 2014: 585)

HANDELN

WISSEN

Wissen 2

(mentale Strukturen, Wissen im subjektiven Sinne)

Explizites Wissen

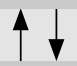

Implizites Wissen
Wissen 3

(Können,

von außen rekonstruierte Logik des Handelns)

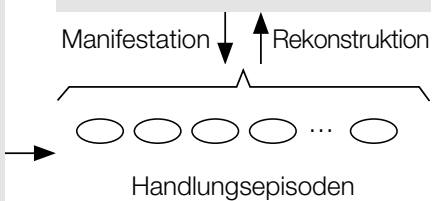

Handlungsepisoden 
Der Zugang „Wissen 1“ integriert das Wissenskonzept über das kodifizierte, systematische, objektive Wissen von Lehrpersonen. Er grenzt Wissen vom Können ab und argumentiert, dass sich das objektive Wissen nicht über die Persönlichkeit, Erfahrung oder reflexive Prozesse beeinflussen lässt. Hier handelt es sich um Wissen, welches mittels Lernen angeeignet wird. Der Zugang „Wissen 2“ definiert sich über ein psychologisches Konstrukt, das subjektive Wissen. Dieses Wissen kann in expliziter oder impliziter Form verankert sein. „Wissen 3“ stützt sich auf ein Erklärungswissen. Dabei wird die Logik des Handelns von außen rekonstruiert, über Äußerungen wie „er weiß, wie es geht“. Es kommt bei dieser Wissensform zu einer Überlappung zwischen Wissen und Können. Neuweg zufolge muss dieses Wissen nicht verbalisiert werden. Vielmehr lassen sich die Handlungsepisoden von außen beobachten und rekonstruieren.

Neuweg (2014) differenziert in seinem dreigliedrigen Zugang zwei Arten, wie Wissen gewusst werden kann. Er verweist auf die von Polanyi (1985) generierten Klassen des expliziten sowie des impliziten Wissens. Basierend auf der von Neuweg vorgebrachten Forderung, LehrerInnenkognitionen zu untersuchen, die LehrerInnenwissen systematisieren, können im Kontext des LehrerInnenwissens weitere Formen diskutiert werden. Diese beziehen sich auf die Formen deklaratives/prozedurales Wissen (vgl. Ryle 1949; Anderson 1982) sowie auf das handlungswirksame gegenüber dem trägen Wissen (vgl. Renkl 1996).

De Jong \& Ferguson-Hessler (1996) entwickelten eine 4x5 Matrix und präsentierten ein weiteres Modell für die Klassifizierung der Wissensformen von Lehrpersonen. Sie definieren den Wissensbegriff über zwei Dimensionen, die der Wissensart sowie die der Wissensqualitäten. Die Wissensart gliedert sich in die von Ryle (1949) und Anderson (1982) definierten Wissensformen, dem prozeduralen und deklarativen Wissen, und ergänzen es mit dem konzeptuellen Wissen. Die Qualitäten des Wissens sind der bierarchische Status, die innere Struktur, der Automatisierungsgrad, die Modalität sowie der Allgemeinheitsgrad.

Bislang gibt es über die Formen und die Qualität von LehrerInnenwissen kein einheitliches Modell. Basierend auf den inhaltlichen Wissensbereichen und kognitiven Formen professionellen Wissens wird in der vorliegenden Forschungsarbeit relevante Klassifizierungen, wie in Abbildung 11 dargestellt, diskutiert. Shulmans Konzept bildet die Grundlage zur Diskussion professionellen Wissens im Kontext dieser Arbeit. Seine Taxonomie wird aufgrund der angeführten Diskussion von Kompetenzmodellen und der unabdinglichen Bedeutung theoretischer sowie handlungsorientierter Wissensbereiche von Lehrpersonen (vgl. Dann et al. 1987: 306ff.; Fried 2002: 12f.; Kunter 2011/2013; Loughran 2019: 3) mit einem handlungsorientierten Wissen ergänzt (vgl. Abbildung 11).

Für die Arbeit relevante kognitive Formen beziehen sich auf die Unterscheidungen zwischen handlungswirksamem/trägem Wissen, explizitem/implizitem Wissen sowie deklarativem/prozeduralem Wissen. Berücksichtigte Wissensqualitäten sind die Stufen der Ausdifferenzierung, die Struktur sowie die Ritualisierung.

Professionelles Wissen von Lehrpersonen wird in Anlehnung an die hier vorgestellten Wissensformen und -qualitäten im weiteren Verlauf der Arbeit diskutiert. Aus professionssoziologischer Perspektive fordert Herzog (1995: 261f.) eine "multiple Wissensbasis“, d.h. unterschiedliche Wissensformen für professionelles Wissen und Handeln zu integrieren (vgl. Herzog 1995: 261f.) und bestätigt damit die Notwendigkeit, professionelles Wissen mehrperspektivisch zu untersuchen. 


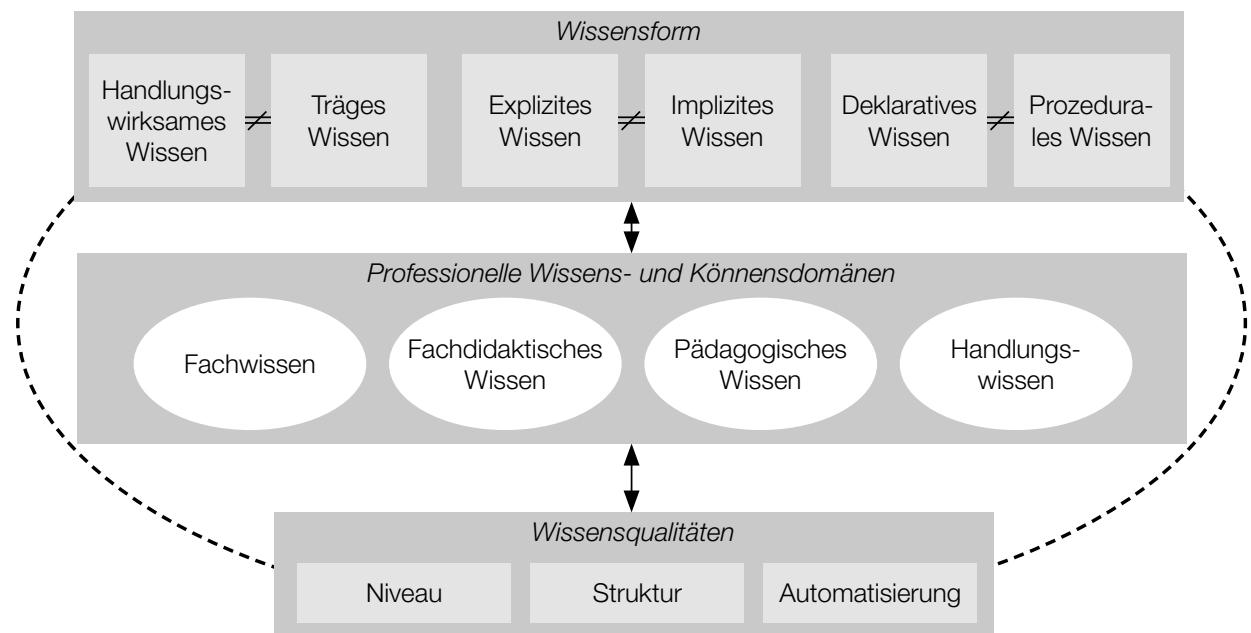

Abb. 11: Wissensmodell professioneller Lehrpersonen

\subsection{Wissensformen}

Im Folgenden werden wesentliche Formen professionellen Wissens diskutiert. Wichtige Formen betreffen das deklarative/prozedurale Wissen (vgl. Ryle 1949; Anderson 1982), das implizite/explizite Wissen (vgl. Polanyi 1985) sowie das handlungswirksame/träge Wissen (vgl. Renkl 1996). Die Wissensformen können nicht getrennt voneinander klassifiziert werden, vielmehr stehen sie, wie in Abbildung 12 dargestellt, in einem Beziehungsgefüge.

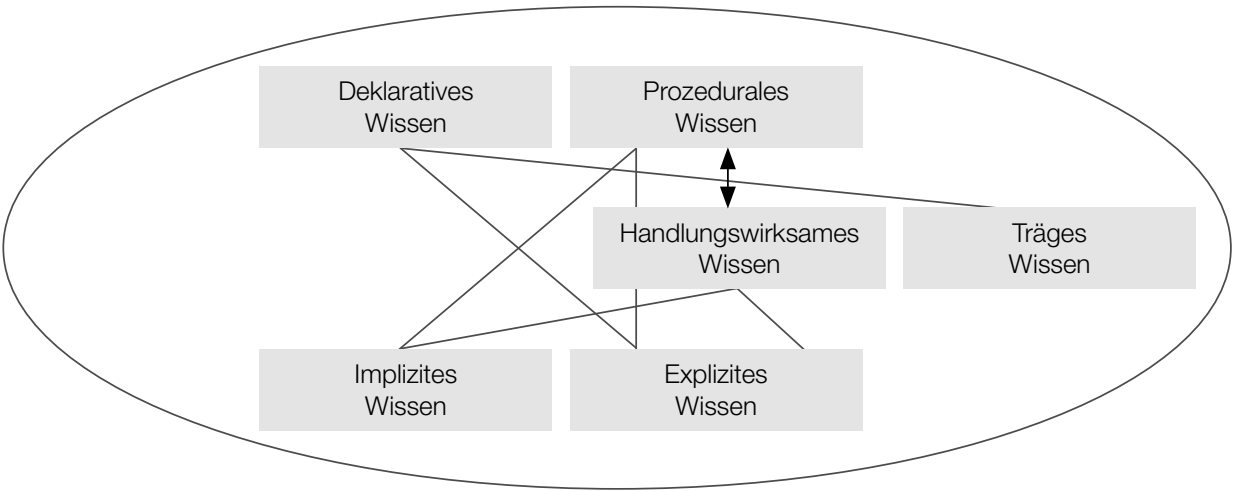

Abb. 12: Beziehungen der Formen pädagogischen Wissens

Die jeweiligen Beziehungsgefüge werden situativ bei der Darstellung der einzelnen Wissensformen beschrieben und ihre Bedeutung diskutiert.

\subsubsection{Deklaratives und prozedurales Wissen}

Ryle (1949) benennt im Kontext der kognitiven Psychologie zwei Arten von Wissen, das deklarative sowie das prozedurale Wissen. Dieses Konzept wurde von De Jong \& Ferguson-Hessler 
(1996) um das konzeptuelle Wissen erweitert. Das deklarative, das konzeptuelle sowie das prozedurale Wissen sind häufige Formen, die gemeinsam mit dem professionellen Wissen von Lehrpersonen sowie in den vorhandenen Operationalisierungen (vgl. Kapitel 4.2) pädagogischen Wissens diskutiert werden.

Das deklarative Wissen („wissen, das ...") bezieht sich auf ein theoretisches Wissen über Begriffe, Fakten und Sachverhalte (vgl. Lenske 2015: 226). Der Erwerb findet über die Wissensvermittlung statt. Es gilt als Voraussetzung für das konzeptuelle sowie prozedurale Wissen (vgl. Anderson 1982; Klieme et al. 2003). Wesentliche Merkmale deklarativen Wissens sind die Verbalisierbarkeit sowie die passende Anwendung in spezifischen Situationen. Es kennzeichnet sich darüber, dass es nicht automatisch in der Praxis angewendet werden kann (vgl. Gruber und Renkl 2000; Anderson \& Krathwohl 2001; König \& Klemenz 2015: 249). Das konzeptuelle Wissen wird in der Literatur häufig als Synonym deklarativen Wissens verstanden. ${ }^{19}$ Es kann jedoch im Vergleich zum deklarativen Wissen als eine inhaltliche Wissensform bezeichnet werden, da es sich nicht nur über theoretisches Wissen, sondern über inhaltliche Schemata und Verknüpfungen charakterisieren lässt (vgl. Rittler-Johnson et al. 2001). Das prozedurale Wissen („wissen, wie ....) wird dem anwendungsorientierten Prozess- und Handlungswissen gleichgesetzt (vgl. Aebli 1983; Putnam 1987). De Jong \& Ferguson-Hessler (1996: 105ff.) beschreiben die Wissensform über kognitive Strukturen, die Handlungsabläufe ermöglichen. Ryle benennt das prozedurale Wissen als das Können (vgl. Ryle 1976: 69ff.). Die Wissensform, wie sie schon Mittelstraß (1998: 19) als Verfügungswissen definiert, charakterisiert sich über vier wesentliche Eigenschaften:

1. Es handelt sich um ein aktions-orientiertes Wissen. Prozedurales Wissen eignen sich Lehrpersonen vorwiegend über die Erfahrung, d.h. über direktes Anwenden theoretischen Wissens und praktisches Handeln, an (vgl. Carter 1990). Prozedurales Wissen kann nicht durch andere vermittelt werden (vgl. Johnston 1992).

2. Das prozedurale Wissen ist personen- und kontextabhängig. Art und Umfang des praktischen Wissens stehen konstitutiv mit der individuellen Lehrperson in Verbindung. Dabei spielt die Motivation, Einstellung und Haltung dieser Lehrperson eine wesentliche Rolle, die die Wissensorganisation sowie das individuelle Handeln leiten (vgl. Pajares 1992: 302ff.; Richardson 1996: 102ff.).

3. Prozedurales Wissen kennzeichnet sich durch explizite sowie implizite Wissensstrukturen. Lehrpersonen sind es nicht gewöhnt, ihr Handeln spezifisch und ganzheitlich zu benennen (vgl. Baumert \& Kunter 2006). Clandini (1986) und Eraut (1994) sprechen bei Lehrpersonen von einer "doing“ anstatt einer „knowledge environment“.

4. Beim prozeduralen Wissen handelt es sich um eine integrative Wissensform. Wesentliche Bestandteile sind wissenschaftliche, formelle, alltägliche Wissensmomente sowie Normen und Werte der Lehrpersonen (vgl. Handal \& Lauvas 1987). Der Prozess der Wissensintegration stützt sich auf die Erfahrung, welche eine Hauptrolle bei der Entwicklung und/oder Weiterentwicklung von praktischem Wissen der Lehrpersonen spielt (vgl. Beijaard \& Verloop 1996: 275ff.).

Explizites und implizites Wissen stehen insofern in Beziehung zueinander, als das deklaratives Wissen von Anderson (1982) als Voraussetzung für prozedurales Wissen beschrieben wird. Der Umwandlungsprozess der Wissensformen vom deklarativen zum prozeduralen Wissen wird mit einer Steigerung der Qualität des Wissens gleichgesetzt, indem wie Klieme et al. (2003) argumentieren,

19 In der vorliegenden Arbeit wird das deklarative und konzeptuelle Wissen im Zusammenhang mit professionellem pädagogischem Wissen als eine Wissensform diskutiert. 
der Transformationsprozess zu einer „Steigerung der professionellen Kompetenz“, d.h. vom Wissen zum Können, führt. Neuweg (2014: 237f.) benennet diesen Prozess als „Integration von Erkenntnissen in subjektiven Denkstrukturen“. Das prozedurale Wissen nimmt im Vergleich zum deklarativen Wissen hinsichtlich der LehrerInnenprofessionalität einen besonderen Stellenwert ein (vgl. Van Driel et al. 2001: 142), da es zu Handlungen führen kann (vgl. Brickhouse 1990). Van Driel et al. (2001) und Klieme et al. (2003) unterstützen die Notwendigkeit der Gestaltung eines handlungs- sowie anwendungsorientierten Unterrichts (vgl. Kapitel 2.5) für qualitativen Unterricht.

\subsubsection{Explizites und implizites Wissen}

In enger Beziehung zum deklarativen und prozeduralen Wissen stehen explizites und implizites Wissen (vgl. Abschnitt 3.1.1). Diese werden von Polanyi (1985) wie Neuweg (1999) als handlungsleitende Kognitionen charakterisiert. Das implizite Wissen, wie bei Mittelstraß das Verfügungswissen (vgl. Mittelstraß 1998: 19), definiert sich über eine mentale Repräsentation, die über ihren Anwendungscharakter automatisiert werden kann. Es lässt sich über ein verdichtetes Erfahrungswissen beschreiben, das zur Handlung sowie zu einer Weiterentwicklung führen kann. „Das Wissen liegt [somit] im Urteilen und Tun." (Molander 1992: 11) Volpert (1992: 63) charakterisiert implizites Wissen als Erfahrungswissen, welches sich im Laufe der Berufsbiografie erweitert (vgl. Abschnitt 3.3). Das explizite Wissen gilt im Vergleich hierzu als eine weniger verdichtete Wissensform (vgl. Bromme 1997: 196ff.).

Explizites und implizites Wissen werden in Anlehnung an Polanyis Definition nicht eindeutig voneinander unterschieden. Explizites Wissen existiert nicht als eigenständiges Konstrukt, sondern enthält Elemente impliziter Strukturen (vgl. Polanyi 1985: 144). Dies unterstreicht die wesentliche Bedeutung des impliziten für das explizite Wissen. Gleichzeitig kann explizites Wissen Neuweg zufolge zu implizitem Wissen transformiert werden (vgl. Neuweg 2000: 199). Für das Professionswissen und damit für das professionelle Handeln von Lehrpersonen ist ein Repertoire an explizitem sowie implizitem Wissen von zentraler Bedeutung.

Implizites Wissen kann im Vergleich zum expliziten nicht verbalisiert werden (vgl. Neuweg 2000: 198). Foppa (1990) bezeichnet es als „nichtberichtbares Wissen“, welches vorwiegend über Handeln sichtbar wird (vgl. Schön 1983; Polanyi 1985; Neuweg 1999). Hackl (2004: 71) beschreibt implizites Wissen als eine passive Wissensform. Hackl (2004: 71) charakterisiert es als eine Form unbewussten Wissens, wie Polanyi (1985: 14), der sagt, „dass wir mehr wissen, als wir zu sagen wissen“. Die zuvor beschriebene Aussage von Foppa (1990), dass implizites Wissen nicht artikuliert werden kann, wird von Polanyi (1985) nur teilweise bestätigt. Er revidiert diese Position, indem er darauf verweist, dass implizite Strukturen nicht vollständig verbalisiert werden können aber reflexive sowie sprachliche Handlungen implizites Wissen zu explizitem transformieren (vgl. Polanyi 1985: 87). Der Transfer von implizitem zu explizitem Wissen findet bei Neuweg (2004: 599) über die Reflexion statt (vgl. Kapitel 5.2). Schon Bohnsack beschreibt (vgl. Kapitel 1.2) das handlungsleitende reflexive Wissen als implizit. Waldenfels (2000: 169) greift auf die Argumentation Polanyis zurück, definiert das implizite Wissen als einverleibte Form und macht deutlich, dass das Wissen ,in den Händen oder in den Füßen [steckt]“ und über den Leib mittels Handeln aufgezeigt werden kann und nicht nur in kognitiver Form existiert.

Oberauer (1993) knüpft an diesen handlungsorientierten Zugang des impliziten Wissens an. Er betont ein Beziehungsgefüge zwischen dem impliziten und dem in Abschnitt 3.1.1 beschriebenen prozeduralen Wissen:

„Wenn jemand in einer bestimmten Situation wiederholt erfolgreich handelt, schreiben wir ihm prozedurales Wissen für diese Situation als Disposition zu erfolgreichem Umgang mit ihr zu. Nur die Tatsache, dass 
erfolgreiches Handeln in dieser Situation erlernt werden kann, legitimiert dabei zunächst die Bezeichnung als ,Wissen'. Die Zuschreibung prozeduralen Wissens ist nur für einen Beobachter möglich. Der Handelnde selbst handelt und erwartet intuitiv, nicht auf der Grundlage expliziter Regeln oder Kriterien. Er ist sich des Gegenstandes seiner Handlung bewußt, aber nicht seines eigenen Wissens.“'(Oberauer 1993: 37)

Oberauer präsentiert den Zusammenhang des impliziten und prozeduralen Wissens, grenzt sie jedoch gleichzeitig voneinander ab. Er argumentiert, dass implizites Wissen nur über wiederholtes, erfolgreiches Handeln sichtbar wird. Das zugrundeliegende Wissen wird bei der handelnden Person nicht bewusst wahrgenommen. Neuweg (1999: 18f.) knüpft an die handlungsbezogene Sichtweise an. Er verweist auf die essenzielle Bedeutung impliziter Strukturen für das professionelle Wissen von Lehrpersonen und argumentiert, dass dieses erst über die Anwendung, das Können und ihr Handeln sichtbar gemacht werden kann. Der Ausübung räumt er dabei einen wichtigeren Stellenwert ein als der expliziten sprachlichen Benennung von Wissensformen von Lehrpersonen. Wie beim deklarativen und prozeduralen Wissen diskutiert (vgl. Abschnitt 3.1.1), wird dem prozeduralen Wissen von Lehrpersonen eine zentrale Bedeutung für das Handeln von Lehrpersonen zugeschrieben. Diese Argumentation lässt sich auf das explizite sowie implizite Wissen übertragen. Wird unter dem deklarativen Wissen das explizite Wissen sowie unter dem prozeduralen Wissen das explizite sowie implizite Wissen verstanden, erlangt das implizite Wissen im Vergleich zum expliziten Wissen aufgrund seiner komplexeren Struktur und stark handlungsleitenden Funktion wesentliche Bedeutung für das professionelle Handeln von Lehrpersonen. Der Diskurs zum expliziten und impliziten Wissen macht deutlich, dass Lehrpersonen nicht in der Lage sind, ihr professionelles Wissen gänzlich zu verbalisieren. Verweisend auf Polanyi (1985) und Foppa (1990) gibt es unterschiedliche Positionen dazu, inwieweit implizites Wissen verbalisiert werden kann. Eine wichtige Argumentation der Arbeit knüpft an das Verständnis von Polanyi (1985), Neuweg (1999) sowie Waldenfels (2000) an, dass sich implizite Wissensformen über das Handeln äußern (vgl. Kapitel 5).

\subsubsection{Handlungswirksames und träges Wissen}

Weitere Wissensformen, die mit dem professionellen Wissen von Lehrpersonen diskutiert werden können, sind das handlungswirksame sowie das träge Wissen (vgl. Abschnitt 3.1). Handlungswirksames Wissen ist Wissen, das Lehrpersonen anleitet, im Unterricht situationsgerecht zu agieren (vgl. Renkl 1996). Für die Anwendung von Wissen wird dem handlungswirksamen Wissen, aufgrund der zentralen Bedeutung der Praxis im LehrerInnenberuf, eine grundlegende Bedeutung zugeschrieben (vgl. Bertschy 2007: 42). Es wird dem in Abschnitt 3.1.1 beschriebenen prozeduralen Wissen gleichgesetzt und charakterisiert sich über die dort beschriebenen Eigenschaften.

Träges Wissen beschreibt im Vergleich zum handlungswirksamen Wissen theoretisches Wissen, welches keine Umsetzungskomponente besitzt. Es wird aufgrund seiner fehlenden handlungswirksamen Entfaltung als nicht wirksames Wissen bezeichnet und grenzt sich vom impliziten Wissen ab. Renkl (1996) unterscheidet in ihrem Modell unterschiedliche Formen trägen Wissens. Diese beziehen sich auf Metaprozesserklärungen, Strukturdefiziterklärungen sowie Situiertheitserklärungen. Metaprozesserklärungen beziehen sich auf vorhandenes Wissen das nicht explizit von Lehrpersonen angewendet wird. Die Strukturdefiziterklärung beschreibt aufgrund falscher Strukturierung die Schwierigkeit, Wissen abzurufen und anzuwenden. Wissen, welches aufgrund fehlenden Kontextwissens nicht angewendet werden kann, bezeichnet sie über die Situiertheitserklärung.

In Anlehnung an dieses Modell beschreiben Gruber \& Renkl (2000) drei Merkmale trägen Wissens, die in einem mehrstufigen Modell erklärt werden: (1) Personen demonstrieren, dass Sie über 
bestimmtes Wissen verfügen und (2) es liegen begründete Annahmen vor, dass das vorhandene Wissen zu kompetentem und situationsgerechtem Handeln eingesetzt werden kann. (3) Trotzdem wenden Personen es nicht an. Dieses Modell versucht zu erklären, wie Lehrpersonen über situationsgerechtes und anwendungsorientiertes Wissen verfügen, es jedoch im Unterricht nicht einsetzen.

Die Ausdifferenzierung von Renkl (1996) sowie Gruber \& Renkl (2000) zeigt bei Lehrpersonen unterschiedliche Ebenen trägen Wissens, die in Situationen durch falsches Wissen oder fehlende Anwendung von Wissen zum Ausdruck kommen. Träges Wissen wird aufgrund vorhandener theoretischer Strukturen mit dem deklarativen Wissen in Verbindung gebracht und als nicht wirksam beschrieben. Es kann argumentiert werden, dass Lehrpersonen, die weniger träges Wissen besitzen, über ein größeres nutzbares Wissensrepertoire verfügen. Dieser fehlende Transformationsprozess wird in der Arbeit in Kapitel 5.2 in Bezug auf die Reflexionsfähigkeit und das pädagogische Handeln diskutiert.

\subsection{Wissensqualitäten}

De Jong und Ferguson-Hessler (1996) definieren im Zusammenhang mit professionellem Wissen von Lehrpersonen unterschiedliche Wissensqualitäten. Im Folgenden werden für die Forschungsarbeit drei wesentliche Wissensqualitäten „Stufen der Ausdifferenzierung“, „Struktur“ und „Ritualisierung“ diskutiert.

Die Wissensqualitäten beeinflussen die Qualität der Wissensformen deklaratives/prozedurales Wissen, handlungswirksames/träges Wissen, implizites/explizites Wissen hinsichtlich ihrer Nachhaltigkeit und ihrer Wirkung (vgl. Abbildung 13).

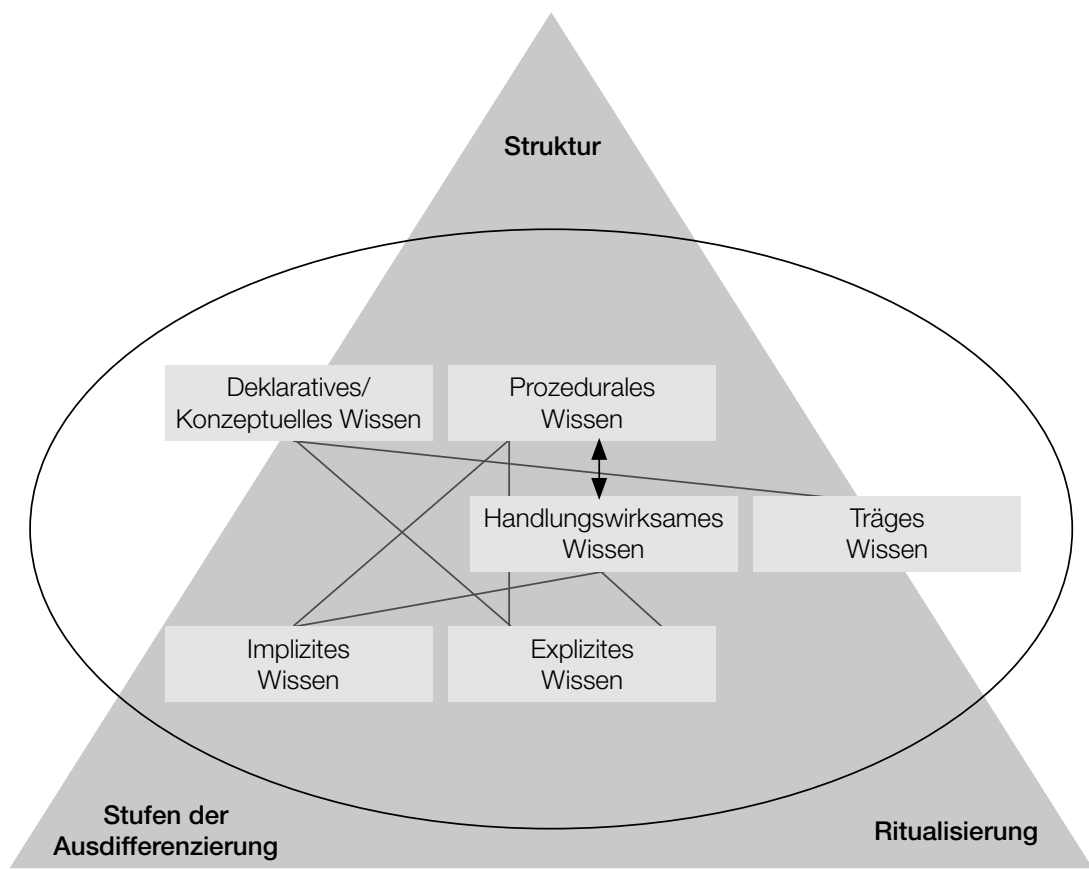

Abb. 13: Zusammenhang zwischen Wissensqualitäten und Wissensformen 
Im Zusammenhang mit dem professionellen Wissen werden sie aufgrund der Forderung von Herzog (1995: 261f.) berücksichtigt, der die Notwendigkeit der Berücksichtigung unterschiedlicher Wissensformen und -qualitäten für die Auseinandersetzung mit professionellem LehrerInnenwissen argumentiert.

\section{Stufen der Ausdifferenzierung}

Die Stufen der Ausdifferenzierung (bei De Jong und Ferguson-Hessler (1996) als Niveau benannt) beschreiben die Tiefenqualität von Wissen. De Jong und Ferguson-Hessler (1996) unterscheiden oberflächiges Wissen sowie Wissen tieferer Qualität. Das oberflächige Wissen bzw. auch Überblickswissen beschreiben sie als Wissen, dessen Strukturen allein nicht nachvollziehbar sind. Es wird mit Reproduktion und Auswendiglernen ohne Urteilsvermögen in Verbindung gebracht. Dieses Wissen ist jedoch insofern relevant, als dass es als Grundlage für den Aufbau einer Tiefenqualität charakterisiert ist. Lehrpersonen mit einem tiefgründigen Niveau können das Wissen mehrperspektivisch betrachten und begründen. Es gilt als verbalisierbar sowie artikulierbar (vgl. De Jong \& Ferguson-Hessler 1996).

\section{Struktur}

Eine weitere Qualität des Wissens ist nach De Jong und Ferguson-Hessler (1996) die Struktur. Die Struktur des Wissens von Lehrpersonen ist qualitätsvoll, wenn die Lehrpersonen über kohärente sowie logische Wissensstrukturen verfügen. Professionelle Lehrpersonen zeichnen sich durch ein vernetztes und gut organisiertes Wissen aus. Das vorhandene Wissen ist sprachlich zugänglich und für die Empfänger nachvollziehbar (vgl. Baumert \& Kunter 2006: 483).

\section{Ritualisierung}

Die dritte Qualität, die Ritualisierung (bei De Jong \& Ferguson Hessler als Automatisierung benannt), definiert sich über eine hoch routinierte Anwendung. Die Qualität der Unterrichtsroutine beeinflusst das situationsspezifische Handeln und Reagieren der Lehrpersonen (vgl. Bromme 1992). Die Automatisierung unterstützt den zuvor beschriebenen Transformationsprozess (vgl. Abschnitt 3.1.1) von deklarativem zu prozeduralem Wissen. ${ }^{20}$ Das routinierte Wissen kann auf explizites sowie implizites Wissen (vgl. Abschnitt 3.1.2) zurückgreifen (vgl. Polanyi 1985).

Das Zusammenspiel der beschriebenen Wissensqualitäten unterstützt die Nachhaltigkeit und positive Wirkung einzelner Wissensformen. Zu oberflächigem Wissen und Wissen tiefer Qualität sind ein strukturelles Wissen sowie die Ritualisierung für ein qualitätsvolles professionelles Wissen von Lehrpersonen hilfreich. Die Aneignung der Wissensformen und -qualitäten von Lehrpersonen findet über einen spezifischen professionellen Lernprozess statt, der im folgenden Abschnitt diskutiert wird.

\subsection{Professionelles Wissen und seine Aneignung}

Die Aneignung professionellen Wissens von Lehrpersonen und der Erwerb berufsspezifischer Fertigkeiten werden anhand verschiedener Stadien (vgl. Abbildung 14) der beruflichen Biografie von Lehrpersonen beschrieben (vgl. Terhart 1990: 247; Reh \& Schelle 2006: 391f.; Voss et al. 2015:

20 Der Transformationsprozess von deklarativem zu prozeduralem Wissen kann mit dem Kompetenzmodell von Gordon (2013) und dem Übergang von einer unbewussten zu einer bewussten Kompetenz verglichen werden. In seinem Zugang beschreibt er vier unterschiedliche Stadien: (1) unbewusste Inkompetenz, (2) bewusste Inkompetenz, (3) bewusste Kompetenz sowie (4) unbewusste Kompetenz. Über das Durchlaufen der vier Stufen erlangt die Lehrperson durch die Aneignung von Fähigkeiten und Fertigkeiten ein fundiertes prozedurales Wissen, das sie handlungsleitend im Unterricht einsetzen kann. 
198). Kunter et al. (2011), Voss et al. (2011) und Loughran (2019: 3) gehen davon aus, dass sich professionelles Wissen im Laufe der Berufsbiografie fortlaufend aufbaut und sich kontinuierlich weiterentwickelt. Die lebenslange professionelle Entwicklung wird im Dokument der EU (2013: 9) als Schlüsselfaktor einer Lehrperson beschrieben: „Good practice increasily views teachers as lifelong learners.“ (European Commission 2013: 33) Die Aneignung pädagogischen Wissens von Lehrpersonen kann in drei Phasen untergliedert werden (vgl. Abbildung 14).

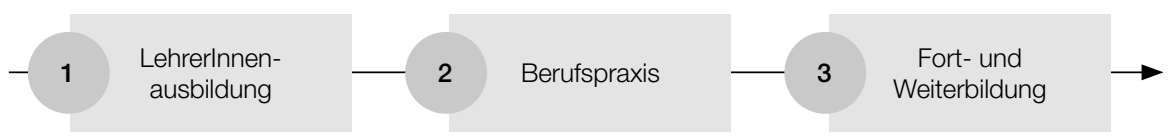

Abb. 14: Stadien der Aneignung professionellen Wissens von Lehrpersonen

Die erste Phase bezieht sich auf die Aneignung professionellen Wissens über die LehrerInnenausbildung. Darauf folgten als die Phasen zwei und drei die berufliche Praxis einer Lehrperson sowie Fort- und Weiterbildungen (vgl. Voss et al. 2015). Herrmann \& Hertramph (2000: 176) betonen die Notwendigkeit der Aneignung professionellen Wissens über die gesamte Berufslaufbahn hinweg. Die berufliche Entwicklung von Lehrpersonen basiert für die Aneignung zeitgerechten Wissens sowie für einen kontinuierlichen Zuwachs von Kenntnissen, Fähigkeiten und Kompetenzen auf einem Prozess des lebenslangen Lernens (vgl. Terhart 1990: 247; Hof \& Rosenberg 2018: 2).

In der Ausbildung werden grundlegende theoretische, bildungswissenschaftliche, pädagogische sowie psychologische Wissenskonzepte erworben. Zentral ist, hinsichtlich der zuvor beschrieben Wissensformen und -qualitäten (vgl. Abschnitt 3.2) der Aufbau des deklarativen sowie des expliziten Wissens oder, wie Mittelstraß argumentiert, des Verfügungswissens mit seinen Formen der Strukturierung, Ritualisierung und Stufen der Ausdifferenzierung. Die während der Ausbildung vermittelten theoretischen Wissenselemente betreffen, zu den spezifischen fachlichen und fachdidaktischen Inhalten einzelner Unterrichtsfächer, allgemeine pädagogische Inhalte wie Lehren, Lernprozesse, Klassenführung, Umgang mit Konflikten, Kommunikation sowie die Anwendung von Methoden, die gängige Themen im Curriculum darstellen (vgl. Hohenstein et al. 2014). Einen besonderen Stellenwert bekommen die in der Ausbildung zu absolvierenden schulpraktischen Ausbildungsanteile. Praktische Erfahrungen helfen dabei, gelernte theoretische Konzepte anzuwenden, und unterstützen die Bildung komplexer Schemata prozeduralen Wissens (vgl. Kothagen \& Kessels 1999; Wang et al. 2011). König et al. (2014a) sowie Voss et al. (2013) vertreten die These, dass schulpraktische Ausbildungsanteile die Weiterentwicklung professioneller pädagogischer Wissensformen fördern. Die Möglichkeit der Theorie-Praxis-Verknüpfung sowie angeleitete Reflexionsprozesse während der Ausbildung unterstützen die Ausbildung kognitiver Fertigkeiten der angehenden Lehrpersonen. ${ }^{21}$ Zudem soll die Ausbildung dabei helfen eigene subjektive Theorien und Bilder kritisch zu hinterfragen und neue Erfahrungsräume für die Weiterentwicklung kognitiver Muster schaffen. Die Kombination aus theoretischen fachlichen und pädagogischen Inhalten sowie ihre Anwendung während der Ausbildung bedingen einen ersten umfangreichen professionellen Wissensaufbau von Lehrpersonen.

21 Die Reflexionsfähigkeit wird im Zusammenhang mit der eigenen Professionalisierung als eine Kernfähigkeit von Lehrpersonen beschrieben. Sie unterstützt den Wissensaufbau und fördert die Veränderung individueller Haltungen und subjektiver Theorien. Der Diskurs und die Bedeutung der Reflexion im Kontext des Professionswissens wird im Abschnitt 5.2 eröffnet. 
Die Berufspraxis wird als weitere Phase für den Erwerb von Professionswissen beschrieben. Lehrpersonen stehen vor der Herausforderung, während der beruflichen Praxis ihr professionelles Wissen weiterzuentwickeln (vgl. Guerriero 2017). Im Dokument der EU (2013: 34) wird die Relevanz der Berufspraxis für die Wissensaneignung hervorgehoben:

„[They] need actively to maintain and further develop their professional competences throughout their careers, in an ongoing professional development process which at the same time supports the individual engagement and professional profiling of teachers."

König et al. (2014a) sowie Schneider \& Plasman (2011) setzen diese Argumentation fort, indem sie auf die Notwendigkeit eines flexiblen Wissens für die professionelle Entwicklung hinweisen. Die berufliche Praxis ist für den Wissenserwerb relevant, da über individuelle Erfahrungen prozedurale Wissensstrukturen aufgebaut werden, die sich wiederum in implizites Wissen transformieren lassen. ${ }^{22}$ Das in der Ausbildung erworbene theoretische Wissen wird in der beruflichen Praxis vertieft. Erfahrungen und soziale Beziehungen zur Umwelt sind zentrale Momente für den Wissenserwerb (vgl. Kapitel 2.4/2.6). Zudem bauen sie über die Berufspraxis eine Beziehung zu ihrem Umfeld (KollegInnen, SchülerInnen und SchulleiterInnen) auf und lernen situationsspezifisches Wissen anzuwenden. Als weiterer unterstützender Faktor für den Wissenserwerb wird von Dunn \& Shriner (1999) das Einholen von Feedback von SchülerInnen, Eltern sowie KollegInnen genannt. Persönliche Entwicklungsaufgaben befördern den Aneignungsprozess professionellen Wissens. Lehrpersonen setzen sich hierfür persönliche Ziele, die den Professionalisierungsprozess beeinflussen (vgl. Buchwald \& Hobfoll 2004).

Eng verknüpft mit der Berufspraxis sind Fort- und Weiterbildungen. Lipowsky (2014: 512) benennt die Fort- und Weiterbildungen als wichtigen Aneignungsort für die Entwicklung professionellen Wissens von Lehrpersonen. Er appelliert an eine kontinuierliche und selbstständige Weiterentwicklung, um mit fortschreitenden gesellschaftlichen Transformationsprozessen professionell und angemessen umgehen zu können. Fort-und Weiterbildungen unterstützen grundlegende theoretische pädagogische Inhalte aufzubauen und zu erweitern. ${ }^{23}$

AutorInnen wie Fuller \& Braun (1975), Bromme (1992) oder Reh \& Schelle (2006) haben sich mit dem Verlauf von Berufsbiografien von Lehrpersonen auseinandergesetzt. Das Modell von Fuller \& Braun (1975) erklärt die professionelle Entwicklung und den damit verbundenen Wissenszuwachs unter Berücksichtigung persönlicher Herausforderungen einer Lehrperson anhand von drei verschiedenen biografischen Stadien. In der ersten Stufe („survival stage") sind Lehrpersonen mit persönlichen Schwierigkeiten für die professionelle Bewältigung des Schulalltags konfrontiert und sind nicht in der Lage, professionell zu handeln. Anschließend folgt die „mastery stage“. Mit dem Erreichen einer beruflichen Stabilisierung können Lehrpersonen persönliche Herausforderungen in den Hintergrund rücken und situationsspezifisch im Unterricht agieren. Die letzte Stufe („routine stage“) zeichnet sich durch professionelles Handeln aus. SchülerInnen und deren individuelle Interessen und Bedürfnisse werden in den Mittelpunkt gerückt, mit dem Ziel, einen bestmöglichsten Lernprozess zu garantieren (vgl. Fuller \& Brown 1975).

22 Die zentrale Bedeutung des prozeduralen sowie des impliziten Wissens für ein professionelles Handeln von Lehrpersonen wird in Kapitel 3 diskutiert.

23 Statistiken der OECD verdeutlichen, wie gering die Bereitschaft von Lehrpersonen zu Fortbildungen ist. Der Besuch von Fortbildungen findet vorwiegend aus Eigeninteresse und von individueller Motivation von Lehrpersonen statt. Hinzu kommt, dass die Forschung zu langfristigen Auswirkungen von Fortbildungen auf die Qualität von Unterricht unzureichend ist (vgl. Herzmann \& König 2016: 167). 
Fuller \& Brown legen im Vergleich zu Bromme ihren Schwerpunkt auf subjektive sowie persönliche Eigenschaften als mitbestimmende Faktoren eines berufsbezogenen Aneignungsprozesses professionellen Wissens. Bromme (1992) unterscheidet in seinem Expertenansatz über einen berufsbiografischen Durchlauf fünf verschiedene Stadien, vom Novizen bis hin zum Experten/ zur Expertin. Lehramtsstudierende am Beginn ihrer Ausbildung werden anhand dieses Modells als Novizen bezeichnet. Novizen sind angehende Lehrpersonen, die über kein systematisches professionelles Wissen verfügen und auf keine Erfahrungswissen zurückgreifen können. Das zweite Stadium definiert Bromme als den „fortgeschrittenen Anfänger“. Zum ersten Mal findet die Aneignung praktischen Wissens über Schulerfahrungen und reflexive Prozesse statt. Die dritte Phase „Stadium des kompetenten Praktikers“ manifestiert sich über flexible Handlungsstrukturen und ermöglicht, Standardsituationen professionell zu meistern. Aufbauend auf diese Phase ist eine Lehrperson mit dem „Stadium des gewandten Praktikers“ fähig, Wissen intuitiv situationsgerecht einzusetzen und zu vernetzen. Die letzte Phase, das „Meister- oder ExpertInnenstadium“, beschreibt Lehrpersonen als routinierte professionelle ExpertInnen, die angemessen, schnell und vielfältig reagieren und handeln (vgl. König 2014a: 616; Bromme 1922: 90ff.). Neuere Studien der LehrerInnenbiografieforschung (vgl. Reh \& Schelle 2000/2006; Hericks 2006) beschreiben den Professionalisierungsprozess nicht nur über die Biografie, sondern legen den Fokus verstärkt auf den Einfluss von Persönlichkeitsmerkmalen, Fertigkeiten und subjektiven Erfahrungen und Theorien der jeweiligen Lehrpersonen. Hericks (2006) zufolge korreliert der Aneignungsprozess des Professionswissen von Lehrpersonen mit deren Reflexionsfähigkeit (vgl. Kapitel 5), dem kooperativen Handeln mit KollegInnen sowie der Nutzung institutioneller Strukturen als Ressource für persönliche, berufsspezifische Ziele. Den Einfluss persönlicher Eigenschaften und Erfahrungen auf den Wissenserwerb betont bereits Schön (1983) in seinem Zugang zur Reflexion. Er geht davon aus, dass Wissen immer im Subjekt (hier: die Lehrperson) steckt und über reflexive Prozesse bewusstgemacht werden kann. ${ }^{24}$ Die Reflexionsfähigkeit von Lehrpersonen nimmt im Prozess der Entwicklung und Weiterentwickelung professionellen Wissens eine wesentliche Stellung ein. Wyss (2008: 9) beschreibt in Anlehnung an Schön (1983) die Lehrpersonen als „reflective practitioner“. Der Wissenserwerb von Lehrpersonen basiert Wyss (2008: 9) zufolge auf der Reflexion gemachter unterrichtlicher Erfahrungen. Messner \& Reusser (2000) verbinden den biografischen Ansatz mit der Reflexionskompetenz und mit Persönlichkeitsmerkmalen, indem sie den biografischen Aneignungsprozess als eine Selbstkonstruktion charakterisieren. Die Reflexionsfähigkeit unterstützt nicht nur den Wissenserwerb. Sie gilt gleichzeitig als Medium für den Übergang vom Handeln zum Wissen (vgl. Kapitel 5). Resümierend kann in Anlehnung an die angeführte Diskussion die Aneignung professionellen Wissens über unterschiedliche biografische Stadien, kooperatives Handeln, subjektive Theorien sowie Persönlichkeitsmerkmale und die Reflexionskompetenz von Lehrpersonen beschrieben werden.

24 Das Modell von Schön (1983) wird in Abschnitt 5.2 im Zusammenhang mit dem Transfer von explizitem zu implizitem Wissen diskutiert. 


\section{Pädagogisches Wissen}

Neben den fachlichen Anforderungen stehen Lehrpersonen täglich vor vielen Herausforderungen, auf die sie angemessen reagieren müssen. Pädagogisches Wissen von Lehrpersonen wird im Kontext der angeführten Transformationsprozesse als zentraler Wissensbereich professionellen Wissens (vgl. Shulman 1986/1987; Bromme 1992; Terhart 1993) sowie als grundlegende professionelle Kompetenz (vgl. Baumert \& Kunter 2006; Kunter et al. 2011; European Commission 2013) von Lehrpersonen beschrieben. Es wird positiv mit der Unterrichtsqualität sowie, als Folge, mit dem Lernerfolg von SchülerInnen in Zusammenhang gebracht (vgl. Baumert et al. 2010; Lenske et al. 2015: 225; Guerriero 2017). Das derzeitige Verständnis pädagogischen Wissens charakterisiert sich über unterschiedliche Zugänge. Im weiteren Verlauf des Kapitels werden Definitionen, Konzeptualisierungen sowie Operationalisierungen pädagogischen Wissens aufgezeigt und diskutiert.

\subsection{Definitionen und Konzeptualisierungen}

Die aktuellen Forschungsbefunde präsentieren ein differenziertes Verständnis pädagogischen Wissens (vgl. Kunter et al. 2011; Voss et al. 2011; König et al. 2011; Baumert \& Kunter 2006/2011). Ein international einheitliches Verständnis stellt nach Blömeke \& Paine (2008: 2027ff.) sowie Voss et al. (2015: 188) bislang eine wesentliche Herausforderung dar. Die Verwendung unterschiedlicher Zugänge spiegelt sich in den diversen angeführten Begrifflichkeiten wider. In der Literatur simultan verwendete Bezeichnungen sind etwa „pädagogisches (Professions-)Wissen“ (z.B. König \& Blömeke 2009), „pädagogisches Unterrichtswissen“ (z.B. König \& Seifert 2012), „pädagogische Kompetenz“ (z.B. Seifert et al. 2009), „erziehungswissenschaftliches Wissen“ (z.B. Blömeke et al. 2008), „adaptive Lehrerkompetenz“ (z.B. Beck et al. 2008), „bildungswissenschaftliches Wissen“ (z.B. Terhart et al. 2012), „professional vision“ (z.B. Seidel \& Stürmer 2014) und „pädagogisch-psychologisches Wissen“ (z.B. Voss \& Kunter 2011). Shulman beschreibt pädagogisches Wissen als „broad principles and strategies of ClassroomManagement and organisation that appear to transcend subject matter, as well as knowledge about learners and learning, assessment, and educational context and purpose" (Shulman 1987: 8). In Anlehnung an Shulmans Verständnis stellen zahlreiche Autoren weitere Definitionen auf (vgl. Wilson et al. 1987; Grossman \& Richert 1988; Fennema \& Franke 1992; Borko \& Putnam 1996; Morine-Deshimer \& Kent 1999; Bromme 2001; Baumert \& Kunter 2006/2011; Voss et al. 2011; König et al. 2011; Kunter et al. 2011). In Tabelle 1 werden die gängigsten Konzeptualisierungen pädagogischen Wissens aus der Fachliteratur dargestellt.

Tab. 1: Beispiele für Konzeptualisierungen pädagogischen Wissen (in Anlehnung an Voss et al. 2015: 192)

\begin{tabular}{ll}
\hline AutorInnen & Definition \\
\hline Wilson et al. & "knowledge of pedagogical principles and techniques that is not bound by topic or \\
$(1987): 114$ & subject matter“
\end{tabular}




\begin{tabular}{|c|c|}
\hline AutorInnen & Definition \\
\hline $\begin{array}{l}\text { Fennema } \\
\& \text { Franke } \\
(1992): 162\end{array}$ & $\begin{array}{l}\text { „teachers' knowledge of teaching procedures such as effective strategies for planning, class- } \\
\text { room routines, behaviour management techniques, classroom organizational procedures, and } \\
\text { motivational techniques“ }\end{array}$ \\
\hline $\begin{array}{l}\text { Borko \& } \\
\text { Putnam } \\
(1996): 675\end{array}$ & $\begin{array}{l}\text { „a teachers' knowledge and beliefs about teaching, learning, and learners that transcend } \\
\text { particular subject matter domain [...] knowledge of various strategies and arrangements for } \\
\text { effective Classroom-Management, instructional strategies for conducting lessons and creating } \\
\text { learning environments, and more fundamental knowledge and beliefs, how they learn, and } \\
\text { how that learning can be fostered by teaching“ }\end{array}$ \\
\hline $\begin{array}{l}\text { Morine- } \\
\text { Deshimer \& } \\
\text { Kent (1999) }\end{array}$ & $\begin{array}{l}\text { 1. Instructional model (teaching method) } \\
\text { 2. Classroom management } \\
\text { 3. Classroom communication }\end{array}$ \\
\hline $\begin{array}{l}\text { Bromme } \\
(2001): \\
15463\end{array}$ & $\begin{array}{l}\text { „[...] area of knowledge that is important for optimizing teaching-learning situations but is } \\
\text { relatively independent from the individual subject. It includes, for example, knowledge on } \\
\text { how to generate and maintain the necessary patterns of interaction and working style for the } \\
\text { planned course of instruction, or knowledge about how to deal with difficult situations and } \\
\text { problem cases“ }\end{array}$ \\
\hline $\begin{array}{l}\text { Baumert } \\
\text { \& Kunter } \\
(2006 / 2011): \\
16 / 39\end{array}$ & $\begin{array}{l}\text { 1. Konzeptuelles bildungswissenschaftliches Grundlagenwissen } \\
\text { 2. Allgemeindidaktisches Konzeptions- und Planungswissen } \\
\text { 3. Unterrichtsführung und Orchestrierung von Lerngelegenheiten } \\
\text { 4. Fachübergreifende Prinzipien des Diagnostizierens, Prüfens und Bewertens } \\
\text { 5. Methodische Grundlagen der empirischen Sozialforschung }\end{array}$ \\
\hline $\begin{array}{l}\text { Voss et al. } \\
(2011) \text {; } \\
\text { König et al. } \\
\text { (2011) }\end{array}$ & $\begin{array}{l}\text { "General pedagogical knowledge consists of pedagogical and psychological components: } \\
\text { 1. Knowledge of classroom management: maximising the quantity of instructional time, } \\
\text { handling classroom events, teaching at a steady place, maintain clear direction in lesson } \\
\text { 2. Knowledge of teaching methods: having a command of various teaching methods, know- } \\
\text { ledge when and how to apply each method } \\
\text { 3. Knowledge of classroom assessment: knowledge of different forms and purposes of forma- } \\
\text { tive and summative assessments, knowledge of how different frames of reference impact } \\
\text { students' motivation } \\
\text { 4. Structure: structuring of a learning objectives and the lesson process, lesson planning and } \\
\text { evaluation. } \\
\text { 5. Adaptivity: dealing with heterogeneous learning groups in the classroom. } \\
\text { 6. Knowledge of learning process: supporting and fostering individual learning progress by } \\
\text { having knowledge of various cognitive and motivational learning processes } \\
\text { 7. Knowledge of individual student characteristics: having knowledge of the sources of stu- } \\
\text { dent cognitive, motivational, and emotional heterogeneity" }\end{array}$ \\
\hline
\end{tabular}

Kunter et al. „das für eine erfolgreiche Gestaltung und Optimierung der Lehr-Lern-Situation in verschie(2011): 194 denen Unterrichtsfächern nötig ist “

Voss et al. 1. Lernen und Lernende:

(2011/2015); - Lernprozesse (Lern-, motivations und emotionspsychologisches Wissen)

Kunter et al. - Unterschiede in den Voraussetzungen der Lernenden (Heterogenität)

(2011/2013) - Altersstufen und Lernbiografien (entwicklungspsychologisches Wissen)

2. Umgang mit der Klasse als komplexes soziales Gefüge:

- Klassenführung/Strukturierung der Klassenprozesse

- Interaktion/Kommunikation und soziale Konflikte

3. Methodisches Repertoire:

- Lehr-Lern-Methoden und Konzepte und deren lernzieladäquate Orchestrierung

- Generelle Prinzipien der Individual- und Lernprozessdiagnostik und Evaluation

4. Gestaltung von Lernumgebungen (räumliche, materiale und mediale Gestaltung) 
Die in der Tabelle angeführten Ansätze stimmen inhaltlich insofern überein, als dass pädagogisches Wissen von allen AutorInnen als fachunabhängiges bzw. generisches Wissen (vgl. Voss et al. 2015: 192) beschrieben wird. Die definitorische Vielseitigkeit repräsentiert sich über individuelle Binnenstrukturen und Schwerpunkte jedes konzeptionellen Ansatzes. Einigkeit herrscht in den verschiedenen Zugängen darüber, dass Klassenführung eine wesentliche planerische Facette pädagogischen Wissens darstellt (vgl. Voss et al. 2015: 191f.). Die jeweiligen AutorInnen präsentieren in Anlehnung an Tabelle 1 in ihren Definitionen unterschiedliche inhaltliche Wissensformen, die im Folgenden in planerische, interaktive (vgl. Voss et al. 2015: 192) sowie theoretische Wissensformen untergliedert werden können (vgl. Tabelle 2).

Tab. 2: Thematische Clusterung der Definitionen pädagogischen Wissens

\begin{tabular}{lll}
\hline Planerische Wissensbereiche & Interaktive Wissensbereiche & Theoretische Wissensbereiche \\
\hline - Classroom-Management & - Interaktion während des & - Lernen \\
- Unterrichtsplanung & Unterrichts & - Philosophie \\
& - Anwendung von Methoden & - Lehren \\
& - Wissen über interaktives & - Wissen über die Lernenden \\
& Lernen & - Umgang mit Schwierigkeiten \\
& - Gestaltung individueller & und Problemen \\
& Lernangebote & - Bildungswissenschaftliche \\
& - Ummunikation im Unterricht & Grundlagen \\
& - Umgang mit Schwierigkeiten & - Allgemeindidaktisches Konzep- \\
& Unterricht & - Methodische Grundlagen \\
& & - Diagnostizieren und Bewerten \\
\hline
\end{tabular}

Zusätzlich zu den planerischen Wissensbereichen, Classroom-Management und Unterrichtsplanung nennen die AutorInnen interaktive und theoretische Wissensformen. Theoretische Wissensformen inkludieren das Wissen über das Lernen, die Philosophie, das Lehren, die Lernenden sowie den Umgang mit Schwierigkeiten und Problemen (vgl. Grossmann \& Richert 1988: 54; Borko \& Putnam 1996: 675; Bromme 2011: 15463). Baumert \& Kunter (2006: 16; 2011: 39), Voss et al. (2011) sowie König et al. (2011) erwähnen weitere notwendige theoretische Wissensbereiche, wie das Wissen über bildungswissenschaftlichen Grundlagen, allgemeindidaktisches Konzeptions- und Planungswissen, Wissen über Diagnostizieren und Bewerten sowie das Wissen zu methodischen Grundlagen.

Interaktive Subfacetten, die in den Definitionen mehrfach erwähnt werden, beziehen sich auf die Anwendung von Methoden, das Aufrechterhalten der Interaktion während des Unterrichts sowie auf Wissen zur Motivation (vgl. Fennema \& Franke 1992: 162; Morine-Deshimer \& Kent 1999; Voss et al. 201/2015; König et al. 2011; Kunter et al. 2011/2013). Vereinzelt verweisen AutorInnen (vgl. Borko \& Putnam 1996; Voss et al. 2011/2015; König et al. 201; Kunter et al. 2011/2013) auf das Wissen zu interaktivem Lernen sowie die Gestaltung individueller Lernangebote. Voss et al. (2011/2015), König et al. (2011), Bromme (2001), Morine-Deshimer \& Kent (1999) sowie Kunter et al. (2011/2013) führen ergänzend Wissensfacetten zu den Themenbereichen „Kommunikation“, „Umgang mit Schwierigkeiten“ sowie „Umgang mit Heterogenität" an.

Im Kontext der Kompetenzorientierung stellen Voss et al. (2011/2015) und Kunter et al. (2011/2013) eine erweiterte Definition pädagogischen Wissens auf. Sie appellieren, deklara- 
tive, konzeptuelle sowie prozedurale Wissensformen in ihrer Konzeptualisierung zu berücksichtigen. Voss et al. (2011) und Kunter et al. (2011) unterscheiden in ihrer Definition zwischen pädagogischem und psychologischem Wissen. Dieses bezieht sich auf das Wissen über Lernprozesse sowie das Wissen über individuelle SchülerInnenmerkmale. Die Wissensbereiche „Organisation“, „Struktur“, „Umgang mit Heterogenität“, „Bewerten“ und „Methoden“ ordnen sie dem pädagogischen Wissen unter. Diese Konzeptualisierung stellt gegenwärtig ein gängiges Verständnis pädagogischen Wissens dar.

Auf nationaler sowie internationaler Ebene gibt es keine einheitliche Definition pädagogischen Wissens. Guerriero (2015) bemerkt die Abhängigkeit pädagogischen Wissens von zugrundeliegenden kulturellen Normen und Aspekten verschiedener Länder. König et al. (2011) argumentieren in diesem Zusammenhang, dass das Wissen über die Unterrichtsdurchführung im Vergleich zu anderen Wissenskategorien wie die „Erziehung“ als kulturunabhängiger Wissensbereich klassifiziert werden kann. Es muss jedoch allgemein in Frage gestellt werden, inwieweit die bestehenden Konzepte, die sich alle vorwiegend auf vorgegebene Standards der KMK (2004) stützen, international über den europäischen Raum hinaus tragfähig sind und inwieweit eurozentristische Modelle international Anwendung finden können.

\subsection{Operationalisierungen}

Im Zuge der Kompetenzorientierung wurden im Rahmen unterschiedlicher Studien Testverfahren zur Erfassung pädagogischen Wissens entwickelt (vgl. Kapitel 1.2 und 2.6). Diese beziehen sich auf Instrumente für die Erfassung pädagogischer Wissensanteile von Lehramtstudierenden sowie Lehrpersonen, die vorwiegend im Unterrichtsfach Mathematik tätig sind (vgl. Voss et al. 2015: 196). Die Studien stützen sich bei den Operationalisierungen vorwiegend auf PapierBleistift-Tests (vgl. König et al. 2014: 76). Vereinzelt wurden in den letzten Jahren Studien für die Erfassung pädagogischen Wissens mithilfe von Videovignetten entwickelt (vgl. Brühwiler et al. 2017; Lenske et al. 2017).

Oelkers (1989) sowie Tenorth (1989) betonen, dass sich pädagogisches Wissen nur über verschiedene Bereiche und Funktionen differenziert beschreiben lässt. Diese Annahme wird in den Testverfahren bestätigt. Alle Studien operationalisieren das Konstrukt mehrdimensional. In Tabelle 3 werden Studien, die pädagogisches Wissen operationalisieren und messbar machen sollen, aufbauend auf unterschiedlichen definitorischen Zugängen und Testinstrumenten vorgestellt (vgl. Hohenstein et al. 2015: 18; Voss et al. 2015: 196f.)

Tab. 3: Übersicht der Studien zu pädagogischem Wissen (in Anlehnung an Voss et al. 2015: 196f.)

\begin{tabular}{|c|c|c|c|c|}
\hline Referenz & Testinstrument & Subskalen & Zielgruppe & $\begin{array}{l}\text { Test- und } \\
\text { Itemformate }\end{array}$ \\
\hline $\begin{array}{l}\text { z.B. König } \\
\text { \& Blömeke } \\
(2010)\end{array}$ & $\begin{array}{l}\text { TEDS-M: Teacher } \\
\text { Education and De- } \\
\text { velopment Study in } \\
\text { Mathematics }\end{array}$ & $\begin{array}{l}\text { 1. Strukturierung von Unter- } \\
\text { richt } \\
\text { 2. Motivierung } \\
\text { 3. Umgang mit Heterogeni- } \\
\text { tät } \\
\text { 4. Klassenführung } \\
\text { 5. Leistungsbeurteilung }\end{array}$ & $\begin{array}{l}\text { Lehramts- } \\
\text { studierende }\end{array}$ & $\begin{array}{l}\text { Papier-Bleistift- } \\
\text { Test; offene und } \\
\text { geschlossene } \\
\text { Testformate }\end{array}$ \\
\hline
\end{tabular}




\begin{tabular}{|c|c|c|c|c|}
\hline Referenz & Testinstrument & Subskalen & Zielgruppe & $\begin{array}{l}\text { Test- und } \\
\text { Itemformate }\end{array}$ \\
\hline $\begin{array}{l}\text { z.B. Voss et } \\
\text { al. }(2011)\end{array}$ & $\begin{array}{l}\text { COACTIV-Instru- } \\
\text { ment zur Erfassung } \\
\text { des pädagogisch-psy- } \\
\text { chologischen Wissens, } \\
\text { entwickelt im Rah- } \\
\text { men des COACTIV- } \\
\text { Referendariatsprojekt }\end{array}$ & $\begin{array}{l}\text { 1. Klassenführung } \\
\text { 2. Lernprozesse } \\
\text { 3. Heterogenität } \\
\text { 4. Unterrichtsmethoden } \\
\text { 5. Leistungsbeurteilung }\end{array}$ & $\begin{array}{l}\text { Referen- } \\
\text { darInnen }\end{array}$ & $\begin{array}{l}\text { Papier-Bleistift- } \\
\text { Test; geschlossene, } \\
\text { offene und videoba- } \\
\text { sierte Items }\end{array}$ \\
\hline $\begin{array}{l}\text { z.B. Blö- } \\
\text { meke et al. } \\
(2008)\end{array}$ & $\begin{array}{l}\text { MT21: Mathematics } \\
\text { Teaching in the 21st } \\
\text { Century }\end{array}$ & $\begin{array}{l}\text { 1. Unterrichtsplanung } \\
\text { 2. Leistungsbeurteilung } \\
\text { 3. Umgang mit sozialen } \\
\text { Ungleichheiten }\end{array}$ & $\begin{array}{l}\text { Lehr- } \\
\text { personen }\end{array}$ & $\begin{array}{l}\text { Paper-Bleistift-Test; } \\
\text { offene Testformate }\end{array}$ \\
\hline $\begin{array}{l}\text { z.B. } \\
\text { Kunina-Ha- } \\
\text { benicht et } \\
\text { al. (2012); } \\
\text { Kunter et } \\
\text { al. (2011) }\end{array}$ & $\begin{array}{l}\text { BilWiss-Instrument } \\
\text { zur Erfassung des } \\
\text { bildungswissenschaft- } \\
\text { lichen Wissens von } \\
\text { Referendar(in), entwi- } \\
\text { ckelt im Rahmen der } \\
\text { BilWiss-Studie } \\
\end{array}$ & $\begin{array}{l}\text { 1. Unterrichtsdidaktik } \\
\text { 2. Schulpädagogik } \\
\text { 3. Bildungstheorie } \\
\text { 4. Lernen/Entwicklung } \\
\text { 5. Diagnostik/Evaluation } \\
\text { 6. Lehrerberuf }\end{array}$ & $\begin{array}{l}\text { Referen- } \\
\text { darInnen }\end{array}$ & $\begin{array}{l}\text { Papier-Bleistift-Test; } \\
\text { Reproduktions-, } \\
\text { Verknüpfungs- und } \\
\text { Anwendungsaufga- } \\
\text { ben (v.a. geschlossen) }\end{array}$ \\
\hline $\begin{array}{l}\text { z.B. Kleick- } \\
\text { mann et al. } \\
(2014)\end{array}$ & $\begin{array}{l}\text { KiL-Instrument } \\
\text { zur Erfassung des } \\
\text { bildungswissenschaft- } \\
\text { lichen Wissen von } \\
\text { Lehramtsstudierende, } \\
\text { entwickelt im Rah- } \\
\text { men des KiL-Projekts }\end{array}$ & $\begin{array}{l}\text { 1. Lehren } \\
\text { 2. Lernen und Entwicklung } \\
\text { 3. Klassenführung } \\
\text { 4. Leistungsbeurteilung } \\
\text { 5. Bildungssystem und Schul- } \\
\text { organisation } \\
\text { 6. Methoden und Ergebnisse } \\
\text { bildungswissenschaftlicher } \\
\text { Forschung } \\
\text { 7. Weiterentwicklung/ } \\
\text { Innovation }\end{array}$ & $\begin{array}{l}\text { Lehramts- } \\
\text { studierende }\end{array}$ & $\begin{array}{l}\text { Papier-Bleistift- } \\
\text { Test; offene und } \\
\text { geschlossene } \\
\text { Formate }\end{array}$ \\
\hline $\begin{array}{l}\text { z.B. Lenske } \\
\text { et al. (2015) }\end{array}$ & $\begin{array}{l}\text { ProwiN-Instrument } \\
\text { zur Erfassung des } \\
\text { Professionswissens von } \\
\text { Lehrkräften in den } \\
\text { Naturwissenschaften, } \\
\text { entwickelt im Rahmen } \\
\text { des Pro-Win-Projekts } \\
\end{array}$ & $\begin{array}{l}\text { 1. Klassenführung } \\
\text { 2. Unterrichtsmethoden } \\
\text { 3. Individuelle Lernprozesse } \\
\text { 4. Leistungsbeurteilung }\end{array}$ & Lehrkräfte & $\begin{array}{l}\text { Papier-Bleistift- } \\
\text { Test, komplexe } \\
\text { multiple-choice } \\
\text { Aufgaben, über } \\
\text { Quasi-Paraverglei- } \\
\text { che ausgewertete }\end{array}$ \\
\hline $\begin{array}{l}\text { z.B. Seifert } \\
\text { et al. (2009) }\end{array}$ & $\begin{array}{l}\text { SPEE-Instrument zur } \\
\text { Erfassung pädagogi- } \\
\text { scher Kompetenzen } \\
\text { von Lehramtsstudie- } \\
\text { rende }\end{array}$ & $\begin{array}{l}\text { Inhaltsdimensionen: } \\
\text { 1. Erziehung und Bildung } \\
\text { 2. Unterricht und allgemeine } \\
\text { Didaktik } \\
\text { 3. Schulentwicklung und } \\
\text { Gesellschaft } \\
\text { Kognitive Anforderungen: } \\
\text { 4. Wissen reproduzieren und } \\
\text { verstehen } \\
\text { 4. Reflektieren, anwenden } \\
\text { und urteilen } \\
\text { 5. Bewerten und entscheiden }\end{array}$ & $\begin{array}{l}\text { Lehramts- } \\
\text { studierende }\end{array}$ & Papier-Bleistift-Test \\
\hline
\end{tabular}




\begin{tabular}{|c|c|c|c|c|}
\hline Referenz & Testinstrument & Subskalen & Zielgruppe & $\begin{array}{l}\text { Test- und } \\
\text { Itemformate }\end{array}$ \\
\hline $\begin{array}{l}\text { z.B. König } \\
\text { und Seifert } \\
(2012)\end{array}$ & $\begin{array}{l}\text { LEK - Längsschnittli- } \\
\text { che Erhebung pädago- } \\
\text { gischer Kompetenzen } \\
\text { von Lehramtsstudie- } \\
\text { renden }\end{array}$ & $\begin{array}{l}\text { 1. Bildungswissenschaftliche } \\
\text { Wissen: } \\
\text { - Erziehung und Bildung } \\
\text { - Unterricht und } \\
\text { Allgemeine Didaktik } \\
\text { - Schulentwicklung und } \\
\text { Gesellschaft } \\
\text { 2. Pädagogischen Unter- } \\
\text { richtwissen: } \\
\text { - Allgemein-didaktisches } \\
\text { Wissen } \\
\text { - Pädagogisch-psycholo- } \\
\text { gisches Wissen }\end{array}$ & $\begin{array}{l}\text { Lehramts- } \\
\text { studierende }\end{array}$ & Papier-Bleistift-Test \\
\hline $\begin{array}{l}\text { z.B. } \\
\text { Guerriero } \\
\text { (2017); } \\
\text { Sonmark et } \\
\text { al. (2017) }\end{array}$ & $\begin{array}{l}\text { ITEL-TKS The } \\
\text { Innovative Teaching } \\
\text { for Effective Learning } \\
\text { Teacher Knowledge } \\
\text { Survey International }\end{array}$ & $\begin{array}{l}\text { 1. Lehren } \\
\text { 2. Lernen } \\
\text { 3. Leistungsbeurtei- } \\
\quad \text { lung }\end{array}$ & $\begin{array}{l}\text { Lehramts- } \\
\text { studierende, } \\
\text { Lehrperso- } \\
\text { nen, } \\
\text { Lehramts- } \\
\text { auszubil- } \\
\text { dende }\end{array}$ & Papier-Bleistift-Test \\
\hline
\end{tabular}

Die in der Tabelle aufgelisteten Untersuchungen werden im Folgenden präsentiert und diskutiert. Studien wie COACTIV, TEDS-M, BilWiss, MT21 und SPEE wurden über Folgestudien im Anschluss weiterentwickelt und hinsichtlich neuer Fragestellungen untersucht (vgl. Abbildung 15).

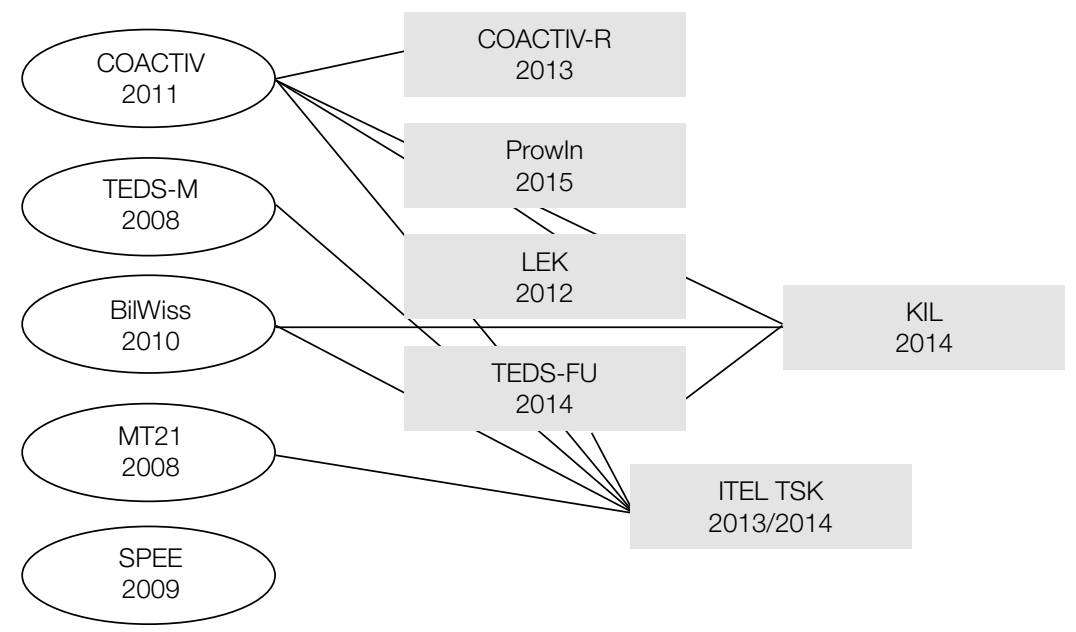

Abb. 15: Übersicht der Studien zur Untersuchung pädagogischen Wissens

Abbildung 15 illustriert die Hauptstudien mit ihren Folgestudien, die im weiteren Verlauf der Arbeit beschrieben werden. Zusammenhänge beziehen sich auf überlappende AutorInnen- 
schaften sowie die Verwendung ähnlicher Konstrukte und Verfahren für die Messung pädagogischen Wissens.

TEDS-M (Teacher Education and Development Study in Mathematics) aus dem Jahr 2008 war eine der ersten Studien, die pädagogisches Wissen im Rahmen einer internationalen vergleichenden Large-scale-Studie maßen (vgl. Blömeke et al. 2009b). Das Hauptziel der Studie war ein internationaler Vergleich der LehrerInnenausbildung bei angehenden Mathematiklehrkräften für die Primar- sowie Sekundarstufe I in 16 Ländern (vgl. Blömeke 2004; Blömeke et al. 2009b; Townsend \& Bates 2007; Blömeke et al. 2010). Für die Messung pädagogischen Wissens wurden in einer Pilotstudie 800 angehende Mathematiklehrkräfte (bei der Testdurchführung befanden sich die TeilnehmerInnen am Ende ihrer Ausbildung) aus Deutschland, den USA und Taiwan getestet (vgl. König \& Blömeke 2010: 275ff.). Die Untersuchung pädagogischen Wissens fand über fünf Wissensbereiche statt: Strukturierung von Unterricht, Motivierung, Umgang mit Heterogenität, Klassenführung und Leistungsbeurteilung (vgl. Blömeke \& König 2010: 179). Diese wurden von Bromme (1997), KMK (2004), Baumert und Kunter (2006) sowie Tenorth (2006) anhand definierter Kernaufgaben von Lehrpersonen bestimmt. Als weitere Kriterien wurden die Befunde aus der Allgemeinen Didaktik (vgl. Heimann et al. 1965; Klafki 1985; Bönsch 2004; Tulodziecki et al. 2004) und der Unterrichtsforschung (vgl. Brophy \& Good 1986; Slavin 1994; Helmke 2003) herangezogen. Zur inhaltlichen Kategorisierung wurden die von Anderson und Krathwohl (2001) revidierten Kriterien „Wissen abrufen bzw. erinnern“, „,verstehen/analysieren“ und „Handlungsoptionen kreieren“ berücksichtigt. Die inhaltlichen sowie kognitiven Facetten bildeten die mehrdimensionale Grundlage für die Operationalisierung pädagogischen Wissens (vgl. König \& Blömeke 2010: 253ff.). Laut den Ergebnissen dieser Studie verfügen Lehrpersonen aus Deutschland und Taiwan über mehr pädagogisches Wissen als jene aus den USA. Blömeke und König (2010: 179) sehen die Ursache darin, dass die LehrerInnenausbildung in diesen Ländern umfangreichere Praxisphasen enthält. Die AutorInnen gehen davon aus, dass die Selbsteinschätzungskompetenzen der Lehrpersonen in den untersuchten Ländern voneinander abweichen.

Das Ziel der Längsschnittstudie COACTIV (Professionswissen von Lehrkräften, kognitiv aktivierender Mathematikunterricht und die Entwicklung von mathematischer Kompetenz) ${ }^{25}$ war die Spezifizierung und Erfassung des Professionswissens von Mathematiklehrkräften mit einem Schwerpunkt auf der Operationalisierung pädagogischen Wissens. Voss et al. (2011: 198f.) betonen, dass dieses Instrument das handlungsnahe pädagogische Wissen von Lehrpersonen misst und deklaratives sowie prozedurales Wissen berücksichtigt. Das Sample bestand aus 746 ReferendarInnen. Die inhaltlichen Dimensionen berücksichtigten vier unterschiedliche Subfacetten: das Wissen über Klassenführung, Lernprozesse, Heterogenität sowie das Wissen über Unterrichtsmethoden (vgl. Voss et al. 2011: 198f.). Im Vergleich zu TEDS-M berücksichtigte die Längsschnittstudie nicht nur allgemein-didaktische Inhalte, sondern schloss psychologische Aspekte des Lehrens und Lernens mit ein. Der Untersuchungsschwerpunkt lag auf einem Vergleich des pädagogischen Wissens von ReferendarInnen mit bzw. ohne LehrerInnenerfahrung. Ergebnisse verdeutlichen, dass angehende Lehrpersonen mit Praxiserfahrung ein umfangreicheres pädagogisches Wissen aufweisen als diejenigen ohne Praxiserfahrung. Bei Lehrpersonen mit Praxiserfahrung konnten spezifischere kognitive Fähigkeiten aufgezeigt werden (vgl. Voss \& Kunter 2011).

25 Die COACTIV-Studie steht in enger Beziehung zur internationalen Vergleichsstudie PISA 2003/2004. Die Durchführung der Testinstrumente fand zeitgleich mit der PISA-Testwelle 2003 und 2004 statt. 
Die Studie Mathematics Teaching in the 21st Century (MT21) der National Science Foundation (vgl. Blömeke et al. 2008) wurde 2008 durchgeführt. Im Rahmen einer Vergleichsstudie zur MathematiklehrerInnenausbildung wurde das pädagogische Wissen operationalisiert. Die Messungen fanden über offene, standardisierte Testverfahren mit 2.628 Lehrpersonen der Sekundarstufe I in Bulgarien, Deutschland, Mexiko, Südkorea, Taiwan und den USA statt (vgl. Blömeke et al. 2009a). Die inhaltlichen Kriterien wurden anhand der vom Kultusministerium (KMK 2004) definierten Aufgabenbereiche von Lehrpersonen definiert. Die untersuchten Dimensionen waren Unterrichtsplanung, Lernzielkontrolle sowie der Umgang mit sozialer Ungleichheit. Blömeke et al. (2008) weisen auf die Verwendung allgemeindidaktischer, pädagogisch-psychologischer sowie bildungssoziologischer Dimensionen. Die AutorInnen untersuchten für die deutsche Stichprobe, inwieweit die drei Wissensbereiche der KMK (2004) von den untersuchten Lehrpersonen realisiert werden. Die Ergebnisse der Studie zeigen, wie die geprüften Merkmale bei den Lehrpersonen vorliegen und in einem engen Zusammenhang zueinander stehen.

Die BilWiss-Studie (Bildungswissenschaftliches Wissen und der Erwerb professioneller Kompetenz in der Lehrerausbildung), durchgeführt im Jahr 2010, hatte die Erfassung bildungswissenschaftlichen Wissens von Lehramtsstudierenden und dessen Bedeutung für den Einstieg in die Berufspraxis in Nordrhein-Westfahlen zum Ziel (vgl. Terhart et al. 2012; vgl. Kunter et al. 2017). Das Testverfahren stützte sich auf standardisierte Papier-Bleistift-Tests. Die Konstrukte wurden anhand einer Delphi-Experten-Befragung von 36 Hochschullehrpersonen sowie 13 SeminarleiterInnen aus dem Referendariat entwickelt. Ziel der Delphi-Studie war es, relevante bildungswissenschaftliche Inhalte für den LehrerInnenberuf zu definieren (vgl. Kunina-Habenicht et al. 2012; Kunter et al. 2017). Die inhaltlichen Schwerpunkte wurden mittels einer Recherche in Lehrbüchern sowie mittels einer Dokumentenanalyse von Studienordnungen festgelegt (vgl. Kunter et al. 2017: 42). Im Rahmen der Delphi-Studie wurden die Lehrpersonen hinsichtlich der Relevanz bildungswissenschaftlicher Inhalte für den späteren LehrerInnenberuf befragt. Die in der Delphi-Studie entwickelten Konstrukte bildeten die Grundlage für das Testinstrument. Das pädagogische Wissen wurde anhand von sechs empirisch begründeten Dimensionen untersucht: Unterrichtsgestaltung, Schulorganisation, Bildungstheorie, Lernen und Entwicklung, Diagnostik und Evaluation, LehrerInnenberuf als Profession (vgl. Kunter et al. 2017: 42). In der Hauptuntersuchung wurden 3.118 LehramtsabsolventInnen in NordrheinWestfalen getestet. Eine Teilstichprobe von 362 Lehrpersonen wurde ein Jahr später mit dem Ziel untersucht, Aussagen über die langfristige Bedeutung des bildungswissenschaftlichen Wissens für das professionelle Handeln zu erlangen. Laut Testformat wurden bei der Durchführung deklarative sowie konzeptuelle Wissenszugänge, unterrichtsrelevante und über den Unterricht hinausgehende bildungsrelevante Themen abgefragt (vgl. Voss et al. 2011: 198f.). Die Ergebnisse verdeutlichen, dass die untersuchten Personen alle sechs definierten Konstrukte als wesentliche Wissensbereiche von Lehrpersonen klassifizieren und diese von ihnen als Erleichterung für den Eintritt in die Berufspraxis beschrieben werden. Die Studie verweist damit auf unterschiedliche erziehungswissenschaftliche, psychologische sowie soziologische Inhalte in der LehrerInnenausbildung für die Aneignung umfangreichen pädagogischen Wissens der Lehramtsstudierenden. Die Frage der Wirksamkeit bildungswissenschaftlichen Wissens konnte im Testverfahren nur unzureichend beantwortet werden (vgl. Kunter et al. 2017: 49ff.).

Die Studie SPEE (Standard-Profil-Evaluation) (vgl. Seifert et al. 2009) hatte die Entwicklung eines psychometrischen Messinstruments zur Erfassung pädagogischer Kompetenz zum Ziel (vgl. Seifert 2009: 98). Es nahmen 430 PhysikstudentInnen aller Semester aus der Lehramts- 
ausbildung teil. Die Itementwicklung wurde in Anlehnung an die Anforderungen der KMK (2004) sowie des Paderborner Curriculums realisiert (vgl. Seifert et al. 2009: 87f.). Untersucht wurden inhaltliche Wissensbereiche sowie kognitive Anforderungsbereiche der Lehrpersonen. Die Inhaltsdimensionen gliederten sich in die Wissensfacetten Erziehung und Bildung, Unterricht und allgemeine Didaktik sowie Schulentwicklung und Gesellschaft (vgl. Seifert et al. 2009: 87f.). Kognitive Anforderungen betrafen Aufgabenstellungen, in denen man Wissen reproduzieren, verstehen, reflektieren, anwenden, bewerten oder entscheiden musste (vgl. Seifert et al. 2009: 88). Die Ergebnisse zeigen, wie Lehrpersonen im Grundstudium besserer Leistungen als aus dem Hauptstudium liefern. Dieses Ergebnis wurde jedoch in der Studie nur unzureichend begründet (vgl. Seifert et al. 2009: 98).

Basierend auf den vorhandenen theoretischen Operationalisierungen der vorgestellten Studien wurden Folgestudien wie das KiL-Projekt, die LEK-Studie, das ProwiN-Projekt, ITEL, COACTIV-R sowie die Studie TEDS-FU entwickelt, die von den erstellten Konstrukten abgeleitet und individuell adaptiert wurden (vgl. Abbildung 15). Diese werden im Folgenden näher beschrieben.

Das KiL-Projekt (Messung professioneller Kompetenz in mathematischen und naturwissenschaftlichen Lehramtsstudiengängen) hatte die Entwicklung eines Tests für die valide Messung pädagogischen Wissens zum Ziel (vgl. Kleickmann et al. 2014). Die Testkonstrukte orientierten sich an den Standards für die LehrerInnenbildung der KMK (vgl. KMK 2004) sowie an weiteren Modellen pädagogischen Wissens der Studien COACTIV, TEDS-M und der Delphistudie BilWiss. Konstrukte bezogen sich auf die sieben Wissensfacetten Lehren, Lernen/ Entwicklung/Motivation, Klassenführung und Umgang mit Konflikten, Leistungsbeurteilung, Bildungssystem und Schulorganisation, Methoden bildungswissenschaftlicher Forschung sowie professionelle Weiterentwicklung und Schulentwicklung (vgl. Kleickmann et al. 2014). Die Konstrukte bezogen sich ähnlich wie bei der BilWiss-Studie auf direkte, unterrichtsnahe Kriterien sowie auf Entwicklungsmomente. Das Sample bestand aus ca. 820 angehenden Lehrpersonen naturwissenschaftlicher Unterrichtsfächer. Die Ergebnisse verdeutlichen, dass die sieben Wissensbereiche pädagogischen Wissens ausreichend reliabel gemessen und somit wesentliche Bereiche pädagogischen Wissens beschrieben werden konnten (vgl. Kleickman et al. 2014; Voss et al. 2015: 18; Kunter et al. 2017).

König und Seifert (2012) erstellten im Rahmen der Längsschnittlichen Erhebung pädagogischer Kompetenzen von Lehramtsstudierenden (LEK) ein weiteres Testinstrument, welches an das TEDS-M Modell angelehnt war. Grundlegendes Ziel der LEK-Studie war es, die Wirksamkeit der LehrerInnenausbildung empirisch zu beforschen. In der Studie wurde untersucht, wie sich pädagogisches Wissen (hier: pädagogisches Professionswissen) im Verlauf der universitären LehrerInnenausbildung der Primarstufe unter Berücksichtigung individuelle Lernvoraussetzung entwickelt. Berücksichtigt wurden Lehramtsstudierende von vier deutschen Universitäten (Universität Erfurt, Universität Köln, Universität Paderborn, Universität Passau). Die Studierenden wurden im Rahmen einer Längsschnittstichprobe im ersten sowie am Ende des vierten Semesters befragt. Der theoretische Bezugsrahmen für die Messung der Wirksamkeit der LehrerInnenausbildung bildete ein Mehrebenenmodell, welches die Indikatoren Vorwissen, pädagogische Vorerfahrungen, Berufswahlmotive, demografische Merkmale sowie erworbene Wissensbereiche während des Studiums berücksichtige (vgl. König 2010b: 62ff.). Für die Messung des Erwerbs pädagogischen Wissen der Studierenden wurden die Konstrukte der TEDS-M Studie (Strukturierung von Unterricht, Motivierung, Umgang mit Heterogenität, Klassenführung und Leistungsbeurteilung) sowie die Berücksichtigung kognitiver Prozesse (erinnern, 
verstehen/analysieren, kreieren) herangezogen. Die individuellen Lernvoraussetzungen wurden mit spezifischen Fragen betreffend Vorerfahrungen, intrinsische sowie extrinsische Berufswahlmotivation sowie der Befragung nach ehemaligen Schulnoten erfasst (vgl. König 2010b: 65). Die Ergebnisse verdeutlichen die essenzielle Bedeutung von individuellen Voraussetzungen, wie intrinsischer Motivation und Erfahrungen angehender Lehrpersonen, im Zusammenhang mit dem Erwerb pädagogischen Wissens während der Lehramtsausbildung (vgl. König 2010b:75). Die AutorInnen berichten von einem Zuwachs an pädagogischem Wissen über die Zeit. Die Lehramtsstudierenden im vierten Semester verfügen über umfangreicheres pädagogisches Wissen als im ersten Semester. Ein starker Zuwachs ist vor allem beim unterrichtsbezogenen Wissen zu verzeichnen (vgl. König und Seifert 2012).

Lenske et al. (2015) entwickelten das ProwiN-Projekt (Professionswissen in den Naturwissenschaften). Ziel der Studie war, Unterschiede und Zusammenhänge zwischen Faktenwissen und anwendungsbezogenem Wissen unter Berücksichtigung von fachlichem, fachdidaktischem und pädagogischem Wissen zu untersuchen und wie sich dieser auf den Zuwachs von SchülerInnen auswirkt (vgl. Lenske et al. 2015: 231f.). In Anlehnung an die COACTIV-Studie wurde pädagogisches Wissen von Lehrpersonen naturwissenschaftlicher Unterrichtsfächer anhand von vier Wissensdimensionen gemessen: Klassenführung, Unterrichtsmethoden, Individualisierung sowie Leistungsbeurteilung und Feedback. Die Testkonstrukte beziehen sich auf didaktische sowie pädagogisch-psychologische Bereiche. Es wird in bei der COACTIV- und in der BillWiss-Studie argumentiert, dass der Test nicht nur deklaratives Wissen, sondern prozedurale Wissenskomponenten unterrichtsnah und anwendungsorientiert erfasst (vgl. Lenske et al. 2015: 225). Die Testung wurde im Rahmen einer Befragung sowie mithilfe von Videovignetten durchgeführt. Das Sample bestand aus 452 Lehrpersonen aus Nordrhein-Westfalen und Bayern mit den Fächern Physik, Chemie und Biologie (vgl. Lenske et al. 2015: 236ff.). Die Ergebnisse konstatieren einen Zusammenhang zwischen den Formen des Professionswissens sowie zwischen dem Faktenwissen und dem anwendungsorientierten Wissen. Die Studie verdeutlicht, wie der Einsatz aller Wissensformen von Lehrpersonen einen Kompetenzzuwachs bei SchülerInnen positiv unterstützt (vgl. Lenske et al. 2015: 240ff.).

Das Projekt Innovative Teaching for Effective Learning - Teacher Knowledge Survey (ITEL TKS) der OECD präsentiert ein weiteres Testverfahren. Die Studie setzte sich mit der Frage auseinander, ob Lehrpersonen über das notwendige professionelle Wissen für die Vermittlung von Fähigkeiten des 21. Jahrhunderts verfügen. Ein Schwerpunkt der Studie lag auf der Überprüfung pädagogischer Wissensanteile (vgl. Guerriero 2015/2017). Die Pilotstudie wurde in fünf OCED-Ländern (Estland, Griechenland, Ungarn, Israel, Slowenien) durchgeführt. Sechs Länder haben die Teilnahme aufgrund einer umstrittenen Konstruktion der Testanlage zurückgezogen. Das Sample bestand bei jedem Teilnehmerland aus über hundert Personen, untergliedert in Lehramtstudierende, Lehrpersonen im Schuldienst sowie Lehramtsauszubildende. Die Konstrukte basierten auf den Studien COACTIV, TEDS-M, BilWiss sowie MT21. Ausgehend von den Studien wurden drei Hauptkategorien sowie für jede Hauptkategorie zwei Subkategorien entwickelt. Die Hauptkategorien bezogen sich auf die Bereiche Lehren, Lernen sowie Leistungsbeurteilung. Das Lehren untergliederte sich in die von Voss et al. (2011) und König et al. (2011) definierten Subdimensionen Lehrmethoden und Unterrichtsplanung sowie Classroom-Management. Die Dimensionen für das Lernen betrafen die Themenbereiche Lernen und Entwicklung sowie affektive-motivationale Aspekte, in Anlehnung an Voss et al. (2011) und König et al. (2011). Die Leistungsbeurteilung wurde über die Kriterien Evaluation und Diagnostik sowie Daten und Forschungskompetenz basierend auf Voss et al. (2011) bestimmt (vgl. 
Guerriero 2015; Sonmark et al. 2017: 11ff.). Das Testgerüst inkludierte als weitere Unterteilungen unterschiedliche Felder. Sie bezogen sich auf eine modifizierte Version der kognitiven Verarbeitung von Anderson und Krathwohl (2001) der Studie TEDS-M (vgl. König 2014b), die Bereiche des Reproduzierens, Verstehens, Analysierens und Interpretierens berücksichtigt, sowie die von Shalem (2014) berücksichtigten Kriterien deklaratives und prozedurales Wissen. Des Weiteren kann Wissen dahingehend beschrieben werden, ob es sich auf einen traditionellen Ansatz bezieht oder ob es sich um einen innovativen, neuen pädagogischen Ansatz handelt, wie Pellegrino und Hilton (2012) definieren (vgl. Sonmark et al. 2017: 17ff.). Den Ergebnissen zufolge scheinen bei den Lehrpersonen die untersuchten Konstrukte vorzuliegen. Die AutorInnen argumentieren in Anlehnung an diesen Befund, dass nach diesem Verständnis die Lehrpersonen über essenzielle Wissensbereiche pädagogischen Wissens des 21. Jahrhunderts verfügen. In Anlehnung an bestehende Studien wurden in den letzten Jahren weitere Testverfahren wie COACTIV-R oder TEDS-FU entwickelt. Die Studie TEDS-FU war ein weiterführendes Projekt der Studie TEDS-M. Das Ziel von TEDS-FU war es, mithilfe von Videovignetten die Erfassung des pädagogischen Kompetenzniveaus von Lehrpersonen und der Kompetenzstruktur im Zusammenhang mit ihrem fachdidaktischen Wissen (vgl. König et al. 2014: 76f.). Das Sample setzte sich aus einer Teilmenge von Mathematiklehrpersonen der TEDS-M-Studie zusammen, die sich im letzten Ausbildungsjahr befanden. Insgesamt wurden 304 Lehrpersonen aus der Primarstufe und der Sekundarstufe I befragt, die am Ende ihrer LehrerInnenausbildung standen. Ergebnisse zeigen, wie über die Berücksichtigung von pädagogischen Kompetenzen und fachdidaktischem Wissen das professionelle Wissen von Lehrpersonen angemessen erfasst werden kann und Gemeinsamkeiten der beiden Formen hervorgehoben werden (vgl. Busse \& Kaiser 2015: 328). Die Pilotstudie COACTIV-R, welche auf der Studie COACTIV aufbaut, zielte darauf $\mathrm{ab}$, mithilfe einer Längsschnittstudie die Kompetenzentwicklung am Ende des Studiums bis nach dem Referendariat zu untersuchen. Das Sample stützte sich auf 856 Lehramtsstudierende der Sekundarstufe I aus den Bundesländern Bayern, Baden-Württemberg, Nordrhein-Westfalen und Schleswig-Holstein (vgl. Kunter 2009: 13; 22). Die untersuchten Lehrpersonen wurden am Ende ihres Studiums sowie nach absolviertem Referendariat bezüglich ihrer erworbenen Kompetenzen in den Bereichen Professionswissen, Überzeugungen und individuelle Werte getestet (vgl. Kunter 2009: 22ff.). Ergebnisse verdeutlichen einen Kompetenzzuwachs in allen Bereichen über die Praxiserfahrungen während des Referendariats.

Hohenstein et al. (2017) entwickelten basierend auf der KIL-Studie ein Instrument mit dem Ziel, pädagogisches und psychologisches Wissen basierend auf einem Sample von 500 Lehramtsstudierenden zu erfassen (vgl. Hohenstein et al. 2017: 106). Sie argumentierten die Testung mit bisherigen unzureichenden Testverfahren, die pädagogisches Wissen maßen. Sie entwickelten in ihrer Studie zehn Konstrukte, die sich auf schulpädagogische sowie pädagogisch-psychologische Inhalte bezogen. Die Auswahl erfolgte über die KMK-Standards sowie die vorhandenen Studien COACTIV, TEDS-M und BilWiss (König et al. 2011; Voss et al. 2011; Kunina-Habenicht et al. 2012). Die Wissensbereiche bezogen sich auf folgende Felder: Lehren, Lernen und Entwicklung, Klassenführung, Leistungsbeurteilung und individuelle Besonderheiten, Bildungssystem und Schulorganisation, Methoden und Ergebnisse bildungswissenschaftlicher Forschung, Reflexion und Weiterentwicklung der eignen Professionalität, Schulentwicklung und Werte sowie Normen und soziale Konflikte (vgl. Hohenstein 2017 et al.: 94f.). Die AutorInnen argumentieren basierend auf den Ergebnissen, dass die Studie bisherige Testverfahren erweitert und alle untersuchten Konstrukte, die pädagogisches Wissen mehrdimensional abbilden, über die Lehrpersonen valide getestet werden konnten. 
Die angeführten Studien zeichnen sich vorrangig durch standardisierte Testverfahren aus. Einzelne AutorInnen argumentieren, dass ihre Studien sich auf die Berücksichtigung deklarativer sowie prozeduraler Wissensformen stützen (vgl. ITEL, COACTIV, BilWiss, ProwiN). Diese Argumentation muss jedoch aufgrund der standardisierten Testformate infrage gestellt werden, da quantitative Testverfahren vorwiegend deklaratives sowie explizites Wissen und nicht das essenzielle Handlungswissen aus der Praxis testen. Die angeführten Testverfahren unterscheiden sich insofern, als sie auf unterschiedliche Wissensformen für die Operationalisierung zurückgreifen (vgl. Voss et al. 2015: 203f.). Gemeinsame Konstrukte, die sich in allen Studien wiederfinden, sind die Klassenführung, die Leistungsbeurteilung sowie der Einsatz von Unterrichtsmethoden. Die Studien SPEE sowie LEK beziehen sich nicht nur auf schulrelevante Formen pädagogischen Wissens, sondern berücksichtigen Konstrukte, die außerschulische Bereiche betreffen. Die SPEE-Studie beinhaltet zum Beispiel im Unterschied zu den anderen Studien die Reflexionsfähigkeit von Lehrpersonen. Konstrukte wie der Umgang mit Heterogenität, Lehren und Lernen werden in den Studien TEDS-M, COACTIV, MT21 sowie BilWiss untersucht. Konstrukte zu theoretischem, bildungswissenschaftlichem Wissen sowie der Schulorganisation sind bei den Studien KiL und LEK relevant.

Kritisch anzumerken ist, dass die Studien vorrangig auf einem Sample von Lehrpersonen aus naturwissenschaftlichen Fächern basieren. Die Ergebnisse der Testverfahren sind in Bezug auf die Generalisierbarkeit anderer Unterrichtsfächer daher kritisch zu sehen. Eine Vielzahl der Studien stützt sich auf die Untersuchung angehender Lehrpersonen. Obwohl in diesem Zusammenhang aufgezeigt werden konnte, dass Lehrpersonen über das Studium ihr pädagogisches Wissen vermehren, ist der Anteil der Untersuchungen mit Lehrpersonen, die im Schulalltag tätig sind, gering. Da die Aneignung pädagogischen Wissens zu einem großen Teil über die Erfahrung während der Praxis stattfindet (vgl. Kapitel 3.3) und dadurch neue Strukturen impliziten Wissens generiert werden (vgl. Kapitel 3.1.2), die für das Handeln von Lehrpersonen zentral sind (vgl. Polanyi 1985; Neuweg 2004), könnten Untersuchungen mit Lehrpersonen im aktiven Schuldienst zu weiteren Befunden führen. In einer weiteren Ausdifferenzierung zeigt sich ein Forschungsdesiderat hinsichtlich der Wirkung und dem Einfluss pädagogischen Wissens auf den Unterricht. Bisherige Testverfahren wurden vorwiegend für die Entwicklung eines Messinstruments für die Überprüfung der pädagogischen Konstrukte und des Wissenszuwachses während der LehrerInnenausbildung entwickelt. Als problematisch zeigt sich bei den Testverfahren insbesondere, dass sich die Verfahren, die im Rahmen internationaler Studien entwickelt wurden, alle auf den deutschsprachigen Raum beziehen. Alle Konstrukte der Testverfahren der Studien COACTIV, TEDS-M, MT21 sowie ITEL basieren auf den von der KMK (2004) vorgegebenen Anforderungsbereiche und Standards von Lehrpersonen, die individuell von den jeweiligen AutorInnen in Anlehnung ihres Forschungsfokus adaptiert wurden. Bei diesen Studien handelt es sich daher um deutsche Studien, die alle miteinander in Beziehung stehen und individuell weiterentwickelt wurden. Die Studien repräsentieren daher keinen international vergleichenden Diskurs. 


\section{Pädagogisches Handeln}

In der vorliegenden Arbeit liegt der Schwerpunkt auf dem pädagogischen Wissen von Lehrpersonen. Dieses steht, wie in Abschnitt 2.1 in der allgemeinen Darstellung zum Wissensbegriffs und in Kapitel 3 in Bezug auf das professionelle Wissen von Lehrperson diskutiert, in enger Beziehung zum pädagogischen Handeln. Wissen konstituiert sich hinsichtlich des Lernens und Lehrens zwischen theoretischen und praktischen Wissensstrukturen (vgl. Neuweg 2004; Koch 2015: 30f.). Das vorliegende Kapitel diskutiert einen theoretischen Zugang pädagogischen Handelns sowie liefert einen Ansatz für die Entfaltung individueller Wissensstrukturen mittels Reflexion auf das professionelle Handeln. Ein weiterer Fokus liegt auf der Entwicklung metareflexiver Prozesse professionsspezifischen Denkens und Handeln von Lehrpersonen. Mead mit seinem Konzept des „Me und „I“ sowie Giddens mit seiner Theorie der sozialen Praxis stellen hierbei den theoretischen Bezugsrahmen dar und erweitern das Verständis zur pädagogischen Interaktion unter Berücksichtigung kognitiver Fähigkeiten von Lehrpersonen und institutionellen Rahmenbedinungen (vgl. Kapitel 5.3).

\subsection{Modelle pädagogischen Handelns}

Gegenwärtig wird das Konzept pädagogisches Handeln im Zusammenhang mit dem Professionalisierungsdiskurs über die Kompetenzorientierung diskutiert (vgl. Weinert 2001a; KMK 2004; König 2010a; Terhart et al. 2014; Blömeke et al. 2015; Ontario 2016). Die in Bildungsstandards definierten Fähigkeiten und Fertigkeiten beschreiben von den Lehrpersonen zu bewältigende Aufgaben (vgl. Messner 2007: 365), die sich auf professionelle theoretische Wissensbereiche sowie ihr berufliches Handlungsfeld, das Können in der Praxis, stützen (vgl. Kapitel 1.2/2.5).

Unter pädagogischem Handeln wird in der formalen Bildung ein sozialer Zugang mit der Intention, Lernen zu ermöglichen und die individuelle Entwicklung zu unterstützen, verstanden (vgl. Petzel 1964: 16; Giesecke 2013: 24). Giesecke (2013: 24) definiert die Sozialität über eine Wechselbeziehung vorhandener Personen im Raum (vgl. Abbildung 16).

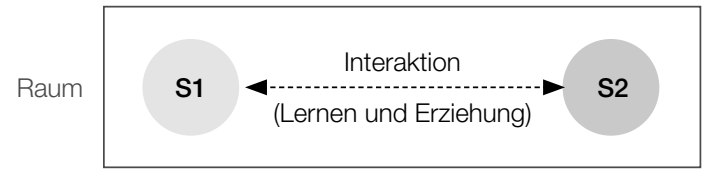

Abb. 16: Pädagogische Interaktion nach Giesecke

Die im Raum vorhandene soziale Interaktion wird stark von den Subjekten beeinflusst, sodass es kein richtiges, sondern, wie Radtke (2000) und Giesecke (2013: 21f.) argumentieren, nur ein angemessenes, vernünftiges und zielorientiertes pädagogisches Handeln geben kann. Diese zugrundeliegende Beziehung bestimmt Giesecke (2013: 105) als pädagogische Interaktion. Pädagogisches Handeln und die damit entstehende pädagogische Interaktion beschreibt schon Petzelt (1964: 16) in seiner Theorie über die Interaktion und den Fokus auf das Lernen. ${ }^{26}$ Das

26 Petzelt (1964) beschreibt diesen Prozess als „Lehrgut“. Er begründet das pädagogische Handeln mit der Notwendigkeit, dass es bei der zugrundeliegenden Interaktion um das Lernen geht. Aufbauend auf diese Grundidee entwickelt er das didaktische Dreieck Lehren-Lernen-Gegenstand und begründet das Lernen über das Lehren unter Berücksichtigung eines Gegenstands. 
Thema der pädagogischen Interaktion wird im folgenden Abschnitt 5.3 aufgrund seiner Relevanz für das pädagogische Wissen diskutiert.

Die Forschungsliteratur beschäftigt sich vielfach mit der Frage, welches Wissen notwendig ist, um professionell zu handeln. Dabei wird auf die in Kapitel 2 sowie in Kapitel 3 vorgestellten Wissensklassifikationen zurückgegriffen. In Anlehnung an die wesentlichen Formen professionellen Wissens wird dem impliziten Wissen eine essenzielle Bedeutung beigemessen (vgl. Kapitel 3). Die bestehenden Befunde zeigen jedoch kein einheitliches Bild hinsichtlich relevanter Wissensformen. Neuweg (1999) betont, dass Lehrpersonen im Unterricht basierend auf implizitem Wissen handeln, das sie häufig nicht verbalisieren können. Er begründet diese Aussage damit, dass der LehrerInnenberuf sich vorwiegend über schnelles Reagieren, Entscheiden und Handeln charakterisiere (vgl. Neuweg 1999). Für diese Handlungsakte werde weniger auf explizites Wissen zurückgegriffen. Als handlungsleitende Wissensform bezeichnet Neuweg (2002) das Erfahrungswissen, das er auf Polanyi’s (1985) Begriff des impliziten Wissens zurückführt. Wird Handlungswissen als verbalisierbar beschrieben, bezieht es sich auf Wissen, welches erst nach der Handlung rekonstruiert werden kann (vgl. Schön 1983; Messner 2007: 267). Ryle (1949), Nolle (2004) sowie Messner (2007) diskutieren in diesem Zusammenhang einen weiteren Zugang. Sie verweisen auf die Notwendigkeit expliziten Wissens von Lehrpersonen für professionelles pädagogisches Handeln. Intelligentes Handeln, so Ryle (1949: 137), kann sich nur über explizites Wissen realisieren. Herzog (1995: 262) sowie Nolle (2004) führen diesen Diskurs fort. ${ }^{27}$ Professionelle Lehrpersonen zeichnen sich Herzog (1995: 262) zufolge unter anderem dadurch aus, dass sie ihre Handlungsmöglichkeiten schon vor einer Handlung explizit kennen.

Terhart (2006: 580ff.) betont Schwierigkeiten und Herausforderungen pädagogischen Handelns, die er über den Begriff der „Nicht-Algorithmizität“ beschreibt. Es gibt keine Rezepte oder technische Vorschriften, die erfolgreiches Handeln von Lehrpersonen garantieren. Vielmehr steht das LehrerInnenhandeln in Relation zu Normen, Haltungen und Motivationen sowie den Gebrauch von Wissen (vgl. Schoenfeld 2010). Hierzu haben Groeben et al. (1988) das Konzept der subjektiven Theorien entwickelt, die das Handeln basierend auf subjektiven Theorien argumentieren. Zentrale handlungsleitende Elemente betreffen in Anlehnung an Groeben et al. (1988), Dann (1994), Baumann (2006) sowie Mikhail (2016) kognitive Strukturen des Wissens sowie zugrundeliegende Haltungen und Überzeugungen ${ }^{28}$ einer Lehrperson. Professionelle Haltung äußert sich nach Rogers (1981) und Schmied (2016: 157) über einen ressourcenorientierten Umgang mit den Personen im Klassenzimmer. Handlungen basieren auf individuellen Werten, Zielen, Ängsten und Hoffnungen der jeweiligen Lehrpersonen. Carl Rogers (1981) beschreibt in seinem personzentrierten Ansatz über das Selbstkonzept des Selbst, wie Kongruenz, Akzeptanz und Empathie zu einer positiven Beziehung und Wertschätzung beitragen (vgl. Keil 2001: 119; Zimring 2006). Kongruenz ist für Rogers, sich selbst treu zu sein, so dass das individuelle Selbstkonzept mit dem eigenen Verhalten übereinstimmt (vgl. Plate 2015: 51). Er

27 Der Transformationsprozess vom impliziten Wissen zum expliziten Wissen kann, wie schon in Abschnitt 3.2.3 im Zusammenhang mit dem Kompetenzmodell von Gordon (2013) dargestellt, als ein Übergang von einer unbewussten Inkompetenz zu einer bewussten Kompetenz beschrieben werden.

28 Haltung und Überzeugung werden hier als Synonyme verwendet. Fend verweist auf die nicht eindeutige Abgrenzung der beiden Begriffe und verwendet den übergeordneten Begriff „pädagogische Grundhaltungen bzw. Grundhaltungen" (vgl. Fend 1998: 308). Fend (2008: 299ff.) stellte in Bezug auf Haltung und Überzeugung die Menschenbilder bzw. das Weltbild in den Mittelpunkt des Interesses. Die Forschungsgrundlage zum Thema Haltung und Überzeugung wird jüngst gemeinsam mit dem Berufsethos von Lehrpersonen klarer formulieren (vgl. Oser 1998; Mikhail 2016). 
verweist auf die Bedeutung der Unterstützung von anderen Menschen und die ressourcenorientierte Begleitung durch aktives Zuhören (vgl. Plate 2015: 51).

Ein weiterer Zugang zum Selbstkonzept, der das Verständnis von Rogers erweitert, ist das Konzept von Mikhail (2006: 206). Er beschreibt das individuelle Selbstkonzept einer Person anhand von drei in Beziehung stehenden Prinzipien - „Bildsamkeit“, „Selbstbestimmung" und „Dialogizität" -, die er in einem didaktischen Dreieck veranschaulicht (vgl. Abbildung 17). Die Selbstbestimmung sowie die Dialogizität zeigen Übereinstimmung mit den von Rogers definierten Selbstkonzepten. Neu ist bei Mikhail das Konzept der Bildsamkeit.

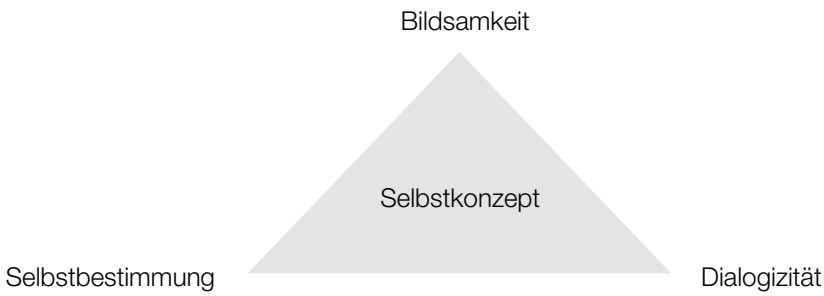

Abb. 17: Selbstkonzept nach Mikhail (in Anlehnung an Mikhail 2006: 206)

Mikhail charakterisiert das Konzept über die ordnungsstiftende Funktion kognitiver Strukturen. Die Bildsamkeit beschreibt er in Anlehnung an Herbarts (1982) Verständnis als „Bildungsfähigkeit“. Dabei unterstreicht er die Bedeutung pädagogischen Handelns für die Bildung. Es geht ihm weniger um die Bestimmung des Menschen, wie er ist, sondern vielmehr darum, wie man ihn sich vorstellen sollte, um pädagogische Interaktionen zu gestalten. Mit dem Konzept der Selbstbestimmung postuliert er, wie auch Rogers, dass jede pädagogische Haltung auf der Entscheidung, dem Denken und der Bewertung der Personen gegenüber begründet ist (vgl. Mikhail 2016: 164). Das Kriterium der Kommunikation, wie es Rogers in seinem Ansatz über das aktive Zuhören beschreibt, wird bei Mikhail über die Dialogizität definiert. Mikhail verweist in seinem Ansatz, dass vor allem über die Kommunikation Beziehungen zwischen Personen geschaffen werden können (vgl. Mikhail 2016: 171). In Anlehnung an Rogers und Mikhail kann eine Lehrperson mithilfe ihres Selbstkonzepts, und vor dem Hintergrund eines ressourcenorientierten Zugangs, eine Beziehung mit den Lernenden auf Augenhöhe entwickeln, welche das pädagogische Handeln fördert. Der Aufbau sowie die (Weiter-)Entwicklung eines Selbstkonzepts von Lehrpersonen können mithilfe der Reflexion stattfinden. Die Reflexionsfähigkeit ist Schwer \& Solzbacher (2014) zufolge für die Realisierung des Selbstkonzepts von Bedeutung und wird von Minnameier (2009: 333) als kognitive Voraussetzung pädagogischer Professionalität charakterisiert. Reflexion wird als unterstützender Faktor für ein verändertes Denken, die Verbesserung von professionellen Fertigkeiten und Fähigkeiten sowie für die Generierung neuen Wissens für das professionelle pädagogische Handeln beschrieben (vgl. Korthagen 2001: 47; Sembill \& Seifried 2009: 345ff.). Helsper (2001: 13) diskutiert in seinem Konzept der doppelten Professionalisierung die wissenschaftlich-reflexive Form, die das Begründen pädagogischer Handlungen ermöglicht. ${ }^{29}$ Reflexion wird als Transfermittel des Wissens für das Handeln sowie für den Aufbau von Wissen von Lehrpersonen betrachtet (vgl. Schön 1983; Giesecke 2013; Radtke 1999; Helsper 2001;

29 Die zweite Form stützt sich auf einen praktischen-pädagogischen Habitus, der sich über Erfahrungen entwickelt. 
Neuweg 2002). Im folgenden Abschnitt werden das zugrundeliegende Verständnis von Reflexion sowie grundlegende Modelle diskutiert.

\subsection{Reflexion und Wissen}

Reflexion wird als Schlüsselkonzept für den Aufbau von Wissen und den Transfer von pädagogischem Wissen zum Handeln beschrieben. Zentral für diesen Prozess ist Korthagen et al. (2001: 47) zufolge eine strukturierte sowie systematische Selbstreflexion. Diese bedingt ein verändertes Denken sowie die Verbesserung von Fertigkeiten und Fähigkeiten. In Anlehnung an Oelkers \& Tenorth (1993: 18) kann über Reflexion neues Wissen generiert werden. Neuweg (2004) verweist spezifisch auf einen Transferprozess von implizitem zu explizitem Wissen, der mittels Reflexion stattfinden kann.

Der Transfer vom Wissen zum Handeln wird in der Forschungsliteratur (vgl. Herbart 1969; Schön 1983; Bauer 1998; Korthagen \& Kessel 1999) mittels unterschiedlicher Modelle und Ansätze beschrieben. Diese beinhalten in der Regel spezifische reflexive Prozesse, die dabei auf vorhandene Wissensstrukturen zurückgreifen und zu einer Veränderung kognitiver Muster und Haltungen führen. Für die Forschungsarbeit wesentliche Modelle, die das zugrundeliegende Transferverständnis von Wissen zum Handeln aufzeigen, basieren auf dem Zugang der reflexiven Praxis von Schön (1983) sowie dem pädagogischen Selbst von Bauer (1998). Beide Zugänge präsentieren auf unterschiedliche Weise, wie kognitive Strukturen und das Bewusstsein der Lehrpersonen, hinsichtlich ihres individuellen (pädagogischen) professionellen Wissens, über Reflexion transformiert werden können. Schön (1983) legt seinen Fokus auf den reflexiven Dialog; wobei Bauer (1998) stärker das Selbst in den Vordergrund rückt.

\section{Reflexive Praxis}

Schön (1983) führt in seinem Buch „Reflective Practitionier“ das Konzept der reflexiven Praxis ein. Die Grundidee seines Modells ist die Gewinnung von Wissen über einen reflexiven Dialog. Dieser Prozess unterstützt einen bewussten und systematischen Aufbau professionellen Wissens (vgl. Herzog 1995: 254). Schön (1983: 49) und Neuweg (1999) beschreiben, wie das handlungsleitende Wissen vorrangig in impliziter Form vorliegt und sich erst über Reflexion direkt beim Handeln zu explizitem Wissen transformieren kann. Ein Ansatz bei Schön ist die Veränderung des Wissens direkt in einer Situation und nicht erst nach Beendigung einer Handlung (vgl. Schön 1983: 49). Er beschreibt den Reflexionsprozess über in sich aufbauende Handlungen: (1) „Wissenin-der-Handlung“, (2) „Reflexion-in-der-Handlung“ sowie (3) „Reflexion-über-die-Handlung“. Das „Wissen-in-der-Handlung“ beschreibt Schön (1983) als praktisches Handeln ohne bewusste Anwendung spezifischer Wissensformen. Denken und Handeln finden unbewusst statt (vgl. Strasser 2006: 137). Die zweite Handlung, „Reflexion-in-der-Handlung“, wird von Schön (1983: 68f.) als „reflexive Konversation mit der Situation beschrieben“. Strasser (2006: 137) vertritt die Position, dass ein Aufbau professioneller Kompetenz vorwiegend ab dieser Handlungsform stattfindet. Der Prozess „Reflexion-in-der-Handlung“ kennzeichnet sich durch Reflexion während einer Handlung. Ausgelöst wird er durch eine Problemsituation, die nicht mehr mit alltäglichem routiniertem Handeln gelöst werden kann. Dieser Bruch leitet einen reflexiven Prozess und das Zurückgreifen auf vorhandenes implizites Wissen ein (vgl. Schön 1983: 63). Die Lehrperson wird, wie Schön (1983: 68f.) beschreibt, „zu einem Forscher im Kontext der Praxis, [...] der eine neue Theorie des spezifischen Falls [konstruiert]". In Abbildung 18 wird der Prozess anhand von sechs verschiedenen Phasen dargestellt. Die Reflexion geschieht bei diesem Phasenmodell direkt im Handlungsverlauf und muss nicht von der Lehrperson verbalisiert werden. 


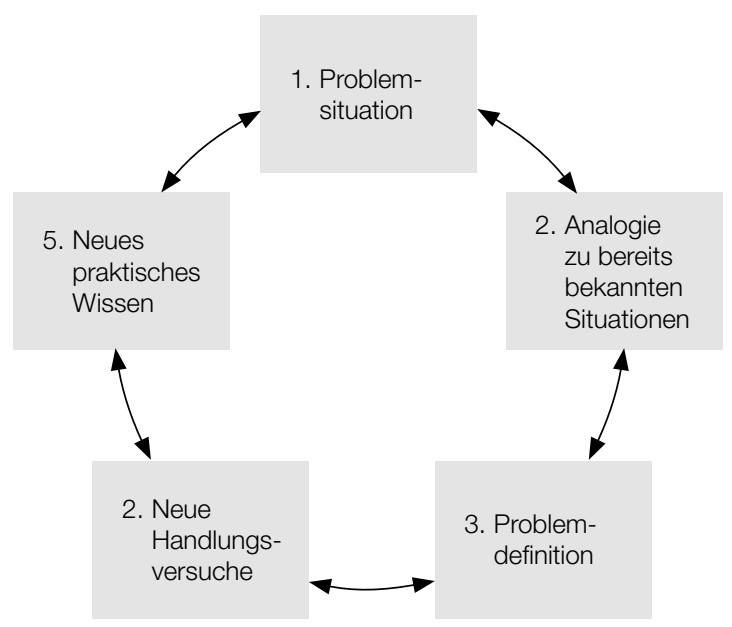

Abb. 18: Prozess Reflexion-in-der-Handlung

Der Reflexionsprozess einer Person beginnt mit dem Auftreten einer Problemsituation. Sie ist nicht mehr in der Lage, auf vorhandene Muster zurückzugreifen (1). Diese Unsicherheit bedingt einen Vergleich zu bereits bekannten Situationen (2). Anschließend wird das Problem von der handelnden Person definiert und nach neuen Handlungsmöglichkeiten gesucht (3). Dieser reflexive Handlungsvorgang führt über neue Handlungsversuche nach Beendigung des Prozesses zu neuen, expliziten, praktischen Wissensstrukturen ( 4 und 5).

Die „Reflexion-über-die-Handlung“ ist im Vergleich zur „Reflexion-in-der-Handlung“ durch das Verbalisieren von Wissensformen charakterisiert. Dazu ist eine gewisse Distanz zur Problemsituation nötig. Die handelnde Person tritt aus einer Situation heraus und nimmt eine Metaperspektive ein, die dabei hilft, Wissen zu analysieren und für das Handeln zu reorganisieren (vgl. Altrichter 2000: 208). Das eigene Handeln wird Altrichter (2000: 9) zufolge von einem dreiteiligen Phasenverlauf unterbrochen und reflexiv betrachtet (vgl. Abbildung 19).

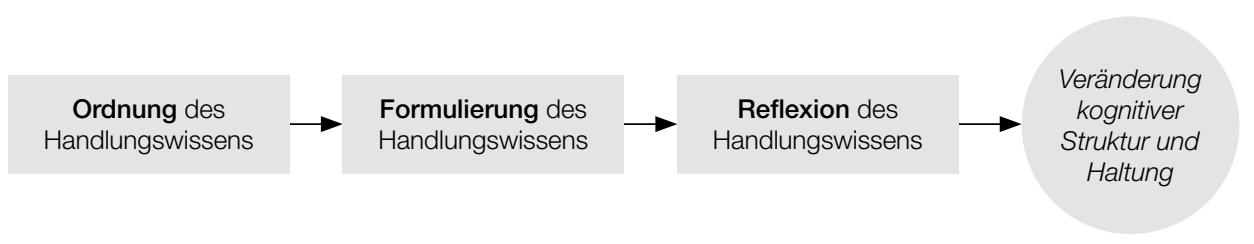

Abb. 19: Prozess Reflexion-über-die-Handlung

Dieser Zustand kann über eine systematische Ordnung, Formulierung und Reflexion des Handlungswissens erreicht werden. Die beschriebene Abweichung der gewohnten Handlungsroutinen unterstützt die Rekonstituierung und Modifikation kognitiver Muster und Haltungen.

Das vorgestellte Modell von Schön versucht, mithilfe des Heraustretens aus einer Situation aufgrund von Problemsituationen und fehlendem Wissen, über Reflexion den Transformationsprozess von implizitem zu explizitem Wissen zu erklären. Schön (1983) argumentiert alle 
drei Handlungen als wesentlich, betont jedoch die „Reflexion-über-die-Handlung“ als wichtigste Form für die Veränderung kognitiver Strukturen. Besitzen Lehrpersonen umfangreiches implizites Wissen, ist das Modell von Schön insofern von Bedeutung, als es einen Prozess für die Entwicklung expliziten Wissens beschreibt. Das Konzept zielt darauf ab, sich eigene Wahrnehmungs- und Deutungsmuster bewusst zu machen und schwer zugängliche Strukturen durch innere und äußere Distanz aufzubrechen (vgl. Heppekausen 2013: 112).

Ein weiterer Faktor, der kognitive Veränderungen herbeiführen kann und der bei Schön unzureichend diskutiert wird, ist die Reflexion über das eigene Bewusstsein. Bauer (1998) entwickelte hierzu ein Modell über das „pädagogische Selbst“, welches das Bewusstsein der Lehrperson für den Transfer von Wissen zum Handeln in den Mittelpunkt des Interesses rückt.

\section{Pädagogisches Selbst}

Das Konzept „pädagogisches Selbst“ von Bauer (1998) beschreibt die Veränderungen kognitiver Strukturen im Zusammenhang mit dem Bewusstsein von Lehrpersonen. Es stützt sich auf neurowissenschaftliche und psychologische Erkenntnisse (vgl. Csikszentmihalyi 1995). Ziel des Zugangs ist es, durch den Aufbau des pädagogischen Selbst professionelles pädagogisches Wissen und Handeln zu entwickeln. Der Orientierungsrahmen sind Werte und Haltungen einer Lehrperson (vgl. Bauer 1998: 346). Das professionelle Selbst setzt sich aus den Werten/Zielen, dem Fachwissen, dem pädagogischen Wissen, dem fachdidaktischen Wissen sowie dem Handlungsrepertoire einer Lehrperson zusammen (vgl. Abbildung 20). Das pädagogische Selbst von Csikszentmihalyi (1995) sowie Bauer (1998: 353) wird als „organisiertes Zentrum, dessen Kern Werte und Ziele darstellen“ beschrieben. Es unterliegt einem ständigen Entwicklungsprozess, über den sich eine Lehrperson über Erfahrungen und Wissensbestände selbstgesteuert fort- und weiterentwickelt (vgl. Marotzki 2007). ${ }^{30}$ Das Steuerungsinstrument für den Aufbau des pädagogischen Selbst ist die Reflexion, die eine Bewusstseinsveränderung bewirkt (vgl. Heppekausen 2013: 113).

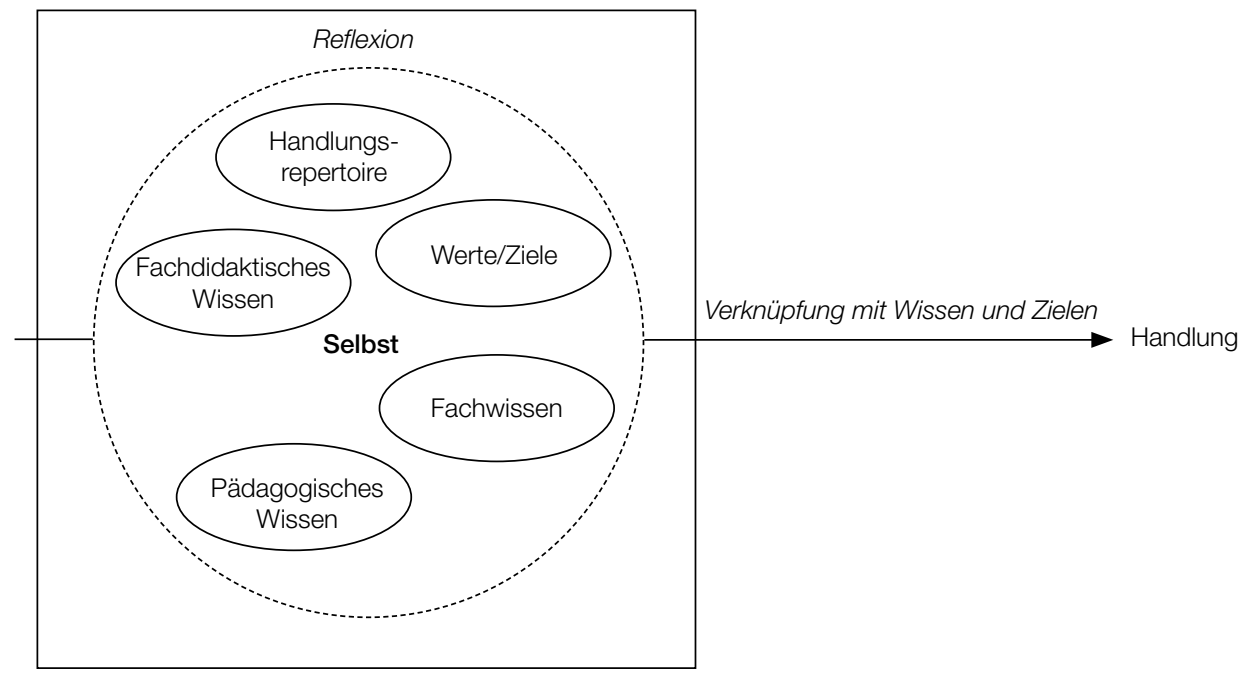

Abb. 20: Pädagogisches Selbst (in Anlehnung an Bauer 1998: 353)

30 Das Konzept pädagogisches Selbst stützt sich auf den Gedanken des lebenslangen Lernens und der Selbstorganisation beruflicher Professionalität im Berufsleben von Lehrpersonen (vgl. Kapitel 1.5). 
Die Reflexion über das eigene Bewusstsein (hier: Selbst) führt dazu, dass die zugrundeliegenden Wissensformen und Haltungen mit explizitem Wissen und individuellen Zielen verknüpft und zu expliziten Handlungen transformiert werden (vgl. Bauer et al. 1996: 14). In Anlehnung an Bauer (1998: 355) entsteht das pädagogische Selbst über die „[...] Unvollkommenheit, wenn diese nicht nur wahrgenommen wird, sondern auch als bearbeitbar erlebt wird“. Das Hinterfragen und dadurch Bewusstmachen individueller Wissensstrukturen hilft den Lehrpersonen, ihr Wissen zu transformieren und es situationsgerecht im Unterricht anzuwenden.

Die Ansätze von Schön (1983) und Bauer (1998) können mit der von Reh und Schelle (2010: 218) entwickelten Formel „Professionalität durch Reflexivität“ zusammengefasst werden. Reflexionsprozesse bedingen eine Entwicklung expliziter kognitiver Strukturen, die professionelles Wissen und Handeln positiv unterstützen. Die Entfaltung expliziter, individueller Wissensstrukturen im professionellen Handeln von Lehrpersonen, wie sie Herzog (1995) und Nolle (2004) fordern, kann über die Reflexion verwirklicht werden. Professionelles (pädagogisches) Wissen und Handeln sowie das Selbstkonzept von Lehrpersonen können sich verändern und weiterentwickeln. Dieser Ansatz wird in Abschnitt 5.3 mit den Theorien von Mead über das „Me“ und das „I“ sowie Giddens' „sozialer Praxis“ weitergeführt. Der Fokus wird auf die Reflexion im Kontext von Interaktionsprozessen gelegt. Parallelen zu Schön (1983) und Bauer (1998) zeigen sich insbesondere bei Mead, der eine Bewusstseinsveränderung sowie eine individuelle (Weiter-)Entwicklung über Reflexionsprozesse anvisiert.

\subsection{Pädagogische Interaktion im Kontext des symbolischen Interaktionismus und der sozialen Praxis}

Das vorliegende Kapitel diskutiert über handlungstheoretische Modelle von Mead und Giddens die Bedeutung von Interaktionsprozessen im Zusammenhang mit pädagogischem professionellem Wissen und Handeln. Die wesentliche Bedeutung von Interaktionen wurde schon in Abschnitt 2.1 im Zusammenhang des mehrperspektivischen Zugangs zu Wissen von Husserl, Mannheim, Luckmann sowie Schütz beschrieben, die das Erkennen von Wissen über Interaktionen argumentieren. Giesecke (2013: 24) verstärkt diese Bedeutung, indem sie pädagogisches Handeln von Lehrpersonen über Interaktionen und Wechselbeziehungen zwischen Lehrpersonen und SchülerInnen beschreibt. Allerdings führt Giesecke in ihrer Diskussion die Prozesse pädagogischer Interaktion nicht vertieft aus. Theorien von Mead und Giddens bieten die Möglichkeit, für ein weiteres vertieftes Verständnis des Kernthemas dieser Arbeit aufzuzeigen, wie man pädagogisches Wissen und Handeln über Interaktionen sichtbar und diskutierbar machen kann.

Handlungstheorien beziehen sich vorrangig auf einen mikrosoziologischen Zugang, der die gesellschaftliche Wirklichkeit und ihre soziale Ordnung über Interaktionsprozesse von AkteurInnen zu erklären versucht (vgl. Krotz 2008: 30f.; Miebach 2010: 15ff.). Wesentlich für die Interaktion sind das Wissen sowie das Handeln einer Person. Wechselbeziehungen zwischen Wissen und Handeln generieren, wie Denninger et al. (1973: 21) argumentieren, einen Wirkungskreislauf, der die zugrundeliegende Bedeutung von Interaktionen aufzeigt. Das Wirkungsgefüge charakterisiert sich wie Neuweg (1999), Waldenfels (2000) oder Balog (2012: 13) argumentieren, über Formen, die durch Handlungen entstehen und gleichzeitig über Handlungen identifizierbar und rekonstruierbar gemacht werden können. Grundlegende Eigenschaft der Handlungen beziehen sich auf die Notwendigkeit intersubjektiver notwendiger Kriterien, mit der Intention eines bestimmten Ziels (vgl. Balog 2012: 13). Interaktionsprozesse 
lassen sich in Anlehnung an dieses Verständnis als strukturgebunden und inhaltlich situationsspezifisch charakterisieren (vgl. Denninger et al. 1973: 21). Handlungen finden innerhalb von Interaktionsprozessen statt. Entscheidend sind die beteiligten Personen, die Handlungen identifizieren.

Der handlungstheoretische Zugang ist für die Auseinandersetzung mit dem pädagogischen Wissen relevant, da pädagogisches Wissen und Handeln über Interaktionsprozesse von Lehrpersonen und SchülerInnen im Unterricht realisiert werden kann und Bedeutung generiert.

Für die Diskussion wird im Folgenden auf die Theorien von Mead, mit seinen Ansätzen des „Me“ und des „I“, und auf die Theorie der „sozialen Praxis“ von Giddens eingegangen. Diese liefern einen ergänzenden Zugang zum pädagogischen Interaktionsdiskurs zwischen Lehrpersonen und SchülerInnen und erweitern das Verständnis von Giesecke (2013).

Mead diskutiert für eine Entwicklung einer Identität, die Interaktion in Bezug zur Umwelt unter Berücksichtigung subjektiver Konzeptionen. Giddens legt den Fokus auf die Dualität von Struktur und Handeln. Er stellt den Interaktionsprozess in einer Zeit-Raum-Konstellation unter Berücksichtigung von Ressourcen und der zugrundeliegenden Regeln in den Mittelpunkt. Giddens misst im Vergleich zu Mead in seinem Ansatz Strukturen einen größeren Stellenwert bei, welche über das Handeln der AkteurInnen selbst generiert werden.

\subsubsection{Mead - „Me“ und „I“}

Mead konzentriert sich in seinem Zugang auf die individuelle Entwicklung (bzw. das Selbst) einer Person. Der Identitätsprozess, welcher sich nur in einer Gemeinschaft handelnder sowie kommunizierender Menschen herausbilden kann (vgl. Preglau 2001: 52f.; Krotz 2008: 50ff.; Vester 2009: 142), wird dabei maßgeblich von gesellschaftlichen Prozessen beeinflusst. Mead präsentiert ein Modell, welches die Identitätsbildung einer Person über soziale Interaktionen beschreibt (vgl. Mead 1934; Vester 2009: 141). Er beschreibt die Gesellschaft als wesentliche Grundlage für die Entwicklung des individuellen Selbst und konzentriert sich auf das menschliche Zusammenleben. Gesellschaft definiert Mead als „Haltung und Reaktionen der anderen dem Individuum gegenüber" (Vester 2009: 144). Er legt ein Modell zugrunde, in dem aus der Interaktion der AkteurInnen heraus soziale Prozesse entstehen. Die beteiligten Personen werden als aktiv Handelnde beschrieben, die über ihr Handeln Interaktionen erzeugen und Wissen rekonstruierbar machen (vgl. Abels 2010: 16). Wesentlicher Faktor bei Mead ist die Sprache, die Erfahrungen einer Gesellschaft und ihr Wissen repräsentiert. Erfahrungen basieren auf zugrundeliegenden Symbolen (wie zum Beispiel Gegenständen, Personen, Gesten oder auch Institutionen), welche über die Sprache sichtbar gemacht werden und den Sinn einer Situation oder eines Handlungszusammenhangs aufzeigen (vgl. Mead 1934: 85; 162f.; Abels 2010: 22). Abels fasst den Zugang Meads wie folgt zusammen:

„Persönlichkeit und soziales Handeln sind durch Symbole geprägt, die im Prozess der Sozialisation erworben werden und im Prozess der Interaktion von den Handelnden wechselseitig bestätigt oder verändert werden." (Abels 2010: 17)

Die Bedeutung der Symbole entwickelt sich aus der Art und Weise, wie eine Person auf das Gegenüber reagiert. Bedeutungen sind, Denniger et al. (1973: 83ff.) zufolge, „soziale Produkte“. Symbole müssen von den jeweiligen Personen interpretiert und reflektiert werden und führen zu einem Prozess des Lernens (vgl. Denninger et al. 1973: 83ff.). Über das Handeln der beteiligten Personen finden soziale Interaktionsprozesse statt. Die einzelnen Interaktionspartner bestimmen die zugrundeliegende Interaktion und generieren durch Rollenübernahmen 
Beziehungsstrukturen. Abels definiert die Rollenübernahme über die Fähigkeit „,on der Position des Anderen aus zu denken“ (vgl. Abels 2010: 22). Die angesprochene Rollenübernahme ist das Kernstück von Meads Handlungstheorie. Sie führt über kommunikative Verständigung über das Handeln und die Perspektiven der Interaktionspartner mithilfe der Reflexion zur Identitätsbildung der AkteurInnen (vgl. Abels 2010: 23). Die Rollenübernahme bedingt, dass eine Person Gegenstand ihrer eigenen Handlung und für sich selbst ein Objekt ist. Sie steuert über ihr Handeln ihre eigene Person sowie das Handeln des Gegenübers. Aus der sozialen Interaktion entwickelt sich ein „Selbst-Objekt“, indem andere Personen das eigene Subjekt definieren (vgl. Denniger et al. 1973: 18). Denniger et al. beschreiben den Prozess einer Perspektivenübernahme anhand „eines imaginären deutenden Subjektes außerhalb der eigenen Person“. Mead entwickelte für die Beschreibung dieses Prozesses das Konzept des „Me“ und des „““ (vgl. Denninger et al. 1973: 17; 92; Vester 2009: 143). Die Handelnden stehen bei diesem Prozess in einer Wechselbeziehung zueinander (vgl. Abbildung 21).

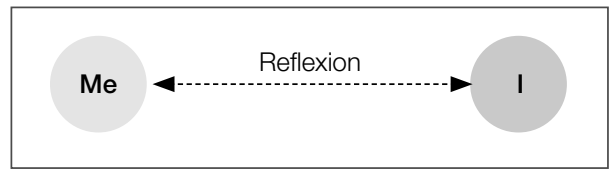

Abb. 21: Interaktionsmodell nach Mead

Mead argumentiert, wenn „eine Person etwas [sagt], so sagt sie es zu sich selbst, was sie zu den Anderen sagt" (Mead 1934: 189). Die angesprochene Selbstbezüglichkeit führt zu einer Identitätsentwicklung, indem das eigene Handeln der Person über die anderen Personen gespiegelt wird (vgl. Mead 1934: 399) oder, wie Abels (2010: 33) argumentiert, „der Einzelne mit den Augen des anderen [sieht]“. Das „Me“ definiert Mead als die Art und Weise, wie beteiligte Personen auf die Handlungen reagieren. Abels (2010: 34) beschreibt es als „reflektiertes Ich“, welches die gesellschaftliche Dimension der Identität repräsentiert. Das eigene Handeln der Person charakterisiert Mead über den Begriff „I“ (vgl. Abels 2013: 33). Abels (2010: 34) sowie Sölch (2014: 200) beschreiben diese Wechselbeziehung über das Einnehmen einer anderen Perspektive, die auf individuelle Haltungen basiert. Abels beschreibt die Einnahme einer neuen Perspektive wie folgt:

„Unter der Perspektive von Identität meint das reflektierte Ich die Seite zugewiesener Identität, die internalisierte Vorstellung von dem Bild, das sich der Andere wahrscheinlich von mir gemacht hat. [...] Das reflektierte Ich enthält die organisierten Werthaltungen, die im Verlauf der Sozialisation erworben werden. Das reflektierte Ich repräsentiert die gesellschaftliche Dimension der Identität. Das ,me' ist das, was das Subjekt über sich selbst im Prozess der Rollenübernahme erfahren hat. Es bezeichnet meine Vorstellung von dem Bild, das der Andere von mir hat, [...]." (Abels 2010: 34).

Diese Übernahme des reflektierten Ichs ist für Mead der Erfolg, wie ein Individuum sein Handeln mit dem erwarteten Handeln der Gesellschaft koordiniert und mit dieser kooperieren kann (vgl. Bonss 2020: 161). Reflexion als kontinuierlicher Prozess führt die Identitäten „Me“ und „I“ zusammen und entwickeln eine stabile Selbstidentität eines Subjekts (vgl. Denninger et al. 1973: 25f.; Vester 2009: 142). Das zugrundeliegende Wissen einer Handlung wird in der Reflexion während des Identitätsprozesses über das „Me“ und das „„“ sichtbar und (weiter-)entwickelt. 
Identitätsbildung einer Person ist, wie oben beschrieben, eng mit gesellschaftlichen Interaktionen verbunden. Dieser Ansatz kann auf die formale Bildung, die Schule, übertragen werden. Schule als Spiegel der Gesellschaft (vgl. Kraler \& Schratz 2012: 89) hat die Aufgabe, mit gesellschaftlichen Prozessen umzugehen und diese adäquat in den Schulalltag zu implementieren. Lehrpersonen und SchülerInnen stehen in einer zwischenmenschlichen Beziehung zueinander und beeinflussen sich gegenseitig in ihrem Lernen und Lehren. Auf das didaktische Dreieck bezogen lassen sich die diskutierten Elemente, wie in Abbildung 22 dargestellt, mit dem pädagogischen professionellen Wissen von Lehrpersonen integrieren. Stehen Inhalt, Lehrpersonen und SchülerInnen in Beziehung, realisiert sich pädagogisches professionelles Wissen in dieser Beziehungskonstellation.

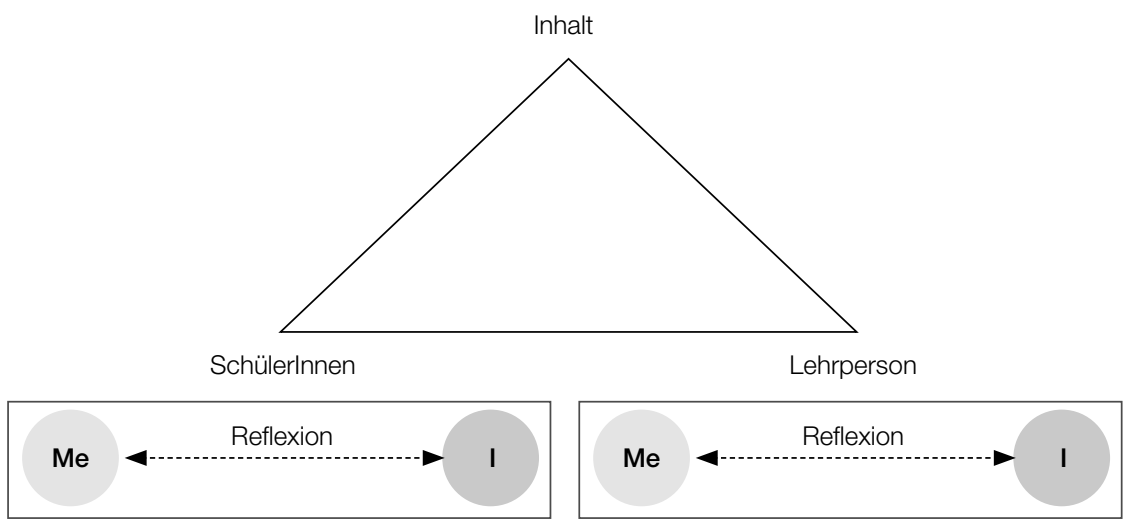

Abb. 22: Didaktisches Dreieck im Kontext von „Me“ und „I“

Pädagogisches Wissen realisiert sich über das Handeln, dem „I“, einer Lehrperson. Dieses steht mit dem Inhalt, welcher über die Sprache zum Ausdruck kommt, mit den SchülerInnen in einem Beziehungsgefüge. SchülerInnen reagieren auf diese Symbole und repräsentieren das Selbst-Objekt der Lehrperson das „Me“. Mithilfe der Reflexion und des gespiegelten „Me“ der SchülerInnen kann pädagogisches Wissen von Lehrpersonen über Reflexion rekonstruiert sowie weiterentwickelt und hinsichtlich den sozialen Vorstellungen angepasst werden. SchülerInnen unterstützen die Lehrpersonen bei der Ausbildung ihrer eigenen professionellen Identität. SchülerInnen repräsentieren das „Me“ und „I“ einer Lehrperson, welche mit Hilfe von Selbstreflexion unter Berücksichtigung sozialer Vorstellungen eine veränderte Identität herbeiführen kann. Das reflektierte Ich der Lehrperson zeigt die zugewiesene Identität, die SchülerInnen spiegeln und wie beteiligte SchülerInnen auf Handlungen reagieren (vgl. Abels 2010: 34). Grundlegender Aspekt ist die Reflexion (vgl. Kapitel 5.2), die Identitäten und Handlungen zusammenführen, greifbar sowie pädagogisches Wissen sichtbar machen.

\subsubsection{Giddens - Soziale Praxis}

Legt Mead den Fokus auf die Identitätsbildung, stellt Giddens (1984) in seiner Strukturierungstheorie zur sozialen Praxis („social theory“) die Struktur und Handlungen in den Mittelpunkt. Er beschreibt eine Dualität von Struktur und Handeln (vgl. Lamla 2003: 45) und erweitertet den Zugang mit einem Konzept der „sozialen Praxis“, in dem, wie bei Mead, die Reflexion als Instrument zur Generierung und Weiterentwicklung von Wissen argumentiert wird. Meads Idee wird von 
Giddens' Theorie über seinen Fokus der Interaktionsprozesse im Zusammenhang von Strukturen ergänzt. Zentral bei Giddens ist sein Strukturbegriff. Jede Handlung setzt Struktur voraus. Strukturen charakterisieren sich über „Regeln“ und „Ressourcen“. Die handelnden Personen werden von Strukturen beeinflusst. Giddens zeigt hier Parallelen zu Habermas, der Normen als handlungsleitend definiert (vgl. Kapitel 2.1). Gleichzeitig gestalten Handelnde die soziale Struktur über ihr individuelles Verhalten (vgl. Welskopp 2001: 104; 108). Strukturen müssen, so Welskopp (2001: 104), „immer durch das Bewußtsein der Handelnden ,hindurch', um reproduziert oder modifiziert zu werden“. Sie dienen als Grundlage für das Handeln sowie als Modus für die Strukturierung gesellschaftlicher Systeme (vgl. Welskopp 2001: 109). „Ressourcen“ beschreibt Giddens über materiale Ressourcen, die mit ihrem Einfluss Macht auf soziale Beziehungen ausüben. Dadurch werden die Möglichkeiten der Handelnden eingeschränkt. Sie stellen gleichzeitig die Mittel bereit, die Handeln ermöglichen. Er unterscheidet zwischen autoritativen und allokativen Ressourcen. Autoritative Ressourcen beziehen sich auf die Koordination des Handelns von Menschen, allokative Ressourcen auf die Kontrolle materieller Produkte bzw. Aspekte der materiellen Welt (vgl. Miebach 2014: 185). Wichtig für den Interaktionsprozess ist die Anwendungsfähigkeit der Ressourcen der beteiligten Personen (vgl. Lamla 2003: 51; Giddens 1984: 33f.). Jede soziale Handlung beschreibt Welskopp (2001: 110), in Bezug auf Giddens, als eine kommunikative, normbezogene und eine machtförmige Dimension. Die Handelnden beziehen sich auf die vorhandenen Regeln und die zur Verfügung stehenden Ressourcen (vgl. Welskopp 2001: 110). Die „Regeln“ definieren sich über soziale und kulturelle Merkmale sozialer Sinnstrukturen, die über die Handlungsfähigkeit zum Ausdruck kommen (vgl. Lamla 2003: 52). Handelnde greifen während ihrer Interaktion unbewusst auf vorhandene Regelsysteme zurück. Giddens postuliert hinsichtlich der sozialen Interaktion, dass Handlungen nicht nur über strukturelle Bedingungen vorgeschrieben werden, sondern diese über das Handeln selbst entstehen (vgl. Reckwitz 1999: 316ff.).

Von grundlegender Bedeutung für Interaktionen ist für Giddens neben Raum, wie auch schon für Mead (1934), die Zeit (vgl. Abbildung 23). Soziale Interaktionen finden in Anlehnung an dieses Verständnis in einer bestimmten Raum-Zeit-Konstellation statt (vgl. Lamla 2003: 46).

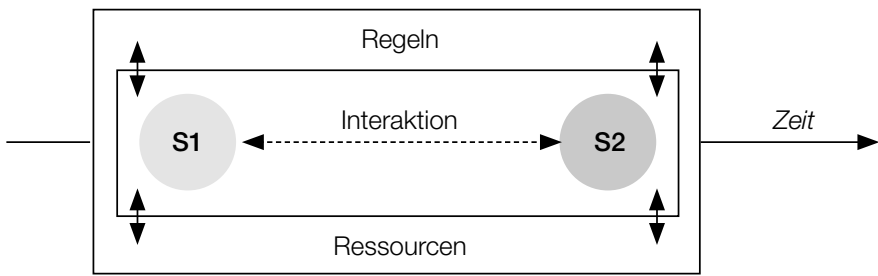

Abb. 23: Soziale Praxis nach Giddens

Der Vollzug einer pädagogischen Handlung wird in Anlehnung an Giddens' Verständnis nicht als „teleologische Aktivität, sondern als ein Tun auf der Grundlage eines praktischen Bewußtseins impliziten Wissens" verstanden (Reckwitz 1999: 316). Wesentlich für die Handlung sind die Bedeutung und das Motiv der handelnden AkteurInnen.

Parallelen zu Mead zeigen sich über die Sprache, die Bedeutung und Beziehung generiert (vgl. Lamla 2003: 47). Die Interaktion über die Sprache und der damit initiierte Handlungskreislauf werden über das Interpretieren und die Reflexion unter Berücksichtigung von Regeln und Ressourcen verstärkt. Reflexive Prozesse der Handelnden („reflexive monitoring of action“) beeinflussen die Aktivitäten der Personen (vgl. Welskopp 2001: 111). Lamla (2003: 13) zufolge 
beschreibt Giddens Menschen als kompetente Individuen und intelligente Beobachter ihrer Umwelt. Wie Mead stützt er sich auf den Gedanken einer Bedeutungsentwicklung über die Interaktion (vgl. Lamla 2003: 14). Die Reflexion der Handlungen führt zu einer Entwicklung diskursiven Wissens zwischen den beteiligten Interaktionspartnern und unterstützt die Weiterentwicklung von Wissen (vgl. Welskopp 2001: 106).

Die beschriebenen Modelle von Mead und Giddens ergänzen sich in ihren konzeptuellen Zugängen. Sie rücken die Interaktion über Sprache in das Zentrum des Interesses. Mead forciert vorwiegend die individuelle Entwicklung, während Giddens den Einfluss von Strukturen für einen Interaktionsprozess beschreibt. Mead und Giddens argumentieren, dass Wissen über das Handeln realisiert und über den Reflexionsprozess weiterentwickelt und angepasst werden kann (vgl. Abels 2010: 16; Welskopp 2001: 111). Interpretation sowie Reflexionen der handelnden Personen erzeugen über den interaktiven Prozess Wissen.

Strukturen spielen im Zusammenhang formaler Bildung eine wesentliche Rolle. Sie beeinflussen das Handeln von Lehrpersonen und SchülerInnen sowie das Lernen und Lehren maßgeblich. Lehrpersonen bilden pädagogisches Wissen unter Berücksichtigung von Strukturen und formen es im Kontext autoritativer und allokativer Ressourcen sowie vorhandener Regeln. Das didaktische Dreieck mit seinen Konstituenten Inhalt (pädagogisches Wissen), Lehrperson und SchülerInnen steht in einem Beziehungsgefüge mit Regeln und Ressourcen (vgl. Abbildung 24).

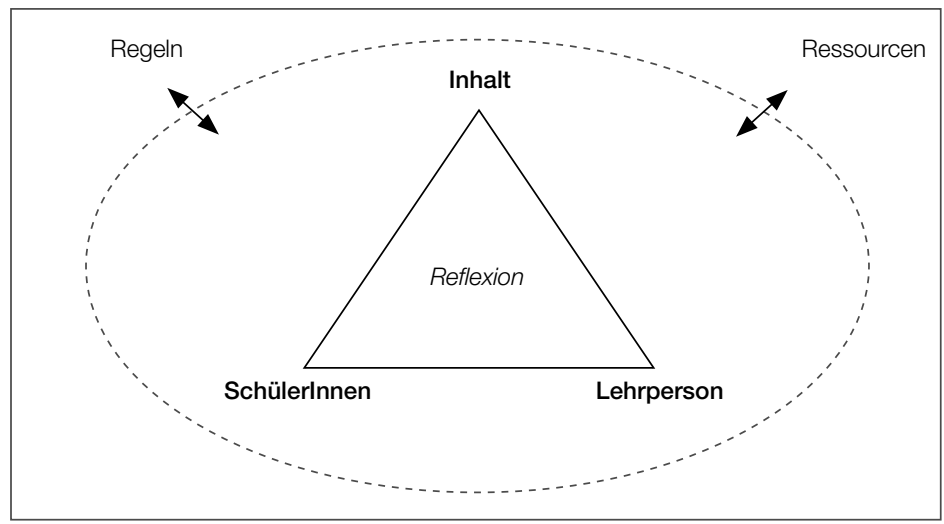

Abb. 24: Didaktisches Dreieck im Kontext der sozialen Praxis von Giddens

Über das Handeln der SchülerInnen und Lehrpersonen wird pädagogisches Wissen unter Berücksichtigung struktureller Regeln und Ressourcen generiert und aufgezeigt. Gleichzeitig entwickeln die Lehrpersonen über einen Interaktionsprozess mit den SchülerInnen eigene Regeln und Ressourcen, die für das Handeln und das Generieren von pädagogischem Wissen grundlegend sind. Lehrpersonen können eigene Strukturen unter Berücksichtigung institutioneller Logiken pädagogischen Wissens aufbauen und entwickeln, die das Lernen und Lehren beeinflussen. Hinzu kommt, dass der Interaktionsprozess in einer bestimmten Raum-Zeit-Konstellation stattfindet, in der pädagogisches Wissen lebt. Dies führt zu einer geschlossenen, in sich bedeutungsvolle Interaktion, die über den wechselseitigen Prozess und mithilfe der Reflexionsfähigkeit der Lehrperson Wissen präsentiert und neues Wissen generiert. 



\section{Empirischer Zugang}

Im Folgenden wird die Diskussion empirisch eröffnet. Es werden die Forschungsfragen sowie das methodologische Vorgehen der vorliegenden Arbeit vorgestellt. Anschließend erfolgt die Darstellung der Ergebnisse der durchgeführten empirischen Untersuchung.

\section{Forschungsfragen}

Ziel der Untersuchung war die Rekonstruktion pädagogischen Wissens aus der Schulpraxis. Bisherige Operationalisierungen verfolgen, insbesondere seit der zweiten empirischen Wende, vorrangig das Ziel, pädagogisches Wissen von Lehrpersonen über quantitative Forschungsansätze zu messen (vgl. Kapitel 4). Ausgehend vom gegenwärtigen Forschungsstand verfolgt die vorliegende Studie das Ziel, den Begriff „pädagogisches Wissen“ um einen rekonstruktiv-qualitativen Zugang aus dem Praxisfeld „Schule“ zu erweitern. Es wird versucht, für die vorliegende Arbeit relevante Ausschnitte in ihrer Heterogenität und unter Berücksichtigung gegenwärtiger sozialer, politischer und wirtschaftlicher Transformationsprozesse zu erfassen. Der Unterrichtsalltag, den Antinomien des pädagogischen Handelns unvorhersehbar machen, kann als durchaus komplex beschrieben werden. Dies bedingt, dass Bildung als solches nicht gemessen werden kann (vgl. Schlömerkemper 2017). Die Rekonstruktion pädagogischen Wissens erfolgt auf dem Verständnis und dem Handeln im Schulalltag von Lehrpersonen und ermöglicht den komplexen Unterrichtsalltag gegenstandsadäquat abzubilden.

Bisherige Untersuchungen von pädagogischem Wissen basieren vorwiegend auf quantitativen Studien, die das Feld nicht zur Gänze erfassen können. Die vorliegende Studie versucht über einen qualitativen Zugang aus der Praxis, der aktuellen Komplexität und den Dynamiken des Schulalltags eine Stimme zu geben und bestehende Befunde zu ergänzen. Da nicht die gesamte Komplexität erfasst werden kann, leistet die vorliegende Untersuchung einen Beitrag, pädagogisches Wissen möglichst gegenstandsadäquat abzubilden.

Diese Arbeit zielt darauf ab, das gegenwärtige Verständnis pädagogischen Wissens sowie seine Herausforderungen für Lehrpersonen aus der Schulpraxis zu rekonstruieren. Ein weiterer Schwerpunkt der Untersuchung liegt auf der Verbindung von Wissen und Handeln. Im Rahmen der Analyse von Unterrichtsbeobachtungen wird pädagogisches Wissen über das Handeln von Lehrpersonen erschlossen. Ein damit zusammenhängendes Ziel ist es zu untersuchen, inwieweit die von den Lehrpersonen genannten Wissensformen in ihrem Handeln beobachtet und ob Übereinstimmungen bzw. Abweichungen kognitiver Muster und pädagogischen Handelns im günstigen Fall aufgezeigt werden können. 
Vor diesem Hintergrund werden in der vorliegenden Untersuchung die folgenden Forschungsfragen behandelt:

1. Was verstehen Lehrpersonen im Schuldienst unter „pädagogischem Wissen“?

2. Wie zeigt sich pädagogisches Wissen im unterrichtlichen Handeln von Lehrpersonen?

3. Welche Übereinstimmungen und Unterschiede zeigen sich zwischen konzeptuellen Mustern pädagogischen Wissens von Lehrpersonen und ihrem konkreten Handeln?

4. Welche aktuellen Herausforderungen für pädagogisches Wissen lassen sich aus der Schulpraxis rekonstruieren?

Die Bearbeitung der Forschungsfragen basiert auf einem rekonstruktiv-qualitativen Zugang mittels ExpertInneninterviews und ethnografischer Unterrichtsbeobachtungen. Dieses Vorgehen ermöglicht eine metatheoretisch gegenstandsbezogene Modellbildung (vgl. Strauss \& Corbin 1996: 148; Przyborski und Wohlrab-Sahr 2008: 43f.) unmittelbar aus der Schulpraxis. 


\section{Methodologisches Vorgehen}

In der vorliegenden Arbeit wird das Feld „Schule“ als komplexes soziales System unter Berücksichtigung bildungswissenschaftlicher, professionsspezifischer und pädagogischer Kontexte interpretiert. Die Arbeit hat zum Ziel, mithilfe eines rekonstruktiven Verfahrens, und unter Berücksichtigung gegenwärtiger Herausforderungen, pädagogisches Wissen von Lehrpersonen mit ihrem faktischen Wissen und Handeln aufzuzeigen und zu rekonstruieren. Die Erfassung des pädagogischen Felds bedarf einer integrativen Sichtweise. Im vorliegenden Fall wird dem über den doppelten Zugang mit Interviews und Beobachtung Rechnung getragen (vgl. Engel 2016: 164). Mittels eines explorativen Vorgangs und der damit verbundenen Generierung von Kategorien wird eine strukturierte und kohärente Darstellung des Felds aus der rekonsturierten Praxis aufgezeigt. Die strukturierte Darstellung über Kategorien wird über Fallstudien zurückgebunden und konkretisiert (vgl. Lamneck 2010: 373ff.). Das Auswertungsverfahren basiert auf der Methode der Grounded Theory von Glaser und Strauss (1967), welche eine auf empirische Daten begründete Theoriegenerierung sozialer Prozesse zum Ziel hat (vgl. Glaser \& Strauss 1967: 5f. ${ }^{31}$ ).

Die Studie lehnt sich an die formulierten Gütekriterien qualitativer Sozialforschung von Strübing et al. (2018: 83ff.) an. Sie beschreiben in ihrem Zugang die Gegenstandsangemessenheit, die textuelle Performanz, die empirische Sättigung, die Originalität sowie die theoretische Durchdringung als zentrale Kriterien qualitativen Arbeitens. ${ }^{32}$

\subsection{Qualitativ-rekonstruktiver Zugang}

Der Anspruch der qualitativ-rekonstruktiven Sozialforschung (vgl. Lamneck 2010: 30f.) gilt als Grundlage des angewendeten methodologischen Zugangs der vorliegenden Forschungsarbeit. Um die soziale Realität möglichst gegenstandsadäquat abzubilden und zu erforschen, liegt der Fokus der Forschungsarbeit auf dem alltäglichen Praxisfeld „Schule“. Der rekonstruktive Zugang ermöglicht das Feld Schule explorativ zu erfassen und neue Modelle pädagogischen Wissens zu generieren. Wie die Gegenstandsangemessenheit von Strübing et al. (2018: 86) beschreibt, können kognitive Muster und dynamisch wechselseitige Prozesse der untersuchten Lehrpersonen abgebildet werden (vgl. Flick 2000: 15ff.; Flick 2008: 49). Im Mittelpunkt des Interesses steht für die gegenstandsadäquate Modellbildung das hypothesen-

31 Die Anfänge der Grounded Theory gehen auf die Ansätze von Strauss und Glaser in den 1960er Jahren zurück. Klassische Texte sind „Awareness of dying“ (1965), „The Discovery of Grounded Theory: Strategies for Qualitative Research“ (1967) sowie die Schrift „Time of Dying“"(1968).

32 Strübing et al. (2018: 85ff.) definieren fünf Gütekriterien qualitativer Sozialforschung. Die Gegenstandsangemessenheit bezieht sich auf die zielorientierte Auswahl des Forschungsvorhabens, des Samples und der Methode auf Basis der vorliegenden Fragestellung. Die empirische Sättigung beschreibt das zu untersuchende Feld, die Breite und Vielfältigkeit des Samples sowie die Intensität der Gewinnung und Analyse der Daten. Sie steht in enger Beziehung mit dem Begriff „theoretisches Sampling“ der Grounded Theory, das in Abschnitt 7.5 diskutiert wird. Kriterien der empirischen Sättigung sind der angemessene Zugang zum Feld, die reflexive Haltung der ForscherIn/des Forschers, ein ausreichendes Sample, der Umfang der Datenerhebung sowie ein interaktivzyklischer Prozess zwischen Datenerhebung und Analyse (vgl. Strübing et al. 2018: 88f.). Die theoretische Durchdringung beschreibt die Tatsache, dass gegenstandsangemessene Forschung ohne Theorie nicht realisierbar ist (vgl. Strübing et al. 2018: 91f.). Bei der textuellen Performanz geht es um die adäquate und nachvollziehbare Darstellung der Forschungsergebnisse. Die Originalität ist der Anspruch, das Gewinnen und das Diskutieren von neuen Erkenntnissen (vgl. Strübing et al. 2018: 94). 
generierende Erkunden des Felds. Dieses wird in der vorliegenden Arbeit systematisch dargestellt und liefert einen Überblick über pädagogisches Wissen. Das Vorgehen ermöglicht für die Studie, relevante professionelle Handlungs-, Orientierungs- und Deutungsmuster sowie Lebens- und Erfahrungsräume zu rekonstruieren (vgl. Von Wensierski 1997: 76; Lamneck 2010: 28). Die dabei entstehenden interdisziplinären Perspektiven ergänzen sich gegenseitig und führen zu einem Gesamtbild des untersuchten Gegenstands (vgl. Flick 2000: 15ff.; Flick 2008: 49). Die Rekonstruktion von subjektiven Perspektiven und Handlungsmustern der Lehrpersonen wird zum Analyseinstrument sozialer Welten (vgl. Flick 1995: 30; Strübing et al. 2018). Von Wensierski und Jakob (1997: 9) charakterisieren das rekonstruktive Vorgehen als eine intersubjektive, wirksame Methode, um handelnde Personen zu verstehen und entsprechend der Realität abzubilden. Zentral für die Rekonstruktion sind die soziale Interaktion und das Handeln von Lehrpersonen. Diese bedingen, wie Wilson (1973/1982), Flick et al. (2000: 20) und Meuser (2006: 140) beschreiben, dass Interpretations- und Konstruktionsprozesse verwirklicht werden können. Im Handeln zeigt sich über Rekonstruktion das Wissen der untersuchten Personen. Dieser methodische Zugang zeigt Parallelen mit den Theorien von Mead (1934) sowie Giddens (1984), die in Kapitel 5.3 der vorliegenden Arbeit diskutiert wurden. Sie stützten sich über einen handlungstheoretischen Zugang auf Modelle, die über Handeln Wissen aufzeigen.

Das rekonstruierte Feld Schule beinhaltet unterschiedliche statische und bewegliche Objekte wie SchülerInnen, Lehrpersonen, Schulleitung, Klassenzimmer, Schulgebäude, externes Fachpersonal sowie Eltern. Das Handeln in diesem Umfeld stellt einen sinnhaften Bezug zur sozialen Realität her und repräsentiert das dynamische Feld Schule, worüber die empirische Sättigung erreicht werden kann (vgl. Strübing et al. 2018: 88). In der vorliegenden Untersuchung beschränkt sich das untersuchte Feld vorwiegend auf Lehrpersonen.

Damit das Feld in der Studie in seiner Fülle wahrgenommen werden kann, ist wie Lamneck (2010: 19ff.) erwähnt und Strübung et al. (2018: 88) bezüglich der empirischen Sättigung und der Gegenstandsangemessenheit fordern, eine offene sowie reflexive Haltung unabdinglich, um neue Entwicklungen, Phänomene und Zusammenhänge zu entdecken und im Verlauf der Forschung neue Modelle zu generieren. Grundvoraussetzung ist eine offene Datenerhebung, um die Komplexität der sozialen Realität zu erfassen (vgl. Flick et al. 2000: 23f.). Für die gegenstandangemessene Erforschung (vgl. Strübing 2018: 86) von pädagogischem Wissen wird in der Studie konkret mit Interviews und Beobachtungen gearbeitet (vgl. Abbildung 25).

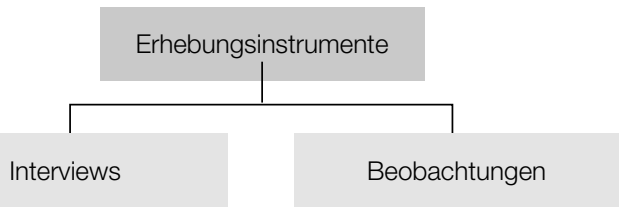

Abb. 25: Datenerhebungsinstrumente der vorliegenden Untersuchung

Der Methodenmix, mit dem Ziel, spezifische Schwächen einer Methode über Stärken einer anderen Methode auszugleichen, kann das Forschungsfeld adäquat abbilden. Können über Interviews nur Einschätzungen und Beschreibungen der befragten Lehrpersonen rekonstruiert werden, erweitern Beobachtungen die Untersuchung, indem konkrete pädagogische Handlungsweisen erfasst werden (vgl. Aeppli et al. 2016: 193). Helmke (2009: 288) misst Beobach- 
tungen ein besonderes Gewicht bei, da über diese die Differenziertheit des Unterrichts sowie dynamische Prozesse und Muster auf dem Feld aufgezeigt und systematisch erfasst werden können.

Der Einsatz von Interviews und Beobachtungen sowie die jeweiligen anschließenden Interpretationen sind im Forschungsprozess eng miteinander verknüpft und bilden ein verzahntes Forschungsinstrument. Dieses garantiert über den zyklischen Prozess die von Strübing et al. (2018: 89f.) geforderte zyklische Interaktion für das Erreichen einer empirischen Sättigung (vgl. Abbildung 26).

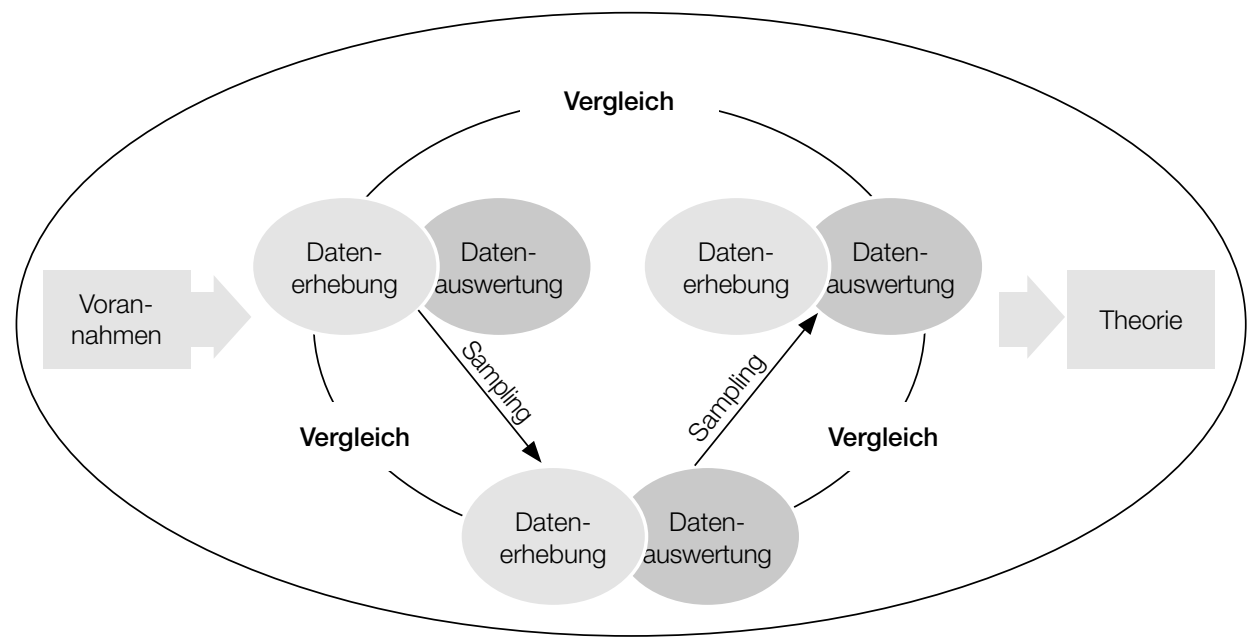

Abb. 26: Forschungsprozess (in Anlehnung an Flick 2007: 128)

Abbildung 26 verdeutlicht, dass der vorliegende Forschungsprozess über einen zirkulären Zugang beschrieben werden kann. Die Datenerhebung mittels Interviews und Beobachtungen und ihre Auswertung stehen in einer zirkulären Beziehung zueinander. Gleichzeitig findet ein permanenter Vergleich mit theoretischen Grundlagen statt (vgl. Flick 2007: 128). Die theoretische Durchdringung als wesentliches Gütekriterium von Strübing et al. (2018: 90f.) wird über diese Verknüpfung erreicht. Über den zyklischen Prozess erfolgt eine kontinuierliche Präzisierung und Modifizierung der generierten Modelle pädagogischen Wissens (vgl. Flick 2007: 126; Lamneck 2010: 80). Die gegenstandsbezogene Modellbildung findet mithilfe eines Verzahnungsprozesses zwischen Datenerhebung, Interpretation und den resultierenden Erkenntnissen statt (vgl. Strauss \& Corbin 1996: 148; Przyborski \& Wohlrab-Sahr 2008: 43f.; vgl. Lamnek 2010: 19ff.).

\subsection{Sample}

Die Auswahl der konkreten Stichproben beruht auf den von Morse (1998: 73) formulierten Kriterien. Diese basieren auf den Kriterien: (1) Verfügung über das notwendige Wissen, ggf. Erfahrung mit dem jeweiligen Thema oder Gegenstand, (2) der Fähigkeit zur Reflexion und Artikulation, (3) dem Vorhandensein von Zeit und (4) der Bereitschaft, an einer Untersuchung teilzunehmen. Charakteristisch für das rekonstruktiv-qualitative Forschen ist das Arbeiten mit 
kleinen Stichproben, die das soziale Feld repräsentieren (vgl. Patton 2002: 230). Das vorliegende Sample besteht aus 26 Lehrpersonen. Diese sind zum Zeitpunkt der Erhebung in unterschiedlichen Schulformen im Schuldienst tätig. Die Lehrpersonen zeigen eine hohe Übereinstimmung mit dem Kriterium der empirischen Sättigung (vgl. Strübing et al. 2018: 89), indem sie alle einen Bezug zum untersuchten Gegenstand (pädagogisches Wissen) aufweisen. Um die Komplexität zu garantieren, stützt sich die Auswahl neben dem Kriterium, das Feld möglichst breit zu erfassen, vor allem auf eine tiefgründige Analyse der einzelnen Personen (vgl. Flick 2007: 167) sowie auf die gegenstandsadäquate Abbildung für die Rekonstruktion pädagogischen Wissens (vgl. Strübing et al. 2018: 88ff.).

Flick (2000: 79ff.) unterscheidet bei der Auswahl der Stichprobe zwei mögliche Vorgehensweisen: das statistische und das theoretische Sampling. Die vorliegende Arbeit orientiert sich an dem Verfahren des theoretischen Samplings, welches sich aus dem Prozess der Grounded Theory ableitet und eine enge Verflechtung zwischen Theorie, Datenerhebung und Auswertung mit dem Ziel der Entwicklung von Modellen beabsichtigt (vgl. Strauss \& Corbin 1996: 148). Die Untersuchung wurde im vorliegenden Prozess abgeschlossen, sobald eine theoretische Sättigung bei den Lehrpersonen erreicht wurde. Diese zeichnet sich dadurch aus, dass kein weiterer Zuwachs der Erkenntnisse für eine Theoriebildung stattgefunden hat (vgl. Strauss \& Corbin 1967: 68; Flick 2007: 213; Przyborski \& Wohlrab-Sahr 2008: 178ff.).

Das untersuchte Sample charakterisiert sich über unterschiedliche soziodemografische Eckdaten. Tabelle 4 gibt einen Überblick über die soziodemografischen Eckdaten der in der Forschungsarbeit untersuchten Lehrpersonen.

Tab. 4: Soziodemografische Daten des ausgewählten Samplings

\begin{tabular}{ccrrlll}
\hline $\begin{array}{l}\text { Per- } \\
\text { son }\end{array}$ & $\begin{array}{l}\text { Ge- } \\
\text { schlecht }\end{array}$ & $\begin{array}{l}\text { Al- } \\
\text { ter }\end{array}$ & $\begin{array}{l}\text { Dienst- } \\
\text { jahre }\end{array}$ & Schultyp & Unterrichtsfächer & Ausbildungsinstitution \\
\hline 1 & w & 26 & 4 & Grundschule & Alle Unterrichtsfächer & Pädagogische Hochschule \\
2 & w & 45 & 20 & Gymnasium & Geschichte/Sport & Universität \\
3 & m & 29 & 5 & Grundschule & Alle Unterrichtsfächer & Pädagogische Hochschule \\
4 & w & 42 & 25 & Gymnasium & Ethik/Deutsch & Universität \\
5 & w & 30 & 7 & Gymnasium & Musik/Ethik & Universität \\
6 & m & 31 & 6 & Neue Mittelschule & Sport/Deutsch & Pädagogische Hochschule \\
7 & m & 63 & 34 & Gymnasium & Sport/Englisch & Universität \\
8 & w & 43 & 20 & Neue Mittelschule & Geschichte/Englisch & Universität \\
9 & m & 31 & 3 & Neue Mittelschule & Englisch/Geografie & Universität \\
10 & w & 29 & 4 & Grundschule & Alle Unterrichtsfächer & Pädagogische Hochschule \\
11 & w & 23 & 2 & Grundschule & Alle Unterrichtsfächer & Pädagogische Hochschule \\
12 & M & 40 & 22 & Gymnasium & Geschichte/Deutsch & Universität \\
13 & m & 37 & 18 & Neue Mittelschule & Englisch/Werken & Pädagogische Hochschule \\
14 & w & 62 & 45 & Neue Mittelschule & Deutsch/Biologie & Pädagogische Hochschule \\
15 & m & 32 & 6 & Gymnasium & Instrumentalerziehung & Universität \\
16 & w & 53 & 26 & Gymnasium & Deutsch/Geschichte & Universität
\end{tabular}




\begin{tabular}{|c|c|c|c|c|c|c|}
\hline $\begin{array}{l}\text { Per- } \\
\text { son }\end{array}$ & $\begin{array}{l}\text { Ge- } \\
\text { schlecht }\end{array}$ & $\begin{array}{l}\text { Al- } \\
\text { ter }\end{array}$ & $\begin{array}{l}\text { Dienst- } \\
\text { jahre }\end{array}$ & Schultyp & Unterrichtsfächer & Ausbildungsinstitution \\
\hline 17 & $\mathrm{~m}$ & 42 & 24 & Neue Mittelschule & Musik/Mathematik & Pädagogische Hochschule \\
\hline 18 & $\mathrm{w}$ & 43 & 14 & Grundschule & Alle Unterrichtsfächer & Pädagogische Hochschule \\
\hline 19 & $\mathrm{~m}$ & 38 & 21 & Grundschule & Alle Unterrichtsfächer & Pädagogische Hochschule \\
\hline 20 & $\mathrm{w}$ & 33 & 6 & Grundschule & Alle Unterrichtsfächer & Pädagogische Hochschule \\
\hline 21 & $\mathrm{w}$ & 29 & 3 & Neue Mittelschule & Kunst/Sport & Pädagogische Hochschule \\
\hline 22 & $\mathrm{w}$ & 33 & 10 & Gymnasium & Mathematik/Physik & Universität \\
\hline 23 & $\mathrm{w}$ & 27 & 1 & Neue Mittelschule & Mathematik/Chemie & Pädagogische Hochschule \\
\hline 24 & $\mathrm{w}$ & 37 & 18 & Grundschule & Alle Unterrichtsfächer & Pädagogische Hochschule \\
\hline 25 & $\mathrm{w}$ & 24 & 2 & Grundschule & Alle Unterrichtsfächer & Pädagogische Hochschule \\
\hline 26 & $\mathrm{w}$ & 42 & 26 & Neue Mittelschule & Physik/Deutsch & Pädagogische Hochschule \\
\hline
\end{tabular}

Wie in Tabelle 4 dargestellt, besteht das vorliegende Sample aus Lehrpersonen unterschiedlicher Schultypen, Unterrichtsfächer, mit unterschiedlichem Alter, einer unterschiedlichen Anzahl von Dienstjahren sowie unterschiedlichem Geschlecht. Über den mehrperspektivischen Zugang und die Berücksichtigung verschiedener soziodemografischen Daten kann die empirische Sättigung, im Sinne von Strübung et al. (2018: 89), garantiert werden. Es werden in der vorliegenden Studie Lehrpersonen aller zentraler Schultypen in Österreich - Grundschule, Neue Mittelschule, Gymnasium (AHS) - berücksichtigt ${ }^{33}$ (vgl. Abbildung 27).

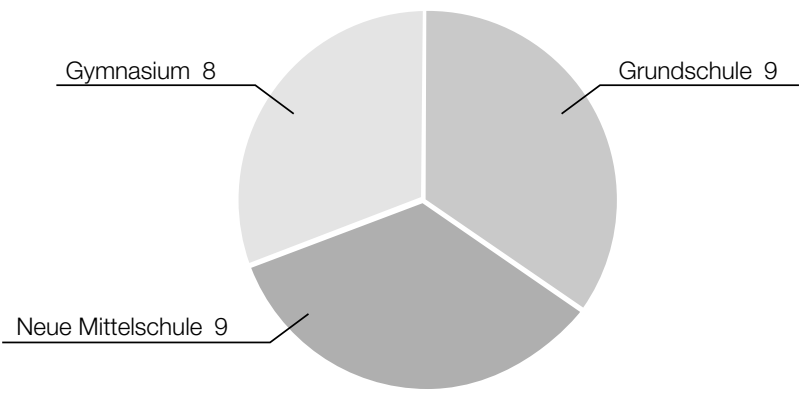

Abb. 27: Überblick Anzahl Lehrpersonen nach Schultyp

Von den ausgewählten 26 Lehrpersonen sind jeweils neun Lehrpersonen aus einer Grundschule sowie einer Neuen Mittelschule und acht aus dem Gymnasialbereich. Die Art der Ausbildung ergibt sich aus ihrer jeweiligen Schulform. Lehrkräfte aus der Grundschule und Neuen Mittel-

33 Das österreichische Schulsystem untergliedert sich in drei verschieden Bereiche: Primarstufe (1. bis 4. Schulstufe), Sekundarstufe I (5. bis 8. Schulstufe) sowie der Sekundarstufe II (9. bis 13. Schulstufe). Die Primarstufe bezieht sich auf den Grundschulbereich. Sekundarstufe I beinhaltet die Neue Mittelschule sowie die Unterstufe der Allgemeinbildenden höheren Schulen (AHS). Die Sekundarstufe II umfasst die Oberstufe der Allgemeinbildenden höheren Schulen (AHS) sowie Berufsbildende höhere Schulen (BHS). In der vorliegenden Studie wird der Fokus auf die Primarstufe der Grundschule, die Sekundarstufe I der Neuen Mittelschule und AHS sowie die Sekundarstufe II der AHS gelegt. Untersuchte AHS-Lehrpersonen der Sekundarstufen I und II werden für ein gemeinsames Verständnis als Lehrpersonen des Gymnasiums bezeichnet. Ab September 2019 werden Neue Mittelschulen zu Mittelschulen umbenannt. Diese Änderung wird in der Forschungsarbeit nicht berücksichtigt. 
schule haben das Lehramtsstudium an einer Pädagogischen Hochschule (früher Pädagogische Akademie), Lehrpersonen aus dem Gymnasium an der Universität absolviert. Es wurden Lehrpersonen unterschiedlichen Alters und mit einer unterschiedlichen Anzahl von Dienstjahren in der vorliegenden Arbeit untersucht (vgl. Abbildung 28).

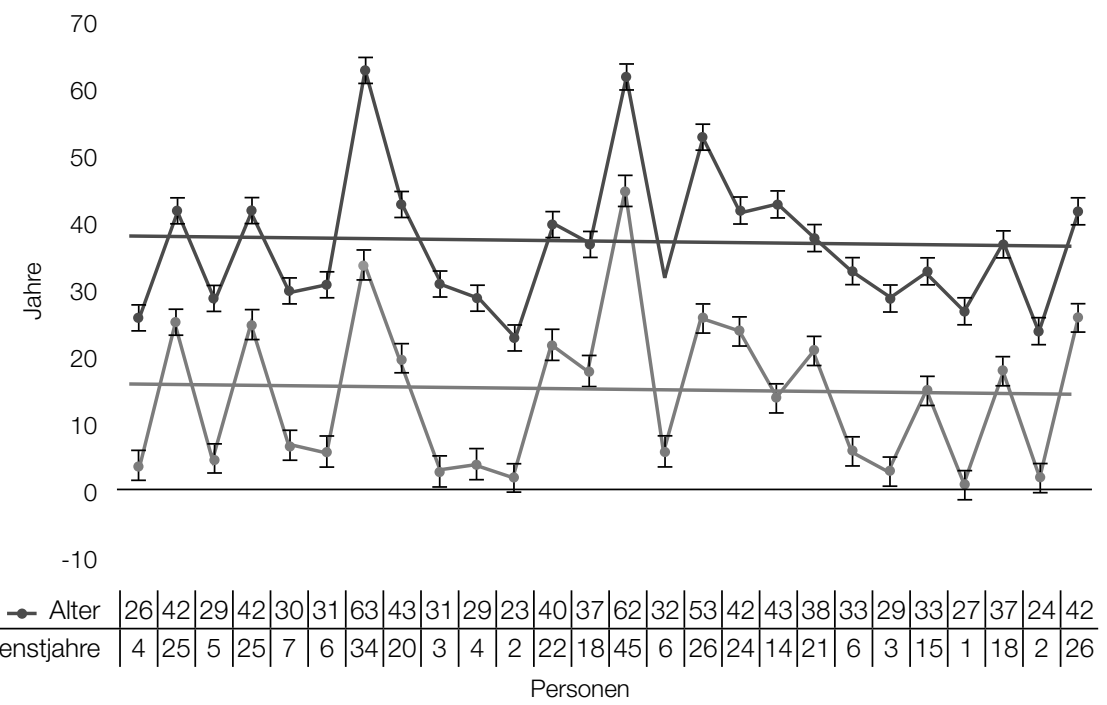

Abb. 28: Überblick Alter und Dienstjahre der Lehrpersonen

In Abbildung 28 sind das Alter und die Dienstjahre der untersuchten Lehrpersonen wiedergegeben. Das Dienstalter der untersuchten Personen reicht von einem Jahr bis zu insgesamt 45 Jahren, der Durchschnittswert des Dienstalters liegt bei 15 Jahren, die Streuung beträgt elf Jahre. Das Alter der untersuchten Personen variiert zwischen 23 und 63 Jahren, das Durchschnittsalter liegt bei 38 Jahren, die Streuung beträgt 10 Jahre.

Um ein fächerübergreifendes Wissen zu rekonstruieren, wurden Lehrpersonen für eine umfassende Rekonstruktion pädagogischen Wissens unterschiedlicher Unterrichtsfächer ausgewählt. Abbildung 29 zeigt die in der Studie berücksichtigten Unterrichtsfächer, kategorisiert nach Schultyp.

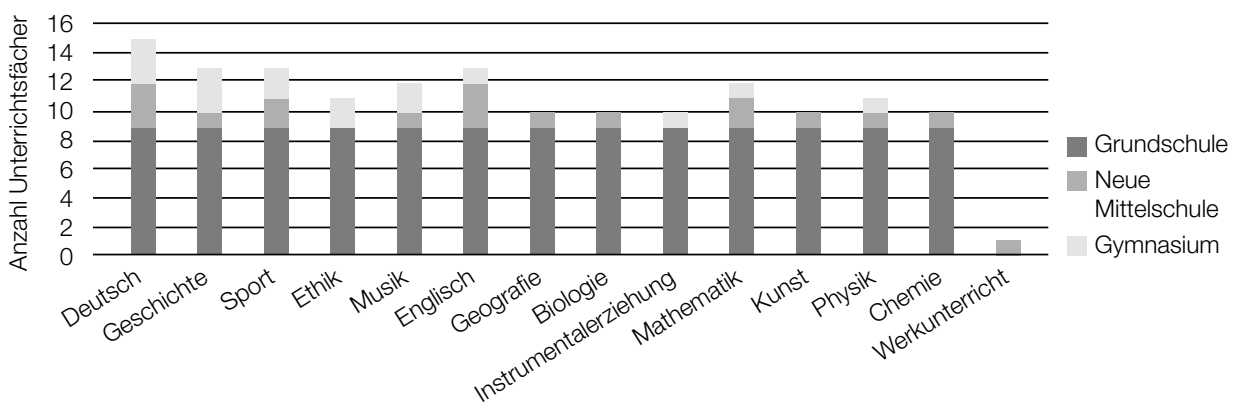

Abb. 29: Überblick Unterrichtsfächer der Lehrpersonen nach Schultyp 
Die untersuchten Gymnasiallehrpersonen verteilen sich auf die Unterrichtsfächer Deutsch, Geschichte, Sport, Musik und Ethik, Englisch, Mathematik, Physik sowie Instrumentalerziehung. Die ausgewählten Lehrpersonen aus der Neuen Mittelschule unterrichten hauptsächlich Englisch, Deutsch, Mathematik sowie Sport. Weitere berücksichtigte Unterrichtsfächer sind Geschichte, Musik, Geografie, Biologie, Kunst, Physik, Chemie und Werken. Grundschullehrpersonen sind Lehrpersonen, die dem KlassenlehrerInnenprinzip folgen. Sie sind generalisiert, d.h. ohne Schwerpunkte, ausgebildet.

Berücksichtigt man das Verhältnis der Geschlechter im LehrerInnenberuf, sind mehr Frauen als Männer im Schuldienst tätig. Diese Verteilung korreliert mit dem vorliegenden Sample (vgl. Abbildung 30).

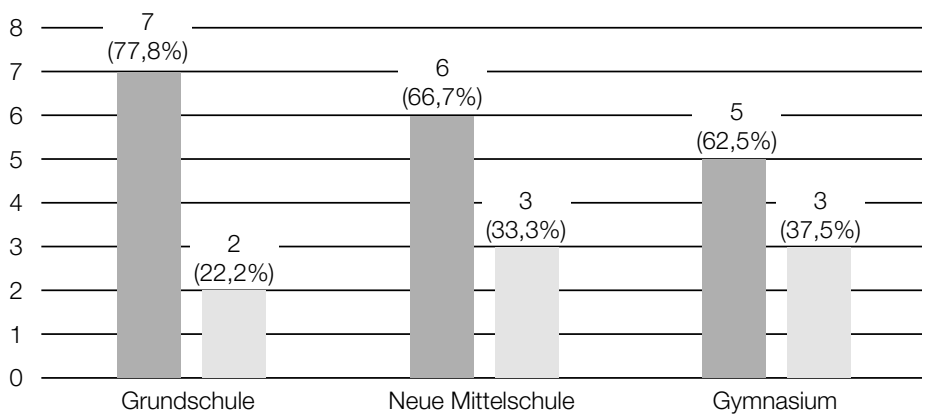

Abb. 30: Überblick Geschlechteranteil der Lehrpersonen nach Schultyp

Die Abbildung zeigt einen Überblick über die Geschlechterverteilung, aufgelistet nach den jeweiligen Schultypen. Von den ausgewählten 26 Personen sind 18 Lehrpersonen weiblich und acht männlich. Der Vergleich mit der Statistik Austria (Schuljahr 2017/2018) zeigt Parallelen mit der hier berücksichtigten Geschlechterverteilung. In allen drei Schultypen ist der Anteil weiblicher Lehrpersonen größer als männliche Lehrpersonen (vgl. Statistik Austria 2019).

Die Anzahl der untersuchten Lehrpersonen variiert je nach Anwendung des Erhebungsinstruments (vgl. Abbildung 31). Bei den Interviews wurden alle in Tabelle 4 abgebildeten Lehrpersonen befragt. Die Feldbeobachtungen stützen sich auf eine Auswahl der Befragten. Przyborski und Wohlrab-Sahr (2008: 76) weisen darauf hin, dass sich das ausgewählte Beobachtungssample vom Gesamtsample unterscheiden kann. Hierfür wurden aus allen drei Schultypen neun der interviewten Lehrpersonen für die Beobachtungseinheiten ausgewählt.

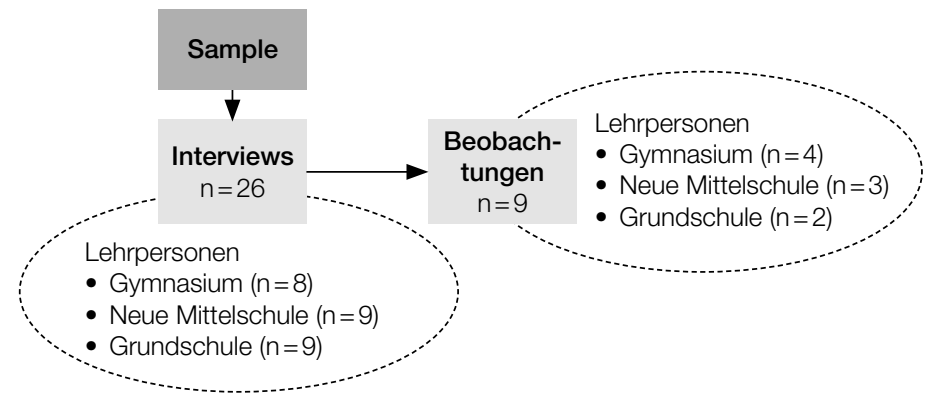

Abb. 31: Überblick Sample Interviews und Beobachtungen 
Von den neun ausgewählten Lehrpersonen wurden zwei Lehrpersonen aus der Grundschule, drei Lehrpersonen aus der Neuen Mittelschule und vier Lehrpersonen aus dem Gymnasium beobachtet. Das Alter der beobachteten Personen variiert zwischen 26 und 63 Jahren, das Dienstalter zwischen drei und 34 Jahren. Es wurden mehr weibliche als männliche Lehrpersonen beobachtet. Sie zeichnen sich durch unterschiedliche Unterrichtsfächer aus. Tabelle 5 gibt einen Überblick über die soziodemografischen Daten der ausgewählten ExpertInnen der Beobachtungen.

Tab. 5: Soziodemografische Daten - Beobachtungssample

\begin{tabular}{clcccl}
\hline Person & Schultyp & Geschlecht & Alter & Dienstjahre & Unterrichtsfächer \\
\hline 1 & Grundschule & w & 26 & 4 & Alle Unterrichtsfächer \\
2 & Grundschule & m & 29 & 5 & Alle Unterrichtsfächer \\
3 & Neue Mittelschule & m & 31 & 6 & Sport/Deutsch \\
4 & Neue Mittelschule & w & 43 & 20 & Geschichte/Englisch \\
5 & Neue Mittelschule & m & 31 & 3 & Englisch/Geografie \\
6 & Gymnasium & w & 42 & 25 & Ethik/Deutsch \\
7 & Gymnasium & w & 30 & 7 & Musik/Ethik \\
8 & Gymnasium & w & 45 & 20 & Geschichte/Sport \\
9 & Gymnasium & m & 63 & 34 & Sport/Englisch \\
\hline
\end{tabular}

Anzahl weiblicher Lehrpersonen $=5$

Anzahl männlicher Lehrpersonen $=4$

Mittelwert Alter $=37$

Mittelwert Dienstjahre $=13$

Um valide Ergebnisse zu erlangen, ist trotz des Kriteriums der Offenheit ein Grad an Standardisierung und Kontrolle essenziell (vgl. Flick 2007: 174f.). Dies kann über eine detaillierte Planung bezogen auf das Sample und den Untersuchungszeitraum sowie durch eine gezielte Auswahl von Erhebungsinstrumenten erreicht werden.

\subsection{ExpertInneninterviews}

Die erste Erhebungsphase wurde mittels themenzentrierter ExpertInneninterviews $(n=26)$ durchgeführt. Der Fokus lag bei der subjektiven Beschreibung und Interpretation des Verständnisses pädagogischen Wissens der untersuchten Personen (vgl. Flick 2007:201; Strübing et al. 2018: 86). Zur Unterstützung der Datenerhebung wurde ein halbstandardisierter Leitfaden entwickelt. Die vorstrukturierten Fragen bieten den Lehrpersonen ausreichenden Freiraum, ihre subjektiven Perspektiven, Annahmen und jeweiligen Expertisen in einem offenen Gespräch darzulegen. Sie unterstützen das von Lamneck (2010: 19ff.) geforderte Prinzip der Offenheit (vgl. Mayring 1999: 51f.; Flick 2007: 195ff.; Hug \& Poscheschnik 2010: 103). Mithilfe der Interviews wurden das individuelle Verständnis pädagogischen Wissens, die subjektive Einschätzung eigenen Handelns sowie die individuelle Umsetzung pädagogischen Wissens im Unterricht erhoben. Dabei standen Gedanken und Konzepte der einzelnen Lehrpersonen im Vordergrund. Die untersuchten Personen verfügen aufgrund ihrer Ausbildung und Tätigkeit im LehrerInnenberuf über einen komplexen Wissensbestand zum Thema und reagieren spontan auf offene Fragen, wodurch eine empirische Sättigung und die Gegenstandsangemessenheit 
der Untersuchung garantiert werden kann (vgl. Flick 2007: 203ff.; Aeppli et al. 2010: 184f.; Strübing et al. 2018: 88).

Die empirische Sättigung sowie die Gegenstandsangemessenheit (vgl. Strübing et al. 2018: 86ff.) wurde im Zuge der durchgeführten halb-standardisierten Interviews an den jeweiligen Schulen der einzelnen Lehrpersonen berücksichtigt (vgl. Lamneck 2010: 354). Die gewohnte Umgebung sorgte für eine angenehme Atmosphäre für die Befragten, was eine größere Tiefe bei den Interviews unterstützte (vgl. Frick 2007: 198). Insgesamt dauerten die Interviews zwischen minimal 30 und maximal 50 Minuten. Die Lehrpersonen hatten vor dem Interview keinen Zugang zu den Fragen und konnten sich nicht auf das Gespräch vorbereiten. Die Gespräche wurden mithilfe eines Diktiergeräts aufgenommen und anschließend anonymisiert und transkribiert (vgl. Gläser \& Laudel 2006: 188f.). Um Vergleichbarkeit sowie die Gegenstandsangemessenheit zu erhöhen, wurden zusätzlich schriftliche Memos nach jedem durchgeführten Interview verschriftlicht (vgl. Gläser \& Laudel 2006: 187f.; Strübing et al. 2018: 86f.). Inhalte, die in den Memos festgehalten wurden, beziehen sich auf die Rahmenbedingungen (Ort, Dauer, Störfaktoren), Bemerkungen zum Gesprächsverlauf sowie eigene Anmerkungen und Gedanken.

Eingeleitet wurden die Interviews mit einer themenspezifischen allgemeinen und erzählgenerierenden Frage. Während des Interviews wurde darauf geachtet, dass alle für die Studie relevanten Fragen behandelt wurden. Zusätzlich wurden Verständnisfragen eingesetzt, die zu einer thematische Tiefe und Klarheit in den Interviews führen. Der Interviewprozess wurde mit der erzählgenerierenden Frage „Was verstehen Sie unter dem Begriffpädagogisches Wissen?" eingeleitet. Lehrpersonen konnten durch die Frage ihre ersten allgemeinen Gedanken und das individuelle Verständnis zum untersuchten Thema verbalisieren. Ausgehend von der Kernfrage wurden sie anschließend anhand spezifischer Fragen bezogen auf das eigene Handeln, das unterrichtsnahe Verständnis pädagogischen Wissens sowie das Wissen im Umgang mit aktuellen Veränderungsprozessen befragt. Lehrpersonen wurden gefordert, basierend auf ihrem Wissen, praxisnahe Fragen zu beantworten. Folgende Fragestellungen bilden das weitere Interviewgerüst:

- Welches pädagogische Wissen brauchen Lehrpersonen Ihrer Erfahrung nach zum Unterrichten?

- Falls kein pädagogisches Wissen im Unterricht vorhanden wäre, wie würde ein Unterricht ablaufen?

- Woran kann man erkennen, dass eine Lehrperson ein fundiertes pädagogisches Wissen besitzt?

- Der Umgang mit sozio-politischen Veränderungsprozessen ist seit einiger Zeit ein essenzielles Thema in der Schule. Welches pädagogische Wissen braucht man ibrer Erfahrung nach im Unterricht, um Veränderungsprozesse in der Klasse adäquat zu berücksichtigen?

- Wenn Sie gegenwärtige und zukünftige Herausforderungen anschauen, welches pädagogische Wissen scheint Ihnen dabei besonders wichtig?

Während der Interviews lag ein thematischer Schwerpunkt auf dem Erwerb und Aufbau pädagogischen Wissens. Das gezielte Fragen danach, wo Lehrpersonen ihr pädagogisches Wissen erworben hätten, fordert sie, sich über den Aneignungszeitraum sowie Aneignungsort Gedanken zu machen.

Zusätzlich zur Rekonstruktion pädagogischen Wissens hat die vorliegende Untersuchung zum Ziel, kognitive Muster mit beobachtetem Handeln zu vergleichen. Um diesen Forschungs- 
schwerpunkt zu berücksichtigen, wurden während der Interviewdurchführung zielgerichtete Fragen an die Lehrkräfte gestellt:

- Welches spezifische pädagogische Wissen wenden Sie in ihrem Unterricht an?

- Wiekann man von außen erkennen, dass Siepädagogisches Wissen beim Handeln berücksichtigen?

Die Lehrpersonen charakterisieren basierend auf diesen Fragestellungen ihr Verständnis pädagogischen Wissen und berichten, wie sie es im Unterricht anwenden. Die digitalen Aufzeichnungen der Interviews sowie die im Anschluss erfassten Memos wurden in einem nächsten Schritt computerunterstützt transkribiert. Für die Transkriptionen wurden wichtige umgangssprachliche Ausdrücke vom Dialekt in das Hochdeutsche gebracht. Sonstige Merkmale eines Interviews, wie Intonationsunterschiede (z.B. Lautstärke, Betonung), Sprachpausen, nonverbale Gesten (z.B. Schmunzeln, Lachen, Stirnrunzeln) und sonstige Geräusche (z.B. das Klingeln der Schulglocke) wurden in den Transkriptionen nicht berücksichtigt.

\subsection{Unterrichtsbeobachtungen}

Das zweite Instrument stützt sich auf ethnografische Beobachtungen, die mit neun Lehrpersonen durchgeführt wurden. ${ }^{34}$ Bloomberg et al. (1993: 123ff.) entwickelten vier grundlegende Merkma-

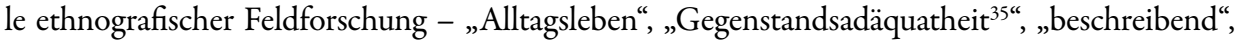
„teilnehmende Beobachtung“ -, die sich auf den vorliegenden Beobachtungsprozess anwenden lassen. Der ethnografische Zugang ermöglicht, die soziale Realität und die Handlungsvorgänge der Lehrpersonen aus der alltäglichen Schulpraxis komplex zu rekonstruieren. Die teilnehmende Beobachtung gewährt einen direkten Einblick in die alltägliche Lebenswelt der Interaktionspartner, um Handlungen und Handlungszusammenhänge zu verstehen und diese gegenstandsadäquat abzubilden (vgl. Bauer et. al 1996: 58; Strübing et al. 2018: 86f.).

Der ethnografische Zugang ermöglicht für die vorliegende Untersuchung, die vorhandenen Wissensbestände und -formen zu rekonstruieren, und lenkt den Blick auf Interaktionen, Handlungsmuster und Diskurse der Lehrpersonen (vgl. Lüders 2000: 390). Für das Erkennen relevanter pädagogischer Handlungsweisen und das pädagogische Wissen ist ein pädagogischer Blick grundlegend (vgl. Schmidt et al. 2016: 7). Dieser unterscheidet zwischen Gegenstandsbestimmung und Auffassung. Diese beziehen sich wiederum auf sichtbare pädagogische Handlungen sowie nicht sichtbare Muster (vgl. Schmidt et al. 2016: 26). In der Forschungsarbeit sind beide Ebenen, Gegenstandsbestimmung und Auffassung, die in einem Wechselverhältnis zueinander stehen, für die Herstellung von Realitätsbezügen und die Rekonstruktion pädagogischer Wissensformen bedeutsam (vgl. Schmidt et al. 2016: 29; Strübing et al. 2018: 86). Pädagogisches Wissen wird über direkte sowie indirekte Symbole und Handlungen rekonstruiert. Für die Gewährleistung der empirischen Sättigung und eine gegenstandsädaquate Darstellung in der vorliegenden Untersuchung ist eine reflexive Haltung gegenüber dem eigenen Vorgehen, der eigenen Wahrnehmung sowie dem Einfluss subjektiver Muster und Wertvorstellungen von essenzieller Bedeutung (vgl. Lüders 2000: 395; Strübing et al. 2018: 89). Mittels reflexiver Haltung, wie sie Strübling (2018: 88) fordert, und der interpersonalen Konsensbildung werden persönliche Urteile sowie selektive pädagogische Blicke verringert und eine, wie von Wulf gefordert, wechselnde Fokussierung des untersuchten Gegenstands angestrebt.

34 Auswahlbegründung des Samplings sowie ihre sozio-demografischen Eigenschaften sind in Abschnitt 7.2 ausführlich beschrieben.

35 Das Kriterium „Gegenstandsadäquatheit“ wird bei Bloomberg et al. (1993: 123ff.) unter dem Begriff „Ganzheitlichkeit" subsumiert. 
Der Verlauf der Unterrichtsbeobachtung orientiert sich an einem von Seidel und Prenzel (2010: 145) entwickelten Phasen-Modell ${ }^{36}$, das in Abbildung 32 dargestellt wird:

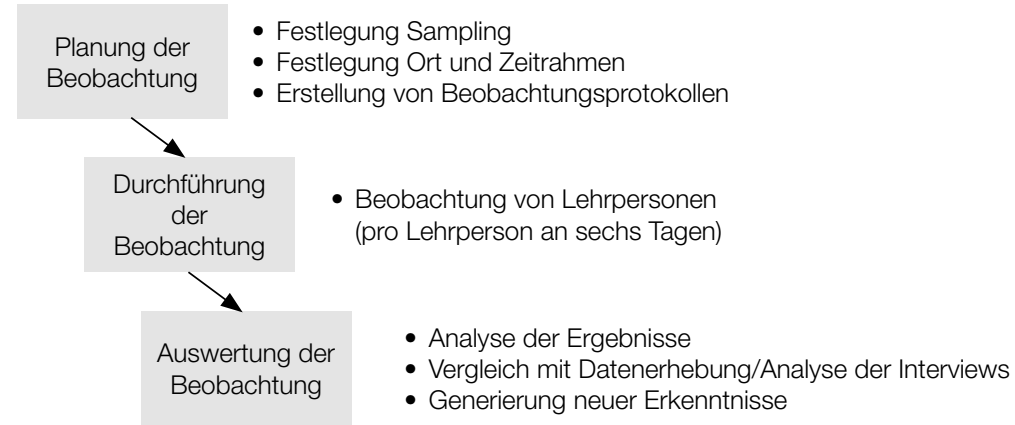

Abb. 32: Beobachtungsablauf - dreistufiges Modell

Der Ablauf der Beobachtungen basiert auf einem dreistufigen Modell, das sich in Planung, Durchführung und Auswertung unterteilt. Im vorliegenden Kapitel wird der Schwerpunkt auf die Planung sowie die Durchführung gelegt. ${ }^{37}$ Der Beobachtungsprozess fordert eine strukturierte Planung und systematisches, transparentes Vorgehen (vgl. Ziefuß 1987: 8f.; Strübing et al. 2018: 86ff.). Die Beobachtungen wurden pro Lehrperson in der Schulzeit an insgesamt sechs Tagen durchgeführt. Die Anzahl der Stunden kam, wie in Abschnitt 7.2 beschrieben, über das Prinzip der theoretischen Sättigung zustande. Kriterium hierfür war, dass kein weiterer Erkenntnisgewinn für die Rekonstruktion pädagogischen Wissens stattgefunden hat. Die Beobachtungsphasen bestanden aus zwei Beobachtungsintervallen (vgl. Abbildung 33).

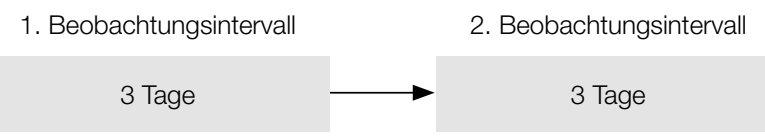

Abb. 33: Beobachtungsintervalle

Im ersten und im zweiten Beobachtungsintervall wurden die Lehrpersonen jeweils an drei aufeinanderfolgenden Tagen im Forschungsfeld „Schule“ beobachtet. Zwischen den Beobachtungsintervallen lag eine Pause von ca. zehn Wochen.

36 Das Modell von Seidel \& Prenzel (2010:145f.) stützt sich auf fünf Phasen der Beobachtungsdurchführung, die im Folgenden dargestellt werden.

- Theorie: Forschungsbereiche, Forschungsfrage, Hypothesen

- Entwicklung: Wahl der Stichprobe, Formulierung der Kodieranleitung

- Vortest: Validierung der Kategorien und Überprüfung der Beobachtungsübereinstimmung an einer Stichprobe

- Kodierung und Datenanalyse: Durchführung der Kodierung

- Interpretation: Interpretation der Ergebnisse, Diskussion auf Basis der Fragestellungen

- Die angeführten Phasen stehen in einem zyklischen Prozess zueinander.

37 Das zu untersuchende Sample für die Unterrichtsbeobachtungen wurde in Abschnitt 7.2 beschrieben. Das Auswertungsverfahren der Unterrichtsbeobachtungen basiert auf dem Verfahren der Grounded Theory. Dieses wird im nachfolgenden Abschnitt diskutiert. 
Die Lehrpersonen wurden in Summe zwischen 14 und 28 Stunden beobachtet. Die Stundenanzahl variiert aufgrund der natürlichen Verteilung der Stundenanzahl jeder Lehrperson an den Beobachtungstagen. Die Gesamtbeobachtungszeit aller ExpertInnen beträgt insgesamt 167 Stunden, der Durchschnittswert liegt bei 18,5 Stunden pro Lehrperson. Die Dauer und der Umfang der Beobachtungen ermöglichen, einen entsprechenden Datenkorpus für die empirische Sättigung zu bilden (vgl. Strübing et al. 2018: 89).

Mithilfe der Unterrichtsbeobachtungen werden in der Studie zwei Forschungsziele verfolgt. Zum Ersten wird eine ergänzende Rekonstruktion pädagogischer Wissensformen zu den durchgeführten Interviewdaten angestrebt. Als Zweites wird untersucht, inwieweit sich die von den Lehrpersonen genannten Wissensbereiche in ihrem unterrichtlichen Handeln beobachten lassen. Die Beobachtung stützte sich auf zwei Komponenten: die strukturierte und die unstrukturierte Beobachtung. Abbildung 34 gibt einen Überblick über die Anwendungsbereiche und Ziele der zwei eingesetzten Beobachtungsinstrumente.

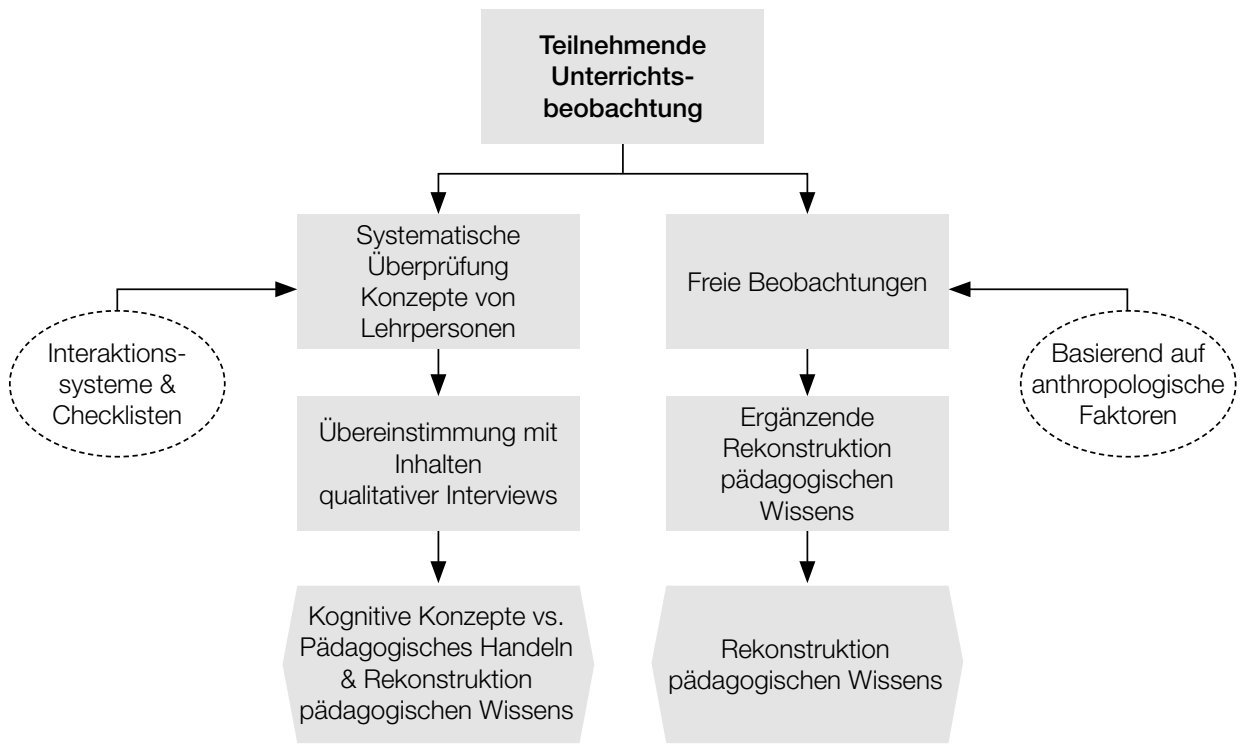

Abb. 34: Überblick Anwendungsbereiche Unterrichtsbeobachtungen

Das strukturierte bzw. systematische Vorgehen hatte zum Ziel, mittels einer kriterienbezogenen Beobachtung, und unter Abarbeiten einer Checkliste, die kognitiven Konzepte der Lehrpersonen aus den Interviews, d.h. wie sie selbst angeben, pädagogisches Wissen im Unterricht anzuwenden, mit ihrem tatsächlichen Handeln zu vergleichen (vgl. Aeppli et al. 2016: 197). Damit ließ sich überprüfen, inwieweit die von den Lehrpersonen in den Interviews genannten Formen pädagogischen Wissens in ihrem unterrichtlichen Handeln beobachtet werden konnte. Mithilfe dieses standardisierten Prozesses können alle wesentlichen Gütekriterien nach Strübing et al. (2018) berücksichtigt werden.

Der zweite Fokus der Beobachtung lag auf einem induktiv offenen, nicht strukturierten Zugang (vgl. Aeppli et al. 2016: 197). Da bei einer theoriegeleiteten, strukturierten Beobachtung die Gefahr besteht, vorrangig bestehende Konzepte von pädagogischem Wissen zu beobachten 
(vgl. Diekmann 2001: 458), wurde der Schwerpunkt bei den Unterrichtsbeobachtungen vor allem auf ein unstrukturiertes, induktives Beobachten gelegt. Das unstrukturierte Verfahren verfolgte das Ziel, Momente, in denen pädagogisches Wissen über Handeln sichtbar wird, festzuhalten und weitere Formen pädagogischen Wissens zu erheben. Laut Helmke (2009: 303) wird nicht die Ganzheit des unterrichtlichen Handelns erfasst, sondern das Auftreten von situativen Verhaltensweisen festgehalten. Verhaltensweisen und Handlungsmuster sowie Interaktionen zwischen Lehrpersonen und SchülerInnen werden im Beobachtungsprozess mit Hilfe ausformulierter Texte, Stichworte oder graphischen Elementen festgehalten (vgl. Bauer et al. 1996: 58; Atteslander 2010: 86ff.). Diese werden mittels Beobachtungsprotokollen aufgezeichnet, die dem Kriterium Validität (vgl. Lüders 2000: 396f.) bzw. der von Strübung et al. (2018: 86ff.) geforderten textuellen Performanz und Gegenstandsangemessenheit gerecht werden.

Die nicht strukturierten Beobachtungen stützten sich auf den methodologischen Zugang der Anthropologie (vgl. Jendrowiak \& Kreuzer: 1982: 83; Tervooren 2014: 55f.). Parallelen der vorliegenden Arbeit zum methodologischen Zugang der Anthropologie zeigen sich über die gegenstandsadäquate Darstellung der sozialen Realitäten von Lehrpersonen. Die unstrukturierte Beobachtung orientiert sich an den anthropologischen Grundkonstituenten Raum, Zeit, Sache, Person und Relation. In der Literatur sowie in der vorliegenden Studie wird vorrangig dem Raum und der Zeit eine große Bedeutung für das pädagogische Wissen und Handeln zugeschrieben (vgl. Göhlich \& Zirfas 2007: 89; Zirfas \& Wulf 2014; Giddens 1984). Personen, die Sache und die Relationen agieren im vorkonstruierten Rahmen von Raum und Zeit.

Basierend auf dem anthropologischen Verständnis wurden während des Beobachtungsprozesses die Elemente „Zeit“, „Objekt“, „Subjekt“, „Beziehungen“ und „Handeln“ unter Berücksichtigung des untersuchten Gegenstands beobachtet (vgl. Abbildung 35).

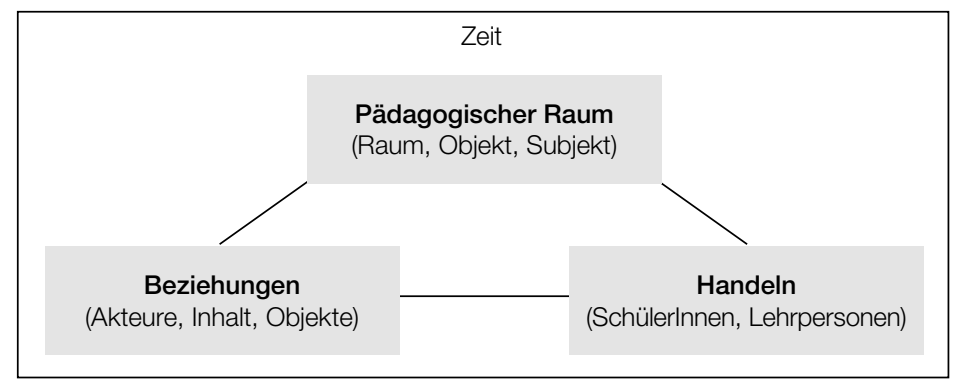

Abb. 35: Beobachtungskategorien basierend auf anthropologischen Grundkonstituenten

Während des ersten Beobachtungsintervalls haben sich folgende Ordnungskategorien für die Rekonstruktion pädagogischen Wissens entwickelt:

- Wie sprechen die AkteurInnen (verbale Kommunikation)?

- Wie handeln die AkteurInnen (situatives Moment)?

- Wie reagieren die AkteurInnen?

- Wie treten sich die AkteurInnen gegenseitig in Kontakt?

- Wie treten die AkteurInnen gegenüber ibrem Anderen auf?

- Wie nutzen die AkteurInnen den Raum?

- Wie ist die Zeitorganisation der AkteurInnen? 
- Wie ist die Strukturierung und Organisation der AkteurInnen?

- Wie ist der Ablauf der AkteurInnen?

- Wie ist die räumliche Gestaltung?

Diese Ordnungskategorien aus dem ersten Beobachtungsintervall lieferten eine Orientierung für das zweite Beobachtungsintervall und unterstützten dabei das strukturierte Vorgehen des Untersuchungsprozesses (vgl. Strübing et al. 2018: 68).

\subsection{Grounded Theory}

Die mit der qualitativ-rekonstruktiven Studie erhobenen Daten aus Interviews und Beobachtungen wurden nach dem Verfahren der Grounded Theory ausgewertet. Die vorliegende qualitativ-rekonstruktive Forschungsmethode sowie das Auswertungsverfahren nach der Grounded Theory ergänzen sich aufgrund des gemeinsamen Ziels der Generierung von Modellen, sodass beide Verfahren adäquat kombiniert werden konnten.

Der Fokus der Grounded Theory liegt auf der systematischen Auswertung empirischer Daten, mit dem Ziel, gegenstandsbezogene Modelle zu generieren. Die vorliegende Arbeit orientiert sich an dem induktiv orientierten Ansatz von Glaser ${ }^{38}$ (2007: 49) sowie an der von Strauss \& Corbin (1994) verfolgte pragmatisch-internalistische Auslegung ${ }^{39}$. Basierend auf den empirischen Daten aus Interviews und Beobachtungen wird ein rekonstruiertes Modell entwickelt. Dieses charakterisiert sich über eine gegenstandsadäquate Abbildung sozialer Prozesse aus der Schulpraxis über das Handeln von Lehrpersonen (vgl. Strauss \& Corbin 1994: 279). Strübing (2008: 38) beschreibt in diesem Zusammenhang den vorliegenden interaktiven Prozess folgendermaßen: „Unsere Realität entsteht [...] in der tätigen Auseinandersetzung mit Elementen der sozialen wie stofflichen Natur, die damit zu Objekten für uns werden und Bedeutung erlangen, die wir uns über Prozesse der Symbolisation anzeigen können." Strübing beschreibt, wie Strauss \& Corbin, die unabdingliche Bedeutung sozialer Prozesse für die gegenstandsadäquate Darstellung. Der Kodierprozess der erhobenen Daten basiert auf einem kontinuierlichen Vergleich. Die Daten werden systematisch verglichen, um die vorliegenden Forschungsfragen (vgl. Kapitel 6) zu untersuchen (vgl. Aeppli et al. 2016: 255). Dieser Prozess unterstützt eine systematische Modellentwicklung pädagogischen Wissens (vgl. Lamneck 2010: 93).

Für die Analyse der Transkripte und Mitschriften wurde MAXQDA zur Auswertung herangezogen (vgl. Kuckartz 2016: 163f.). Das Auswerten der Daten mittels des theoretischen Kodierens wurde in mehrere Schritte eingeteilt (vgl. Abbildung 36).

38 Glaser (2015: 4) beschreibt die induktive Herangehensweise so: „GT is just a simple inductive model that can be used on any data type and with any theoretical perspective. It is just a general inductive model, or paradigm, if you will, that is sufficiently general to be used at will by any researchers in any field, any department and any data type." Die wahrheitsgetreuten Erkenntnisse können laut Glaser direkt aus den Ergebnissen rekonstruiert werden. Die Daten sind in ihrem Entstehungszusammenhang klar benennbar und müssen nicht mehr hinterfragt werden (vgl. Glaser 2001: 145; Glaser 2002: 2).

39 Der pragmatische Ansatz von Strauss und Corbin wird maßgeblich von Deweys pragmatischem Ansatz beeinflusst. Er stützt sich auf die Argumentation, dass Gedanken, Ideen und Einstellungen über das soziale Handeln generiert werden. Die existierende Realität manifestiert sich nicht über abstrakte Theorien. Wesentliches Kriterium für die Darstellung der Realitäten ist der Bezug zur praktischen Relevanz. 


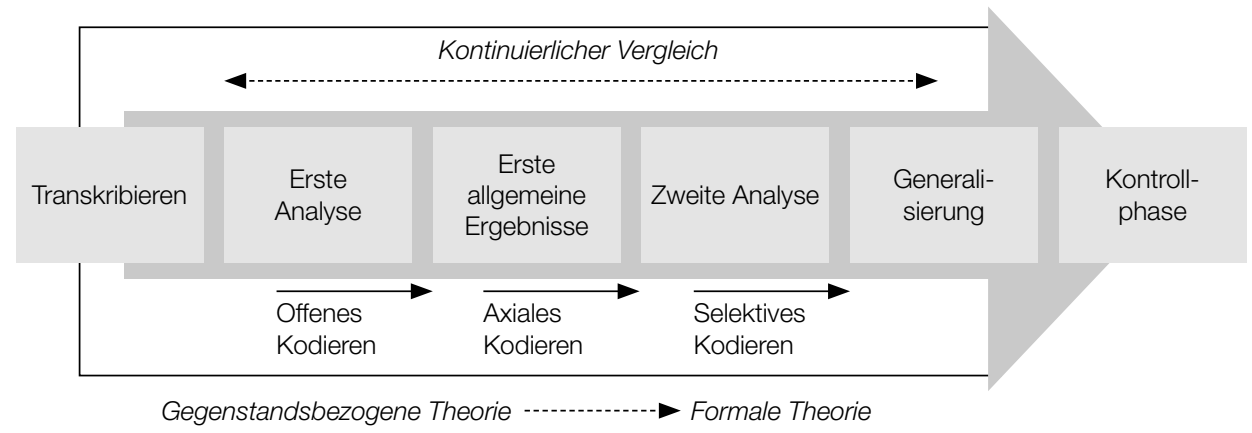

Abb. 36: Datenauswertungsprozess - theoretisches Kodieren

Aufbauend auf den vorliegenden Transkripten aus Interviews und Beobachtungen fanden Kodierprozesse in unterschiedlichen Arbeitsphasen statt. Der erste Analysezugang bezog sich auf ein offenes und axiales Verfahren, wohingegen das selektive Verfahren erst gegen Ende der Analyse Anwendung fand. Beim Kodieren wurden die zugrundeliegenden empirischen Daten aufgebrochen, interpretiert und auf neue Art und Weise zusammengefügt (vgl. Strauss \& Corbin 1996: 39ff.; Flick 2002: 259). In der von Strauss \& Corbin (1996: 47ff.) definierten Generalisierungsphase werden die generierten Kategorien von gegenstandsbezogenen Theorien zu formalen Theorien weiterentwickelt. Formale Theorien charakterisieren sich im Vergleich zu den gegenstandsbezogenen Theorien über einen hohen Standardisierungsgrad und bilden die Ergebnisse der vorliegenden Untersuchung (vgl. Lamneck 2010: 92f.; 368f.; Strübing et al. 2018). In der vorliegenden Arbeit war der Anspruch aufgrund des rekonstruktiven explorativen Zugangs in dieser Phase geringer. Es fand keine Entwicklung formaler Theorien, sondern eine erste Modellbildung statt und garantiert das Kriterium einer textuellen Performanz (vgl. Strübing et al. 2018). Dieses Modell wurde anschließend im Zusammenhang mit den Forschungsfragen und theoretischen Grundlagen diskutiert (vgl. Kapitel 10-12). Die Datenanalyse sowie die Integration und Interpretation sind in der vorliegenden Arbeit getrennt dargestellt. Allerdings enthält die Datenanalyse einzelne erste interpretative Merkmale. Ein weiterer methodologischer Zugang für die Ergebnisdarstellung sind Fallstudien. Ergebnisse aus Interviews und Beobachtungen werden zusammengeführt. Die Bedeutung sowie die Darstellung von Fallstudien wird im folgenden Abschnitt beschrieben. Die Darstellung der Ergebnisse entspricht den von Strübing et al. (2018) geforderten Gütekriterien textuelle Performanz sowie Originalität. Die Forschungsergebnisse werden nachvollziehbar mit Hilfe von Belegstücken in der vorliegenden Arbeit präsentiert und dienen als Basis für eine theoretische Diskussion sowie der Gewinnung neuer Erkenntnisse.

\subsection{Fallstudie}

Eine weitere Form der Ergebnisdarstellung findet in der vorliegenden Forschungsarbeit über Fallstudien statt. Die Fallstudien präsentieren individuelle kognitive Muster und Handlungsformen pädagogischen Wissens ausgewählter Lehrpersonen. Empirische Ergebnisse werden systematisch zusammengeführt und auf die untersuchte Praxis zurückgeführt.

Der Mehrwert für die Berücksichtigung von Fallstudien in der Forschungsarbeit liegt in der detaillierten, systematischen Erklärung innerer Strukturen und typischer Handlungsmuster der 
untersuchten Lehrpersonen (vgl. Brüsemeister 2008: 97). Damit wird dem zugrundeliegenden Prinzip des methodologischen Vorgehens der Forschungsarbeit, der gegenstandsadäquate Darstellung des Untersuchungsfelds, zusätzlich Genüge getan. Abels (1975: 339) betont die essenzielle Bedeutung von einzelnen personenbezogenen Untersuchungen für die Darstellung von Realitäten und argumentiert:

„Statt uns auf immer abstraktere Generalisierungen zu konzentrieren, die wir mit immer größeren Datenerhebungen zu finden hoffen, sollten wir versuchen, in intensiven Fallstudien Material zu sammeln, das Aussagen über konkrete Wirklichkeit und Wahrnehmung dieser Wirklichkeit durch konkrete Personen zulässt." (Abels 1975: 330)

Die Darstellung der vorliegenden Fallstudien stützt sich auf die von Stake (1995: 47f.) sowie Senger und Österle (2004: 3) definierten Kriterien: Ganzheitlichkeit, das empirische Arbeiten, den interpretativen Prozess sowie das Merkmal der Empathie. Diese Kriterien lassen sich wie folgt auf das Forschungsprojekt übertragen: Die Ganzheitlichkeit bezieht sich auf die Darstellung aller wesentlichen Forschungsschwerpunkte der durchgeführten Studie über die Fallstudien, die für die Arbeit von großer Relevanz sind. Dem Kriterium „empirisches Arbeiten“ wird mit dem qualitativen Zugang sowie der Rekonstruktion der Unterrichtspraxis mit Interviews und Beobachtungen genügt. Interpretative Prozesse beziehen sich auf die gegenstandsadäquate Darstellung sozialer Prozesse über die Rekonstruktion. Die Empathie wird in der vorliegenden Untersuchung über eine personenorientiere und realitätsnahe Darstellung der kognitiven Konzepte und des Handelns der Lehrpersonen berücksichtigt.

Insgesamt werden drei der untersuchten Lehrpersonen bei der Präsentation der Fallstudien dargestellt. Die Auswahl bezieht sich auf Lehrpersonen, die den untersuchten Gegenstand adäquat abbilden. Hierfür werden Lehrpersonen aus jedem Schultyp (Grundschule, Neue Mittelschule und Gymnasium) ausgewählt, die verallgemeinerbare Muster präsentieren (vgl. Hartfiel 1982: 160). Die Fallstudien werden mit einer kurzen allgemeinen Beschreibung der untersuchten Lehrperson eingeleitet. Anschließend werden, anhand individueller kognitiver Schemata, das Verständnis einzelner Lehrpersonen sowie deren Handlungsmuster mithilfe von Interviewausschnitten und Grafiken illustriert. Über die Fallstudien werden erste Verbindungen zwischen den Ergebnissen aus Interviews und Beobachtungen hergestellt. 


\section{Ergebnisse und Analyse}

Im vorliegenden Kapitel werden zentrale Ergebnisse der Studie in vier unterschiedlichen Abschnitten dargestellt (vgl. Abbildung 37).

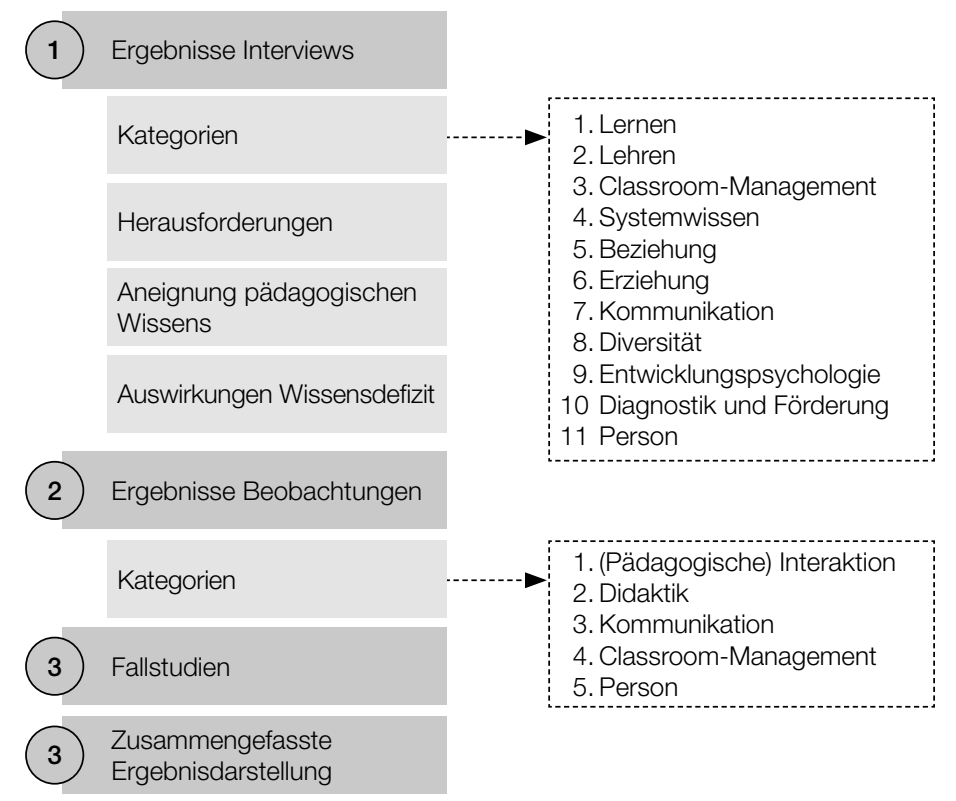

Abb. 37: Übersicht Aufbau Ergebnisdarstellung

Zuerst wird das rekonstruierte Verständnis pädagogischen Wissens aus den Interviews präsentiert. Weitere Interviewergebnisse beziehen sich auf genannte Herausforderungen, den Erwerb pädagogischen Wissens sowie die Auswirkungen von Wissensdefiziten auf den Unterricht. Der zweite Abschnitt beinhaltet die Darstellung pädagogischen Wissens basierend auf den Unterrichtsbeobachtungen. Wie im vorherigen Abschnitt 7.5 beschrieben, werden die Ergebnisse aus den Beobachtungen und Interviews mittels der rekonstruierten Kategorien und Subkategorien dargestellt.

Die Fallstudien präsentieren individuelle kognitive Muster und Handlungsformen pädagogischen Wissens ausgewählter Lehrpersonen. Empirische Ergebnisse werden hierbei systematisch zusammengeführt und erste Verbindungen zwischen den Interview- und Beobachtungsergebnissen hergestellt.

Abschließend erfolgt eine Zusammenführung der Ergebnisse und eine Präsentation generierter Kategorien aus den empirischen Daten. Die Zusammenführung der Ergebnisse mit den diskutierten theoretischen Grundlagen sowie die Darlegung und Diskussion der Bedeutung für die LehrerInnenbildung erfolgt in der abschließenden Integration und Interpretation der vorliegenden Forschungsarbeit in Kapitel III. 


\subsection{Kategorien pädagogischen Wissens aus den Interviews}

Der vorliegende Abschnitt präsentiert die aus den Interviews rekonstruierten Ergebnisse pädagogischen Wissens von Lehrpersonen. Es zeigt sich bei einem Großteil der Befragten, dass sie Schwierigkeiten hatten, auf die Einführungsfrage „Was verstehen Sie unter dem Begriff pädagogisches Wissen?" ad hoc zu antworten:

„Was ist pädagogisches Wissen? Das ist eine gute Frage. Jetzt muss ich erst mal überlegen [...] Das ist schwer zu definieren." (It 5)

„Unter pädagogischem Wissen, ach, was verstehe ich unter pädagogischem Wissen? Das ist eine gute Frage. Die Frage ist sehr spontan gestellt, aber das ist gut so. In Bezug auf die Schule ist der Begriff breit gefasst [...].“ (It 6)

Die Ergebnisse verdeutlichen, dass die Lehrpersonen den Begriff trotz anfänglicher Schwierigkeiten im Verlauf des Interviews präzisieren konnten. Sie definieren pädagogisches Wissen als ein fachunabhängig generisches Wissen, welches sich vorrangig auf den Umgang mit SchülerInnen bezieht.

„Es geht um den Umgang mit den Kindern und Jugendlichen, wie man pädagogisch wertvoll mit ihnen umgeht. Ich muss ehrlich sagen ich habe noch nie über den Begriff pädagogisches Wissen nachgedacht, aber für mich ist es mehr das Zwischenmenschliche als das Fachliche. [...]." (It 3)

„Pädagogisches Wissen ist ein fachunabhängiges Wissen. [...] Ich verstehe darunter das Soziale bzw. pädagogische Kompetenzen von Lehrpersonen." (It 26)

Wie im Zitat angeführt, verbinden die Lehrpersonen mit dem Begriff pädagogisches Wissen das Zwischenmenschliche. Eine Lehrperson erzählt, dass sie am Ende des Schuljahres müde sei (It 9). Sie bezieht das Müdesein nicht auf das Vorbereiten, Unterrichten und Korrigieren, sondern betont eine soziale Müdigkeit aufgrund des permanenten pädagogischen Handelns im Unterricht.

Die Rekonstruktion verdeutlicht, dass Lehrpersonen das pädagogische Wissen unter Berücksichtigung einer lern- sowie lehrseitigen Perspektive charakterisieren. Zentrales Moment sind die SchülerInnen, die als wichtigste Bezugspersonen für das pädagogische Wissen hervorgehoben werden.

„Das pädagogische Wissen sollte sich heutzutage an den SchülerInnen orientieren. Natürlich geht es hier um pädagogisches Wissen von Lehrpersonen, aber ohne dass man die SchülerInnen mitdenkt, ist dieses Wissen in der heutigen Gesellschaft und im Verständnis von gegenwärtigen Lern- und Lehrtheorien wertlos. SchülerInnen müssen selbst das Lernen und die Welt verstehen“ (It 25)

„Ich muss zwar Wissen welche Aufgaben ich mache und wie ich den Unterricht plane, allerdings sollte der Fokus dann auf dem Lernen und den individuellen Lernerfahrungen von SchülerInnen liegen. Sie müssen eigenständig durch Erfahrungen Lernen. [...] Ich muss hierfür wissen was SchülerInnen brauchen, um dieses Ziel zu erreichen." (It 1)

Bei einer lernseitigen Orientierung wird das Lernen von SchülerInnen in den Mittelpunkt des Interesses gestellt. Das Handeln einer Lehrperson soll sich am individuellen Lernprozess von SchülerInnen orientieren und das entdeckende sowie erfahrende Lernen unterstützen. Im Vergleich hierzu stellt eine lehrseitige Orientierung „[...] das Lehren ins Zentrum des Unterrichts“ (It 20). Die richtige Planung steht im Vordergrund. 
Die SchülerInnen nehmen nicht nur als Bezugspersonen pädagogischen Wissens eine wichtige Stellung ein. Die Befragten beschreiben, wie SchülerInnen als „Spiegel“ (It 9) fungieren, die über ihr Verhalten rückmelden, inwieweit die Lehrpersonen pädagogisches Wissen anwenden.

„Die SchülerInnen spiegeln pädagogisches Wissen der Lehrpersonen. Sie zeigen über ihr Verhalten, inwieweit Lehrpersonen pädagogisches Wissen anwenden.“ (It 3)

„[...] man kann das an den Kindern selber erkennen. Wenn ein Kind gerne in die Schule kommt und gerne auf die Lehrperson zugeht, dann ist da schonmal ganz viel an pädagogischem Wissen. Man erkennt pädagogisches Wissen von Lehrpersonen ansonsten an dem Verhalten der SchülerInnen im Klassenzimmer." (It 9)

Die generierte, lern- sowie lehrseitige Charakterisierung pädagogischen Wissen wird von einer Interviewpartnerin (It 13) mit spezifischem didaktischem Wissen ergänzt. Sie betont den signifikanten Einfluss pädagogischen Wissens auf das zugrundeliegende Beziehungsgefüge von Lerngegenstand, SchülerInnen und Lehrperson. Das pädagogische Wissen ist nach Argumentation der Interviewten konstitutiv für die ganzheitliche Entfaltung des didaktischen Dreiecks in der Unterrichtssituation verantwortlich.

Die Befragten definieren Grundlagen- und Erfahrungswissen als Grundlage aller Wissenskategorien pädagogischen Wissens. Grundschullehrpersonen und Lehrpersonen der Neuen Mittelschule fokussieren verstärkt das Erfahrungswissen, während Gymnasiallehrpersonen häufiger das Grundlagenwissen benennen.

„Für pädagogisches Wissen brauche ich generell Theorie- und Erfahrungswissen. Das ist die Grundlage. Auf diesem baut sich dann weiteres Wissen auf." (It 4)

„Die Grundlage von pädagogischem Wissen ist einerseits das theoretische Wissen, das sozusagen aus der Literatur oder über Ausbildung bzw. Weiterbildungen generiert wird. Zweitens ist es ein intuitives Wissen oder Erfahrungswissen. Meine Professionalisierung besteht darin, dass ich immer wieder neues theoretisches Wissen mit meinem Erfahrungswissen verknüpfe [...].“ (It 18)

Das Erfahrungswissen entwickelt sich aus der Praxis. Die Interviewten berichten von einem „aus dem Bauch heraus handeln“ (It 4) oder benennen es mit „pädagogisches Gespür“ (It 7). Dieses Wissen ist personenbezogen und über routinierte Handlungen sowie individuelle Praxiserfahrungen und Haltungen der Lehrpersonen charakterisiert. Es kann sich dabei um bewusste sowie um unbewusste Wissensaspekte handeln. Das Grundlagenwissen beschreiben die Lehrpersonen als grundlegendes theoretisches Wissen:

„Grundlegend ist das Theoriewissen über pädagogisches Wissen. Natürlich hat jede Lehrperson über langjährige Praxiserfahrungen Wissen. Allerdings um diesem Wissen einen Wert zu geben und tatsächlich zu Wissen was man tut, ist das Theoriewissen von Nöten." (It 25)

Die Lehrpersonen erläutern die essenzielle Bedeutung von Grundlagenwissen für die Aneignung und das situationsgerechte Anwenden von fundiertem professionellem pädagogischem Wissen. Lehrpersonen betonen, dass sich dieses Wissen nur über die selbstständige, permanente Auseinandersetzung mit wissenschaftlichen Erkenntnissen und Theorien aufbaut und weiterentwickelt. Eine Lehrperson nennt es „State of the Art sein“ (It 17) und hebt die Bedeutung der Aneignung des Grundlagenwissens über wissenschaftliche Literatur sowie den Besuch von Fortbildungen hervor. 
$\mathrm{Zu}$ der hier angeführten allgemeinen Charakterisierung pädagogischen Wissens wurden aus den Interviews spezifische Kategorien rekonstruiert. Insgesamt lässt sich das Gesamtbild anhand von elf Kategorien darstellen (vgl. Abbildung 38).

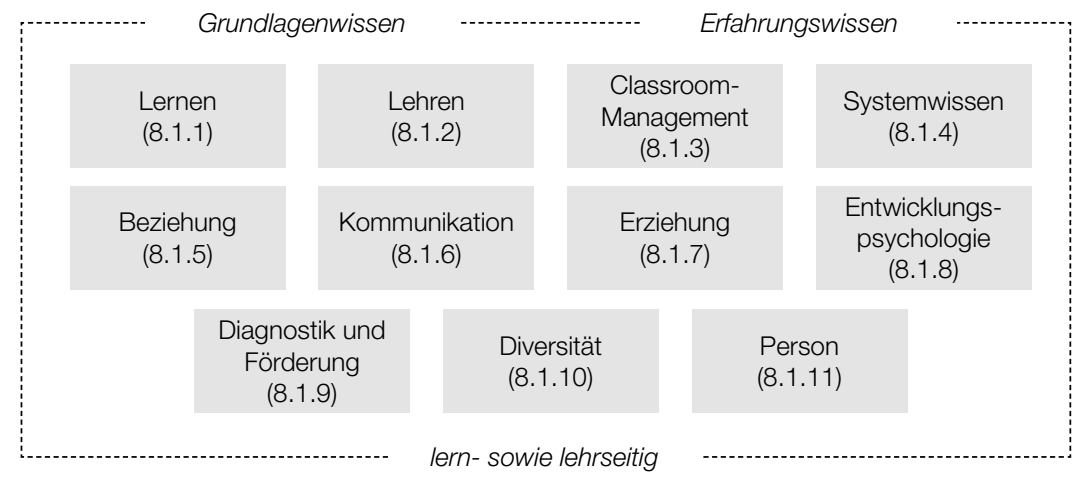

Abb. 38: Überblick Interviewkategorien

Die aus den Interviews rekonstruierten Kategorien beziehen sich auf das Lernen, Lehren, Classroom-Management, Systemwissen, Beziehungen, Kommunikation, Erziehung, Entwicklungspsychologie, Diversität, Diagnostik und Förderung sowie auf das Wissen über die Person. Sie bilden das Gesamtverständnis pädagogischen Wissens der Befragten. Diese Wissenskategorien werden im folgenden Teil zur Diskussion gestellt. Die Reihenfolge richtet sich nach der Berücksichtigung wechselseitiger Beziehungen einzelner Kategorien. Die Lehrpersonen charakterisieren, wie am Anfang des Kapitels beschrieben, das pädagogische Wissen als lern- sowie lehrseitige Wissensform. Diese Wissensformen werden im weiteren Verlauf als Ordnungskategorie für die Beschreibung der Kategorien herangezogen.

\subsubsection{Lernen}

Wissen über Lernen wird von den Interviewten als wesentliche und grundlegende Form pädagogischen Wissens beschrieben. Diese wird anhand von drei Subkategorien charakterisiert: Lernentwicklung, Lernatmosphäre und Grundlagenwissen über Lernen (vgl. Abbildung 39). Die Kategorien beziehen sich auf lern- sowie auf lehrseitiges Wissen.

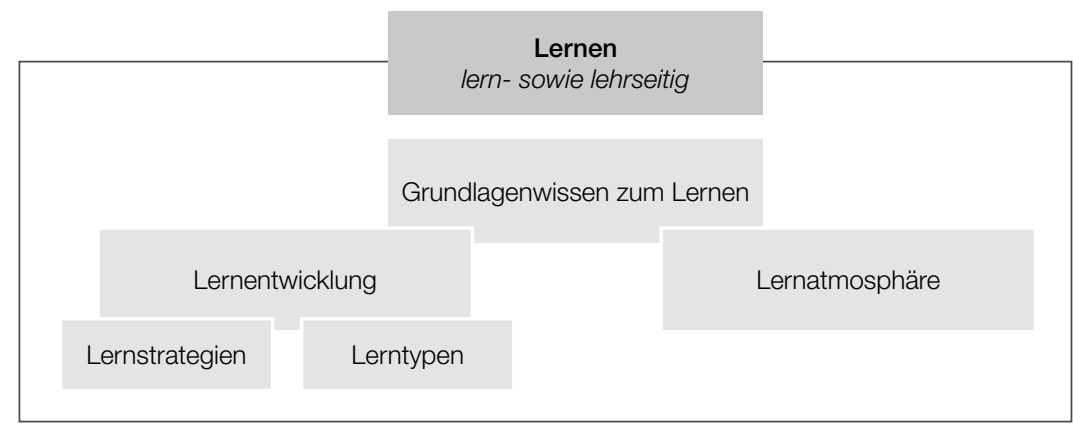

Abb. 39: Subkategorien Lernen 
Die Interviewten betonen die wichtige Bedeutung des Grundlagenwissens. Dieses gilt als Basis der weiteren angeführten Subkategorien Lernentwicklung und Lernumgebung. Die Interviewten vertreten die Position, dass Lehrpersonen ein generelles, fundiertes und aktuelles Grundlagenwissen über die Bedeutung und Zugänge zum Lernen besitzen müssen. Sie artikulieren unterschiedliche Zugänge und Definitionen zum Thema Lernen. Sie konstruieren einen für sich passenden Definitionsrahmen:

„Selbstständiges Lernen über Erfahren steht im Vordergrund, das von den Lehrpersonen angeleitet und begleitet werden muss." (It 10)

„Wenn sich die SchülerInnen in einer Klasse wohlfühlen, fällt ihnen das Lernen leichter. Als Lehrkraft muss ich versuchen die Interessen der Kinder zu berücksichtigen. Auch muss ich sie stetig motivieren und anleiten, dass sie Selbstverantwortung für ihr eigenes Lernen übernehmen. Und lernen heißt entdecken, erkunden, erforschen und ausprobieren." (It 9)

Die Befragten definieren den Begriff Lernen mehrdimensional. Sie beschreiben es über das Aufgreifen von SchülerInneninteressen, Motivation, das Anleiten und die Unterstützung eines selbstständigen Lernprozesses und betonen das anwendungsorientierte und selbstentdeckende Lernen.

Eine Interviewte hebt in ihrer Definition das Umdenken als ein weiteres Merkmal hervor. Sie versteht unter Lernen das Verändern und Anpassen eigener Perspektiven und Haltungen:

„Lernen muss Spaß machen, es muss eine Interaktion da sein. Sie müssen das Gelernte anwenden und Kompetenzen entwickeln, die sie nicht nur in der Schule anwenden sollen. Ich glaube da braucht es von Seiten der Lehrperson sehr viel Wissen darüber, was Lernen bedeutet. Kinder müssen lernen umzudenken und neue Perspektiven und Haltungen einnehmen. Erst wenn man das erreicht, lernen die SchülerInnen." (It 12)

Die Befragten stützen sich auf die Annahme, dass das Grundlagenwissen zum Thema Lernen im Hinblick auf einen nachhaltigen Lernprozess essenziell ist. Sie definieren nachhaltiges Lernen über einen lebensnahen und lebenslangen Lernprozess, der SchülerInnen im alltäglichen Handeln positiv unterstützt und handlungsfähig macht:

„Lehrpersonen benötigen ein theoretisches Wissen über Lernen. Erst wenn man unterschiedliche Zugänge zum Thema Lernen kennt und weiß wie die SchülerInnen lernen, ist es möglich nachhaltige Lernprozesse zu unterstützen. Damit meine ich, dass sie nicht nur für mich lernen, sondern für das Leben." (It 24)

Die Lernentwicklung ist eine weitere rekonstruierte Subkategorie zum Thema Lernen. Diese thematisiert das allgemeine Wissen über die Entwicklung von Kindern sowie die individuelle Unterstützung von personenbezogenen und fachlichen Fähigkeiten und Fertigkeiten für einen bestmöglichen Lernprozess bei SchülerInnen.

„Wissen über die persönliche und fachliche Entwicklung der Kinder, um das Lernen bestmöglich zu unterstützen. Lehrpersonen müssen die SchülerInnen für diesen Prozess begleiten und anleiten." (It 23)

Lehrpersonen haben die Aufgabe, SchülerInnen in ihrem persönlichen sowie fachlichen Prozess zu unterstützen. Sie müssen über individuelle Faktoren einzelner SchülerInnen Bescheid wissen, um personalisiertes individuelles Lernen zu fördern. Ein wichtiger Wissensbereich ist nach Angaben der Lehrpersonen das Wissen über Lernstrategien. Diese werden für die Unterstützung individuellen Lernens als wichtig erachtet: 
„Wenn ich SchülerInnen etwas beibringen möchte, muss ich Lernstrategien einsetzen. [...] Das ist sehr faszinierend. Ich muss den Kindern Lernstrategien zeigen. Sie haben wenig Wissen wie sie effektiv lernen können. Deshalb baue ich die Lernstrategien in meinen Unterricht ein und weise die SchülerInnen ständig darauf hin. [...] So kann jeder für sich selbst herausfinden und ausprobieren, mit welcher Strategie er am besten klar kommt. Kinder und Jugendliche sind sehr kreativ, wenn sie individuell wählen können.“ (It 9)

„Für das unterstützen einer Lernentwicklung bei SchülerInnen braucht es spezifische Lernstrategien. Diese beziehen sich zum Beispiel auf das Definieren individueller Lernziele oder die Förderung problemlösungsorienteres Lernen." (It 22)

Wie der Interviewauszug verdeutlicht, kann personalisiertes Lernen mithilfe von Lernstrategien unterstützt werden. Darunter verstehen die Lehrpersonen den Einsatz unterschiedlicher entwicklungsfördernder Strategien. Diese betreffen nach Angaben der Befragten das individuelle Definieren von Lernzielen, sich Zeit für einzelne SchülerInnen zu nehmen, den SchülerInnen für gemeinsames Arbeiten und Diskutieren Raum zu geben, Feedback zu geben, SchülerInnen zu beobachten, Möglichkeiten zu schaffen, in denen SchülerInnen sich zurückziehen können und das problemlösungsorientierte Lernen zu fördern. Es zeigt sich, dass vorwiegend Lehrpersonen der Neuen Mittelschule und der Grundschule das individuelle Lernen betonen.

Spezifische Ziele, die über das individualisierte Arbeiten und das Anwenden gezielter Lernstrategien erreicht werden, sind nach Angaben der Lehrpersonen die folgenden vier:

- SchülerInnen erlernen Selbstständigkeit

- Stärken und Schwächen können besser berücksichtigt und gefördert werden

- SchülerInnen können ihre eigene Lernstrategien für sich entdecken

- SchülerInnen können ihrem eigenen Lerntempo nachgehen

Als weitere Form für eine spezifische Unterstützung der Lernentwicklung benennen die Interviewten das Wissen über Lerntypen. Sie fordern Wissen zu kognitiven Verarbeitungsprozessen:

„Es muss einem bewusst sein, dass es unterschiedliche Lerntypen gibt. Jeder Mensch ist verschieden und individuell. Man muss in einer Klasse lernen zu erkennen welche Lerntypen man vor sich sitzen hat, um den Unterricht bestmöglich vorbereiten und abhalten zu können. Es müssen unterschiedliche Sinne angeregt werden - visuell, auditiv oder kinästhetisch.“ (It 19)

In den Interviews werden drei unterschiedliche Lerntypen - visuell, auditiv, kinästhetisch - angesprochen. Die Förderung unterschiedlicher Lerntypen kann den Lernprozess von SchülerInnen positiv beeinflussen.

Relevantes Wissen zum Thema Lernen inkludiert nach Angaben der Befragten die Subkategorie Lernatmosphäre. Sie definieren, wie in Abbildung 40 dargestellt, unterschiedliche Kriterien einer Lernatmosphäre, die das Lernen positiv unterstützt.

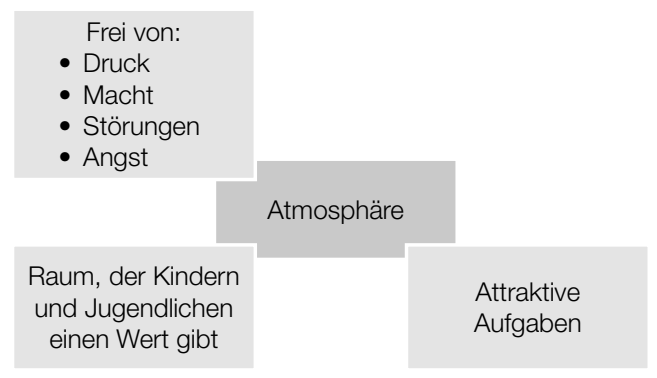

Abb. 40: Verständnis Lernatmosphäre 
Die Lernatmosphäre ist laut den Interviewten von unterschiedlichen Faktoren abhängig. Von grundlegender Bedeutung ist eine Atmosphäre, die den Kindern und Jugendlichen Wertschätzung entgegenbringt. Diese betrifft die Akzeptanz unterschiedlicher Persönlichkeiten sowie die Ermöglichung individueller Lernprozesse. Zusätzlich garantiert eine Umgebung ohne Druck, Machtgefälle, Störungen und Angst eine Atmosphäre, in der sich die SchülerInnen wohlfühlen können. Das Thema Umgang mit Druck zeigt sich vorwiegend über die Aussagen von Gymnasiallehrpersonen. Von den Befragten werden didaktische Kriterien genannt, die positiv auf die Lernatmosphäre einwirken. Sie sprechen über den Einsatz von attraktiven Aufgaben. Diese zeichnen sich durch interessante, herausfordernde, kreative sowie problemlösungsorientierte Aufgaben aus, die das selbstständige Lernen fördern.

Zusammenfassend verdeutlicht die beschriebene Kategorie eine stark ausgeprägte lernseitige Orientierung. Dabei stehen nicht die Interessen der Lehrperson, sondern diejenigen der Lernenden im Vordergrund, damit ein individuelles, wertvolles, nachhaltiges und selbstständiges Lernen ermöglicht wird. In enger Beziehung zum Lernen steht die Kategorie Lehren. Diese wird im nächsten Abschnitt dargestellt.

\subsubsection{Lehren}

Das Wissen über Lehren wird von den Befragten über sieben lern- sowie lehrseitige Subkategorien beschrieben: professionelles pädagogische Fachwissen, didaktisches Wissen, Unterrichtsvorbereitung und Unterrichtsdurchführung, psychologisches Wissen, Wissen über Macht, Wissen über Fehlerkultur, Wissen über die Lernstandserhebung sowie motivationales Wissen. Das didaktische Wissen untergliedert sich in vier weitere Subkategorien, das Wissen zu Methodik, Raum, Medien sowie zu Sozialformen (vgl. Abbildung 41).

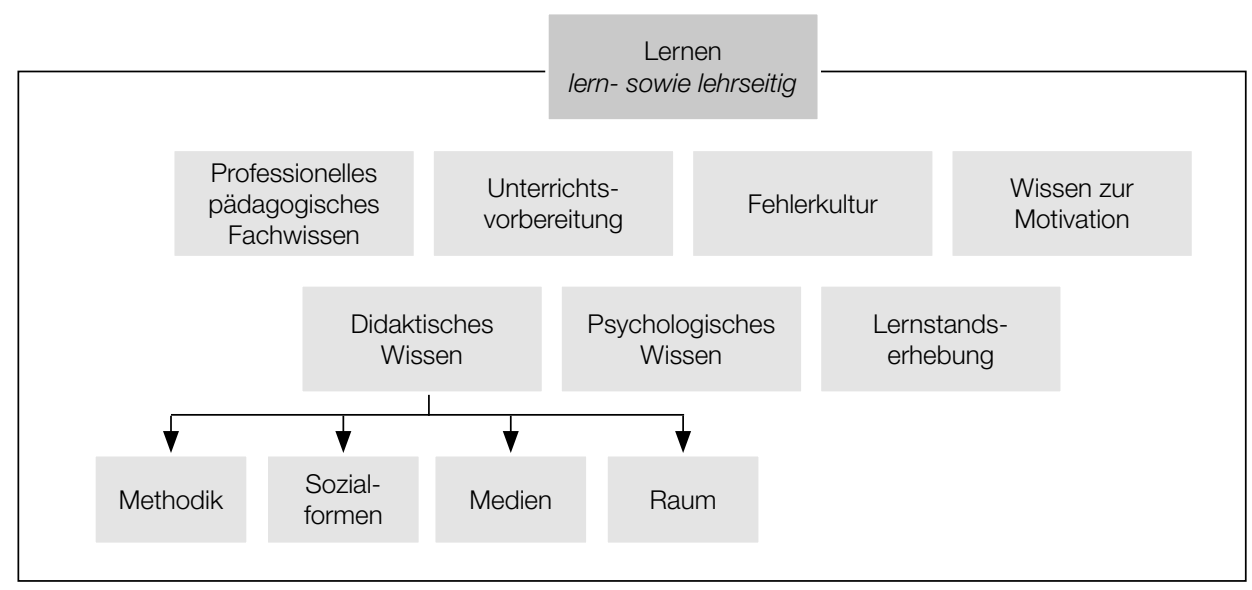

Abb. 41: Subkategorien Lehren

Das professionelle pädagogische Fachwissen ist laut den Interviewten das Grundlagenwissen über pädagogische (Unterrichts-)Konzepte (z.B. offener Unterricht, personalisierter Unterricht, Frontalunterricht, Projektklassen, Freiarbeit, handlungsorientierter Unterricht, problemorientierter Unterricht, kompetenzorientierter Unterricht, Begrifflichkeiten sowie Professionalisierungsmodelle und Handlungskonzepte: 
„Es ist wichtig, Kenntnisse im Bereich der Wissenschaft zu besitzen. Das beinhaltet zum Beispiel wissenschaftliche Erkenntnisse im Bereich Unterrichtskonzepte, wie zum Beispiel offener Unterricht [...]. Hier gibt es ja unterschiedliche Strömungen.“ (It 5)

Die Interviewten sehen das Grundlagenwissen als Basis für erfolgreiches Lehren. Als wesentliche Aufgabe beschreiben sie, „Kinder und Jugendliche zu mündigen, aktiven Aktanten der gegenwärtigen und zukünftigen Gesellschaft“ heranzubilden (It 23).

Im Zusammenhang mit Lehren benennen fast alle Interviewten die Subkategorie didaktisches Wissen. Es steht laut den Befragten in einer engen Beziehung zum professionellen pädagogischen Fachwissen:

„Unter Berücksichtigung theoretischer Grundlagen formt sich das didaktische Wissen. Darunter versteht man das Didaktische, wie man etwas aufbereitet, so dass SchülerInnen es verstehen." (It 1)

Die Befragten verstehen unter Didaktik die Aufbereitung von Unterrichtsinhalten. Eine Lehrperson nennt explizit die Wichtigkeit „[...] zu wissen, wie man den SchülerInnen kognitiv, sozial und zwischenmenschlich" (It 7) etwas beibringen kann. Das didaktische Ziel steht im Vordergrund:

„Die Sinnfrage warum wir etwas wie machen, etwas einen Wert geben. Die Frage der didaktischen Grundhaltung, was will ich denn eigentlich, ist bei der Didaktik wesentlich." (It 26)

Sie benennen spezifische Merkmale, die von einer Lehrperson für eine professionelle didaktische Aufbereitung eines Unterrichts berücksichtigt werden sollten (vgl. Abbildung 42). Diese beziehen sich auf die Merkmale Offenheit der Lehrperson, Berücksichtigung der Fähigkeiten und Fertigkeiten der SchülerInnen sowie auf den Unterrichtsprozess.

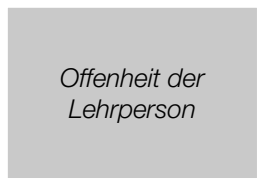

\section{Fähigkeiten und \\ Fertigkeiten der \\ Schülerlnnen \\ berücksichtigen}

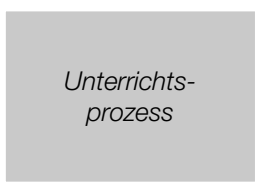

- Raum für spontanes Agieren berücksichtigen

- Verschiedene Herangehensweisen ausprobieren

- Ansprechen unterschiedlicher Schülerlnnen

- Neue bzw. verschiedene Unterrichtskonzepte ausprobieren und integrieren

- Unterschiedliche Kompetenzen fördern

- Selbstständiges Arbeiten unterstützen

- Talente fördern

- Schwächere SchülerInnen bewusst im Unterricht integrieren

- Stärken und Schwächen der Schülerlnnen (er-)kennen

- Erklären vom Leichten zum Schweren

- Theorie in der Praxis anwenden (Praxisbezüge herstellen)

- Altersgerechtes Unterrichten

- Unterschiedliche Sinne anregen: Denken, Greifen, Überlegen

Abb. 42: Wissensformen im Bereich der Didaktik

Wissen der Didaktik ist nach Angaben der Befragten das Methodenwissen, das Wissen zu Sozialformen, zu Medien und Raum. Mithilfe des Methodenwissens kann ein abwechlungsreicher Unterricht angeboten werden: 
„Man sollte versuchen vielseitig zu agieren, um möglichst viele SchülerInnen anzusprechen. [...] Mit vielseitig agieren meine ich einfach, dass man möglichst viel ausprobiert, verschiedene Sozialformen und verschiedene Methoden im Unterricht anwendet." (It 7)

Das Methodenwissen hat das vorrangige Ziel, „[...] nachhaltige Neugierde bei SchülerInnen zu entwickeln“ (It 25). Die Befragten meinen, dass für einen nachhaltigen Lerneffekt eine Auswahl unterschiedlicher Methoden wichtig ist. Der Einsatz verschiedener Methoden ist vor allem an Gymnasien ein wichtiges Thema:

„Unter pädagogischem Wissen verstehe ich, was man für Methoden weiß oder was man kennengelernt hat, um zu lernen und um zu wissen, wie es den Lernenden dabei geht, sie richtig für ein nachhaltiges Lernen anzuwenden [...].“ (It 14)

Eng verbunden mit dem Wissen zu Methoden ist die Berücksichtigung unterschiedlicher Sozialformen. Sie unterstützen den Lehrpersonen zufolge die Kooperations- und Interaktionsfähigkeit in einer Klasse. Genannte Sozialformen beziehen sich auf die Partnerarbeit, die Gruppenarbeit sowie die Integration von Auflockerungs- und Konzentrationsübungen in den Unterricht. Zusätzlich zur Förderung von sozialen Kompetenzen streben die Befragten an, ihre SchülerInnen über Zusammenarbeit besser kennenzulernen. Für die Umsetzung von unterschiedlichen Methoden und Sozialformen ist das Wissen zum Raum hilfreich:

„Der Raum spielt eine wichtige Rolle. Man sollte wissen ob der Raum für das Lehren und Lernen geeignet ist. Ja einmal von seiner Atmosphäre, Akustik oder wegen seiner Größe. Der Raum hat etwas mit der Gestaltung von Lehr- und Lernprozessen zu tun." (It 5)

Vor allem Lehrpersonen der Grundschule war der Raum wichtig. Sie legten Wert auf eine sorgfältig vorbereitete Umgebung und eine sinnvolle Raumnutzung. Zusätzlich zu den fix im Raum platzierten Plätzen haben die SchülerInnen die Möglichkeit, sich im Unterricht frei zu bewegen. Zum Medienwissen nehmen vorrangig Lehrpersonen des Gymnasiums und der Neuen Mittelschule Stellung. Sie sprechen sich generell für den Einsatz verschiedener Medien im Unterricht aus und möchten SchülerInnen für Medien sensibilisieren. Im Detail werden jedoch keine konkreten Vorschläge für den Einsatz von Medien genannt.

Die Subkategorie Unterrichtsvorbereitung stellt eine weitere rekonstruierte Subkategorie zum Thema Lernen dar. Diese zeigt Übereinstimmung mit dem rekonstruierten Verständnis der Kategorie Lernen sowie der Kategorie Classroom-Management. ${ }^{40}$ Themenschwerpunkte, die hinsichtlich des Lehrens genannt werden, sind das Definieren von Lernzielen, das Erstellen von Materialien sowie das Berücksichtigen von Interessen und Vorlieben der SchülerInnen bei der Unterrichtsplanung. Die Befragten bezeichnen die Unterrichtsvorbereitung als wichtiges Hilfsmittel für einen strukturierten und qualitätsvollen Unterricht:

„Die Unterrichtsplanung ist für einen qualitätsvollen Unterricht wichtig. Über das Planen eines Unterrichts und der Definition von Lernzielen, kann ein zielgerichteter und systematischer Unterricht sattfinden." (It 24)

„Bei der Unterrichtsplanung müssen individuelle Faktoren der SchülerInnen berücksichtigt werden. Darunter zählen methodische Vorlieben, Themenschwerpunkte oder auch die Berücksichtigung unterschiedlicher Lerntypen." (It 11)

40 Die Wissenskategorie Classroom-Management wird in Abschnitt 8.1.3 ausführlich beschrieben. 
Wie das Zitat verdeutlicht, ist eine schülerorientierte Ausrichtung bei der Erstellung einer Unterrichtsplanung gefordert. Es sollen methodische sowie inhaltliche Kriterien auf die SchülerInnen abgestimmt werden.

Für das Lehren wurde von den Interviewten die Subkategorie psychologisches Wissen angeführt. Sie definieren das psychologische Wissen über Wissen zu Macht, Druck, Angst sowie den Umgang mit Stereotypen.

„Lehrpersonen sollten im Zusammenhang mit dem Lehren ein psychologisches Wissen besitzen. Zum Beispiel die Frage der Machtausübung von Lehrpersonen oder wie viel Druck lässt man bei den Kindern entstehen. Die dadurch entstehende Überforderung und Angst der SchülerInnen sollte man dabei immer berücksichtigen." (It 26)

„Psychologisches Wissen wie man mit Stereotypen im Klassenzimmer umgeht sollte ein zentrales Wissen von Lehrpersonen sein.“ (It 2)

Das psychologische Wissen charakterisiert sich über eine hohe Beeinflussungsdynamik von Macht, Druck, Stereotypen, Angst und Überforderung zwischen Lehrpersonen und SchülerInnen (vgl. Abbildung 43).

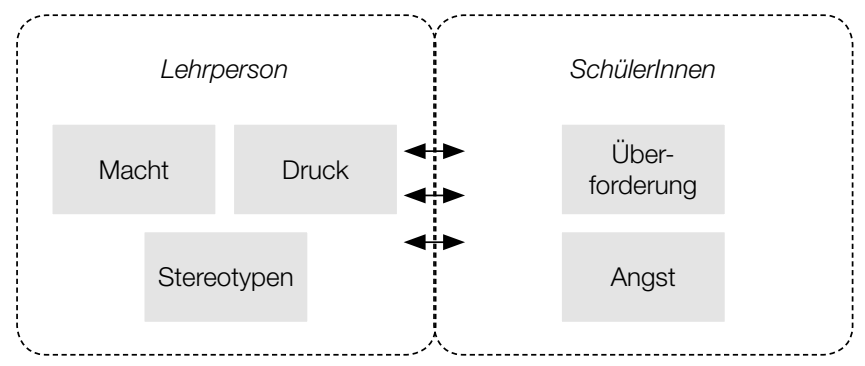

Abb. 43: Beeinflussungsdynamik von psychologischem Wissen

Die Befragten beschreiben fehlendes Wissen über die bestehende Wechselbeziehung psychologischen Wissens zwischen Lehrpersonen und SchülerInnen als „pädagogisches Halbwissen“ (It 25). Unzureichendes Wissen kann bei SchülerInnen zu Scham und Minderwertigkeitsgefühlen führen. Die Interviewten betonen einen pädagogisch wertvollen Umgang mit Macht:

„Man sollte seine Macht als Lehrperson kontrollieren können. Ja, dass man die Kinder in Situationen so behandelt, dass sie nicht beschämt und bloßgestellt werden. Ich muss wissen wie ich mit Ihnen in gewissen Situationen umgehe, wie ich handle, vielleicht wie die SchülerInnen darauf reagieren. Das fängt damit an, dass man nicht mit dem Rotstift Klassenarbeiten der SchülerInnen korrigiert und hört damit auf, dass man als Lehrperson keine Leistungen aberkennt." (It 5)

„Macht sollte von einer Lehrperson nicht ausgenutzt werden. Diese führt schnell bei SchülerInnen zu Angst und Überforderung." (It 1)

Mit dem Thema Macht ist das Wissen um Druck verbunden. Die Interviewten stellen sich hierzu während des Interviews indirekte Fragen: Wie belastbar sind Kinder? Welcher Druck (Leistungsdruck, Zeitdruck) ist angemessen? Wie viel Druck sollte man als Lehrperson ausüben? Die Befragten heben hervor, dass es wichtig ist, sich diese Grundfragen permanent zu stellen, um Drucksituationen im Unterricht einschätzen und vermeiden zu können. In diesem Zusammen- 
hang wird das Wissen um Angst und Überforderung erwähnt. Die Interviewten vertreten die Position, dass Lehrpersonen wissen müssen, welche Verhaltensweisen und Situationen SchülerInnen verängstigen bzw. überfordern.

Der Umgang mit Stereotypen wird von den Interviewten als weitere Wissensform psychologischen Wissens beschrieben:

„Lehrpersonen haben viele Vorurteile und zum Teil ein Stereotypendenken. Das ist bevor sie in die Klasse kommen in ihren Köpfen verankert [...]. Dieses aufzuzeigen und zu hinterfragen, wäre einmal ein wichtiger Aspekt, der unbedingt beim pädagogischen Wissen beachtet werden muss.“ (It 22)

Lehrpersonen aller Schulformen berichten von Zuschreibungen und Stereotypendenken, die den Unterrichtsalltag mitprägen. Die in den Interviews genannten Stereotypen sind beispielsweise: „Mädchen sind besser in Sprachen“, „Jungs sind besser in naturwissenschaftlichen Fächern", "Jungs sind oft faul" (It 11). Die Befragten vertreten die Position, dass es notwendig ist, die eigenen Haltungen und Handlungen zu hinterfragen, um Stereotypen im Unterricht bestmöglich zu reduzieren. Ein unbewusster Umgang mit diesen kann zu Überforderung und Druck aufseiten der SchülerInnen führen.

Als eine weitere Subkategorie zum Thema Lehren wurde das Wissen zu einer Fehlerkultur rekonstruiert. Konkret wünscht man sich eine Kultur, in der Fehler toleriert und Angstsituationen vermieden werden. Ziel soll sein, gemeinsam Fehler aufzugreifen und zu bearbeiten, damit die SchülerInnen ihre Fehler erkennen und Fehler selbstständig vermeiden können. Den Befragten ist es wichtig, dass ihre SchülerInnen „aus Fehlern lernen“ (It 25) können. Hierzu wurden in den Interviews keine genauen Handlungsstrategien genannt.

Die Lernstandserhebungen sind eine weitere rekonstruierte Subkategorie zum Thema Lehren. Eine Lehrperson schildert die Bedeutung der Leistungen von SchülerInnen:

„Hier ist das Wissen zentral, wie man wertschätzend Klassenarbeiten von SchülerInnen bewertet.“ (It 24)

Aus den Interviews lassen sich vier Merkmale für eine Lernstandserhebung rekonstruieren (vgl. Abbildung 44)

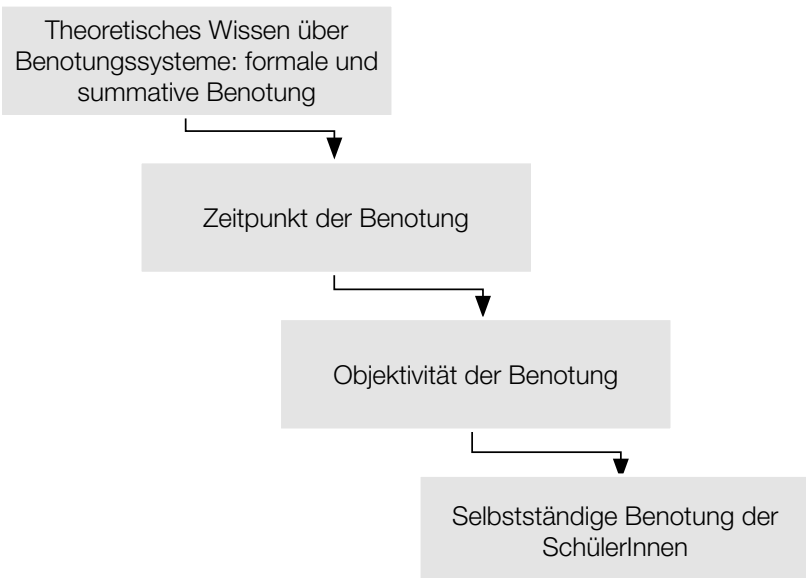

Abb. 44: Wissensbereiche von Lernstandserhebungen 
Die Interviewten betonen die Notwendigkeit von Grundlagenwissen bezogen auf formale oder summative Benotungssysteme:

„Ich mache lange keine Tests mehr, weil somit das Lernen nicht gefördert wird. Das dadurch erlernte Fachwissen ist eventuell eine Woche bzw. an diesem einen Tag vorhanden. [...] Wir erarbeiten lieber zusammen ein Thema und danach präsentieren die SchülerInnen ihr Wissen. Das wird dann sehr individuell umgesetzt. Entweder können sie ein Referat vorbereiten oder sie entwickeln selbst eine Idee. Ich sage einfach - ihr müsst mir beweisen, dass ihr über das Thema X Bescheid wisst.“ (It 9)

Effiziente Beurteilungsformate werden von den Befragten über die Kriterien transparente Notengebung, Mix aus summativen und formativen Benotungen, Berücksichtigung mündlicher wie schriftlicher Leistungen, Arbeiten mit Punktesystemen, regelmäßiges Feedback oder Einfließen von Sozialleistungen und Engagement beschrieben. Ein weiteres angesprochenes Merkmal der Lernstanderhebung ist der Benotungszeitpunkt. Die befragten Lehrpersonen betonen, die Lernstandserhebung nicht zum Hauptbestandteil des Unterrichts zu machen. Permanentes Prüfen und Kontrollieren wird als negativ wahrgenommen. Die Objektivität wird für den Benotungsprozess als grundlegendes Kriterium beschrieben. Allerdings betonen die Interviewten die Objektivität als herausfordernd und problematisch, da es ihnen oftmals schwer fällt, neutral und sympathiefrei zu bewerten. Sie erwähnen als einen weiteren Punkt die Bedeutung der Selbstbenotung der SchülerInnen. Darunter definieren die Befragten die Kompetenz der SchülerInnen, ihre Leistungen eigenständig zu benoten und ihre Leistungen einschätzen zu können.

Als letzte Subkategorie zum Thema Lernen thematisieren Gymnasiallehrpersonen das Wissen zur Motivation. Um die Motivation bei SchülerInnen zu steigern, wird das Eingehen auf Wünsche, Vorlieben und Bedürfnisse der SchülerInnen hervorgehoben:

„Ich mache sehr viel Gruppenarbeit mit dem Hintergrund, dass sie sich eigene Gedanken zu dem Thema machen. Ich motiviere sie kreativ zu sein. Es ist wichtig herauszufinden, wie die Motivation bei den SchülerInnen ist. Manche arbeiten nur, weil sie es lernen müssen andere wiederrum, weil sie das Thema wirklich interessiert." (It 15)

Eine Lehrperson benennt spezifische Strategien, wie sie die Motivation im Unterricht fördern kann. Sie nennt das Loben sowie den Spaß im Unterricht:

„Ich motiviere durch Lob und einen lockeren Umgang. Was sehr hilfreich ist, sind schauspielerische Eigenschaften. Wenn man Dinge übertreibt, wird das ganze etwas aufgelockert. Ich versuche witzig zu sein, übertreibe gerne und bringe sie so zum Lachen." (It 16)

Weitere genannte Strategien für die Förderung eines motivierenden Unterrichts beziehen sich auf Neugierde wecken, Kribbeln auslösen, Zubören, Anerkennung von Leistungen, Kommunikation mit SchülerInnen, Hilfestellungen anbieten und einen abwechslungsreichen Unterricht anbieten.

Theoretische Wissensgrundlagen über extrinsische bzw. intrinsische Motivation wird von den Lehrpersonen ebenso vorausgesetzt. Der intrinsischen Motivation für das Lernen wird eine wesentliche Bedeutung zugeschrieben:

„Es gibt zwei Arten von Motivation - die intrinsische und extrinsische. Als Lehrperson muss ich wissen, wie ich beide Formen im Unterricht adäquat einsetze und fördere. Für das Lernen ist vor allem die intrinsische Motivation bei den SchülerInnen zentral.“ (It 6) 
Die Kategorie Lehren beeinflusst nach Angaben der Interviewten maßgeblich das Lernen von SchülerInnen. Lernen und Lehren stehen in einer starken Wechselbeziehung zueinander. Von Bedeutung für Lehren und Lernen ist das Classroom-Management. Dieses wird im folgenden Abschnitt dargestellt.

\subsubsection{Classroom-Management}

Das Classroom-Management stellt eine weitere rekonstruierte Kategorie pädagogischen Wissens dar. Diese umfasst die Subkategorien Leadership, Störungen, Unterrichtsplanung und Ablauf. Letzterer Punkt gliedert sich zusätzlich in die Bereiche Unterrichtsvorbereitung, Unterrichtsdurchführung sowie Unterrichtsnachbereitung (vgl. Abbildung 45):

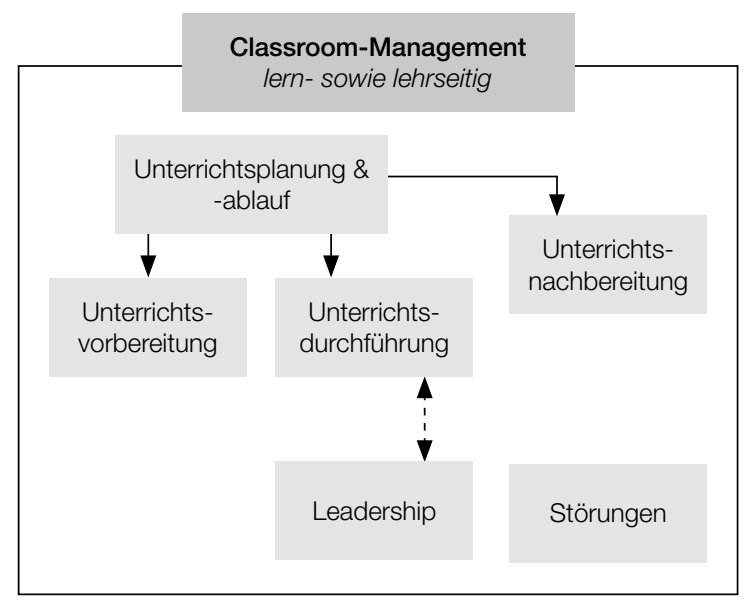

Abb. 45: Subkategorien Classroom-Management

Die Befragten führen hinsichtlich des Classroom-Managements das Wissen zur Planung von Unterrichtsabläufen an.

„Die Organisation benötigt ein zentrales pädagogisches Wissen. Ich beziehe das jetzt einmal auf den Unterricht. Da geht es doch zuerst einmal generell um die Unterrichtsplanung und den Ablauf des Unterrichts. Dazu gehören dann die Unterrichtsvorbereitung, Unterrichtsdurchführung und die Unterrichtsnachbereitung [...]. (It 23)

Ziel ist eine spezifische Unterrichtsvorbereitung. Diese umfasst das Definieren von Lernzielen, das Wissen über den gezielten Einsatz von Unterrichtsmethoden, die Berücksichtigung des Kontextes und die Anwendung von unterschiedlichen Unterrichtssettings.

Im Zusammenhang mit der Unterrichtsplanung wird die Notwendigkeit zeitlicher Ressourcen betont, in denen Lehrpersonen mithilfe ihres „pädagogischen Gespürs“ spontan auf die Bedürfnisse der SchülerInnen reagieren und entsprechend agieren können:

„Pädagogisches Gespür ist die Klassenstruktur zu erfassen und leiten zu können, zu schauen, wie es ihnen geht und wie sie untereinander agieren. Das einfach wahrzunehmen, anzusprechen, in der Gruppe anzusprechen und soziale Kompetenz und Intelligenz zu fördern. Das zum Thema machen, das ist ein Mangel.“ (It 9) 
Rekonstruierte Merkmale einer Unterrichtsdurchführung beziehen sich auf das Zeitmanagement sowie auf einen strukturierten Ablauf. Ergänzend argumentieren die Befragten, dass es wichtig ist, flexibel in der eigenen Agenda zu sein, um auf die SchülerInnen reagieren zu können:

„Ich versuche immer spontan auf die Gegebenheiten im Unterricht zu reagieren und nicht ein Programm durchzudrücken, das ich mir überlegt habe. Wenn sie müde sind und keine Lust mehr haben, versuche ich über ganz einfache Auflockerungsübungen, die sie gar nicht wahrnehmen sollen, sie wieder zu erreichen und zu motivieren. Ich erkläre den SchülerInnen nicht lange, was wir hier machen, damit keine Unsicherheit aufkommt." (It 14)

Die Interviewpartner beschreiben als wichtiges Merkmal der Unterrichtsdurchführung das transparente Arbeiten:

„Es ist wichtig, dass die Lehrpersonen transparent Arbeiten. Die SchülerInnen müssen über die Erwartungen von Lehrpersonen Bescheid wissen und Unterrichtsabläufe verstehen." (It 15)

SchülerInnen können durch transparentes Arbeiten dem Unterricht besser folgen. Sie kennen die Erwartungen der Lehrpersonen und können mit diesen angemessen umgehen.

Das Wissen zur Unterrichtsnachbereitung wurde von den Interviewten im Zusammenhang mit der Unterrichtsplanung beschrieben. Dabei zeigt die Analyse, dass die Lernleistungsdokumentation nicht nur im Rahmen der Unterrichtsdurchführung, sondern auch bei der Unterrichtsnachbereitung stattfinden sollte. Einige Befragte unterstreichen die Notwendigkeit, Unterrichtsstunden im Anschluss zu analysieren, zu hinterfragen und zu reflektieren. Dies kann über das Einholen von Feedback der SchülerInnen, den Austausch mit KollegInnen bzw. über Selbstreflexion stattfinden. Wie die Lehrpersonen im Interviewauszug darlegen, vertreten sie die Position, dass die eigene Professionalität nur dann weiterentwickelt werden kann, wenn man eigene Handlungs- und Denkweisen hinterfragt:

„Ein Unterricht braucht eine Nachbereitung. Hierfür gibt es unterschiedliche Möglichkeiten. Ich denke es ist wichtig, dass man nach dem Unterrichten die Stunde hinterfragt und reflektiert. Nur dann ist es möglich sich weiterzuentwickeln." (It 22)

Das Wissen zu Störungen im Unterrichtsablauf ist eine weitere Subkategorie zum Thema Classroom-Management. Die Interviewten verweisen auf externale sowie internale Störungen (vgl. Abbildung 46).

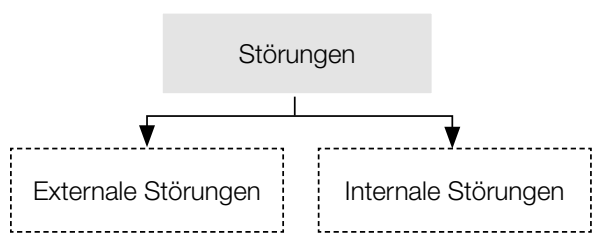

Abb. 46: Internale und externale Störungen im Unterricht

Externale Störungen beziehen sich auf Einflüsse, die von anderen Personen ausgehen, z.B. wenn eine Kollegin/ein Kollege ins das Klassenzimmer kommt, um Informationen zu verkünden, wenn SchülerInnen miteinander reden oder ein Handy klingelt. Der Umgang mit diesen Stö- 
rungen verlangt von Seiten der Lehrperson Fertigkeiten und Fähigkeiten, um situationsgerecht reagieren zu können. Internale Störungen werden von den Interviewten als solche Faktoren definiert, die von den Lehrpersonen selbst ausgehen. Diese können durch Situationen wie eine schlechte Unterrichtsvorbereitung, unpassendes Zeitmanagement, Vergesslichkeit oder hektisches Agieren auftreten.

Das Wissen zu Leadership ist die letzte von den Befragten benannte Subkategorie des Classroom-Managements. Wie in Abschnitt 8.1.2 erwähnt, zeigt die Analyse Überschneidungspunkte mit den Kriterien der Unterrichtsdurchführung aus der Kategorie Lehren. Schnittstellen betreffen das Wissen zur transparenten Führung sowie das spontane Reagieren und Agieren auf Situationen im Klassenzimmer. Die Interviewten bennen weitere Aspekte, die eine professionelle Führung kennzeichnen. Grundlegend ist die „soziale Kompetenz, um eine Klasse leiten zu können“ (It 9). Im Zuge der Rekonstruktion ergaben sich sechs Merkmale einer professionellen Führung:

- Führen auf Augenhöhe: Die Führung einer Lehrperson sollte auf Augenhöhe mit den SchülerInnen stattfinden.

- Klare Strukturen im Unterricht schaffen: SchülerInnen müssen die Aufgaben, Regeln und Rituale, die im Klassenzimmer herrschen, kennen. Die Lehrpersonen müssen einen Blick auf das Ganze haben, um die Klasse zusammenzuhalten und zu organisieren.

- Wissen zur soziale Handlungsfähigkeit: Zentraler Aspekt ist das Wissen darüber, wie eine Lehrperson über Handlungs- und Kommunikationsstrategien zu einem sozial ausgleichenden Klassenklima beitragen kann. Spaß und Freude sind essenzielle Faktoren eines Unterrichts. Spontanes, situationsgerechtes Reagieren ist eine wesentliche Eigenschaft für die soziale Handlungsfähigkeit.

- Strukturelles Wissen: Strukturelles Wissen bezieht sich auf strukturelle sozio-demografische Merkmale einer Klasse wie das Alter, das Geschlechterverhältnis oder die Herkunft.

- Wissen über Zuständigkeiten: Lehrpersonen müssen wissen, welche Zuständigkeiten sie im System Schule übernehmen müssen. Es wird vorausgesetzt, dass sie ihre Aufgaben- und Verantwortungsbereiche kennen und sich mit diesen identifizieren können.

- Wissen über Gruppendynamiken: Das Wissen über Gruppendynamiken bezieht sich auf das Wissen über den Einfluss von Einzelpersonen, vorherrschende Spannungsverhältnisse sowie Gruppenzusammenschlüsse.

Das Wissen zum Classroom-Management wird von allen Interviewten als grundlegender Wissensbereich für einen strukturierten Ablauf des Unterrichts benannt. Die genannten Subkategorien gehören vorwiegend zur lehrseitigen Perspektive. Die Befragten beschreiben ClassroomManagement als einen Bereich des pädagogischen Wissens, der sich auf das Lernen und Lehren auswirkt. Die Befragten greifen bei ihrem Wissen zum Classroom-Management auf Systemwissen zurück. Dieses wird im folgenden Abschnitt beschrieben.

\subsubsection{Systemwissen}

Das Systemwissen wird als eine lehrseitige Kategorie rekonstruiert. Die Interviewten berichten von unterschiedlichen Bereichen, die spezielles Systemwissen für das Lehren voraussetzen. Diese betreffen das Wissen um Gesellschaft, Bildungspolitik, Schulstruktur und Curriculum (vgl. Abbildung 47). 


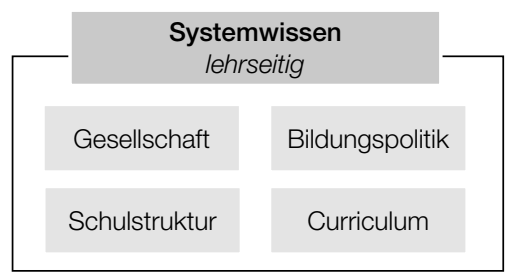

Abb. 47: Subkategorien Systemwissen

Das Wissen von Gesellschaft bezieht sich auf kontinuierliche gesellschaftliche Entwicklungen sowie darauf, diese angemessen im Unterricht zu integrieren. Die Befragten verweisen in den Interviews auf eine wechselseitige Beziehung zwischen Schule und der Gesellschaft. Die Schule wird von ihnen als „Spiegel der Gesellschaft“ (It 14) beschrieben. Dies bedingt, dass Lehrpersonen mit gesellschaftlichen Anforderungen und Veränderungen im Schulalltag adäquat umgehen müssen. Aktuell relevante Themen beziehen sich zum Beispiel auf die Zunahme der Heterogenität, die Veränderung von Familienstrukturen sowie Themen der Mobilität. Die Interviewten beschreiben, wie spezifisches Wissen für den angemessenen Umgang mit neuen Anforderungsbereiche notwendig ist:

„Die Gesellschaft verändert sich. Die Schule und Lehrpersonen müssen damit umgehen. Wir brauchen ein umfangreicheres spezifisches Wissen darüber, wie wir neue Anforderungen in den Schulalltag implementieren." (It 16)

Als eine Subkategorie des Systemwissens benennen die Interviewten das bildungspolitische Wissen. Es ist laut den Befragten treibender Motor neuer Reformen und Veränderungen. Sie benennen in diesem Zusammenhang ihre Rolle als „Player im System“ (It 10) und heben die Abhängigkeit vom System hervor. „State of the Art“ zu sein (It 13) in Bezug auf aktuelle bildungspolitische Prozesse wird als wesentlich erachtet. Die Befragten bleiben bei ihren Ausführungen an der Oberfläche und gehen nicht ins Detail.

Eine weitere rekonstruierte Kategorie ist das Wissen zur Schulstruktur. Die Interviewten beschreiben, wie jede Schule über eigene Regeln verfügt. Diese beeinflussen maßgeblich das Handeln der Lehrpersonen. Genannte Faktoren beziehen sich auf die Dauer von Unterrichtsstunden, die Häufigkeit der Pausen, die Stundenverteilung am Tag sowie die Infrastruktur, wie Räume, verfügbare Materialien und Geräte an einer Schule.

Im Zuge der Interviews wurde das Wissen über Curricula von Lehrpersonen der Neuen Mittelschule und des Gymnasiums angesprochen:

„Politik und Verwaltung beeinflussen Inhalte und Unterrichtsweisen die wir als Lehrpersonen berücksichtigen müssen. Es gibt das Curriculum als vorgegebenes Instrument dieser zwei Instrumente, deren Inhalte wir umsetzen müssen." (It 24)

Die Befragten erläutern in den Interviews, dass sie sich beim Unterrichten auf curriculare Vorgaben stützen. Sie verweisen auf die Pflicht, „die dort vorgegebenen Inhalte ordnungsgemäß umzusetzen, um so die Kompetenz der SchülerInnen der Schulstufe entsprechend aufzubauen und zu fördern“ (It 22). Das Wissen über Anforderungen und Inhalt des Curriculums und die zu erbringenden Erziehungsaufgaben wird als grundlegend beschrieben. 


\subsubsection{Beziehungen}

Die im Rahmen der Interviews gewonnen Ergebnisse verdeutlichen das lern- sowie lehrseitige Wissen von Beziehungen. Die Befragten vertreten die Position, dass sich das Lehren und das Lernen nur unter Berücksichtigung von Beziehungen realisieren kann. Diese werden von den Lehrpersonen anhand den Subkategorien Wissen um die Person, Umgang sowie das Wissen zur Kooperation (vgl. Abbildung 48) beschrieben.

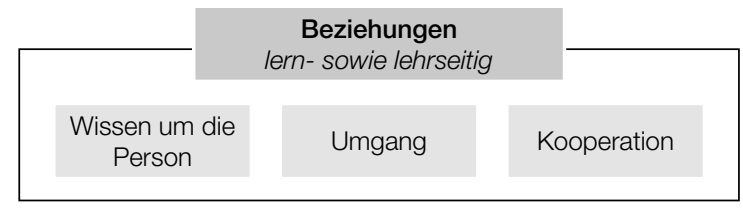

Abb. 48: Subkategorien Beziehung

Wissen um die Person definiert sich an dem Interesse an SchülerInnen. Die Interviewpartner benennen dabei, wie in Abbildung 49 veranschaulicht, das Wissen über Kulturen, Sprachen, Religionen, Familienmitglieder, die jeweiligen familiären Rollen sowie allgemeine Lebenseinstellungen und Hobbies.

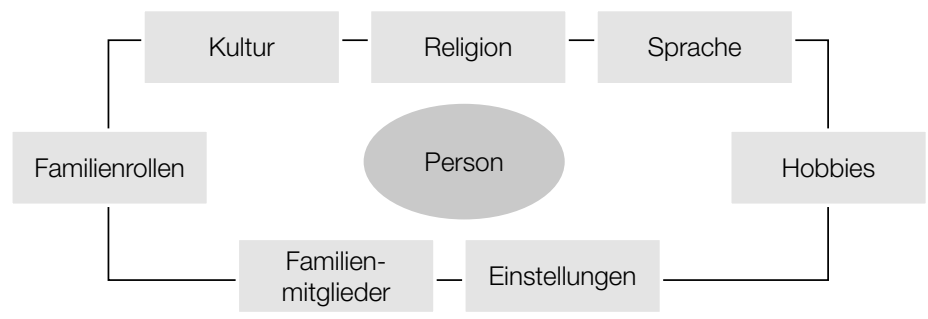

Abb. 49: Private Hintergründe von Kindern und Jugendlichen

Die Interviewten betonen, dass es von Vorteil sein kann, wenn SchülerInnen ihre Lehrpersonen zu einem gewissen Grad persönlich kennen. Wichtig erschien ihnen das Erzählen persönlicher Geschichten aus ihrem Leben:

„Eine gute Beziehung ist für mich, wenn ich Interesse an den Kindern, und zwar an jedem Einzelnen, zeige. Und wenn ich jetzt die Paul Nitsche zitiere, mit ihren ganzen Strategien wie sie sagt: Die Klasse spiegelt dich. Da steckt so viel drin. Das stimmt. So wie ich mit den Kindern umgehe, so gehen sie untereinander um, so gehen sie mit mir um." (It 9)

Eine von den Interviewten genannte Subkategorie der Kategorie Beziehungen ist der Umgang von Personen. Es wird in den Interviews beschrieben, wie Lehrpersonen mit ihrem eigenen Verhalten den Umgang in einer Klasse beeinflussen können. Alle Befragten sind sich einig, dass das Wissen über einen pädagogisch wertvollen Umgang im Klassenzimmer einen wichtigen Bestandteil pädagogischen Wissens bildet. Abbildung 50 skizziert, was sie unter einem pädagogisch wertvollen Umgang verstehen: 


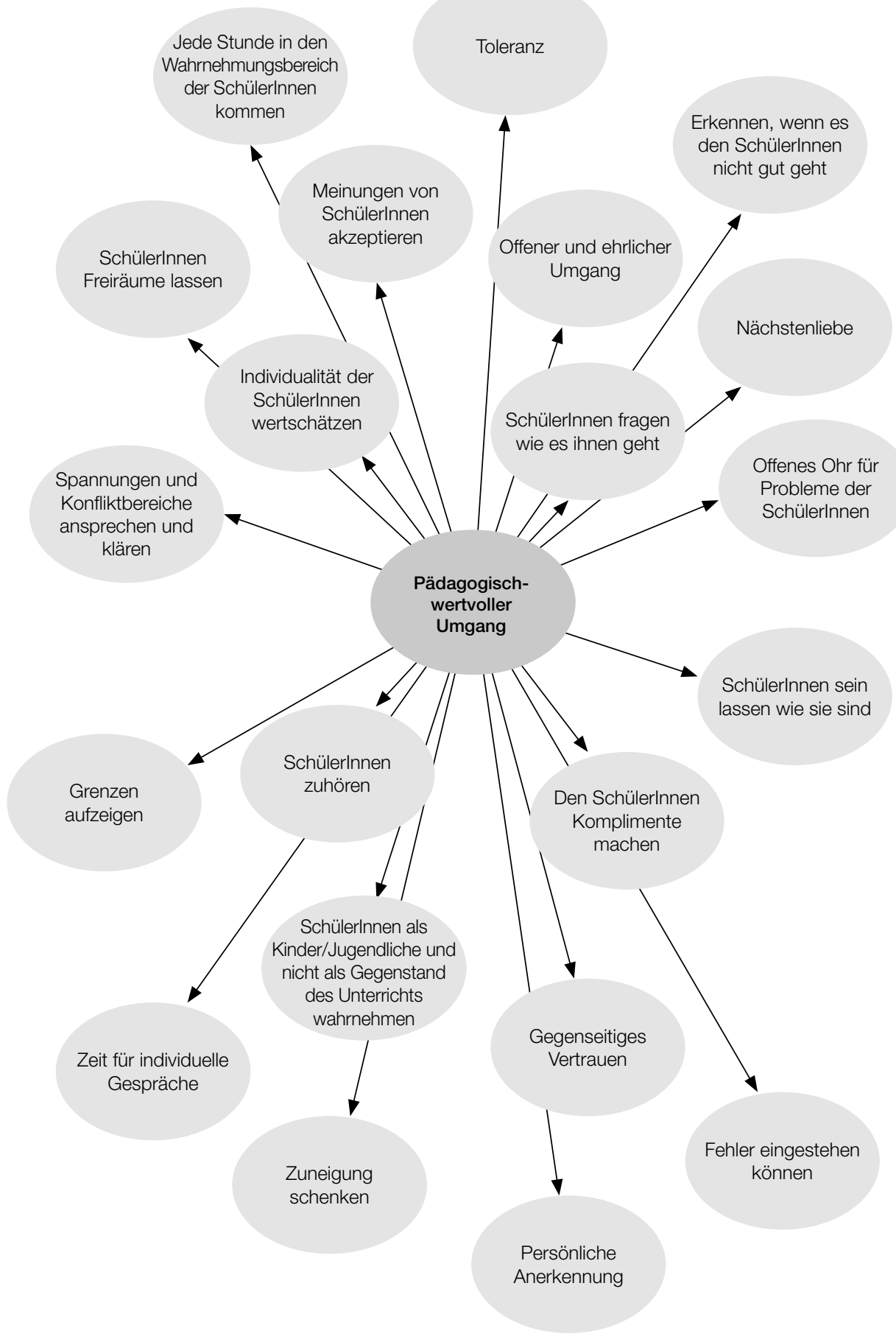

Abb. 50: Gesamtverständnis eines pädagogisch wertvollen Umgangs 
Ein pädagogisch wertvoller Umgang charakterisiert sich über ein mehrdimensionales Verständnis. Dieses bezieht sich, wie in der Abbildung 50 dargestellt, auf Merkmale wie strukturelle Rahmenbedingen, das Interesse an SchülerInnen sowie Aspekte des gemeinsamen Arbeitens. Die Befragten erwähnen, dass SchülerInnen über ihr Verhalten aufzeigen, inwieweit ein pädagogisch wertvoller Umgang stattfindet:

„Man merkt sofort am Verhalten von den SchülerInnen, ob ein wertvoller Umgang im Klassenzimmer stattfindet. Ich bin der Meinung, dass wenn SchülerInnen Gefühle zulassen und sich nicht bei negativen/peinlichen Situationen schämen, ist ein guter Umgang vorhanden." (It 15)

Die Kooperation stellt eine weitere rekonstruierte Subkategorie der Hauptkategorie Beziehungen dar. Diese umfasst nicht nur die Kooperation zwischen Lehrpersonen und SchülerInnen, sondern auch die Kooperation zwischen Lehrpersonen und Erziehungsberechtigten, Schulleitung, KollegInnen, externem Fachpersonal sowie jene zwischen den SchülerInnen. Eine Interviewte berichtet, wie über die Kooperation und Zusammenarbeit mit unterschiedlichen AkteurInnen pädagogisches Wissen aufgezeigt werden kann (It 1). Der Schwerpunkt der Kooperation lag bei den Interviews mit den Gymnasiallehrpersonen auf der Zusammenarbeit zwischen den Lehrpersonen. Ein Interviewpartner spricht jedoch von einer LehrerInnenkultur, die über Einzelkämpfer charakterisiert ist und in der jede/jeder „ein eigenes Süppchen kocht" (It 14).

„Jede Lehrperson macht hinter sich die Klassenzimmertür zu und keiner weiß was dahinter passiert. Wir sollten eine weitaus offenere Kultur haben, in der wir keine Einzelkämpfer sind, sondern gemeinsam an einem Strang ziehen." (It 25)

Die Befragten fordern in den Interviews bessere Kooperation und glauben, dass sich so die Qualität von Lern- und Lehrprozessen steigern lässt. Der Austausch untereinander wird als gewinnbringende Ressource für das LehrerInnendasein argumentiert. Die genannten Austauschformate beziehen sich auf eine (fächerübergreifende) Zusammenarbeit, das Abstimmung von sozialen Themen, den Austausch mit KollegInnen und das gemeinsame Erstellen von Unterlagen:

„Das ist sehr spannend für mich, weil Lehrpersonen ganz unterschiedliche Zugänge zum Lernen und Lehren haben. Gerade über den Austausch von kleinen Unterrichtssequenzen, wo man findet, dass man sie gut gemacht hat, kann man sehr viel dazulernen." (It 14)

Um dieses Ziel zu erreichen, erwähnen die Interviewten die Bedeutung einer kooperativen Haltung. Eine gewinnbringende Zusammenarbeit verlangt die Fähigkeit, die eigenen Bedürfnisse zurückzustellen und neue Sichtweisen einzunehmen. Dies wird jedoch von den Befragten als herausfordernd gesehen.

Um eine gute Kooperation zwischen KollegInnen zu fördern, wird ein regelmäßiger Austausch für nötig erachtet. Dabei sollen nicht nur Problemfälle und Schwierigkeiten besprochen werden, essenziell ist laut den Befragten der Austausch von Erfolgserlebnissen und sogenannten Sparkling Moments ${ }^{41}$. Sie argumentieren, dass der Austausch untereinander die Möglichkeit bietet, neue Handlungsstrategien und Verhaltensweisen kennenzulernen, die ihre eigene Professionalität weiterentwickeln. Eine Interviewte betont den Austausch über neue wissenschaftliche Theorien und Studien:

41 Bedeutung in diesem Zusammenhang: prägende, nachhaltige Momente. 
„Wir sollten uns viel öfters über neue wissenschaftliche Theorien oder Studien austauschen. Das würde einen großen Mehrwert bringen. Erst dadurch erhält man einen vielfältigeren Weitblick über den aktuellen Forschungsstand und kann neue Perspektiven und Möglichkeiten in den Unterricht integrieren.“ (It 23)

Wie das Zitat verdeutlicht, sehen die Befragten einen Mehrwert in der Zusammenarbeit. Im Mittelpunkt des Interesses steht die Zusammenarbeit von sozialen und pädagogischen Feldern, um Gruppendynamiken, Klassengemeinschaften sowie die eigene Professionalität zu stärken. Um einen regelmäßigen Austausch zu ermöglichen, geben die Interviewten neben dem Engagement der Lehrpersonen die Notwendigkeit einer Unterstützung von der Schulleitung als wichtig an. Diese muss laut Interviewten über eine gute Führungskompetenz verfügen. Damit Teamprozesse und Austauschplattformen im Schulalltag nachhaltig implementiert werden können, benennen die Befragten sechs wesentliche Merkmale:

- das Engagement aller beteiligten AkteurInnen

- das wertfreie Sammeln von Ideen und Anregungen

- die sachliche Bewertung von Aussagen

- Wertschätzung

- gemeinsames Entwickeln von Zielvorstellungen und Vereinbarungen

- Raum für Geselligkeit, Humor und Ironie

Diese Merkmale fördern nach Abgaben der Interviewten die Kooperationen zwischen Lehrpersonen.

Lehrpersonen insbesondere des Gymnasiums und der Neuen Mittelschule erwähnen das TeamTeaching. Sie benennen spezifisches pädagogisches Wissen, das für den Einsatz von Team-Teaching von Bedeutung ist. Ziel ist laut den Befragten eine individuelle Förderung der SchülerInnen, die durch räumliche und zeitliche Ressourcen garantiert werden kann. Damit es nicht zur sogenannten „Heizungsdidaktik“ ${ }^{2}$ kommt, werden wesentliche Parameter genannt, die eine zielführende Zusammenarbeit unterstützen (vgl. Abbildung 51).

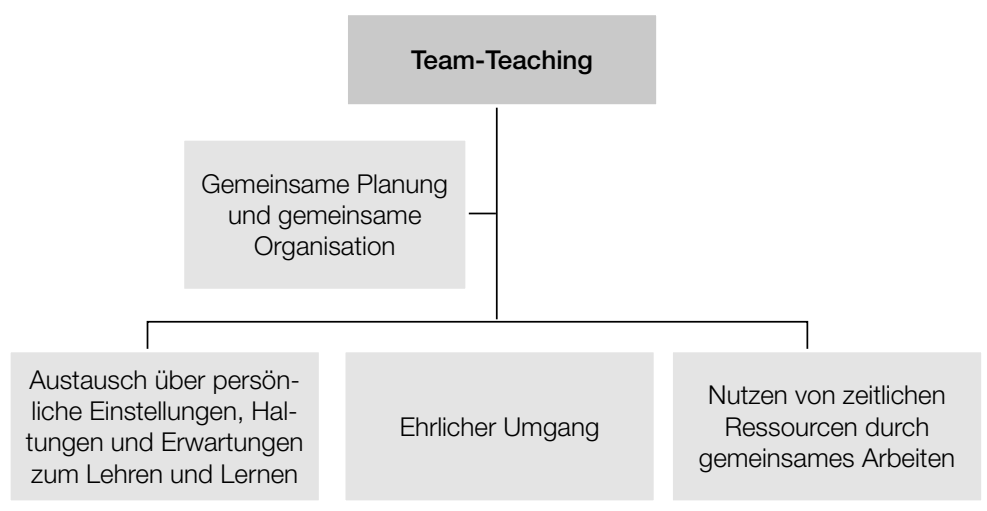

Abb. 51: Verständnis zielführender Team-Teaching-Prozesse im Unterricht

42 Während des Team-Teachings unterrichtet eine Lehrperson, während die zweite Lehrperson an der Heizung steht und dem Unterrichtsgeschehen zuschaut. Nur eine Lehrperson führt und organisiert den Unterricht. Die zweite Lehrperson greift nur ein, wenn es nötig ist. Die Verantwortung und Planung liegt nur bei einer Lehrperson. Jede Stunde übernimmt eine andere Lehrperson die Führungsrolle. 
Wie in Abbildung 51 dargestellt, beziehen sich wesentliche Parameter von Team-Teaching-Prozessen auf die gemeinsame Planung und Organisation, den Austausch über Haltungen und Erwartungen zum Lehren und Lernen, einen ehrlichen Umgang sowie das Nutzen von zeitlichen Ressourcen durch gemeinsames Arbeiten.

Als weitere Kooperationsstruktur benennen die Interviewten die Zusammenarbeit mit den Eltern. Sie heben einen regelmäßigen Austausch zwischen Lehrperson und Erziehungsberechtigten hervor. Verhaltensmuster, Rollenstrukturen, Problemfelder oder positive Erfahrungen von Kindern und Jugendlichen sollten besprochen werden. Die Anliegen und Erwartungen der Eltern sollten Lehrpersonen bei ihren Entscheidungsfindungen im Zusammenhang mit den SchülerInnen nicht außer Acht lassen. Gefordert wird eine offene und wertschätzende Beziehung zwischen Lehrpersonen und Erziehungsberechtigten. Diese kann laut Aussage den Lehrpersonen nur über regelmäßige Treffen entstehen.

In Bezug auf die Kooperation mit SchülerInnen charakterisieren die Interviewten diese über einen offenen, respektvollen und ehrlichen Umgang, Mitspracherecht der SchülerInnen sowie Verlässlichkeit:

„Wir sollten uns gegenseitig nichts vormachen, sondern offen und ehrlich zueinander sein. Für mich ist es oft sehr faszinierend, dass sie diese Kooperation annehmen. Sie zeigen Respekt mir gegenüber und kommen immer wieder zu mir, da sie meine Aufmerksamkeit brauchen und wir sozial zusammen kooperieren müssen. Ich habe oft das Gefühl, dass sie alle liebes- und aufmerksamkeitsbedürftig sind." (It 9)

Die Zusammenarbeit beeinflusst das Beziehungsgefüge zwischen Lehrpersonen und SchülerInnen. Wichtig erscheint den Befragten, eine Zeit spezifisch für den Austausch in den Schulalltag zu integrieren, um eine gegenseitige Beziehung aufzubauen.

Die letzte angesprochene Kooperation bezieht sich auf die Zusammenarbeit mit externen Fachleuten:

„Die Zusammenarbeit mit externen Fachleuten erweist sich als immer wichtiger. Die unterstützenden Hilfsleute erleichtern es mir im Unterricht auf Problemsituationen und ProblemschülerInnen zu reagieren. Ich weiß vor allem, dass ich nicht allein gelassen werde und das ist schon Sicherheit genug". (It 14)

Die Befragten berichten, wie die Zusammenarbeit mit externen Fachleuten das LehrerInnendasein erleichtert, indem sie gezielt auf Unterstützungsmöglichkeiten zurückgreifen können. Besonderes Gewicht wird TherapeutInnen, Beratungslehrpersonen sowie den SchulärztInnen zugeschrieben. Die Interviews verdeutlichen, dass die befragten Lehrpersonen aus der Grundschule und der Neuen Mittelschule im Vergleich zum Gymnasium öfter auf die Hilfestellungen von externem Fachpersonal zurückgreifen.

Beziehungen werden für den Lernprozess von SchülerInnen sowie für die Lehrpersonen als essenzielles pädagogisches Wissen beschrieben. Sie fördern das Lernen und erweitern über Austauschprozesse eigene Denkweisen. Eine weitere Kategorie, die sich auf die Beziehung auswirken kann, ist die Kommunikation. Diese wird im folgenden Abschnitt dargestellt.

\subsubsection{Kommunikation}

Im Rahmen der Interviews konnte als Kategorie pädagogischen Wissens das lern- sowie lehrseitige Wissen zur Kommunikation rekonstruiert werden. Der Wissensbereich gliedert sich in die Subkategorien Wissen über Kommunikationsstrategien, Kommunikationsregeln sowie Gesprächsfübrung (vgl. Abbildung 52). 


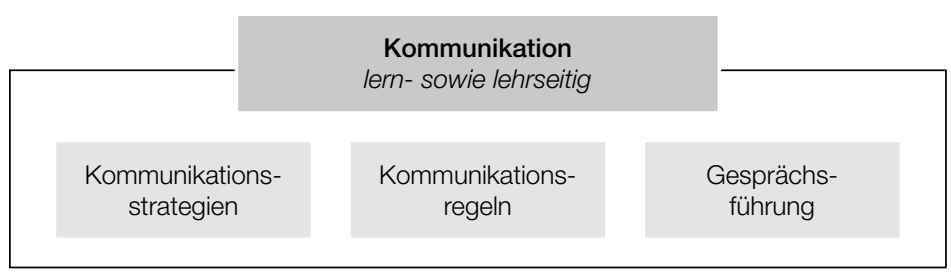

Abb. 52: Subkategorien Kommunikation

Die Befragten berichten in den Interviews von Kommunikationsstrategien, mit denen sie verbal oder nonverbal mit den SchülerInnen in Kontakt treten. Eine Lehrperson erklärt, dass sie über Kommunikation die SchülerInnen für sich gewinnt „[... indem sie auf sie eingeht, schaut wie es ihnen geht, sich Zeit nimmt für jeden Schüler [/jede Schülerin] “ (It 9). Eine Interviewte berichtet, wie sie nonverbal mithilfe ihrer Körperhaltung kommuniziert:

„Ich erreiche die SchülerInnen über die Sprache, $40 \%$ sind davon nonverbal. Ich stehe an einer bestimmten Stelle im Klassenzimmer und die Kinder wissen genau was ich von ihnen verlange. Wenn ich am Waschbecken stehe, dann wissen sie, dass sie ihre Sachen zur Seite räumen und leise an ihren Platz zurückkehren sollen. Diese Dinge habe ich natürlich am Anfang des Schuljahres mit den SchülerInnen geübt.“" (It 4)

Die Rekonstruktion verdeutlich die Wirkung der Kommunikation auf andere Kategorien. Laut den Interviewten beziehen sich die Einflussfaktoren vor allem auf die Wissenskategorien Lehren, Lernen oder auf das Wissen zu Beziehungen. Die Interviewten verweisen dabei auf eine „Beziehung des Wissens [...] das sich nicht allein als pädagogisches Wissen [entwickelt]“ (It 24), sondern sich nur über die Berücksichtigung mehrerer Kategorien ganzheitlich vollziehen kann. Die direkte Kommunikation charakterisiert sich nach Angabe der Lehrenden aller Schulformen über permanentes Nachfragen. Explizit genannte verbale Kommunikationsstrategien betreffen neben dem direkten Ansprechen und Nachfragen das regelmäßige Feedback:

„SchülerInnen sollen nicht nur von der Lehrperson Feedback bekommen, sondern sich gegenseitig Feedback geben. Dies ist jedoch sehr schwierig. Die Feedbackkultur hat viel zu wenig Raum. Die SchülerInnen können das nicht. Sie sagen mir dann es war alles gut, weil sie wissen, dass ich das hören will.“ (It 14)

Die Ergebnisse zeigen, dass Feedback individuell, zwischen SchülerInnen und Lehrpersonen sowie allgemein, in der Gruppe durchgeführt wird. Es umfasst Rückmeldungen zu Leistungen, Verhaltens- und Handlungsweisen. Auf diesem Weg erhalten SchülerInnen nicht nur Feedback, sondern können sich aktiv äußern:

„Feedback von den SchülerInnen einholen ist etwas Wichtiges [...] Und vor allem muss man auf Rückmeldungen hören, da habe ich persönlich ganz viel gelernt." (It 18)

Dieses Instrument bieten Lehrpersonen die Möglichkeit, Feedback von den SchülerInnen einzuholen.

Die Subkategorie Kommunikationsregeln ist Teil der Hauptkategorie Kommunikation. Der Schwerpunkt liegt laut den Befragten auf einer gewaltfreien, ehrlichen und respektvollen Kommunikation: 
„Es fängt damit an, wie ich einen Schüler/eine Schülerin anspreche, wie er/sie mich anspricht. Wie ich mit Ihnen kommuniziere, also mit Respekt, aber doch irgendwie locker. Ich versuche einen lockeren Umgang mit Ihnen zu haben." (It 7)

Sie vertreten die Ansicht, dass vor allem Regeln für eine wertschätzende Kommunikation ein gemeinsames Arbeiten unterstützen. Sie verstehen darunter folgendes: SchülerInnen ausreden lassen, auf Fragen vernünftige Antworten geben, sich nicht gegenseitig verpetzen, nicht lästern oder binter dem Rücken schlecht reden.

Die Gesprächsführung stellt eine zusätzliche rekonstruierte Subkategorie dar. Als wichtiges Kriterium einer gelungenen Gesprächsführung wird angegeben, dem Gesprächspartner mit Respekt auf Augenhöhe zu begegnen, damit sich das Gegenüber verstanden fühlt. Um gegenseitiges Verstehen zu ermöglich, ist das Anwenden einer altersgerechten Sprache essenziell. Weitere rekonstruierte Merkmale schließen das vorurteilsfreie Gespräch sowie das richtige Fragen mit ein.

Die lern- sowie lehrseitige Kommunikation unterstützt im Unterricht das Lernen, Lehren und Beziehungen. Professionelle Kommunikation kann den Unterrichtsverlauf positiv beeinflussen. In enger Beziehung mit der Kommunikation steht die Erziehung. Diese wird im folgenden Abschnitt beschrieben.

\subsubsection{Erziehung}

Die Gesamtanalyse zeigt, dass die Befragten der Kategorie Erziehung eine wichtige Rolle zusprechen. Im Mittelpunkt steht die Autonomie der Kinder und Jugendlichen, die an eine selbstentwickelnde Erziehungsform anknüpft. Rekonstruierte lernseitige Subkategorien beziehen sich auf das Wissen zu Ressourcen. Aus lehrseitiger Perspektive kommen sie über das Wissen zu Werten und Normen in den Interviews zum Ausdruck (vgl. Abbildung 53).

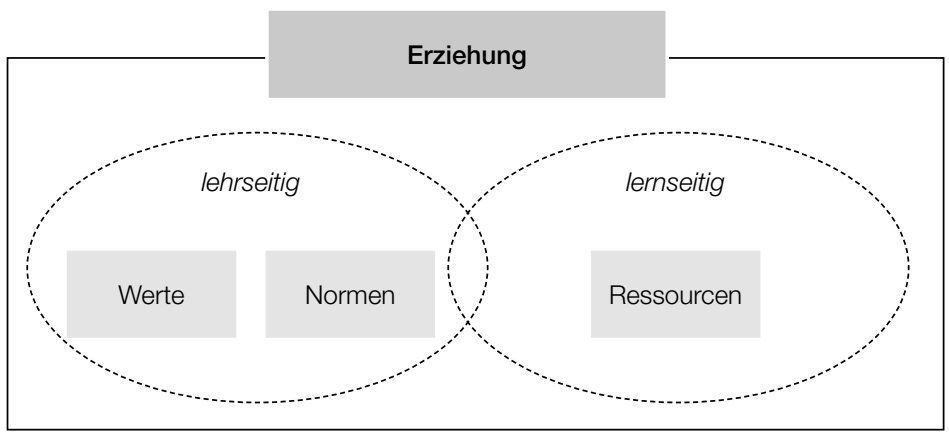

Abb. 53: Subkategorien Erziehung

Die Befragten berichten in den Interviews, wie sie Kinder zu offenen und mündigen Personen erziehen müssen. Sie argumentieren die Notwendigkeit, ein Bewusstsein für die Welt zu schaffen, in der ehrlich und wertschätzend miteinander umgegangen wird und Kinder und Jugendliche ihre eigene Autonomie entwickeln und verwirklichen können:

„Kinder und Jugendliche brauchen mehr Raum sich selbstständig entfalten zu können, ohne dass ständig jemand ihnen im Nacken sitzt und ihnen sagt was zu tun ist. Sie müssen durch Unterstützung von uns lernen, wie man am besten in der Welt und Gesellschaft agiert. Ehrlichkeit und Wertschätzung sind wesentliche Eigenschaften die man den SchülerInnen vermitteln muss.“ (It 23) 
Wie das Zitat zum Ausdruck bringt, sollen sich SchülerInnen durch ressourcenorientiertes Anleiten selbst verwirklichen. Die lernseitige Betrachtung verlangt von den Lehrpersonen, den SchülerInnen Raum für individuelle Gestaltungs- und Entwicklungsmöglichkeiten zu geben, damit diese ihren individuellen Weg einschlagen können.

In den Interviews erwähnen die Lehrenden aller Schulformen die Vermittlung von grundlegenden Werten und Normen. Diese beziehen sich auf die Sensibilisierung der SchülerInnen für eine offene Haltung gegenüber unterschiedlichen Kulturen, Sprachen sowie Geschlechtern:

„Die Kinder und Jugendlichen sollen zu mündigen Personen heranwachsen, die in der Gesellschaft ihren Platz finden. Allerdings ist das Wissen über Werte und Normen, sowie über Regeln des Zusammenlebens wichtig, um Grenzen zu kennen.“ (It 24)

Lehrpersonen sollen den SchülerInnen ein Verständnis über Grenzen und Regeln in der Gesellschaft vermitteln. Schule übernimmt einen Erziehungsauftrag. Lehrpersonen sind gefordert, wesentliche Normen und Werte zu vermitteln sowie die SchülerInnen zu mündigen Menschen zu erziehen. Zu der Kategorie Erziehung benennen die Befragten entwicklungspsychologische Wissensbereiche, die den Erziehungsauftrag positiv unterstützen. Die Hauptkategorie Entwicklungspsychologie wird im folgenden Abschnitt beschrieben.

\subsubsection{Entwicklungspsychologie}

Das entwicklungspsychologische Wissen wurde als eine Hauptkategorie pädagogischen Wissens rekonstruiert. Diese bezieht sich auf das lern- sowie lehrseitige Wissen über Entwicklungsstufen (vgl. Abbildung 54). ${ }^{43}$

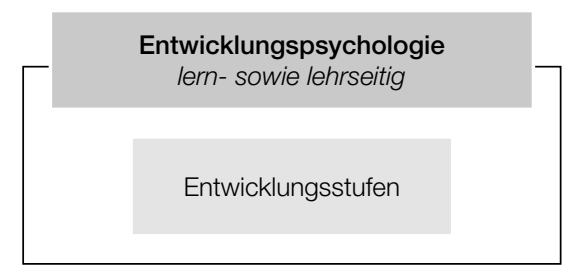

Abb. 54: Subkategorien Entwicklungspsychologie

Das Wissen zu Entwicklungsstufen wird von den Befragten als wichtig beschrieben. Sie verstehen darunter Entwicklungsmerkmale und ihre Bedürfnisse, bezogen auf eine bestimmte Altersstufe:

„Das Wissen über Entwicklungsstufen von Kinder ist zentrales pädagogisches Wissen von Lehrpersonen. In welcher Altersstufe kann ich was erwarten. Natürlich auch die Schülerseite, was brauchen die Kinder, welche Bedürfnisse sollte man als Lehrpersonen erfüllen können." (It 20)

Die Interviewten der Grundschule und Neuen Mittelschule fordern Wissensstrukturen zu einzelnen Entwicklungsstufen und den damit verbundenen Verhaltensmustern sowie Bedürfnis-

43 In Abschnitt 8.1.1 zur Kategorie Lernen wurden lernpsychologische Eigenschaften erklärt. Diese grenzen sich insofern von der Kategorie Entwicklungspsychologie ab, als dass sie sich vorwiegend auf Entwicklungsstufen beziehen. Der Schwerpunkt des lernpsychologischen Wissens in Abschnitt 8.1.1 liegt auf dem Lernen. 
sen. Wichtig erschien den Befragten, „dass man den jungen Menschen als jemanden ansieht, der in einer Entwicklung ist und nicht mit den Leistungen und Fähigkeiten von Erwachsen messen kann“ (It 13).

In Verbindung mit den Entwicklungsstufen benennen die Interviewten das Wissen über Krankheitsbilder, die in den unterschiedlichen Etappen einer Entwicklung auftreten können. Sie beschreiben die Notwendigkeit eines umfassenden Verständnisses von möglichen Defiziten und Krankheitsbildern:

„Wir sollten uns vielmehr mit möglichen Krankheitsbilder und Defiziten auskennen. Vor allem auch zu wissen, wie man diese erkennt und wie man mit ihnen umgeht." (It 15)

Eine wesentliche Fertigkeit einer Lehrperson ist, Krankheiten erkennen zu können und zu wissen, wie man angemessen mit diesen umgehen sollte.

Das Wissen zu Entwicklungsstufen wird von den Lehrpersonen für eine adäquate Unterstützung und Förderung vorausgesetzt. Sie können mithilfe ihres spezifischen Wissens angemessen auf Bedürfnisse der SchülerInnen reagieren und das Lernen der SchülerInnen bestmöglich unterstützten. Die starke Forderung nach der Berücksichtigung diverser Strukturen verstärkt die Bedeutung des Wissens, um individuelles Lernen bestmöglich stattfinden zu lassen. Das Verständnis der Kategorie Diversität wird im folgenden Abschnitt dargestellt.

\subsubsection{Diversität}

Die Diversität stellt eine weitere Kategorie pädagogischen Wissens dar. ${ }^{44}$ Wie aus der Rekonstruktion hervorgeht, präsentieren vorrangig Grundschullehrpersonen sowie Lehrpersonen der Neuen Mittelschule theoretische Ansätze für das Verständnis des Begriffs Diversität. Eine Befragte erklärt, was sie unter Diversität versteht:

„Wir haben immer wieder SchülerInnen mit körperlicher und geistiger Behinderung sowie verhaltensauffällige Kinder. Verhaltensauffällig jetzt nicht nur, dass ich merke, da ist etwas anders, sondern die wirklich negativ orientiert sind, die zerstören wollen. Wir haben sehr viele leistungsstarke SchülerInnen, die extreme Freude am Lernen haben. Wir haben große Unterschiede zwischen den Eltern, die einfach die Schule anders sehen. Wir haben SchülerInnen mit unterschiedlichen Hobbys, Altersstufen, Geschlechtern, Sprachen, Kulturen und Religionen." (It 10)

Beschreiben einige Lehrpersonen ein klares Konzept zum Thema Diversität, argumentieren andere über unzureichendes Wissen:

„Der Umgang mit Heterogenität ist in unserem Studium viel zu kurz gekommen. Es geht nicht nur um verschiedene Muttersprachen oder Behinderungsgrade, sondern es geht darum, dass der eine seinen Stimmbruch als zwölffähriger bekommt und der andere mit vierzehn und wie man damit umgeht. Entwicklungsfelder von Kindern und die Unterschiede beim Lernen“. (It 14)

Die Lehrpersonen verweisen in den Interviews auf gegenwärtige, gesellschaftliche Veränderungen, die sich in der Schule widerspiegeln. Sie definieren den Begriff über die Berücksichtigung individueller Persönlichkeiten, Kulturen, Geschlechter, Potenziale, Schwächen sowie Leistungen von SchülerInnen.

44 Einzelne Inhalte zur Kategorie Diversität wurden in den vorherigen Kategorien vereinzelt angesprochen und diskutiert. Da die Lehrpersonen das Thema Diversität in den Interviews sehr häufig erwähnen, wird es aufgrund seiner essenziellen Bedeutung für die Lehrpersonen als eigene Kategorie angeführt. 
Ingesamt konnten drei lern- sowie lehrseitige Subkategorien rekonstruiert werden: das Grundlagenwissen, das Wissen zum Bewusstsein für Diversität sowie das Wissen zu Umsetzungsstrategien (vgl. Abbildung 55).

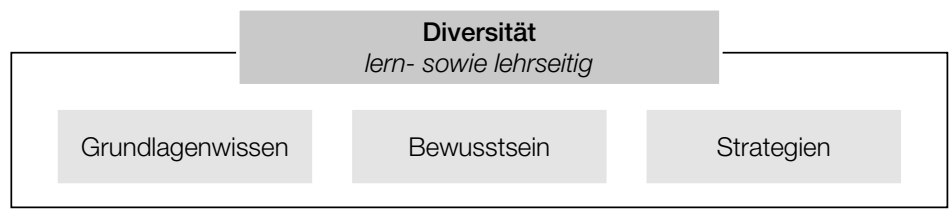

Abb. 55: Subkategorien Diversität

Die Interviewten beschreiben sechs allgemeine Aspekte des Grundlagenwissen zur Diversität:

- Bedeutung von Diversität

- Wissen über Begrifflichkeiten und Abgrenzungen von Heterogenität, Inklusion, Diversität

- Wissen über unterschiedliche Kulturen, Religionen, Sprachen

- Geschlechterspezifisches Wissen

- Wissen über Menschenrechte

- Wissen über Migrationspädagogik

Nur wenige Lehrpersonen nannten explizite Strategien, mit welchen sie das Thema Diversität im Unterricht berücksichtigen. Abbildung 56 illustriert die genannten Umsetzungsstrategien.

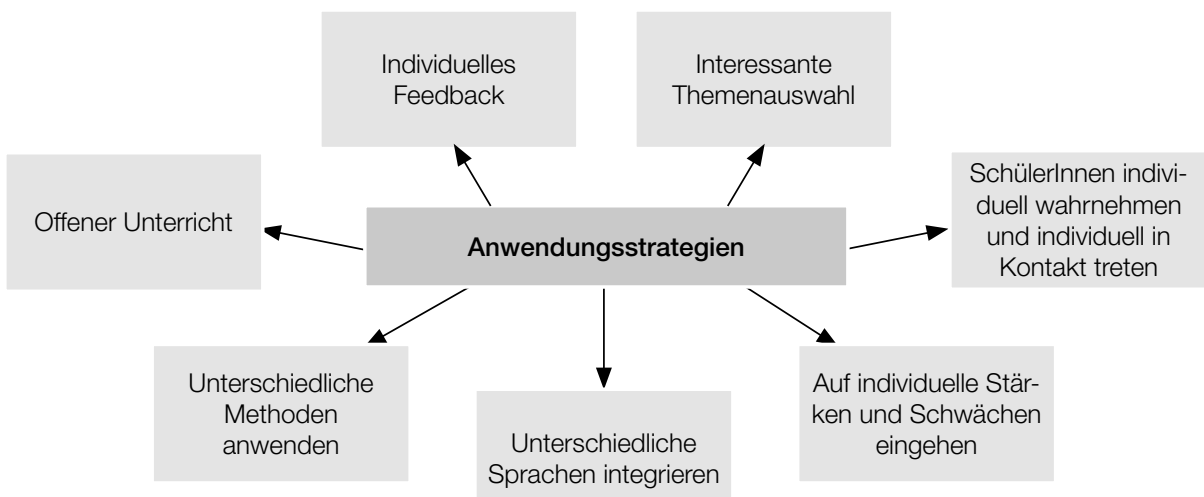

Abb. 56: Umsetzungsstrategien der Kategorie Diversität im Unterricht

Die Umsetzungsstrategien beziehen sich auf das individuelle Feedback, eine interessante Themenauswahl, die Umsetzung eines offenen Unterrichts, die Anwendung unterschiedlicher Methoden, die Integration unterschiedlicher Sprachen, das Wahrnehmen individueller SchülerInnen und die Berücksichtigung von Stärken und Schwächen.

Als weitere Subkategorie wird das Wissen zum Bewusstsein im Kontext der Diversität genannt:

„Das Wissen über Diversität ist momentan das Wichtigste [...] Ich habe den Leitspruch: Jeder Mensch ist anders. Und das muss ich ihnen erklären und den SchülerInnen aber auch mir als Lehrperson das Bewusstsein schaffen, was es bedeutet." (It 9) 
Die Befragten beschreiben die Notwendigkeit eines Bewusstseins im Zusammenhang mit Unterschiedlichkeiten. Allerdings gingen sie mit ihren Ausführungen nicht weiter ins Detail.

Die Kategorie Diversität zeigt sich im Kontext gegenwärtiger Transformationsprozesse, und mit dem Fokus auf dem Lernen, als eine wesentliche Kategorie pädagogischen Wissens. In enger Beziehung damit stehen diagnostische Maßnahmen. Diese werden im Anschluss beschrieben.

\subsubsection{Diagnostik und Förderung}

Eine lern- sowie lehrseitig rekonstruierte Wissenskategorie ist die Diagnostik und Förderung. Das Verständnis diagnostischen Wissens basiert laut den Interviewten auf dem Wissen zu Gewalt, Gruppendynamiken, Unterstützung sowie Konflikten (vgl. Abbildung 57).

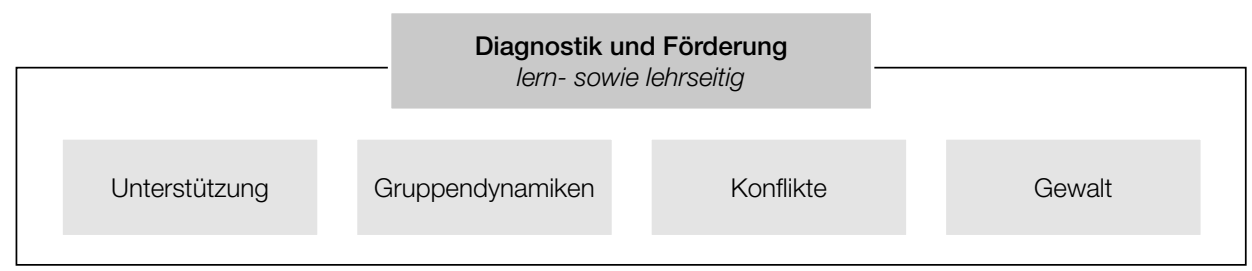

Abb. 57: Subkategorien Diagnostik und Förderung

Unter dem Wissen zur Unterstützung verstehen die Interviewten aller Schulstufen und Schulformen grundsätzlich die Kenntnis spezifischer Unterstützungsmöglichkeiten für SchülerInnen. Diese beziehen sich auf die Förderunge von Stärken und Talenten bzw. die Berücksichtigung von Schwächen oder Defiziten. Die Befragten berichten, wie sie dies über eine ressourcenorientierte Unterstützung erreichen können:

„Wir müssen die SchülerInnen unterstützen. [...] Von uns wird eine ressourcenorientierte Unterstützung gefordert, die den Kindern nicht nur ihre Schwächen sondern ihre Stärken aufzeigt. Wir müssen mehr mit Stärken und Potenzialen arbeiten." (It 17)

Lehrpersonen fordern eine stärkenorientierte Unterstützung, die an Potenziale der SchülerInnen anknüpft und diese nachhaltig fördert. Spezifische Handlungen werden von den Befragten nicht genannt.

Unter dem diagnostischen Wissen wird als Subkategorie das Wissen zu Gruppendynamiken angeführt:

„Jede Gruppe oder Klasse ist anders. Sie lernen anders, brauchen einen anderen Zugang und verhalten sich anders gegenüber mir. Das war am Anfang sehr schwer für mich damit klarzukommen. Ich habe aber sehr schnell gemerkt, dass das was ich in einer Klasse mache und wie ich mich in einer Klasse verhalte, nicht auf alle Klassen übertragen kann." (It 25)

Der Interviewauszug verdeutlicht das Interesse der Lehrpersonen, mit unterschiedlichen Gruppen und individuellen Zugängen zu arbeiten. Sie betonen die Unterschiedlichkeit jeder Klasse, die unterschiedliches Vorgehen und Arbeiten voraussetzt.

Die letzte Subkategorie bezieht sich auf das Wissen zu Konflikten. Darunter verstehen die Befragten Grundlagenwissen zu Konfliktformen sowie Handlungsstrategien. Praxisrelevantes Wissen wird in den Interviews nur mit wenigen Sätzen erwähnt. Die Interviewpartner beschreiben, wie Fort- und Weiterbildungen ihr Wissen in diesem Bereich vergrößert: 
„Wie geht man mit Konflikten und Störungen um? Dies hat mich eine lange Zeit beschäftigt. Ich habe dann eine Weiterbildung zum Thema Mediation gemacht. Seither kann ich sagen, ich kenne passende Handlungsmöglichkeiten, die ich bei Konflikten oder Störungen einbringen kann. Persönlich hat mich diese Weiterbildung beeinflusst [..." (It 21)

Laut den Interviewten ist vor allem die Fähigkeit, in der Klasse vorhandene Probleme und Konflikte zu erkennen, wichtig. Genannte Lösungsstrategien beziehen sich auf das direkte, beschämungsfreie Ansprechen und eine personalisierte Kommunikation.

Eng verbunden mit dem Thema Konflikte berichten die Befragten der Neuen Mittelschule und des Gymnasiums über das Thema Gewalt. Die Interviewten erzählen von einzelnen Gewaltakten in der Schule und fordern eine bessere Ausbildung im Umgang mit dieser Problematik. Thematisiert werden dabei fehlendes Wissen über Gewaltprävention und Mobbing. Praxisrelevante Lösungsansätze werden in den Interviews wenig genannt. Eine Lehrperson des Gymnasiums benennt spezifisch die Mediation sowie die Anwendung von Kommunikationstechniken.

„Ich habe meine eigene Methode, wie ich mit Konflikten und Gewalt umgehe. [...] Ich führe bei Problemen eine Mediation durch. Sie hat sich als eine effiziente Möglichkeit erwiesen, wie man mit Gewalt in der Schule umgehen kann." (It 18)

Die Kategorie Diagnostik und Förderung zeigt, wie über einen ressourcenorientierten Zugang und spezifische Handlungsstrategien effiziente Maßnahmen zur Unterstützung und Förderung getroffen werden können. Für die professionelle Unterstützung beschreiben die Befragten als Voraussetzung, mit der eigenen (Lehr-)Person im Reinen zu sein. Die Kategorie Person wird mit ihren Subkategorien im nächsten Abschnitt beschrieben.

\subsubsection{Person}

Ein thematischer Schwerpunkt pädagogischen Wissens ist das Wissen der Lehrperson über die eigene Person.

„Für mich ist ein ganz wesentlicher Aspekt, dass die Lehrperson selber weiß wie man reflektiert und wie man sich weiterentwickeln kann. Einfach sich als Lehrperson immer wieder selber kritisch zu hinterfragen, um dauernd in einem gewissen Lernprozess zu bleiben und zu schauen wie man seinen Unterricht gestaltet und ob es noch passt.“ (It 19)

Lehrpersonen vor allem des Gymnasiums berichten von der signifikanten Bedeutung der Reflexion. Von zentraler Bedeutung ist in diesem Zusammenhang laut den Befragten die Selbst- sowie die Fremdreflexion durch KollegInnen, Schulleitung, Freunde oder die Familie. Insgesamt konnten im Zuge der Rekonstruktion drei Subkategorien - Wissen zur Person, Haltung, Weiterentwicklung - konzeptualisiert werden, welche alle in einer Wechselbeziehung zur Reflexionsfähigkeit stehen (vgl. Abbildung 58).

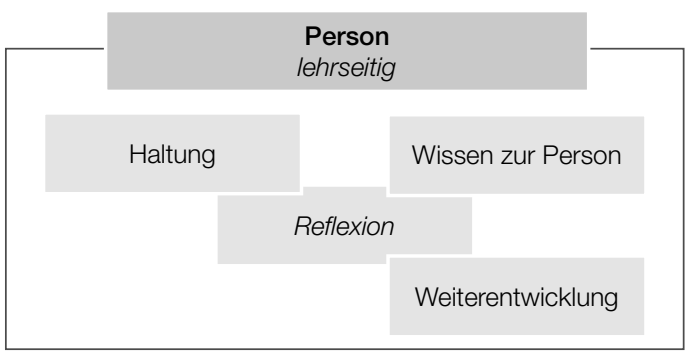

Abb. 58: Subkategorien Person 
Beim Wissen zur Person beschreiben die Befragten die permanente Bewusstseinsbildung und Reflexion der eigenen (LehrerInnen-)Persönlichkeit. Sie betonen, dass es nicht die eine vorgegebene (LehrerInnen-)Persönlichkeit gibt, sondern sich jede Lehrperson durch individuelle Eigenschaften auszeichnet. Die Interviewten nennen dabei unterschiedliche Wissensmerkmale einer (LehrerInnen-)Persönlichkeit, die in Abbildung 59 dargestellt werden.

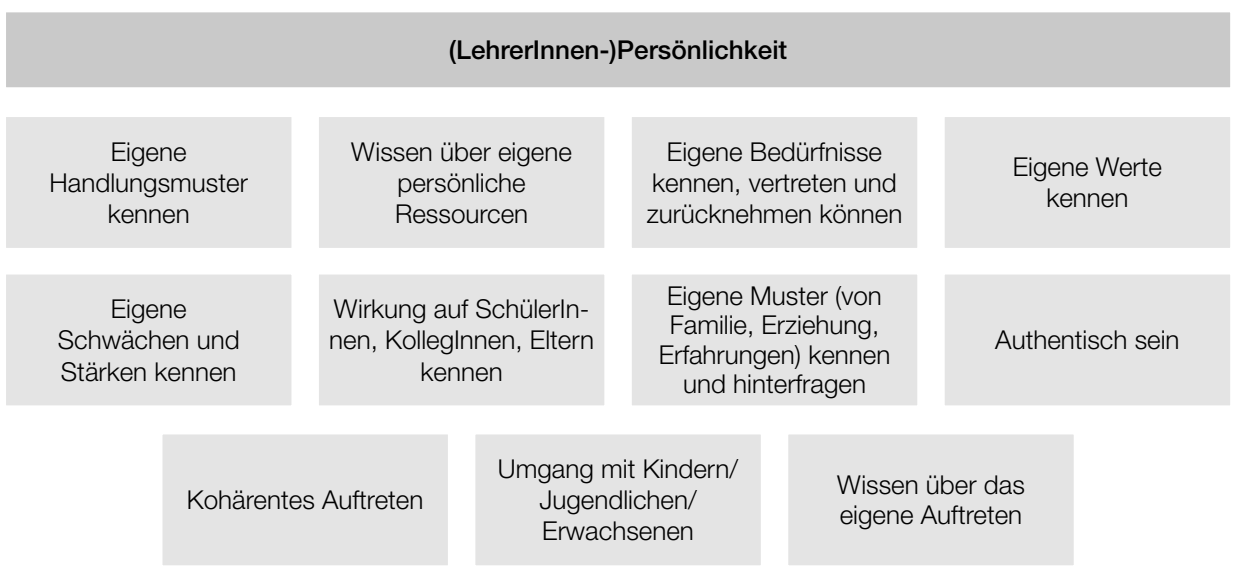

Abb. 59: Merkmale einer (LehrerInnen-)Persönlichkeit

Die von den Lehrpersonen genannten Merkmale beziehen sich auf die Bereiche eigene Bedürfnisse, individuelle Ressourcen sowie auf das Bewusstsein über eigene Stärken und Schwächen. Gleichzeitig werden Umgangsmuster und die eigene Wirkung auf andere Personen erwähnt. Als ein Kriterium zu persönlichen Wissensmerkmalen legen die Befragten einen besonderen Wert auf die Haltung zum Lehrberuf. Es zeigt sich anhand der gewonnenen Ergebnisse, dass eine große Anzahl von ihnen die Freude am Beruf, die Freude am Arbeiten mit Kindern und Jugendlichen, aber auch die Freude der Vorbildfunktion als wesentlich charakterisieren. Die Befragten sprechen in Verbindung mit der Haltung über veränderte Rollenbilder. Sie beschreiben, wie sich die Rolle einer Lehrperson in den letzten Jahren gewandelt hat:

„Ich bin der Meinung, ich verkörpere jetzt schon einen Lehrer der Zukunft. Für mich ist es nicht so, dass ich in eine Klasse gehe und Stoff durchziehe. Man sollte Freund und Kollege sein, aber immer in MaBen. Wichtig ist, sich ihnen anzunähern und in Gesprächen mehr über sie zu erfahren. Die Klassische Lehrerrolle von früher gibt es heute kaum noch.“ (It 11)

Die Befragten betonen, wie sie nicht nur für die Stoffvermittlung zuständig sind, sondern die Rolle von Eltern, Therapeuten oder Freizeitpartnern einnehmen müssen. Es wird von ihnen eine zunehmend offene Haltung gefordert:

„Man braucht eine lockere Haltung und einen lockeren Umgang. Sehr hilfreich ist es manchmal, wenn man etwas schauspielert. Wenn man Sachen übertreibt, oder ja, dann wird das Ganze etwas aufgelockert. Ich sage lustige Dinge, übertreibe, bringe sie zum Lachen.“ (It 16)

Weitere Attribute der Haltung betreffen das Belastungsniveau. Dabei steht die eigene Gesundheit im Mittelpunkt. Die Interviewten berichten von der Notwendigkeit, dem Druck von au- 
Ben, durch Eltern, die Gesellschaft, die Bildungspolitik, die Schulleitung, oder durch die KollegInnen, standhalten zu können. Zusätzlich müssen sie entscheiden, welche Probleme sie „mit zu sich nach Hause nehmen“ (It 25). Die Interviewten nennen Eigenschaften von Lehrpersonen, die für die eigene Gesundheit von Vorteil sein können. Sie verweisen auf Kritikfähigkeit, einen offenen Geist, Optimismus, auf die Fähigkeit, nicht alles persönlich zu nehmen und zu versuchen, sich nicht mit anderen zu vergleichen.

Die letzte rekonstruierte Subkategorie der Person bezieht sich auf die Weiterentwicklung. Diese beinhaltet laut den Befragten die kognitive sowie die persönliche Entwicklung. Wichtig ist die Bereitschaft zu Fortbildungen, der Austausch mit KollegInnen, die Aneignung von aktuellem Grundlagenwissen sowie die Auseinandersetzung mit Forschungsliteratur, um nicht an alten, traditionellen Mustern festzuhalten.

Die Kategorie Person zeigt, wie das Wissen über sich selbst das Wohlbefinden und das professionelle Auftreten von Lehrpersonen unterstützt. Es wirkt sich dieses auf das Lehren und Lernen aus und beeinflusst nach Angaben der Befragten weitere Kategorien wie Beziehung, Erziehung, Diagnostik und Kommunikation.

\subsection{Herausforderungen für pädagogisches Wissen}

Im vorliegenden Abschnitt werden die von den Interviewten benannten Herausforderungen für pädagogisches Wissen aufgezeigt. Sie gliedern sich in sieben Wissenskategorien. Diese beziehen sich, wie in der Abbildung 60 dargestellt, auf Diversität, Erziehung, Lernen/Lehren, Systemwissen, psychologisches Wissen, Medien und zur eigenen (Lehr-)Person. ${ }^{45}$

Diversität
- Umgang mit
Heterogenität
- Wissen über
didaktische
Konzepte
- Zusammenarbeit mit
externem Personal
- Umgang mit Vielfalt
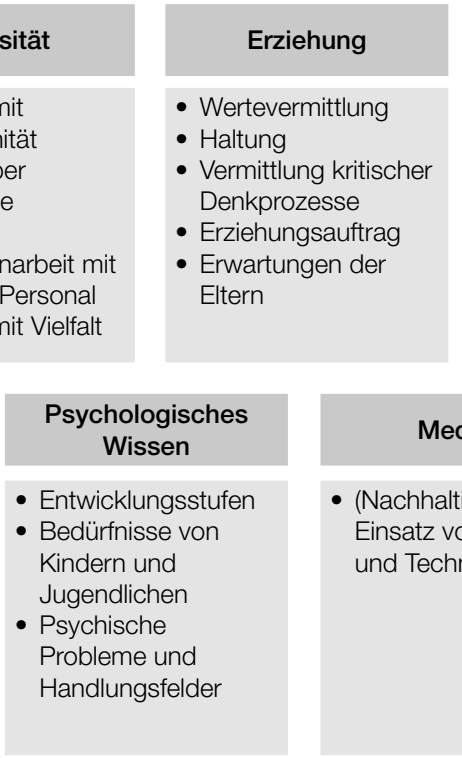
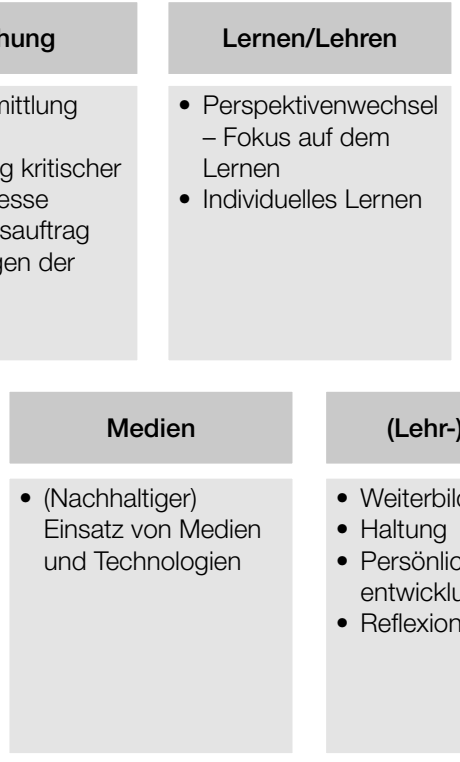

\section{Systemwissen}

- Bildungspolitische Veränderungen

- Schulinterne Veränderungen

Abb. 60: Übersicht zu den Herausforderungen für pädagogisches Wissen

45 Die angeführten Kategorien zu Herausforderungen für pädagogischen Wissen beziehen sich auf die in Abschnitt 8.1 rekonstruierten Kategorien aus den Interviews. Die Lehrpersonen beschreiben zu den hier angeführten Kategorien spezifische Herausforderungen. Diese werden bei der allgemeinen Ergebnisdarstellung nicht diskutiert. 
Das Thema Diversität wird von Lehrpersonen aller Schulformen als eine große Herausforderung beschrieben. Lehrpersonen thematisieren in den Interviews die Zunahme einer „bunten Gesellschaft“: „es wird immer bunter und gemischter“ (It 3). Sie betonen die Notwendigkeit zentraler Handlungsstrategien, wie mit neuen gesellschaftlichen Anforderungen im Unterricht umgegangen werden kann:

„Was bei uns ein großes Thema ist, ist die Multikulturalität, die Pluralität. Wie gehe ich damit um? Wir erleben jetzt die Unterschiedlichkeit ganz anders als früher. Da braucht es viel Wissen, Gespür und die Bereitschaft, dass man kulturelle Vielfalt zum Inhalt machen kann." (It 6)

Herausforderungen beziehen sich ebenso auf fehlendes Wissen bezogen auf didaktische Unterrichtskonzepte, wie man das Thema Diversität im Unterricht integrieren kann:

„Das sind neue Herausforderungen und da gehört mehr pädagogisches Wissen her und mehr Wissen über Strukturen des Unterrichts. Ich würde einfach sozusagen offenere Lernphasen, Projektunterricht verpflichtend überall einführen, weil da kann man Differenz hereinbringen [...].“ (It 18)

Laut den Befragten beziehen sich die Herausforderungen auf einen Umgang mit speziellen, vielfältigen Bedürfnissen der SchülerInnen hinsichtlich Sprache, Lernen, Motivation und kulturelle Unterschiede und die adäquate Berücksichtigung im Unterrichtsalltag.

Zum Thema Diversität wird von den Lehrenden zusätzlich die Schwierigkeit der Kooperation und der Integration von sonderpädagogischem Fachpersonal erwähnt:

„Es gibt viele Fördermaßnahmen, aber es ist schwierig, dass sie genehmigt werden und man unterstützt wird." (It 1)

Nach Angaben der Befragten sind die Organisation und die Zusammenarbeit mit sonderpädagogischem Fachpersonal mit großen Mühen verbunden. „Wir haben zu wenig Zeit und Geld.” (It 21) Sie berichten von fehlenden zeitlichen sowie finanziellen Ressourcen.

Herausforderungen werden von den Befragten im Zusammenhang mit dem Thema Erziehung beschrieben. Die zuvor angesprochene zunehmende Vielfältigkeit und Multikulturalität fordert von den Lehrpersonen, ihre Erziehungsformen und Muster zu überdenken. Sie erwähnen in diesem Zusammenhang die Schwierigkeit, passende Werte und Haltungen zu vermitteln, um allen Menschen in der Gesellschaft offen zu begegnen.

„Ich glaube, dass es wichtig ist, den SchülerInnen für ihr Leben mitzugeben, dass sie generell einen guten, respektvollen Umgang mit anderen Personen führen, und einen offenen, ja keine Vorurteile. Wissen wie etwas zu werten ist, ob man und wie man Dinge glauben kann.“ (It 7)

Weitere Schwierigkeiten beziehen sich auf die Anleitung kritischer Denkprozesse und die Unterstützung der Selbstentfaltung. Immer größere Anforderungen werden hinsichtlich des Erziehungsauftrags gestellt. Die Befragten berichten, wie sie nicht nur für das Lernen der SchülerInnen verantwortlich sind, sondern unterschiedliche Funktionen, wie jene von Eltern, Freunden oder FreizeitpartnerInnen, übernehmen:

„Ich glaube, dass man sich noch enger mit den Kindern beschäftigen muss. Dass man als Lehrer für viele Kinder schon Papa, Mama, Therapeut und FreizeitpartnerIn ist. Weil viele die familiäre Situation nicht mehr haben, so wie man sie von früher kennt. Dass die Mutter daheim ist beim Kind und der Vater kommt nach der Arbeit und dann macht man was gemeinsam. Das gibt es nicht mehr. Deshalb braucht es Freundlichkeit. SchülerInnen sind Menschen und das ist unsere Zukunft. Man sollte sie auf die richtige Spur lenken." (It 11) 
Beim Thema Erziehung wird von den Interviewten berichtet, wie Erziehungsberechtigte immer größere Erwartungen in die Lehrpersonen stellen. Sie erwarten von den Lehrpersonen eine angemessene Wertevermittlung und die Aneignung notwendiger Fähigkeiten.

Von den Lehrpersonen werden aufgrund des Pragmatic Shift vom Lehren zum Lernen neue Denkweisen zum Lernen und Lehren gefordert. Das Einnehmen neuer Perspektiven wird von den Lehrpersonen als herausfordernd beschrieben. Einzelne Lehrende erwähnen die Schwierigkeit, alte, traditionelle LehrerInnenrollen und kognitive Muster zu reduzieren:

„Das Lernen bei den Kindern lassen, nicht meinen, ich als Lehrperson weiß was ein Kind zum Lernen braucht, sondern die Verantwortung beim Kind lassen, das ist aus meiner Sicht eine große Herausforderung. Nicht ich bereite den Unterricht vor und weiß was gut ist, sondern man muss gemeinsam herausfinden was gut ist und was SchülerInnen [...]. (It 5)

Lehrpersonen aller Schulformen berichten, wie das Lernen von den SchülerInnen selbst bestimmt werden sollte. Dieser Prozess wird über gemeinsames Arbeit und eine individuelle Förderung unterstützt. In diesem Zusammenhang bemängeln die Befragten die unzureichende Geduld einiger Lehrpersonen, die den lernseitigen Blick verhindert:

„Oft fehlt es an Geduld bei den Lehrpersonen, den Kindern die Möglichkeit zu bieten, Dinge so lange zu versuchen, bis man etwas richtig macht" (It 5).

Die Lehrpersonen berichten, dass neue wissenschaftliche Erkenntnisse, innovative Methoden oder neue Unterrichtsformate unzureichend in den Unterricht implementiert werden und das Ziel eines individuellen Unterrichts lückenhaft bis gar nicht umgesetzt wird.

Als eine weitere Herausforderung für pädagogisches Wissen wird der Umgang mit Medien rekonstruiert. Rasche technologische Entwicklungen und der permanente Gebrauch von Medien stellt Lehrpersonen aller drei Schulformen vor neue Aufgaben.

„Eine Herausforderung ist, da Kinder sehr viel mit Technologien zu tun haben, wie kann ich das nützen in der Schule? Konstruktiv." (It 17)

Sie sind gefordert, neue Technologien in ihren Unterricht zu implementieren sowie die SchülerInnen hinsichtlich eines kritischen Mediengebrauchs zu erziehen. Dies verlangt eine mediengeschulte Haltung und Erziehung. Sie betonen dabei ihre Pflicht, einen Beitrag für den sinnstiftigen, nachhaltigen Einsatz von Medien zu leisten. Sie berichten jedoch von unzureichendem Wissen über die Bedienung und die Möglichkeiten von Technologien sowie von einer ineffizienten Implementierung in den Unterricht.

Weitere Schwierigkeiten zeigen sich in den Interviews im Bereich psychologische Wissensstrukturen.

„Man sollte viel mehr Einblick in das psychologische Wissen haben. Zu wissen, was etwas für ein Kind bedeutet, was in einem Kind vorgeht. Weil wenn ich das weiß, kann man schon ganz anders mit dem Kind arbeiten." (It 1)

„Ich glaube es wird wichtiger, dass Lehrer viel mehr im psychologischen Bereich ausgebildet werden. Wenn ich jetzt meine Klasse die ich als KV betreue anschaue, ich habe vier SchülerInnen, die stationäre Aufenthalte in der Psychiatrie gehabt haben und wo es in der Familie wahnsinnig drunter und drüber geht." (It 12)

Lehrpersonen sprechen sich für mehr Wissen im Bereich Förderung und Unterstützung sowie das Anwenden passender Handlungsstrategien aus: 
„Zum Beispiel Wissen über Lernförderung. Auch die Therapiemöglichkeiten. Was für Möglichkeiten habe ich eigentlich? Was für Möglichkeiten hat das Kind? Welche Hilfe kann ich mir als Lehrperson holen? Was heißt das Defizit, was ist das Krankenbild? Oder, dass man die Geschichte von dem Kind besser versteht, sei es physisch wie psychisch.“ (It 1)

Die Interviewten erwähnen spezifische lernförderliche Maßnahmen sowie Wissen zu Therapiemöglichkeiten und Beratungsgespräche. Sie fordern umfangreicheres Wissen, wie sie mit Defiziten und Krankheiten im Unterricht umgehen sollen, damit Lernen bestmöglich gefördert werden kann.

Die Befragten benennen im Bereich Systemwissen weitere Herausforderungen für das pädagogische Wissen. Sie berichten, wie sich bildungslandschaftliche Veränderungen und Entwicklungen auf Handlungsmöglichkeiten von Lehrpersonen auswirken. Sie erzählen von der Herausforderung, die von der Bildungspolitik vorgeschriebenen Gesetze und Reformen adäquat in den Unterricht zu integrieren. In diesem Zusammenhang erwähnen vorwiegend die Gymnasiallehrpersonen und Lehrpersonen der Neuen Mittelschule die pädagogischen Herausforderungen, sich permanent den neuen Bedingungen und Vorschriften anzupassen. Beispiele betreffen zum Beispiel das Team-Teaching, Ganztagsschulen, neue Benotungsverfahren oder die Implementierung neuer didaktischer Konzepte. Eine Lehrperson berichtet von den Schwierigkeiten, die durch das Implementieren von Ganztagsschulen entstehen:

„Und diese Ganztagsklassen. Es geht immer mehr dazu hin, im Rahmen der Gesamtschule, dass man ein Ganztageskonzept anbietet, wo die Kinder dann wirklich den ganzen Tag aufgehoben sind. Sowohl schulisch oder privat. Die Kinder gehen abends nach Hause und die Eltern können ihrem Beruf nachgehen. Ich muss dann plötzliche die Rolle der Eltern übernehmen und bin für viel mehr verantwortlich als nur für das Lernen." (It 2)

Die Interviewten bemängeln, wie sie durch neue Strukturen, wie hier die Implementierung von Ganztagsschulen, neue und weitere Aufgaben übernehmen müssen, die über das Lernen hinausgehen.

Eine befragte Person erzählt in den Interviews von den Schwierigkeiten und Ängsten von Lehrpersonen, die durch neue didaktische Anforderungen und Leistungsbenotungskonzepte entstehen:

„Ich denke zusätzlich, dass ganz viel offener Unterricht ein wichtiges Thema wird [...]. Und da sehe ich einfach an unserer Schule, dass Lehrpersonen nicht mit dem in Kontakt gekommen sind und schon älter sind. Die haben einfach einige gewisse Angst davor was kommt und überrumpelt werden." (It 3)

Lehrpersonen fühlen sich oftmals durch Veränderungen überfordert und allein gelassen. Sie benötigen mehr Wissen, wie sie mit didaktischen Konzepten und neuen Vorgaben umgehen sollen.

Die letzte Herausforderung bezieht sich auf den Wissensbereich (Lehr-)Person. Die Befragten thematisieren die Schwierigkeit fachlicher Weiterbildung und sprechen von einer unzureichenden professionellen Haltung, die für eine persönliche Weiterentwicklung nötig ist. Im Gespräch zu Weiterbildungen beschweren sich Lehrpersonen über zu geringe interne Fortbildungsprogramme und Fortbildungsmöglichkeiten:

„Bei uns an der Schule gibt es keine Fortbildungen. Ich denke es gibt schon Fortbildungen und ich würde mir erwarten, dass diese als Lehrperson verpflichtend werden." (It 3)

Eine Lehrperson vertritt die Position, dass nicht genügend Fortbildungen angeboten werden. Besonders die Interviewten der Neuen Mittelschule und des Gymnasiums verweisen auf die Not- 
wendigkeit von Fortbildungen für Lehrpersonen, um sich fachlich sowie persönlich weiterzuentwickeln und den Anforderungen gerecht zu werden. Eine untersuchte Person stützt sich auf die Argumentation, dass nur über Fortbildungen Wissenslücken bewusst gemacht werden können:

„Es muss sichtbar gemacht werden, was alles in der Schule für Wissen verlangt und benötigt wird.“ (It 8) Hinsichtlich der Haltung benennen die Befragten unzureichende Reflexionsfähigkeit von Lehrpersonen und das fehlende Hinterfragen von Situationen (It 13). Eine Befragte fordert: „Man braucht hier offenes und kreatives Denken." (It 5) Einzelne Interviewte berichten von voreiligen Zuschreibungen und Vorurteilen, die sie nicht ablegen können:

„Das lässt dem Menschen keine Chance, dass er anders sein kann und ich denke mir, Menschen können anders sein und werden. Und sie einzukasteln, nur durch den ersten Eindruck, finde ich äußerst bedenklich und pädagogisch sehr fragwürdig. Das passiert leider immer noch viel zu oft." (It 13)

Sie fordern, eine offene Haltung einzunehmen, tolerant und offen zu sein:

„Lehrer brauchen eine Arbeitshaltung und einen offenen Geist. Haltung und Werte. Toleranz und Offenheit und für sich selber einstehen zu können." (It 9)

Die Befragten erzählen, wie sie des Öfteren an ihre Grenzen stoßen. Sie argumentieren, dass sie durch gegenwärtige technologische und gesellschaftliche Entwicklungen, welche ihren Unterricht beeinflussen, ihr Wissen stetig weiterentwickeln müssen.

Die angeführten Herausforderungen beziehen sich auf unterschiedliche Kategorien pädagogischen Wissens. Es zeigt sich, dass Lehrpersonen im Zusammenhang mit dem Begriff pädagogisches Wissen unterschiedliche Schwierigkeiten thematisieren. Diese korrelieren stark mit gegenwärtigen gesellschaftlichen Entwicklungen, auf die Lehrpersonen angemessen reagieren müssen. Spezifische Maßnahmen für die Überwindung der genannten Herausforderungen werden von den Lehrpersonen nicht benannt.

\subsection{Aneignung pädagogischen Wissens}

Die Lehrpersonen berichten in den Interviews über unterschiedliche Aneignungsprozesse und -orte pädagogischen Wissens. Sie verweisen auf vier verschiedene zeitliche Etappen. Diese beziehen sich auf Kindheit, Jugend, Studium und Berufsleben (vgl. Abbildung 61).

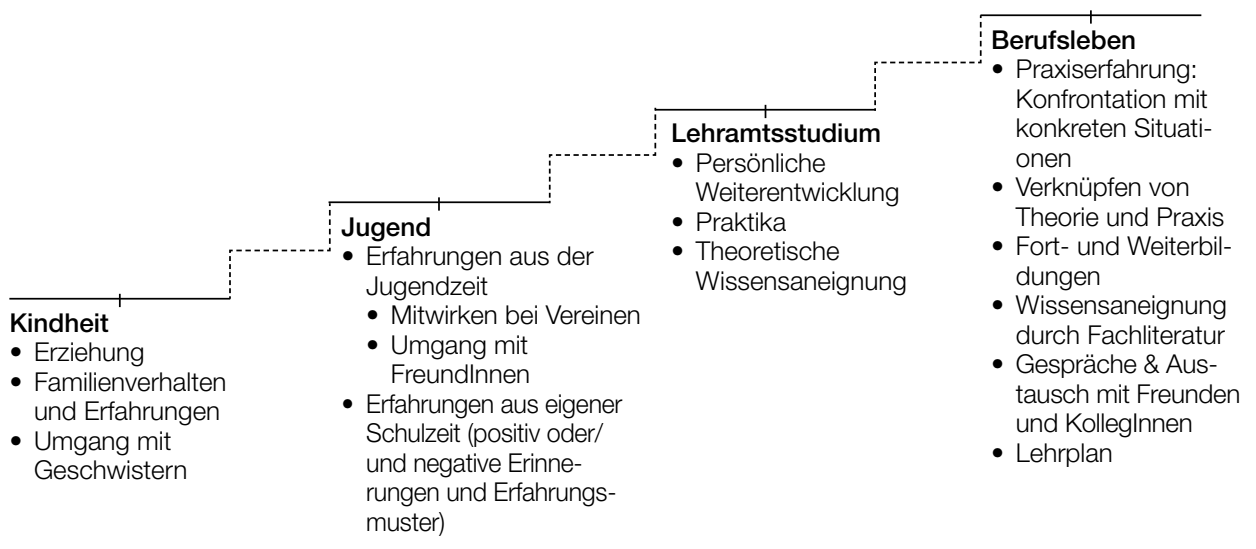

Abb. 61: Aneignungsetappen pädagogischen Wissens 
In den Interviews wird die Aneignung pädagogischen Wissens während der Kindheit und Jugend als bedeutend beschrieben. Die Befragten berichten aus der eigenen Erziehung, dem Umgang mit Geschwistern oder aus der Erfahrung in Vereinen übernommenen pädagogischen Handlungsmustern. Einzelne Lehrpersonen verweisen auf die Aneignung von Wissensaspekten über Freundschaften in der Jugend:

„Ich habe mein pädagogisches Wissen durch die Lebenserfahrungen angeeignet. Erstens bin ich die Älteste bei den Geschwistern, habe viel auf die Geschwister aufgepasst, war dann immer die Verantwortliche, die viele Unternehmungen mit den Kleinen gemacht hat. Das ist viel praktisches Wissen. Ich habe Wissen aus meiner eigenen Erziehung, auf das ich immer wieder zurückgreife." (It 17)

„Ich habe pädagogisches Wissen durch Freundschaften angeeignet. Hier muss man ja schon pädagogisches Handeln, damit Freundschaften funktionieren. Wir waren alle in einem Sportverein und hatten auch mit anderen Jugendlichen zu tun. Hier war pädagogisches Wissen gefragt, damit wir überhaupt alle zusammen in Trainings und Ausflügen gut miteinander umgehen konnten." (It 6)

Vorhandene Wissensbereiche entwickeln sich laut den Interviewten aus den individuellen Erfahrungen während der eigenen Schulzeit. Vor allem positive Erfahrungen werden von ihnen weitergeführt. In Erinnerung gebliebene, negative Erfahrungen beeinflussen das pädagogische Wissen, sodass sie im Voraus Wissensfelder und Handlungsstrategien aufbauen, damit sie ihre negativen Erfahrungen nicht wiederholen.

Eine weitere zeitliche Ebene, die von den Befragten genannt wurde, ist die Aneignung pädagogischen Wissens durch das Studium. Einzelne Lehrpersonen beschreiben, wie sie didaktische, pädagogische und bildungswissenschaftliche Wissensbereiche über das Studium aneignen konnten:

„Die didaktischen Sachen wie ich wann was mache, habe ich über die Ausbildung gelernt.“ (It 13)

„An der Universität haben wir einige theoretische Grundlagen mitbekommen, auf die man zurückgreifen konnte." (It 24)

Als gewinnbringend haben einige Befragte die mit dem Studium einhergehenden schulpraktischen Ausbildungsanteile erwähnt, die ihnen vor allem beim Aufbau einer eigenen LehrerInnenpersönlichkeit geholfen haben:

„Ich hatte im Studium einige Praktika. Hier konnte ich das erste Mal als Lehrpersonen agieren. Allerdings muss ich sagen habe ich in dieser Zeit mich hauptsächlich persönlich weiterentwickeln können.“ (It 16)

Die Mehrzahl der Interviewten weist jedoch auf einen wenig bedeutenden Aneignungsprozess pädagogischen Wissens während des Studiums hin:

„Auf keinen Fall während meiner Ausbildung an der pädagogischen Hochschule.. (It 15)

„Ich kann mich nicht erinnern, dass ich mir im Studium pädagogisches Wissen aneignen konnte. Ahja, da war was mit Kommunikationsmodellen. Vielleicht das?“ (It 3)

„Da war bestimmt was. Aber ich kann mich nicht mehr genauer erinnern. Wir mussten Unterrichtsplanungen erstellen und viele Reflexionstexte schreiben." (It 23)

Die Lehrpersonen betonen, wie während des Studiums wenig bis keine Aneignung pädagogischen Wissens erfolgte. Sie können kein spezifisches Wissen benennen, sondern stellen einzelne Vermutungen auf.

Die Ebene des Berufslebens wird von den Interviewten als die wichtigste Stufe für den Erwerb pädagogischen Wissens argumentiert. Ihre Aussagen unterstreichen damit gleichzeitig die 
grundlegende Bedeutung des Erfahrungswissens. Sie argumentieren, dass die Aneignung pädagogischen Wissens vorrangig auf Praxiserfahrung zurückzuführen ist:

„Mein pädagogisches Wissen habe ich in der Praxis erworben. Vor allem durch die gemachten positiven und negativen Erfahrungen. In der Uni war die Ausbildung sehr theoretisch und die Praktika sehr kurz. Erst über die Erfahrung und den ganzheitlichen Blick auf den LehrerInnenberuf weiß man, welches Wissen man braucht und lernt so neue Handlungswege." (It 24)

Laut den Interviewten ist die Vernetzung der Praxiserfahrungen mit theoretischen Inhalten zentrales Moment für den pädagogischen Wissensaufbau. Dieses konnten sie sich vorwiegend durch Fort- und Weiterbildungen sowie Fachliteratur aneignen. Die Befragten benennen vor allem die Fort- und Weiterbildungen als wichtige Quelle pädagogischen Wissens:

„Ich wusste nie wirklich wie man mit Problemen in der Klasse umgehen soll. Dann habe ich mir bewusst Fortbildungen gesucht und das wichtigste war für mich die Schulmediation. Weitere Fortbildungen waren zum Beispiel über offenen Unterricht, Gruppendynamiken und Lernpsychologie.“ (It 6)

Die Lehrpersonen berichten, wie sie durch das Besuchen von Fort- und Weiterbildungen pädagogisches Wissen vertiefen und festigen konnten. Im Zusammenhang mit dem Berufsleben werden von den Lehrpersonen Gespräche mit Freunden und KollegInnen für den Wissensaufbau als fruchtbar und hilfreich empfunden:

„Auf keinen Fall während meiner Ausbildung an der pädagogischen Hochschule. Ich habe mir das pädagogische Wissen im Laufe der Zeit selbst angeeignet, über Selbsterfahrung, über Fortbildung, über KollegInnen, über Schulleitung und Lehrpersonen aus dem Freundeskreis, über viele Gespräche mit diesen Personen. Über den Austausch von Erfahrungen kann ich mir neues Wissen über das Handeln in bestimmten Situationen aneignen." (It 10)

Sie erwähnen, wie sie sich über den Austausch und das Teilen von Erfahrungen neues pädagogisches Wissen aneignen konnten. Der Aneignungsprozess wurde durch den Lehrplan beeinflusst:

„[...] und über den Lehrplan. Der ist einfach vorgegeben und sagt mir was die Kinder am Ende können müssen. Und mit den ganzen Kompetenzen und Bildungsstandards in der vierten Klasse müssen alle Kinder gewisse Standards erreichen. Und da haben wir schon vorgaben, die man erreichen soll." (It 2)

Der Lehrplan fordert Lehrpersonen, sich mit pädagogischem Wissen auseinanderzusetzen. Der Erwerb pädagogischen Wissens verläuft nach Angaben der Lehrpersonen in unterschiedlichen zeitlichen Etappen. Als wichtiger Aneignungsort wird das Berufsleben bzw. die Berufspraxis hervorgehoben. Weitere unterstützende Inhalte pädagogischen Wissens konnten, wie die Befragten beschreiben, in der Kindheit und Jugend erworben werden. Das Studium wird von den Interviewten als eher unwichtig bis nichtig beschrieben. Nur wenige konnten spezifisches pädagogisches Wissen benennen, welches sie in der Ausbildung erworben haben. Als zweitranging ist die Aneignung über wissenschaftliche Theorien.

\subsection{Auswirkungen von Defiziten pädagogischen Wissens}

Im folgenden Kapitel werden von den Lehrpersonen beschriebene Auswirkungen von Defiziten pädagogischen Wissens dargestellt. Insgesamt konnten sieben Bereiche rekonstruiert werden, in denen Wissenslücken zum Tragen kommen. Diese beziehen sich, wie in Abbildung 62 veranschaulicht, auf das Classroom-Management, die Beziehung, die individuelle Unterstützung und Förderung, die Kommunikation, das Lernen, das Lehren sowie Aspekte bezogen auf die Rolle der Lehrperson. 


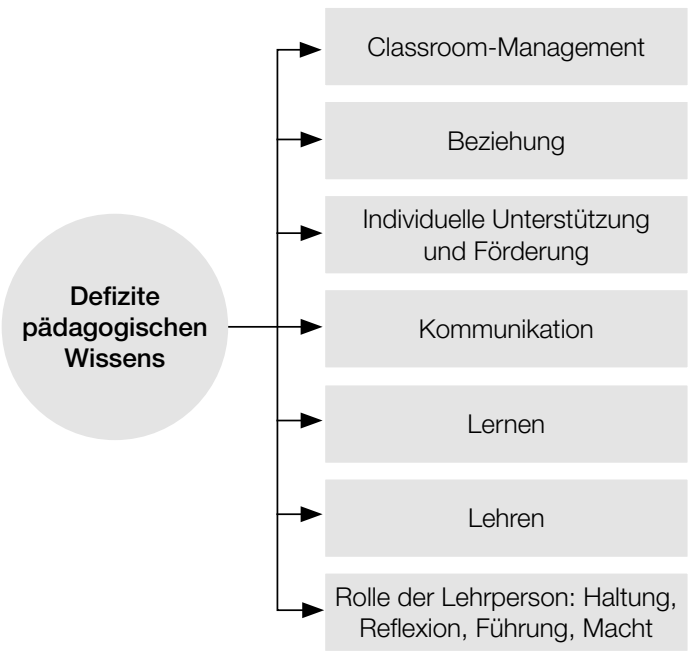

Abb. 62: Wissenslücken pädagogischen Wissens

Die Befragten beschreiben, wie ein Unterricht mit unzureichendem pädagogischem Wissen „sehr chaotisch“ (It 2,1,8) und ohne Classroom-Management ablaufen würde: „Der Unterricht findet ohne Struktur und Organisation statt." (It 25) Eine Lehrperson beschreibt den Unterricht sogar als „furchtbar“ (It 18). Auswirkungen auf pädagogisches Wissen beziehen sich auf ein unstrukturiertes Arbeiten, das Fehlen eines roten Fadens sowie schlechte Organisation.

Auswirkungen von Wissenslücken werden im Bereich Beziehung beschrieben. Die Interviewten bemängeln fehlende Beziehungen zwischen Lehrperson und SchülerInnen, die zu einem einseitigen, beziehungslosen und interaktionslosen Unterricht führen:

„Dann schaut der Unterricht wie vor 30 Jahren aus, als ich noch in die Schule gegangen bin. Totaler Input, Vorlesen, Mitschreiben, Tafel abschreiben, Prüfen." (It 10)

„Das ist dann militärischen Ablauf von Lernen und Abprüfen. Die Kinder gehen nach vier Jahren aus der Schule und können nichts, weder menschlich noch schulisch“ (It 11).

Eine weitere Lehrperson charakterisiert Beziehungen ohne pädagogisches Wissen als eine „Lehrer-Schulbuch-Beziehung“ (It 8). Es kann keine persönliche wie fachliche Beziehung zu den SchülerInnen aufgebaut werden. Dies wirkt sich nach Angaben der Lehrpersonen negativ auf das Lernen und Lehren aus.

Eine Interviewte assoziiert mit der Beziehung das „pädagogische Gespür“ (It 9). Sie verweist auf diese Notwendigkeit, um überhaupt mit SchülerInnen arbeiten zu können:

„Ein pädagogisches Gespür ist eine Grundvoraussetzung um mit SchülerInnen zu arbeiten. Pädagogisches Gespür ist die Klassenstruktur zu erfassen, dann die Wahrnehmung, wie es den Kindern geht wie sie drauf sind, wie sie untereinander agieren. Das einfach wahrzunehmen, in der Gruppe und einzeln anzusprechen und soziale Kompetenz und Intelligenz fördern." (It 9)

Die Lehrperson nennt spezifische Merkmale eines pädagogischen Gespürs. Diese beziehen sich auf den Umgang, das Wohlbefinden der SchülerInnen sowie den generellen Überblick einer Lehrperson. Bei fehlendem pädagogischem Gespür können die beschriebenen Merkmale nicht umgesetzt werden. 
Weitere Auswirkungen von pädagogischen Wissenslücken beziehen sich auf das Lernen. Die Befragten berichten als Folge von Wissensdefiziten von einem langweiligen Unterricht, einem Vorpredigen von Inhalten sowie fehlenden didaktischen Maßnahmen, die das individuelle Lernen unterstützen:

„Ich glaube auf den Unterricht bezogen, wäre es ein sehr fader Unterricht. Es wäre nur ein Vermitteln von irgendwelchen Inhalten, ein Vorpredigen von Wissen das einfach nicht bei den Kindern ankommt. Lehrpersonen stehen vor der Klasse, wissen vielleicht irgendwann selber nicht mehr genau was sie machen. Die Kinder können sich nicht entfalten. Ich denke da würde es keine Beziehung geben. Das menschliche, die Interaktion, das individuelle Bedürfnis wird nicht berücksichtigt." (It 3)

$\mathrm{Zu}$ der im Zitat angesprochenen fehlenden individuellen Entfaltung beschreiben die Interviewten weitere Merkmale für die Umsetzung personalisierten Lernens. Aufgrund unzureichenden pädagogischen Wissens können individuelle Bedürfnisse von SchülerInnen im Unterricht nur ungenügend berücksichtigt werden:

„Auswirkungen fehlenden pädagogischen Wissens sind für mich, dass man bei schwierigen Kindern einfach darüberfährt. Sonderlinge werden dabei einfach nicht berücksichtigt und gefördert. Es findet kein individueller Unterricht statt. Lehrpersonen sind nicht in der Lage auf SchülerInnen und deren Bedürfnisse und Lernen einzugehen." (It 10)

Aus den Interviews geht hervor, dass Lehrpersonen bei unzureichendem pädagogischem Wissen ein unprofessionelles Verständnis von Lernen befürchten:

„Ohne pädagogisches Wissen fehlt ein Verständnis zum Thema „Lernen“. Ich weiß nicht welche Zugänge es gibt und nicht wie ich das Lernverhalten von einzelnen SchülerInnen fördern und unterstützen kann“. (It 24)

In den Interviews wird ein thematischer Bezug zum Thema Lehren im Zusammenhang mit der Vermittlung von Inhalten und Methoden angesprochen. Nach Angaben der Interviewten ist es nicht möglich, effizient zu lehren und die passenden Unterrichtsmethoden, Materialien und Lehrmethoden anzuwenden, wenn das nötige pädagogische Wissen nicht vorhanden ist:

„Ich glaube nicht, dass eine Lehrperson dann bestmöglich entscheiden kann, welche Methode sie anwenden soll oder welcher Stoff unterrichtsrelevant ist. Das wirkt sich auf die Qualität des Lehrens aus." (It 14)

Fehlendes pädagogisches Wissen wirkt sich laut den Befragten auf die Kommunikation zwischen Lehrpersonen und SchülerInnnen aus. Fehlende Kommunikationsstrukturen führen dazu, dass der Unterricht nur oberflächlich abgehalten wird, was die soziale Interaktion in der Klasse und den Verlauf des Unterrichts beeinträchtigt:

„Die Kommunikation in der Klasse wird über fehlendes pädagogisches Wissen gestört. Die Kommunikation findet nur oberflächlich statt und führt zu einer unzureichenden sozialen kommunikativen Interaktion. Eine schlechte Kommunikation beeinflusst den Verlauf des Unterrichts und den Arbeitsprozess." (It 25)

Zu den hier aufgezeigten lern- sowie lehrseitigen Auswirkungen skizzieren die Befragten spezifische Bereiche, die das Wissen einer Lehrperson explizit beeinflussen. Fehlendes pädagogische Wissen zeigt sich laut Angaben der Interviewten im Zusammenhang mit der Reflexionskompetenz:

„Das pädagogische Wissen führt dazu, dass man in der Lage ist Muster zu hinterfragen. Ich muss mir ja irgendwie meiner Blindenflecke bewusst sein. Das pädagogische Wissen hilft mir dabei die Blindenflecke sichtbar zu machen. Dafür sollte mich das pädagogische Wissen befähigen, in meinem Handeln durch Selbstreflexion, mich hinzuführen." (It 6) 
Defizite pädagogischen Wissens wirken sich auf die Selbstreflexion aus. Lehrpersonen haben eine unzureichende Reflexionskompetenz, um eigene Muster und Handlungsweisen zu hinterfragen. Eine befragte Person spricht im Interview weitere Folgen an. Diese beziehen sich auf das Führen einer Klasse sowie auf den Umgang mit Macht:

„Wie kann man ohne pädagogisches Wissen überhaupt wissen wie man richtig eine Klasse führt? Das fehlt dann komplett. Der Zusammenhang zwischen Führen und Macht denke ich wird dann komplett ignoriert. Gerade das ist doch wichtig, dass wir die alten Gedanken von Machtstrukturen in die Klassen weitertragen und weiterführen." (It 6)

Eine weitere mögliche Auswirkung von fehlenden Wissensstrukturen ist eine unprofessionelle Haltung zum LehrerInnenberuf. Pädagogische Wissensstrukturen befähigen, eine pädagogische Haltung einzunehmen und diese vorzuleben. Dies betrifft vor allem den Umgang mit SchülerInnen. Eine Person berichtet in diesem Zusammenhang, wie „erschrocken“ (It 18) sie war zu sehen, wie manche mit ihren SchülerInnen umgehen. Sie erzählt von Beschämungen sowie ungerechten Schuldzuweisungen.

Die angeführten Beispiele zeigen unterschiedliche Auswirkungen und verdeutlichen die wesentliche Bedeutung pädagogischen Wissens für einen strukturierten, individualisierten, abwechlunsgreichen und professionellen Unterricht. In Abbildung 63 werden die genannten Auswirkungen von unzureichendem pädagogischem Wissen noch einmal zusammengefasst dargestellt.
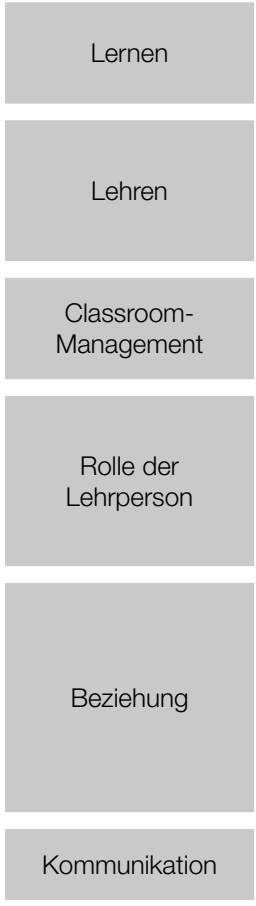

Individuelle Unterstützung und Förderung
- Differenzierung findet nicht statt

- Kein theoretisches Wissen über Lernen

- Keine Kenntnis von Lernstrategien und deren Anwendung

- Wissenslücken im Bereich professionellen pädagogischen Fachwissens und didaktischen Wissens

- Langweiliger Unterricht durch Vorpredigten von Inhalten

- Kein Wissen über Leistungsbeurteilungen

- Keine strigente Unterrichtsplanung: Lücken in der Unterrichtsvorbereitung und chaotische Umsetzung

- Fehlende Organisation und Struktur

- Reflexionsfähigkeit nicht vorhanden, eigene Muster und Handlungsweisen werden nicht hinterfragt

- Fehlende Vorbildfunktion für Schülerlnnen

- Fehlendes Verständnis von Führung

- Fehlendes Verständnis vom Umgang mit Macht

- Schülerlnnen können sich nicht entfalten

- Keine individuelle Förderung

- Keine Interaktion zwischen SchülerInnen und Lehrpersonen

- Probleme beim Umgang mit Schülerlnnen, KollegInnen und Eltern

- Keine Zusammenarbeit und Kooperation zwischen Schülerlnnen, Eltern und Kolleginnen

- Fehlendes Wissen über die Person

- Fehlen von Kommunikationsstrategien und Regeln

- Brüche in der Gesprächsführung

- Problemsituationen werden nicht erkannt und können nicht gelöst werden

- Fehlendes Wissen über Unterstützungs- und Fördermöglichkeiten

- Konflikt- und Mobbinganfälligkeit erhöht

- Neid und Konkurrenzdenken im Vordergrund

Abb. 63: Auswirkungen von Wissenslücken auf den Unterricht 
Wissensdefizite spiegeln sich auf lern- sowie lehrseitiger Ebene im Unterricht wider. Auswirkungen beziehen sich auf die Beziehungsebene, den Umgang sowie das Classroom-Management. Weitere rekonstruierte Auswirkungen von Wissenslücken pädagogischen Wissens sind die fehlende individuelle Unterstützung und Förderung, ein unzureichendes Verständnis von Lernen und Lehren, ein unprofessioneller Umgang mit Gruppendynamiken und Kommunikation, unzureichendes Führen sowie ein unprofessionelles Verhalten von Lehrpersonen.

\subsection{Kategorien pädagogischen Wissens aus den Beobachtungen}

Das vorliegende Kapitel präsentiert die rekonstruierten Kategorien pädagogischen Wissens aus den durchgeführten Unterrichtsbeobachtungen. Im Rahmen der Beobachtungen hat sich gezeigt, wie pädagogisches Wissen über die Interaktion zwischen Lehrpersonen und SchülerInnen rekonstruiert werden konnte. Spezifische Beispiele werden bei der Darstellung einzelner Kategorien beschrieben.

Eine unabdingliche Bedeutung wird im Zusammenhang mit pädagogischem Wissen dem Raum zugeschrieben. Beobachtungen verdeutlichen, wie pädagogisches Wissen auf Basis der jeweiligen Strukturen und Konfigurationen einer Schule beeinflusst wird. Der Raum wurde im Rahmen der empirischen Datenerhebung als Grundlage für das pädagogische Wissen rekonstruiert. $\mathrm{Zu}$ der hier angeführten allgemeinen Charakterisierung pädagogischen Wissens wurden über die Beobachtungen spezifische Kategorien aufgezeigt. Insgesamt lässt sich das Gesamtbild anhand von fünf Kategorien darstellen (vgl. Abbildung 64).

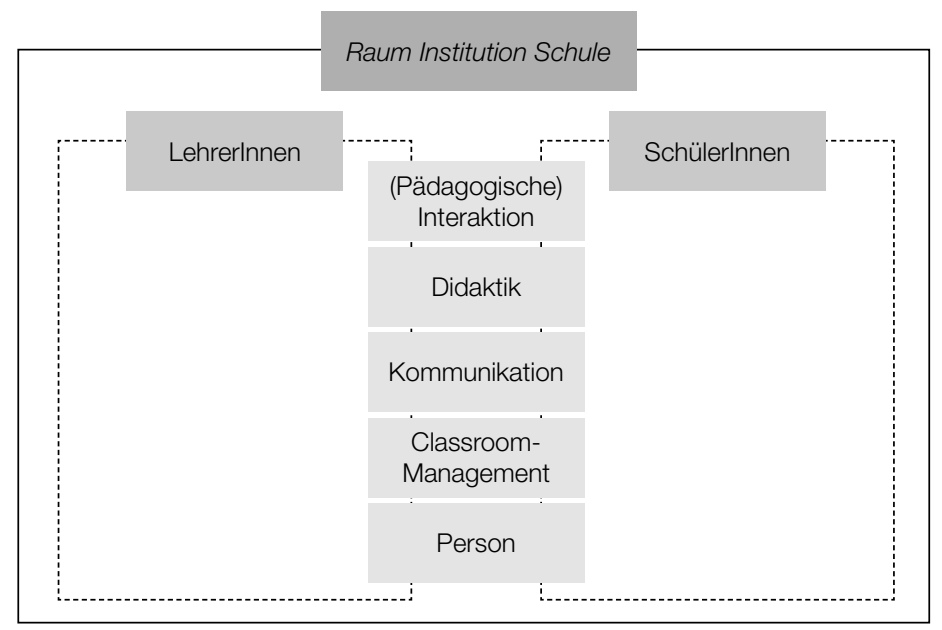

Abb. 64: Kategorien pädagogischen Wissens aus den Beobachtungen

Die rekonstruierten Kategorien pädagogischen Wissens beziehen sich auf die (pädagogische) Interaktion, die Kommunikation, das Classroom-Management, die Didaktik sowie die im Unterricht beteiligten Personen, SchülerInnen und LehrerInnen. Diese Wissenskategorien werden im folgenden Abschnitt zur Diskussion gestellt. Hierfür werden, wie schon bei der Darstellung der Interviewkategorien, die Ordnungskategorien lernseitig und lehrseitig herangezogen. ${ }^{46}$

46 Die Definition der Begriffe lern- und lehrseitig ist in Abschnitt 8.1 zu finden. 


\subsection{1 (Pädagogische) Interaktion}

Die (pädagogische) Interaktion wurde als eine Hauptkategorie pädagogischen Wissens rekonstruiert. Rekonstruierte Kategorien beziehen sich lern- sowie lehrseitig auf das Wissen zu Erziebung, Kooperation, Haltung und Emotionen, Vielfalt, Gruppendynamik, Umgang mit Problemen sowie Unterstützung und Förderung (vgl. Abbildung 65).

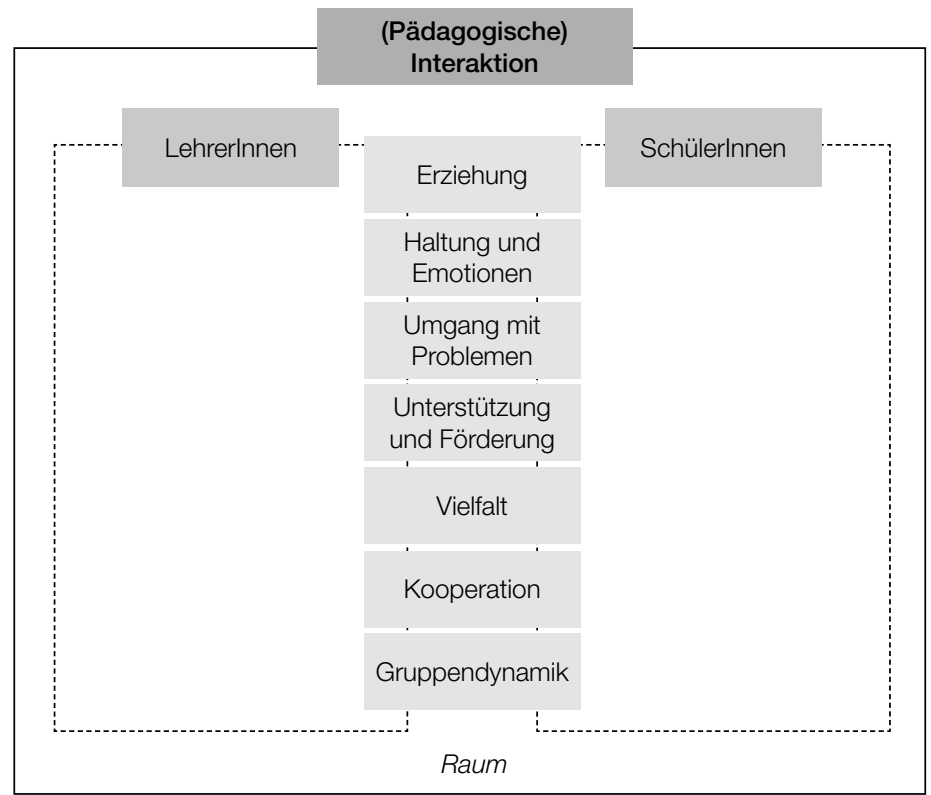

Abb. 65: Kategorien (pädagogischer) Interaktion

Die (pädagogische) Interaktion zeigt sich über das Handeln zwischen SchülerInnen und Lehrpersonen über die in der Grafik angeführten Kategorien. Die Reihenfolge basiert auf der Berücksichtigung wechselseitiger Beziehungen einzelner Kategorien. Diese werden im Folgenden mit ihren jeweiligen Subkategorien zur Diskussion gestellt.

\subsubsection{Erziehung}

Das Thema Erziehung beschreibt sich über die Subkategorien Werte und Regeln (vgl. Abbildung 66).

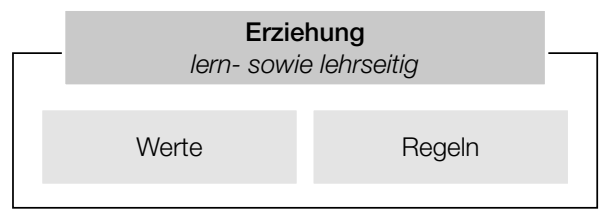

Abb. 66: Subkategorien Erziehung

Im Rahmen der Beobachtung hat sich der Umgang mit Regeln gezeigt. Diese beziehen sich explizit auf den Unterricht und umfassen Regeln für das tägliche Leben. Rekonstruierte Beispiele 
betreffen die Nutzung von Handys im Unterricht, das Wippen mit dem Stuhl, den Umgang mit Arbeitsmaterialien sowie das Aufzeigen bei Wortmeldungen. Regeln außerhalb des Klassenzimmers sind die Pünktlichkeit, Begrüßung und Verabschiedung, das aktive Zuhören, eine angemessene Körperhaltung, einen respektvollen Umgang und das leise Arbeiten in einer Gruppe. Weitere Regeln, die über die Beobachtungen rekonstruiert wurden, beziehen sich auf die Kommunikation, wie zum Beispiel Feedback und Regeln einer respektvollen Kommunikation ${ }^{47}$.

Die Beobachtungen zeigen, wie Grundschullehrpersonen und Lehrpersonen der Neuen Mittelschule die SchülerInnen verstärkt im Umgang mit Regeln sensibilisieren, indem diese explizit vermittelt werden. Im Gymnasium hingegen wird das Wissen zu Regeln vorausgesetzt. SchülerInnen werden bei Nichteinhalten der Regeln auf Verstöße hingewiesen.

In enger Beziehung zu den Regeln steht die Wertevermittlung. Es zeigt sich, wie die Wertevermittlung von eigenen Wertvorstellungen einer Lehrperson beeinflusst wird, und wie Letztere individuelle Wertvorstellungen in die Klasse trägt. Die Vermittlung von Werten im Klassenzimmer definiert sich über Formeln wie Bitte oder Danke, über Ehrlichkeit, sich entschuldigen können, kritisches Denken, Offenheit gegenüber Vielfalt, Umwelterziehung (z.B. Mülltrennung), die Ernsthaftigkeit von Themen erkennen oder über den Kommunikationsprozess untereinander (ausreden lassen und/oder Beschimpfungen). In allen Schulformen wird explizit auf Werte, die in der gegenwärtigen Gesellschaft wichtig sind, hingewiesen. SchülerInnen zeigen, wie gewillt sie sind, Verantwortung für sich und die Gesellschaft zu übernehmen und wesentliche Werte im Unterricht zu berücksichtigen.

Die Rekonstruktion macht sichtbar, wie Lehrpersonen ein kohärentes und stringentes Verhalten im Umgang mit Werten und Regeln vorleben. Dieses Verhalten wirkt sich auf SchülerInnen aus. Sie übernehmen vorgelebte Muster und Verhaltensweisen. Rekonstruierte Beispiele beziehen sich auf eine angemessene Kommunikation, auf das Arbeitsverhalten der SchülerInnen, die Verwendung der Formeln Bitte oder Danke oder einen ehrlichen Umgang miteinander.

Die Erziehung zu Werten und Regeln kann von Haltungen und Emotionen einer Lehrperson beeinflusst werden. Diese werden im folgenden Abschnitt beschrieben.

\subsubsection{Haltung und Emotionen}

Als eine weitere Kategorie der (pädagogischen) Interaktion wurde das Wissen zu Haltung und Emotionen rekonstruiert. Diese untergliedert sich in die Subkategorien Angst, Nähe und Distanz, Wertschätzung sowie Fürsorge (vgl. Abbildung 67).

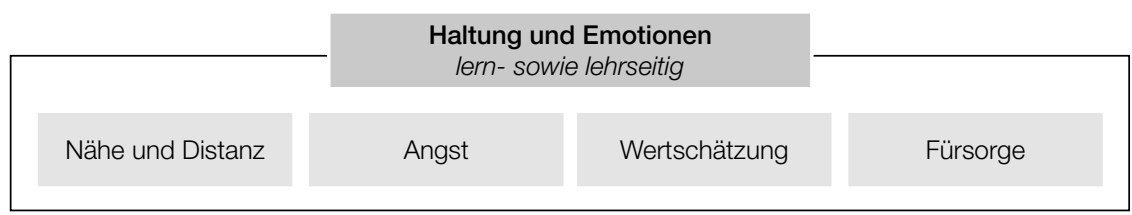

Abb. 67: Subkategorien Haltung und Emotionen

Das Thema Nähe und Distanz beschreibt, wie die beteiligten AkteurInnen individuell für sich ein Abstands- bzw. Näheverhältnis aufbauen. Die Beobachtungen zeigen, wie Lehrpersonen dies über direktes, aber auch indirektes Verhalten erreichen. Rekonstruierte Beispiele beziehen

47 Die Kommunikation wird wegen ihrer besonderen Stellung als eigenständige Kategorie pädagogischen Wissens in Abschnitt 8.5.3 beschrieben. 
sich auf das Ansprechen mit Vor- oder Nachname, das Preisgeben privater Informationen, den Austausch von persönlichen Geschichten oder körperliche Aspekte, wie die Hand auf die Schulter legen. SchülerInnen offenbaren über individuelle Verhaltensmuster ihre eigenen persönlichen Grenzen, die von den Lehrpersonen im Unterricht respektiert werden sollten. Es zeigt sich, wie vor allem jüngere SchülerInnen aus der Grundschule Nähe zu ihren Lehrpersonen suchen, indem sie diese umarmen und anfassen. Körperliche Nähe wird in der Neuen Mittelschule und im Gymnasium seltener. Nähe zwischen Lehrpersonen und SchülerInnen kommt in beiden Schulformen durch Gespräche über persönliche Themen und Geschichten zum Ausdruck.

Der Umgang mit Wertschätzung zeigt sich auf allen Schulstufen. Lehrpersonen der Grundschule und der Neuen Mittelschule planen bewusst Zeit für die Sensibilisierung der SchülerInnen im Umgang mit Wertschätzung ein. Bewusst geplante Zeitfenster werden für die Anwendung von Kooperationsspielen, Gespräche mit Beratungspersonen, die Begrüßung jedes einzelnen Schülers/jeder einzelnen Schülerin oder für persönliche Geschichten und Beiträge eingesetzt. Wertschätzung zeigen Gymnasiallehrpersonen gegenüber SchülerInnen, indem sie sich zum Beispiel bei ihnen für ihre Aufmerksamkeit bedanken, fragen, wie es den SchülerInnen geht, Partizipationsmöglichkeiten anbieten, ihre Stärken und Schwächen annehmen und sich Situationen und Verhalten erklären lassen, bevor sie über Leistungen und Verhaltensformen urteilen. Aufseiten der SchülerInnen zeigt sich, wie sie sich über spezifisches Verhalten gegenseitig wertschätzen. Rekonstruierte Beispiele beziehen sich auf das gegenseitige Akzeptieren, Interesse zeigen (Vorlieben, Interessen, Hobbies, Kultur), einen respektvollen Umgang, darauf, kein Konkurrenzverhalten vorzuleben und keine MitschülerInnen zu verpetzen.

Die Wertschätzung zwischen SchülerInnen und Lehrpersonen zeigt sich über einen respektvollen Umgang und das Respektieren und Annehmen von individuellen Wertvorstellungen der jeweiligen Lehrpersonen. Beispiele aus den Beobachtungen betreffen das Anerkennen von persönlichen Eigenschaften von Lehrpersonen, wie zum Beispiel der Sprache, körperlicher Auffälligkeiten, individueller Verhaltensmuster, wie Räuspern und Wortwiederholungen, und persönlicher Vorstellungen und Vorlieben zu Themen und Methoden.

Ein weiteres Thema der Kategorie Haltung und Emotionen ist das Thema Angst. Lehrpersonen repräsentieren in den Beobachtungen explizit, mit welchen bewussten Handlungsstrategien den SchülerInnen das Gefühl der Angst genommen bzw. dieses verringert wird (vgl. Abbildung 68).

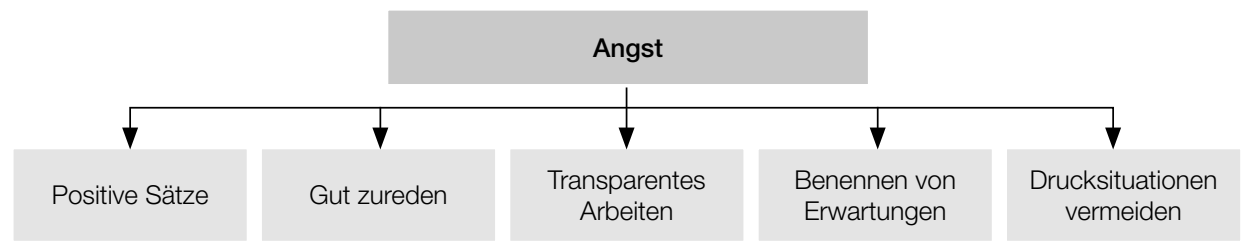

Abb. 68: Handlungsstrategien zur Reduktion lernseitiger Angstsituationen

Beobachtete Handlungsstrategien zur Reduktion von lernseitigen Angstsituationen sind das Gut-Zureden der Lehrpersonen, das Formulieren von positiven Sätzen, das transparente Arbeiten, das Vermeiden von Drucksituationen oder das Äußern von Erwartungen und Vorstellungen. Es zeigt sich, wie über den Einsatz der beschriebenen Strategien Ängste von SchülerInnen reduziert werden können. 
Zur Kategorie Haltung und Emotionen wurde das Thema der Fürsorge rekonstruiert. SchülerInnen, aber auch Lehrpersonen durchleben in einer Unterrichtsstunde unterschiedliche Gefühlslagen. Beobachtungen illustrieren, wie die betroffenen AkteurInnen situativ mit diesen Gefühlen umgehen und angemessen reagieren können. SchülerInnen aus der Grundschule benötigen $\mathrm{Zu}$ neigung und Betreuung vonseiten der Lehrperson. Auf Ebene der Neuen Mittelschule sowie des Gymnasiums beschränkt sich der Umgang mit den Gefühlen auf das Trösten, Gut-Zureden, den SchülerInnen Mut zu machen oder die Nervosität vor Prüfungen und angespannten Situationen zu nehmen. Es zeigt sich, wie SchülerInnen Gefühlslagen von Lehrpersonen respektieren. Bemerken die SchülerInnen, dass eine Lehrperson nicht gut gelaunt ist, konnte rekonstruiert werden, wie sie diese darauf ansprechen oder es akzeptieren, indem sie damit umgehen.

Das Thema Haltung und Emotionen zeigt, wie Lehrpersonen mit diesem Wissen eine wertschätzende Beziehung aufbauen und angemessen handeln können, indem Gefühlslagen und persönliche Empfindungen berücksichtigt werden. Ein adäquates Handeln konnte auch im Wissen zum Umgang mit Problemen rekonstruiert werden.

\subsubsection{Umgang mit Problemen}

Der Umgang mit Problemen wurde als weitere Kategorie im Bereich der (pädagogischen) Interaktion rekonstruiert. Es lassen sich, wie in Abbildung 69 dargestellt, die Subkategorien Handlungsstrategien und Prävention identifizieren.

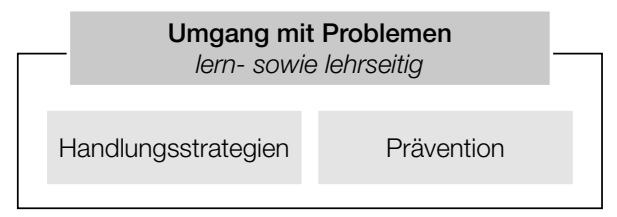

Abb. 69: Subkategorien Umgang mit Problemen

Beobachtungen zeigen, dass Lehrpersonen spezifisches Wissen anwenden, um Probleme zu erkennen und Handlungsstrategien indizieren zu können. Folgende Problemsituationen konnten rekonstruiert werden:

- SchülerInnen kommen zu spät in den Unterricht.

- SchülerInnen halten sich nicht an Abgabetermine von Arbeiten.

- SchülerInnen verhalten sich im Unterricht laut und sind unkonzentriert.

- SchülerInnen sind müde und schlafen im Unterricht.

- SchülerInnen spielen mit dem Handy.

- SchülerInnen streiten untereinander.

- SchülerInnen arbeiten nicht mit.

- SchülerInnen kommen mit zerrissenen Kleidungsstücken in die Schule.

- SchülerInnen haben keine Unterrichtsmaterialien dabei.

- SchülerInnen ärgern sich untereinander und beschimpfen sich.

Es wird versucht, Problemsituationen über direktes und offenes Ansprechen in der Klasse oder über ein Vier-Augen-Gespräch zu lösen. Spezifische Handlungsstrategien im Umgang mit Problemen beziehen sich auf den Einsatz von Mediationen oder Rollenspielen. Eine präventive Maßnahme, die beobachtet werden konnte, ist die Förderung einer Zusammenarbeit mit Beratungspersonen, die regelmäßig die Klasse besuchen und mit den SchülerInnen Themen wie den 
Umgang mit Problemen bespricht. Dabei werden SchülerInnen im Umgang untereinander sowie über alternative Handlungsmöglichkeiten bei Problemen geschult. Beobachtungen zeigen, wie SchülerInnen oftmals versuchen, selbstständig Probleme zu lösen, indem sie sich gegenseitig auf Verhaltensweisen ansprechen.

Es konnte rekonstruiert werden, wie das Wissen zum Umgang mit Problemen einen wertvollen Umgang untereinander schafft. Die Lehrpersonen übernehmen eine wichtige Funktion, indem sie versuchen, über bewusste Strategien Problemsituationen präventiv zu verringern. Unterstützung und Förderung ist ein essenzielles Thema und wurde als weitere Kategorie aus den Beobachtungen rekonstruiert.

\subsubsection{Unterstützung und Förderung}

Eine weitere Form der (pädagogischen) Interaktion ist das lern- sowie lehrseitige Wissen über Unterstützung und Förderung. Diese wird in die Subkategorien diagnostische Kompetenz und Handlungsstrategien gegliedert (vgl. Abbildung 70).

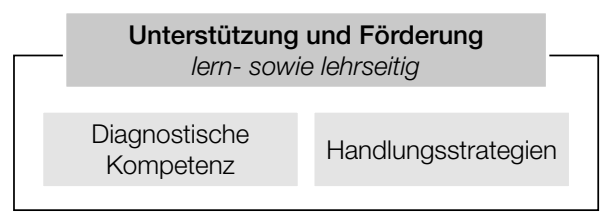

Abb. 70: Subkategorien Unterstützung und Förderung

Über die Beobachtungen zeigt sich die Fähigkeit von Lehrpersonen zu erkennen, wann SchülerInnen fachlich sowie persönlich Unterstützung benötigen, und fallspezifisch darauf reagieren zu können. Unterstützungsmöglichkeiten zeigen sich über die Zusammenarbeit mit externem Fachpersonal, das Führen von individuellen Gesprächen bei persönlichen sowie fachlichen Problemen und einer spezifischen Lernförderung über das Anbieten von unterschiedlichen Lerntipps. Es konnte beobachtet werden, wie Lehrpersonen die SchülerInnen unterstützen, indem sie ihnen Glück wünschen, an Leistungen der Kinder glauben und ihnen Mut zusprechen.

Weitere Unterstützungsstrukturen konnten im Umgang mit Transitionen festgestellt werden. Die Felduntersuchungen verdeutlichen, wie vor allem Grundschullehrpersonen versuchen, den SchülerInnen einen Übergang in eine weiterführende Schule zu erleichtern. Es wird Zeit für spezielle Übungen, Arbeitsaufträge und Gespräche geschaffen, in denen die SchülerInnen auf Strukturen (z.B. Prüfungssituationen) der Neuen Mittelschule oder des Gymnasiums vorbereitet werden.

Unterstützung und Förderung findet in der Schule auf unterschiedlichen Ebenen statt. Die Rekonstruktion macht deutlich, wie Lehrpersonen versuchen, SchülerInnen persönlich sowie fachlich für ein bestmögliches Lernen und eine individuelle Entwicklung zu unterstützen. Aufgrund zunehmend diverser Strukturen im Klassenzimmer ist eine Förderung sowie Unterstützung im Zusammenhang mit dem Thema Vielfalt von großer Bedeutung. Der Umgang mit Vielfalt wird aufgrund seiner wichtigen Bedeutung als eigenständige Kategorie im folgenden Abschnitt beschrieben.

\subsubsection{Vielfalt}

Beobachtungen zeigen das Thema der kulturellen Vielfalt. Die Vielfalt beschreibt den Umgang mit unterschiedlichen kulturellen Hintergründen und Sprachunterschieden im Unterricht (vgl. Abbildung 71). 


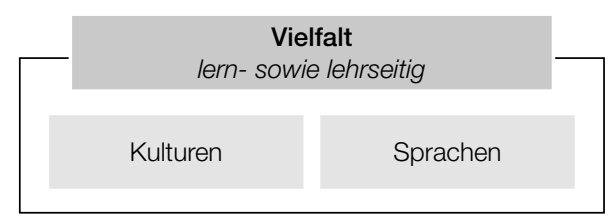

Abb. 71: Subkategorien Vielfalt

Die Datenerhebung illustriert, wie Lehrpersonen spezifische Maßnahmen für den Umgang mit Vielfalt im Zusammenhang mit den Themen Sprache und Kultur in einer Klasse einsetzen. Die Ergebnisse stützen sich vorwiegend auf Beobachtungen aus Grundschulen. Grundschullehrpersonen integrieren kulturelle Vielfalt in ihren Unterricht, indem sie diese als positiv hervorheben. Im Zuge der Beobachtungen konnten verschiedenste Veranstaltungen und Austauschformate festgehalten werden, zum Beispiel das Mitbringen unterschiedlicher Speisen aus aller Welt, das Organisieren von Sprachencafés, das Anhören von Musikstücken unterschiedlicher Sprachen oder die Möglichkeit, Texte in unterschiedlichen Sprachen zu verfassen. Im Gymnasium zeigten sich hierzu keine spezifischen Maßnahmen.

Pädagogisches Wissen wurde bei den Lehrpersonen darüber rekonstruiert, dass sie explizite Strategien anwenden, damit sich SchülerInnen mit verschiedenen Sprachen und Kulturen auseinandersetzen. Es bedarf vonseiten der Lehrpersonen eines angemessenen didaktischen Handelns. Eine weitere Form von Austausch findet über das Wissen zu Kooperation statt. Diese wird im weiteren Verlauf der Arbeit beschrieben.

\subsubsection{Kooperation}

Die Kooperation ist eine weitere rekonstruierte Kategorie der Hauptkategorie (pädagogische) Interaktion. Diese charakterisiert sich über das Wissen zu Zusammenarbeit und Austausch zwischen unterschiedlichen AkteurInnenn (vgl. Abbildung 72).

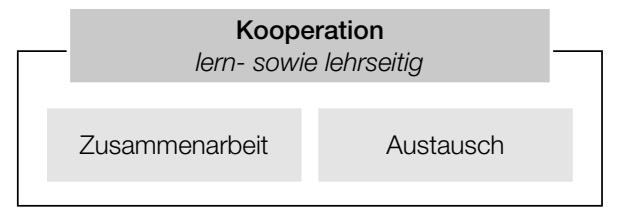

Abb. 72: Subkategorien Kooperation

Die folgende Abbildung zeigt die rekonstruierten Kooperationsmuster im Schulalltag, ausgehend von den AkteurInnen, den SchülerInnen und Lehrpersonen (vgl. Abbildung 73).

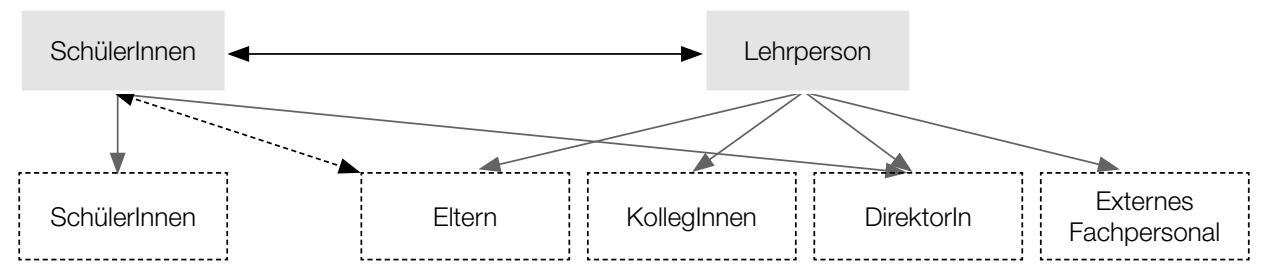

Abb. 73: Kooperationsmuster zwischen den AkteurInnen 
Kooperationen konnten zwischen SchülerInnen und Lehrpersonen, zwischen Lehrpersonen und Erziehungsberechtigten sowie mit externem Fachpersonal und zwischen den SchülerInnen untereinander rekonstruiert werden. Die Beobachtungen veranschaulichen, wie die Zusammenarbeit und der Austausch eng in Verbindung stehen.

Die Kooperation zwischen SchülerInnen und Lehrpersonen lässt sich auf fachlicher sowie persönlicher Ebene beobachten. Fachlich spiegelt sich die Kooperation zum Beispiel in der Auswahl der Themen, Inhalte, Materialien und Methoden wider. Wenn die AkteurInnen im Unterricht kooperieren, zeigen Beobachtungen, wie sich die Lehrpersonen bei der Themenwahl, der Methodenwahl und den Aufgabenstellungen an den Interessen und Vorlieben der SchülerInnen orientieren. Auf personaler Ebene zeichnet sich die Kooperation über das Erzählen persönlicher Geschichten zwischen Lehrpersonen und SchülerInnen aus. Es zeigen sich weitere Kooperationen über eine freie Meinungsäußerung. Beobachtungen illustrieren, wie SchülerInnen Wünsche und Anliegen äußern und über einen partizipatorischen Prozess den Schulalltag aktiv mitgestalten. Im Rahmen der Rekonstruktion konnte demonstriert werden, wie SchülerInnen Lehrpersonen bitten, gewisse Themenbereiche im Unterricht zu behandeln, Sportveranstaltungen zu organisieren oder Ausflüge gemeinsam zu gestalten. Es konnte beobachtet werden, wie SchülerInnen großes Engagement zeigen, aktiv bei (außer)schulischen Veranstaltungen mitzuwirken und Aufgaben zu übernehmen. SchülerInnen melden sich freiwillig, um zum Beispiel beim Aufbau von Veranstaltungen in der Schule, beim Tag der offenen Tür, beim Servieren von Getränken, beim Maturaball oder nur bei kleinen, unterstützenden Aufgaben mitzuhelfen.

Die Rekonstruktion macht sichtbar, wie SchülerInnen die Lehrperson als BeraterIn sehen und bei Anliegen, Schwierigkeiten und Problemen um Rat fragen. Aufseiten der Lehrperson ist für diese Wechselbeziehung das Interesse an Problemen und Anliegen der SchülerInnen und die Bereitschaft, ihnen Ratschläge zu geben, Voraussetzung.

Die Kooperation zwischen SchülerInnen wird über die Zusammenarbeit zwischen den SchülerInnen präsentiert. Beobachtungen belegen, dass diese miteinander kooperieren und eine in sich geschlossene Gruppendynamik entwickeln, in der sie kooperativ zusammenarbeiten, sich unterstützen und aufeinander Rücksicht nehmen. Es zeigt sich, wie sie Leistungen gegenseitig anerkennen, indem sie bei Präsentationen applaudieren oder sich gegenseitig loben und Mut zusprechen.

Weitere rekonstruierte Kooperationen der Lehrpersonen beziehen sich auf die Zusammenarbeit mit Eltern, Schulleitung, KollegInnen und externem Fachpersonal. Die Beobachtungen skizzieren eine intensive Zusammenarbeit zwischen einzelnen Lehrpersonen. Diese verdeutlichen die essenzielle Bedeutung des direkten Kontakts für einen fachlichen und inhaltlichen Austausch. Rekonstruierte Formen beziehen sich auf einen fächerübergreifenden Unterricht, den inhaltlichen Austausch zwischen den KollegInnen, den Austausch von Ideen und Materialien sowie die gegenseitige Unterstützung bei Problemen und Unklarheiten. In der Neuen Mittelschule und der Grundschule findet als weitere Form der Zusammenarbeit zwischen den Lehrpersonen eine intensive Zusammenarbeit über das Team-Teaching in den Hauptfächern statt. Rekonstruierte Formen der Zusammenarbeit mit den Eltern charakterisiert sich über regelmäßige Austauschformate wie zum Beispiel Elternsprechtage, Kinder-Eltern-LehrerInnengespräche oder Schoolworld Cafés. In Bezug auf das Fachpersonal illustrieren Beobachtungen, wie Lehrpersonen aus der Grundschule und der Neuen Mittelschule vielfältige Ressourcen zur Verfügung stehen, um sich Unterstützung zu holen, damit sie bedarfsorientiert agieren können.

Beobachtungen deuten darauf hin, dass die Schulleitungen einzelner Schulen sich verstärkt einen direkten Kontakt mit SchülerInnen und Lehrpersonen bemühen. Informationen und An- 
liegen werden nicht über Dritte, sondern persönlich weitergegeben. Schulleitungen kennen SchülerInnen mit Namen, kommen während des Unterrichts in die Klassen, um Informationen direkt weiterzugeben. Es wird die Zusammenarbeit zwischen SchülerInnen und Schulleitung gefördert.

Das Wissen zu Kooperation verstärkt die Zusammenarbeit und den Austausch zwischen einzelnen AkteurInnen und unterstützt damit positiv das Lernen und Lehren. Kooperationen zwischen SchülerInnen können explizit durch Wissen über Gruppendynamiken gefördert werden. Das Wissen zu Gruppendynamik wird im weiteren Verlauf beschrieben.

\subsubsection{Gruppendynamik}

Als eine weitere Kategorie der (pädagogischen) Interaktion wurde das Wissen zu Gruppendynamik rekonstruiert. Beobachtungen illustrieren, wie in jeder Klasse unterschiedliche individuelle Rollen vorhanden sind, die Dynamiken im Klassenzimmer beeinflussen (vgl. Abbildung 74).

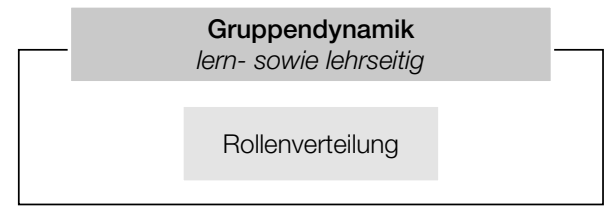

Abb. 74: Subkategorien Gruppendynamik

Pädagogisches Wissen erleichtert das Erkennen unterschiedlicher Dynamiken sowie den Umgang mit unterschiedlichen Einflüssen von Rollen der AkteurInnen. Einzelne SchülerInnen illustrieren, wie sie über ihr Verhalten unterschiedliche AkteurInnen gewinnbringend zusammenführen oder Ausgrenzungs-, Neid- sowie Mobbingverhalten vorleben. Präventiv gesetzte Maßnahmen von Lehrpersonen beziehen sich auf Gespräche mit SchülerInnen und Eltern sowie die aktive Integration von Betreuungslehrpersonen, die SchülerInnen über Mobbing und Ausgrenzung informieren und sensibilisieren. Aus den Beobachtungen geht hervor, wie Lehrpersonen unterstützende Maßnahmen veranlassen, um eine stabile Gruppendynamik zu fördern. Zusätzlich konnten Kooperationsspiele und das Anwenden unterschiedlicher Sozialformen rekonstruiert werden, die die Gemeinschaft einer Klasse stärken.

Die Rekonstruktion macht sichtbar, wie einzelne Personen mit ihrem Verhalten einen großen Einfluss auf das Lehren und Lernen aller Beteiligten haben können. In diesem Zusammenhang ist spezifisches Eingreifen vonseiten der Lehrpersonen notwendig, um Dynamiken, die sich negativ auf das Lernen und Lehren auswirken, zu verhindern bzw. professionell damit umgehen zu können.

\subsubsection{Didaktik}

Das Wissen zur Didaktik stellt eine rekonstruierte Hauptkategorie pädagogischen Wissens dar. Beobachtungen illustrieren, dass die von der Lehrperson angewendeten didaktischen Konzepte die Interaktion sowie den Ablauf des Unterrichts beeinflussen. Teile der Didaktik sind den Beobachtungen zufolge Wissensbereiche zu Themen, Unterrichtsstrategien und Konzepten und lernpsychologischem Wissen (vgl. Abbildung 75). 


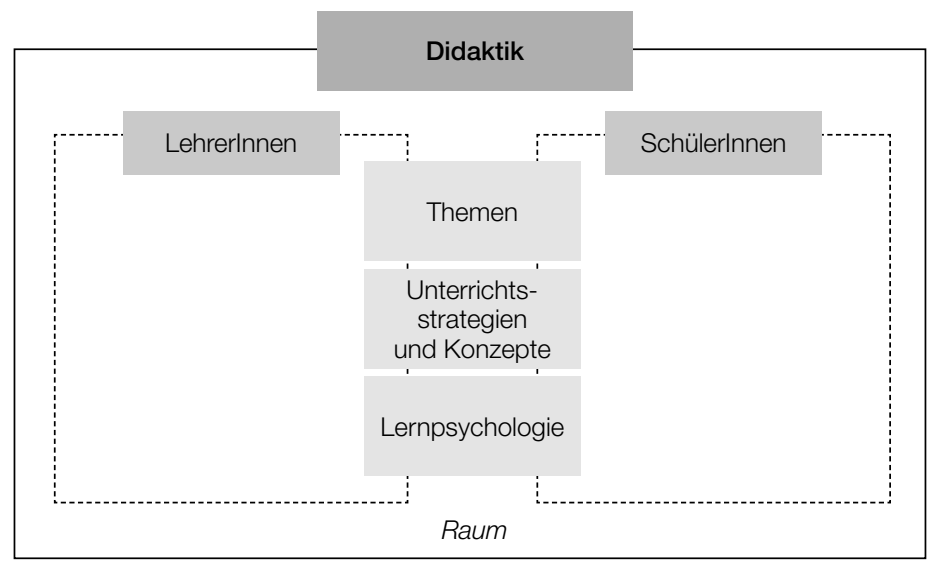

Abb. 75: Kategorien der Didaktik

Das pädagogische Wissen zu Didaktik zeigt sich über das Handeln zwischen SchülerInnen und Lehrpersonen über die in der Grafik angeführten Kategorien. Als Erstes werden allgemein didaktische Kategorien beschrieben. Anschließend erfolgen Inhalte betreffend psychologische Aspekte. Diese werden im Folgenden mit ihren jeweiligen Subkategorien zur Diskussion gestellt.

\subsubsection{Themen}

Die Beobachtungen skizzieren im Zusammenhang mit der Didaktik das Wissen über die Themenwahl (vgl. Abbildung 76).

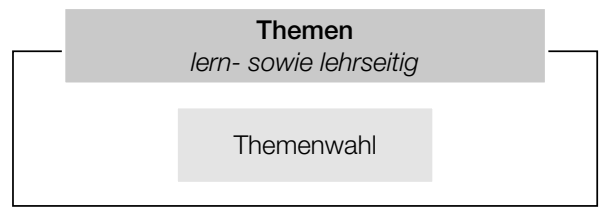

Abb. 76: Subkategorien Themen

Beobachtungen illustrieren, inwieweit SchülerInnen sich bei der Themenwahl beteiligen können. Themen werden in allen drei Schulformen vorrangig von den Lehrpersonen vorgegeben. Die Rekonstruktion verdeutlicht, wie den SchülerInnen die Möglichkeit angeboten wird, individuelle Inhalte über Präsentationen, Referate und Projektarbeiten mitzubestimmen. Pädagogisches Wissen lässt sich darüber beobachten, wie Lehrpersonen versuchen, interessennahe Themen in den Unterricht zu integrieren. Hierfür verwenden sie für das Abfragen von Interessen und Vorlieben spezifische Methoden, wie Fragebögen oder das Schreiben von Texten.

$\mathrm{Zu}$ dem Wissen zur Themenwahl konnte weiteres spezifisches pädagogisches Wissen zur Didaktik rekonstruiert werden. Dieses bezieht sich auf den Einsatz unterschiedlicher Strategien und Konzepte. 


\subsubsection{Unterrichtsstrategien und Konzepte}

Als weitere Wissenskategorie geht das Wissen zu Unterrichtsstrategien und Konzepte aus den Beobachtungen hervor. Dieses charakterisiert sich über die Subkategorien Methodik, Aufgabenentwicklung, Sozialformen, Leistungsbeurteilung, Einsatz von Medien, Differenzierung sowie Feedback (vgl. Abbildung 77).

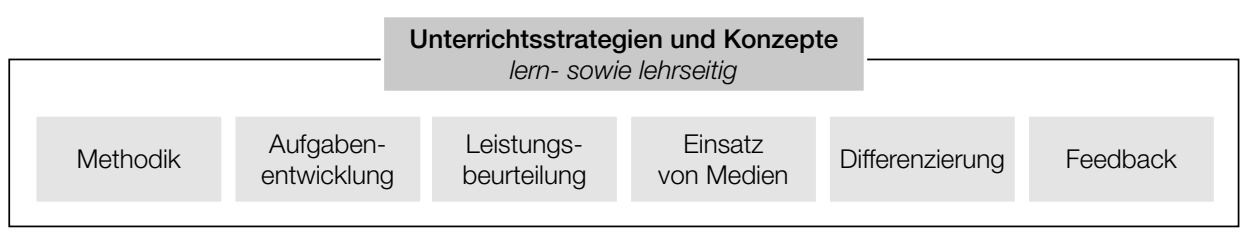

Abb. 77: Subkategorien Unterrichtsstrategien und Konzepte

Pädagogisches Wissen wird im Einsatz unterschiedlicher Methoden im Unterricht sichtbar. Die Beobachtungen verdeutlichen, wie Lehrpersonen unterschiedliche Methoden integrieren. Gängige rekonstruierte Methoden im Gymnasium sind der Frontalunterricht sowie Methoden, die unterschiedliche Sozialformen, wie Einzelarbeit, Partnerarbeit und Gruppenarbeit, fördern. Das Schulbuch dient als Hauptmedium für die Anleitung von unterschiedlichen Aufgaben. Innovative Momente sind Präsentationen von SchülerInnen und Projektarbeiten, die von der Lehrperson verteilt werden. Ein anderes Bild präsentiert die Neue Mittelschule. Hier zeigen sich zum Frontalunterricht und der Förderung unterschiedlicher Sozialformen regelmäßige Freiarbeitsstunden in den Hauptfächern. In der Grundschule werden vorrangig offene Lernformate angeboten, die im Rahmen von Doppelstunden stattfinden. Das individuelle Lernen steht im Vordergrund. Auf dieser Ebene konnten keine spezifischen Maßnahmen bezogen auf die Integration verschiedener Sozialformen rekonstruiert werden.

Die Beobachtungen verdeutlichen Wissensbereiche zur Aufgabenentwicklung. Es konnte beobachtet werden, wie Lehrpersonen Aufgaben unterschiedlicher Qualitäten in ihrem Unterricht anwenden. Ein paar wenige Lehrpersonen arbeiten unterschiedlich schwierige Aufgaben aus, mit denen sie es schaffen, verschieden starke SchülerInnen anzusprechen. Sie fragen dabei nicht nur das Faktenwissen ab, sondern fördern das anwendungsorientierte und problemlösungsorientierte Lernen. Vor allem im Zusammenhang mit offenen Lernformaten konnte rekonstruiert werden, wie Lehrpersonen verstärkt den Fokus auf handlungsorientierte Aufgaben legen, die unterschiedliche kognitive Ebenen wie das Abfragen, Vergleichen, Diskutieren und Kreieren berücksichtigen.

Als eine weitere Subkategorie konnte das Wissen zur Leistungsbeurteilung rekonstruiert werden. Dieses macht sichtbar, wie die Lehrpersonen unterschiedliche Verfahren für die Lernstandserhebungen anwenden. Sie beziehen sich auf summative sowie formative Verfahren. Beobachtungen verdeutlichen, wie Lehrpersonen während des Schuljahrs mit einem Punktsystem und weniger mit Noten arbeiten. Dieser Zugang verringert die Gefahr, einem ständigen Notendruck ausgesetzt zu sein. Es konnte beobachtet werden, wie der Fokus verstärkt auf die Selbstbenotung von SchülerInnen gelegt wird. Sie sollen ihre eigenen Leistungen einschätzen und benoten. Als weitere Methode der Selbstbewertung zeigt sich die Anwendung expliziter Reflexionsphasen am Ende des Unterrichts. SchülerInnen werden von den Lehrpersonen angeleitet, ihren individuellen Lernprozess nach der Unterrichtsstunde zu dokumentieren. Pädagogisches Wissen wird darüber sichtbar, dass Lehrpersonen unterschiedliche Leistungsbeurteilungsmöglichkeiten anbieten. 
Pädagogisches Wissen kommt zusätzlich über den spezifischen Einsatz von Medien zum Ausdruck. Rekonstruierte Beispiele beziehen sich auf den Einsatz von Beamern, Computern sowie das Nachschlagen von Wörtern im Fremdsprachenunterricht auf dem Handy oder dem Tablet. Beobachtungen verdeutlichen, wie Lehrpersonen über den passenden Einsatz von verschiedenen Medien den Unterricht abwechslungsreich gestalten.

Als eine wichtige Subkategorie wurde das Wissen zu Strategien für die Berücksichtigung von Differenzierung im Unterricht rekonstruiert. In Abbildung 78 werden spezifische beobachtete Strategien dargestellt.

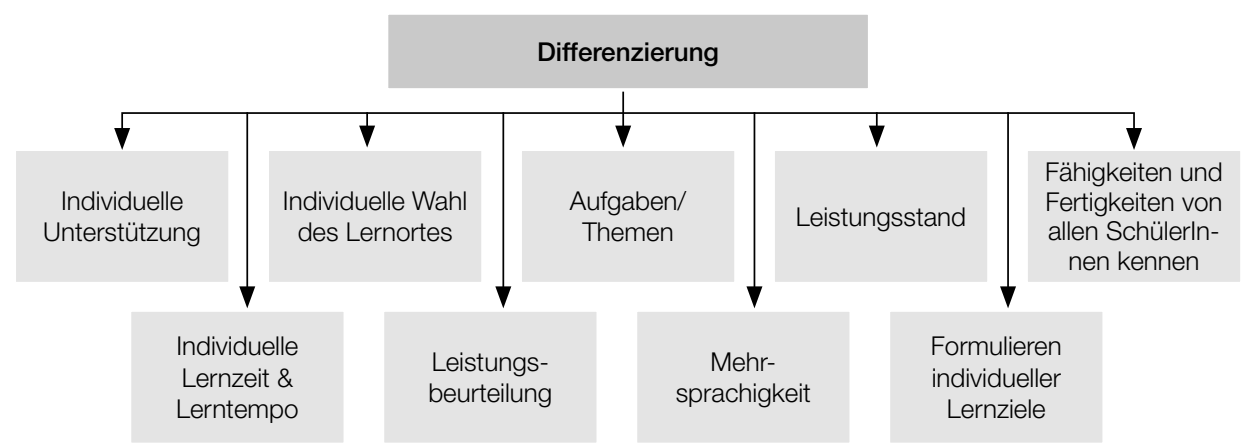

Abb. 78: Strategien für die Berücksichtigung von Differenzierung im Unterricht

Beobachtete Formen beziehen sich auf Strategien wie die individuelle Unterstützung, das Setzen individueller Lernziele, die Berücksichtigung unterschiedlicher Leistungsbeurteilungen, die Anwendung unterschiedlicher Aufgaben und Themen, die Förderung der Mehrsprachigkeit, das Anknüpfen am individuellen Leistungsstand sowie das Kennen von Fähigkeiten und Fertigkeiten der SchülerInnen. Beobachtungen verdeutlichen, wie vor allem in der Grundschule und der Neuen Mittelschule diese Strategien zur Differenzierung eingesetzt werden. Weitere spezifische Differenzierungsstrategien der Grundschule beziehen sich zum Beispiel auf das Bilden heterogener Tischgruppen, sodass SchülerInnen unterschiedlicher Lernniveaus miteinander arbeiten und sich gegenseitig helfen und unterstützen konnten.

Eine weitere rekonstruierte Subkategorie ist das Wissen zu Feedback. Beobachtungen zeigen pädagogisches Wissen, indem Lehrpersonen in einem partizipativen Unterricht Feedback einsetzen. Sie schaffen Raum, um den SchülerInnen mündliche sowie schriftliche Feedbackmethoden näherzubringen und diese nachhaltig einzusetzen. Sie kommen beispielsweise bei Präsentationen oder Rückmeldungen zu Leistungen zur Anwendung. Feedback wird entweder vor der gesamten Klasse oder in Einzelgesprächen gegeben.

Die angeführten Bereiche pädagogischen Wissens unterstützen das individuelle, selbstständige und anwendungsorientierte Lernen der SchülerInnen. Zusätzlich zu spezifisch eingesetzten didaktischen Strategien und Konzepten befördert lernpsychologisches Wissen das Lehren und Lernen.

\subsubsection{Lernpsychologisches Wissen}

Über die Beobachtungen konnten lernpsychologische Wissensformate rekonstruiert werden. Diese untergliedern sich in die Wissenskategorien Fehlerkultur, Emotionen und Stereotypen (vgl. Abbildung 79). 


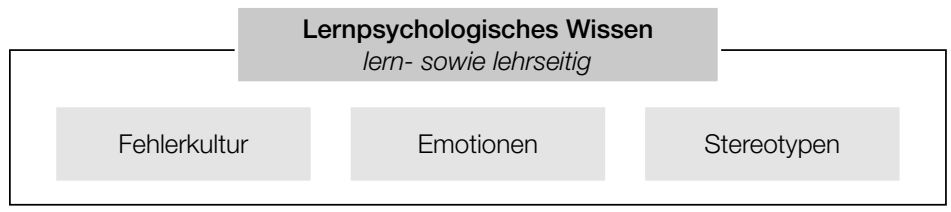

Abb. 79: Subkategorien lernpsychologischer Wissensformate

Die Lehrpersonen verdeutlichen pädagogisches Wissen über einen offenen Umgang mit Fehlern. Sie versuchen, gemeinsam mit den SchülerInnen fehlerhafte Antworten und Aufgaben zu verbessern, und geben ihnen Raum für die eigenständige Verbesserung. Dabei geben sie, wenn nötig, spezifische Hilfestellungen und Tipps. Über das eigenständige Bearbeiten der Aufgaben zeigen die SchülerInnen, wie sie sogenannte „Aha-Effekte“ erleben. Diese kommen zum Beispiel über Aussagen wie „ah, jetzt verstehe ich es“ oder über eine positive Mimik und Gestik, wie zum Beispiel ein Lächeln, zum Ausdruck.

Eine weitere rekonstruierte Subkategorie bezieht sich auf das Wissen zu Emotionen. Beobachtungen illustrieren, wie persönliches Interesse, Betroffenheit und Glücksgefühle dazu führen, dass SchülerInnen engagierter und motivierter am Unterricht teilnehmen und sorgfältiger sowie selbstständiger die einzelnen Arbeitsaufträge erledigen und vertiefend arbeiten. Dies wird im Unterricht über unterschiedliche Methoden erreicht. Rekonstruierte Beispiele betreffen den Einsatz von lebensnahen Aufgaben, die Berücksichtigung aktueller wichtiger Themenbereiche sowie die Besprechung emotionaler Themen.

Der Umgang mit Stereotypen wurde als weitere Kategorie pädagogischen Wissens rekonstruiert, indem Lehrpersonen einen bewussten Umgang mit diesen im Unterricht vorleben. Es zeigt sich, wie Lehrpersonen konkret versuchen, SchülerInnen in Bezug auf Stereotypen zu sensibilisieren. Wurden vonseiten der SchülerInnen stereotypische Inhalte erwähnt, zeigen die Beobachtungen, wie Lehrpersonen sofort darauf reagierten. Im Rahmen dieser Beobachtung wurden Widersprüche sichtbar, da rekonstruiert werden konnte, wie Lehrpersonen selbst Streotypen im Unterricht äußern. Diese werden in Abbildung 80 dargestellt.

Gleiche Leistungen werden schlechter bewertet, wenn sie von Mädchen erbracht werden

Mädchen sind verlässlicher und ordentlicher
Übertragung von Einzelverhalten der Schülerlnnen auf das gesamte Schulleben

Mädchen haben mehr Gepäck bei Schulausflügen
Assoziationen mit Namen

Jungs sind belastbarer als Mädchen

Abb. 80: Rekonstruierte Stereotypen aus dem Schulalltag

Wie in der Abbildung dargestellt, konnten unterschiedliche Formen von Stereotypen im Schulalltag festgehalten werden. Diese beziehen sich vorwiegend auf Unterschiede hinsichtlich Geschlecht, Assoziationen zu Namen oder das Übertragen von Einzelfällen auf die gesamte Klasse. Inwieweit den Lehrpersonen dieses Verhalten bewusst ist, konnte im Zuge der Rekonstruktion nicht beobachtet werden. 
Mithilfe des lernpsychologischen Wissens können Lehrpersonen die SchülerInnen und das Lernen unterstützen, indem sie ressourcenorientiert an ihren Fehlern arbeiten und das Denken über Stereotypen verringern. Das Berücksichtigen von Gefühlslagen hilft, einen motivierenden Unterricht zu gestalten, der schülerInnenorientiert aufgebaut ist.

\subsubsection{Kommunikation}

Die Kommunikation wird aufgrund ihrer wesentlichen Rolle als eine eigenständige Beobachtungskategorie formuliert. Als Teile der Kommunikation wurden die Sprache, die Körpersprache und die Kommunikationsinteraktion beobachtet (vgl. Abbildung 81).

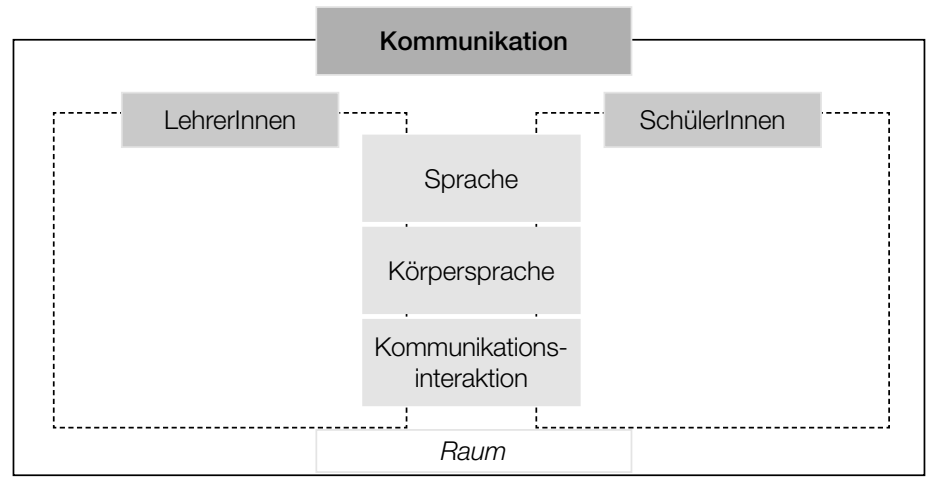

Abb. 81: Kategorien der Kommunikation

Das pädagogische Wissen zur Kommunikation stützt sich auf die in Abbildung 81 dargestellten Kategorien. Am Anfang wird das Wissen zur Sprache dargestellt. Anschließend werden spezifischere Wissensbereiche bezogen auf die Körpersprache sowie die Kommunikationsinteraktion behandelt. Diese werden im Folgenden mit ihren jeweiligen Subkategorien zur Diskussion gestellt.

\subsubsection{Sprache}

Pädagogisches Wissen wird über das Wissen zur Wirkung von Sprache und ihren Einfluss auf den Unterricht sichtbar. Es konnten in diesem Zusammenhang unterschiedliche Eigenschaften der Sprache und ihre Wirkung auf SchülerInnen rekonstruiert werden (vgl. Abbildung 82).

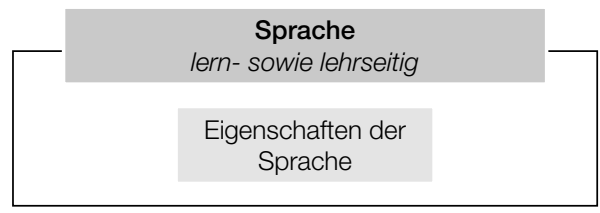

Abb. 82: Subkategorien Sprache

Pädagogisches Wissen kann über den Einsatz einer bewussten Sprache aufgezeigt werden. Lehrpersonen verdeutlichen, wie darüber unterschiedliche Ergebnisse erzielt werden können. Rekonstruierte Wirkungsbereiche werden in Abbildung 83 dargestellt. 


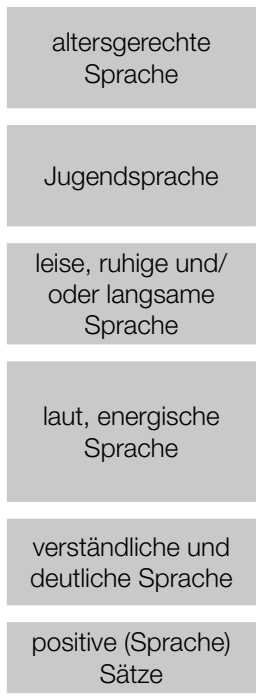

- Schülerlnnen können dem Unterrichtsverlauf folgen

- Schülerlnnen verstehen den Inhalt

- Schülerlnnen und Lehrpersonen befinden sich auf gleicher Kommunikationsebene

- SchülerInnen fühlen sich wahrgenommen und geschätzt

- Schülerlnnen und Lehrpersonen befinden sich auf gleicher Kommunikationsebene

- Schülerlnnen sprechen gerne mit Lehrperson

- Schülerlnnen verhalten sich leise

- Schülerlnnen reden leise miteinander

- Schülerlnnen wirken weniger gestresst und ruhiger

- Schülerlnnen verhalten sich lauter

- Schülerlnnen reden lauter miteinander

- Schülerlnnen fühlen sich direkt angesprochen, Aufmerksamkeit stark auf Lehrperson gerichtet

- Schülerlnnen verstehen die Lehrperson und können dem Unterrichtsverlauf folgen

- Wertschätzung gegenüber den Schülerlnnen

- Positive Grundhaltung im Klassenzimmer wird vermittelt

Abb. 83: Rekonstruierte Wirkungsbereiche der Sprache

Beobachtete Spracheigenschaften beziehen sich auf eine altersgerechte Sprache, eine Jugendsprache, eine ruhige und langsame Sprache, eine laute und energische Sprache, eine verständliche Sprache sowie die Verwendung positiver Sätze. Diese illustrieren, wie in Abbildung 83 dargestellt, bei den SchülerInnen unterschiedliche rekonstruierte Wirkungen.

Wissen um die bewusste Anwendung von Sprache kann gebraucht werden, um erwünschte Situationen zu schaffen und Verhaltensweisen der SchülerInnen zu beeinflussen. Die Reaktionen der SchülerInnen verdeutlichen eine Wechselbeziehung zwischen ihrem Verhalten und dem Sprachgebrauch. Beobachtungen veranschaulichen, wie SchülerInnen das Kommunikationsverhalten der Lehrpersonen widerspiegeln. Es lässt sich beobachten, dass sich SchülerInnen in ihrem Verhalten an die Lehrperson anpassen und Sprachmuster übernehmen.

\subsubsection{Körpersprache}

Aus den Beobachtungen geht als weitere Wissenskategorie der Kommunikation die Körpersprache hervor. Diese bezieht sich auf die Subkategorien Mimik, Gestik sowie Körperausdruck (vgl. Abbildung 84).

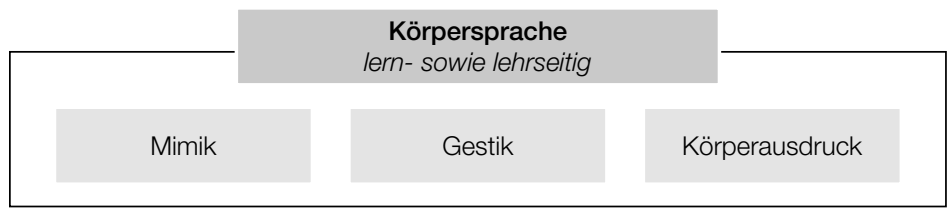

Abb. 84: Subkategorien Körpersprache

Pädagogisches Wissen wird über einen bewussten Einsatz von Mimik und Gestik aufgezeigt. Meist setzen Lehrpersonen Mimik und Gestik aus dem Affekt heraus oder aus Gewohnheit 
ein. Sie bewirken, dass der Unterricht lebendiger wirkt. Im Zuge der Beobachtungen konnten bewusste Anwendungsstrategien rekonstruiert werden. Wie Beobachtungen demonstrieren, werden sie von den Lehrpersonen eingesetzt, um wichtige Inhalte sowie Verhaltensweisen und Anweisungen zu unterstützen. Ein rekonstruiertes Beispiel ist das Gestikulieren bei Störungen. Lehrpersonen reagieren auf Lärm mit der Geste, den Finger vor den Mund zu halten. Weitere Beobachtungen beziehen sich auf Gestik und Mimik im Umgang mit Fehlern. Wenn Lehrpersonen oder SchülerInnen sich gegenseitig auf Fehler hinweisen, ist dies meistens mit einer Geste, zum Beispiel mit dem Hochziehen der Arme oder Schultern, verbunden. SchülerInnen und Lehrpersonen gestikulieren mit den Händen und verwenden ausdrucksstarke Mimik, wenn sie wichtige Inhalte im Unterricht betonen möchten. Das gilt für Inhalte des Unterrichts, eigene Positionen und Stellungnahmen.

Der Körperausdruck von SchülerInnen und Lehrpersonen, wie sie im Klassenzimmer stehen und sitzen, spiegelt die Haltung der jeweiligen Personen wider. Pädagogisches Wissen zeigt sich aufseiten der Lehrperson den Beobachtungen zufolge darin, dass Lehrpersonen eine motivierende Körperhaltung einnehmen. Sitzen sie hinter dem Pult, konnte beobachtet werden, wie SchülerInnen dadurch öfter ihren Kopf auf den Tisch legten. Diese Beobachtung verweist auf die Notwendigkeit, dass Lehrpersonen wissen, wie ihr Körperausdruck auf SchülerInnen wirkt. Die Kommunikation ist ein wichtiger Bereich pädagogischen Wissens, da sie, wie Beobachtungen bestätigen, über spezifischen Einsatz große Wirkung erzielen kann. Das spezifische Anwenden von Körpersprache unterstützt die allgemeine Wirkung und verstärkt Inhalte und Bedeutungen. Kommunikation kann verbal oder nonverbal stattfinden. Dies wird im folgenden Abschnitt über die Subkategorie Kommunikationsinteraktion beschrieben.

\subsubsection{Kommunikationsinteraktion}

Als weiteres Merkmal der Kommunikation kann die Interaktion beschrieben werden. Diese bezieht sich auf die verbale und nonverbale Kommunikation (vgl. Abbildung 85).

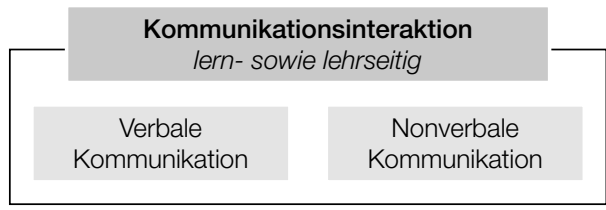

Abb. 85: Subkategorien Kommunikationsinteraktion

Die nonverbale Kommunikation charakterisiert sich, wie im vorherigen Abschnitt beschrieben, über die Anwendung von Gestik und Mimik. Vor allem in der Grundschule hat sich die nonverbale Kommunikation als wesentliches Instrument herauskristallisiert. SchülerInnen wissen bei verschiedenen Verhaltensmustern der Lehrpersonen sowie beim Einsatz von unterschiedlichen Hilfsmitteln, wie Musik oder einer Klingel, was von ihnen erwartet wird. In der Grundschule konnten folgende nonverbale Kommunikationsstrategien auf SchülerInnen- sowie auf LehrerInnenseite rekonstruiert werden:

- Die Lehrperson oder die SchülerInnen spielen Musik ab. $\rightarrow$ SchülerInnen fangen an, ihre Unterrichtsmaterialien wegzuräumen und setzen sich ruhig an ihren Platz.

- Die Lehrperson setzt sich in die Mitte des Klassenzimmers auf einen Teppich. $\rightarrow$ SchülerInnen setzen sich ebenfalls auf den Teppich und bilden einen Kreis. 
- SchülerInnen notieren ihren Namen auf die Tafel. $\rightarrow$ Die Lehrperson weiß, dass der/die SchülerIn Hilfe benötigt.

- SchülerInnen stellen sich an das Pult. $\rightarrow$ Die Lehrperson weiß, dass eine Schülerin/ein Schüler Unterstützung benötigt.

Die ausgewählten Beispiele verdeutlichen den Umgang mit nonverbalen Kommunikationsmitteln im Klassenzimmer. Wie aus den Beobachtungen hervorgehen, werden diese kohärent angewendet, damit sie ihre Bedeutung und Wirkungsbereiche verstärken. Nonverbale Kommunikationsstrukturen im Gymnasium und in der Neuen Mittelschule beziehen sich auf den bewussten Umgang mit einem starren Blick bei Störungen oder Unzufriedenheiten aufseiten der Lehrperson. Die nonverbale Kommunikation wurde vorrangig in Verbindung mit negativ konnotierten Situationen (unzufriedene Aussagen und Handlungsakte) eingesetzt.

Die direkte Kommunikation präsentiert sich im direkten Ansprechen von Problemen, Unklarheiten, Positionen und Erwartungen. Die Rekonstruktion veranschaulicht, wie ein Unterricht, der sich auf traditionelle Muster und Interaktionen stützt, eine direkte Kommunikation weniger einsetzt als Unterrichtsformate, in denen SchülerInnen partizipieren können. Ein offenes Kommunikationsverhalten zeichnet sich durch das Preisgeben von Erwartungen und Meinungen sowie das Ansprechen von Unklarheiten aus. Traditionelle Unterrichtsformen hingegen schränken die SchülerInnen in ihrem Kommunikationsverhalten ein. Es konnte beobachtet werden, dass Erwartungen und Positionen von SchülerInnen nur selten vor der gesamten Klasse und gegenüber der Lehrperson angesprochen wurden.

Verbunden mit dem Wissen über verbale Kommunikation ist Wissen über das Zuhören. Lehrpersonen machen deutlich, dass für die verbale Kommunikation das Zuhören wichtig ist. Lehrpersonen fühlen sich verantwortlich, dass sich alle Beteiligten in der Klasse gegenseitig zuhören. Hat die Lehrperson das Gefühl, die anderen AkteurInnen hören nicht zu, wird die Kommunikation unterbrochen und erst dann fortgesetzt, wenn alle beteiligten Personen der Kommunikation folgen.

Weitere Wissensformate werden bei der verbalen Kommunikation über die Einnahme der gleichen Kommunikationsebene ersichtlich. Lehrpersonen stellen sich auf eine Kommunikationsebene mit den SchülerInnen, um wertschätzend und respektvoll zu kommunizieren. Beispiele sind das Reden auf gleicher Augenhöhe, der Einsatz von leicht verständlichen Wörtern und altersgerechter Sprache.

Um die Wirkung verbaler Kommunikation zu verstärken, suchen die Beteiligten Blickkontakt. Dieser führt bei den Kommunikationspartnern dazu, dass sie sich angesprochen fühlen und der Kommunikation aufmerksam folgen.

Die verbale sowie die nonverbale Kommunikation können als wichtige Interaktionen beschrieben werden, die das Lernen und den Umgang miteinander positiv unterstützen. Es zeigt sich jedoch, wie SchülerInnen vor allem im Bereich nonverbaler Kommunikation sensibilisiert werden müssen, um Verhaltensweisen und Handlungen einer Lehrperson zu verstehen.

\subsubsection{Classroom-Management}

Pädagogisches Wissen konnte im Zuge der Unterrichtsbeobachtungen im Umgang mit dem Classroom-Management rekonstruiert werden. Beobachtungen belegen, dass diese Wissenskategorie über eine vorwiegend lehrseitige Orientierung charakterisiert ist. Das ClassroomManagement kann in die Kategorien Ablauf, Leadership, Agieren und in der Organisation von Störungen (vgl. Abbildung 86) gegliedert werden. 


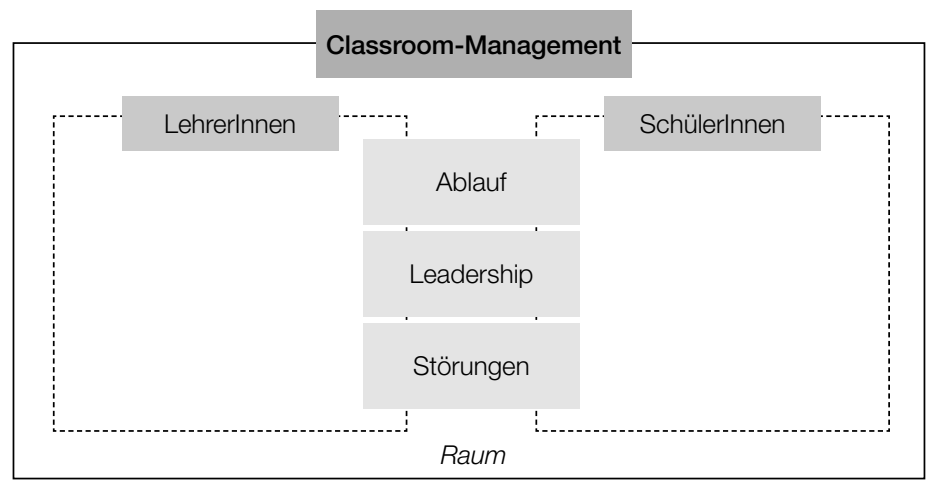

Abb. 87: Kategorien des Classroom-Managements

Das pädagogische Wissen zum Classroom-Management untergliedert sich über die in der Grafik angeführten Kategorien. Als Erstes werden allgemeine Wissensbereiche zum Ablauf und zu Leadership beschrieben. Anschließend erfolgt die Darstellung eines spezifischen Wissens im Zusammenhang mit Störungen.

\subsubsection{Ablauf}

Das Wissen über den Ablauf korreliert mit dem Wissen zu Planung, Zeitmanagement, Raumnutzung, Struktur sowie zum Umgang mit Ritualen (vgl. Abbildung 87).

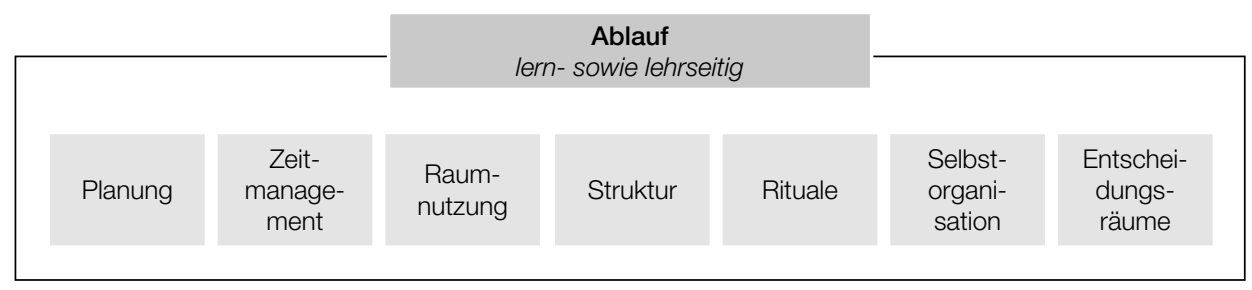

Abb. 87: Subkategorien Ablauf

Pädagogisches Wissen kommt über einen systematischen und kohärenten Ablauf zum Ausdruck. Aus den Beobachtungen geht hervor, dass Lehrpersonen ihren Unterricht im Hinblick auf Inhalte und Zeitmanagement vorab planen. Sie zeigen, wie sie bewusst ausgewählte Inhalte unterrichten sowie spezifische Methoden anwenden. Es wurde im Zuge der Rekonstruktion beobachtet, wie Lehrpersonen aller Schulformen bewusst Zeitfenster für Anliegen und Fragen der SchülerInnen einplanen. Zum Beispiel gibt es vor Prüfungen und wichtigen Abgaben Unterrichtsstunden, in denen Unklarheiten vorab besprochen werden. Die bewusste Bereitstellung von Zeitfenstern für derartige Zwecke verhindert laut den Beobachtungen, dass SchülerInnen über permanentes Fragen den Unterrichtsverlauf stören.

Eine offene und partizipatorische Struktur im Umgang mit Inhalten und Zeitressourcen wird in der Grundschule sowie teilweise in der Neuen Mittelschule sichtbar. SchülerInnen haben dort ein Mitsprachrecht bei der Auswahl der Inhalte, Methoden und beim Zeitmanagement. Offene Unterrichtsmethoden werden angewendet. 
Im Rahmen der Beobachtungen konnten unterschiedliche strukturelle Muster des Unterrichtsverlaufs aufgezeigt werden (vgl. Abbildung 88).

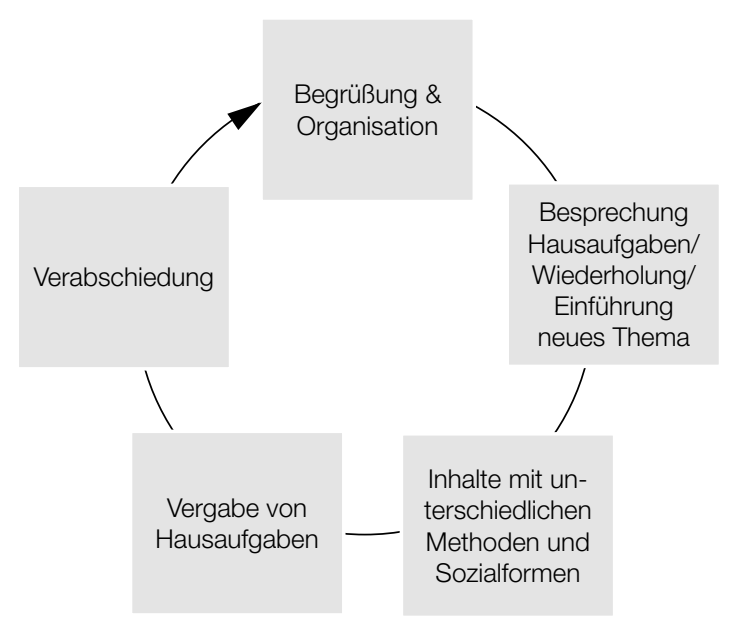

Abb. 88: Strukturelle Muster eines Unterrichtsverlaufs

Struktur I wurde vorwiegend im Gymnasium sowie teilweise in der Neuen Mittelschule beobachtet. Ein Mix aus Struktur I und Struktur II zeigte sich in der Neuen Mittelschule. Die zweite Struktur war in der Grundschule beobachtbar. Korrelierten die Unterrichtsstrukturen stark mit den formalen Bedingungen der jeweiligen Schulform, illustrieren die Beobachtungen, wie vor allem Gymnasiallehrpersonen über das Anwenden offener Unterrichtsformate vorgegebene Strukturen aufbrechen.

Beobachtungen veranschaulichen, wie ein ritualisiertes Vorgehen den Unterricht auf allen Schulebenen charakterisiert. Rekonstruierte Rituale von einzelnen Schulformen werden in Tabelle 6 zusammengefasst. 
Tab. 6: Rituale nach Schulformen

\begin{tabular}{lll}
\hline \multicolumn{1}{c}{ Grundschule } & \multicolumn{1}{c}{ Neue Mittelschule } & \multicolumn{1}{c}{ Gymnasium } \\
\hline $\begin{array}{l}\text { Freiarbeit: in der Reihe warten, } \\
\text { um den Lehrpersonen Aufgaben } \\
\text { zu zeigen bzw. Fragen zu stellen }\end{array}$ & $\begin{array}{l}\text { Beim Verlassen des Klassenzim- } \\
\text { mers Name auf Tafel schreiben }\end{array}$ & $\begin{array}{l}\text { SchülerInnen stehen auf, sobald } \\
\text { Lehrperson in die Klasse kommt }\end{array}$ \\
\hline $\begin{array}{l}\text { Aufzeigen bei Fragen und münd- } \\
\text { lichen Beiträgen }\end{array}$ & $\begin{array}{l}\text { Aufzeigen bei Fragen und münd- } \\
\text { lichen Beiträgen }\end{array}$ & $\begin{array}{l}\text { Aufzeigen bei Fragen und } \\
\text { mündlichen Beiträgen }\end{array}$ \\
\hline $\begin{array}{l}\text { Musik: SchülerInnen räumen } \\
\text { ihre Unterlagen weg und setzen } \\
\text { sich an ihren Platz }\end{array}$ & $\begin{array}{l}\text { Musik: SchülerInnen räumen ihre } \\
\text { Unterlagen weg und setzen sich an } \\
\text { ihren Platz, SchülerInnen müssen }\end{array}$ & $\begin{array}{l}\text { Läuten in die Klasse. Begrüßung } \\
\text { findet mündlich statt. }\end{array}$ \\
& $\begin{array}{l}\text { von der Lehrperson vorgegebene } \\
\text { Reflexionsfragen beantworten }\end{array}$ \\
\hline $\begin{array}{l}\text { Lehrperson schon vor Stunden- } \\
\text { beginn in der Klasse, jedes Kind } \\
\text { wird mit Handschlag begrüßt }\end{array}$ & $\begin{array}{l}\text { SchülerInnen stehen auf, sobald } \\
\text { Lehrperson in die Klasse kommt }\end{array}$ \\
\hline $\begin{array}{l}\text { Begrüßung: gemeinsames Vorle- } \\
\text { sen von Datum, Tag, Wetter }\end{array}$ & $\begin{array}{l}\text { Begrüßung am Morgen in einem } \\
\text { Sesselkreis, organisatorische }\end{array}$ \\
& $\begin{array}{l}\text { Inhalte werden besprochen und } \\
\text { individuelle Arbeiten können }\end{array}$ \\
& vorgestellt werden \\
\hline Lehrperson liest aus dem Buch & Wenn SchülerInnen Unterstüt- & \\
vor, während SchülerInnen essen & zung benötigen, schreiben sie & \\
& ihren Namen auf die Tafel & \\
\hline Entspannungsphasen während & & \\
des Unterrichts & & \\
\hline
\end{tabular}

Spezifisch angewendete Rituale konnten in der Grundschule und der Neuen Mittelschule aufgezeigt werden. Es wurde sichtbar, wie Lehrpersonen täglich dieselben Handlungen ausführen, die den Unterrichtsverlauf beeinflussen. Ein beobachbares Ritual der Grundschule und der Neuen Mittelschule ist das Arbeiten mit Musik. Wie die in Tabelle 6 aufgelisteten Rituale demonstrieren, ist das Arbeiten mit Musik für die Grundschule und die Neue Mittelschule typisch. Im Gymnasium ist die Art und Weise der Begrüßung ritualisiert. Die Analyse zeigt anhand zielgerichteter Implementierung und Durchführung von Ritualen im Unterrichtsalltag pädagogisches Wissen. Eine weitere Subkategorie des Classroom-Managements ist das Wissen um die Raumnutzung. Im Zuge der Feldforschung konnten zwei unterschiedliche Settings beobachtet werden, die mögliche Raumnutzungen widerspiegeln (vgl. Abbildung 89):
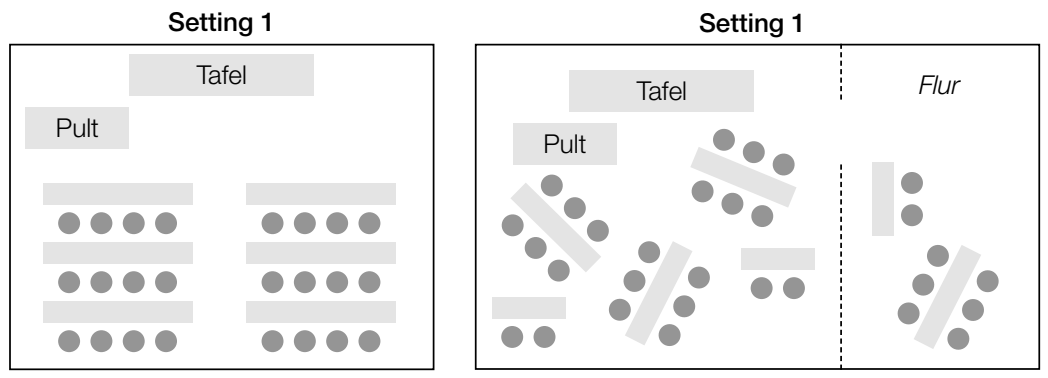

Abb. 89: Raumnutzung 
Setting 1 charakterisiert sich über linear angeordnete Sitzplätze. Dieses Bild zeigte sich vorwiegend im Gymnasium. Setting 2 weist offene, dynamische und flexible Strukturen auf und wurde in der Grundschule und der Neuen Mittelschule sichtbar. Die Unterschiede zwischen den beiden Settings betreffen die Tischanordnung im Klassenzimmer sowie das Miteinbeziehen von weiteren Räumlichkeiten (Setting 1), wie etwa des Flurs. Beobachtungen legen nahe, dass das Setting die Bewegungen im Klassenzimmer stark beeinflusst (vgl. Abbildung 90):

Setting 1

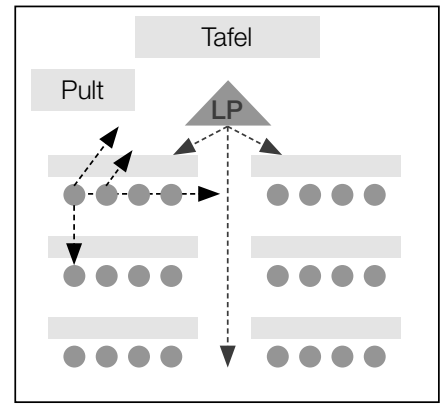

Abb. 90: Bewegungen der AkteurInnen im Raum
Setting 1

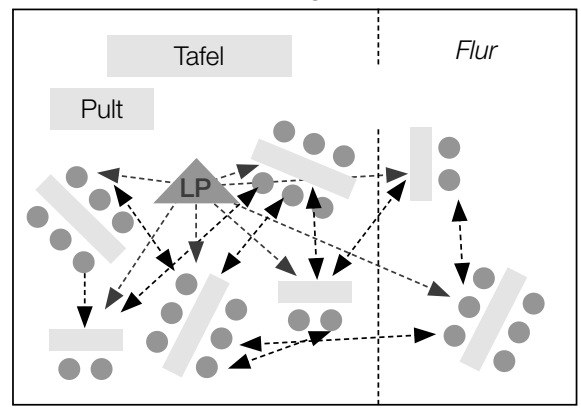

Wie in Abbildung 90 veranschaulicht, bewegt sich die Lehrperson in Setting 1 vorrangig im Bereich vor der Tafel. Einzelne Wege betreffen den Mittelgang sowie den Weg zwischen den Tischen. Die SchülerInnen sitzen linear hintereinander in Tischreihen. Setting 2 lässt aufgrund seiner dynamischen Anordnung verschiedene Bewegungsmuster zu. Die Lehrpersonen und SchülerInnen in Setting 2 haben neben schon angesprochenen Bewegungsmustern die Möglichkeit, sich freier im Raum zu positionieren. Das Wissen über die Wirkung der Bewegungen im Raum hat sich als Teil pädagogischen Wissens gezeigt. Die unterschiedlichen Bewegungsmuster der Lehrpersonen wirken sich auf das Verhalten der SchülerInnen im Unterricht aus. Dieses ist in Setting 1 auf starre Strukturen beschränkt. Der Wirkungsbereich der einzelnen SchülerInnen beschränkt sich bei dieser Raumstruktur auf ihre unmittelbaren SitznachbarInnen und die Tischreihen vor und hinter ihnen. Aufgrund der Anordnung der Tische ist der Blick der SchülerInnen permanent zur Lehrperson gerichtet.

Wie die Abbildung des zweiten Settings illustriert, können dynamische Prozesse stattfinden. Diese Raumstruktur bietet den SchülerInnen die Möglichkeit, auf unterschiedliche Weisen mit verschiedenen SchülerInnen in Kontakt zu treten. Zusätzlich haben sie die Möglichkeit, eine zweite Räumlichkeit (hier den Flur) mitzubenutzen. Ist der Fokus im Setting 1 auf die Nutzung der Tafel als zentrales Medium konzentriert, welches die verbale Kommunikation der Lehrperson unterstützt, konnte im Zuge der Rekonstruktion beobachtet werden, wie Lehrpersonen im Raumsetting 2 die Tafel weniger verwendete. Die direkte Interaktion zwischen SchülerInnen und Lehrperson steht bei diesem Format im Vordergrund.

Wie Beobachtungen verdeutlichen, fördert die offene Raumstruktur dynamische Prozesse. Pädagogisches Wissen kann über die Anwendung und die Implementierung unterschiedlicher Raumstrukturen aufgezeigt werden. Für eine optimale Raumnutzung ist ein wichtiger Wissensbereich das professionelle Führen. Die Kategorie Leadership wird im folgenden Abschnitt mit ihren Subkategorien dargestellt. 


\subsubsection{Leadership}

Eine weitere Wissensform ist das Wissen zu Leadership. Es wurden das Wissen über Prozesse rekonstruiert (vgl. Abbildung 91).

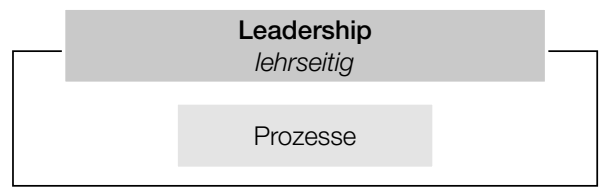

Abb. 91: Subkategorien Führung

Im Zuge der Rekonstruktion konnte in allen Schulformen unterschiedliches Wissen zu Prozessen im Zusammenhang mit Leadership sichtbar gemacht werden, die im Folgenden dargestellt werden:

(1) Kommunikationsprozesse: Kommunikationsprozesse beinhalten das Anleiten von Diskussionsrunden unter SchülerInnen, das Anleiten von mündlichen Beiträgen sowie das Anleiten von selbstständigen Diskursen und Präsentationen der SchülerInnen.

(2) Unterrichtsprozesse: Das Führen von Unterrichtsprozessen bezieht sich auf das Anleiten von Arbeitsschritten, Arbeitsphasen, Pausen, Ritualen und das Anwenden und Erklären verschiedener Methoden. Im Zusammenhang mit den Unterrichtsprozessen hat sich eine Dreiteilung der Arbeitsphasen in Inhalt, Erholung und Bewegung ergeben. Es hat sich gezeigt, dass nicht nur der Inhalt, sondern die Erholung mittels Auflockerungsübungen und Bewegung den Unterricht lebendiger machen und dadurch den Unterrichtsprozess positiv beeinflussen.

(3) Kognitive Prozesse: Das Führen von kognitiven Prozessen wird über das Anleiten von Denkprozessen bei SchülerInnen sichtbar, indem sie zum Nachdenken angeregt sowie zum Arbeiten angeleitet werden. Dabei ist auf die Integration unterschiedlicher Aufgabenformate - und die damit korrelierende Förderung unterschiedlicher Kompetenzen - achtzugeben.

(4) Unterstützungsprozesse: Die Lehrpersonen demonstrieren, wie sie Unterstützungsarrangements zielgerichtet führen und dadurch Schwierigkeiten bei SchülerInnen überwinden.

(5) Organisationsprozesse: Diese betreffen Administration, Schulausflüge, Veranstaltungen, Exkursionen, die Überprüfung der Anwesenheit und außerschulische Aktivitäten.

(6) Gruppeneinteilungen: Das Führen zeigt sich bei der Einteilung von Gruppenarbeiten zwischen SchülerInnen.

(7) Individuelle Ökonomieprozesse: SchülerInnen passen sich den von der Lehrperson vorgegebenen Strukturen an und ordnen sich diesen unter.

(8) Regeln: Regeln werden von der Lehrperson im Unterricht vorgelebt. Beispiele betreffen zum Beispiel die Pünktlichkeit, das Mitbringen von Arbeitsunterlagen, das Begrüßen und Verabschieden und/oder das Benützen von Handys.

Die angeführten Führungsbereiche setzen für die adäquate Umsetzung spezifisches professionelles Wissen im Bereich Leadership voraus. Dieses bezieht sich, wie in der Auflistung angeführt, auf verschiedene strukturelle sowie kognitive Wissensbereiche. Lehrpersonen benötigen hierfür einen umfassenden Überblick über Aufgaben und Prozesse und müssen angemessen agieren.

\subsubsection{Agieren}

Eine rekonstruierte Wissensform, bezogen auf das Classroom-Management, ist das lehrseitige Agieren. Diese umfasst das Wissen über das Abwägen von Situationen sowie das Wissen über Handlungsakte (vgl. Abbildung 92). 


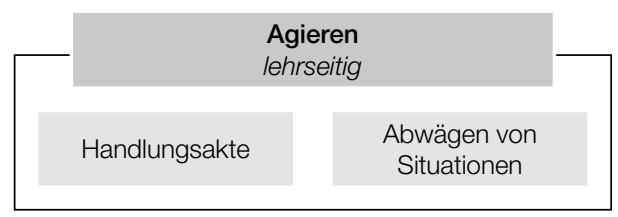

Abb. 92: Subkategorien Agieren

Pädagogisches Wissen wird über das spontane, angemessene Reagieren auf unterschiedlichste Situationen im Unterricht sichtbar. Drei rekonstruierte Handlungsbeispiele, die das situationsgerechte Handeln von Lehrpersonen aufzeigen, werden im Folgenden erläutert:

Handlungsbeispiel 1: Maturastreik in der Schule: SchülerInnen tanzen zu der lauten Musik, die vom Schulhof ins Klassenzimmer tönt, auf ihren Stühlen. Sie sind sehr unruhig und unkonzentriert. Der Lehrer ermahnt die SchülerInnen und bittet um Ruhe. Er hält an seinem Unterrichtsplan fest. Als er merkt, dass die SchülerInnen nicht mehr zuhören und ihm keine Antworten auf seine Fragen geben, klappt er das Schulbuch zu. Anstatt den Unterrichtsstoff durchzudrücken, beendet er den Unterricht und spielt fachspezifische Spiele mit den SchülerInnen. Sie hören auf, auf den Stühlen zu tanzen und spielen mit.

2 Handlungsbeispiel 2: Die Lehrperson präsentiert vor der Klasse. Eine Schülerin fängt plötzlich an zu weinen. Die Lehrperson nimmt die Schülerin wahr, unterbricht den Vortrag und gibt der Klasse einen Arbeitsauftrag für eine Gruppenarbeit. Er geht mit der Schülerin aus dem Klassenzimmer, während die anderen SchülerInnen arbeiten.

3

Handlungsbeispiel 3: Ein Schüler hat die Aufgabe, zum Einstieg in die Unterrichtsstunde eine Präsentation zu halten. Der Schüler kommt an diesem Tag nicht zum Unterricht. Die Lehrperson reagiert spontan und übernimmt den Einstieg ins neue Thema.

Die Beispiele skizzieren unterschiedliche Situationen, in denen die Lehrpersonen eingreifen und in kurzer Zeit Handlungsentscheidungen treffen und umsetzen müssen. Beobachtungen illustrieren pädagogisches Wissen, indem sie dies professionell umsetzen. Spezifisches Handeln und Agieren wird im Schulalltag besonders bei Störungen gefordert. Das Wissen zu Störungen wird im nächsten Abschnitt beschrieben.

\subsubsection{Störungen}

Die letzte rekonstruierte Kategorie des Classroom-Managements ist das Wissen zum Umgang mit Störungen im Unterricht. Das Wissen äußert sich über Handlungsmöglichkeiten sowie präventiv gesetzte Maßnahmen von Lehrpersonen (vgl. Abbildung 93).

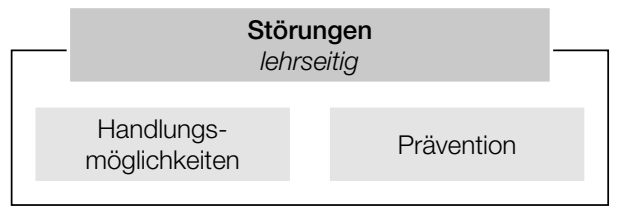

Abb. 93: Subkategorien Störungen 
Pädagogisches Wissen wurde über den Umgang mit Störungen und spezifisch eingesetzten präventiven Maßnahmen aufgezeigt. Rekonstruierte Störungen des Unterrichtsverlaufs, die über das Verhalten von SchülerInnen entstehen, sind vor allem Gespräche zwischen SchülerInnen, das Spielen mit dem Handy, das Essen und das verspäte Eintreten in den Unterricht. Rekonstruierte lehrseitige Unterbrechungen sind Störungen von externen Lehrpersonen, die unangekündigt in die Klasse kommen. Lehrpersonen können direkt mit Störungen umgehen und haben Handlungsmöglichkeiten parat. Störungen werden vorwiegend mittels direkter Kommunikation angegangen. Sie wird hier als ein Instrument im Umgang mit Störungen eingesetzt. ${ }^{48}$

Rekonstruierte präventive Maßnahmen, die Störungen im Unterricht verringern, sind das Auseinandersetzen von SchülerInnen oder das Anbieten eines interessennahen Unterrichts. Lehrpersonen demonstrieren, wie sie bewusst pädagogisches Wissen einsetzen, um Störungen präventiv zu vermeiden.

Da Störungen, wie Beobachtungen skizzieren, einen zentralen Bestandteil des Schulalltags darstellen, ist pädagogisches Wissen im Umgang mit diesen wichtig, um den Unterrichtsablauf so wenig wie möglich zu unterbrechen. Lehrpersonen können durch bewusstes Eingreifen und Handeln vorab präventive Maßnahmen setzen.

\subsubsection{Personen}

Als eine weitere Hauptkategorie pädagogischen Wissens wurde das Wissen zur Person rekonstruiert. Dieses gliedert sich in die Subkategorien Rolle, Bedeutung und Funktion (vgl. Abbildung 94).

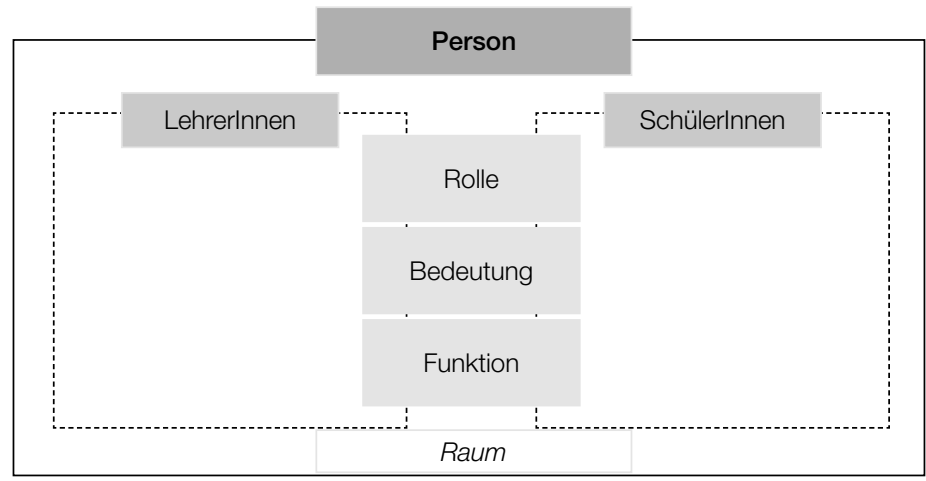

Abb. 94: Kategorien der Personen

Das pädagogische Wissen zur Person zeigt sich über die in der Grafik angeführten Kategorien. Als Erstes wird auf die allgemeine Rolle von SchülerInnen und Lehrpersonen eingegangen. Anschließend werden die Wissensbereiche zur Bedeutung und Funktion der jeweiligen Personen behandelt. Die Analyse verdeutlicht Wechselbeziehungen zwischen den einzelnen AkteurInnen (vgl. Abbildung 95).

48 Beispiele hierzu können in Abschnitt 8.5.3 bei der Wissenskategorie Kommunikation nachgelesen werden. 


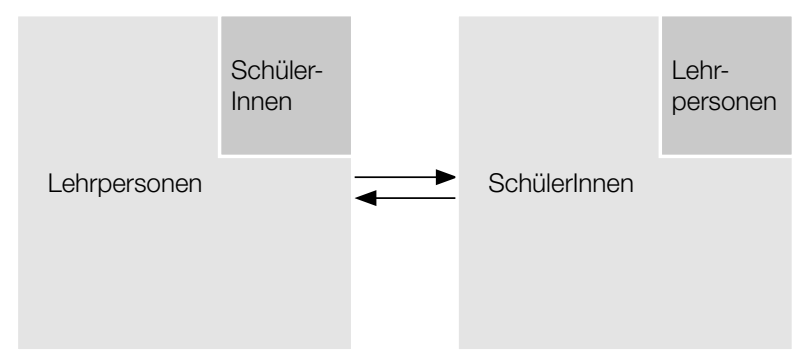

Abb. 95: Realisierung von Wechselwirkungen im Unterricht

Wie die Abbildung veranschaulicht, zeichnen sich Wechselwirkungen zwischen Lehrpersonen und SchülerInnen über ein Abhängigkeitsverhältnis aus. Beobachtungen machen deutlich, wie die AkteurInnen ihre eigene Person und ihr Verhalten immer an das Gegenüber anpassen. SchülerInnen und Lehrpersonen beeinflussen damit das gegenseitige Handeln sowie die Rolle der einzelnen Personen. Im Folgenden werden die Kategorien zum Wissen zur Person mit ihren jeweiligen Subkategorien zur Diskussion gestellt.

\subsubsection{Rolle}

Die Ergebnisse veranschaulichen, wie sich pädagogisches Wissen über die Rolle der einzelnen AkteurInnen zum Ausdruck kommt. Diese bezieht sich laut Beobachtungen lehr- sowie lernseitig auf die Subkategorien Auftreten, Einstellungen sowie Räume jeder individuellen Person (vgl. Abbildung 96).

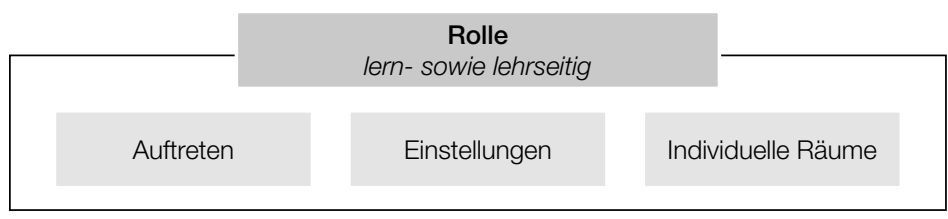

Abb. 96: Subkategorien Rolle

Über Beobachtungen konnte das Auftreten einzelner Personen im Klassenzimmer rekonstruiert werden. Für ein angemessenes Auftreten ist es nötig, dass sich Lehrpersonen und SchülerInnen gegenseitig kennen. Rekonstruierte Beispiele betreffen in diesem Zusammenhang den Umgang mit Sarkasmus, Witzen, Ironie oder den Umgang mit Äußerungen. SchülerInnen reagieren unterschiedlich auf Kommentare und Witze reagieren. Dies verdeutlicht die essenzielle Bedeutung des Wissens über einen bewussten Umgang mit Aussagen und Scherze.

Beobachtungen illustrieren, wie das Auftreten einzelner Personen die Zufriedenheit und/oder die Unzufriedenheit widerspiegelt. Es zeigt sich, wie SchülerInnen und Lehrpersonen fröhlich und zufrieden wirkten, Ruhe und Gelassenheit ausstrahlten, während andere sich wiederum hektisch und laut verhielten. Es konnten Zusammenhänge zwischen dem Auftreten einzelner Personen und dem Verhalten anderer rekonstruiert werden. Diese werden in Abbildung 97 dargestellt. 


Ruhiges Verhalten der Akteure
$\begin{aligned} & \text { Lehruhiges Verhalten der Akteure } \\ & \text { in der Klasse auf }\end{aligned}$

Abb. 97: Wirkungsmuster Auftreten der AkteurInnen

Die Abbildung veranschaulicht die Auswirkungen von ruhigem bzw. unruhigem Verhalten der AkteurInnen in einer Klasse. Lehrpersonen sorgen über bewusst positiv formulierte Sätze für eine angenehme Situation. Positives sprachliches Auftreten beinhaltet etwa Aussagen wie „Ich freue mich, nach dem Wochenende wieder hier zu sein“ und „Ich freue mich auf die Projektarbeit mit euch“. Wenn ein Schüler/eine Schülerin krank war, kommentiert die Lehrperson die Anwesenheit im Unterricht mit „Schön, dass du wieder da bist“. Pädagogisches Wissen zeigt sich über ein bewusstes Auftreten, um Wirkungsbereiche hervorzurufen bzw. verhindern zu können. Es wird die wichtige Bedeutung eines authentischen Auftretens einer Lehrperson hervorgehoben. Folgende Merkmale eines authentischen Auftretens konnten während den Beobachtungen rekonstruiert werden: Umgang mit eigenen Schwächen, Fehler eingestehen, eigene Meinungen vertreten und hinterfragen, Neugierde hinsichtlich Lernen und Lehren, Flexibilität, Motivation vorleben sowie Benennen von Schwierigkeiten.

Eine weitere Subkategorie der Rolle ist das Wissen zu Einstellungen. Wie Abbildung 98 skizziert, stehen die beteiligten Personen in einem Wechselverhältnis, in dem sich Einstellungen und Positionen gegenseitig beeinflussen.

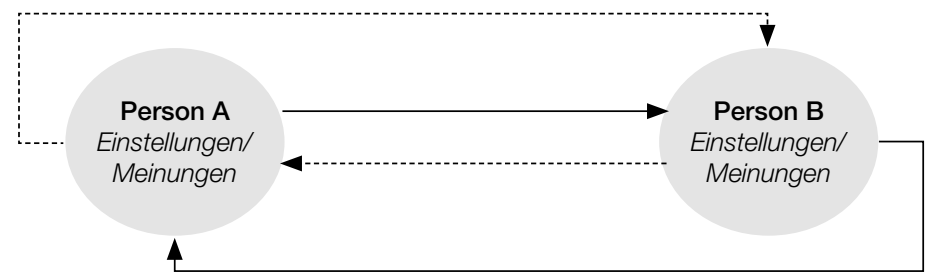

Abb. 98: Wechselverhältnis und Wirkung von Einstellungen und Positionen

Folgende Strukturen konnten während der Beobachtungen sichtbar gemacht werden: Person A benennt eine persönliche Einstellung gegenüber einer anderen Person. Diese reagiert darauf mit Akzeptanz oder Ablehnung, verbindet die geäußerten Positionen mit ihren eigenen und teilt der Person A ihre persönliche Einstellung mit. Der Prozess von Akzeptanz bzw. Ablehnung findet anschließend bei Person A statt. Über das Kennenlernen weiterer Positionen und Einstellungen fängt sie an, eigene Einstellungen zu hinterfragen. Daraufhin reagiert die Person und überträgt eine neue Position an Person B. Pädagogisches Wissen wird im dargestellten Wechselverhältnis insofern sichtbar, als dass die AkteurInnen eigene Einstellungen bewusst und angemessen äußern sowie Einstellungen anderer akzeptieren. 
Pädagogisches Wissen kann durch das Schaffen individueller Räume der AkteurInnen beobachtet werden. Die Felduntersuchungen haben gezeigt, wie SchülerInnen und Lehrpersonen gleichermaßen private Räume konstruieren, eigene Grenzen vorgeben und eine individuelle Haltung bezüglich des Austauschs persönlicher Informationen vertreten. Pädagogisches Wissen konnte vor allem darüber rekonstruiert werden, wie die AkteurInnen persönliche Räume erkennen und akzeptieren. Lehrpersonen und SchülerInnen sprechen direkt an, wenn sie über ein bestimmtes Thema nicht kommunizieren möchten.

Wie über die Rekonstruktion sichtbar gemacht werden kann, beeinflussen AkteurInnen das Lehren und Lernen über ihre Rolle. Die AkteurInnen stehen im Unterricht in einer engen Beziehung zueinander und beeinflussen mit ihrem Auftreten, ihren Einstellungen und dem Schaffen individueller Räume den Unterricht sowie das Verhalten der Personen. Mit dem Wissen über die Rolle ist das Wissen über die Bedeutung der eigenen Person verbunden.

\subsubsection{Bedeutung}

Eine weitere rekonstruierte Kategorie im Zusammenhang mit dem Wissen zur Person ist die Bedeutung. Dabei steht das eigene Sein im Zentrum des Interesses (vgl. Abbildung 99).

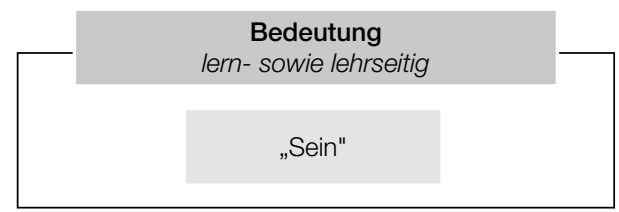

Abb. 99: Subkategorien Bedeutung

Lehrpersonen und SchülerInnen nahmen während der Beobachtungen individuelle Persönlichkeiten in der Klasse ein. Unterschiedlich rekonstruierte Eigenschaften beziehen sich auf Merkmale wie zurückhaltend und ruhig, aufgeweckt, offen, autoritär. Auffallend war, dass AkteurInnen, die offen sind, stärker mit anderen in Kontakt treten als zurückhaltende Persönlichkeiten. Im Zusammenhang mit den SchülerInnen wird aufgezeigt, wie Lehrpersonen diese des Öfteren in Rollen stecken, die nicht der Realität entsprechen. Rekonstruierte Rollenzuschreibungen beziehen sich auf Bilder wie Klassenclown, Streber, Mitläufer, Politiker oder Außenseiter. Beobachtungen illustrieren, wie SchülerInnen den Zuschreibungen ausgesetzt sind. Pädagogisches Wissen wird darüber aufgezeigt, dass Lehrpersonen in der Lage sind, Zuschreibungen zu hinterfragen, bzw. diese im Unterricht vermeiden.

Die Beobachtungen belegen, wie Personen Wünsche und Bedürfnisse äußern, um ihr individuelles Sein zu verwirklichen. Pädagogisches Wissen kann in diesem Zusammenhang darüber rekonstruiert werden, dass Lehrpersonen bewusst Räume für die Äußerungen von Wünschen und Bedürfnissen schafften. Jede Person im Klassenzimmer kann so sein, wie sie ist und ihre Persönlichkeit zeigen. Persönlichkeiten beeinflussen den Unterricht sowie die Beziehungen zwischen den einzelnen Personen. Das Sein kommt über die Funktion einer Person zum Ausdruck. Das Wissen zur Funktion wird im Folgenden Abschnitt beschrieben.

\subsubsection{Funktion}

Die letzte rekonstruierte Kategorie pädagogischen Wissens bezieht sich auf die Funktion der einzelnen AkteurInnen. Diese definiert sich über das Interesse am Beruf (vgl. Abbildung 100). 


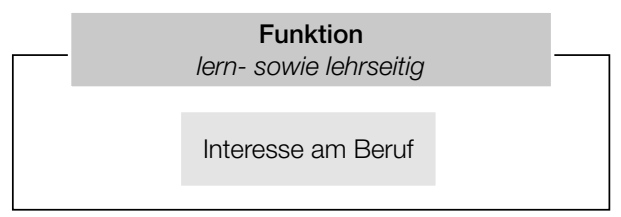

Abb. 100: Subkategorien Funktion

Wie die Ergebnisdarstellung aufzeigt, korreliert das Interesse am Beruf mit dem Auftreten der Personen. Dieses definiert sich über die Entfaltung der eigenen Persönlichkeit, ein authentisches Auftreten sowie Engagement. Lehrpersonen äußern Interesse, indem sie neue didaktische Konzepte implementieren, den Unterricht abwechslungsreich mit dem Einsatz von unterschiedlichen Methoden und Medien gestalten sowie Ausflüge organisieren. SchülerInnenseitig konnte Interessen gegenüber der Schule über ihre aktive Mitarbeit, den Wunsch nach Veranstaltungen oder das Interesse am Fach rekonstruiert werden. Pädagogisches Wissen verkörpert sich darin, wie die beobachteten Personen ihr Interesse am Beruf zeigen und dieses bewusst für erfolgreiches Lernen und Lehren anwenden. Es konnte rekonstruiert werden, wie SchülerInnen ein erhöhtes Interesse am Unterricht illustrieren, wenn die Lehrperson authentisch auftritt und engagiert ist.

Es zeigt sich, wie das Interesse am Beruf über das authentische Auftreten und Engagement einen abwechslungsreichen und motivierenden Unterricht beeinflusst. Das Interesse am Beruf hilft nicht nur, neue didaktische Konzepte anzuwenden, sondern sich fachlich wie persönlich weiterzuentwickeln.

Die Kategorie Person präsentiert, wie das Wissen zur Rolle, Bedeutung und Funktion das professionelle Auftreten einer Lehrperson unterstützt und das Verhalten von SchülerInnen sowie Lernprozesse maßgeblich beeinflusst. 


\section{Fallstudien}

Das vorliegende Kapitel präsentiert individuelle Muster pädagogischen Wissens und Handelns von drei Lehrpersonen aus den Schulformen Grundschule, Neue Mittelschule und Gymnasium. Hierbei werden empirische Ergebnisse systematisch zusammengeführt. Die Fallstudien werden mit einer kurzen Personenbeschreibung eingeleitet. Anschließend folgt die personenbezogene Darstellung kognitiver Muster und Handlungsformen. Zudem wird präsentiert, inwieweit kognitive Konzepte von Lehrpersonen in ihrem unterrichtlichen Handeln beobachtet werden konnten.

\subsection{Fallstudie 1 - Lehrperson Grundschule}

Die erste Fallstudie bezieht sich auf eine männliche Lehrperson, die in der Grundschule unterrichtet. Max ist 29 Jahre alt und seit fünf Jahren im Schuldienst tätig. Er unterrichtet alle gängigen Fächer in einer Grundschule. Seine Ausbildung absolvierte er an einer Pädagogischen Hochschule. Max besucht regelmäßig Fortbildungen und hat eine Montessori-Ausbildung absolviert.

Max beschreibt pädagogisches Wissen als mehrdimensionales Wissen und charakterisiert es, wie mittels Abbildung 101 dargestellt, über fünf verschiedene Bereiche: didaktisches Wissen, Handlungswissen, Wissen über die Person, Wissen über Lernen sowie Wissen über Diversität.

Berufsleben: Praxiserfahrung, Fort- und Weiterbildungen, Fachliteratur

\section{Aneignung}

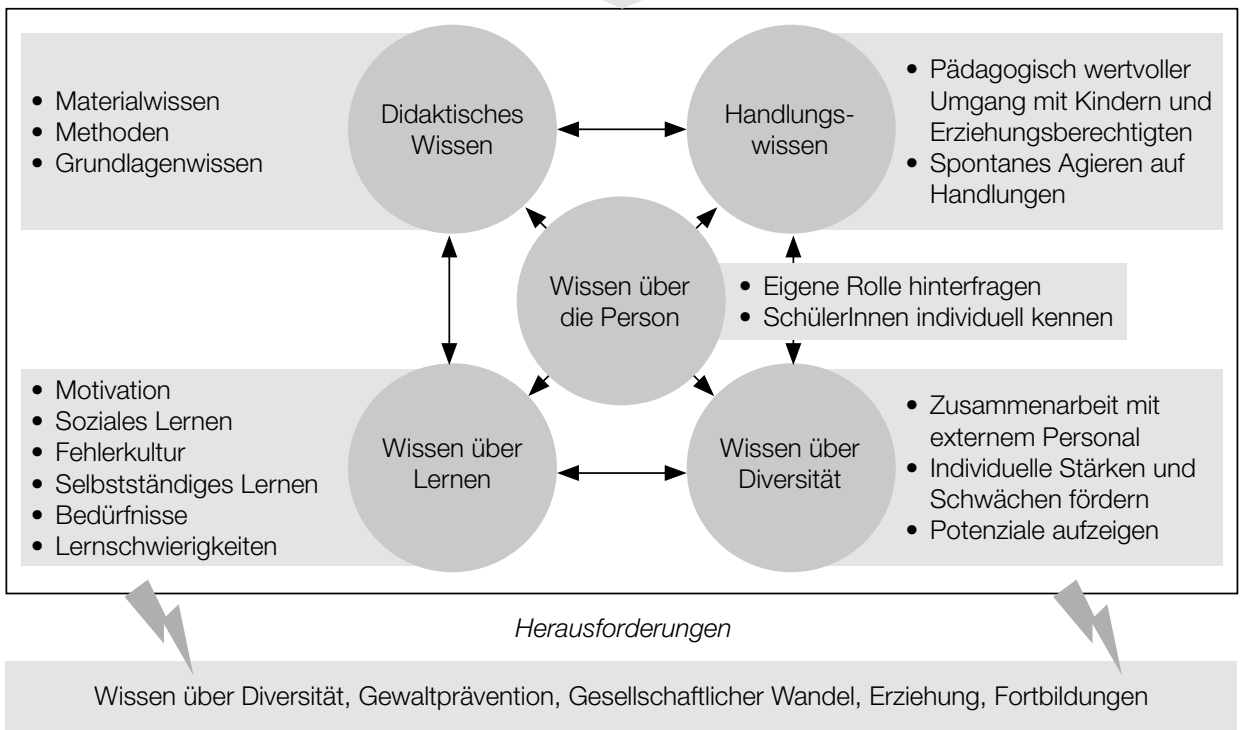

Abb. 101: Individuelles Verständnis pädagogischen Wissens - Lehrperson Grundschule 
Didaktisches Wissen beschreibt er über Materialwissen, Wissen zu Methoden sowie ein didaktisches Grundlagenwissen. Das Handlungswissen bezieht sich auf das spontane Agieren im Unterricht sowie einen wertvollen pädagogischen Umgang mit SchülerInnen und Erziehungsberechtigten. Das Wissen über die eigene Person charakterisiert Max anhand der Selbstreflexion und beschreibt bezogen auf SchülerInnen die Notwendigkeit, Interesse an Kindern zu zeigen. Weitere Kategorien sind das Wissen zum Lernen sowie das Wissen zur Diversität. Genannte Merkmale, die der Kategorie Lernen zugeordnet werden, sind Wissen zur Motivation, das soziales Lernen, der Umgang mit Fehlern, die Bedürfnisse der SchülerInnen kennen, der Umgang mit Lernschwierigkeiten sowie die Förderung des selbstständigen Lernens. Das Wissen zur Diversität charakterisiert er über die Zusammenarbeit mit externem Personal, die Förderung von Stärken und Schwächen einzelner SchülerInnen sowie das Wissen zu Potenzialen. Er präsentiert ein mehrdimensionales Verständnis pädagogischen Wissens, welches sich vorwiegend auf didaktische Methoden und Handlungsweisen bezieht, die das Lernen sowie die individuelle Förderung unterstützen.

Max beschreibt spezifische Aneignungsorte sowie Herausforderungen für pädagogisches Wissen. Als wichtigen Aneignungsorte nennt er das Berufsleben, Praxiserfahrungen, Fort- und Weiterbildungen sowie die Auseinandersetzung mit Fachliteratur. Darüber hinaus verweist er auf die Berücksichtigung des Lehrplans:

„So wie ich jetzt im Klassenverband unterrichten soll habe ich in der Praxis erworben. Die didaktischen Sachen, wie ich jetzt wann was mache, das habe ich dann über die Ausbildung gelernt, über Fort- und Weiterbildungen oder über den Lehrplan. Der ist einfach vorgegeben und sagt mir was die Kinder am Ende können müssen. Und mit den ganzen Kompetenzen und Bildungsstandards, da haben wir in der vierten Klasse müssen alle Kinder gewisse Standards erreichen - und da haben wir schon vorgaben, die man erreichen soll." (It 2)

Die von ihm beschriebenen Herausforderungen für pädagogisches Wissen beziehen sich auf Wissensbereiche über Diversität, Gewaltprävention, gesellschaftlicher Wandel, Erziehung und Fortbildungen:

„Ich glaube Wissen über Diversität ist wichtig. Hinsichtlich Diversität auch Gewaltprävention an Schulen. [...] Und auch diese Ganztagsklassen. Es geht immer mehr dazu hin, im Rahmen der Gesamtschulen, dass man ein Ganztageskonzept anbietet wo die Kinder dann wirklich den ganzen Tag aufgehoben sind. Sowohl schulisch oder Freizeit abgedeckt wird, dass die Kinder abends nach Hause gehen und Eltern die ihrem Beruf nachgehen können." (It 2)

Max nennt spezifisches pädagogisches Handeln, welches er im Unterricht anwendet. Er fasst zehn Handlungen zusammen, die in Tabelle 7 dargestellt werden. Die Tabelle zeigt zu den genannten Handlungsfeldern, inwieweit diese während der Unterrichtsbeobachtungen aufgezeigt werden konnten.

Tab. 7: Sichtbarkeit pädagogischen Wissens im Handeln von Lehrpersonen der Grundschule

\begin{tabular}{ll}
$\begin{array}{l}\text { Wie zeigt sich pädagogisches Wissen } \\
\text { im Handeln der Lehrperson? }\end{array}$ & $\begin{array}{l}\text { Beobach- } \\
\text { tungen }\end{array}$ \\
\hline $\begin{array}{l}\text { Lehrperson berücksichtigt unter- } \\
\text { schiedliche Niveaus in der Klasse }\end{array}$ & $\begin{array}{l}\text { Alle SchülerInnen haben die Möglichkeit, durch } \\
\text { die Anzahl der unterschiedlichen Übungen, für } \\
\text { sich passende, dem Niveau entsprechende Aufga- } \\
\text { ben auszuwählen. }\end{array}$ \\
\hline $\begin{array}{l}\text { Lehrperson fördert individuelles } \\
\text { Arbeiten }\end{array}$ & $\begin{array}{l}\text { Lehrperson schafft Raum, indem die SchülerInnen } \\
\text { individuell lernen können. }\end{array}$ \\
\hline
\end{tabular}




\begin{tabular}{|c|c|c|}
\hline $\begin{array}{l}\text { Wie zeigt sich pädagogisches Wissen } \\
\text { im Handeln der Lehrperson? }\end{array}$ & $\begin{array}{l}\text { Beobach- } \\
\text { tungen }\end{array}$ & Anmerkungen \\
\hline $\begin{array}{l}\text { Lehrperson fördert selbstständiges } \\
\text { Arbeiten der SchülerInnen - benöti- } \\
\text { gen die SchülerInnen Unterstützung, } \\
\text { können sie diese jederzeit von der } \\
\text { Lehrperson erhalten }\end{array}$ & bestätigt & $\begin{array}{l}\text { Die Lehrperson schafft einen Rahmen, in dem die } \\
\text { SchülerInnen selbstständig arbeiten können. Bei } \\
\text { Fragen können sie jederzeit auf die Lehrperson zu- } \\
\text { kommen. Die Lehrperson bietet den SchülerInnen } \\
\text { individuelle Unterstützung bzw. korrigiert fertige } \\
\text { Übungen. }\end{array}$ \\
\hline $\begin{array}{l}\text { Die SchülerInnen fühlen sich wohl } \\
\text { und es geht ihnen gut }\end{array}$ & bestätigt & $\begin{array}{l}\text { Es kann beobachtet werden, wie die SchülerInnen } \\
\text { gerne in der Klasse sind, Spaß haben, sich respekt- } \\
\text { voll verhalten und sich nicht verstellen müssen. }\end{array}$ \\
\hline Einsatz von verschiedenen Materialien & bestätigt & $\begin{array}{l}\text { Die Lehrperson bereitet für dieselben Inhalte eine } \\
\text { Vielzahl an Materialien vor, die die SchülerInnen } \\
\text { nach belieben auswählen können. }\end{array}$ \\
\hline $\begin{array}{l}\text { SchülerInnen müssen etwas leisten } \\
\text { (Ansatz von Lehrperson: das Beste aus } \\
\text { jedem Kind herausholen) }\end{array}$ & bestätigt & $\begin{array}{l}\text { Leistungen von SchülerInnen werden von der Lehr- } \\
\text { person dokumentiert, individuell gesetzte Ziele } \\
\text { müssen erreicht werden, individuelle Gespräche } \\
\text { finden statt. }\end{array}$ \\
\hline $\begin{array}{l}\text { Den SchülerInnen für das Lernen } \\
\text { individuell Zeit geben }\end{array}$ & bestätigt & $\begin{array}{l}\text { Die SchülerInnen können sich ihre Zeit für das } \\
\text { Lernen selbstständig einteilen. }\end{array}$ \\
\hline $\begin{array}{l}\text { SchülerInnen das Gefühl geben, dass } \\
\text { niemand perfekt ist }\end{array}$ & bestätigt & $\begin{array}{l}\text { Lehrperson legt einen großen Wert auf die Ver- } \\
\text { mittlung von Toleranz und einer offenen Haltung. } \\
\text { Jede Person wird ganzheitlich betrachtet. Die } \\
\text { Lehrperson kommuniziert dieses Anliegen laut in } \\
\text { der Klasse. }\end{array}$ \\
\hline $\begin{array}{l}\text { SchülerInnen lernen voneinander } \\
\text { (z.B. ältere und jüngere SchülerInnen } \\
\text { arbeiten zusammen) }\end{array}$ & bestätigt & $\begin{array}{l}\text { Alters- und jahrgangsgemischte Klassen charakte- } \\
\text { risieren die Klassenstrukturen in der Schule. Dies } \\
\text { ermöglicht, dass ältere SchülerInnen den jüngeren } \\
\text { SchülerInnen helfen und SchülerInnen voneinan- } \\
\text { der lernen können. }\end{array}$ \\
\hline $\begin{array}{l}\text { Selbstständiges Arbeiten fördern: Bei } \\
\text { Fragen zuerst die MitschülerInnen } \\
\text { fragen und danach die Lehrperson }\end{array}$ & $\begin{array}{l}\text { nicht } \\
\text { bestätigt }\end{array}$ & $\begin{array}{l}\text { Es zeigt sich wie SchülerInnen als erstes die Lehr- } \\
\text { person um Unterstützung bitten. SchülerInnen } \\
\text { fragen sich nur dann gegenseitig um Hilfe, wenn } \\
\text { die Lehrperson den Prozess einleitet. }\end{array}$ \\
\hline
\end{tabular}

Die Tabelle veranschaulicht, wie neun der genannten pädagogischen Handlungen explizit im Unterricht beobachtet werden konnten. Ein von Max genannter Wissensbereich konnte über die Beobachtungen nicht aufgezeigt werden. Er präsentiert keine signifikanten Unterschiede zwischen kognitiven Mustern und seinem Handeln. Dies zeigt, dass Max sein Handeln explizit benennen kann. Die Beobachtungen verdeutlichen zusätzlich, wie weitere pädagogische Handlungsformen rekonstruiert werden konnten. Abbildung 102 veranschaulicht, welche Kategorien von ihm genannt und umgesetzt sowie welche mit Hilfe von der Beobachtung zusätzlich rekonstruiert wurden. Als Basis werden die rekonstruierten Wissenskategorien aus den Unterrichtsbeobachtungen (vgl. Kapitel 10.5) herangezogen. Von den Lehrpersonen genannte Wissensbereiche werden in der Abbildung kursiv markiert, zusätzlich rekonstruierte Kategorien sind in dicker Schrift dargestellt. 
- Erziehung

- Kooperation

- Haltung und Emotionen

- Umgang mit Problemen

- Unterstützung/Förderung

- Gruppendynamik

- Vielfalt
- Ablauf

- Leadership

- Störungen

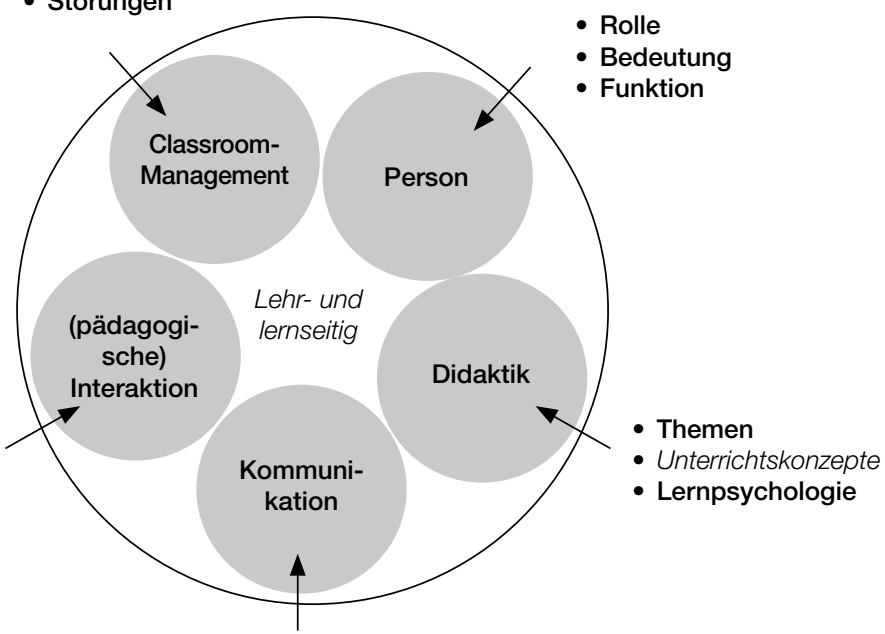

- Sprache

- Kommunikationsinteraktion

- Körpersprache

Abb. 102: Vergleich kognitiver Muster und pädagogischen Handelns

Wie in Abbildung 102 veranschaulicht, benennt Max in den Interviews zu den Kriterien (pädagogische) Interaktion, Classroom-Management und Didaktik spezifisches pädagogisches Wissen zu Unterrichtskonzepten, Erziehung, Kooperation, Unterstützung/Förderung, Vielfalt sowie Ablauf. Max zeigt über sein Handeln, wie er weiteres pädagogisches Wissen in den Bereichen Person und Didaktik, wie zum Beispiel das Wissen zu Leadership, Störungen, Haltung und Emotionen, Umgang mit Problemen oder Körpersprache, anwendet. Die weiteren beobachteten Kategorien sind in der Abbildung dargestellt.

Die personenbezogene Darstellung von Max präsentiert, inwieweit kognitive Muster mit seinem pädagogischen Handeln übereinstimmen bzw. wie diese voneinander abweichen. Er verdeutlicht, wie die von ihm genannten Formen pädagogischen Wissens zum Großteil mit seinem Handeln übereinstimmen. Abweichungen beziehen sich auf die Anwendung von Wissen, die er nicht explizit benennen kann.

Max zeigt ein mehrdimensionales Verständnis pädagogischen Wissens, welches er vorwiegend aus der Berufspraxis sowie Fort- und Weiterbildungen erworben hat. Er verdeutlicht ein Bewusstsein über das pädagogische Wissen und sein Handeln. Dennoch demonstriert die Einzeldarstellung sowie der Vergleich, wie er umfassendes implizites Wissen besitzt, welches er nicht explizit benennen kann. Im folgenden Abschnitt wird Paula, eine Lehrperson aus der Neuen Mittelschule, dargestellt, die unterschiedliche kognitive Muster im Vergleich zu Max aufweist.

\subsection{Fallstudie 2 - Lehrperson Neue Mittelschule}

Die zweite Fallstudie bezieht sich auf eine Lehrperson, die in der Neuen Mittelschule unterrichtet. Paula ist weiblich, 43 Jahre alt und seit 20 Jahren im Schuldienst tätig. Ihre Unterrichtsfächer sind Geschichte und Englisch. Paula absolvierte ihre Ausbildung an einer Pädagogischen Hochschule. 
Sie zeigt großes Interesse an wissenschaftlicher Literatur und neuen Erkenntnissen im Bereich Lernen und absolvierte zahlreiche Fortbildungen zu Themenbereichen wie Umgang und Beziehungen im Klassenzimmer.

Paula charakterisiert pädagogisches Wissen vorwiegend über das soziale Handeln zwischen Lehrpersonen und SchülerInnen:

„Pädagogisches Wissen, das ist für mich eine Klasse führen zu können. Also ich sehe mich als sozial Handelnde und meine Aufgabe und meine Position als Lehrperson ist sozial zu agieren. Ich brauche dazu Wissen und Handlungsfähigkeit, um eine Klasse sozial auszugleichen und eine Klasse zu führen und zu schauen, dass sie sich untereinander zu einer guten Mannschaft entwickeln." (It 9)

In Abbildung 103 wird das rekonstruierte Verständnis pädagogischen Wissens von Paula dargestellt:

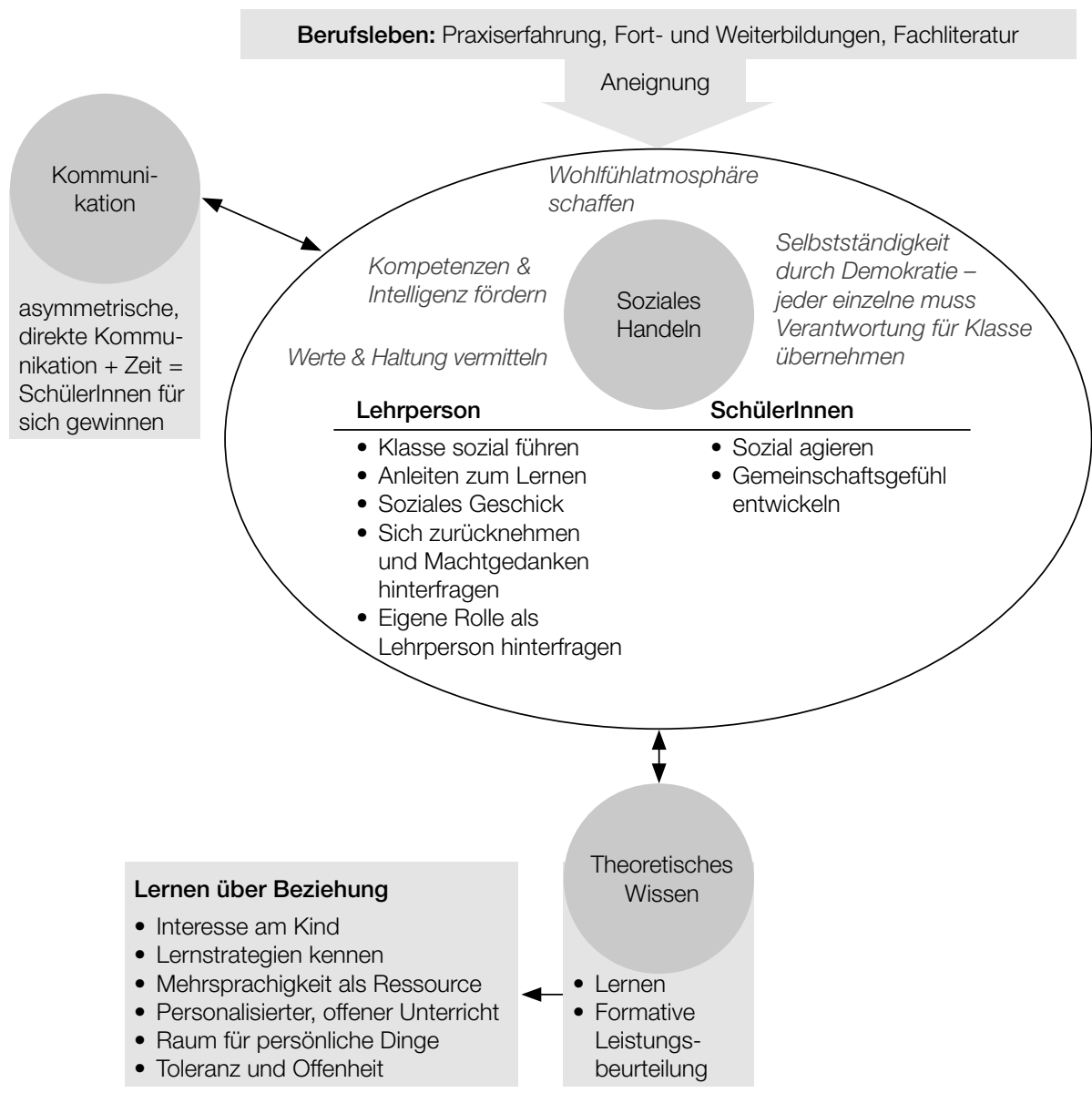

Herausforderungen

Offenheit und Toleranz, Wissen über Werte und Haltung, Wissen über Lernen, eigen Rolle und Zufriedenheit der Lehrperson

Abb. 103: Individuelles Verständnis pädagogischen Wissens - Lehrperson Neue Mittelschule 
Sie definiert für sich lern- sowie lehrseitiges Wissen über das soziale Handeln. Lernseitige Wissensbereiche beziehen sich auf das Wissen über soziales Agieren oder die Entwicklung eines Gemeinschaftsgefühls. Lehrseitig charakterisiert sie es über die Wissensbereiche soziales Führen, Anleiten zum Lernen, soziales Geschick, die Bedeutung der eigenen Rolle als Lehrperson sowie das Thema Macht. Zu den spezifischen lern- sowie lehrseitigen Wissensbereichen nennt Paula vier allgemeine Wissenskomponenten, die für soziale Handeln grundlegend sind: Werte-und Haltungsvermittlung, Kompetenz und Intelligenz fordern, Wohlfühlatmosphäre schaffen, Übernehmen von Verantwortung.

„Ich probiere in der Klasse Demokratie zu vermitteln, Demokratie in die Klasse zu bringen, so dass sie Verantwortung für ihre Klasse haben. Auch für die Klassenorganisation. Und das sind sehr oft kleine Dinge. Ich schicke sie oft einkaufen, dann brauchen wir um gut zu lernen und strukturiert zu lernen Materialien, ganz egal welche ob das Papier ist oder Locher. Und man muss immer schauen, dass es vorhanden ist. Irgendwann sind die Kinder so weit, dass sie die Dinge selber holen und organisieren. Ihnen Jobs zu geben, ganz egal ob er der Energie-Manager ist oder der Boden-Manager. Also sie organisieren im Grunde genommen ihre Klasse selber." (It 9)

Wissen ist Paula zufolge eng mit der Kategorie soziales Handeln verbunden. Dieses definiert sie über das Wissen zur Kommunikation und zum Grundlagenwissen. Das Wissen zur Kommunikation beinhaltet Wissensstrukturen über die asymmetrische, direkte Kommunikation sowie die Einplanung von Zeitressourcen für die Verwirklichung von Kommunikation im Unterricht. Das Grundlagenwissen stützt sich bei ihr auf das Wissen aus Fachliteratur sowie die Kompetenz, auf dieses zurückgreifen zu können:

„Ich kann jetzt, wenn ich den Gerhalt Witter lese oder den Joachim Bauer, da sind die Grundideen teilweise sowas von gleich, wie lernen funktioniert und wie kann ich einen Fremdsprachenunterricht aufbauen. Ich denke einfach zu wissen, dass man auf Fachliteratur zurückgreifen muss." (It 9)

Spezifisches Grundlagenwissen bezieht sich nach Angaben von Paula auf das Wissen zum Lernen sowie zur formativen Leistungsbeurteilung. Sie vertritt die Position, dass Lernen nur über Beziehung stattfindet, die über das Einnehmen einer Haltung als „Coach“ erreicht werden kann:

„Lernen geht für mich einfach nur über Beziehung. Das ist für mich dieser Kunstgedanke. Wenn ich kein Interesse am Kind habe und nicht in der Lage bin eine Beziehung aufzubauen und dem Kind zu zeigen, dass ich da bin um mit ihm zu lernen dann glaube ich findet lernen asymmetrisch statt. Dass ich irgendwo da oben bin und ich presse ihnen das Wissen so wie früher, also ich definiere meine Rolle als Lehrer anders. Ich bin gar nicht gerne im Mittelpunkt, sondern ich bin eher der Coach zum Lernen und ich gebe ihnen die Mittel, die sie brauchen zum Lernen." (It 9)

Als spezifische Aneignungsorte pädagogischen Wissens benennt sie das Berufsleben, die Praxis, Fort- und Weiterbildungen sowie die Auseinandersetzung mit Fachliteratur.

„Ich habe pädagogisches Wissen aus der Praxis. Über Fort und Weiterbildungen. [...]. Wichtig ist für mich das Lesen von Fachliteratur." (It 9)

Von Paula genannte Herausforderungen für pädagogisches Wissen betreffen das Wissen zum Umgang mit Werten, die Weiterentwicklung der eigenen Haltung, das Wissen zum Lernen, das Wissen zur eigenen Rolle und Zufriedenheit sowie das Wissen, wie SchülerInnen zu offenen und toleranten Menschen erzogen werden können:

„Lerne deinen Schülern Haltung und Werte, damit sie Erfolg haben und sich ein bisschen von dem Fachwissen lösen. [...] Haltung und Werte. Toleranz, Offenheit und für sich selber einstehen. Die Ge- 
sellschaft ist so bunt und man muss schauen ein Platz zu finden wo man sich wohl fühlt, ein Beruf wo man zufrieden ist, weil dann kann ich einen offenen Geist haben. Wenn ich das nicht habe, glaube ich, stößt man bald an unsere gesellschaftlichen Grenzen." (It 9)

Paula benennt spezifisches Handeln, wie pädagogisches Wissen in ihrem Unterricht aufgezeigt werden kann. Sie beschreibt sechs verschiedene Formen, die in Tabelle 8 dargestellt werden. $\mathrm{Zu}$ den genannten Bereichen pädagogischen Wissens zeigt die Tabelle, inwieweit die Merkmale aus den Unterrichtsbeobachtungen beobachtet werden konnten.

Tab. 8: Sichtbarkeit pädagogischen Wissens im Handeln von Lehrpersonen der Neuen Mittelschule

\begin{tabular}{|c|c|c|}
\hline $\begin{array}{l}\text { Wie zeigt sich pädagogisches Wis- } \\
\text { sen im Handeln der Lehrperson? }\end{array}$ & $\begin{array}{l}\text { Beobach- } \\
\text { tungen }\end{array}$ & Anmerkungen \\
\hline Lehrperson kann sozial agieren & bestätigt & $\begin{array}{l}\text { Lehrperson zeigt während den Beobachtungen, wie } \\
\text { sie sozial in der Klasse agiert. Die soziale Interaktion } \\
\text { bezieht sich auf unterschiedliche Bereiche: Lernen, } \\
\text { Gruppe, individuelle Person, Bewusstseinsbildung, } \\
\text { Vermittlung von Werten und Normen. }\end{array}$ \\
\hline $\begin{array}{l}\text { Lehrperson kann Klasse führen: } \\
\text { weiß wie jede/jeder Einzelne lernt } \\
\text { und was er zum Lernen braucht }\end{array}$ & bestätigt & $\begin{array}{l}\text { Lehrperson führt die Klasse. Sie weiß wie jedes } \\
\text { einzelne Kind lernt, welche Schwächen und Stärken } \\
\text { es hat und wendet individuelle Förderungen und } \\
\text { Lernstrategien an. }\end{array}$ \\
\hline $\begin{array}{l}\text { Lehrperson schafft eine gute } \\
\text { Atmosphäre in der sie sich wohl } \\
\text { fühlt und die Gruppe als Einheit } \\
\text { zusammenhält }\end{array}$ & $\begin{array}{l}\text { nicht } \\
\text { bestätigt }\end{array}$ & $\begin{array}{l}\text { Lehrperson legt sehr starken Wert auf den Zusammen- } \\
\text { halt der Klasse und sorgt für eine gute Atmosphäre. Es } \\
\text { konnten zahlreiche Probleme wie Mobbing, Ausschluss- } \\
\text { verhalten und Probleme zwischen den SchülerInnen } \\
\text { sowie unter den Lehrpersonen beobachtet werden. }\end{array}$ \\
\hline $\begin{array}{l}\text { Lehrperson ist Teil der Gruppe. Sie } \\
\text { steht nicht vorne und referiert }\end{array}$ & bestätigt & $\begin{array}{l}\text { Lehrperson steht selten vor der Klasse und referiert. } \\
\text { Die meiste Zeit ist sie aktiver Teil der Klasse, die das } \\
\text { individuelle Lernen unterstützt. }\end{array}$ \\
\hline $\begin{array}{l}\text { SchülerInnen können gelerntes } \\
\text { wiedergeben }\end{array}$ & $\begin{array}{l}\text { teilweise } \\
\text { bestätigt }\end{array}$ & $\begin{array}{l}\text { SchülerInnen müssen jeden Tag aufschreiben, was sie } \\
\text { in den jeweiligen Stunden fachlich sowie sozial ge- } \\
\text { lernt haben. Es zeigt sich, wie ein paar SchülerInnen } \\
\text { nicht in der Lage sind Gelerntes zu verbalisieren. }\end{array}$ \\
\hline $\begin{array}{l}\text { SchülerInnen sind für ihr Lernen } \\
\text { selbst verantwortlich }\end{array}$ & $\begin{array}{l}\text { teilweise } \\
\text { bestätigt }\end{array}$ & $\begin{array}{l}\text { Lehrperson gibt den SchülerInnen sehr viel Freiraum, } \\
\text { in dem die SchülerInnen selbstständig für ihr Lernen } \\
\text { verantwortlich sind. Beobachtungen demonstrieren, } \\
\text { wie einige Kinder es nicht schaffen selbstständig zu } \\
\text { lernen, sondern stark auf die Lehrperson fixiert sind. }\end{array}$ \\
\hline
\end{tabular}

Insgesamt benennt Paula sechs spezifische Handlungen. Es konnten drei der genannten Handlungen beobachtet werden. Zwei weitere Kriterien wurden teilweise sichtbar. Eine von ihr genannte Handlung konnte nicht aufgezeigt werden. Die Ergebnisse verdeutlichen Wahrnehmungsunterschiede zwischen kognitiven Mustern und ihrem pädagogischen Handeln. Sie beschreibt spezifisches Wissen, das sie im Unterricht anwendet, welches über die Beobachtungen nicht bzw. nur teilweise rekonstruiert werden kann. 
Beobachtungen verdeutlichten eine Fülle weiteren pädagogischen Wissens. Abbildung 104 veranschaulicht, welche Wissenskategorien von Paula genannt und umgesetzt (kursiv) und welche während der Beobachtung zusätzlich rekonstruiert wurden (dick gedruckte Felder). Letztere beziehen sich auf die Kategorien (pädagogische) Interaktion, Classroom-Management, Person und Didaktik.

- Erziehung

- Kooperation

- Haltung und Emotionen

- Umgang mit Problemen

- Unterstützung/Förderung

- Gruppendynamik

- Vielfalt

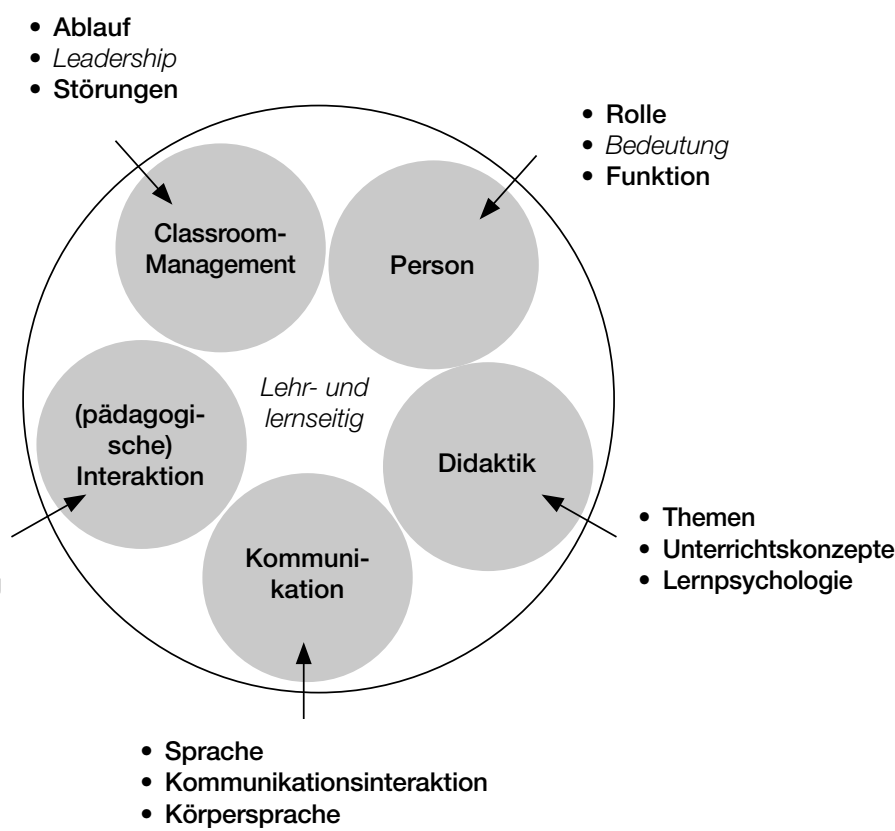

Abb. 104: Vergleich kognitiver Muster und pädagogischen Handelns

Beobachtungen veranschaulichen, wie Paula spezifisches pädagogisches Wissen aus den Kategorien (pädagogische) Interaktion, Classroom-Management und Person benennt. Es konnte für alle drei genannten Kriterien weiteres Wissen rekonstruiert werden. Weitere Wissensbereiche von Paula, die von ihr nicht benannt wurden, sind das Wissen zur Kommunikation und zur Didaktik.

Paula präsentiert, inwieweit kognitive Muster mit ihrem pädagogischen Handeln übereinstimmen bzw. von diesem abweichen. Personenbezogene Abweichungen, die bei der Untersuchung festgehalten wurden, beziehen sich auf folgende Aspekte:

1. Die Lehrperson besitzt mehr pädagogisches Wissen, als sie verbalisieren kann.

- Vorhandene Wissensstrukturen können nicht vollständig explizit benannt werden.

2. Von den sechs angeführten Handlungen können zwei Formen nur zum Teil beobachtet und eine Kategorie nicht beobachtet werden.

- Es besteht eine Lücke zwischen ihren Annahmen und ihrem tatsächlichen Handeln.

3. Die Lehrperson beschreibt spezifisches Wissen, das sie im Unterricht anwendet, welches über die Beobachtungen nicht rekonstruiert werden kann.

- Es bestehen Abweichungen zwischen explizitem Handeln und ihren Annahmen. 
Paula präsentiert ein mehrdimensionales Verständnis pädagogischen Wissens, welches sie über die Fachliteratur, die Berufspraxis sowie Fort- und Weiterbildungen erwerben konnte. Ihr Fokus liegt auf Wissen zum sozialen Handeln. Die Darstellung illustriert zu den Übereinstimmungen zwischen kognitiven Mustern und pädagogischen Handlungen auch Diskrepanzen. Dies verdeutlicht prototypisch, wie sie umfangreiches implizites Wissen besitzt. Es wird über die individuelle Darstellung aufgezeigt, wie Paula Wissen explizites Wissen benennt, welches über die Beobachtung nicht rekonstruiert werden konnte.

\subsection{Fallstudie 3 - Lehrperson Gymnasium}

Fallstudie 3 bezieht sich auf eine Lehrperson, die im Gymnasium unterrichtet. Anna ist weiblich, 45 Jahre alt und seit 20 Jahren im Schuldienst tätig. Ihre Unterrichtsfächer sind Geschichte und Sport. Die Ausbildung absolvierte sie an einer Universität. Im Laufe ihres Berufslebens hat sie zahlreiche Fort- und Weiterbildungen absolviert. Anna ist sehr engagiert und nimmt über Schulentwicklungsprojekte aktiv am Schulleben teil.

Das Verständnis pädagogischen Wissens von Anna gliedert sich in zwei Arten von Wissen, das Grundlagenwissen sowie das Erfahrungswissen (vgl. Abbildung 105).

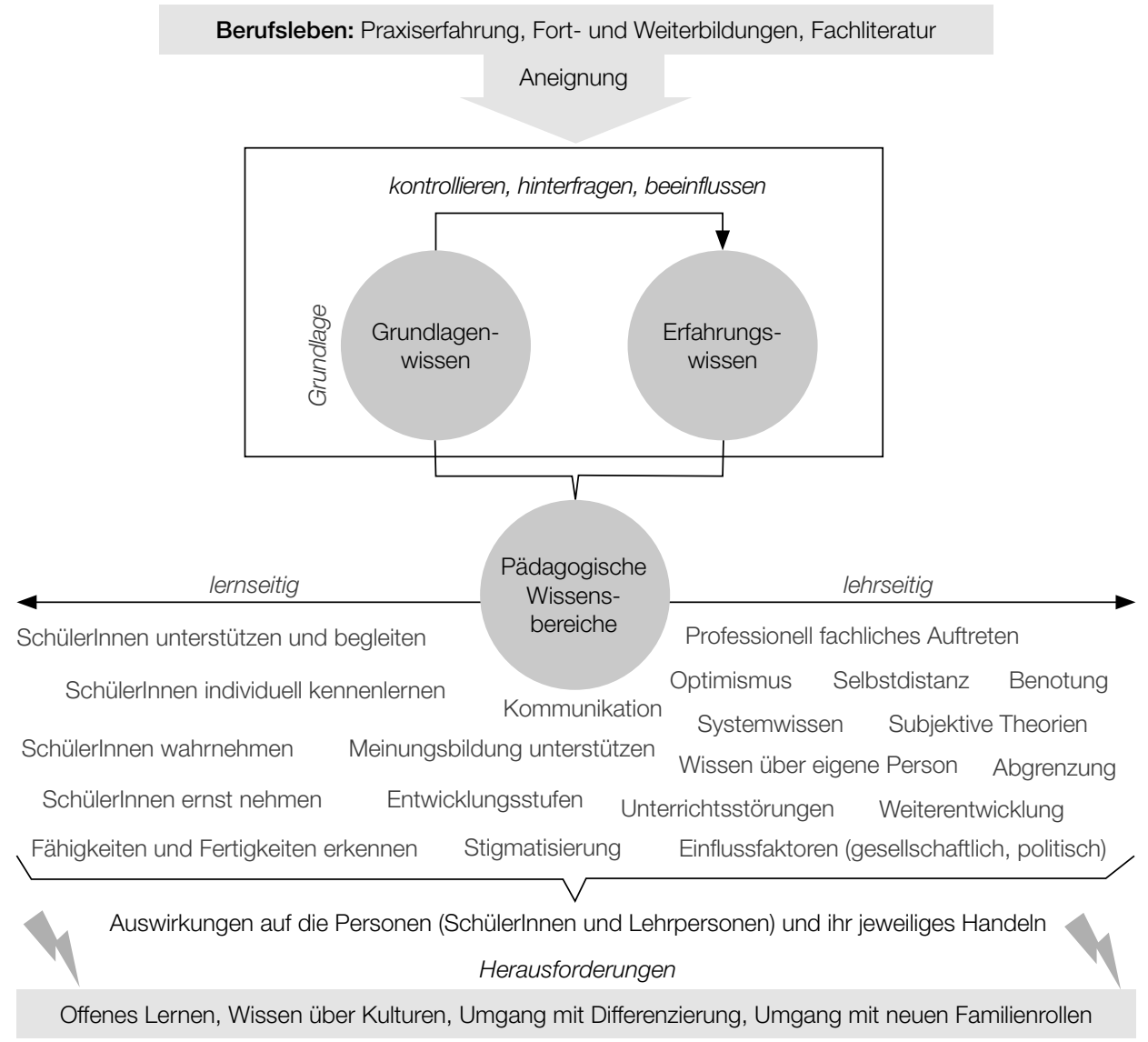

Abb. 105: Individuelles Verständnis pädagogischen Wissens - Lehrperson Gymnasium 
Grundlagenwissen und Erfahrungswissen werden von Anna als Grundlage pädagogischen Wissens beschrieben. Diese stehen in einem Verhältnis zueinander:

„Pädagogisches Wissen ist für mich zweigeteilt. Einmal ist es das Wissen um eine Sache. Da geht es um Fachwissen. Viel Einzelwissen über etwas, über pädagogische Bereiche und pädagogisches Wissen heißt für mich die Erfahrung die für mich miteinfließt über das Unterrichten." (It 13)

Paula zufolge kontrolliert, hinterfragt und beeinflusst Grundlagenwissen das Erfahrungswissen. Definitorisch bezieht sich das Grundlagenwissen nach ihrer Aussage auf theoretische Formen pädagogischen Wissens. Das Erfahrungswissen stützt sich auf die in der Erfahrung gewonnenen pädagogischen Wissenselemente.

Auf Basis dieser Wissensformen entwickeln sich lern- sowie lehrseitige Wissensbereiche. Sie benennt unterschiedliche Kategorien, die in Abbildung 105 veranschaulicht werden. Diese beziehen sich auf das Wissen zur Rolle einer Lehrperson, zum Lehren und Lernen bis hin zu den Wissensbereichen Erziehung, Systemwissen, Beziehung, Diagnostik und Förderung. Sie hebt die Notwendigkeit hervor, Menschen zu mögen, und legt ihren Schwerpunkt vor allem auf Bedürfnisse und unterstützende Maßnahmen:

„Ich denke, dass es sehr wichtig ist, dass man Menschen mag. Ich muss bzw. man muss junge Leute als jemanden sehen, ja das sind Menschen die nicht fertig sind, die in einer Entwicklung sind und die man nicht messen kann, mit dem was ich als Erwachsene dann tun soll oder können soll. Sie sind in einem Entwicklungsprozess. Man muss sich immer wieder sagen, dass sie das jetzt noch gar nicht wissen können, sondern da helfe ich und stehe ihnen bei. Da versuche ich mit Fördermaßnahmen zu begleiten." (It 13)

Anna spricht neben dem lernseitigen Fokus explizit die Abhängigkeit vom und die Wirkung des Systems und dessen Einfluss auf das pädagogische Wissen an:

„Die ganze Organisation,Schule‘ was kommt auf uns zu auf Seiten der Verwaltung, Ministerium, politischer Seite. Also die Faktoren werden einfach zunehmend mehr spürbar. Das fließt alles mit ein, das hat eine Wirkung auf eine Lehrperson. Diese nimmt man auf in irgendeiner Weise mit in die Klasse hinein. Wenn man das abstrakt sieht und eine Distanz dazu hat, dann hat es trotzdem eine Wirkung auf den Beruf und auf das pädagogische Wissen." (It 13)

Sie beschreibt, wie pädagogisches Wissen nicht nur von den einzelnen Lehrpersonen abhängig ist. Sie verweist bei ihrer Aussage auf den Einfluss struktureller Rahmenbedingungen und benennt spezifisch die Verwaltung sowie bildungspolitische Prozesse.

Im Interview werden als spezifische Aneignungsorte pädagogischen Wissens die Praxis sowie Fort- und Weiterbildungen hervorgehoben:

„Pädagogisches Wisse habe ich mir hauptsächlich in der Praxis angeeignet. Im Umgang mit den SchülerInnen. Ich konnte hier mein theoretisches Wissen mit der Praxis verknüpfen. Ja dann war da noch die Ausbildung [...]. Die didaktischen Sachen wie ich wann was mache, habe ich über die Ausbildung gelernt." (It 13)

Von Anna genannte Herausforderungen beziehen sich auf die Umsetzung didaktischer Strukturen, wie offenes Lernen, das Wissen zu Kulturen, den Umgang mit Differenzierung sowie den Umgang mit gesellschaftlichen Wandlungsprozessen:

„Ich stehe jeden Tag wieder vor der neuen Herausforderung gesellschaftliche Prozess im Unterricht zu berücksichtigen. Der Umgang mit Diversität und Kultur, die Personalisierung oder auch neue didaktische Formen, wie das offene Lernen [...].“ (It 13) 
Sie benennt während des Interviews spezifisches Handeln, an dem pädagogisches Wissen sichtbar gemacht werden kann. Anna stützt sich auf acht verschiedene Formen, die in Tabelle 9 dargestellt sind. Zu den genannten Formen pädagogischen Wissens zeigt die Tabelle, inwieweit die Merkmale während den durchgeführten Unterrichtsbeobachtungen beobachtet werden konnten.

Tab. 9: Sichtbarkeit pädagogisches Wissen im Handeln von Gymnasiallehrpersonen

\begin{tabular}{|c|c|c|}
\hline $\begin{array}{l}\text { Wie zeigt sich pädagogisches Wis- } \\
\text { sen im Handeln der Lehrperson? }\end{array}$ & $\begin{array}{l}\text { Beobach- } \\
\text { tungen }\end{array}$ & Anmerkungen \\
\hline $\begin{array}{l}\text { SchülerInnen beim Lernen beglei- } \\
\text { ten und verstehen, durch perma- } \\
\text { nentes nachfragen }\end{array}$ & bestätigt & $\begin{array}{l}\text { Die Lehrperson fragt sehr oft bei Aussagen nach, was } \\
\text { SchülerInnen denken oder meinen, um sie besser zu } \\
\text { verstehen. }\end{array}$ \\
\hline $\begin{array}{l}\text { SchülerInnen objektiv und ohne } \\
\text { Druck bewerten }\end{array}$ & $\begin{array}{l}\text { teilweise } \\
\text { bestätigt }\end{array}$ & $\begin{array}{l}\text { Objektive Benotung hat sich bestätigt. Allerdings } \\
\text { wurde beobachtet, wie die Lehrperson jede Stunde } \\
\text { Leistungen einzelner SchülerInnen unangekündigt } \\
\text { mündlich sowie schriftlich abfragt. SchülerInnen } \\
\text { stehen dadurch permanent unter einem Notendruck. }\end{array}$ \\
\hline $\begin{array}{l}\text { Entwicklungsstand und Lernstand } \\
\text { der SchülerInnen kennen }\end{array}$ & $\begin{array}{l}\text { teilweise } \\
\text { bestätigt }\end{array}$ & $\begin{array}{l}\text { Die Lehrperson hat einen Überblick, wie sich die } \\
\text { einzelnen SchülerInnen im Unterricht beteiligen und } \\
\text { welche Inhalte Sie wissen bzw. gelernt haben. Durch das } \\
\text { frontale Unterrichten wird nicht auf individuelle Lern- } \\
\text { und Entwicklungsstände eingegangen. Die Klasse wird } \\
\text { als kollektiv unterrichtet. }\end{array}$ \\
\hline $\begin{array}{l}\text { Die Wahrnehmung jedes einzelnen } \\
\text { als Individuum }\end{array}$ & $\begin{array}{l}\text { teilweise } \\
\text { bestätigt }\end{array}$ & $\begin{array}{l}\text { Die Lehrperson kreiert im Unterricht Raum für indi- } \\
\text { viduelle Fragen und Anliegen. Personalisiertes Lernen } \\
\text { findet nicht statt. }\end{array}$ \\
\hline $\begin{array}{l}\text { Anwendung unterschiedlicher } \\
\text { Methoden }\end{array}$ & bestätigt & $\begin{array}{l}\text { Lehrperson arbeitet hauptsächlich mit dem Schul- } \\
\text { buch. Zusätzlich greift die Lehrperson auf eine } \\
\text { Vielzahl unterschiedlicher Methoden (Konzentrati- } \\
\text { onsübungen, Gruppenarbeiten, etc.) zurück. }\end{array}$ \\
\hline Spontanes agieren & bestätigt & $\begin{array}{l}\text { Die Lehrperson agiert spontan auf Reaktionen und } \\
\text { Meinungen von SchülerInnen und kann fachlich gut } \\
\text { argumentieren. }\end{array}$ \\
\hline $\begin{array}{l}\text { Störungen abwägen und Hand- } \\
\text { lungsstrategien bewusst einsetzen } \\
\text { durch direktes Ansprechen sowie } \\
\text { die selbstständige Lösungskompe- } \\
\text { tenz der SchülerInnen fördern }\end{array}$ & bestätigt & $\begin{array}{l}\text { Lehrperson entscheidet bewusst für sich, auf welche } \\
\text { Störungen sie reagiert und welche sie ignoriert. Dabei } \\
\text { spricht sie Störungen direkt an und benennt sie vor } \\
\text { der Klasse und gibt den SchülerInnen Raum um } \\
\text { Störungen zu beheben. }\end{array}$ \\
\hline $\begin{array}{l}\text { Lehrperson stellt die eigene Person } \\
\text { in den Mittelpunkt. Eigene Haltun- } \\
\text { gen und Bedürfnisse werden in der } \\
\text { Klasse offen angesprochen }\end{array}$ & bestätigt & $\begin{array}{l}\text { Die Lehrperson schafft persönliche Grenzen und ach- } \\
\text { tet darauf, dass Bedürfnisse und Unzufriedenheiten } \\
\text { von ihr direkt angesprochen werden. }\end{array}$ \\
\hline
\end{tabular}

Es konnten fünf von acht beschriebenen Handlungen von Anna beobachtet werden. Drei der angegebenen Merkmale zeigten sich nur teilweise. Diese Abweichungen verdeutlichen Unterschiede zwischen kognitiven Mustern und ihrem Handeln, die jedoch, wie die Analyse illustriert, in diesem Beispiel sehr gering ausfallen. 
Anna macht sichtbar, wie sie mehr pädagogisches Wissen anwendet, als sie explizit genannt hat. Ausgehend von der Gesamtdarstellung der Kategorien der Beobachtungen wird in Abbildung 106 dargestellt, welche Wissensbereiche Anne explizit während des Interviews benannt hat (kursiv) und welche zusätzlichen Formen über Beobachtungen aufgezeigt werden konnten (fett gedruckte Kategorien).

- Erziehung

- Kooperation

- Haltung und Emotionen

- Umgang mit Problemen

- Unterstützung/Förderung

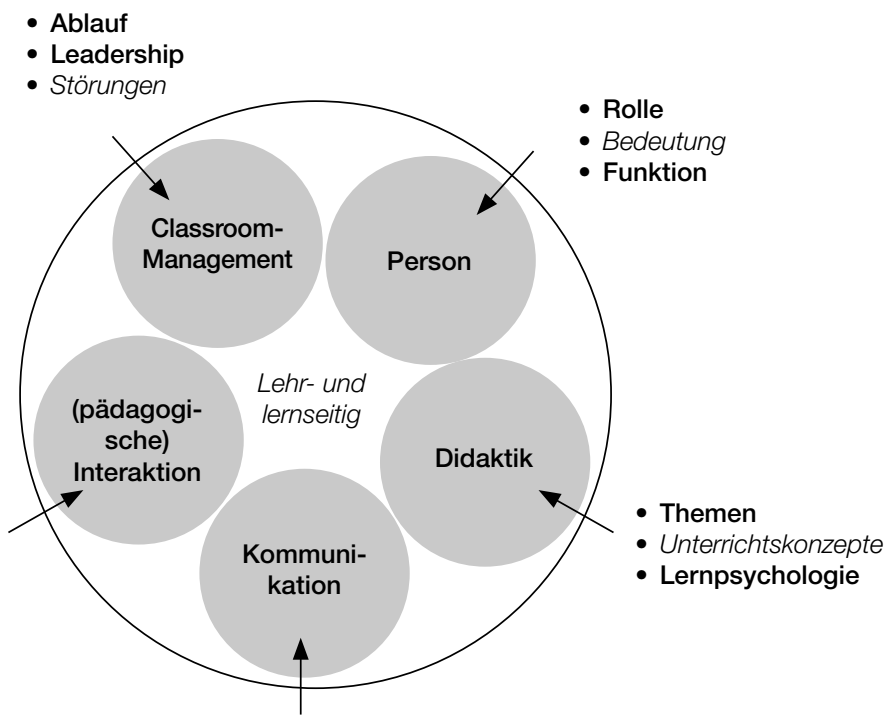

- Sprache

- Körpersprache

- Kommunikationsinteraktion

Abb. 106: Vergleich kognitiver Muster und pädagogischen Handelns

Anna benennt aus allen fünf rekonstruierten Beobachtungskategorien - (pädagogische) Interaktion, Classroom-Management, Person, Didaktik, Kommunikation - Wissensbereiche, die mithilfe der Beobachtungen größtenteils bestätigt werden konnten. Die von ihr nicht genannten Wissensbereiche beziehen sich, wie die Abbildung darstellt, auf alle der hier dargestellten Kategorien. Zum Beispiel wurde in der Kategorie Kommunikation die Interaktion von der Lehrperson nicht benannt. Über die Beobachtungen wurde zusätzlich Wissen zur Sprache und zur Körpersprache sichtbar. Bei der Kategorie Didaktik wurde das Wissen zu Themen, Lerninteraktion und Lernpsychologie nicht erwähnt. Weitere Abweichungen können in der Abbildung 106 abgelesen werden. Die Darstellung von Anna zeigt auf, inwieweit kognitive Muster und ihr pädagogisches Handeln übereinstimmen bzw. voneinander abweichen. Rekonstruierte Abweichungen betreffen folgende Aspekte:

1. Von acht explizit genannten Handlungen konnten drei nur teilweise beobachtet werden.

Es werden geringe Abweichungen zwischen kognitiven Mustern und ihren Annahmen rekonstruiert.

2. Die Rekonstruktion verdeutlicht die Anwendung von weiteren Wissensfeldern, die die Lehrperson nicht explizit benennt. 
Anna charakterisiert pädagogisches Wissen über ein mehrdimensionales Verständnis, welches sie sich über die Praxis sowie Fort- und Weiterbildungen angeeignet hat. Die Darstellung verdeutlicht zu den Übereinstimmungen kognitiver Muster und pädagogischer Handlungsstrukturen Wahrnehmungsunterschiede im Zusammenhang ihres Wissens und Handelns. Anna verfügt wie die Lehrpersonen in den Fallstudien 1 und 2 über umfangreiches implizites Wissen.

Die dargestellten Lehrpersonen aus den Schultypen Grundschule, Neue Mittelschule und Gymnasium verdeutlichen unterschiedliche lern- sowie lehrseitige Charakterisierungen pädagogischen Wissens und geben protypisch einen Einblick in individuelle Muster und ihr pädagogisches Handeln. Alle drei Lehrpersonen charakterisieren pädagogisches Wissen über ein mehrperspektivisches Verständnis. Sie lassen sich über unterschiedliche Wissensbereiche abgrenzen. Parallelen beziehen sich auf Wissen, das eine ressourcenorientierte individuelle Unterstützung sowie erfolgreiches Lernen unterstützt. Erfahrungsbezogenes Wissen sowie Grundlagenwissen wird von allen drei Lehrpersonen benannt. Personenspezifische Schwerpunkte beziehen sich zum Beispiel auf das Wissen zur Kommunikation, zur Didaktik oder das Wissen zur eigenen Person. Alle dargestellten Lehrpersonen geben an, wie sie pädagogisches Wissen über die Praxis sowie Fort- und Weiterbildungen erworben haben. Eine dargestellte Person benennt zusätzlich die Aneignung über Fachliteratur und den Lehrplan. Herausforderungen, die in allen drei Fallbeispielen genannt wurden, beziehen sich auf neue Anforderungen, die eng mit gegenwärtigen sozialen Transformationsprozessen verbunden sind. Diese betreffen das Wissen zu Diversität, das Wissen zu Kulturen sowie den Umgang mit gesellschaftlichen Wandlungsprozessen. Weitere Herausforderungen sind das Wissen zum Lernen, didaktisches Wissen sowie die Vermittlung von Werten, die offene Menschen heranbilden. Es zeigen sich zwischen den Lehrpersonen hinsichtlich ihrer Herausforderungen für das pädagogische Wissen Parallelen sowie Abweichungen.

Die personenbezogene Darstellung präsentiert, inwieweit individuelle Muster und pädagogisches Handeln übereinstimmen bzw. voneinander abweichen. Es konnte rekonstruiert werden, wie die Lehrpersonen Wissen benennen, welches sich in ihrem Handeln konkret zum Ausdruck kommt. In diesen Bereichen sind keine Abweichungen zwischen ihren Mustern und ihrem Handeln erkennbar. Auffällig ist, dass alle Lehrpersonen über umfangreiches implizites Wissen verfügen. Diese Annahme wird dadurch bestätigt, dass sie mehr pädagogisches Wissen anwenden, als sie explizit benennen. Weitere Abweichungen beziehen sich auf die Tatsache, dass Lehrpersonen explizites Wissen benennen, welches jedoch nicht beobachtet werden konnte. 


\section{Zusammengefasste Ergebnisdarstellung aus den rekonstruierten Kategorien pädagogischen Wissens}

Das vorliegende Kapitel führt die aus der Datenerhebung generierten Kategorien der Interviews und Beobachtungen (vgl. Kapitel 8) zusammen und dient als Grundlage für die Integration und Interpretation der Ergebnisse in Kapitel III.

Mithilfe des qualitativ-rekonstruktiven Zugangs und des Auswertungsverfahrens konnten, wie in den vorherigen Abschnitten dargestellt, unterschiedliche Kategorien pädagogischen Wissens aus den Interviews und Beobachtungen rekonstruiert werden. Im Folgenden werden das rekonstruierte Verständnis pädagogischen Wissens, die Unterschiede und Übereinstimmungen der Kategorien zu pädagogischem Wissen abhängig von den Erhebungsinstrumenten sowie schulformspezifische Rekonstruktionsschwerpunkte diskutiert.

\subsection{Verständnis pädagogischen Wissens}

Ziel des Kapitels ist eine synaptische Darstellung der rekonstruierten Ergebnisse pädagogischen Wissens. Die Ergebnisse aus den Interviews verdeutlichen, wie Lehrpersonen mithilfe ihres Grundlagenwissens und ihres Erfahrungswissens pädagogisches Wissen charakaterisieren. Fällt es ihnen anfangs schwer, ad hoc auf die Frage „Was verstehen Sie unter dem Begriff pädagogisches Wissen?“ zu antworten, konnten sie dies mithilfe der Frage „Wie wenden Sie pädagogisches Wissen im Unterricht an?" basierend auf ihren Erfahrungen, ihren subjektiven Theorien und teilweise ihrem theoretischen Wissen den Begriff definieren und präzisieren.

Wie zu Beginn der Ergebnisdarstellung mit Interviewauszügen illustriert und mit Hilfe der Unterrichtsbeobachtungen aufgezeigt, wird pädagogisches Wissen in einer Wechselbeziehung zwischen der Lehrperson und den SchülerInnen realisiert. Diese bezieht sich in der vorliegenden Arbeit auf zwei Interaktionsprozesse. Die Lehrpersonen argumentieren, dass ihr pädagogisches Wissen über einen Spiegelungseffekt von SchülerInnen sichtbar werden kann, indem sie über ihr Handeln pädagogisches Wissen repräsentieren. Als einen weiteren Interaktionsprozess verdeutlichen die empirischen Daten, wie pädagogisches Wissen über die Interaktion zwischen SchülerInnen und Lehrpersonen rekonstruiert und aufgezeigt werden konnte. Pädagogisches Wissen lebt von einer Wechselbeziehung zwischen den beteiligten AkteurInnen, welche von der Klassenstruktur sowie vorhandener institutioneller Strukturen und Rahmenbedingungen beeinflusst wird.

Ergänzend wird für die Realisierung pädagogischen Wissens die lernseitige Perspektive diskutiert. Liegt der Fokus der Arbeit auf dem pädagogischen Wissen von Lehrpersonen, verdeutlichen sie über Interviews und Beobachtungen, wie die untersuchten Personen das pädagogische Wissen unter Berücksichtigung der SchülerInnen charakterisieren und ausrichten. Es ist in Anlehnung an die Befunde ein lern- und lehrseitiges Wissen. Bei der Lernseitigkeit stehen nicht die Lernfragen oder Aufgaben von Lehrpersonen im Mittelpunkt, sondern die Auseinandersetzung mit der Welt und dem individuellen Lernprozess. Lehrpersonen versichern ihren Unterricht auf das individuelle, erfahrungsbezogene Lernen auszurichten und legen ihre didaktischen Konzepte an daran an.

Wie die Analyse des Datenmaterials verdeutlicht, wird pädagogisches Wissen als fachunabhängiges Wissen mehrdimensional konzeptualisiert. Die in der Studie rekonstruierten Kategorien pädagogischen Wissens aus den Interviews und Beobachtungen lassen sich über sieben generierte Wissensfelder darstellen (vgl. Abbildung 107). 


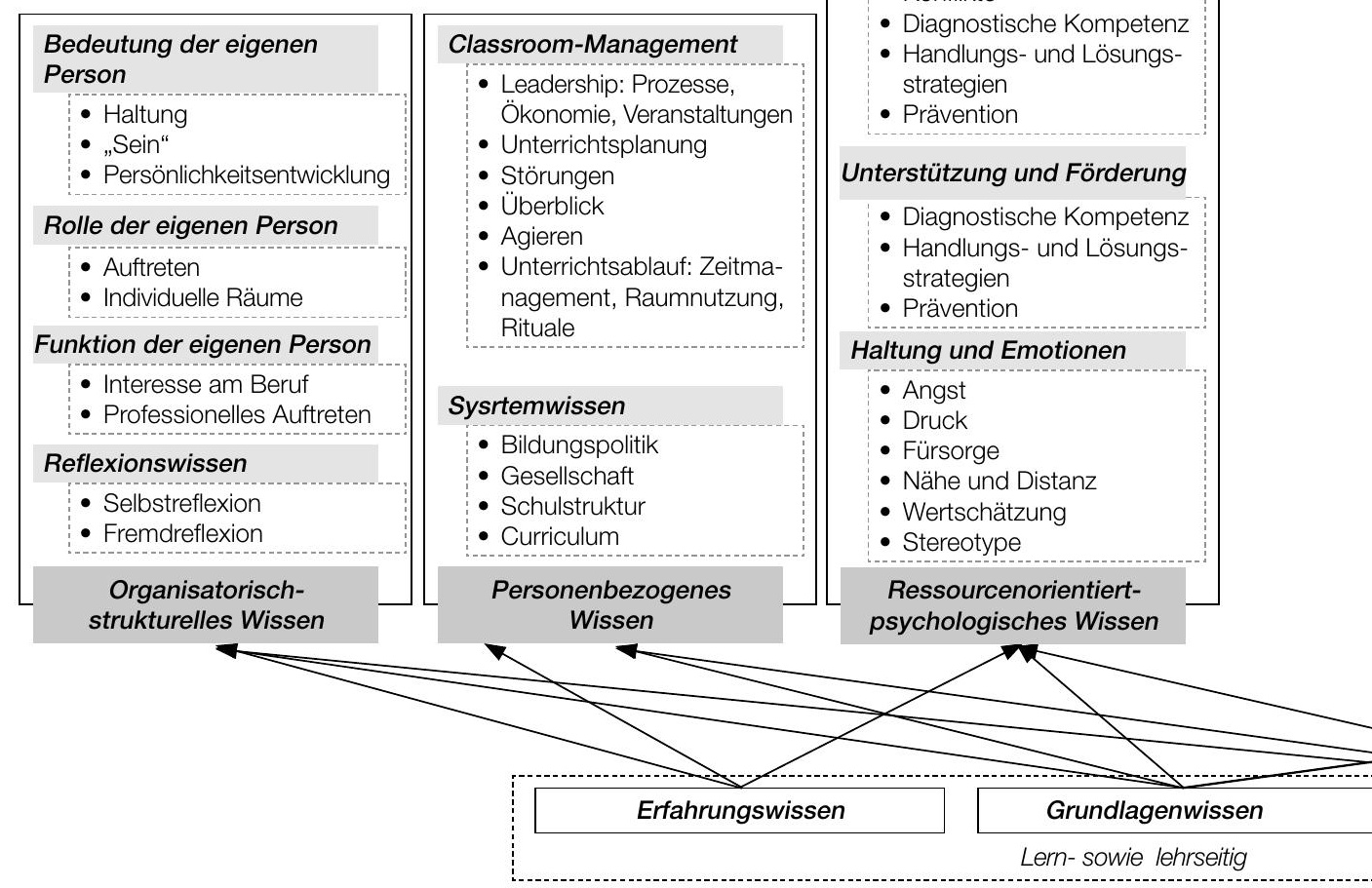

Abb. 107: Matrix pädagogischen Wissens

Es zeigt sich, wie drei der Wissensfelder - Grundlagenwissen, Erfahrungswissen, Wissen zum institutionellen Raum - für die weiteren Formen grundlegend sind. Sie bilden die Grundlage für die Wissensfelder personenbezogenes Wissen, organisatorisch-strukturelles Wissen, interaktionales Wissen sowie ressourcenorientiert-psychologisches Wissen. Lehrpersonen argumentieren, dass ohne die grundlegenden Wissensfelder pädagogisches Wissen nur unzureichend ausgebildet und angewendet werden kann. Der institutionelle Raum bezieht sich auf schulinterne strukturelle Regeln sowie auf bildungspolitische Anforderungen auf Makro- und Mesoebene. Das Erfahrungswissen stützt sich auf erfahrungsbezogene pädagogische Wissensbereiche aus der Berufspraxis sowie Vorerfahrungen, die in der Kindheit, Jugendzeit oder im Studium stattgefunden haben.

Das personenbezogene Wissen untergliedert sich in die Wissenskategorien Bedeutung, Rolle und Funktion der Lehrperson sowie das Reflexionswissen. Das organisatorisch-strukturelle 


\begin{tabular}{|c|c|c|}
\hline Beziehung & Erziehung & Lernen \\
\hline $\begin{array}{l}\text { - Beziehungsgefüge } \\
\text { - Umgang }\end{array}$ & \multirow{3}{*}{$\begin{array}{l}\text { - Werte } \\
\text { - Regeln } \\
\text { - Disziplin } \\
\text { - Bewusstseinsbildung } \\
\text { - Ressourcen }\end{array}$} & \multirow{3}{*}{$\begin{array}{l}\text { - Lernentwicklung } \\
\text { - Lernatmosphäre } \\
\text { - Lerntypen } \\
\text { - Lerninteraktion } \\
\text { Kommunikation }\end{array}$} \\
\hline Lehren & & \\
\hline \multirow{6}{*}{\begin{tabular}{|l} 
- Motivationales Wissen \\
- Lernstandserhebung \\
- Fehlerkultur \\
- Didaktik \\
- Raum, \\
- Digitalisierung \\
- Methodik \\
- Sozialformen, \\
- Lernpsychologie \\
- Unterrichtsstrategien \\
- Und -konzepte \\
- Unterrichtsvorbereitung
\end{tabular}} & & \\
\hline & Gruppendynamik & \multirow{4}{*}{$\begin{array}{l}\text { - Strategien } \\
\text { - Regeln } \\
\text { - Gesprächsführung } \\
\text { - Interaktion (verbale- und } \\
\text { nonverbale Kommunikation) } \\
\text { - Sprache (Eigenschaft } \\
\text { Sprache) } \\
\text { - Körpersprache (Gestik, } \\
\text { Mimik, Körperausdruck) }\end{array}$} \\
\hline & $\begin{array}{l}\text { - Rollenverteilung } \\
\text { - Gruppendynamik }\end{array}$ & \\
\hline & Kooperation & \\
\hline & $\begin{array}{l}\text { - Zusammenarbeit } \\
\text { - Austausch }\end{array}$ & \\
\hline & & \\
\hline
\end{tabular}

\section{Wissen zum institutionellen Raum}

Wissen beinhaltet die Wissensbereichen Classroom-Management und Systemwissen zusammen. Das interaktionale Wissen charakterisiert sich über die meisten Wissenskategorien. Dies verdeutlicht die zentrale Bedeutung von Interaktionsprozessen für pädagogisches Wissen. Inhalte im Bereich des interaktionalen Wissens sind Erziehung, Beziehung, Kommunikation, Gruppendynamik, Kooperation, Lernen und Lehren. Das ressourcenorientiert-psychologische Wissen setzt sich aus den Komponenten Entwicklungspsychologie, Diversität/Vielfalt, Konfliktmanagement, Unterstützung und Förderung sowie das Wissen über Haltung und Emotionen. Die jeweilige Beschreibung der Kategorien sind in Abschnitt 8.1 sowie 8.5 bei der Ergebnisdarstellung beschrieben und wird hier nicht mehr aufgegriffen.

Die Befunde illustrieren Wechselwirkungen pädagogischen Wissens zwischen den dargestellten Wissenskategorien. Diese zeigen sich vorwiegend über die Interviewergebnisse, die Lehrpersonen explizit benennen. Vereinzelt konnten über die Beobachtungen weitere Wechselwirkungen rekonstruiert werden. In Abbildung 108 werden die Kategorien und ihre Wechselwirkungen zusammengefasst dargestellt. 
Abbildung 108 illustriert, mit welchem Wissen einzelne Wissensbereiche in Beziehung stehen. Kategorien, die auf Basis der Befunde Wirkungen gegenüber anderen zeigen, sind die Kommunikation, die Beziehung, das Classroom-Management, die Gruppendynamik, das Lehren, das psychologische Wissen, das Reflexionswissen, das Systemwissen, das Lernen und die Diversität. Einzelne Wissenskategorien wirken sich vorrangig auf die Kategorien Lernen und Lebren aus. Weitere Kategorien, die von anderen Kategorien abhängen, sind die Kommunikation, die Beziehung, die Kooperation, die Unterstützung und Förderung, das Classroom-Management, die Haltung und Emotionen, die Diversität, die Rolle der eigenen Person sowie die Reflexionsfähigkeit.

Die rekonstruierten Wechselwirkungen verdeutlichen Abhängigkeitsverhältnisse zwischen den einzelnen Kategorien. Ist eine Kategorie nur unzureichend ausgebildet, kann dies die Qualität des pädagogischen Wissens beeinflussen. Die Abhängigkeitsverhältnisse verweisen auf die wesentliche Bedeutung eines umfangreichen pädagogischen Wissens für professionelles Handeln.

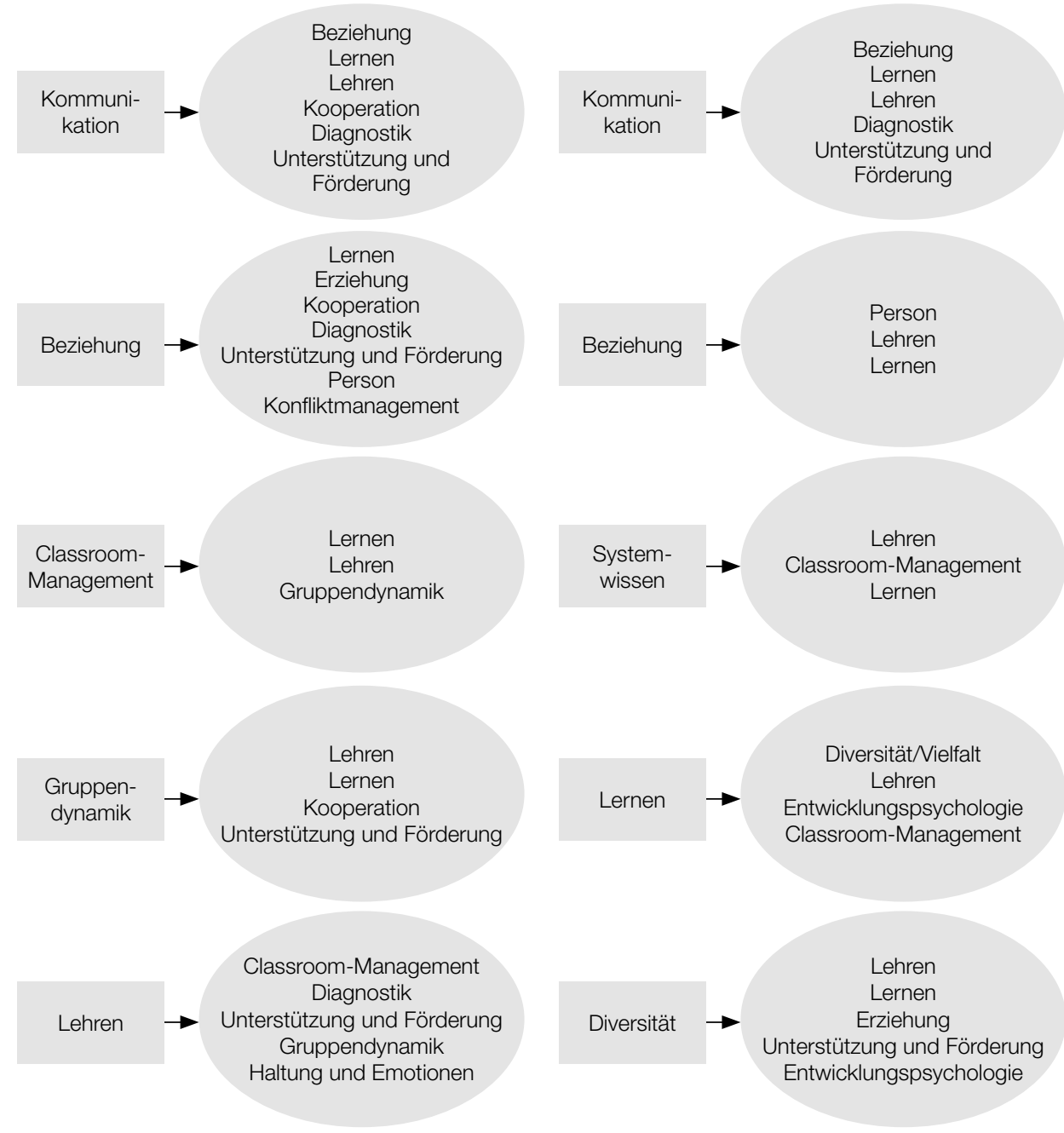

Abb. 108: Wechselwirkungen pädagogischen Wissens 
Basierend auf den Befunden konnten über eine weitere Ausdifferenzierung unterschiedliche Häufigkeiten rekonstruierter Kategorien pädagogischen Wissens festgestellt werden. Es zeigt sich, wie sich die einzelnen Kategorien häufiger bzw. weniger häufig in der Analyse abbilden (vgl. Abbildung 109).

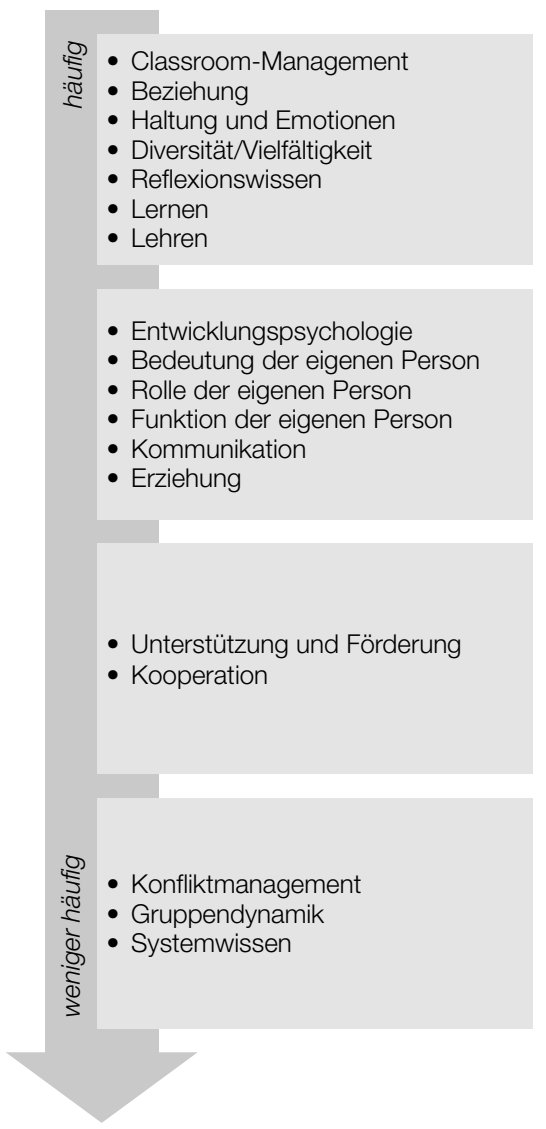

Abb. 109: Häufigkeiten spezifischer rekonstruierter Kategorien pädagogischen Wissens

Die Kategorien, die am häufigsten rekonstruiert werden konnten, sind das Classroom-Management, die Beziehung, die Haltung und Emotionen, die Diversität, die Reflexionswissen und das Lehren und Lernen. Kategorien, die des Öfteren in der Analyse sichtbar wurden, betreffen das Wissen über die Entwicklungspsychologie, die Bedeutung/Rolle/Funktion der Lehrperson, die Kommunikation und die Erziehung. Weniger häufig konnten das Wissen zu Unterstützung und Förderung sowie Kooperation aufgezeigt werden. Am wenigsten häufig wurden die Kategorien Konfliktmanagement, Gruppendynamik und Systemwissen rekonstruiert. Die Anzahl der Häufigkeiten der rekonstruierten Kategorien ist ein Hinweis, wie einzelne Kategorien für die untersuchten Lehrpersonen und ihren Unterricht zentraler sind als andere. Die Bedeutung der Anzahl der Nennung der Kategorien sowie die Bedeutung der Wechselwirkungen pädagogischen Wissens werden in Abschnitt 11.1 im Zusammenhang mit den theoretischen Grundlagen diskutiert. 
Zusammenfassend kann festgehalten werden, dass sich pädagogisches Wissen über ein mehrperspektivisches lern- sowie lehrseitiges Verständnis charakterisiert. Sieben Wissensbereiche konnten entwickelt werden, die das Verständnis der untersuchten Personen gegenstandsadäquat abbilden. Drei Wissensfelder, das Grundlagenwissen, das Erfahrungswissen und das Wissen zum institutionellen Raum, sind für die weiteren Formen (personenbezogenes Wissen, organisatorisch-strukturelles Wissen, interaktionales Wissen, ressourcenorientiert-psychologisches Wissen) grundlegend. Die Rekonstruktion von Wechselwirkungen und Häufigkeiten einzelner Kategorien repräsentieren, wie einzelne Wissensbereiche für die Lehrpersonen eine wesentlichere Bedeutung im Unterrichtsalltag einzunehmen scheinen als andere sowie ein umfangreiches mehrperspektivisches pädagogisches Wissen nötig ist, damit sich pädagogisches Wissen ganzheitlich im Unterricht realisieren kann.

\section{2 Übereinstimmungen und Unterschiede der rekonstruierten Kategorien zwischen den Erhebungsinstrumenten}

Das vorliegende Kapitel präsentiert rekonstruierte Übereinstimmungen und Unterschiede zwischen den Erhebungsinstrumenten. Die Ergebnisse unterscheiden, ergänzen und/oder überschneiden sich bezüglich der Inhalte der rekonstruierten Kategorien pädagogischen Wissens ${ }^{49}$. Im Folgenden werden die Kategorien aus den Interviews und den Beobachtungen anhand der einzelnen Wissensbereiche personenbezogenes Wissen, organisatorisch-strukturelles Wissen, interaktionales Wissen und ressourcenorientiert-psychologisches Wissen separat präsentiert, um aufzuzeigen, inwieweit sich die Ergebnisse aus den jeweiligen Erhebungsinstrumenten ergänzen bzw. voneinander abweichen (vgl. Abbildungen 110, 111, 112, 113).

Der Vergleich der rekonstruierten Kategorien zwischen den Interviews und Beobachtungen im Bereich des ressourcenorientiert-psychologischen Wissens zeigt unterschiedliche Inhalte. Aus der Analyse der Interviews konnten die Kategorien Entwicklungspsychologie, Diversität und Diagnostik und Förderung rekonstruiert werden. Die Beobachtungen verdeutlichen im Vergleich zu den Interviewergebnissen vier Kategorien pädagogischen Wissens: Vielfalt, Umgang mit Problemen, Unterstützung und Förderung sowie Haltung und Emotionen. Die Kategorien Diversität und Vielfalt decken sich bei den Interviews und Beobachtungen und wurden über die Interviews umfangreicher konzeptualisiert. Die bei den Interviews generierte Kategorie Entwicklungspsychologie zeigt sich während den Beobachtungen über die Unterstützung und Förderung. Inhaltlich ergänzen die Beobachtungen die Interviewergebnisse und differenzieren das Verständnis weiter aus. Die Beobachtungen ergänzen die Matrix insofern, als dass sie handlungsbezogene Wissensbereiche aufzeigen, die bei den Interviews von den Lehrpersonen nicht erwähnt wurden. Die Kategorie Diagnostik und Förderung der Interviews wurde bei den Beobachtungen ausdifferenzierter über die Kategorien Umgang mit Problemen sowie Unterstützung und Förderung rekonstruiert. Die Kategorie Haltung und Emotionen bei den Beobachtungen wurde über die Interviews im Zusammenhang mit dem ressourcenorientiert-psychologischen Wissen nicht rekonstruiert.

49 In diesem Zusammenhang können Grenzen der Arbeit aufgezeigt werden. Dies bezieht sich auf die Sichtbarkeit pädagogischen Wissens über die Beobachtungen. Diese Diskussion wird in Kapitel 14 „Grenzen der vorliegenden Arbeit" angeführt. 


\begin{tabular}{|c|}
\hline Interviewmatrix \\
\hline $\begin{array}{l}\text { Grundlagen- und } \\
\text { Theoriewissen }\end{array}$ \\
\hline $\begin{array}{l}\text { Ressourcenorientiert- } \\
\text { psychologisches Wissen }\end{array}$ \\
\hline Entwicklungspsychologie \\
\hline - Entwicklungsstufen \\
\hline Diversität \\
\hline $\begin{array}{l}\text { - } \text { Bedeutung } \\
\text { - Formen } \\
\text { - Strategien } \\
\text { - Umgang mit Kulturen } \\
\text { und Sprachen }\end{array}$ \\
\hline Diagnostik und Förderung \\
\hline $\begin{array}{l}\text { - Gewalt } \\
\text { - Konflikte } \\
\text { - Förderung/Unterstüt- } \\
\text { zung } \\
\text { - Gruppendynamik }\end{array}$ \\
\hline
\end{tabular}

\begin{tabular}{|c|}
\hline Beobachtungsmatrix \\
\hline $\begin{array}{l}\text { Grundlagen- und } \\
\text { Theoriewissen }\end{array}$ \\
\hline $\begin{array}{l}\text { Ressourcenorientiert- } \\
\text { psychologisches Wissen }\end{array}$ \\
\hline Vielfalt \\
\hline $\begin{array}{l}\text { - Umgang mit Kulturen } \\
\text { und Sprachen }\end{array}$ \\
\hline Umgang mit Problemen \\
\hline $\begin{array}{l}\text { - Diagnostische Kom- } \\
\text { petenz } \\
\text { - Handlungs- und } \\
\text { Lösungsstrategien } \\
\text { - Prävention }\end{array}$ \\
\hline $\begin{array}{l}\text { Unterstützung und } \\
\text { Förderung }\end{array}$ \\
\hline $\begin{array}{l}\text { - Diagnostische } \\
\text { Kompetenz } \\
\text { - Handlungs- und } \\
\text { Lösungsstrategien } \\
\text { - Prävention }\end{array}$ \\
\hline Haltung und Emotionen \\
\hline $\begin{array}{l}\text { - Angst } \\
\text { - Druck } \\
\text { - Fürsorge } \\
\text { - Nähe und Distanz } \\
\text { - Wertschätzung }\end{array}$ \\
\hline
\end{tabular}

\begin{tabular}{|c|}
\hline Integrative Matrix \\
\hline $\begin{array}{l}\text { Grundlagen- und } \\
\text { Theoriewissen }\end{array}$ \\
\hline $\begin{array}{l}\text { Ressourcenorientiert- } \\
\text { psychologisches Wissen }\end{array}$ \\
\hline Entwicklungspsychologie \\
\hline - Entwicklungsstufen \\
\hline Diversität/Vielfalt \\
\hline $\begin{array}{l}\text { - Bedeutung } \\
\text { - Formen } \\
\text { - Strategien } \\
\text { - Umgang mit Kulturen } \\
\text { und Sprachen }\end{array}$ \\
\hline Konfliktmanagement \\
\hline $\begin{array}{l}\text { - Gewalt } \\
\text { - Konflikte } \\
\text { - Diagnostische Kom- } \\
\text { petenz } \\
\text { - Handlungs- und } \\
\text { Lösungsstrategien } \\
\text { - Prävention }\end{array}$ \\
\hline $\begin{array}{l}\text { Unterstützung und } \\
\text { Förderung }\end{array}$ \\
\hline $\begin{array}{l}\text { - Diagnostische Kom- } \\
\text { petenz } \\
\text { - Handlungs- und } \\
\text { Lösungsstrategien } \\
\text { - Prävention }\end{array}$ \\
\hline Haltung und Emotionen \\
\hline $\begin{array}{l}\text { - Angst } \\
\text { - Druck } \\
\text { - Fürsorge } \\
\text { - Nähe und Distanz } \\
\text { - Wertschätzung }\end{array}$ \\
\hline
\end{tabular}

Abb. 110: Vergleich rekonsturierter Kategorien zwischen den Interviews und Beobachtungen im Bereich ressourcenorientiert-psychologisches Wissen

Der Vergleich zwischen den Erhebungsinstrumenten zeigt auf, dass sich Kategorien aus den Interviews in den Beobachtungskategorien wiederfinden. Beobachtungen ergänzen das Verständnis pädagogischen Wissens. Die Kategorie Diversität wurde in den Interviews von den Lehrpersonen detaillierter beschrieben, als sie beobachtet werden konnte. Abweichende Strukturen zeigen sich hinsichtlich des organisatorisch-strukturellen Wissens (vgl. Abbildung 111). 


\begin{tabular}{|l|}
\hline \multicolumn{1}{|c|}{ Interviewmatrix } \\
Grundlagen- und Raum \\
Theoriewissen \\
Organisatorisch- \\
strukturelles Wissen \\
Classroom-Management \\
- Leadership \\
- Umgang mit Stö- \\
rungen \\
- Unterrichtsplanung \\
- Unterrichtsablauf (Vor- \\
bereitung, Durchfüh- \\
rung, Nachbereitung) \\
Systemwissen \\
- Bildungspolitik \\
- Gesellschaft \\
$\bullet$ Schulstruktur \\
Curriculum
\end{tabular}
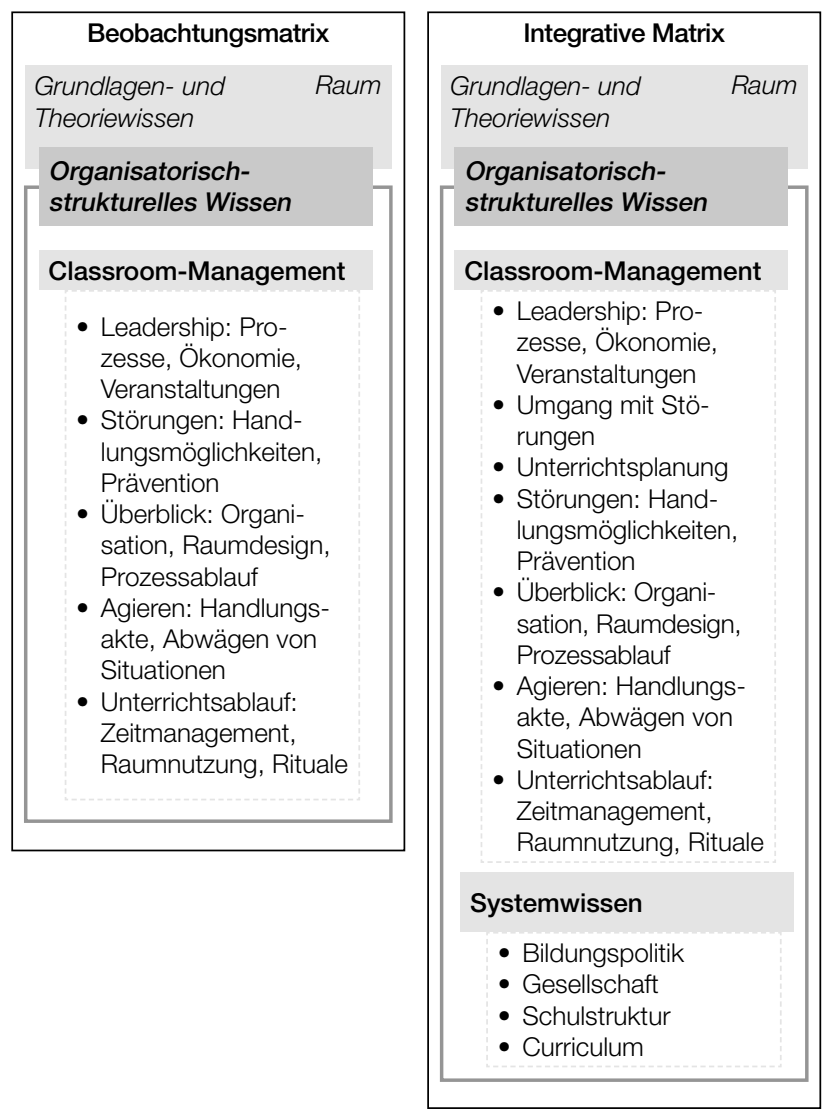

Abb. 111: Vergleich rekonsturierter Kategorien zwischen den Interviews und Beobachtungen im Bereich organisatorisch-strukturelles Wissen

Der Vergleich der rekonstruierten Kategorien zwischen den Interviews und Beobachtungen im Bereich des organisatorisch-strukturellen Wissens (vgl. Abbildung 111) präsentiert über die Rekonstruktion aus unterschiedlichen Erhebungsinstrumenten zum Teil abweichende Ergebnisse. Über die Interviews konnten die Kategorien Classroom-Management und Systemwissen rekonstruiert werden. Bei den Beobachtungen zeigt sich nur die Kategorie Classroom-Management. Diese wurde in einer weiteren Ausdifferenzierung über ergänzende Subkategorien konzeptualisiert.

Der Vergleich illustriert, wie Lehrpersonen beim organisatorisch-srukturellen Wissen mehr Wissen verbalisieren, als über die Beobachtungen sichtbar wurde. Es wird deutlich, wie weiteres spezifischeres Wissen über die Beobachtungen im Bereich des Classroom-Managements aufgezeigt werden konnte. Diese beziehen sich zum Beispiel auf spezifische Handlungsmöglichkeiten und präventive Maßnahmen im Bereich Störungen oder auf das Agieren in unterschiedliche Situationen. Weiteres Wissen konnte über das Zeitmanagement, der Umgang mit Rituale, das Wissen zum Überblick wie die Organisation, das Raumsetting oder zu Prozessabläufe beobachtet werden. Abweichende Strukturen präsentiert die Kategorie personenbezogenes Wissen (vgl. Abbildung 112). 


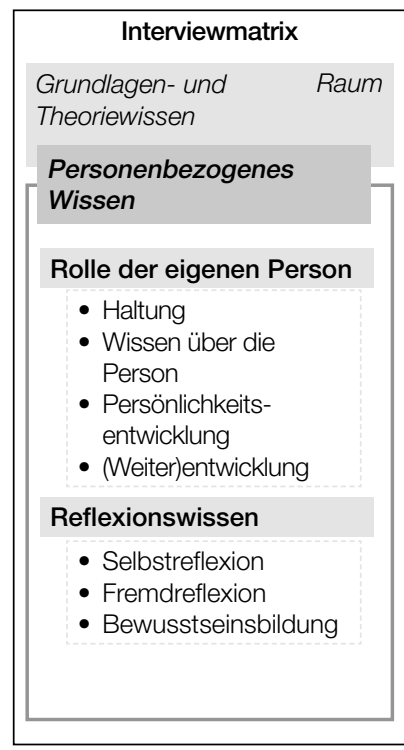

\begin{tabular}{|c|}
\hline Beobachtungsmatrix \\
\hline $\begin{array}{l}\text { Grundlagen- und } \\
\text { Theoriewissen }\end{array}$ \\
\hline $\begin{array}{l}\text { Personenbezogenes } \\
\text { Wissen }\end{array}$ \\
\hline $\begin{array}{l}\text { Bedeutung der eigenen } \\
\text { Person }\end{array}$ \\
\hline • „Sein“ \\
\hline Rolle der eigenen Person \\
\hline $\begin{array}{l}\text { - Einstellung } \\
\text { - Auftreten } \\
\text { - Individuelle Räume } \\
\text { - Individuelle Erfah- } \\
\text { rungen }\end{array}$ \\
\hline $\begin{array}{l}\text { Funktion der eigenen } \\
\text { Person }\end{array}$ \\
\hline $\begin{array}{l}\text { - Interesse am Beruf } \\
\text { - Professionelles } \\
\text { Auftreten }\end{array}$ \\
\hline
\end{tabular}

\begin{tabular}{|l|}
\hline \multicolumn{1}{|c|}{ Integrative Matrix } \\
Grundlagen- und Raum \\
Theoriewissen \\
\hline Personenbezogenes \\
\hline Wissen \\
\hline Bedeutung der eigenen \\
Person \\
• Haltung \\
• "Sein“ \\
- Persönlichkeits- \\
entwicklung \\
\hline Rolle der eigenen Person \\
• Einstellung \\
- Auftreten \\
- Individuelle Räume \\
- Individuelle Erfah- \\
rungen
\end{tabular}

Abb. 112: Vergleich rekonsturierter Kategorien zwischen den Interviews und Beobachtungen im Bereich personenbezogenes Wissen

Der Vergleich zwischen den generierten Kategorien aus den Interviews und Beobachtungen aus der Kategorie personenbezogenes Wissen veranschaulicht, wie über Beobachtungen das Verständnis pädagogischen Wissen erweitert werden konnte. Mithilfe von Interviews wurden die Kategorien Rolle der eigenen Lehrperson und das Reflexionswissen rekonstruiert. Die Beobachtungen differenzieren das Verständnis weiter aus und ergänzen die Matrix anhand der Kategorien Bedeutung der eigenen Person und Funktion der eigenen Person. Pädagogisches Wissen wurde über das Interesse am Beruf, das professionelle Auftreten und Einstellungen der Lehrpersonen sichtbar. Im Zusammenhang mit der Kategorie Rolle der eigenen Person konnte beobachtet werden, wie Lehrpersonen sich bewusst Räume schaffen, in denen sie professionell handeln können. Der Vergleich zwischen den rekonstruierten Kategorien aus den Interviews und Beobachtungen verdeutlicht, wie Beobachtungen das Verständnis pädagogischen Wissens erweitern. Ein ähnliches Bild wird hinsichtlich des interaktionalen Wissens aufgezeigt (vgl. Abbildung 113). 


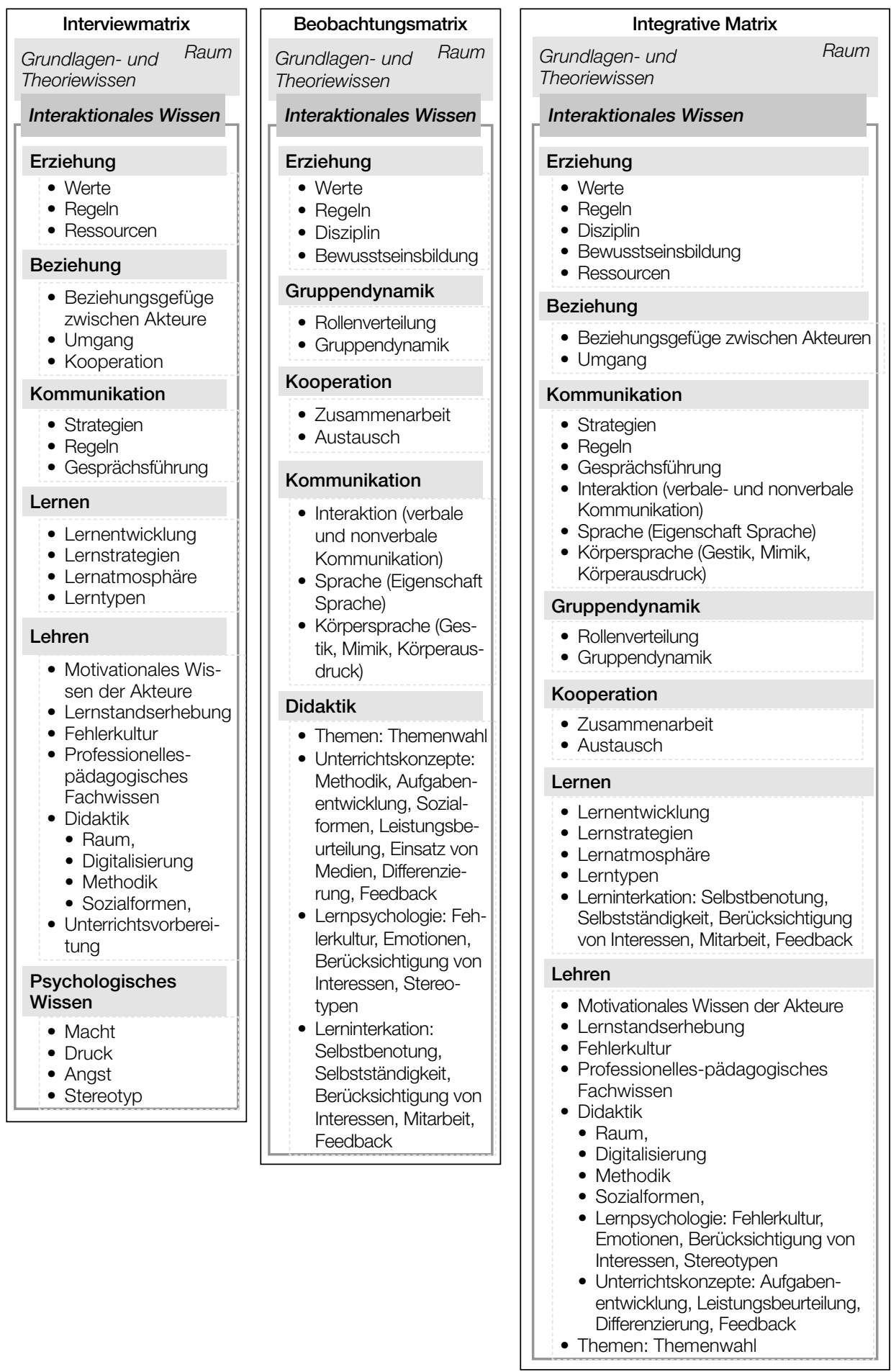

Abb. 113: Vergleich rekonsturierter Kategorien zwischen den Interviews und Beobachtungen im Bereich interaktionales Wissen 
Der Vergleich im Bereich des interaktionalen Wissens zwischen den generierten Kategorien aus den Interviews und Beobachtungen skizziert, wie der Einsatz von zwei Instrumenten das vorliegende Verständnis pädagogischen Wissens erweitert. Das interaktionale Wissen setzt sich aus den Kategorien Erziehung, Beziehung, Kommunikation, Lernen, Lehren sowie psychologisches Wissen zusammen. Die Kategorien Lernen und Lehren werden über die Beobachtungen mit der Kategorie Didaktik ergänzt. Dieses erweitert das Verständnis anhand Wissen zur Themenwahl, zum spezifischen Umgang mit Methoden und Medien, zur Leistungsbeurteilungen, zur Lerninteraktionen sowie zu Lernpsychologischem Wissen. Das gleiche Muster lässt sich bei den Kategorien Erziehung (Disziplin, Bewusstseinsbildung) und Kommunikation (Körpersprache, Interaktion) feststellen. Beobachtungen erweitern das Verständnis pädagogischen Wissens. Die Kategorie psychologisches Wissen wurde nur bei den Interviews im Bereich interaktionales Wissen rekonstruiert. Das Wissen über Beziehung, welches im Rahmen der Interviews rekonstruiert wurde, zeigt sich bei den Beobachtungen über unterschiedlichen Kategorien wie Kooperation, Didaktik, Kommunikation und Erziehung. Dieser Vergleich illustriert, wie Beobachtungen das Verständnis des interaktionalen Wissens erweitern und zusätzliche Kategorien aufzeigen.

Zusammengefasst kann festgehalten werden, dass der angeführte Vergleich der rekonstruierten Kategorien aus Interviews und Beobachtungen den Mehrwert der einzelnen empirischen Zugänge hervorhebt. In jedem Wissensbereich konnte über den Einsatz unterschiedlicher Instrumente das Verständnis pädagogischen Wissens erweitert und damit das Forschungsziel, eine gegenstandsadäquate Abbildung, unterstützt werden. Insgesamt lassen sich drei Muster unterscheiden:

1. Mithilfe der Beobachtung konnte eine reichhaltigere Ausdifferenzierung pädagogischen Wissens bei den Lehrpersonen aufgezeigt werden.

2. Es hat sich ein hohes Maß an kategorialer Kohärenz in der Rekonstruktion basierend auf den beiden empirischen Zugängen gezeigt.

3. Einzelne Kategorien aus den Interviews konnten über die Beobachtungen nicht rekonstruiert werden.

Die rekonstruierten Muster werden hinsichtlich ihrer Bedeutung in Abschnitt 11.2 noch einmal aufgegriffen und unter Berücksichtigung theoretischer Grundlagen diskutiert.

\subsection{Schulformspezifische Rekonstruktionsschwerpunkte}

Ziel des vorliegenden Abschnitts ist die Darstellung schulformspezifischer Rekonstruktionsschwerpunkte pädagogischen Wissens der untersuchten Schulformen. Diese werden in Abbildung 114 veranschaulicht.

Rekonstruktionsschwerpunkte der Grundschule beziehen sich auf die Wissensbereiche zur Diversität, und den Umgang mit Regeln sowie das didaktisches Wissen (z.B. über die Durchführung eines offenen Unterrichts), das Wissen zu den SchülerInnen und zum Classroom-Management sowie das Raumdesign und den Überblick von Lehrpersonen. Lehrpersonen der Neuen Mittelschule charakterisieren sich über die Rekonstruktionsschwerpunkte Benotungssysteme und didaktische Wissensbereiche, bezogen auf den Frontalunterricht oder auf offene Unterrichtsformate.

Die Lehrpersonen aus der Grundschule und der Neuen Mittelschule skizzieren basierend auf der Datengrundlage übereinstimmende Rekonstruktionsschwerpunkte pädagogischen Wis- 
sens. Themen wie das Lernen, mit dem Fokus auf dem selbstständigen, anwendungsorientierten, personalisierten Lernen, die Sprache und Körpersprache, die Aufgabenentwicklung sowie das Wissen zur Zusammenarbeit mit KolegInnen und Eltern werden von den Lehrpersonen der beiden Schulformen hervorgehoben.

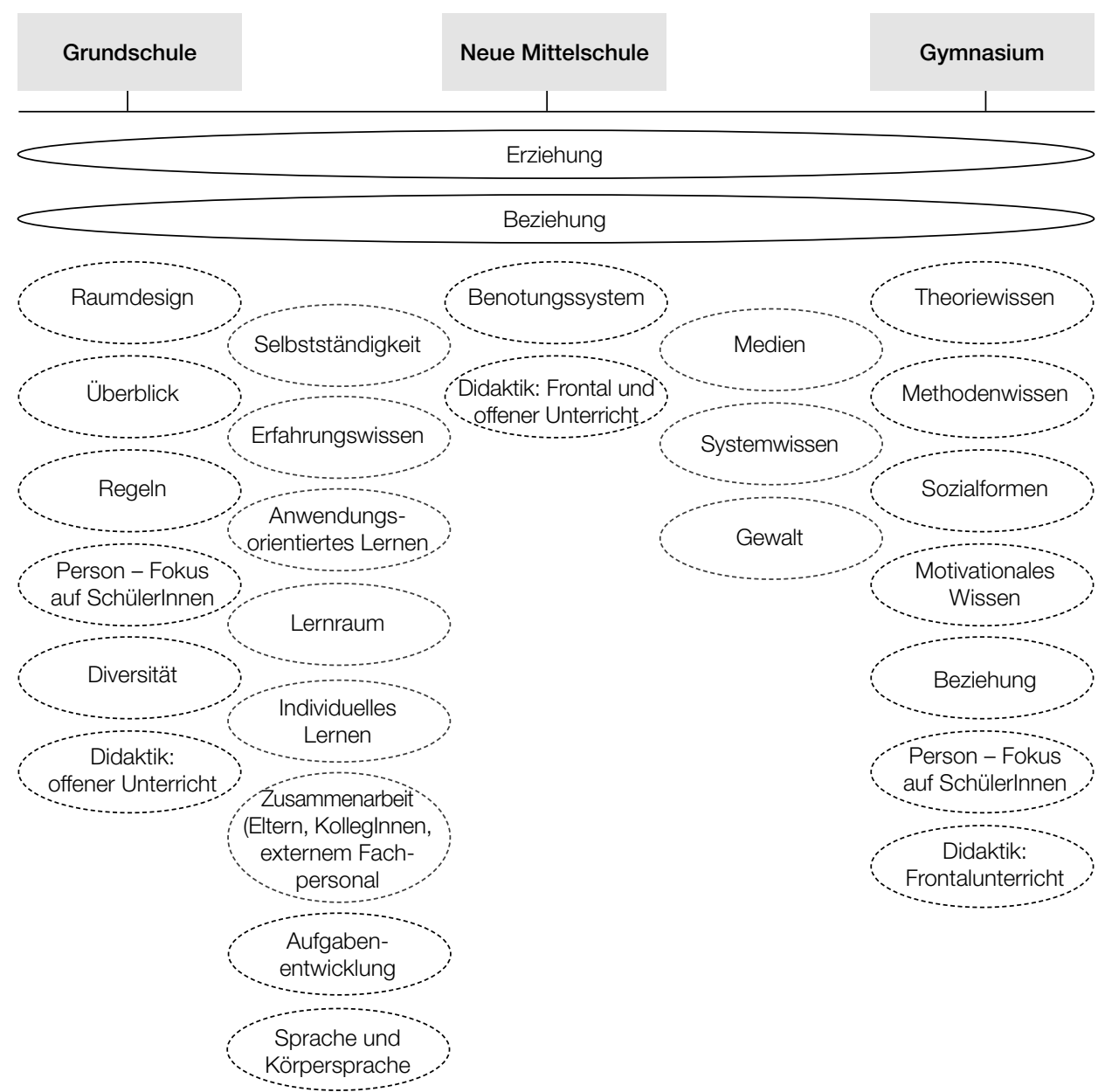

Abb. 114: Schulformspezifische Rekonstruktionsschwerpunkte pädagogischen Wissens

Schwerpunkte der Gymnasiallehrpersonen beziehen sich auf das Grundlagenwissen, das Methodenwissen, das Wissen über unterschiedliche Sozialformen sowie das Wissen zur eigenen (Lehr-)Person. Die Datenerhebung illustriert, wie Lehrpersonen aus der Grund- und der Neuen Mittelschule den Fokus vermehrt auf die SchülerInnen richten. Im Vergleich hierzu stellen die Gymnasiallehrpersonen ihre eigene (Lehr-)Person ins Zentrum des Interesses. Es zeigt sich, wie sie das eigene individuelle Wohlbefinden und ihre Rolle als Lehrperson kontinuierlich hinterfragen. Eine weitere Ausdifferenzierung bei den Gymnasiallehrpersonen bezieht sich auf den Rekonstruktionsschwerpunkt Didaktik mit dem Fokus auf frontale Unterrichtsettings. Inno- 
vative Momente präsentieren sich über den Einsatz unterschiedlicher Methoden und Sozialformen. Gemeinsame Rekonstruktionsschwerpunkte der Neuen Mittelschule und des Gymnasiums beziehen sich auf die Kategorien Medien, Gewalt und Systemwissen.

Die Kategorie Beziehung sowie die Kategorie Erziehung haben sich als grundlegende und wichtige Wissensbereiche aller Schulformen herauskristallisiert. Im Zuge der Rekonstruktion konnte festgestellt werden, wie Lehrpersonen aus den untersuchten Schulformen diese Wissensbereiche unterschiedlich in ihren Unterricht integrieren. Ist das Wissen über Beziehung in allen drei Schulen wichtig, präsentieren die Befunde, wie die einzelnen Schulformen sich innerhalb der Kategorie unterscheiden. Lehrpersonen aus den Schulformen Grundschule und Neue Mittelschule streben vorwiegend die persönliche Beziehung mit den SchülerInnen an. In Relation zeigen die Gymnasiallehrpersonen, wie sie die Beziehung vorrangig über fachliche Aspekte definieren. Mögliche Ursachen finden sich laut Befunden zum Beispiel darin, dass Gymnasiallehrpersonen im Vergleich zu den Lehrpersonen der anderen zwei Schulformen nur für wenige Stunden in einer Klasse unterrichten und deshalb eine weniger intensive Beziehung zu den SchülerInnen aufbauen können. Eine weitere Ursache bezieht sich laut den Befragten auf das Alter der SchülerInnen. Sie beschreiben, wie es mit jüngeren SchülerInnen leichter sei, eine persönlichere Beziehung aufzubauen.

Die Kategorie Erziehung wird in der Grundschule und der Neuen Mittelschule über bewusste Handlungsakte in den Unterricht integriert. Wesentliche Themengebiete sind in Anlehnung an die Befunde gesellschaftliche Werte, die Haltung der SchülerInnen und das Einhalten von Regeln im Unterricht. Auf Gymnasialebene wird die Kategorie Erziehung über einzelne spezifische Merkmale sichtbar. Diese beziehen sich auf die Pünktlichkeit, den Umgang mit Regeln oder das Erziehen zur Selbstständigkeit und zu kritischem Denken.

Die Darstellung macht deutlich, wie Lehrpersonen der Neuen Mittelschule und der Grundschule die Schwerpunkte auf didaktisches Wissen legen. Das selbstständige, anwendungsorientierte und personalisierte Lernen stehen im Zentrum des Interesses. Lehrpersonen aus dem Gymnasium legen ihre Schwerpunkte auf das Grundlagenwissen, das Anwenden von spezifischen Methoden sowie das Wissen über die eigene (Lehr-)Person. Die Kategorien Erziehung und Beziehung sind bei allen drei Schulformen zentral. Allerdings zeigen die befragten Personen der jeweiligen Schulformen ein unterschiedliches Verständnis. Die Bedeutung der Schwerpunkte der einzelnen Schulformen für das pädagogische Wissen von Lehrpersonen werden in Abschnitt 11.1 diskutiert. Im Folgenden werden die rekonstruierten Ergebnisse der empirischen Untersuchung in Verbindung mit den theoretischen Grundlagen integriert und diskutiert. 



\section{Integration und Diskussion}

Im vorliegenden Kapitel werden die Ergebnisse aus Kapitel II im Zusammenhang mit den Fragestellungen und den in der Arbeit diskutierten Grundlagen diskutiert.

Mithilfe des qualitativ-rekonstruktiven Zugangs wurde versucht, pädagogisches Wissen aus der Schulpraxis unter Berücksichtigung bildungswissenschaftlicher, professionsspezifischer und pädagogischer Kontexte zu rekonstruieren und das komplexe heterogene Praxisfeld über kognitive Konzepte und pädagogisches Handeln gegenstandsadäquat abzubilden. Im Zusammenhang mit dem qualitativ-rekonstruktiven Zugang ist zu erwähnen, dass es grundsätzlich nicht möglich ist, ein Feld zu Gänze darzustellen. Dies ist, wie Brüslisauer (2008: 56) im Zusammenhang mit dem Wissensbegriff aufzeigt, schon wissenschaftstheoretisch grundsätzlich nicht möglich (vgl. Kapitel 2.1). Der methodologische Zugang versucht, über die Rekonstruktion des Unterrichtsalltags notwendige Bedingungen pädagogischen Wissens mittels Interviews und Beobachtungen zu konzeptualisieren. Die signifikante Bedeutung der Rekonstruktion wird auch von Neuweg (2014: 585) hervorgehoben, der argumentiert, dass die Logik des Wissens und Handelns von außen rekonstruiert werden sollte.

Der Methodenmix von Interviews und Beobachtungen erweist sich in der vorliegenden Studie als hilfreich. Die Ergebnisse aus den Beobachtungen zeigen eine reichhaltigere Ausdifferenzierung pädagogischen Wissens. Dieses Ergebnis illustriert Übereinstimmung mit der Argumentation von Helmke (2009: 288), der den Beobachtungen einen wesentlichen Stellenwert für die differenzierte Darstellung dynamischer Muster und Prozesse zuschreibt. Beispiele hierfür werden in Abschnitt 11.1 illustriert.

Im Folgenden werden die Ergebnisse aus der vorliegenden Studie zusammen mit den theoretischen Grundlagen anhand der Abschnitte „Konzeptualisierung pädagogischen Wissens“, „Befunde zur Bedeutung pädagogischen Wissens für Lehrpersonen“ und „Einflussfaktoren auf pädagogisches Wissen“ diskutiert.

\section{Konzeptualisierungen pädagogischen Wissens}

Der Abschnitt hat zum Ziel, jene zentralen Konzeptualisierungen pädagogischen Wissens zu diskutieren, die in der vorliegenden Studie rekonstruiert werden konnten.

Mithilfe der Interviews konnten die untersuchten Personen über einen reflexiven Prozess den Begriff „pädagogisches Wissen“ charakterisieren. Die Ergebnisse aus den Interviews verdeutlichen (vgl. Kapitel 8.1), wie Lehrpersonen mithilfe ihres Grundlagenwissens sowie praktischen Wissens pädagogisches Wissen beschreiben. Es zeigt sich, wie schwer es ihnen fällt, spontan auf die Frage „Was verstehen Sie unter dem Begriff pädagogisches Wissen?“" zu antworten. Mithilfe ihrer Erfahrungen, ihrer subjektiven Theorien und der Verknüpfung mit ihrem Grundlagenwissen konnten sie 
den Begriff im Verlauf des Interviews präzisieren. Letzteres bezieht sich vor allem auf bildungspolitische und schulinterne Anforderungen und Regeln, curriculare Bestimmungen sowie auf theoretische Grundlagen von pädagogischen Wissensfeldern. Es konnten über die Analyse unterschiedliche Qualitäten (Stufen der Ausdifferenzierung, Struktur, Ritualisierung) pädagogischen Wissens von den untersuchten Lehrpersonen (vgl. Kapitel 3.2) rekonstruiert werden, die an das Verständnis von De Jong und Ferguson-Fessler (1996) anknüpfen. Einige der untersuchten Personen zeigen in Anlehnung an die Wissensqualitäten Stufen der Ausdifferenzieung und Struktur Unterschiede hinsichtlich ihres strukturierten, vernetzten und mehrperspektivischen Verständnisses. Aus den Befunden kann aufgezeigt werden, wie die Qualität Ritualisierung für alle Lehrpersonen von großer Bedeutung ist. Darin zeigt sich eine Übereinstimmung mit der oben diskutierten Tatsache, dass Lehrpersonen pädagogisches Wissen vorwiegend über ihre rekonstruierten Erfahrungen und die damit eng in Beziehung stehenden Handlungen und Rituale charakterisieren können.

Ergebnisse belegen, wie das pädagogische Wissen in der vorliegenden Arbeit auf einem theoriebasierten und handlungsorientierten Wissensbegriff basiert (vgl. Abbildung 115). Das professionelle Wissen setzt sich im Kontext gegenwärtig diskutierter Anforderungen (vgl. Terhart 2013; Guierriero 2017; Leicht et al. 2018, Schratz 2019) von Lehrpersonen über pädagogisches Handeln und über das von Mittelstraß geforderte Verfügungs- und Orientierungswissen zusammen (vgl. Kapitel 1.2), das nicht nur das prozedurale und deklarative Wissen, sondern in einer weiteren Ausdifferenzierung das Wissen um Ziele und Maxime integriert (vgl. Mittelstraß 1998).

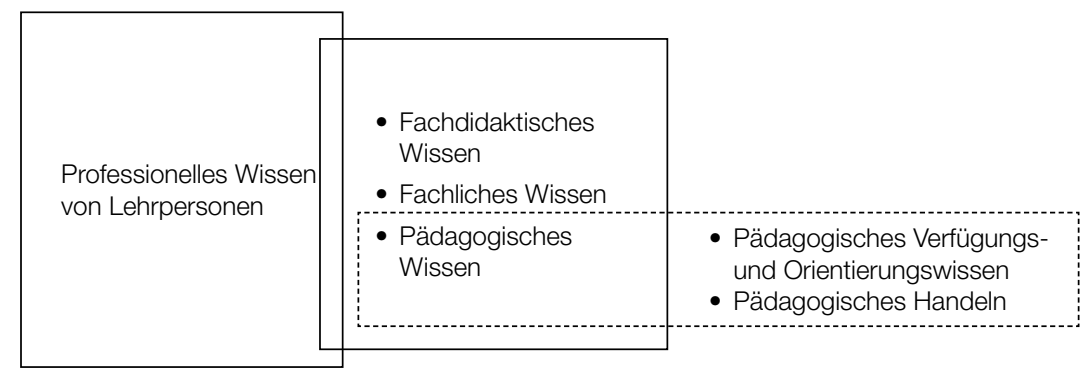

Abb. 115: Theoriebasiertes und handlungsorientiertes Verständnis pädagogischen Wissens aus der vorliegenden Studie

Wissen und Handeln stehen in konstitutiver Beziehung (vgl. Kapitel 5). Für eine adäquate Darstellung der Unterrichtsrealität sind, wie schon Bromme (1992: 10) mit seinem Expertenansatz postulierte, die Berücksichtigung kognitiver Muster und das Handeln sowie der dabei entstehende Blick in die Unterrichtspraxis von wesentlicher Bedeutung. Dies unterstreichen Oelkers \& Tenorth (1991: 19), indem sie Wissen über die Struktur und ihren Gebrauch definieren, sowie Neuweg (2004: 603), der die Möglichkeit aufzeigt, durch einen Blick in die Praxis Wissen und seine Effekte differenzierter und ganzheitlich darzustellen.

Der handlungsorientierte Zugang pädagogischen Wissens lässt sich insbesondere über soziale Interaktionsprozesse zwischen SchülerInnen und Lehrpersonen beobachten. Darüber können Rückschlüsse auf pädagogisches Wissen gezogen werden, die unter Bezugnahme der Konzepte von Mead (1934), Giddens (1984) und Giesecke (2013) abgeleitet werden.

Die Befunde zeigen bei Mead (1934) über ein Konzept des „Me“ und des „I“ sowie über den Zugang der sozialen Praxis von Giddens (1984) Übereinstimmung. Pädagogisches Wissen von Lehrpersonen realisiert sich, wie die Befunde illustrieren, über verbale und nonverbale Interaktionen mit SchülerInnen (vgl. Mead 1934; Giddens 1984; Giesecke 2013). Die Interaktion zwischen Schü- 
lerInnen und Lehrpersonen bedingt eine Entwicklung gemeinsamer Symbole, die pädagogisches Wissen repräsentieren. Dieser Prozess ermöglicht, pädagogisches Wissen in einer bestimmten Raum-Zeit-Konstellation sichtbar zu machen (vgl. Giddens 1984) ${ }^{50}$. Beobachtungen und Interviewergebnisse illustrieren, wie die Lehrpersonen im Klassenzimmer unter Berücksichtigung struktureller Einflussfaktoren wie Ressourcen und Regeln pädagogisch handeln. Pädagogisches Wissen realisiert sich unter Berücksichtigung gesellschaftlicher Anforderungen, schulspezifischer Regelungen und Normen, curricularer Vorgaben sowie des Selbstkonzepts der Lehrperson (vgl. Abbildung 116). Der Interaktionsprozess bedingt, dass jede Lehrperson gemeinsam mit den SchülerInnen unter Berücksichtigung gesellschaftlicher und institutioneller normativer Strukturen und Regeln individuell ihre eigene Umwelt, Beziehung und Identität (vgl. Mead 1934; Giddens 1984; Knoblauch 2005) entwickelt. Es zeigt sich, dass dieser Prozess von den zu unterrichteten SchülerInnen abhängig ist. Pädagogisches Wissen kann sich je nach Klasse und SchülerInnen unterscheiden.

Der Interaktionsprozess ist nicht nur hinsichtlich des Aufzeigens von Wissen relevant. Interaktionen pädagogischen Wissens im Klassenzimmer können, wie die Lehrpersonen in den Interviews betonen, über das Verhalten der SchülerInnen gespiegelt werden. Es zeigen sich hier Parallelen mit dem Modell von Mead (vgl. Kapitel 5.3.1). Seine Idee der Perspektivenübernahme wird durch die Befunde aus Interviews und Beobachtungen deutlich. Spiegelungseffekte, die während der Beobachtungen festgehalten wurden, betreffen die Kategorien Person, Kommunikation, (pädagogische) Interaktion, Didaktik und Classroom-Management. SchülerInnen fungieren als Spiegel pädagogischen Wissens von Lehrpersonen, die über ihr Handeln das Wissen der Lehrepersonen aufzeigen. Spezifische Beispiele hierfür können in Abschnitt 8.5 nachgelesen werden. Die Zusammenführung der Identitäten des „Me“ und des „““ gelingt Mead (1934) zufolge über einen Prozess der Reflexion (vgl. Abbildung 116).

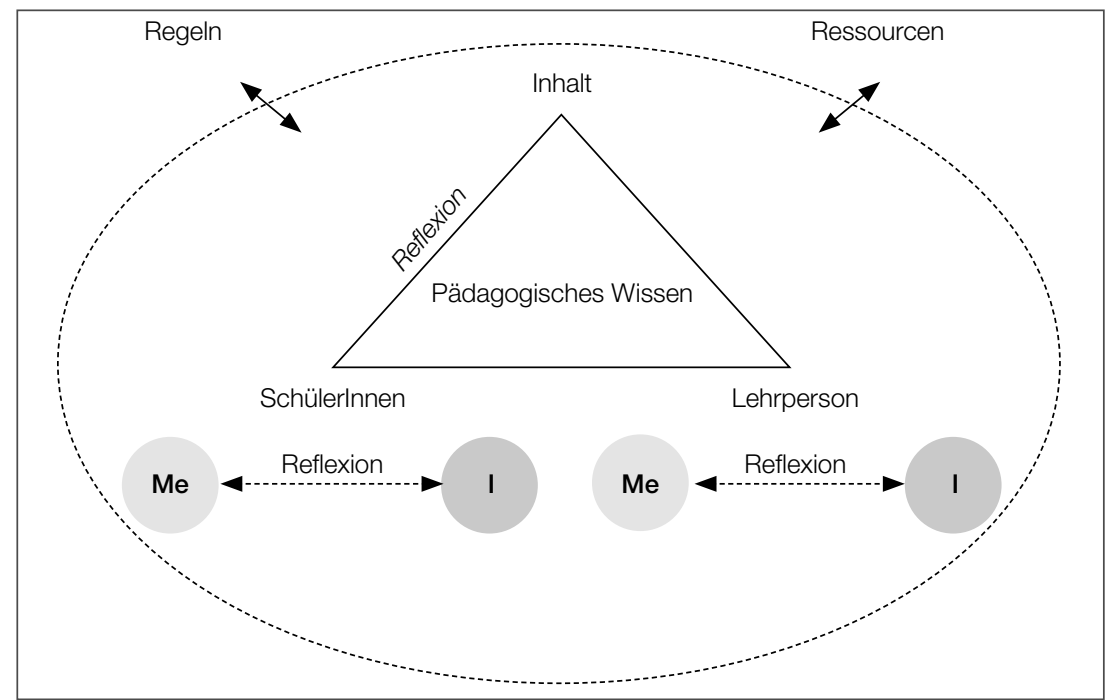

Abb. 116: Rekonstruierter Interaktionsprozess pädagogischen Wissens

50 Rosa (2013) verweist im Zusammenhang mit den technischen Beschleunigungsprozessen auf die Veränderung und Wahrnehmung von Raum und Zeit. Diese beeinflussen das soziale Leben einer Gesellschaft. Schule bzw. die Lehrpersonen müssen auf die Weiterentwicklung von Raum und Zeit angemessen reagieren und ihre Wissensbestände unter Berücksichtigung der Strukturen weiterentwickeln. 
Inwieweit dieser bei den untersuchten Personen bewusst oder unbewusst stattfinden konnte, wurde bei den Beobachtungen nicht festgestellt. Basierend auf den Aussagen der Lehrpersonen kann angenommen werden, dass einige der befragten Lehrpersonen, in Anlehnung an den $\mathrm{Zu}$ gang von Mead, die Reflexionskompetenz besitzen.

Die Reflexionsfähigkeit wird bei Mead, Giddens sowie Neuweg als wichtige Eigenschaft betont, um Handlungsweisen aufzuzeigen und pädagogisches Wissen weiterzuentwickeln (vgl. Mead 1934; Giddens 1984; Neuweg 2014). Sie gilt in Anlehnung an die angeführten handlungstheoretischen Zugänge von Schön (1983), Bauer (1998) und Korthagen (2001) als Schlüsselkompetenz für eine Weiterentwicklung professionellen Wissens. Dieser Ansatz zeigt Parallelen mit dem lebenslangen professionellen Lernen von Lehrpersonen, welches in Kapitel 13 diskutiert wird. Die Reflexionsfähigkeit der Lehrpersonen wird vorwiegend in den Interviews sichtbar. Über die Einnahme einer reflexiven Erzählperspektive charakterisieren sie den Begriff „pädagogisches Wissen“ und benennen spezifische Handlungen, wie sie es im Unterricht anwenden. Wissen wird bei diesem Prozess über verbale und nonverbale Sprache aufgezeigt (vgl. Polanyi 1985; Waldenfels 2000). Der Reflexionsprozess ermöglicht, dass Lehrpersonen über das Benennen ihrer Konzeptualisierungen ihr eigenes pädagogisches Wissen explizit transformieren und weiterentwickeln.

$\mathrm{Zu}$ den beschriebenen Wechselwirkungen nehmen die SchülerInnen in einer weiteren Konzeptualisierung pädagogischen Wissens eine grundlegende Bedeutung ein. Die Lehrpersonen modellieren das pädagogische Wissen als eine lehr- sowie lernseitige Wissensform. Liegt der Fokus der Arbeit auf dem pädagogischen Wissen von Lehrpersonen, verdeutlichen diese, wie sie pädagogisches Wissen mithilfe eines ressourcenorientierten Zugangs unter Berücksichtigung eines bestmöglichen individuellen selbstentdeckenden Lernprozesses und einer individuellen persönlichen Entwicklung von SchülerInnen charakterisieren. Der Unterricht wird nicht nur durch Aufgaben von Lehrpersonen bestimmt, sondern er orientiert sich am Lernprozess der SchülerInnen. Der didaktische Ansatz zeigt Übereinstimmung mit der von Schratz (2009) argumentierten Lernseitigkeit für einen gegenwartsorientierten Unterricht. Die Analyse der Interview- und Beobachtungsdaten verdeutlicht, wie Lehrpersonen den Fokus auf das Lernen ausrichten und die Öffnung der Schule, die Schratz fordert (vgl. Kapitel 2.5), in ihren Konzeptualisierungen pädagogische Wissens berücksichtigen (vgl. Schratz 2019).

\subsection{Kategorien pädagogischen Wissens}

Die in der Forschungsarbeit generierten Kategorien (vgl. Kapitel 8 und 10) werden im Folgenden mit den Grundlagen in Verbindung gebracht und diskutiert. Die Kategorisierung stützt sich auf das mehrperspektivische Verständnis von Wulf (1994: 7), der anthropologisch pädagogisches Wissen mittels unterschiedlicher theoretischer sowie praktischer Formen charakterisiert. Fauser et al. (2007: 10) unterstützen diesen Ansatz und beschreiben, wie für die Unterstützung des Lernens ein mehrperspektivisches Wissen notwendig ist.

Die Ergebnisse der vorliegenden Studie erweitern die vorhandenen Forschungsbefunde zum pädagogischen Wissen (vgl. Kapitel 4). Übereinstimmungen der Ergebnisse mit dem bestehenden Forschungsstand betreffen die Charakterisierung pädagogischen Wissens als ein fachunabhängiges Wissen (vgl. Kunter et al. 2011: 194; Voss et al. 2011; König et al. 2011; Baumert \& Kunter 2011: 39) sowie das mehrdimensionale Verständnis pädagogischen Wissens (vgl. Gudjons 2000; Kunter 2006; Voss et al. 2015; Kunter et al. 2017). Die Analyse der Daten zeigt auf, wie 
Lehrpersonen unabhängig von ihren Unterrichtsfächern pädagogisches Wissen über ein mehrdimensionales Verständnis konzeptualisieren.

Der existierende Forschungsstand präsentiert pädagogisches Wissen (vgl. Shulman 1987, Borki \& Putnam 1996, Morine-Deshimer \& Kents, Bromme; Baumert \& Kunter 2006/2011; Voss et al. 2011; König et al. 2011) anhand planerischer, interaktiver sowie theoretischer Wissensbereiche (vgl. Kapitel 4.1). Diese drei Bereiche finden sich im Verständnis von pädagogischem Wissen der vorliegenden Arbeit wieder. Es zeigt sich eine differenzierte und teilweise eine von den bestehenden Modellen abweichende Strukturierung. Das in der Studie rekonstruierte pädagogische Wissen ergänzt das gängige existierende Modell der planerischen, interaktiven und theoretischen Wissensbereiche. Die Ergänzungen beziehen sich auf die Konzeptualisierung von Formen des Wissens, die Lehrpersonen als Grundlage pädagogischen Wissens charakterisieren, sowie auf eine Erweiterung der bestehenden drei Bereiche des planerischen, interaktiven und theoretischen Wissens.

In der vorliegenden Forschungsarbeit (vgl. Kapitel 10.1) lässt sich pädagogisches Wissen über sieben Formen rekonstruieren (vgl. Abbildung 107). Drei dieser Formen, das Grundlagenwissen, das Erfahrungswissen und das Wissen zum institutionellen Raum, werden als Grundlage der weiteren Formen pädagogischen Wissens modelliert. Die Forschungsbefunde verdeutlichen, dass ohne die grundlegenden Wissensbereiche pädagogisches Wissen sich nicht vollständig ausbilden kann. Sie beschreiben den institutionellen Raum über schulinterne, strukturelle Wissensbereiche, über Regeln und Normen sowie über politische Vorgaben. Das Erfahrungswissen bzw. Orientierungswissen stützt sich auf die Wissensbereiche, die jede Person individuell über Berufserfahrung oder Erfahrungen aus Kindheit/Jugend sowie während der Ausbildung sammelt. Das Grundlagenwissen bzw. das von Mittelstraß so benannte Verfügungswissen (vgl. Mittelstraß 1989: 19) bezieht sich auf das explizite theoretische und handlungsorientierte Wissen von Lehrpersonen.

Die vier weiteren Formen pädagogischen Wissen sind das personenbezogene Wissen, das organisatorisch-strukturelle Wissen, das interaktionale Wissen sowie das ressourcenorientiert-psychologische Wissen. Die einzelnen Kategorien sind in Abbildung 107 dargestellt und werden in Kapitel 8 ausführlich beschrieben.

Die Studie verdeutlicht, dass mithilfe eines qualitativ-rekonstruktiven Zugangs die einzelnen Wissenskategorien differenziert sowie mehrperspektivisch abgebildet werden können. Parallelen zeigen sich zu den existierenden Modellen über planerische und interaktive Wissensfelder. Weitere ausdifferenzierte Felder pädagogischen Wissens beziehen sich auf das personenbezogene und das ressorcentorientiert-psychologische Wissen. Das theoretische Wissen wird in der vorliegenden Konzeptualisierung nicht wie bei Voss et al. (2015) modelliert. Die Analyse der Interviewdaten verdeutlicht ein erweitertes Verständnis von Grundlagenwissen. Dieses wird, wie oben diskutiert, als Basis für die Wissensformen von Lehrpersonen definiert. Die interviewten Lehrpersonen betonen die essenzielle Bedeutung von Theoriewissen für das situationsgerechte Anwenden professionellen pädagogischen Wissens.

Konzeptionierungen von Shulman (1987), Bromme (2001), Baumert und Kunter (2006/2011), Voss et al. (2011/2015), König et al. (2011) oder Kunter et al. (2011/2013), die pädagogisches Wissen anhand der Kategorien Lehren, Lernen, Motivation, Entwicklungspsychologie, Kommunikation, Interaktion, Classroom-Management, Wissen über Methoden, Klassenführung, Diagnostik, Leistungsbeurteilung und Wissen über die Lernumgebung sowie Umgang mit Heterogenität definieren, finden sich in der rekonstruierten Matrix der vorliegenden Forschungsarbeit wieder (vgl. 
Abbildung 107), konnten zum Teil jedoch differenzierter und umfangreicher in der vorliegenden Arbeit definiert werden. Zusätzliche Wissensfelder, die in der vorliegenden Arbeit über die Analyse aufgezeigt werden konnten und die existierenden Modelle ergänzen, beziehen sich neben den zwei Wissensfeldern personenbezogenes Wissen und ressourcenorientiert-psychologisches Wissen auf den einzelnen Kategorien Systemwissen, Erziehung, Beziehung, Gruppendynamik, Kooperation, Konfliktmanagement und (entwicklungs-)psychologische Wissensbereiche.

Die Erweiterung gängiger Modelle pädagogischen Wissens kann anhand unterschiedlicher Kriterien argumentiert werden. Von Bedeutung ist die Rekonstruktion aus dem Unterrichtsalltag mittels Interviews und Beobachtungen sowie die heterogene Datenbasis hinsichtlich der Schulformen und der Unterrichtsfächer (vgl. Kapitel 7.1 und 7.2), die eine vielschichtige Perspektive ermöglichen (vgl. Helmke 2009; Neuweg 2014). Bisherige Untersuchungen basieren, wie in Abschnitt 4.2 diskutiert, vorrangig auf standardisierten Testverfahren mit (angehenden) Lehrpersonen naturwissenschaftlicher Unterrichtsfächer, die nur ein eingeschränktes Modell abbilden. Die Rekonstruktion von Wissen und Handeln ermöglicht pädagogisches Wissen umfassend zu erfassen.

Im Rahmen der Studie konnten rekonstruierte Häufigkeiten einzelner Kategorien pädagogischen Wissens sowie Wirkungsbereiche von Kategorien aufgezeigt werden (vgl. Kapitel 10.1). Die Kategorien, die am häufigsten rekonstruiert wurden, beziehen sich auf das Classroom-Management, die Beziehung, die Haltung und Emotionen, die Diversität, das Reflexionswissen und das Lehren und Lernen. Kategorien, die des Öfteren in der Analyse sichtbar wurden, betreffen das Wissen über die Entwicklungspsychologie, die Bedeutung/Rolle/Funktion der Lehrperson, die Kommunikation und die Erziehung. Weniger häufig konnte das Wissen zu Unterstützung und Förderung sowie Kooperation aufgezeigt werden. Eine geringe Anzahl rekonstruierter Kategorien bezieht sich auf Konfliktmanagement, die Gruppendynamik und das Systemwissen. Gründe für die Häufigkeiten einzelner Kategorien werden mittels zweier Faktoren interpretiert: der individuellen Wissensbereiche der befragten Lehrpersonen sowie der zentralen Bedeutung einzelner Wissensbereiche für den Unterricht. Wissensbereiche, die von den Lehrpersonen am häufigsten genannt werden, verdeutlichen ihre wesentliche Bedeutung und ihre Aktualität für den Unterricht.

Die Wirkungsbereiche beziehen sich, wie in Abbildung 109 dargestellt, auf unterschiedliche Wissenskategorien pädagogischen Wissens. Kategorien, die auf Basis der Befunde aus dem Datenmaterial Wirkungen gegenüber anderen zeigen, sind die Kommunikation, die Beziehung, das Classroom-Management, die Gruppendynamik, das Lehren, das psychologische Wissen, das Reflexionswissen, das Systemwissen, das Lernen und die Diversität. Die angeführte Abbildung (vgl. Abbildung 109) illustriert, mit welchen Kategorien einzelne Wissensbereiche in Beziehung stehen. Es wird ersichtlich, dass sich einzelne Wissenskategorien tark auf die Kategorien Lernen und Lehren auswirken. Dieses Ergebnis zeigt Parallelen zum aktuellen Forschungsstand, der diskutiert, wie pädagogisches Wissen den Lernerfolg sowie die Unterrichtsqualität beeinflusst (vgl. Baumert et al. 2010; Lenske et al. 2015: 225; Guerriero 2017). Weitere Wirkungsbereiche beziehen sich auf Kommunikation, Beziehung, Kooperation, Unterstützung und Förderung, Classroom-Management, Haltung und Emotionen, Diversität, die Rolle der eigenen Person sowie die Reflexionsfähigkeit. Die rekonstruierten Wechselwirkungen verdeutlichen Abhängigkeitsverhältnisse zwischen den einzelnen Kategorien. Ist eine Kategorie nur unzureichend ausgebildet, kann die Qualität pädagogischen Wissens von Lehrpersonen beeinflusst werden. ${ }^{51}$ Die Ab-

51 Die Auswirkungen von Wissensdefiziten pädagogischen Wissens werden in Kapitel 12 bei den Befunden zur Bedeutung pädagogischen Wissens diskutiert. 
hängigkeitsverhältnisse zeigen die Notwendigkeit eines mehrperspektivischen umfangreichen pädagogischen Wissens für professionelles Handeln. Die Ergebnisse liefern einen Forschungszuwachs im Zusammenhang mit der Wirksamkeit pädagogischen Wissens, der bislang als Forschungsdesiderat beschrieben wird.

Die Analyse der zugrundeliegenden Daten skizziert schulformspezifische Rekonstruktionsschwerpunkte pädagogischen Wissens (vgl. Abbildung 116). Das rekonstruierte Wissen von Lehrpersonen der Neuen Mittelschule und der Grundschule (vgl. Kapitel 10.3) mit dem didaktischen Schwerpunkt auf selbstständigem, anwendungsorientiertem, personalisiertem Lernen zeigen Parallelen zu Kochs (2015: 89ff.) Ansatz, wie Wissen sich von den Lernenden angeeignet werden kann. Koch beschreibt, wie mittels unterschiedlicher Lernprozesse der Aneignungsprozess stattfindet und illustriert Übereinstimmung mit den didaktischen Zielen des selbstständigen, anwendungsorientierten Lernens (vgl. Schratz 2009; Helmke 2009, Terhart 2013). Wissen kann in Anlehnung an Holzkamp (2004: 29) und Koch (2015) nur dann angeeignet werden, wenn die SchülerInnen motiviert sind zu lernen und eigenständig Handlungsstrategien entwickeln. Die Schwerpunkte der Grundschule und der Neuen Mittelschule verfolgen diese Ansätze über didaktische Zugänge, wie den offenen Unterricht, und zeigen Parallelen zum diskutierten Verständnis des Lernbegriffs (vgl. Koch 2015). Die Schwerpunkte des Gymnasiums beziehen sich auf das Grundlagenwissen, das Methodenwissen und das Wissen über die eigene (Lehr-)Person, wie das Wohlbefinden und die eigene Rolle. Es können Parallelen zu Koch hergestellt werden, der die Notwendigkeit eines gewussten Wissens einer Lehrperson argumentiert (vgl. Kapitel 2.5). Dieses wird über die beschriebenen Schwerpunkte dargestellt. Innovative Momente des Gymnasiums präsentieren sich vorwiegend über verschiedene methodische Einsatzbereiche, die das Lernen und die persönliche Entwicklung der SchülerInnen, wie sie Rogers und Mikhail fordert, unterstützen und einen ressourcenorientierten Zugang verfolgen (vgl. Rogers 1981; Mikhail 2016). Übereinstimmungen können bei den Gymnasiallehrpersonen explizit zu Mikhails Zugang der Bildsamkeit festgestellt werden, der wie die Befragten die Bildungsfähigkeit der SchülerInnen als wesentlich beschreibt (vgl. Mikhail 2016).

Die Kategorien Erziehung und Beziehung, die sich in allen drei Schulformen als zentral erweisen, stehen in enger Beziehung mit dem von Herbart (1890) beschriebenen Konzept des erzieherischen Unterrichts (vgl. Kapitel 2.5). Die Kategorien Erziehung und Beziehung werden in den jeweiligen Schulstufen unterschiedlich im Unterrichtsalltag berücksichtigt (vgl. Kapitel 10.3 und 11.1), verfolgen jedoch in ihrer Anwendung den beschriebenen Ansatz des erzieherischen Unterrichts. In der Untersuchung bestätigt sich die essenzielle Bedeutung der Erziehung und der Beziehung für den Unterricht und das Lernen, wie theoretisch formuliert wurde (vgl. Herbart 1890; Gruschka 2011; Esslinger-Hinz \& Sliwska 2011; Koch 2015). Ohne Beziehung zur Umwelt kann Lernen nicht ermöglicht werden (vgl. Göhlich und Zirfas 2007: 7). Mithilfe ihres pädagogischen Wissens haben die Lehrpersonen, wie sie in den Interviews betonen (vgl. Kapitel 8.1.7), die Aufgabe, die SchülerInnen zu handlungsfähigen und kommunikativen TeilnehmerInnen gesellschaftlicher Prozesse heranzubilden (vgl. Fend 2006). Parallelen zu Kant (1977), Fend (2006) und Mikhail (2016) die eine Erziehung zur Selbstständigkeit fordern können aufgezeigt werden.

In Anlehnung an Eagleton (2006), der die Schule als Ort der Wissenstradierung beschreibt, stehen Lehrpersonen in einem Spannungsfeld zwischen Tradierung und Innovation. Sie haben die Aufgabe, unter Berücksichtigung bestehender Traditionen neue zukunftsorientierte Wissensbestände zu vermitteln. Soziale, ökonomische sowie politische Transformationsprozesse 
bedingen eine Bedeutungsverschiebung des Faktenwissens hin zu einem handlungsorientierten, anwendungsorientierten Wissen sowie die Vermittlung von Schlüsselkompetenzen (vgl. Helmke 2009) und generischen lernseitigen Kompetenzen (vgl. Schratz 2019). Die schulformspezifischen Rekonstruktionsschwerpunkte zeigen, dass sich die Umsetzung der Anforderungen eher in den Schulformen Grundschule und Neue Mittelschule zeigt. Ursachen für die schulformspezifischen Rekonstruktionsschwerpunkte lassen sich über institutionelle Strukturen und über die Lehrpersonen selbst erklären. Institutionelle Strukturen, politische Vorgaben sowie schulinterne Regeln und curricular festgelegte Bildungs- und Erziehungsaufgaben in den einzelnen Schulformen bedingen, dass Lehrpersonen einzelne Wissensbereiche in den Fokus des Interesses rücken. Eine weitere Ausdifferenzierung zeigt, wie berufsbezogene Möglichkeiten, wie Fort- und Weiterbildungen, sowie individuelle Überzeugungen und die Motivation von Lehrpersonen das vorliegende Verständnis pädagogischen Wissens beeinflussen (vgl. Kapitel 13).

Die Datenanalyse skizziert, wie in Abschnitt 8.2 dargestellt, über die Interviews beschriebene Herausforderungen für pädagogisches Wissen. Diese beziehen sich auf die Wissensbereiche $D i$ versität, Erziehung, Lernen/Lehren, Systemwissen, Haltung und Emotionen, Medien und dem Wissen über die eigene (Lehr)Person. Die rekonstruierten Herausforderungen illustrieren $\mathrm{Zu}-$ sammenhänge mit den in der Arbeit diskutierten gegenwärtigen soziale, ökonomischen und politischen Entwicklungen und den damit einhergehenden Anforderungen von Lehrpersonen. Die Schule ist gefordert, gesellschaftliche Prozesse im Unterrichtsalltag zu berücksichtigen (vgl. Kraler \& Schratz 2012: 89). Hierzu illustrieren die Ergebnisse, dass es von den Lehrpersonen als schwierig und herausfordernd beschrieben wird, in kurzer Zeit auf Transformationsprozesse und den Anforderungen bezogen auf Themen wie Diversität, Medien, Erziehung und Lernen zu reagieren. Aus den Aussagen der Lehrpersonen lassen sich fehlende Fort- und Weiterbildungen, eine fehlende Haltung zum LehrerInnenberuf, wechselnde institutionelle Rahmenbedingungen und mangelnde Unterstützungsstrukturen als Ursachen ausmachen.

Resümierend lässt sich festhalten, dass die entwickelten Kategorien pädagogischen Wissens gängige existierende Modelle über weitere Konzeptualisierungen ergänzen. Pädagogisches Wissen wird von den untersuchten Lehrpersonen als lehr- sowie lernseitiges mehrdimensionales Wissen charakterisiert. Die generierten Kategorien pädagogischen Wissens stehen in Wechselwirkung zueinanderstehen und zeigen gegenseitige Abhängigkeiten. Im Kontext gegenwärtiger Transformationsprozesse und der daraus entstehenden neuen Anforderungen verdeutlicht die Rekonstruktion, dass Lehrpersonen aus der Grundschule und der Neuen Mittelschule mit ihrem pädagogischen Wissen diesen eher gerecht werden. Alle Schulformen streben einen ressourcenorientierten Zugang an, der das Lernen und die Entwicklung zu einer selbstständig handelnden Person unterstützt. Die diskutierten Häufigkeiten und Wechselbeziehungen pädagogischen Wissens verstärken die Bedeutung einzelner Formen des Wissens für einen professionellen und nachhaltigen Unterricht. Die Rekonstruktion macht deutlich, wie einzelnes Wissen im Unterrichtsalltag für die Lehrpersonen relevanter scheinen mag als andere. Die Darstellung von Herausforderungen für pädagogisches Wissen skizziert, dass Lehrpersonen im Kontext kontinuierlicher Transformationsprozesse vor zahlreichen Herausforderungen stehen. Lehrpersonen betonen die Schwierigkeit, schnell und angemessen auf Veränderungsprozesse reagieren zu können.

Im folgenden Abschnitt wird der Fokus auf die Unterschiede bzw. Übereinstimmungen zwischen konzeptionellen Darstellungs- und Begründungsmustern pädagogischen Wissens und der konkreten Handlungsebene diskutiert. 


\section{2 Übereinstimmungen und Unterschiede zwischen konzeptionellen Mustern pädagogischen Wissens und der Handlungsebene}

Dieser Abschnitt hat zum Ziel, die rekonstruierten Unterschiede bzw. Übereinstimmungen zwischen konzeptionellen Mustern pädagogischen Wissens von Lehrpersonen und ihrem konkreten Handeln mit dem expliziten/impliziten, deklarativen/prozeduralen und handlungswirksamen/trägen Wissen (vgl. Kapitel 3.1) zu diskutieren. Mithilfe des qualitativ-rekonstruktiven Zugangs und des Einsatzes von Interviews und Beobachtungen konnten in der vorliegenden Studie kognitive Muster und pädagogisches Handeln untersucht werden. Einen besonderen Mehrwert brachten die Unterrichtsbeobachtungen, die sich für das Gewinnen von differenzierten Erkenntnissen aus dem Schulalltag als besonders wertvoll gezeigt haben (vgl. Helmke 2009: 288). Im Folgenden werden die rekonstruierten Unterschiede zwischen Unterrichtsbeobachtungen und Interviews anhand von drei Mustern (vgl. Kapitel 10.2) diskutiert.

1. Mithilfe der Beobachtungen konnte eine reichhaltigere Ausdifferenzierung pädagogischen Wissens bei den Lehrpersonen aufgezeigt werden.

2. Es hat sich ein hohes Maß an kategorialer Kohärenz in der Rekonstruktion, basierend auf den beiden empirischen Zugängen, gezeigt.

3. Einzelne Kategorien aus den Interviews konnten über die Beobachtungen nicht rekonstruiert werden.

Muster 1 bezieht sich auf Abweichungen zwischen explizitem und implizitem Wissen. Die Ergebnisse zeigen bei den untersuchten Personen, wie sie weniger pädagogisches Wissen verbalisieren können, als sie in der Praxis anwenden. Diese Annahme wird über Fallstudien (vgl. Kapitel 9) weiter spezifiziert und auf Personenebene dargestellt. Im theoretischen Diskurs zeigt sich Uneinigkeit (vgl. Kapitel 3.1.2), inwieweit implizites Wissen von Lehrpersonen verbalisiert werden sollte (vgl. Polanyi 1985; Foppa 1990; Hackl 2004). Die vorliegende Forschungsarbeit kommt zu ähnlichen Ergebnissen wie Polanyi (1985: 87) und Neuweg (2004: 599). Implizites Wissen kann in Anlehnung an die Ergebnisse (vgl. Kapitel 9 und 10.2) nicht vollständig verbalisiert werden. Es wird über das Handeln, der zugrundeliegenden Interaktionen sowie dem körperlichen Ausdruck sichtbar (vgl. Mead 1934; Waldenfels 2000: 169). Die Befragten argumentieren in den Interviews die Notwendigkeit, Wissen verbalisieren zu können. Sie decken sich in ihren Aussagen mit Ryle (1949), Herzog (1995) wie Nolle (2004), die für professionelles Wissen und Handeln explizites Wissen voraussetzen. Basierend auf den Ergebnissen aus der Analyse besitzen die Lehrpersonen mehr implizites als explizites Wissen. Wird in Anlehnung an die angeführte Diskussion mehr explizites pädagogisches Wissen von ihnen argumentiert, lassen sich bei den untersuchten Personen konzeptuelle Unterschiede darstellen. Die Ergebnisse folgen dem vorhandenen Diskurs über die unterschiedliche Bedeutung von implizitem Wissen von Lehrpersonen.

Muster 2 verdeutlicht hohe Übereinstimmung in der Rekonstruktion. Die Befunde zeigen keine Unterschiede zwischen konzeptueller Darstellungsebene und Handlungsebene und zeigen parallelen mit Ryle (1949). Diese Lehrpersonen können genanntes theoretisches Wissen in der Praxis anwenden und kennzeichnen sich über keine Abweichungen hinsichtlich ihrer kognitiven Muster und pädagogischen Handlungsformen.

Bezogen auf Muster 3 konnten Unterschiede in Bezug auf das deklarative und prozedurale Wissen bei Lehrpersonen rekonstruiert werden. Sie benennen pädagogisches Wissen, welches sie in der Praxis anwenden. Dabei konnten jedoch über die Beobachtungen nicht alle genannten Wissensbereiche verifiziert werden (vgl. Fallstudie 2, Kapitel 9). Forschungsergebnisse von Gruber 
\& Renkl (2000) beschreiben theoretisches Wissen, welches in der Praxis keine Anwendung findet, als träges Wissen (vgl. Kapitel 3.1.3). Ungeklärt bleibt an dieser Stelle, inwieweit es sich bei den untersuchten Personen um träges Wissen handelt. Das vorliegende Untersuchungssetting ist zu begrenzt, um träges Wissen identifizieren zu können. Es wären hierfür weitere Untersuchungen notwendig.

Bezugnehmend auf die Frage, was wir (hier: Lehrpersonen) überhaupt wissen können (vgl. Kapitel 2.3), verweisen Kant (1781), Hume (1975) sowie Hoerster (2010) auf das Wissen über den Gebrauch des eigenen Verstands, über Erfahrungen und Reflexion. Dieser Zugang zeigt Parallelen zu den untersuchten Personen, die pädagogisches Wissen in den Interviews vorwiegend über unterschiedliche Erfahrungen aus Kindheit/Jugend/Studium/Beruf und Reflexion charakterisieren. Sie argumentieren, wie Heidenreich (2002) und Brüslisauer (2008) bestätigen, dass sie nicht alles wissen können. Es besteht jedoch die Anforderung, dass sie ein situationsspezifisches und angemessenes pädagogisches Wissen (vgl. Radtke 2000; Helmke 2009) unter Berücksichtigung kultureller gesellschaftlcher Bedingungen (vgl. Heidenreich 2002: 1; Hoerster 2010: 85) besitzen, um ihren Erziehungs- und Bildungsauftrag umzusetzen und professionell auf die gegenwärtigen Anforderungen reagieren zu können (vgl. Bromme 192).

Rekonstruierte Herausforderungen betreffen in diesem Zusammenhang vor allem den Transfer von implizitem zu explizitem sowie vom deklarativen zum prozeduralen Wissen. Die Rekonstruktion demonstriert ein mangelndes Bewusstsein bezüglich unterschiedlicher Wissensformen, ihrem Umgang dieser sowie ihrem Transfer. Der Transfer von Wissen wird im weiterführenden Verlauf (vgl. Kapitel 13) noch einmal aufgegriffen und aufgrund seiner wesentlichen Bedeutung ausführlich diskutiert.

Die kognitiven Muster und das pädagogische Handeln zeigen Unterschiede und Übereinstimmungen pädagogischen Wissens hinsichtlich des expliziten/impliziten Wissens, deklarativen/ prozeduralen Wissens und trägen/handlungswirksamen Wissens. Forschungsergebnisse verdeutlichen konzeptuelle Unterschiede. Die Befunde illustrieren eine Übereinstimmung diskutierter Grundlagen hinsichtlich der Notwendigkeit expliziten Wissens. Allerdings konnte über die Rekonstruktion festgestellt werden, dass bislang das implizite Wissen für das pädagogische Handeln von Lehrpersonen wesentlich ist. Da immer ein Teil implizites Wissen bei Lehrpersonen vorhanden ist (vgl. Polanyi 1985; Waldenfels 2000; Neuweg 2004), ist die Vernetzung von explizitem und implizitem Wissen grundlegend. Je größer das explizite Wissensrepertoire, desto fundierter ist nach Angaben der untersuchten Personen das pädagogische Wissen und Handeln.

Basierend auf der Datengrundlage werden die konzeptuellen Unterschiede über fehlende Fortund Weiterbildungen, unzureichende Unterstützungsmöglichkeiten und zu wenig Reflexionskompetenz bei einzelnen Lehrpersonen beschrieben (vgl. Ryle 1976: 69ff.; Dann 1994; Baumann 2006; Schoenfeld 2010; Mikhail 2016). 


\section{Befunde zur Bedeutung pädagogischen Wissens für Lehrpersonen}

Der vorliegende Abschnitt diskutiert studienbasierte Befunde zur Bedeutung pädagogischen Wissens für Lehrpersonen. Der Fokus liegt auf der Diskussion im Zusammenhang gegenwärtiger Transformationsprozesse und des didaktischen Dreiecks. Forschungsergebnisse von Baumert et al. (2010), Lenske et al. (2015) und Guerriero (2017) untermauern die positive Wirkung pädagogischen Wissens auf SchülerInnenleistungen, die Unterrichtsqualität sowie die persönliche Entwicklung der SchülerInnen. Im Folgenden werden weitere rekonstruierte Wirkungen für den Unterricht dargestellt.

Wie bereits in den Arbeiten von Kraler \& Schratz (2012: 89) diskutiert, gilt die Schule als Spiegel der Gesellschaft. Diese Argumentation wird von den untersuchten Personen in der vorliegenden Studie fortgeführt. Die Befunde aus der empirischen Erhebung verdeutlichen, dass pädagogisches Wissen nicht isoliert von der Gesellschaft und gegenwärtigen Transformationsprozessen betrachtet werden kann, sondern einem kontinuierlichen Veränderungsprozess unterliegt. Die Lehrpersonen berichten in den Interviews (vgl. Kapitel 8.1.4), wie auch Schratz und Schrittesser (2003: 181) verweisen, dass sie als Instrumente gesellschaftlichen Interesses fungieren und kulturelle Werte repräsentieren.

Pädagogisches Wissen wird im Zusammenhang mit den angeführten gegenwärtigen sozialen, wirtschaftlichen und politischen Transformationsprozessen und der im Zentrum stehenden Vermittlung notwendiger Fähigkeiten und Fertigkeiten (vgl. Ontario 2016) von den untersuchten Lehrpersonen als zentraler professioneller Wissensbereich beschrieben (vgl. Kapitel 1.2). Lehrpersonen bestätigen die Argumentation von Shulman (1968/67) sowie Bromme (1992), die pädagogisches Wissen als eine wesentliche professionelle Wissensform konzeptualisieren. Sie argumentieren, dass es keine Lehrperson ohne pädagogisches Wissen geben kann. Der ökonomische Zugang (vgl. Kapitel 1.2) beschreibt das Wissen gegenwärtig als Ressource, die angemessenes Handeln ermöglicht. Das Konzept des Humankapitals für professionelles pädagogisches Wissen und Handeln kann in diesem Zusammenhang übertragen werden.

Gegenwärtige Anforderungen, die Lehrpersonen mithilfe ihres pädagogischen Wissens erfolgreich im Schulalltag umsetzten sollen, beziehen sich nach Angaben von Helmke (2009), Conwey et al. (2009), Terhart (2013) sowie Guierriero (2017), oder wie Schratz (2019) im Zusammenhang mit generischen Kompetenzen fordert, auf unterschiedliche Wissensbereiche. Das Wissen über die Gestaltung von individuellen Lernprozessen, die Umsetzung von anwendungsorientiertem und problemlösungsorientiertem Lernen, der Umgang mit Heterogenität, eine professionelle Haltung, persönliche Weiterentwicklungen, Schulentwicklung, Kooperationen, der Umgang mit gesellschaftlichen Erwartungen, die Aneignung von Soft Skills, angemessene Leistungsbeurteilungen, Technologien und das Classroom-Management steht im Mittelpunkt (vgl. Kapitel 1.2 und 2.5). Der Vergleich mit den rekonstruierten Kategorien pädagogischen Wissens zeigt auf (vgl. Abbildung 107), dass alle geforderten Wissensbereiche bei den untersuchten Lehrpersonen aufgezeigt werden konnten. Sie verdeutlichen damit, dass sie gegenwartsadäquates pädagogisches Wissen besitzen. Das Vermitteln von Soft Skills und die Implementierung neuer Anforderungen in den Unterricht können über das rekonstruierte gegenwartsbezogene Wissen begünstigt werden. Allerdings zeigt sich in diesem Zusammenhang, wie in Abschnitt 11.1 beschrieben, dass die Lehrpersonen der einzelnen Schulformen der Um- 
setzung der Anforderungen unterschiedlich stark nachkommen und mit Herausforderungen zu kämpfen haben.

Paquay und Wagner (2001) definieren sechs Bereiche einer professionellen Lehrrolle über die Merkmale „reflective agent“, „knowledgeable expert“, „skilful expert“, „classroom actor“, „social agent“ und „lifelong learner“. Die in der Studie rekonstruierten Kategorien zeigen, dass sich diese Bereiche vollständig in der generierten Matrix pädagogischen Wissens (vgl. Kapitel 10.1) abbilden. Über die Unterrichtsbeobachtungen konnte sichtbar gemacht werden, dass die untersuchten Personen im Klassenzimmer unterschiedliche Rollen einnehmen und damit die hier angeführten Merkmale einer gegenwärtigen professionellen Lehrperson repräsentieren.

Pädagogisches Wissen ist nicht nur für einen zeitgemäßen Unterricht und für die Realisierung gegenwärtiger diskutierter Anforderungen von Relevanz. Die Rekonstruktion zeigt, wie es die Beziehungen zwischen Lehrperson, SchülerInnen und Lerngegenstand beeinflusst. Auf Basis theoretischer Konzepte (vgl. Hume 1979; Edelmann 2000: 113; Gropengrießer 2003: 35) und in Anlehnung an die Ergebnisse der vorliegenden Studie, ist die Beziehung für das Lernen unabdinglich (vgl. Göhlich und Zirfas 2007: 7). Die Qualität der Konstellation des didaktischen Dreiecks und die zugrundeliegende Beziehung, die in einem guten Unterricht realisiert werden kann, können über das pädagogische Wissen befördert werden (vgl. Abbildung 117).

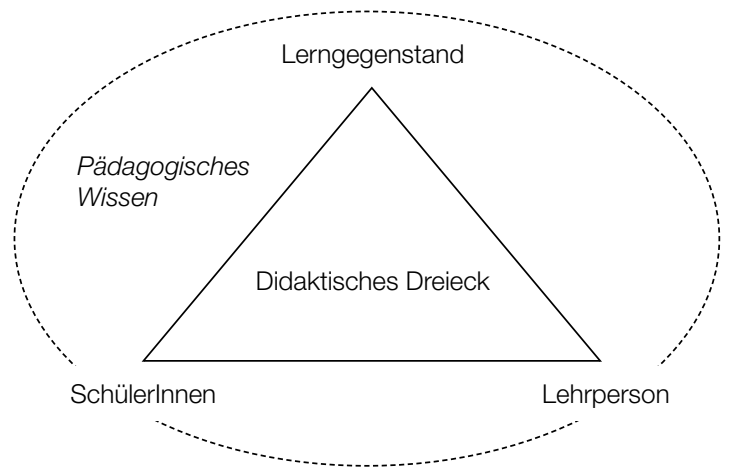

Abb. 117: Verhältnis pädagogisches Wissen und didaktisches Dreieck

Die Befunde illustrieren, dass sich über pädagogisches Wissen einzelne Prozesse im didaktischen Dreieck besser entfalten können und das Beziehungsgefüge sowie die Lernprozesse von SchülerInnen verstärkt werden (vgl. Baumert et al. 2010; Guerriero 2017). Wird pädagogisches Wissen in der vorliegenden Studie (vgl. Kapitel 11.1), wie von Schratz (2009) gefordert, über eine lernseitige Perspektive konzeptualisiert, unterstützt der lernseitige Fokus das Beziehungsgefüge zwischen Lehrperson, SchülerInnen und Lerngegenstand. Abbildung 118 veranschaulicht, wie die lernseitige Perspektive pädagogischen Wissens über fehlendes pädagogisches Wissen gestört werden kann. 


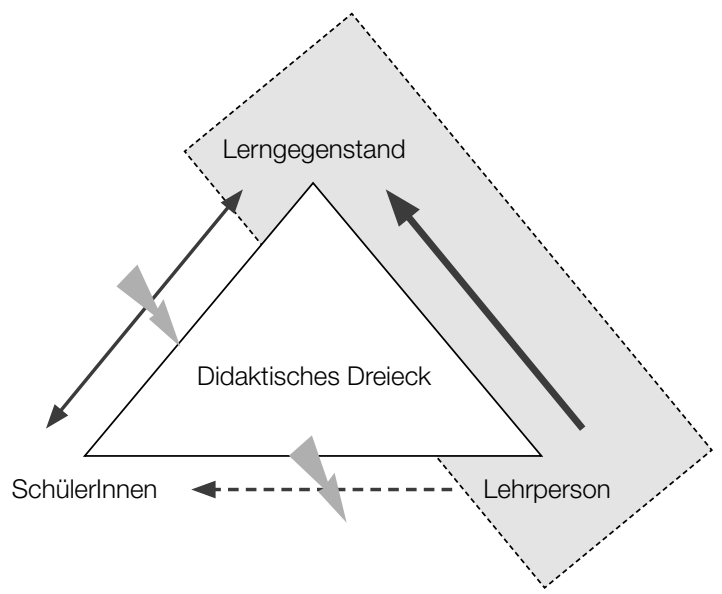

Abb. 118: Auswirkungen von fehlendem pädagogischem Wissen auf das didaktische Dreieck

Defizite pädagogischen Wissens können zu einer einseitigen lehrseitigen Beziehung führen, bei der sich die Lehrpersonen, ohne Rücksichtnahme auf die SchülerInnen, vorrangig auf die Vermittlung des Lerngegenstands konzentriert. Individuelle Förderungen sowie der explizite Einsatz von Lerninteraktionen werden nicht berücksichtigt. Vielmehr liegt der Fokus auf der Vermittlung der Inhalte. Dies bedingt aufseiten der SchülerInnen eine fehlende Beziehung zur Lehrperson sowie zum Lerngegenstand und führt zu einem eingeschränkten Lernprozess (vgl. Petzelt 1964; Koch 2015).

In Anlehnung an die Diskussion zum didaktischen Dreieck können in einer weiteren Ausdifferenzierung Auswirkungen von Wissensdefiziten pädagogischen Wissens auf soziale Interaktionen analysiert werden (vgl. Mead 1934; Giddens 1984; Giesecke 2013). Die vorliegende Arbeit diskutiert die Realisierung pädagogischen Wissens über soziale Interaktionsprozesse in Anlehnung an Mead (1934), Giddens (1984) und Giesecke (2013). Dieser Prozess kann bei unzureichender Ausbildung pädagogischen Wissens nicht über die sozialen Beziehungen aufgezeigt werden. Die zentralen InteraktionspartnerInnen, die SchülerInnen, entwickeln mit dem Lerngegenstand und der Lehrperson einen Interaktionsprozess. Soziale Interaktionen führen Petzelt (1964) zufolge zu einem Lernprozess. Dieser wird bei unzureichenden Interaktionen verringert. Der Interaktionsprozess nimmt in Anlehnung an das Modell des „Me“ und des „I“ von Mead (1934) eine wichtige Bedeutung für die Lehrperson ein. Über einen Interaktionsprozess kann mithilfe von Reflexion und den SchülerInnen pädagogisches Wissen einer Lehrperson gespiegelt werden. Fehlende Beziehungsstrukturen zwischen SchülerInnen und Lehrpersonen verhindern die Identifikationsbildung pädagogischen Wissens einer Lehrperson.

Weitere Bedeutungen pädagogischen Wissens für den Unterricht werden über die Ergebnisse des Kapitels „Auswirkungen von Defiziten pädagogischen Wissens“ (vgl. Kapitel 8.4) aufgezeigt. Lehrpersonen skizzieren in den Interviews, wie ein Unterricht mit unzureichendem pädagogischem Wissen sich gestaltet. Dieser ist handlungsarm, einseitig und reflexionsarm und bildet damit die von Fend (2006) und Schratz (2019) geforderten Fähigkeiten und Fertigkeiten nur unzureichend ab. Die untersuchten Lehrpersonen selbst benennen diesen Zustand als „pädagogisches Halbwissen“ (It 25) (vgl. Kapitel 8.1.2). Die Wissensdefizite können mit dem Verständnis von Weinstein und Weinstein (1978: 151ff.) argumentiert werden. Sie gehen da- 
von aus, dass bestimmtes Wissen nicht in unserer Aufmerksamkeit liegt. ${ }^{52}$ Das vorhandene Nichtwissen kann bei den Lehrpersonen aufgrund fehlenden theoretischen Wissens über Beziehungsstrukturen und dessen Auswirkungen argumentiert werden.

52 Die Diskussion über das Nichtwissen ist nicht Gegenstand der vorliegenden Arbeit. Pädagogisches Wissen lebt jedoch, wie die Lehrpersonen aufzeigen, in einem Spannungsverhältnis zwischen Wissen, Halbwissen und Nichtwissen. Für den Lehrberuf und pädagogisches Wissen zentral ist die Argumentation von Radtke (2000) und Giesecke (2013), die darauf verweisen, dass Wissen immer situationsspezifisch im Sinne des Erziehungs-und Bildungsziels ausgerichtet werden muss und sich im Sinne eines lebenslangen Lernprozesses kontinuierlich weiterentwickeln muss. 


\section{Einflussfaktoren auf pädagogisches Wissen}

Das Ziel dieses Kapitels ist die Darstellung von Einflussfaktoren auf das pädagogische Wissen, die im Rahmen der Studie rekonstruiert werden konnten. Wie in der Einleitung beschrieben (vgl. Kapitel 1), können unterschiedlichste LehrerInnenpersönlichkeiten erfolgreich unterrichten. Dieser Ansatz lässt sich auf das pädagogische Wissen übertragen. Lehrpersonen verdeutlichen, wie Bromme (1992) schon argumentiert, individuelle Konzepte pädagogischen Wissens, die sich in ihren Zugängen und Charakterisierungen unterscheiden. Die Analyse der einzelnen Lehrpersonen, wie in den Fallstudien auf Individualebene exemplarisch dargestellt (vgl. Kapitel 9) bestätigt, dass sich jede Lehrperson über unterschiedliche Zugänge und Schwerpunkte pädagogischen Wissens charakterisieren lässt.

Pädagogisches Wissen basiert auf individuellen Wissensstrukturen, die über unterschiedliche Faktoren beeinflusst werden. Einflussfaktoren, betreffen die individuellen Erfahrungen einer Lehrperson, die Reflexionsfähigkeit, das Selbstkonzept einer Lehrperson, gesellschaftliche Entwicklungen, strukturelle institutionelle Rahmenbedingungen sowie die Ausbildung, Fort- und Weiterbildungen von Lehrpersonen (vgl. Abbildung 119).

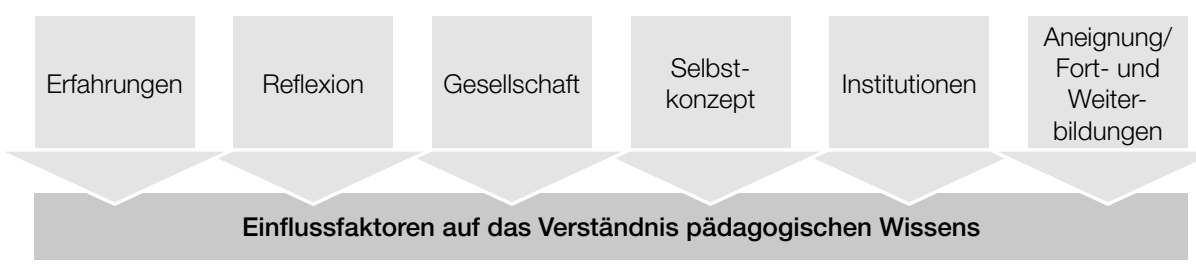

Abb. 119: Einflussfaktoren auf pädagogisches Wissen

Lehrpersonen müssen pädagogisches Wissen aufgrund gesellschaftlicher Entwicklungen stetig weiterentwickeln. Sie sind gefordert, Veränderungsprozesse und die damit verbundenen Herausforderungen in den Schulalltag zu integrieren (vgl. Eagleton 2006: 14ff.). Das Wissen von Lehrpersonen befindet sich in einem stetigen Wandel (vgl. Gudjons 2000: 33ff.). Gegenwärtige Einflussfaktoren werden Terhart (2013: 55f.) zufolge in unterschiedliche Bereiche eingeteilt. Diese beziehen sich auf die zunehmende geografische, soziale und kulturelle Mobilität, das Streben nach der Gestaltung eines selbstbestimmten aktiven Lebens sowie die zunehmende Bedeutung von umfassenden Schlüsselqualifikationen und kommunikativen Kompetenzen (vgl. Kapitel 2.1). Weitere relevante Einflussfaktoren auf die Schule beziehen sich auf technologische Entwicklungen und die Wissenskonjunktur (vgl. Thiel 2007: 153; Eriksen 2001: 3ff.). Die Lehrpersonen berichten in den Interviews von gesellschaftlichen, politischen, ökonomischen Einflussfaktoren, die ihr Wissen verändern. Lehrpersonen als Spiegel der Gesellschaft (vgl. Kraler \& Schratz 2012: 89) müssen auf die Wandlungsprozesse und die damit einhergehenden neuen Herausforderungen reagieren, die nach Angaben der Lehrpersonen pädagogisches Wissen beeinflussen. Inwieweit Lehrpersonen gegenwärtiges Wissen umsetzen, wurde in Kapitel 12.2 diskutiert.

Als weiterer Einflussfaktor konnten aus der Studie die institutionellen Rahmenbedingungen rekonstruiert werden. Wie in Abschnitt 10.3 dargestellt, beeinflussen institutionelle Vorgaben und curriculare Anforderungen die rekonstruierten Kategorien pädagogischen Wissens. Bildungspolitische Verordnungen sowie individuelle, strukturelle Rahmenbedingungen und cur- 
riculare Vorgaben der jeweiligen Schulen wirken sich auf pädagogische Wissensbereiche (zum Beispiel Team-Teaching, Bewertung oder didaktische Konzepte) der einzelnen Lehrpersonen aus. Diese beziehen sich auf (neue) Regelungen und Vorgaben sowie interne Schulprozesse. Die institutionellen Rahmenbedingungen nehmen in der vorliegenden Studie eine wesentliche Bedeutung ein. Sie werden als Grundlage für die rekonstruierten Kategorien pädagogischen Wissens modelliert (vgl. Kapitel 10.1 und 11.1).

Ein weiterer Einflussfaktor auf pädagogisches Wissen ist das Selbstkonzept von Lehrpersonen. Unterschiedliche Zugänge (vgl. Giddens 1984/1977; Rogers 1981, Groeben 1988; Platon 2005; Augustinus 2005; Schoenfeld 2010; Mikhail 2016) unterstützen den Ansatz, dass pädagogisches Wissen und Handeln relational mit der Haltung sowie den Einstellungen einer Person in Verbindung stehen. Die Selbstbildung erweist sich für die Entwicklung einer pädagogischen Haltung als zentral (vgl. Groeben 1988; Mikhail 2016: 42ff.). Wie auch Groeben (1988) diskutiert, präsentieren die Ergebnisse, wie das pädagogische Wissen der Lehrpersonen und ihr Handeln stark von eigenen Einstellungen und Haltungen abhängig ist. Weitere Parallelen zeigen sich zwischen den Befunden und der Forderung von Rogers (1981) und Schmied (2016) nach einem ressourcenorientierten Umgang. Die von Rogers (1981) konzipierte Dreiteilung Kongruenz, Akzeptanz sowie Empathie sowie Mikhail (2016), der an diesem Konzept anknüpft und das Kriterium der Bildsamkeit ergänzt (vgl. Kapitel 5.1), präsentieren eine pädagogische Haltung mit dem Ziel, das Lernen und die Entwicklung der Kinder zu unterstützen. Wie sich über die Befunde dargestellt, wird für die Weiterentwicklung der Haltung an die individuelle Motivation der Lehrpersonen appelliert. Es zeigt sich über die empirische Untersuchung, dass Lehrpersonen die eine pädagogische Haltung besitzen, einen wertvollen pädagogischen Umgang pflegen und qualitativ hochwertigen Unterricht geben. Diese Lehrpersonen hinterfragen über Reflexion ihr eigenes Denken und Handeln und tauschen sich eher mit KollegInnen aus. Es lässt sich rekonstruieren, wie diese bei einer ausgeprägteren pädagogischen Haltung ein komplexeres mehrdimensionales, gegenwartsadäquates Verständnis sowie eine höhere Qualität (vgl. Kapitel 11) pädagogischen Wissens besitzen (vgl. Klieme et al. 2003; De Jong \& Ferguson-Hessler 1996)

Die Reflexionsfähigkeit beeinflusst das pädagogische Wissen von Lehrpersonen. Übereinstimmungen zeigen sich zu Cochran-Smith und Lytle (1999), Neuweg (2002), Minnameier (2009: 333) sowie Sembill und Seilfried (2009: 345f.). Diese beschreiben die Reflexionsfähigkeit als notwendige kognitive Voraussetzung pädagogischer Professionalität und definieren sie als handlungsleitend. Die Reflexionsfähigkeit bezieht sich in der Studie auf unterschiedliche Ebenen der Reflexion (vgl. Abbildung 120).

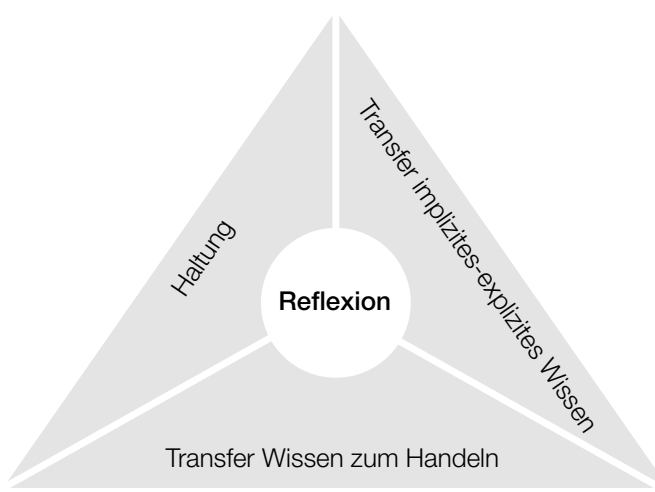

Abb. 120: Ebenen der Reflexion als Einflussfaktor auf pädagogisches Wissen 
Eine Ebene bezieht sich auf die Reflexion und ihren Einfluss auf die Haltung, wie oben beschrieben wurde. Die weiteren Ebenen sind der Transfer vom Wissen zum Handeln sowie der Transfer zwischen explizitem und implizitem Wissen. Der Transferprozess unterstützt, wie Bauer (1998), Schön (1983), Cochran-Smith und Lytle (1999), Klieme et al. (2003) und Gropengießer (2003) in ihren Modellen diskutieren, eine Steigerung des professionellen Lernens einer Lehrperson.

Der Transfer vom Wissen zum Handeln wurde in der vorliegenden Studie mithilfe von Interviews aufgezeigt. Über eine reflexive Auseinandersetzung mit dem Begriff „pädagogisches Wissen“ wird über das Nachdenken neues Wissen generiert (vgl. Neuweg 2014). Die Frage, wie Lehrpersonen das pädagogische Wissen charakterisieren sowie im Unterricht anwenden, bedingt, dass sie ihr eigenes Wissen und Handeln reflektieren sowie neue Wissensbereiche generieren. Dieses Ergebnis verdeutlicht Parallelen mit den diskutierten Grundlagen von Mead (1934), Schön (1983), Giddens (1984), Bauer (1998) und Cochran-Smith und Lytle (1999). Sie beschreiben, wie über einen reflexiven Prozess und die Einnahme einer Metaperspektive pädagogisches Wissen sichtbar gemacht werden kann. Mithilfe eines reflexiven Dialogs, wie ihn Schön (1983) argumentiert, generieren Lehrpersonen neues Wissen, welches sie in ihrem Unterricht anwenden können.

Ein weiterer Prozess, der durch Reflexion unterstützt wird, ist der Transfer vom impliziten zum expliziten Wissen. Reflexion ermöglicht, wie Mead bei seinem Modell von „Me“ und „I“ diskutiert, wie über Reflexion implizites Wissen sich zu explizitem Wissen entwickeln kann. Zu ähnliche Ergebnissen kamen Schön (1983) sowie Bauer (1996) im Zuge ihres reflexiven Modells. Lehrpersonen verdeutlichen, wie das Ausbrechen aus einer Situation und die Rekonstruktion aus einer Metaperspektive eigene Wahrnehmungs- und Deutungsmuster unterstützen und implizites Wissen zu explizitem Wissen entwickeln (vgl. Schön 1983). Implizites Wissen kann über verkörpertes Wissen mit Hilfe der Reflexion zu explizites Wissen transformiert werden (vgl. Neuweg 1999; Waldenfels 2000). Diese Prozesse werden insofern als wichtig argumentiert, als Herzog (1995) und Nolle (2004) für professionelles Handeln explizites Wissen voraussetzen. Die Reflexionsfähigkeit wird in Anlehnung an die Diskussion in der vorliegenden Arbeit als wesentlicher Einflussfaktor für die Qualität pädagogischen Wissens von Lehrpersonen argumentiert.

Pädagogisches Wissen wird über den Aneignungsort und die biografischen individuellen Erfahrungen einer Lehrperson beeinflusst. Sie zeigen Übereinstimmung mit dem Prinzip des selbstgesteuerten Lebenswegs von Beck (1986: 216) sowie der Ichwerdung von Giddens (1997: 301). Wie in Abschnitt 8.3 aufgezeigt und in den Fallstudien (vgl. Kapitel 9) differenziert dargestellt, berichten die Interviewten von unterschiedlichen Aneignungsorten pädagogischen Wissens und beschreiben den Aneignungsprozess anhand von vier Stadien. Der erste Erwerb findet bei einigen Lehrpersonen (Stadium 1) in der Kindheit und im Jugendalter über Erziehung, über Einflüsse der Familie, über das Freizeitverhalten und über eigene Erfahrungen aus der Schulzeit statt. Obwohl es sich um keinen professionsspezifischen Wissenserwerb handelt (vgl. Kapitel 2.4) greifen sie auf frühe Erinnerungen und das dort erworbene Wissen zurück. Vor allem Erfahrungen und Wissen aus der Kindheit oder Jugendzeit wie Schulerfahrungen sind für das eigene pädagogische Wissen relevant. Das Studium (Stadium 2) wird hinsichtlich pädagogischer Wissensbestände als sekundär eingeschätzt. Hier zeigen sich Abweichungen zum bestehenden Forschungsstand. Lehrpersonen argumentieren, dass sie sich über das Studium nur vereinzelt pädagogisches Wissen aneignen konnten. In den Grundlagen wird über das Studium ein grundlegender Aufbau theoretischer, bildungswissenschaftlicher, pädagogischer sowie psychologischer Wissenskonzepte diskutiert (vgl. Hohenstein et al. 2014). Insbesondere der 
Wissensaufbau des deklarativen sowie expliziten Wissens sollte Hohenstein zufolge über die Ausbildung und Schulpraktika unterstützt werden (vgl. Hohenstein et al. 2014). Diskutieren Korthagen \& Kessels (1999) sowie Wang et al. (2011) die schulpraktischen Erfahrungen während des Studiums als besonders bedeutend, wurde diese Annahme von den Befragten nur vereinzelt geteilt. Ursachen der fehlenden Bedeutung der Ausbildung können im Zusammenhang unzureichend vernetzter konzeptueller Modelle pädagogischen Wissens, die im Studium angeeignet wurden sowie unzureichender Erfahrungs- und Reflexionsräumen, argumentiert werden. Zentraler Aneignungsort professionellen pädagogischen Wissens ist nach Angaben der Lehrpersonen das Berufsleben (Stadium 3). Über Praxiserfahrungen, den Austausch mit Freunden und KollegInnen sowie die Wissensaneignung von Grundlagenwissen konnten sie ihr Wissen erweitern. Sie verweisen nicht spezifisch auf einen lebenslangen professionellen Lernprozess (vgl. European Commission 2013), betonen jedoch die essenzielle Bedeutung der Praxiserfahrungen sowie Fort-und Weiterbildung und zeigen Übereinstimmung mit Keller-Schneider \& Hericks (2014: 394), die ein höheres Kompetenzniveau und eine Erweiterung und Neustrukturierung des Denkens über Erfahrungen betonen. Praktisches Wissen sowie das grundlegende implizites Wissen kann weiter ausgebaut werden (vgl. König et al. 2014a; Schneider \& Plasman 2011). Die Studie skizziert, wie die untersuchten Personen pädagogisches Wissen mithilfe von Wissen, welches sie über Fort- und Weiterbildungen gewonnen haben, konzeptualisieren (Stadium 4). Lehrpersonen, die in ihrer beruflichen Laufbahn regelmäßig Fortbildungen absolvierten, konnten höhere Wissensqualitäten und einen professionelleren Umgang mit den Formen des Wissens vorweisen als jene ohne Fortbildungen. Sie verdeutlichen hohe Übereinstimmung mit den Grundlagen bezüglich der relevanten Bedeutung von Fort- und Weiterbildungen für einen professionellen Lernprozess (vgl. Lipowsky 2014: 514). In einer weiteren Ausdifferenzierung berichten die Lehrpersonen, wie über das Einholen von Feedback von SchülerInnen, Eltern sowie KolegInnen das Wissen weiterentwickelt werden kann (vgl. Dunn \& Shriner 1999). Die Aneignung über Erfahrung wird von allen untersuchten Personen als der zentrale Aneignungsort beschrieben. Sie stützen sich mit ihrer Argumentation auf Kant (1781) und Hume (1978), die den Wissenserwerb über die Erfahrung hervorheben. Die Lehrpersonen nennen nicht explizit den lebenslangen professionellen Lernprozess, zeigen jedoch über ihre Aussagen, dass sie diesem Prinzip folgen.

In Anlehnung an das rekonstruierte Verständnis beschreiben sie die kontinuierliche professionelle Weiterentwicklung sowie die Reflexion für pädagogisches Wissen und Handeln als wesentlich. Schratz und Schrittesser (2013: 185) unterstützen diesen Ansatz und postulieren das kontinuierliche Verlernen, Umlernen und Neulernen, um aktuelles und zeitgemäßes professionelles pädagogisches Wissen zu besitzen und den Bildungs- und Erziehungsauftrag professionell umzusetzen. 


\section{Grenzen der vorliegenden Arbeit}

Im Rahmen der vorliegenden Einzelstudie lassen sich die Ergebnisse hinsichtlich ihrer Verallgemeinerbarkeit diskutieren. Die qualitativ-rekonstruktive Studie stützt sich auf eine spezifische Stichprobe aus einem bestimmten Untersuchungsgebiet. Eine Generalisierung der Befunde für alle Lehrpersonen kann dadurch nicht garantiert werden. Dies wird durch die Tatsache, dass eine qualitative Untersuchung allgemein eine Verallgemeinerung der Werte ausschließt, verstärkt. Zudem handelt es sich bei der Ergebnisdarstellung um keine Erstellung einer Systematik von pädagogischem Wissen. Vielmehr steht die Rekonstruktion kognitiver Strukturen aus der Schulpraxis und das Handeln von Lehrpersonen im Mittelpunkt des Interesses. Es handelt sich hierbei um ein klar spezifiziertes Feld, dem vorhandene latente Strukturen zugrunde liegen, und die Beantwortung der Forschunsgfragen durch das vorliegende Forschungsdesign zulassen. Das Sample, welches die Generalisierbarkeit aufgrund dem Prinzip der theoretischen Sättigung ermöglicht, unterstützt die Evidenz der Forschungsarbeit und liefert eine repräsentative Datengrundlage.

Der qualitativ-rekonstruktive Zugang und das Arbeiten nach dem Prinzip der Grounded Theory bieten die Möglichkeit, soziale Realitäten direkt aus der Unterrichtspraxis zu rekonstruieren und aufzuzeigen. Es zeigen sich im Rahmen des methodologischen Zugangs in diesem Zusammenhang jedoch, wie eigene Sichtweisen und Interpretationen Ergebnisse beeinflussen können. Potenzielle Fehlerquellen dieser Arbeit resultieren vor allem aus den Beobachtungen, die durch subjektive Wahrnehmungen beeinflusst werden können. Das Arbeiten in größeren Gruppen könnten das qualitative Arbeiten und das Rekonstruieren von Kategorien begünstigen sowie Fehlerquellen verringern. Der qualitativ-rekonstruktive Zugang kann als ein gewinnbringender Zugang für Konzeptualisierungen pädagogischen Wissens aus dem Unterrichtsalltag argumentiert werden und bedingt eine Weiterentwicklung des Wissenbestandes. Weiterführende nationale sowie internationale Studien müssen für ergänzende Konzeptualisierungen pädagogischen Wissens als sinnvoll erachtet werden, um gegenstandsadäquates Wissen aufzuzeigen.

In der vorliegenden Arbeit werden die rekonstruierten Ergebnisse basierend auf den dargestellten theoretischen Zugängen und Modellen diskutiert. Zusammenhänge und Abweichungen zwischen den theoretischen Grundlagen und den rekonstruierten Konzeptualisierungen werden aufgezeigt. Diese sind insofern eingeschränkt, als dass sie sich nur auf die im empirischen Zugang angeführten theoretischen Grundlagen beziehen. Hinzu kommt, dass in der Arbeit nicht auf alle theoretischen Grundlagen Bezug genommen werden kann und eine weitere Diskussion im Zusammenhang mit unterschiedlichen Themenbreichen, wie bei den Forschungsdesideraten aufgezeigt, sinnvoll erscheint. 


\section{Resümee und Ausblick}

Das Ziel der Arbeit war die Rekonstruktion pädagogischen Wissens von Lehrpersonen aus der Unterrichtspraxis. Über einen qualitativ-rekonstruktiven Zugang konnten kognitive Muster und pädagogisches Handeln gegenstandsadäquat direkt aus dem Unterrichtsalltag aufgezeigt werden. Wie Bruner (1996), Neuweg (2004) oder Loughran (2019) argumentieren, ist die Vernetzung von Theorie und Praxis zentrales Moment für eine gegenstandsadäquate Ergebnisdarstellung der Unterrichtspraxis. Der Einsatz unterschiedlicher Erhebungsinstrumente für die Datengewinnung ermöglicht es, den Unterrichtsalltag differenziert abzubilden und das Feld Schule mit ihren wesentlichen AkteurInnen und Prozessen in der vorliegenden Arbeit zu untersuchen. Die Studie inkludiert Lehrpersonen, die im Untersuchungszeitraum im Schuldienst tätig sind und sich über unterschiedliche soziodemografische Eckdaten (Geschlecht, Alter, Dienstjahre, Schulfächer und Schulformen) kennzeichnen.

Das Verständnis pädagogischen Wissens stützt sich auf einen theoretischen sowie handlungsorientierten Wissensbegriff. In der vorliegenden Studie konnten in einer Ausdifferenzierung zentrale Formen professionellen Wissens von Lehrpersonen, deklaratives/prozedurales (vgl. Ryle 1949), explizites/implizites (vgl. Polanyi 1985), handlungsorientiertes/träges Wissen (vgl. Renkl 1996) und zentrale Wissensqualitäten wie „Stufen der Ausdifferenzierung“, „Struktur“ und „Ritualisierung“ (vgl. De Jong \& Ferguson-Hessler 1996), mit untersucht werden.

Die Lehrpersonen können, basierend auf ihren gemachten Erfahrungen, über reflexive Prozesse pädagogisches Wissen definieren und stützten sich bei der Charakterisierung auf theoretisches sowie hauptsächlich auf das Erfahrungswissen. Dieser Ansatz zeigt Übereinstimmungen mit der von Kant (1781), Hume (1975) und Hoerster (2010) angestoßenen Diskussion, die Wissen über Erfahrungen hervorhebt.

Pädagogisches Wissen wird als ein wichtiger Wissensbereich professionellen Wissens von Lehrpersonen beschrieben, dem aufgrund stetiger Veränderungsprozesse und der zentralen Bedeutung von Fähigkeiten und Fertigkeiten des 21. Jahrhunderts eine große Relevanz zukommt. Das gegenwärtige Verständnis pädagogischen Wissens basiert vorwiegend auf psychometrischen Testverfahren (z.B. COACTIV, TEDS-M, LEK, MT21, ProWin), die nach der zweiten empirischen Wende durchgeführt wurden und pädagogisches Wissen konzeptualisieren. Die standardisierten Studien kennzeichnen sich darüber, dass sie vor allem das deklarative Wissen über vorgegebene Konstrukte untersuchen und die alltägliche Unterrichtspraxis nur unzureichend darstellen (vgl. Kraler et al. 2017). Die vorliegende Studie versucht, über die Rekonstruktion pädagogischen Wissens gegenstandsadäquat aus der Unterrichtspraxis abzubilden und das bestehende Forschungsfeld unter Bezugnahme unterschiedlicher Formen des Wissens über weitere konzeptuelle Zugänge zu ergänzen. Diese zeigen eine differenzierte und teilweise eine von den bestehenden Modellen abweichende Strukturierung.

Das pädagogische Wissen der untersuchten Lehrpersonen charakterisiert sich über ein mehrdimensionales Verständnis, das in einem Beziehungsgefüge zwischen Verfügungs- und Orientierungswissen (vgl. Mittelstraß 1998) und pädagogischem Handeln steht. Dieses spiegelt Mittelstraß zufolge eine moderne Gesellschaft und somit zeitgemäßes Wissen von Lehrpersonen wider. Insgesamt konnten auf Basis des Datenmaterials sieben Formen pädagogischen Wissens rekonstruiert werden (vgl. Abbildung 121). 


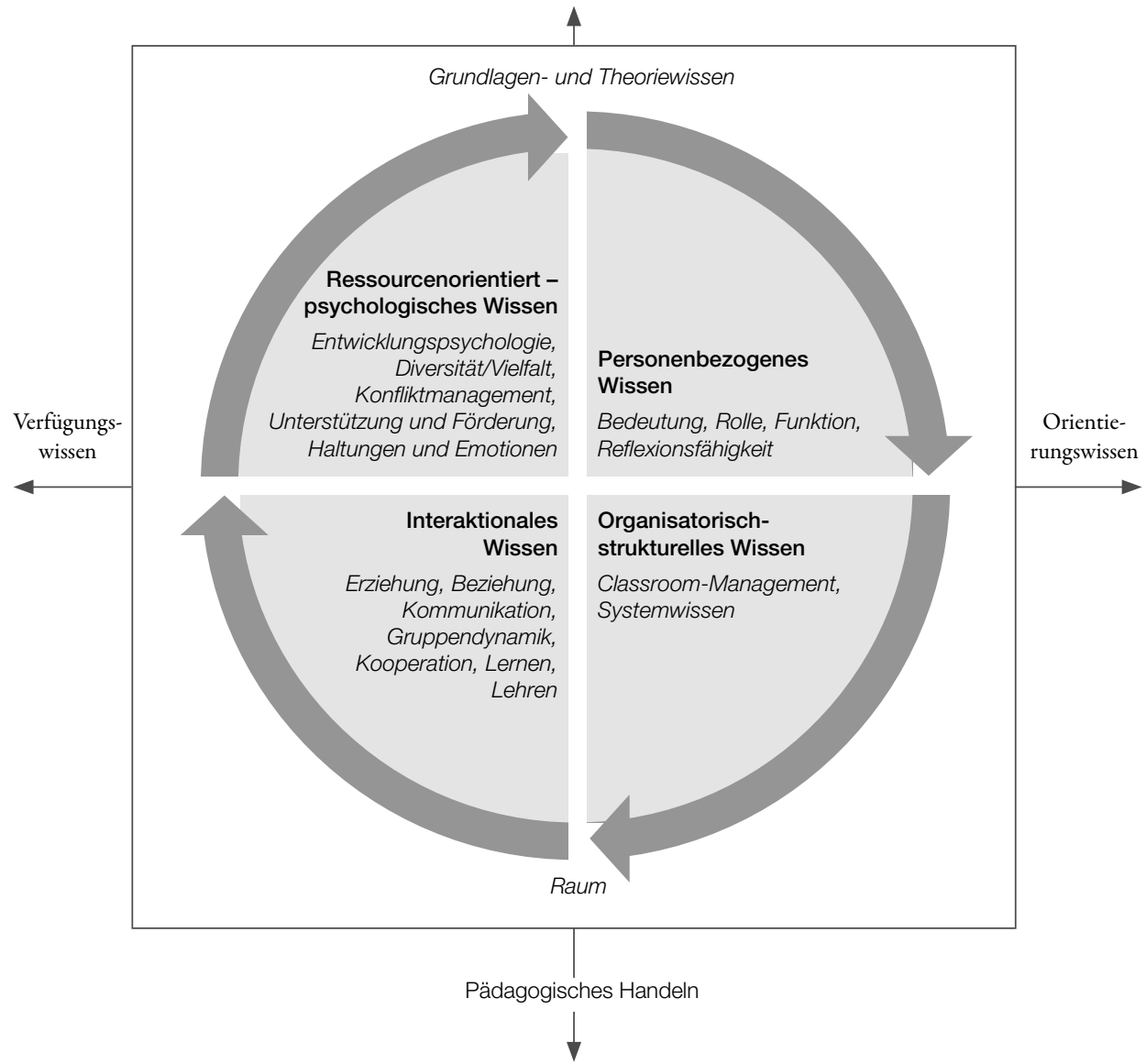

Abb. 121: Felder pädagogischen Wissens von Lehrpersonen

Drei der rekonstruierten Formen, das Grundlagenwissen, das Erfahrungswissen und das Wissen zum institutionellen Raum, werden als Basis der Formen pädagogischen Wissens konzeptualisiert und sind für die Verwirklichung der anderen Wissensfelder konstitutiv. Die weiteren Wissensfelder beziehen sich auf personenbezogenes Wissen, organisatorisch-strukturelles Wissen, interaktionales Wissen sowie ressourcenorientiert-psychologisches Wissen. Das personenbezogene Wissen untergliedert sich in die Wissenskategorien Bedeutung, Rolle und Funktion der Lehrperson sowie das Reflexionswissen. Das organisatorisch-strukturelle Wissen setzt sich aus den Wissensbereichen Classroom-Management und Systemwissen zusammen. Wissen im Bereich des interaktionalen Wissens sind Erziehung, Beziehung, Kommunikation, Gruppendynamik, Kooperation, Lernen und Lehren. Unter ressourcenorientiert-psychologisches Wissen kann Entwicklungspsychologie, Diversität/Vielfalt, Konfliktmanagement, Unterstützung und Förderungen sowie Wissen über Haltung und Emotionen konzeptualisiert werden.

Zentrales Ergebnis der vorliegenden Arbeit ist der konzeptuelle Zugang eines interaktiven Prozesses (vgl. Mead 1934; Giddens 1984; Giesecke 2013) zwischen Lehrpersonen und SchülerInnen, über den sich pädagogisches Wissen realisieren kann. Über Interaktionsprozesse wird 
mittels Symbolen, wie der Sprache, und unter Berücksichtigung struktureller Bedingungen (vgl. Giddens 1984) Wissen generiert und aufgezeigt. Pädagogisches Wissen realisiert sich in einem Spannungsfeld sozialer Interaktion zwischen den beteiligten AkteurInnen, die über ihr Handeln eigenes pädagogisches Wissen entwickeln. In einer weiteren Ausdifferenzierung zeigt sich, wie der Interaktionsprozess die Identitätsbildung pädagogischen Wissens einer Lehrperson unterstützt. Die SchülerInnen fungieren als Spiegel pädagogischen Wissens einer Lehrperson. In einem Perspektivenwechsel, wie Mead in seinem Modell über das „Me“ und das „I“ (vgl. Mead 1934) diskutiert, wird mithilfe reflexiver Prozesse die pädagogische Identität der Lehrpersonen aufgezeigt.

Eine weitere Modellierung bezieht sich auf die lern- sowie lehrseitige Perspektive pädagogischen Wissens. Pädagogisches Wissen orientiert sich, wie Schratz (2009) in seinem didaktischen Modell beschreibt, an einem lernseitigen Zugang. Die Ergebnisse verdeutlichen, wie das pädagogische Wissen schülerInnenorientiert ausgerichtet ist und über eine lernseitige Perspektive das Beziehungsgefüge pädagogischen Wissens zwischen Lehrperson, SchülerInnen und Lerngegenstand verstärkt.

Die rekonstruierten Kategorien pädagogischen Wissens stehen in einer Beziehung zueinander. Die vorliegende Studie illustriert Wechselbeziehungen und Wirkungsbereiche einzelner Kategorien. Wissensfelder, die in einem engen Wirkungsverhältnis zueinander stehen, sind das Lehren und Lernen, gefolgt von der Kommunikation, der Beziehung, der Kooperation, der Unterstützung und Förderung, dem Classroom-Management, der Haltung und Emotionen, der Diversität, der Rolle einer Lehrperson sowie der Reflexionsfähigkeit.

Zu von den Wechselbeziehungen präsentieren die Ergebnisse Häufigkeiten einzelner Wissensbereiche, die für die Lehrpersonen essenzielles Wissen ihres Unterrichtsalltags aufzeigen. Von großer Relevanz zeigen sich das Wissen über das Classroom-Management, die Beziehung, die Haltung und Emotionen, die Diversität, das Reflexionswissen, das Lernen, über das Lehren, die Entwicklungspsychologie, die Bedeutung, Rolle und Funktion der Lehrperson sowie die Kommunikation und die Erziehung.

Pädagogische Wissensbereiche müssen sich Fend (2006) zufolge für den Erhalt einer Gesellschaft im Kontext der gesellschaftlichen und kulturellen Entwicklungen kontinuierlich weiterentwickeln. Schule als Haus des Wissens (vgl. Koch 2015: 67) hat die Aufgabe, SchülerInnen hinsichtlich des Bildungs- und Erziehungsauftrags (vgl. Herbart 1982) zu unterrichten und zu unterstützen. Der Vergleich mit gegenwärtigen Konzepten (vgl. Helmke 2009; Guerriero 2017; European Commission 2018; Schratz 2019), die spezifische Wissensbereiche für einen gegenwartsorientierten Unterricht unter Berücksichtigung der sozialen, ökonomischen und politischen Transformationsprozesse definieren, zeigt, dass die untersuchten Personen über pädagogisches Wissen verfügen. Aus der Rekonstruktion geht hervor, dass Lehrpersonen die Wissensbereiche zwar benennen können, jedoch in ihrer Umsetzung auf Schwierigkeiten stoßen. Rekonstruierte Herausforderungen beziehen sich vorwiegend auf gegenwärtige Themenbereiche wie Diversität, Erziehung, Lernen/Lehren, Systemwissen, psychologisches Wissen, Medien sowie Wissen über die eigene (Lehr-)Person. Schule als gesellschaftlicher Resonanzraum ist gefordert, Wandlungsprozesse in den Unterrichtsalltag zu integrieren und angemessen mit neuen Anforderungen umgehen zu können (vgl. Bauer at al. 1996: 5f.; Kraler \& Schratz 2012: 89). Dieser Prozess wird von den Lehrpersonen als schwierig beschrieben. Sie beklagen sich über zu wenig Unterstützung und berichten, wie sie einem gesellschaftlichen Druck ausgesetzt sind. Fehlende Fort- und Weiterbildungen, ein mangelndes Selbstkonzept sowie eine unzureichende Unterstützung werden von ihnen als Hindernisse argumentiert, die sie daran hindern, den Un- 
terrichtsalltag im Kontext grundlegender Themenbereiche professionell zu gestalten und den Herausforderungen gerecht zu werden. Die Unterschiede zwischen den Schulformen verdeutlichen, wie in diesem Zusammenhang die Lehrpersonen aus der Volksschule und der Neuen Mittelschule die aktuellen, relevanten Wissensbereiche in ihrem Unterricht eher umsetzen als Lehrpersonen des Gymnasiums. Für ein fundiertes pädagogisches Wissen ist daher eine weitere Professionalisierung notwendig, um den geforderten Übergang von „good practice zu next practice“ (vgl. Kraler \& Schratz 2012: 92) im Unterrichtsalltag erfolgreich zu meistern. Die Studie zeigt in diesem Zusammenhang die Relevanz eines kontinuierlichen professionellen lebenslangen Lernprozesses auf. Zentral für den professionellen Wissenserwerb sind in Anlehnung an die Ergebnisse die Erfahrungen außerhalb der Schule und aus der Berufspraxis sowie fachliche und persönliche Fort-und Weiterbildungen (vgl. Kant 1791; Hume 1975; Hoerster 2010). Als weniger bedeutsam für die Aneignung pädagogischen Wissens wurde die Ausbildung erachtet. Aus der Rekonstruktion zeigen sich Unterschiede und Übereinstimmungen zwischen den Konzepten von pädagogischem Wissen der Lehrpersonen und ihrem pädagogischen Handeln. Unterschiedliche Muster konnten aufgezeigt werden. Diese präsentieren Abweichungen hinsichtlich ihres deklarativen und prozeduralen sowie expliziten und impliziten Wissens. Die Ergebnisse aus den Interviews mit den Lehrpersonen verdeutlichen, dass sie weniger pädagogisches Wissen verbalisieren können, als sie in der Praxis anwenden. Die Gespräche weisen auf eine Menge an implizitem Wissen hin (vgl. Polanyi 1985; Waldenfels 2000; Neuweg 2014). Unterschiede zwischen deklarativem und prozeduralem Wissen beziehen sich auf genannte Wissensbereiche bzw. wie pädagogisches Wissen im Unterricht angewendet wird, konnten aber in den Beobachtungen nicht bestätigt werden.

Ressourcen für die Überwindung der Wahrnehmungsunterschiede und für den Transfer vom Wissen zum Handeln, auf die sich die Arbeit stützte, waren das Transferinstrument Reflexion sowie das Selbstkonzept einer Lehrperson (vgl. Schön 1983; Bauer 1998; Korthagen \& Kessel 1999). Wissen führt mithilfe von Reflexion zum Handeln. Dieses kann über Reflexion Wissen generieren. Dieser Ansatz stimmt mit der Argumentation von Dann (2000: 83) überein. Er beschreibt, wie Handeln über Wissen gesteuert und Wissen über Handlungen bestärkt bzw. verändert wird. Unterschiedliche konzeptuelle Ansätze, wie jene von Mead (1934), Schön (1983), Giddens (1984) und Bauer (1996) Neuweg (1999), illustrieren, wie über einen strukturellen und reflexiven Prozess Wissen aufgezeigt und weiterentwickelt werden kann. Das Selbstkonzept sowie subjektive Theorien (vgl. Rogers 1981; Groeben 1988; Bauer 1998; Mikhail 2006) und die zugrundeliegende Haltung von Lehrpersonen unterstützt den Transformationsprozess von Wissen und kann als wesentlicher positiver Einflussfaktor für pädagogisches Wissen charakterisiert werden. Lehrpersonen mit einem ausgeprägten professionellen Selbstkonzept zeigen höhere Wissensqualitäten hinsichtlich der Struktur, der Stufen der Ausdifferenzierung und der Ritualisierung. Das Selbstkonzept wird als signifikanten Einflussfaktor pädagogischen Wissens diskutiert. Wichtig ist die von Rogers (1981) und Mikhail (2016) beschriebene professionelle Haltung, die einen ressourcenorientierten Umgang mit SchülerInnen sowie ihre Selbstbildung verfolgt.

In Anlehnung an den mehrperspektivischen Diskurs über Wissen und Nichtwissen ist es nicht möglich, alles zu wissen und eine bestimmte Kategorisierung pädagogischen Wissens als richtig zu postulieren. Vielmehr ist es wichtig, Wissen situationsspezifisch und angemessen anzuwenden (vgl. Radtke 2000; Helmke 2009; Giesecke 2013) und zu wissen, wie Lehrpersonen ihr Wissen und ihr Selbstkonzept im Sinne des Bildungs- und Erziehungsauftrags kontinuierlich professionalisieren und weiterentwickeln können. 


\section{Forschunsgdesiderate}

Die vorliegende Studie verdeutlicht Themenfelder, die Potenzial für weiterführende Forschung aufzeigen. Ein interessanter Themenbereich im Kontext der LehrereInnenbildung bezieht sich auf die Bedeutung der Aneignung pädagogischen Wissens während des Studiums. Die untersuchten Lehrpersonen argumentieren, dass die Ausbildung nur einen geringen Teil zur professionellen Entwicklung pädagogischen Wissens beiträgt. Für die LehrerInnenbildung wäre es bedeutend, mögliche Ursachen für dieses Ergebnis zu rekonstruieren. Ein weiterer Forschungsbereich ergibt sich aus der Argumentation der Lehrpersonen, dass unzureichende Fort- und Weiterbildungen angeboten werden, die sie in ihrer professionellen Weiterentwicklung unterstützen. Wenn die Realität zeigt, dass zahlreiche Fortbildungsmöglichkeiten für Lehrpersonen angeboten werden, wäre ein Fokus auf die Rekonstruktion von Ursachen für die Abweichung zwischen Angebot und Nachfrage der Lehrpersonen zu richten.

Die Verknüpfung von Grundlagen mit den vorliegenden Befunden zu pädagogischem Wissen illustriert ein Potenzial für die Diskussion weiterer theoretischer Grundlagen. Mögliche theoretische Diskussionen beziehen sich auf das Thema „Nichtwissen“ und „subjektive Theorien“. Die Diskussion dieser Themenbereiche wird in der vorliegenden Arbeit unzureichend mit dem pädagogischen Wissen in Verbindung gebracht.

Im Zusammenhang mit der Diskussion soziologischer und erkenntnistheoretischer Zugänge in Verbindung mit pädagogischem Wissen ergeben sich weitere Desiderate. Zeigen die Ergebnisse die zentrale Bedeutung von Interaktionsprozesse für die Realisierung pädagogischen Wissens, könnten diese im Zusammenhang mit weiteren handlungstheoretischen Zugänge analysiert und zusätzlich mit den Theorien von Mead (1938) und Giddens (1984) vertieft werden.

Im Kontext formaler Bildung entstehen vor dem Hintergrund der Forschungsarbeit weitere Themengebiete in Verbindung mit dem Bildungsbegriff sowie mit dem Kompetenzbegriff. Interessante Fragestellungen beziehen sich auf den Zusammenhang zwischen der Bedeutung pädagogischen Wissens im Sinne des Bildungsauftrags von Schule und der gegenwärtigen Kompetenzdebatte. Zudem wird das Thema Lernen in der vorliegenden Arbeit über kognitionspsychologischen und biographischen Zugängen aufgegriffen. Ein hier nicht berücksichtigter Fokus liegt auf der Vernetzung von Lerntheorien, die vertieft im Zusammenhang mit der Aneignung pädagogischen Wissens diskutiert werden können. Ein weiteres essentielles Thema, das Potenzial für weiterführende Forschung aufzeigt, ist die Abweichung zwischen kognitiven Mustern pädagogischen Wissens von Lehrpersonen und ihrem Handeln. Transfermöglichkeiten wurden in der vorliegenden Arbeit mit Hilfe metakognitiver Entwicklungsprozesse argumentiert, die noch weiter spezifiziert und diskutiert werden können.

Die Pilotstudie und der qualitativ-rekonstruktive Zugang illustrieren, wie pädagogische Konzepte über die Rekonstruktion des Unterrichtsalltags gegenstandsadäquat abgebildet werden können. Diese Erkenntnis fordert für die Zukunft weiterführende nationale und internationale qualitative Studien, die sich mit pädagogischem Wissen von Lehrpersonen beschäftigen. 


\section{Verzeichnisse}

\section{Literaturverzeichnis}

Abels, H. (1975): Lebensweltanalyse von Fernstudenten. Qualitative Inhaltsanalyse - theoretische und methodische Überlegungen. Hagen: Werkstattbericht.

Abels, H. (2010): Interaktion, Identität, Präsentation - Kleine Einführung in interpretative Theorien der Soziologie. Wiesbaden: Springer.

Abels, H. (2013): Ethnomethodologie. In: Kneer, G. \& Schoer, S. (Hrsg.): Handbuch Soziologische Theorien. Wiesbaden: Verlag für Sozialwissenschaften. S. 87-110.

Adorno, T. (1959): Theorie der Halbbildung. In: Gesammelte Schriften Band 8. Soziologische Schriften I. S. 93-121.

Aebli, H. (1983): Zwölf Grundformen des Lehrens. Stuttgart: Klett-Cotta.

Aeppli, J. et al. (2016): Empirisches wissenschaftliches Arbeiten - Ein Studienbuch für Bildungswissenschaften 4. Auflage. Bad Heilbrunn: Julius Klinkhardt.

Altrichter, H. (2000): Handlung und Reflexion bei Donald Schön. In: Neuweg, G.H (Hrsg,).: Wissen, Können, Reflexion. Innsbruck: Studienverlag. S. 201-222.

Altrichter, H. \& Posch, P. (2007): LehrerInnen und Lehrer erforschen ihren Unterricht. 4. Auflage. Düsseldorf: Dirk Krüll.

Anderson, J.R. (1982): Acquisition of cognitive skills. In: Psychological Review, 89, S. 369-406.

Anderson, L.W. \& Krathwohl, D.R. (2001): A taxonomy for learning, teaching, and assessing: A revision of Bloom's taxonomy of education objectives. New York: Longman.

Atteslander, P (2010): Methoden der empirischen Sozialforschung. Berlin: Erich Schmidt Verlag.

Augustinus, A. (2005): De magistro/Über den Lehrer. Lateinisch/Deutsch. Stuttgart: Reclam.

Ausubel, D.P. (1974): Psychologie des Unterrichts. 2 Bd. Weinheim: Beltz.

Balog, A. (2012): Soziologie und die „Theorie des Handelns“. In: Schülein J., Mozetič G. (Hrsg.): Handlung. Wiesbaden: Springer. S. 11-38.

Bauer, K.-O. (1998): Pädagogisches Handlungsrepertoire und pädagogisches Selbst von Lehrerinnen und Lehrern. In: Zeitschrift für Pädagogik, 3, S. 343-359.

Bauer, K.-O. et al. (1996): Pädagogische Professionalität und Lehrerarbeit. Eine qualitativ empirische Studie über professionelles Handeln und Bewußtsein. München: Juventa.

Bauer, J. (2007): Lob der Schule. Hamburg: Hoffmann und Campe.

Baumann, P. (2006): Erkenntnistheorie. Stuttgart/Weimar: J.B. Metzler.

Baumert, J. et al. (2010): Teachers' mathematical knowledge, cognitive activation in the classroom and student progress. In: American Educational Research Journal, 47 (1), S. 133-180.

Baumert, J. \& Kunter, M. (2006): Stichwort: Professionelle Kompetenz von Lehrkräften. In: Zeitschrift für Erziehungswissenschaft, 9 (4), S. 469-520.

Baumert, J. \& Kunter, M (2011): Das Kompetenzmodell von COACTIV. In: Kunter, M. et al. (Hrsg.): Professionelle Kompetenz von Lehrkräften. Ergebnisse des Forschungsprogramms COACTIV. Münster: Waxmann. S. 29-53.

Beck, U. (1986): Risikogesellschaft. Auf dem Weg in eine andere Moderne. Frankfurt am Main: Suhrkamp.

Beck, K. (1994): Medien und die soziale Konstruktion von Zeit. Über die Vermittlung von gesellschaftlicher Zeitordnung und sozialen Zeitbewußtsein. Berlin: Opladen.

Beck, U. (1996): Wissen oder Nicht-Wissen? Zwei Perspektiven reflexiver Modernisierung. In: Beck U.\& Giddens, A. (Hrsg.): Reflexive Modernisierung. Frankfurt: Suhrkamp. S. 289-315.

Beck, E. et al. (Hrsg.) (2008): Adaptive Lehrkompetenz. Analyse und Struktur, Veränderung und Wirkung handlungssteuerndes Lehrerwissens. Münster: Waxmann.

Bell, D. (1968): The measurement of knowledge and technology. In: Sheldon, E. \& Moore, W. (Hrsg.): Indicators of Social Change. Hartford: Concepts and Methods. S. 145-246.

Bell, D. (1973): The Coming of Post-Industrial Society. New York: Basic Books.

Beijaard, D. \& Verloop, N. (1996): Assessing teachers' practical knowledge. In: Studies in Educational Evaluation, S. 22, S. 275-286.

Berger, P. \& Luckmann, T. (1980): Die gesellschaftliche Konstruktion der Wirklichkeit. Eine Theorie der Wissenssoziologie. Frankfurt a.M.: Fischer.

Bergmann, J. (1991): Studies of work/Ethnomethodologie. In: Flick U. et al (Hrsg.): Handbuch qualitative Sozialforschung. München: Beltz. S. 269-272. 
Bergmann, J. (2000): Konversationsanalyse. In: Flick, U. et al. (Hrsg.): Qualitative Forschung. Ein Handbuch. Reinbek: Rowohlt-Taschenbuch-Verlag S. 542-537.

Bertschy, B. (2007): Pädagogisches Wissen problemorientiert prüfen. In: Beiträge zur Lehrerinnen- und Lehrerbildung, 25 (1), S. 37-50.

Bifie (2019): Bildungsstandards und kompetenzorientierter Unterricht.[ https://www.bifie.at/bildungsstandards-undkompetenzorientierter-unterricht/; zugegriffen am 07.08.2019]

Bloomberg, J. et al. (1993): Ethnographic Field Methods and their Relation to Design. In: Douglas Schuler (Hrsg.): Participatory Design: Principles and Practices. Hillsdale: Erlbaum. S. 123-155.

Blömeke, S. (2004): Empirische Befunde zur Wirksamkeit der Lehrerbildung. In: Blömeke, S. et al. (Hrsg.): Handbuch Lehrerbildung. Bad Heilbrunn: Klinkhardt. S. 59-91.

Blömeke, S. et al. (2008): Messung des erziehungswissenschaftlichen Wissens angehender Lehrerinnen und Lehrer. In: Blömeke, S. et al. (Hrsg.): Professionelle Kompetenz angehender Lehrerinnen und Lehrer. Wissen, Überzeugungen und Lerngelegenheiten deutscher Mathematikstudierender und -referendare. Erste Ergebnisse zur Wirksamkeit der Lehrerausbildung. Münster: Waxmann. S. 171-194.

Blömeke, S. et al. (2009a): Differentielle Item-Analysen zur Entwicklung professioneller Kompetenz angehender Lehrkräfte während der Lehrerausbildung. In: R. Mulder et al. (Hrsg.): Professionalität von Lehrenden - Zum Stand der Forschung. Weinheim: Beck. S. 311-332.

Blömeke, S. et al. (2009b): TEDS-M. Messung von Lehrerkompetenzen im internationalen Vergleich. In: ZlatkinTroitschankskaia et al. (Hrsg.): Lehrerprofessionalität - Bedingungen, Genese, Wirkung und Messung. Weinheim: Beltz. S. 181-210.

Blömeke, S. et al. (2010): TEDS-M 2008 Primarstufe: Ziele, Untersuchungsgrundlage und zentrale Ergebnisse. In: Blömeke, S. et al. (Hrsg.): TEDS-M 2008 - Professionelle Kompetenz und Lerngelegenheiten angehender Primarstufenlehrkräfte im internationalen Vergleich. Münster: Waxmann. S. 11-38.

Blömeke, S. et al. (2015): Beyond dichotomies: Competence viewed as a continuum. In: Zeitschrift für Psychologie, 223 (1), S. 3-13.

Blömeke, S. \& König, J. (2010): Messung des pädagogischen Wissens: Theoretischer Rahmen und Teststruktur. In: Blömeke, S. et al. (Hrsg.): TEDS-M 2008 - Professionelle Kompetenz und Lerngelegenheiten angehender Mathematiklehrkräfte für die Sekundarstufe 1 im internationalen Vergleich. Münster: Waxmann. S. 253-273.

Blömeke, S. \& Paine, L. (2008): Getting the fish out of water: Considering benefits and problems of doing research on teacher education at an international level. In: Teaching and Teacher Education, 24 (4), S. 2027-2037.

Böhme, J. (2014): Handlungsraum. In: Zirfas, J. \& Wulf, C. (Hrsg.): Handbuch Pädagogische Anthropologie. Wiesbaden: Springer Verlag. S. 423-432.

Bönsch, M. (2004): Intelligente Unterrichtsstrukturen. Eine Einführung in die Differenzierung. Baltmannsweiler: Schneider Hohengehrer.

Bonß, W. et al. (2020): Handlungstheorie. Eine Einführung. Bielefeld: Transcript Verlag.

Borko, H. \& Putnam, R.T. (1996): Learning to teach. In: Berliner, D.C. \& Calfee, R.C. (Hrsg.): Handbook of educational psychology. New York: Macmillan. S. 673-708.

Bormann, I. \& Haan, G. (2008): Kompetenzen der Bildung für nachhaltige Entwicklung: Operationalisierung, Messung, Rahmenbedingungen, Befunde. Wiesbaden: Springer.

Böschen, S. (2005): Reflexive Wissenspolitik: Zur Formierung und Strukturierung von Gestaltungsöffentlichkeiten. In: Bogner, A.; Torgersen, H. (Hrsg.): Wozu Experten? Wiesbaden: VS, S. 241-263.

Brickhouse, N.W. (1990): Teachers' belief about the nature of science and their relationship to classroom practice. In: Journal of Teacher Education, 41, S. 53-62.

Bromme, R. (1992): Der Lehrer als Experte: zur Psychologie des professionellen Wissens. Bern: Huber.

Bromme, R. (1997): Kompetenzen, Funktionen und unterrichtliches Handeln des Lehrers. In: Weinert, F.E. (Hrsg.): Enzyklopädie der Psychologie: Psychologie des Unterrichts und der Schule, Bd. 3. Göttingen: Hogrefe. S. 177-212.

Bromme, R. (2001): Teachers expertise. In: Smelser, N.J. \& Baltes, P.B. (Hrsg.): International encyclopedia of the social behavioral science Bd. 26. Oxford: Elsevier. Amsterdam: Elsevier. S. 15459-15465.

Brophy, J. \& Good, T.L. (1986): Teacher behaviour and student achievement. In: Wittrock, M.C. (Hrsg.): Handbook of research on teaching. London: Macmillan. S. 328-375.

Bruner, J. (1996): The culture of education. Cambridge, MA: Harvard University Press.

Brügelmann, H. (2015): Vermessene Schulen - standardisierte Schüler. Zu Risiken und Nebenwirkungen von PISA, Hattie, VerA und Co. Beltz: Weinheim.

Brühwiler, C. et al. (2017): Welches Wissen ist unterrichtsrelevant? In: Zeitschrift für Bildungsforschung, 7, S. $209-228$.

Brülisauer B. (2008): Was können wir wissen? Grundprobleme der Erkenntnistheorie. Stutthart: Kohlhammer.

Brüsemeister, T. (2008): Qualitative Forschung. Ein Überblick. Wiesbaden: Verlag für Sozialwissenschaften.

Buchwald, P. \& Hobfoll, S. E (2004): Burnout aus ressourcenorientierter Perspektive. In: Psychologie in Erziehung und Unterricht, 51 (4), S. 247-257. 
Buck, G. (1985): Herbarts Grundlegung der Pädagogik. Heidelberg: Springer.

Buck, G. (1989): Lernen und Erfahrung. 3. Aufl. Darmstadt: Springer.

Burghardt, D. (2014): Psychischer Raum. In: Zirfas, J. \& Wulf, C. (Hrsg.): Handbuch Pädagogische Anthropologie. Wiesbaden: Springer Verlag. S. 403-412.

Busse, A. \& Kaiser, G. (2015): Wissen und Fähigkeiten in Fachdidaktik und Pädagogik. Zur Natur der professionellen Kompetenz von Lehrkräften. In: Zeitschrift für Pädagogik, 61(3), S. 328-344.

Carter, K. (1990): Teachers' knowledge and learning to teach. In: Houston, W.R. (Hrsg.): Handbook of research on teacher education. New York: Macmillan. S. 255-296.

Clandinin, D.J. (1986): Classroom practice: Teacher images in action. London: Falmer Press.

Cochran-Smith, M., \& Lytle, S. L. (1999): Relationships of knowledge and practice: Teacherlearning communities. In: A. Iran-Nejad \& P.D. Pearson (Hrsgs.): Review of research in education, 24, S. 249-305.

Conway, P.F. et al. (2009): Learning to Teach and its Implications for the Continuum of Teacher Education: A NineCountry Cross-National Study. Report Commissioned by the Teaching Council. Ireland: University College Cork and Teaching Council of Ireland.

Csikszentmihalyi, M. (1995): Dem Sinn des Lebens eine Zukunft geben. Eine Psychologie für das dritte Jahrhundert. Stuttgart: Klett-Cotta.

Dann, H.-D. et al. (1987): Subjektive Theorien und erfolgreiches Handeln von Lehrer/-innen bei Unterrichtskonflikten. In: Unterrichtswissenschaft, 15, S. 306-320.

Dann, H.-D. (1994): Pädagogisches Verstehen: Subjektive Theorien und erfolgreiches Handeln von Lehrkräften. In: Reusser, K. \& Reusser-Weyeneth, M. (Hrsg.): Verstehen. Psychologischer Prozess und didaktische Aufgaben. S. 163-182.

Dann, H.-D. (2000): Lehrerkognitionen und Handlungsentscheidungen. In: Schweer, M.K.W. (Hrsg.): Lehrer-Schüler-Interaktion. Opladen: Leske + Budrich. S. 79-108.

Denninger, E. et al. (1973): Alltagswissen, Interaktion und gesellschaftliche Wirklichkeit. Symbolischer Interaktionismus und Ethnomethodologie. Bielefelder Soziologen: Rowohlt.

De Jong, T. \& Ferguson-Hessler, M. (1996): Types and qualities of knowledge. In: Educational Psychologist, 31, S. $105-113$.

Dewey, J. (1915/1964): Demokratie und Erziehung. Eine Einleitung in diephilosophische Pädagogik. Braunschweig: Westermann.

Diekmann, A. (2001): Empirische Sozialforschung. Grundlagen, Methoden, Anwendung. Rowohlt Taschenbuch Verlag: Reinbek.

Drews, F. (2009): Menschliche Willensfreiheit und göttliche Vorsehung bei Augustinus, Proklos, Apuleius und John Milton . Band 1. Frankfurt: Ontos Verlag.

Dunn, T.G. \& Shriner, C. (1999): Deliberate practice in teaching. What teachers do for self-improvement. In: Teacher and Teacher Education, 15 (6), S. 631-651.

Eagleton, T. (2009): Was ist Kultur. München: C.H. Beck.

Ecarius, J. \& Oliveras, R. (2014): Sozialer Raum. In: Zirfas, J. \& Wulf, C. (Hrsg.): Handbuch Pädagogische Anthropologie. Springer Verlag: Wiesbaden. S. 413-422.

Edelmann, W. (2000): Lernpsychologie. Braunschweig: Beltz.

Engel, J. (2016): Pädagogische Blicke zwischen inneren und äußeren Bildern. In: Schmidt, F. et al. (Hrsg.): Pädagogische Blicke. Weinheim und Basel: Beltz. S. 165-192.

Enzelberger, S. (2001): Sozialgeschichte des Lehrerberufs. Gesellschaftliche Stellung und Professionalisierung von Lehrerinnen und Lehrern von den Anfängen bis zur Gegenwart. Weinheim/München: Juventa.

Eraut, M. (1994): Developing professional knowledge and competences. London: Falmer Press.

Eriksen, T. (2001): Tyranny of the Moment. Fast and Slow Time in the Information Age. London: Pluto Press.

Esslinger-Hinz, I. \& Sliwka, A. (2011): Schulpädagogik. Weinheim/Basel: Beltz.

Etymoligisches Wörterbuch Duden (2019): Lexikon - Wissen. [https://www.duden.de/rechtschreibung/etymologisch/Wissen; zugegriffen am 24.02.2019]

European Commission (2013): Supporting teacher competence development - for better learnig outcomes. [http://ec.europa. eu/assets/eac/education/experts-groups/2011-2013/teacher/teachercomp_en.pdf; zugegriffen am 23.05.2019].

European Commission (2015): The Teaching Profession in Europe - Practices, Perceptions and Policies. Eurydice Report. Luxemburg: Publications Office of the European Union.

European Commission (2018): European ideas for better learning: the governance of school education system. Brüssel: ET 2020 Working Group Schools. [https://www.schooleducationgateway.eu/downloads/Governance/2018-wgs6Full-Final-Output.pdf; zugegriffen am 22.05.2019]. 
Fauser, P. et al. (2007): Was für Schulen! Gute Schule in Deutschland. Seelze-Velber: Kallmeyer.

Feger, H. (1983): Planung und Bewertung wissenschaftlicher Beobachtungen. In: Feger, H. \& Bredenkamp, J. (Hrsg.): Datenerhebung. Enzyklopädie der Psychologie. Bd. 1: Forschungsmethoden. Göttingen: Hoegrefe. S. 1-75.

Fend, H. (1998): Qualität im Bildungswesen. Schulforschung zu Systembedingungen, Schulprofilen und Lehrerleistung. Weinheim: Juventa.

Fend, H. (2006): Neue Theorie der Schule. Einführung in das Verstehen von Bildungssystemen. Wiesbaden: Springer.

Fend, H. (2008): Neue Theorie der Schule. Einführung in das Verstehen von Bildungssystemen. Wiesbaden: Springer.

Fennema, E. \& Franke, M.L. (1992): Teacher's knowledge and its impact. In: Grows, D.A. (Hrsg.): Handbook of research on mathematics teaching and learning: A project of the National Council of Teachers of Mathematics. New York: Macmillan. S. 147-164.

Fenstermacher, G.D. (1994): The knower and the known. The nature of knowledge in research on teaching. In: DarlingHammond, L. (Hrsg.): Review of Research in Education. Washington, DC: American Educational Research Association. S. 3-56.

Flick, U. (1995): Qualitative Forschung. Reinbek: Rowohlt Taschenbuch Verlag.

Flick, U. (2000): Qualitative Forschung. Theorie, Methoden, Anwendung in Psychologie und Sozialwissenschaften. Reinbek: Rowohlt Taschenbuch Verlag.

Flick, U. (2007): Qualitative Sozialforschung - Eine Einführung. Rowohlt Taschenbuch Verlag. Reinbek bei Hamburg.

Flick, U. (2008): Triangulation. Eine Einführung. Wiesbaden: Springer.

Flick, U. et al. (2000): Was ist qualitative Forschung? In: Flick, U. et al. (Hrsg.): Qualitative Forschung. Ein Handbuch. Reinbek: Rowohlt Taschenbuch Verlag, S. 11-29.

Foppa, K. (1990): Über Regeln der sprachlichen Kommunikation und die Schwierigkeiten, nichtberichtbares Wissen zu erfassen. In: Bulletin der Schweizer Psychologen, 4, S. 3-12.

Fried, L. (2002): Pädagogisches Professiosnwissen und Schulentwicklung - Eine systemtheoretische Einführung in Grundkategorien der Schultheorie. Weinheim/München: Juventa.

Fuller, F.F. \& Brown, O.H. (1975): Becoming a Teacher. In: Ryan, K. (Hrsg.): Teacher Education, 74 Yearbook oft he NSSE, Part II, Chicago. S. 25-52.

Funke, J. \& Spinath, B. (2014): Die PISA-Studien: EintransdisziplinäresProjektverändertdieBildungswelt.In: Jüttemann, G. (Hrsg.): Entwicklungender Menschheit. Humanwissenschaften in der Perspektive der Integration. Lengerich: Pabst. S. 137-144.

Garfinkel, Harold (1967a): What is ethnomethodology? In: Garfinkel, H. (Hrsg): Studies in ethnomethodology. Englewood Cliffs: Prentice-Hall. S. 1-34.

Garfinkel, H. (1967b): Studies in Ethnomethodology. Englewood Cliffs: Prentice-Hall.

Geiger, D. \& Schreyögg, G. (2003): Wenn alles Wissen ist, ist Wissen am Ende nichts?! In: Deutsche Betriebswirtschaft, 63, S. 7-22.

Geißler, E. (1970): Herbarts Lehre vom erziehenden Unterricht. Heidelberg: Quelle \& Meyer.

Giddens, A. (1984): The Constitution of Society: Outline of the Theory of Structuration. Oxford: Polity Press.

Giddens, A. (1997): Die Konstruktion der Gesellschaft. Grundzüge einer Theorie der Strukturierung. Frankfurt a.M.: Campus.

Giesecke, H. (2013): Pädagogik als Beruf. Grundformen pädagogischen Handelns. Weinheim/Basel: Belz Juventa.

Giles, J. \& Middleton, T. (2008): Studying Culture. A Practical Introduction. Oxford: Blackwell.

Glaser, B. (2001): The Grounded Theory: Issues and Discussions. Mill Valley, California: Sociology Press.

Glaser, B. (2002): Constructivst Grounded Theory? In: Forum Qualitativer Sozialforschung/Forum: Qualitative Social Research, 3 (3), Art. 23.

Glaser, B. (2007): Remodeling Grounded Theory. In: Mey, G. \& Mruck, K. (Hrsg.): Grounded Theory Reader. Zentrum für Historische Sozialforschung. Köln: Zentrum für Historische Sozialforschung. S. 47-68.

Glaser, B. (2015): The impact of Symbolic Interaction on Grounded Theory. In: Grounded Theory Review, 4 (2), S. 1-22.

Glaser, B. \& Strauss, A.L. (1965): Awareness of dying. New York: Aldine.

Glaser, B. \& Strauss, A.L. (1967): The Discovery of Grounded Theory. Strategies for Qualitative Research. New York: Aldine. Glaser, B. \& Strauss, A.L. (1968): Time of Dying. New York: Aldine.

Gläser, J. \& Laudel, G. (2006): Experteninterviews und qualitative Inhaltsanalyse. Wiesbaden: Springer.

Göhlich, M. \& Zirfas, J. (2007): Lernen. Ein pädagogischer Grundbegriff. Stuttgart: Kohlhammer.

Gordon, T. (2013): Gute Beziehungen: Wie sie entstehen und stärker werden. Stuttgart: Klett.

Gottschalk-Mazouz, N. (2007): Was ist Wissen? Überlegungen zu einem Komplexbegriff an der Schnittstelle von Philosophie und Sozialwissenschaften. In: Ammon, S. et al. (Hrsg.): Wissen in Bewegung. Dominanz, Synergien und Emanzipation in den Praxen der „Wissenensgesellschaft". Weilerswirt: Velbrück. S. 21-40.

Groeben, N. et al. (1988): Das Forschungsprogramm Subjektive Theorien. Eine Einführung in die Psychologie des reflexiven Subjekts. Tübingen: Franke. 
Gropengießer, H. (2003): Lernen und Lehren - Thesen und Empfehlunen zu einem professionellen Verständnis. In: Gehirn und Lernen, 3, S. 29-39.

Grossmann, P.L. \& Richert, A.E. (1988): Unknowledged knowledge growth: A re-examination of the effects of teacher education. In: Teaching and Teacher Education, 4(1), S. 53-62.

Gruber, H. \& Renkl, A. (2000): Die Kluft zwischen Wissen und Handeln: Das Problem des trägen Wissens. In: Neuweg, G.H. (Hrsg.): Wissen- Können-Profession. Ausgewählte Verhältnisbestimmungen. Innsbruck: Studienverlag. S. $155-174$.

Gruber, H. \& Stamouli, E. (2009): Intelligenz und Vorwissen. In: Wild, E. \& Mölle, J. (Hrsg.): Pädagogische Psychologie. Heidelberg: Springer. S. 27-46.

Gruschka, A. (2011): Verstehen lehren. Ein Plädoyer für guten Unterricht. Stuttgart: Reclam.

Gudjons, H. (2000): Belastungen und neue Anforderungen. Aspekte der Diskussion um Lehrer und Lerherinnen in den 80er und 90er Jahren. In: Bastian, J. et al. (Hrsg.): Professionalisierung im Lehrerberuf. Opladen: Springer. S. 33-51.

Gudjons, H. (2006): Pädagogisches Grundwissen. Bad Heilbrunn: Klinkhardt.

Guerriero, S. (2015): Directorate for education and skills centre for educational Research and innovation (CERI) governing board. [http://www.oecd.org/officialdocuments/publicdisplaydocumentpdf/?cote=EDU/CERI/CD/ $\mathrm{RD}(2014) 3 / \mathrm{REV} 1 \&$ doclanguage $=$ en; zugegriffen 16.01.2019]

Guerriero, S. (2017): Teachers' Pedagogical Knowledge and the Teaching Profession. Background Report and Project Objectives. OECD.

Hackl, B. (2004): Explizites und implizites Wissen. In: Hackl, B. \& Neuweg, G.-H.: Zur Professionalisierungpädagogischen Handelns. Österreichische Gesellschaft für Bidlunsgforschung Band 1. Münster. LIT Verlag. S. 69-112.

Hamlyn, D.W. (1970): The Theory of Knowledge. London/Basingstoke: The Macmillan Press.

Handal, G. \& Lauvas, P. (1987): Promoting reflective teaching: Supervision in action. Milton Keynes: SHRE and Open University Press.

Hargreaves, D. (2003): From Improvement to Transformation. Keynot bei der ICSE Conference 2003 „Schooling the Knowledge Society"von 05.-08.01.2003.

Hartfiel, G. (1982): Wörterbuch der Soziologie. Stuttgart: Kröner.

Hascher, T. (2014): Forschung zur Wirksamkeit der Lehrerbildung. In: Terhart, E. et al. (Hrsg.): Handbuch der Forschung zum LehrerInnenberuf. Münster: Waxmann. S. 542-571.

Hatti, J. (2003): Teachers Make a Difference. What is the Research Evidence? Camberwell, Victoria: ACER.

Haun, M. (2003): Handbuch Wissensmanagement. Wiesbaden: Springer.

Hebestreit R. (2013): Die Transformation der Gesellschaft zur Wissensgesellschaft. In: Hebestreit; R. (Hrsg.): Partizipation in der Wissensgesellschaft. Wiesbaden: Springer. S. 29-61.

Heidenreich, M. (2002): Merkmale der Wissensgesellschaft. [http://www.sozialstruktur.uni-oldenburg.de/dokumente/ blk.pdf; zugegriffen am 01.03.2019]

Heimann, P. et al. (Hrsg.) (1965): Unterricht - Analyse und Planung. Hannover: Schroedel.

Hellekmaps, S. \& Musloff, H. (2014): Forschung zur Geschichte und Entwicklung des Lehrerberufs in der frühen Neuzeit. In: Terhart, E. et al. (Hrsg.): Handbuch der Forschung zum Lebrerberuf. Münster: Waxmann. S. 34-51.

Helmke, A. (2003): Unterrichtsqualität erfassen, bewerten, verbessern. Seelze-Velber: Kallmeyer.

Helmke, A. (2009): Unterrichtsqualität und Lehrerprofessionalität - Diagnose, Evaluation und Verbesserung des Unterrichts. Seelze-Velber: Klett.

Helmke, A \& Schrader, W.-F. (2006): Lehrerprofessionalität und Unterrichtsqualität - Lehrerprofessionalität und Unterrichtsqualität. In: Schulmagazin, 9, S. 5-12.

Helsper, W. (2001): Praxis und Reflexion: Die Notwendigkeit einer „Doppelten Professionalisierung“ des Lehrers. In: Journal für Lehrerinnen und Lehrerfortbildung,1(3) S. 7-15.

Henting, H. (1996): Die Schule und die Lehrerbildung neu denken. In: Hänsel, D. \& Hubert, L. (Hrsg.): Lehrerbildung neu denken und gestalten. Weinheim: Beltz. S. 17-38.

Henting, H. (2006): Bewärhung. Von der nützlichen Erfabrung nützlich zu sein. München: Hanser Henting.

Heppekausen, J. (2013): Beobachtung, Selbstbeobachtung und Reflexion in der Lernbegleitung. In: Coelen, H. \& Müller-Naendrup, B. (Hrsg.): Studieren in Lernwerkstätten. Wiesbaden: Springer. S. 109-126.

Herbart, J. (1969): Kleine pädagogische Schriften. Düsseldorf: Küpper.

Herbart, J. (1982): Über die ästhetische Darstellung der Welt als das Hauptgeschäft der Erziehung [1804]. In: Pädagogische Schriften. Band 1: Kleinere pädagogische Schriften. Stuttgart: Walter Asmus.

Herbart, J. (1890): Pädagogische Schriften Band 1 Hrsg. 5. Auflage neu bearbeitet von E. Salwürk, Langensalza.

Hericks, U. (2006): Professionalisierung als Entwicklungsaufgabe - Eine rekonstruktive Studie zur Berufseingangsphase von Lehrerinnen und Lehrer. Wiesbaden: Springer.

Herrmann, U. \& Hertramph, H. (2000): Zufallsroutinen oder reflektierte Praxis? Herkömmliche Wege in den Berufseinstieg von Lehrern und notwendige Alternativen. In: Beiträge zur Lehrerbildung,18 (2), S. 172-191. 
Herzmann, P. \& König, J. (2016): Lehrerberuf und Lehrerbildung. Bad Heilbrunn: Klinkhardt.

Herzog, W. (1995): Reflexive Praktika in der Lehrerinnen- und Lehrerbildung. In: Beiträge zur Lehrerbildung, 13 (3), S. 253-273.

Hoerster, N. (2010): Waskönnen wir wissen? Philosophische Grundfragen. München: Verlag C.H. Beck.

Hof, C. \& Rosenberg, H. (Hrsg.) (2018): Lernen im Lebenslauf. Einführung in den Band. In: Hof, C. \& Rosenberg, H. (Hrsg.): Lernen im Lebenslauf - Theoretische Perspektiven und empirische Zugänge. Theorie und Empirie Lebenslangen Lernens. Wiesbaden: Springer. S. 1-12.

Hohenstein, F. et al. (2014): Sind die bildungswissenschaftlichen Standards für die LehrerInnenausbildung in den Curricula der Hochschulen angekommen? In: Zeitschrift für Erziehungswissenschaft, 17 (3), S. 497-507.

Hohenstein, F. et al. (2015): Pädagogisches Wissen von Lehrkräften. In: Zeitschrift für Erziehungswissenschaft, 18, S. 183-186.

Hohenstein, F. et al. (2017): Erfassung von pädagogischem und psychologischem Wissen in der Lehramtsausbildung: Entwicklung eines Messinstruments. In: Zeitschrift für Pädagogik,1, S. 91-113.

Höhne, T. (2007): Der Leitbegriff Kompetenz als Mantra neoliberaler Bildungsreform. Zur Kritik seiner semantischen Weitläufigkeit und inhaltlichen Kurzatmigkeit. In: Pongratz, L. et al. (Hrsg.): Bildung - Wissen - Kompetenz. Bielefeld: Janus Presse. S. 30-43.

Holzkamp, K. (2004): Wider den Lehr-Lern-Kurzschluß. Interview zum Thema $>$ Lernen<. Zuerst erschienen in Rolf Arnold (Hrsg.) Lebendiges Lernen. In: Faulstich, P. \& Ludwig, J.(Hrsg.): Expansives Lernen. Hohengehren: Scheider. S. 29-38.

Horvath, W. (2009): Auf der Suche nach den guten Leherinnen und Lerher. In: Schrittesser, I. (Hrsg.): Professionalität und Professionalisierung. Frankfurt: Peter Lang. S. 21-36.

Howaldt, J. et al. (2004): Neuorientierung des Wissensmanagements - Paradoxien und Dysfunktionalitäten im Umgang mit der Ressource Wissen. Wiesbaden: Deutscher Universitäts-Verlag.

Hug, T. \& Poscheschnik, G. (2010): Empirisch Forschen - Die Planung und Umsetzung von Projekten im Studium. Konstanz: UVK Verlagsgesellschaft mbH.

Hume, D. (1975): A Treatise of Human Natur. Oxford: University Press.

Illeris, K. (2007): How We learn: Learning and Non-learning in School and Beyond. London/New York: Routledge.

Illeris, K. (2018): A comprehensive understanding of human learning. In: Illeris, K. (Hrsg.): Contemporary theories of learning. New York: Routledge. S. 7-20.

Jendrowiak, H.-W. \& Kreuzer, K. (1982): Anthropologische Grundlagen des Unterrichts. Düsseldorf: Schwann.

Johnston, S. (1992): Images: A way of understanding the practical knowledge of student teachers. In: Teaching and Teacher Education, 8, S. 123-136.

Jost, G. (2003): Biographische Selbstorganisation. In: Zeitschrift für Soziologie der Erziehung und Sozialisation, 1, S. 85-94.

Jüssen, G. et al. (2006): Thomas von Aquin: über den Lehrer/De magsitro. Hamburg: FelixMeiner Verlag.

Kahl, R. (1999): Der Neugierologe. In: GEOWISSEN, 1, S. 106-109.

Kant, I. (1781): Kritik der reinen Vernunft. Auflage 1. Leipzig: Reclam.

Kant, I. (1977): Über Pädagogik. In: Kant, I. (Hrsg.): Werke in 12 Bänden. Von Wilhelm Weischedel, Bd.12. Frankfurt am Main: Suhrkamp. S. 691-761.

Keck, R.W. (2009): Zur Geschichte der Schule. In: Blömeke et al. (Hrsg.): Handbuch Schule. Regensburg: KlinkhartUTB. S. 157-161.

Keil, W. (2001): Klientenzentrierter Therapietheorie. In: Frenzel, P. et al. (Hrsg.): Klienten-/Personzentrierte Psychotherapie - Kontexte, Konzepte, Konkretisierungen. Wien: Facultas Universitätsverlag. S. 119-146.

Keller-Schneider, M. \& Hericks, U. (2014): Forschung zum Berufseinstieg. Übergang von der Ausbildung in den Beruf. In: Terhart, E. et al. (Hrsg.): Handbuch der Forschung zum LehrerInnenberuf. Münster: Waxmann. S. 386-407.

Kemnitz, H. (2014): Forschung zur Geschichte und Entwicklung des Lehrerberufs vom 18. Jahrhundert bis zur Gegenwart. In: Terhart, E. et al. (Hrsg.): Handbuch der Forschung zum Lehrerberuf. Münster: Waxmann. S. 52-72.

Kemnitz, H. (2011): Forschung zur Geschichte und Entwicklung des Lehrerberufs vom Ende des 18. Jahrhunderts bis zur Gegenwart. In: Terhart, H. et al. (Hrsg.): Handbuch der Forschung zu Lehrerberuf. Münster: Waxmann. S. 34-51.

Kintzinger, M. (2014): Forschung zur Geschichte und Entwicklung des Lehrerberufs vom Mittelalter bis zum Ende des 17. Jahrhunderts. In: Terhart, E. et al. (Hrsg.): Handbuch der Forschung zum Lebrerberuf. Münster: Waxmann. S. $15-33$.

Klafki, W. (1985): Neue Studien zur Bildungstheorie und Didaktik. Beiträge zur kritisch-konstruktiven Didaktik. Weinheim: Belz.

Kleining, G. (1982): Umriß zu einer Methodologie qualitativer Sozialforschung In: Kölner Zeitschrift für Soziologie und Sozialpsychologie, 34, S. 224-253. 
Kleickmann, T. et al. (2014): Professionswissen von Lehramtsstudierenden der mathematisch-naturwissenschaftlichen Fächer- Testentwicklung im Rahmen des Projekts KiL. In: Unterrichtswissenschaft, 42(3), S. 280-288.

Klieme, E. et al. (Hrsg.) (2003): Bildungsforschung: Bd. 1. Zur Entwicklung nationaler Bildungsstandards. Eine Expertise. Bonn: Bundesministerium für Bildung und Forschung.

KMK (2004): Sekretariat der Ständigen Konferenz der Kultusminister der Länder in der Bundesrepublik Deutschland. Standards für die Lehrerbildung: Bildungswissenschaften. Beschluss der Kultusministerkonferenz vom 16.12.2004. [https://www.kmk.org/fileadmin/veroeffentlichungen_beschluesse/2004/2004_12_16-Standards-Lehrerbildung. pdf; zugegriffen am 15.01.2019]

Knoblauch, H. (2005): Wissenssoziologie. Konstanz: UVK Verlagsgesellschaft.

Knorr, C. (2002): Wissenskulturen. Ein Vergleich naturwissenschaftlicher Wissensformen. Frankfurt: Suhrkamp.

Koch, L. (2015): Lehren und Lernen - Wege zum Wissen. Paderborn: Ferdinand Schönigh.

Kocher, M. (2014): Selbstwirksamkeit und Unterrichtsqualität. Münster: Waxmann.

Korthagen, F.A.J. et al. (2001): Linking practice and theory: the pedagogy of realistic teacher education. Mahawah, $\mathrm{Nj}$ : Lwarence Erlabum Associates.

Korthagen, F.A.J. \& Kessels, J.P.A.M. (1999): Linking theory and practice: changing the pedagogy of teacher education. In: Educational Research, 28 (4), S. 4-17.

Kortz, F. (2008): Handlungstheorien und Symbolischer Interaktionismus als Grundlage kommunikationswissenschaftlicher Forschung. In: Winter, C. et al. (Hrsg.): Theorie der Kommunikations- und Medienwissenschaft. Wiesbaden: Springer. S. 29-48.

König, J. (2010a): Lehrerprofessionalität - Konzepte und Ergebnisse der internationalen und deutschen Forschung am Beispiel fachübergreifender, pädagogischer Kompetenzen. In: König, J. \& Hofmann, B. (Hrsg.): Professionalität von Lehrkräften. Was sollen Lehrkräfte im Lese- und Schreibunterricht wissen und können? Berlin: DGLS. S. 40-105.

König, J. (2010b): Längsschnittliche Erhebung pädagogischer Kompetenzen von Lehramststudierenden (LEK). Theoretischer Rahmen, Fragestellungen, Untersuchungsanalse und erste Ergebnisse zu Lernvoraussetzungen von angehenden Lehrkräften. In: Lehrerbildung auf dem Prüfstand, 3(1), S. 56-83.

König, J. (2014a): Forschung zum Erwerb von pädagogischem Wissen angehender Lehrkräfte in der Lehrerausbildung. In: Terhart, E. et al. (Hrsg.): Handbuch der Forschung zum Lehrerberuf. Münster/New York: Waxmann. S. 615-641.

König, J. (2014b): “Designing an International Instrument to Assess Teachers' General Pedagogical Knowledge (GPK): Review of Studies, Considerations, and Recommendations", Technical paper prepared for the OECD Innovative Teaching for Effective Learning (ITEL) -Phase II Project: A Survey to Profile the Pedagogical Knowledge in the Teaching Profession (ITEL Teacher Knowledge Survey), Paris, OECD, [http://www.oecd.org/officialdocuments/publicdisplaydocum entpdf/?cote=EDU/CERI/CD/RD(2014)3/REV1\&doclanguage=en; zugegriffen am 16.01.2019.]

König, J. et al. (2011): General pedagogical knowledge of further middle school teachers: On the complex ecology of teacher education in the United States, Germany, Taiwan. In: Journal of Teacher Education, 62(2), S. 188-201.

König, J. et al. (2014): Is teachers' general pedagogical knowledge a premise for noticing and interpreting classroom situations? A video-based assessment approach. In: Teaching and Teacher Education, 38, S. 76-88.

König, J. \& Blömeke, S. (2009): Pädagogisches Wissen von angehenden Lehrkräften: Erfassung und Struktur von Ergebnissen der fachübergreifenden Lehrerausbildung. In: Zeitschrift für Erziehungswissenschaft, 12 (3), S. $499-527$.

König, J. \& Blömeke, S. (2010): Messung des pädagogischen Wissens: Theoretischer Rahmen und Teststruktur. In: TEDS-M 2008 Primarstufe: Ziele, Untersuchungsgrundlage und zentrale Ergebnisse. In: Blömeke, S. et al. (Hrsg.): TEDS-M 2008 - Professionelle Kompetenz und Lerngelegenheiten angehender Primarstufenlehrkräfte im internationalen Vergleich. Münster: Waxmann. S. 253-274.

König, J. \& Seifert, A. (Hrsg.) (2012): Lehramtsstudierende erwerben pädagogisches Professionswissens: Ergebnisse der Längsschnittstudie LEK zur Wirksamkeit der erziehungswissenschaftlichen Lehrerausbildung. Münster: Waxmann.

König, J. \& Klemenz, S. (2015): Der Erwerb von pädagogischem Wissen bei angehenden Lehrkräften in unterschiedlichen Ausbildungskontexten: Zur Wirksamkeit der Lehrerausbildung in Deutschland und Österreich. In: Zeitschrift für Erziehungswissenschaft, 18, S. 247-277.

Kuckartz, U. (2016): Qualitative Inhaltsanalyse. Methoden, Praxis, Computerunterstützung. Weinheim; Basel: Beltz Juventa.

Kunina-Habenicht, O. et al. (2012): Welche bildungswissenschaftliche Inhalte sind wichtig in der Lehrerbildung? Ergebnisse einer Delphi-Studie. In: Zeitschrift für Erziehungswissenschaft, 15 (4), S. 649-682.

Kunter, M. (2009): COACTIV-R: Kompetenzerwerb von Lehramtskandidat(inn)en im Vorbereitungsdienst. Berlin: MaxPlank-Institut für Bildungsforschung.

Kunter, M. et al. (Hrsg.) (2011): Professionelle Kompetenz von Lehrkräften. Ergebnisse des Forschungsprogramms COAC$T I V$. Münster: Waxmann.

Kunter, M. et al. (2013): Cognitive activation in the mathematics classroom and professional competences of teachers. Results from the COACTIV-Projects. New York: Springer. 
Kunter, M. et al. (2017): Bildungswissenschaftliches Wissen und professionelle Kompetenz in der Lehramtsausbildung - Ergebnisse des Projekts BilWiss. In: Entwicklung von Professionalität pädagogischen Personals, S. 37-54.

Kübler, H.-D. (2005): Mythos Wissensgesellschaft - Gesellschaftlicher Wandeln zwischen Information, Medien und Wissen. Eine Einführung. Wiesbaden: Springer.

Kübler, H-D. (2010): Medien: Faktoren, Reflexion und Archiv gesellschaftlichen Wandels. In: Engelhardt, A. \& Kajetzke, L. (Hrsg.): Handbuch Wissensgesellschaft. Bielefeld: Transcript Verlag. S. 171-182.

Kraler, C. (2011): Selbstähnlichkeiten in der LehrerInnenbildung. In: Kraler et al. (Hrsg.): Kulturen der Lehrerbildung. München/Berlin: Waxmann. S. 41-74.

Kraler, C. \& Schratz, M. (2012): From Best Practice to Next Practice: A Shift through Research-Based Teacher Education. In: Reflecting Education, 8 (2), S. 88-125.

Krauss, S. et al. (2008): Die Untersuchung des professionellen Wissens deutscher Mathematik-Lehrerinnen- und Lehrer im Rahmen der COACTIV-Studie. In: Journal für Mathematikdidaktik, 29, S. 223-258.

Krohn, W. (1997): Rekursive Lernprozesse: Experimentelle Praktiken in der Gesellschaft. Das Beispiel der Abfallwirtschaft. In: Rammert, W. \& Bechmann, G. (Hrsg.): Technik und Gesellschaft. Jahrbuch 9: Innovation - Prozesse, Produkte, Politik. Frankfurt a.M.: Campus Verlag. S. 65-89.

Krohn, W. (2006): Wissen ist Macht - Francis Bacon. [http://www.uni-bielefeld.de/soz/personen/krohn/wissen_ist_ macht.pdf; zugriffen am 04.05.2019]

Krümmel, F. (1987): Zur Bestimmung der Formel: Pädagogik als „Theorie einer Praxis“. In: Blankertz, H. (Hrsg.): Die Theorie-Praxis-Diskussion in der Erziehungswissenschaft. Weinheim: Beltz.

Lamla, J. (2003): Anthony Giddens. New York/Frankfurt: Campus Verlag.

Lamneck, S. (2010): Qualitative Sozialforschung. Beltz: Weinheim.

Lange, H. (2008): Vom Messen zum Handeln: „empirische Wende“ der Bildungspolitik? In: Recht der Jugend und Bildungswesen, 56, S. 7-15.

Leicht, A. et al. (2018): Issues and trends in Education for Sustainable Development. UNESCO Publishing.

Lenske, G. et al. (2015): Pädagogisch-psychologisches Professionswissen von Lehrkräften: Evaluation des ProwiN-Tests. In: Zeitschrift für Erziehungswissenschaft, S. 225-245.

Lenske, G. et al. (2017): Zum Einfluss des pädagogisch-psychologischen Professionswissens auf die Unterrichtsqualität und das situationale Interesse der Schülerinnen und Schüler. In: Zeitschrift für Bildungsforschung, 7, S. 229-253.

Lenz, W. (2005): Bildung im Wandel. Wien: Studientexte.

Lipowsky, F. (2006): Auf den Lehrer kommt es an. Empirische Evidenzen für Zusammenhänge zwischen Lehrerkompetenzen, Lehrerhandeln und dem Lernen der Schüler. In: Zeitschrift für Pädagogik, 51, S. 47-70.

Lipowsky, F. (2014): Theoretische Perspektiven und empirische Befunde zur Wirksamkeit von Lehrerfort- und -weiterbildung. In: Terhart, E. et al. (Hrsg.): Handbuch der Forschung zum LehrerInnenberuf. Münster: Waxmann. S. 511-541.

Loughran, M. (2019): Pedagogical reasoning: the foundation of the professionalknowledge of teaching. In: Teachers and Teaching, DOI:10.1080/13540602.2019.1633294.

Lüders, C. (2000): Beobachten im Feld und Ethnographie. In: Flick, et al. (Hrsg): Qualitative Forschung - Ein Handbuch. Reinbeck: Rowohlt. S. 284-401.

Luhmann, N. (1997): Die Gesellschaft der Gesellschaft. Frankfurt: Suhrkamp.

Maasen, S. (1999): Wissenssoziologie. Bielefeld: Transcript.

Marotzki, W. (2007). Qualitative Biographieforschung. In Flick, U. et al. (Hrsg.): Qualitative Forschung. Ein Handbuch. Reinbek: Rowohlt. S. 175-186.

Mayring, P. (1999): Einführung in die qualitative Sozialforschung. Weinheim: Psychologie Verlags Union.

McDonalds, J.P. (1992): Teaching: Making sense of an uncertain craft. New York Teachers College Press.

McLuhan, M. (1967): The Medium is the Massage.UK: Penguin Books.

Mead, G. (1934): Geist, Identität und Gesellschaft. Mit einer Einleitung herausgegeben von Charles W. Moriris. Frankfurt am Main: Suhrkamp.

Merton, R. (1972): Wissenschaft und demokratische Sozialstruktur. In: Weingart, P. (Hrsg.): Wissenschaftssoziologie. Wissenschaftliche Entwicklung als sozialer Prozess. Frankfurt. S. 45-59.

Mescheder, B. \& Sallach, C. (Hrsg.) (2012): Wissen und Wissensmanagement. Berlin/Heidelberg: Springer.

Messner, H. (2007): Vom Wissen zum Handeln - vom Handeln zum Wissen. Zwei Seiten einer Medaille. In: Beiträge zur Lehrerinnen- und Lehrerbildung. S. 364-376.

Messner, H. \& Reusser, K. (2000): Berufliches lernen als lebenslanger Prozess. In: Beiträge zur Lehrerbildung 18 (3), S. 277-294.

Meuser, M. (2006): Rekonstruktive Sozialforschung. In: Bohnsack, R. et al. (Hrgs.): Hauptbegriffe Qualitativer Forschung. Opladen: Verlag Barbara Budrich, S. 140-142.

Miebach, B. (2014): Soziologische Handlungstheorie. Wiesbaden: Springer. 
Mikhail, T. (2016): Pädagogisch handeln - Theorie für die Praxis. Paderborn: Ferdinand Schöningh GmbH.

Minnameier, G. (2009): Kognitive Voraussetzungen der Entwicklung von pädagogischer Professionalität. In: ZlatkinTroitschanskaia et al. (Hrsg.): Lehrerprofessionalität - Bedingungen, Genese, Wirkungen und ihre Messung. Weinheim/Basel: Beltz. S. 333-344.

Mittelstraß, J. (1989): Glanz und Elend in den Geisteswissenschaften. Oldenburger Universitätsreden: Bibliotheks- und Informationssysteme.

Mittelstraß, J. (1998): Information oder Wissen - vollzieht sich ein Paradigmenwechsel? In: Physikalische Blätter, 54 (5), S. 445-447.

Mittelstraß, J. (2001): Wissen und Grenzen. Baden-Baden: Suhrkamp.

Moegling, K. (2010): Die Kompetenzdebatte - Zum Verhältnis von Bildung und Kompetenzorientierung. In: Schulpädaogik heute, 1, S. $1-16$.

Molander, B. (1992): Tacit Knowledge and Silenced Knowledge. Fundamental Problems and Controversies. In: Göranzon, B. \& Florin, M. (Hrsg.): Skill and Education. Reflection and Experience. London: Springer. S. 9-31.

Morine-Deshimer, G. \& Kent, T. (1999): The complex nature and sources of teachers' pedagogical knowledge. In: GessNewsome, J. \& Lederman, N.G. (Hrsg.): Examining pedagogical content knowledge. Dordrecht: Kluwer Academic Publisher. S. 21-50.

Morse, J. (1998): Designing Funded Qualitative Research. In N. Denzin, \& Y. Lincoln (Hrsg.): Strategies of Qualitative Inquiry. Thousand Oaks, CA: Sage Publication. S. 56-85.

Müller de Menezes, R. (2012): Soziale Arbeit in der Sozialbilfe - Eine qualitative Analyse von Fallbearbeitungen. Wiesbaden: Springer.

National Research Council (2012): Education for life and work: Developing transferable knowledge and skills in the 21st century. Report Breid. Washington, DC: National Academies Press. [https://www.nap.edu/ressource/13398/dbasse_070895.pdf; zugegriffen am 28.01.2019]

Neumann, S. (2012): Wie kann Forschung Praxis verändern? Der Beitrag ethnigraphischen Wissens zur Qualitätsentwicklung in der Kinderbetreuung. In: Forum für Politik, Gesellschaft und Kultur, 322, S. 35-37.

Neuweg, G.-H. (1999): Könnerschaft und implizites Wissen. München/Berlin: Waxmann.

Neuweg, G.-H. (2000): Mehr lernen, als man sagen kann: Konzepte und didaktische Perspektiven impliziten Lernens. In: Unterrichtswissenschaft, 8, S. 197-217.

Neuweg, G.-H. (2002): Lehrerhandeln und Lehrerbildung im Lichte des Konzepts des impliziten Wissens. In: Zeitschrift für Pädagogik, 48 (1), S. 10-29.

Neuweg, G.-H. (2004): Könnerschaft und implizites Wissen. Münster/New York: Waxmann.

Neuweg, G.-H. (2014): Das Wissen der Wissensvermittler. Problemstellungen, Befunde und Perspektiven der Forschung zum Lehrerwissen. In: Terhart, E. et al. (Hrsg.): Handbuch der Forschung zum LehrerInnenberuf. Münster: Waxmann. S. 583-614.

Nolle, A. (2004): Evaluation der universitären Lehrerinnen-und Lehrerausbildung: Erhebung zur pädagogischen Kompetenz von Studierenden der Lehramtsstudiengänge. München: M-Press.

Nyle, B. et al. (2004): How Large Are Teacher Effects? In: Educational Evaluation and Policy Analysis, 26 (3), S. $237-257$.

Oberauer, K. (1993): Prozedurales und deklaratives Wissen und das Pradigma der Informationsverarbeitung. In: Sprache und Kognition 12,1, S. 30-43.

OECD (2005): Teachers matter. Attracting, developing and retaining effective teachers. Paris: OECD

Oelkers, J. (1989): Die große Aspiration. Zur Herausbildung der Erziehungswissenschaft im 19. Jahrhundert. Darmstadt: Wissenschaftliche Buchgesellschaft.

Oelkers, J. \& Tenorth, E. (Hrsg.) (1991): Pädagogisches Wissen. In: Zeitschrift für Pädagogik, 27. Beiheft. Weinheim und Basel: Beltz. S. 12-35.

Oelkers, J. \& Tenorth, E. (1993): Pädagogisches Wissen als Orientierung und als Problem. In: Oelkers, J. \& Tenorth, E.(Hrsg.): Pädagogisches Wissen. Weinheim/Basel: Beltz. S. 13-38.

Ontario (2016): $21^{\text {st }}$ Century Competencies - Foundation Document for Discussion. [http://www.edugains.ca/resources21CL/About21stCentury/21CL_21stCenturyCompetencies.pdf; zugegriffen am 25.02.2019]

Oser, F. (1998): Ethos - die Vermenschlichung des Erfolgs. Zur Psychologie und Berufsmoral von Lehrpersonen. Opladen: Leske \& Budrich.

Pajares, M.F. (1992): Teachers' belief and educational research: Cleaning up a messy construct. In: Review of Educational Research, 62, S. 307-332.

Paquay, L. \& Wagner, M.-C. (2001): Compétences professionnelles privilégiées dans les stages et en vidéo-formation. In: Paquay, L. (Hrsg.): Former des enseignants professionnels. Quelles stratégies? Quelles compétences? Bruxelles: De Broeck.

Patton, Michael Quinn (2002): Qualitative Research \& Evaluation Methods. Thousand Oaks: Sage Publications. 
Pellegrino, J.W. \& Hilton, M. (Hrsg.) (2012): Teaching, learning and assessing 21st century: Developing Transferable Knowledge and Skills in the 21st Century. Washington, DC: National Academies Press.

Peskoller, H. (2014): Körperlicher Raum. In: Zirfas, J. \& Wulf, C. (Hrsg.): Handbuch Pädagogische Anthropologie. Wiesbaden: Springer. S. 395-402.

Pestalozzi, J. (1961): Wie Gertrud ihre Kinder lehrt. Ein Versuch, den Müttern Anleitung zu geben, ihre Kinder selber zu unterrichten, in Briefen. Bad Heilbrunn: Klinkhardt.

Petzelt, A. (1964): Grundzüge systematischer Pädagogik. Freiburg: Lambertus-Verlag.

Pfister, J. (2016): Texte zur Wissenschaftstheorie. Stuttgart: Reclam.

Plate, M. (2015): Grundlagen der Kommunikation. Göttingen: Vadenhoeck \& Ruprecht LLC.

Platon (2005): Werke in acht Bänden. Griechisch und Deutsch. Übersetzung von Friedrich Schleiermacher. Darmstadt: Gunther Eigler.

Plümacher, M. (2012): Erfahrung und Wissen. Husserls Forschung zum impliziten Wissen. In: Rivista internazionale difilosofia e psicologia, 3 (2), S. 135-146.

Polanyi, M. (1985): Implizites Wissen. Frankfurt: Suhrkamp.

Preißer, R. (2003): Berufsbiographische Selbstorganisation, biographisches Lernen, Selbstsozialisation- Herausforderung für die Erwachsenenbildung? In: Gehirn und Lernen, Report 3. S. 20-28.

Przyborski, A. \& Wohlrab-Sahr, M. (2008): Qualitative Sozialforschung. München: Oldenbourg Wissenschaftsverlag.

Putnam, R.T. (1987): Structuring and adjusting content for students: A study of live and simulated tutoring of addition. In: American Educational Research Journal, 24 (1), S. 13-48.

Rabl, C. (2014): Partiale Perspektiven - Zur Reformulierung eines bildenden Umgangs mit Wissen. Baltmannsweiler: Schneider Verlag.

Radtke, F.-O. (1999): Lehrerbildung an der Universität: zur Wissensbasis pädagogischer Professionalität. Frankfurt: Fachbereich Erziehunsgwissenschaft.

Radtke, F.-O. (2000): Professionalisierung der Lehrerbildung durch Automatisierung, Entstaatlichung, Modularisierung. [https://www.sowi-online.de/sites/default/files/radtke.pdf; zugegriffen am 05.02.2019]

Raithel, J. et al. (2009): Einführung Pädagogik. Wiesbaden: Springer.

Reckwitz, A. (1999): Anthony Giddens. In: Kaesler, D. (Hrsg.): Klassiker der Soziologie. Band II. Von Talcott Parsons bis Anthony Giddens. München: Beck. S. 311-337.

Reetz L. \& Tramm T. (2000): Lebenslanges Lernen aus der Sicht einer berufspädagogisch und wirtschaftspädagogisch akzentuierten Curriculumforschung. In: Achtenhagen F. \& Lempert W. (Hrsg): Lebenslanges Lernen im Berufseine Grundlegung im Kindes- und Jugendalter. Wiesbaden: Springer. S. 69-120.

Reh, S. \& Schelle, C. (2000): Biographie und Professionalität. Die Reflexivität Biographischer Erzählungen. In: Bastian, J. et al. (Hrsg.): Professionalisierung im LehrerInnenberuf. Von der Kritik der Lerherrolle zur pädagogischen Professionalität. Opladen: Leske + Budrich. S. 107-124.

Reh, S. \& Schelle, C. (2006): Biographieforschung in der Schulpädagogik. In: Krüger, H. \& Marotzki, W. (Hrsg.): Handbuch erziehungswissenschaftlicher Biographieforschung. Wiesbaden: Verlag für Sozialwissenschaften. S. 391412.

Renkl, A. (1996): Träges Wissen: Wenn Erlerntes nicht genutzt wird. In: Psychologische Rundschau, 47, S. 78-92.

Rheinberg, F. et al. (2001): Die Erziehenden und Lehrenden. In: Krapp, A. \& Weidenmann, B.: Pädagogische Psychologie. Hemsbach: Beltz. S. 271-354.

Richardson, V. (1996): The role of attitudes and beliefs in learning to teach. In: Sikula, J. (Hrsg.): Handbook of research on teacher education. New York: Macmillan. S. 102-109.

Rittelmeyer, C. (2014): Architektonischer Raum. In: Zirfas, J. \& Wulf, C. (Hrsg.): Handbuch Pädagogische Anthropologie. Wiesbaden: Springer Verlag. S. 387-394.

Rittler-Johnson, B. et al. (2001): Developing conceptual understanding and procedural skill in mathematics: An iterative process. In: Journal of Education Psychology, 93(2), S. 346-362.

Rogers, C. (1981): Die Grundlagen eines personenzentrierten Ansatzes. In: Rogers, C. (Hrsg.): Der neue Mensch. Stuttgart: Klett-Cotta. S. 65-84.

Rosa, H. (2013): Beschleunigung und Entfremdung. Berlin: Suhrkamp.

Roth, G. (2003): Warum sind Lehren und Lernen so schwierig? In: Gehirn und Lernen, Report 3, S. 87-98.

Ryle, G. (1949): The Concept of the Mind. Stuttgart: Reclam.

Ryle, G. (1976): Improvisation. In: Mind, 337, S. 68-83.

Schlömerkemper, J. (2017): Pädagogische Prozess ein antinomischer Deutung: Begriffiche Klärungen und Entwürfe für Lernen und Lehren. Weinheim/Basel: Belt Juventa.

Schmidt, F. (2016): Pädagogische Wahrnehmbarkeitsräume. Historisch-anthropologische Annäherung an die Verfasstheit pädagogischer Blicke. In: Schmidt, F. et al. (Hrsg.) (2016): Pädagogische Blicke. Weinheim und Basel: Beltz Juventa. S. 54-71. 
Schmidt, F. et al. (Hrsg.) (2016): Pädagogische Blicke. Weinheim und Basel: Beltz Juventa.

Schmidt, S. (2003): Was wir vom Lernen zu wissen glauben. In: Gehirn und Lernen, 3, S. 40-50.

Schmied, S. (2016): Anregung zur Entwicklung von professioneller pädagogischer Haltung bei PädagogInnen im Kindergarten. In: Wissenschaftliches Journal österreichischer fachhochschul-studiengänge soziale Arbeit, 16, S. 157-169.

Schneider, R.M. \& Plasman K. (2011): Science teacher learning progressions. A review of science teachers' pedagogical content knowledge development. In: Review of Educational Research, 81 (4), S. 530-565.

Schoenfeld, A.H. (2010): How we think: A theory of goal-oriented decision making and its educational applications. New York: Routledge.

Schön, D. (1983): The reflective practitioner. How professionals think in action. New York: Basic Books.

Schratz, M. \& Schrittesser, I. (2003): Was müssen Lehrerinnen und Lehrer in Zukunft wissen und können? In: Berner, H. \& Isler, R. (Hrsg.): Lehrer-Identität, Lehrer-Rolle, Lehrer Handeln. Professionswissen für Lehrerinnen und Lebrer. Hohengehren: Schneier Verlag. S. 177-200.

Schratz, M. (2009): „Lernseits“ von Unterricht. Alte Muster, neue Lebenswelten - was für Schulen? In: Lernende Schule, 46-47. S. 16-21.

Schratz, M. (2013): Alle reden von Kompetenz, aber wie!? In: Lernende Schule 15(58), S. 17-20

Schratz, M. (2019): Schule im 21. Jahrhundert. In: Harring, M. et al.: Handbuch Schulpädagogik. New York/Münster: Waxmann. S. 41-53.

Schulz-Schaeffer, I. \& Böschen, S. (2003): Wissenschaft in der Wissensgesellschaft. Wiesbaden: Springer.

Schurz, G. (1995): Vorlesung Erkenntnistheorie. [http://www.philosophie.hhu.de/fileadmin/redaktion/Fakultaeten/ Philosophische_Fakultaet/Philosophie/Lehrstuhl_Prof._Dr._Schurz/Schurz/scripts/ErkSkri.pdf; zugriffen am 05.05.2019]

Schütz, A. \& Luckmann, T. (1988): Strukturen der Lebenswelt, Bd.1. 3 Aufl. Frankfurt/M.: Suhrkamp.

Schwer, C. \& Solzbacher, C. (2014): Professionelle pädagogische Haltung: Historische, theoretische und empirische Zugänge in einem viel strapazierten Begriff. Bad Heilbrunn: Julius Klinkhardt.

Seichter, S. (2014): Pädagogische Beziehungsformen. In: Zirfas, J. \& Wulf, C. (Hrsg.): Handbuch Pädagogische Anthropologie. Wiesbaden: Springer Verlag. S. 227-236.

Seidel, T. \& Prenzel, M. (2010): Beobachtungsverfahren: Vom Datenmaterial zur Datenanalyse. In: Holling, B. \& Schmitz, B. (Hrsg.): Handbuch der Psychologischen Methoden und Evaluation. Göttingen: Hogrefe. S. 139-152.

Seidel, T. \& Stürmer, K. (2014): Modelling the structure of professional vision in pre-service teachers. In: American Educational Research Journal, 51 (4), S. 739-771.

Seifert, A. et al. (2009): Entwicklung und psychometrische Überprüfung eines Messinstrumentes zur Erfassung pädagogischer Kompetenz in der universitären Lehrerbildung. In: Lebrerbildung auf dem Prüfstand, 2(1), S. 82-103.

Sembill, D. \& Seifried, J. (2009): Konzeptionen, Funktionen und intentionale Veränderungen von Sichtweisen. In: Zlatkin-Troitschanskaia et al. (Hrsg.): Lehrerprofessionalität - Bedingungen, Genese, Wirkungen und ihre Messung. Weinheim/Basel: Beltz. S. 334-354.

Senger, E., \& Österle, H. (2004): PROMET-Business Engeneering Case Studies 2.0. St. Gallen: Universität St. Gallen.

Shalem, Y. (2014): „What binds professional judgement? The case of teaching“. In: Young, M. \& Muller, J. (Hrsg.): Knowledge, Expertise and the Professions. London: Routledge.

Shavelson, R.J. (2013): On an Approach to Testing and Modeling Competences. In: Educational Psychologist, 48, (2), S. 73-86.

Shulman, L.S. (1986): Paradigms and research programs in the study of teaching: A contemporary perspective. In: Wittrock, M.C. (Hrsg.): Handbook of research on teaching. New York: Macmillan. S. 3-36.

Shulman, L.S. (1987): Knowledge and teaching: Foundations of the new reform. In: Havard Educational Research, 57, S. 1-22.

Simmel, G. (1992): Das Geheimnis und die geheime Gesellschaft. In: Soziologie - Untersuchung über die Formen der Vergesellschaftung. Frankfurt: Suhrkamp. S. 383-455.

Slavin, R.E. (1994): Quality, appropriateness, incentive, and time: A model of instructional effectiveness. In: International Journal of Educational Research, 21, S. 141-157.

Smithon, M. (1985): Toward a Social Theory of Ignorance. In: Journal for the Theory of Social Behaviour 10, S. 157-168.

Smithon, M. (1989): Ignorance and Uncertainy. Emerging Paradigms. New York/Berlin: Springer.

Sölch, D. (2014): Prozessphilosophien. München: Verlag Karl Alber.

Sonmark, K. et al. (2017): „Understanding teachers' pedagogical knowledge: report on an international pilot study“, In: OECD Education Working Papers, No. 159, OECD Publishing, Paris. [http://dx.doi.org/10.1787/43332ebd-en; zugegriffen am 16.01.2019]

Sothayapetch, P. et al. (2013): Primary school teachers' interviews regarding Pedagogical Content Knowledge (PCK) and General Pedagogical Knowledge (GPK). In: European Journal of Science and Mathematics Education, 1 (1), S. 84-105.

Stake, Robert E. (1995): The Art of Case Study Research. London/New Delhi: Thousand Oaks. 
Statistik Austria (2019): Lehrerinen und Lehrer im Schuljahr 2017/2018 im Vollzeitäquivalenten nach Schultypen. [https://www.statistik.at/web_de/statistiken/menschen_und_gesellschaft/bildung/schulen/lehrpersonen/index.html; zugegriffen am 14.08.2019]

Stehr, N. (2000): Die Zerbrechlichkeit moderner Gesellschaften. Weilerswist: Velsbrück.

Stehr, N. (2001): Wissen und Wirtschaften. Die gesellschaftlichen Grundlagen der modernen Ökonomie. Frankfurt: Suhrkamp.

Strasser, J. (2006): Erfahrung und Wissen in der Beratung. Theoretische und empirische Analysen zum Entstehen professionellen Wissens in der Erziehungsberatung. Göttingen: Cuvillier Verlag.

Strauss, A.L. (1991): Grundlagen qualitativer Sozialforschung. Datenanalyse und Theoriebildung in der empirischen soziologischen Forschung. München: Wilhelm Fink.

Strauss, A.L. (1998): Grundlagen qualitativer Sozialforschung. Paderborn: Fink.

Strauss, A.L. \& Corbin, J. (1994): Grounded Theory Methodology. An Overview. In: Denzin, N.K. (Hrsg.): Handbook of Qualitative Research. New York: Sage Publication. S. 273-285.

Strauss, A.L. \& Corbin, J. (1996/2010): Grounded Theory. Grundlagen Qualitativer Sozialforschung. Weinheim: Beltz Psychologie Verlags Union.

Strübing, J. (2008): Grounded Theory. Zur sozialtheoretischen und epistemologischen Fundierung des Verfahrens der empirisch begründeten Theoriebildung. 2. Auflage. Wiesbaden: Springer.

Strübing, J. et al. (2018): Gütekriterien qualitativer Sozialforschung. Ein Diskussionsanstoß. In: Zeitschrift für Soziologie, 47 (2), S. 83-100.

Stuckert, G. (1980): Das Lernen des Lernens. In: Geppert, K. \& Preuß, E. (Hrsg.): Selbständiges Lernen. Bad Heilbrunn: Lenske + Budrich. S. 87-95.

Tapp, Ch. (2016): Philsophische Gotteserekenntnis nach Thomas von Aquin. In: Phil.Jahrbuch 123. S. $426-442$.

Teacher Task Force (2018): International Task Force on Teacher for Education 2030. Strategic Plan 2018-2021. [http:// www.teachersforefa.unesco.org/v2/index.php/en/ressources/file/395-strategic-plan-2018-2021; zugegriffen am 28.01.2019]

Tenorth, H.-E. (1989): Professionstheorie für Pädagogen? In: Zeitschrift für Pädagogik, 35, S. 809-824.

Tenorth, H-E. (2006): Professionalität im LehrerInnenberuf. Ratlosigkeit der Theorie, gelingende Praxis. In: Zeitschrift für Erziehungswissenschaft, 9, S. 580-597.

Terhart, E. (1990): Sozialwissenschaftliche Theorie- und Forschungsansätze zum Beruf des Lehrers: 1970-1990. In: Zeitschrift für Sozialisationforschung und Erziehungssoziologie, 10, S. 235-254.

Terhart, E. (1993): Pädagogisches Wissen. Überlegungen zu seiner Vielfalt, Funktion und sprachlichen Form am Beispiel des Lehrerwissens. In: Oelkers, J. \& Tenorth, H.E.(Hrsg): Pädagogisches Wissen. Weinheim: Belz. S. 129-14.

Terhart, E. (1998): Lehrerberuf: Arbeitsplatz, Biographie, Profession. In: Altrichter, H. et al. (Hrsg.): Handbuch zur Schulentwicklung. Innsbruck. Studienverlag. S. 560-585.

Terhart, E. (2001): LehrerInnenberuf und Lehrerbildung. Forschungsbefunde, Problemanalysen, Reformkonzept. Weinheim: Beltz.

Terhart, E. (2006): Professionalität im LehrerInnenberuf. In: Zeitschrift für Erziehungswissenschaft, 9 (4), S. 580-597.

Terhart, E. (2012): Wie wirkt Lehrerbildung? Forschungsprobleme und Gestaltungsfragen. In: Zeitschrift für Bildungsforschung. S. 3-21.

Terhart, E. (2013): Erziehungswissenschaft und Lehrerbildung. Münster: Waxmann.

Terhart, E. et al. (2012): Bildungswissenschaftliches Wissen und Erwerb professioneller Kompetenz in der Lehramtsausbildung - Eine Kurzdarstellung des BilWiss-Projekts. In: Lehrerbildung auf dem Prüfstand, 5 (1), S. 96-106.

Terhart, E. et al. (2014): Handbuch der Forschung zum Lehrberf. Münster: Waxmann.

Tervooren, A. (2014): Empirie. In: Zirfas, J. \& Wulf, C. (Hrsg.): Handbuch Pädagogische Anthropologie. Wiesbaden: Springer Verlag. S. 55-64.

Thiel, F. (2007): Stichwort: Umgang mit Wissen. In: Zeitschrift für Erziehungswissenschaft, 10 (2), S. 153-169.

Townsend, T. \& Bates, R. (Hrsg.) (2007): Handbook of Teacher Education. Globalization, Standards and Professionalism in Time and Change. Amsterdam: Springer.

Tulodziecki, G. et al. (2004): Gestaltung von Unterricht. Eine Einfübrung in die Didaktik. Bad Heilbrunn: Klinkhardt.

Van Driel, J. et al. (2001): Professional Development and Reform in Science Education: The Role of Teachers' Practical Knowledge. In: Journal of Research in science teaching, 38 (2), S. 137-158.

Vester, H.-G. (2009): Kompendium der Soziologie II: Die Klassiker. Wiesbaden: Springer.

Volpert, W. (1992): Wie wir handeln - was wir können. Ein Disput als Einführung in die Handlungspsychologie. Heidelberg: Asanger.

Voss, T. et al. (2011): Assessing teacher candidates' general pedagogical/psychological knowledge: Test construction and validation. In: Journal of Educational Psychology, 103(4), S. 952-969.

Voss, T. et al. (2013): Development of teacher candidates' Classroom-Management knowledge during a practical induction phase. Vortrag auf der 15th Biennial Conference European Association für Research on Learning and Instruction, München. 
Voss, T. et al. (2014): Die Bedeutung des pädagogisch-psychologischen Wissens von angehenden Lehrkräften für die Unterrichtsqualität. In: Zeitschrift für Pädagogik, 60 (2), S. 84-201.

Voss, T. et al. (2015): Stichwort Pädagogisches Wissen von Lehrkräften- Empirische Zugänge und Befunde. In: Zeitschrift für Erziehungswissenschaften, 18, S. 187-223.

Voss, T. \& Kunter, M. (2011): Pädagogisch-psychologisches Wissen von Lehrkräften. In: Kunter, M. et al. (Hrsg.): Professionelle Kompetenz von Lehrkräften - Ergebnisse des Forschungsproramms COACTIV. Münster: Waxmann. S. 93-214.

Wagenknecht, A. (1992): Das geistige Etwas. [http://achimwagenknecht.de/Augustin/a71dasgeistigeetwas.htm; zugegriffen am 04.05.2019]

Waldenfels, B. (2000): Das leibliche Selbst. Vorlesungen zur Phänomenologie des Leibes. Frankfurt a.M.: Suhrkamp.

Wang, J. et al. (2011): Quality teaching and teacher education. A kaleidoscope of notions. In: Journal of Teacher Education, 62 (4), S. 331-338.

Wehling, P. (2001): Jenseits des Wissens? Wissenschaftliches Nichtwissen aus soziologischer Perspektive. In: Zeitschrift für Soziologie, 6, S. 465-484.

Wehling, P. (2003): Das Recht auf Nichtwissen in der Humangenetik - ein „Irrläufer“ in der Wissensgesellschaft? In: Allmendinger, Jutta (Hrg.): Entstaatlichung und soziale Sicherheit. Verhandl. des 31. Kongresses der Dt. Gesellsch. für Soziologie in Leipzig 2002. 2 Bände und CD-ROM. Opladen: Leske + Budrich. (CD-ROM).

Weinberg, J. (2000): Über die Entstehung von Wissen und lernen aus Alltäglichkeit und Erinnerung. In: Report 45, S. 116-122.

Weinert, F. (1997): Grenzen der Entwicklung des Humankapitals aus Sicht der psychologischen Lerntheorie. In: Clar, G. et al. (Hrsg.): Humankapital und Wissen: Grundlagen einer nachhaltigen Entwicklung. Berlin: Springer. S. $137-156$.

Weinert, F.E. (1998): Entwicklung im Kindesalter - Bericht über eine Längsschnittstudie. Weinheim: PVU.

Weinert, F. (2001a): Concept of Competences: A Conceptual Clarification. In: Rychenj, D.S. \& Salganik, L.H. (Hrsg.): Defining and Selecting Key Competences, Göttingen: Hogrefe. S. 45-56.

Weinert, F. (2001b): Leistungsmessungen in Schulen. Weinheim und Basel: Beltz.

Weinstein, D. \& Weinstein, M. (1978): The Sociology of Nonknowledge: A Paradgim. In: Jones, R.A. (Hrsg.): Research in the Sociology of Knowledge, Sciences and Art, Vol. 1. New York: JAI Press. S. 151-166.

Welskopp, T. (2001): Die Dualität von Struktur und Handeln. Anthony Giddens' Strukturierungstheorie als „praxeologischer" Ansatz in der Geschichtswissenschaft. In: Geschichte und Gesellschaft, Sonderheft Vol. 19, Struktur und Ereignis. S. 99-119.

Wensierski, H. von \& Jakob, G. (1997): Rekonstruktive Sozialpädagogik. Sozialwissenschaftliche Hermeneutik, Fallverstehen und sozialpädagogisches Handeln - eine Einführung. In: Jakob, G. \& Von Wensierski, H. (Hrgs.): Rekonstruktive Sozialpädagogik. Konzepte und Methoden sozialpädagogischen Verstehens in Forschung und Praxis. Weinheim: Juventa Verlag, S. 7-22.

White, H. (2008): Idenity and Control - How Social Formations Emerge. Princeton: University Press.

Wilke, H. (2002): Dystopia. Studien zur Krisis des Wissens in der modernen Gesellschaft. Frankfurt: Suhrkamp.

Wilson, T.P. (1973): Theorien der Interaktion und Modelle soziologischer Erklärung. In Arbeitsgruppen Bielefelder soziologischer Erklärung (Hrsg.): Alltagswissen, Interaktion und gesellschaftliche Wirklichkeit. Bd. 1: Symbolischer Interaktionismus und Ethnomethodologie. Reinbeck: Rowohlt. S. 54-79.

Wilson, S.M. et al. (1987): „150 different ways“ of knowing: Representations of knowledge in teaching. In: Calderhead, J. (Hrsg.): Exploring teachers' thinking. London: Cassell. S. 104-124.

Wilson, T.P. (1982): Qualitative oder quantitative Methoden in der Sozialforschung. In: Kölner Zeitschrift für Soziologie und Sozialpsychologie, 34, S. 487-508.

Witzel, A. (1982): Verfahren der qualitativen Sozialforschung. Überblick und Alternativen. Frankfurt: Campusverlag.

Wright, S. P. et al. (1997): Teacher and Classroom Context Effects on Student Achievement: Implications for Teacher Evaluation. In: Journal of Personnel Evaluation in Education 11, S. 57-67.

Wulf, C. (1994): Zur Einleitung: Grundzüge einer historisch-pädagogischen Anthropologie. In: Wulf, C. (Hrsg.): Pädagogische Anthropologie. Weinheim/Basel: Beltz, S. 7-21.

Wulf, C. (Hrsg.) (1997): Auge. In: Vom Menschen. Handbuch Historische Anthropologie. Weinheim, Basel: Beltz. S. 446-458.

Wyss, C. (2008): Zur Reflexionsfähigkeit und -praxis der Lehrperson. In: Bildungsforschung 5, S. 1-15.

Ziefuß, H. (1987): Methoden der Unterrichtsbeobachtung. Braunschweig: Westermann Verlag.

Zimring, F. (2006): Carl Rogers - originally published in prospects: the quarterly review of comparative education. In: International Bureau of Education, 24 (3/4), S. 411-422.

Zirfas, J. \& Wulf, C. (2014): Handbuch Pädagogische Anthropologie. Wiesbaden: Springer.

Zymek, B. (2008): Geschichte des Schulwesens und des Lehrerberufs. In: Helsper, W. \& Böhme, J. (Hrsg.): Handbuch der Schulforschung. Wiesbaden: Verlag für Sozialwissenschaften Springer. S. 203-138. 


\section{Abbildungsverzeichnis}

Abb. 1: Überblick zentrale Themenbereiche der Forschungsarbeit

Abb. 2: Anforderungen Lehrpersonen (in Anlehnung an Conway et al. 2009; Terhart 2013:

56ff.; Guerriero 2017)

Abb. 3: Fertigkeiten des 21. Jahrhunderts (in Anlehnung an National Research

Council 2012: 2)

Abb. 4: Interdisziplinärer Zugang zum Wissensbegriff in der vorliegenden Arbeit $\ldots \ldots \ldots \ldots \ldots \ldots . \quad 24$

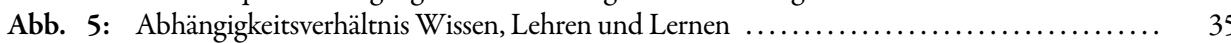

Abb. 6: Verhältnis zwischen Wissen, Lehren, Lernen und Erziehung . ................... 36

Abb. 7: Generische Kompetenzen des lernseitigen Wissens von Lehrpersonen

(in Anlehnung an Schratz 2019: 45f.)

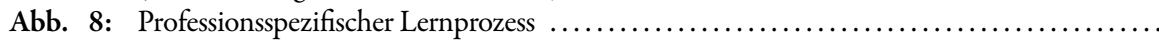

Abb. 9: Theoretisches Rahmenmodell professioneller Kompetenz (Kunter et al. 2011/2013) ..... 44

Abb. 10: Konzept des LehrerInnenwissens (Neuweg 2014: 585) ....................... 45

Abb. 11: Wissensmodell professioneller Lehrpersonen .............................. 47

Abb. 12: Beziehungen der Formen pädagogischen Wissens ............................ 47

Abb. 13: Zusammenhang zwischen Wissensqualitäten und Wissensformen .................. 51

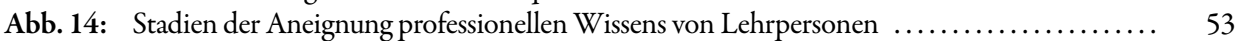

Abb. 15: Übersicht der Studien zur Untersuchung pädagogischen Wissens . . . . . . . . . . . . . . . 61

Abb. 16: Pädagogische Interaktion nach Giesecke ................................. 68

Abb. 17: Selbstkonzept nach Mikhail (in Anlehnung an Mikhail 2006: 206) ................ 70

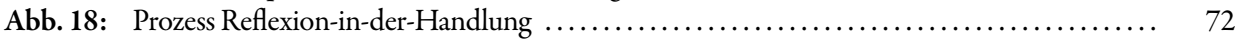

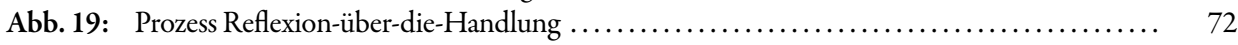

Abb. 20: Pädagogisches Selbst (in Anlehnung an Bauer 1998: 353) ..................... 73

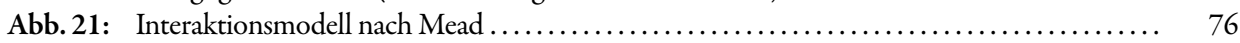

Abb. 22: Didaktisches Dreieck im Kontext von „Me“ und „I“ ......................... 77

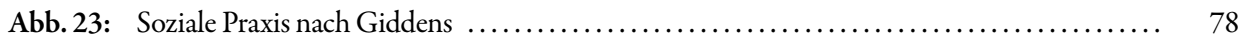

Abb. 24: Didaktisches Dreieck im Kontext der sozialen Praxis von Giddens . .................. 79

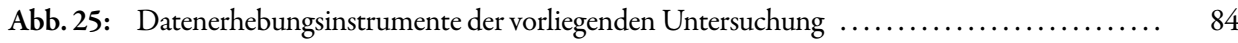

Abb. 26: Forschungsprozess (in Anlehnung an Flick 2007: 128) ........................ 85

Abb. 27: Überblick Anzahl Lehrpersonen nach Schultyp ............................ 87

Abb. 28: Überblick Alter und Dienstjahre der Lehrpersonen $\ldots \ldots \ldots \ldots \ldots \ldots \ldots \ldots \ldots \ldots \ldots \ldots \ldots$

Abb. 29: Überblick Unterrichtsfächer der Lehrpersonen nach Schultyp .................... 88

Abb. 30: Überblick Geschlechteranteil der Lehrpersonen nach Schultyp .................... 89

Abb. 31: Überblick Sample Interviews und Beobachtungen ........................... 89

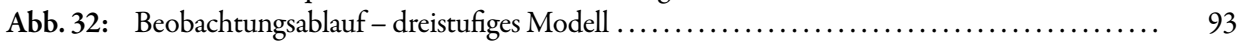

Abb. 33: Beobachtungsintervalle ............................................ 93

Abb. 34: Überblick Anwendungsbereiche Unterrichtsbeobachtungen .................... 94

Abb. 35: Beobachtungskategorien basierend auf anthropologischen Grundkonstituenten .......... 95

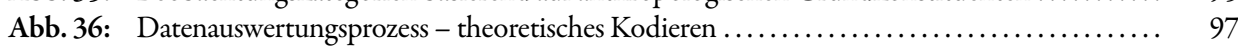

Abb. 37: Übersicht Aufbau Ergebnisdarstellung ................................... 99

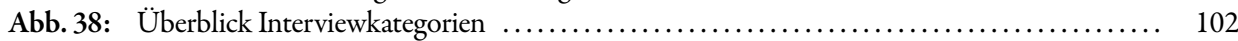

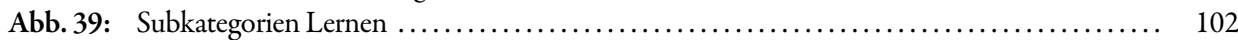

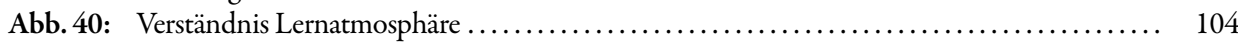

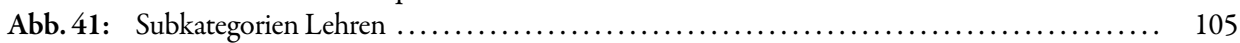

Abb. 42: Wissensformen im Bereich der Didaktik ................................... 106

Abb. 43: Beeinflussungsdynamik von psychologischem Wissen .......................... 108

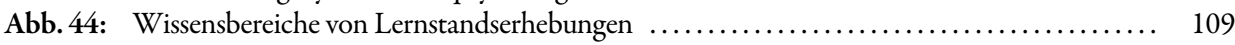

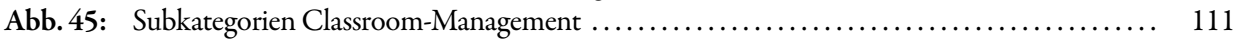

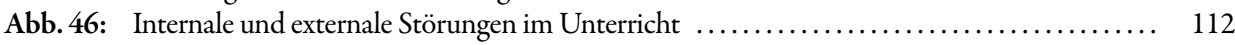

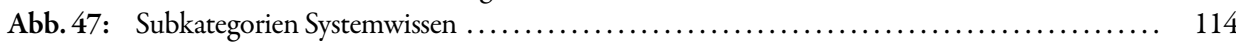




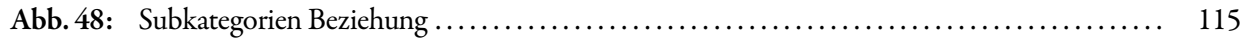

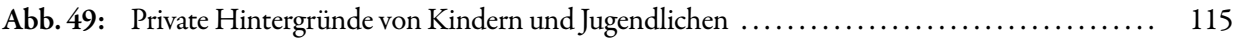

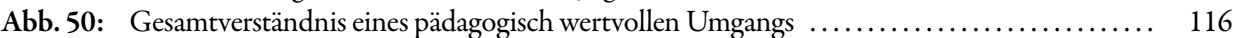

Abb. 51: Verständnis zielführender Team-Teaching-Prozesse im Unterricht $\ldots \ldots \ldots \ldots \ldots \ldots \ldots \ldots, 118$

Abb. 52: Subkategorien Kommunikation ....................................... 120

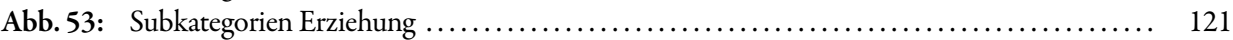

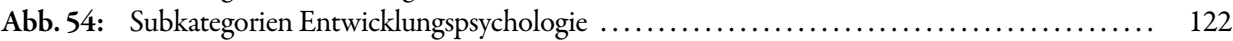

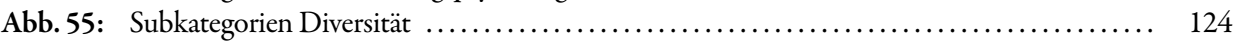

Abb. 56: Umsetzungsstrategien der Kategorie Diversität im Unterricht $\ldots \ldots \ldots \ldots \ldots \ldots \ldots \ldots \ldots . \ldots \ldots$

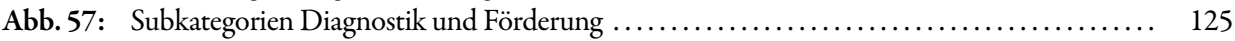

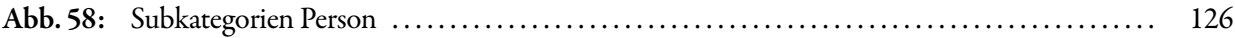

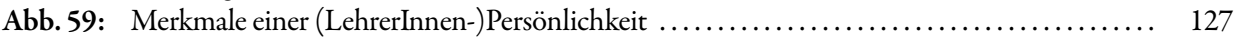

Abb. 60: Übersicht zu den Herausforderungen für pädagogisches Wissen . . . . . . . . . . . . . . . 128

Abb. 61: Aneignungsetappen pädagogischen Wissens ................................ 132

Abb. 62: Wissenslücken pädagogischen Wissens ................................... 135

Abb. 63: Auswirkungen von Wissenslücken auf den Unterricht ......................... 137

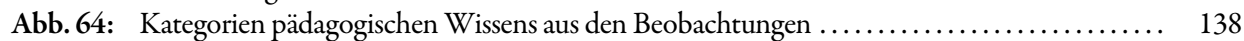

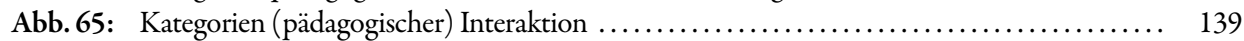

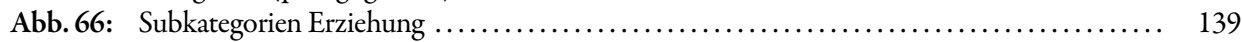

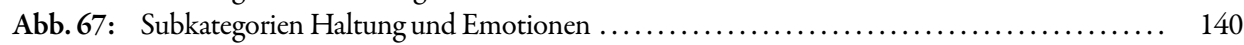

Abb. 68: Handlungsstrategien zur Reduktion lernseitiger Angstsituationen ................. 141

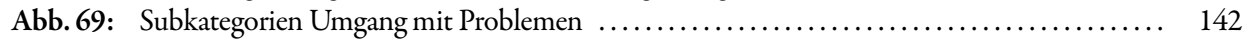

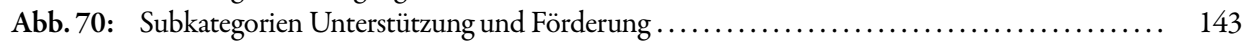

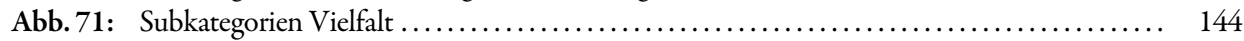

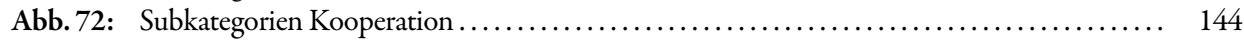

Abb. 73: Kooperationsmuster zwischen den AkteurInnen ............................ 144

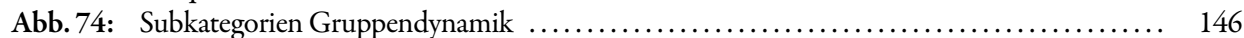

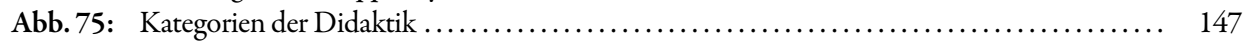

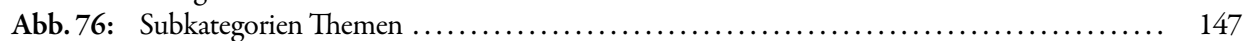

Abb. 77: Subkategorien Unterrichtsstrategien und Konzepte .......................... 148

Abb. 78: Strategien für die Berücksichtigung von Differenzierung im Unterricht .............. 149

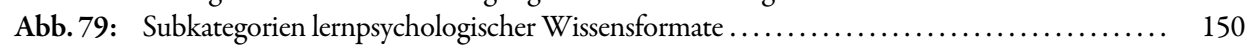

Abb. 80: Rekonstruierte Stereotypen aus dem Schulalltag ........................... 150

Abb. 81: Kategorien der Kommunikation ..................................... 151

Abb. 82: Subkategorien Sprache ......................................... 151

Abb. 83: Rekonstruierte Wirkungsbereiche der Sprache ............................. 152

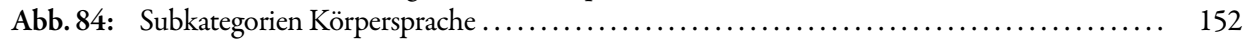

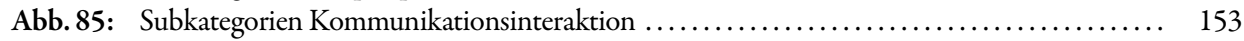

Abb. 87: Kategorien des Classroom-Managements .................................. 155

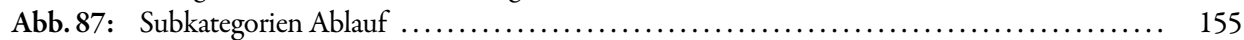

Abb. 88: Strukturelle Muster eines Unterrichtsverlaufs ............................. 156

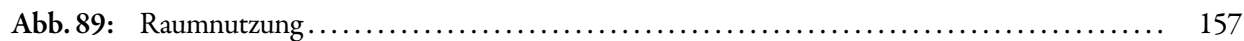

Abb. 90: Bewegungen der AkteurInnen im Raum .................................. 158

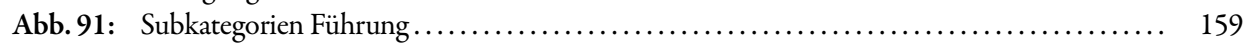

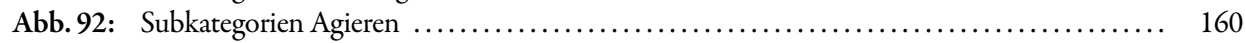

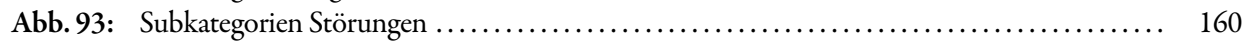

Abb. 94: Kategorien der Personen ........................................... 161

Abb. 95: Realisierung von Wechselwirkungen im Unterricht .......................... 162

Abb. 96: Subkategorien Rolle ............................................. 162

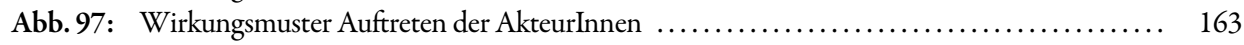

Abb. 98: Wechselverhältnis und Wirkung von Einstellungen und Positionen ................ 163

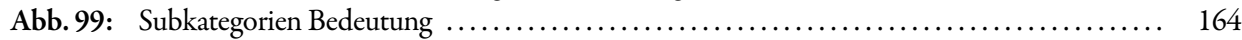




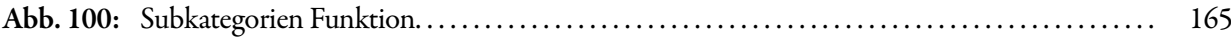

Abb. 101: Individuelles Verständnis pädagogischen Wissens - Lehrperson Grundschule........... 166

Abb. 102: Vergleich kognitiver Muster und pädagogischen Handelns ....................... 169

Abb. 103: Individuelles Verständnis pädagogischen Wissens - Lehrperson Neue Mittelschule ...... 170

Abb. 104: Vergleich kognitiver Muster und pädagogischen Handelns .................... 173

Abb. 105: Individuelles Verständnis pädagogischen Wissens - Lehrperson Gymnasium ........... 174

Abb. 106: Vergleich kognitiver Muster und pädagogischen Handelns $\ldots \ldots \ldots \ldots \ldots \ldots \ldots \ldots \ldots \ldots \ldots$

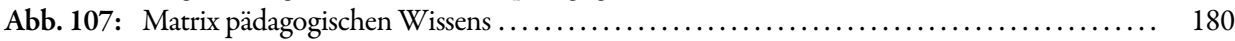

Abb. 108: Wechselwirkungen pädagogischen Wissens .............................. 182

Abb. 109: Häufigkeiten spezifischer rekonstruierter Kategorien pädagogischen Wissens ........... 183

Abb. 110: Vergleich rekonsturierter Kategorien zwischen den Interviews und Beobachtungen im Bereich ressourcenorientiert-psychologisches Wissen ..................... 185

Abb. 111: Vergleich rekonsturierter Kategorien zwischen den Interviews und Beobachtungen im Bereich organisatorisch-strukturelles Wissen .......................... 186

Abb. 112: Vergleich rekonsturierter Kategorien zwischen den Interviews und Beobachtungen im Bereich personenbezogenes Wissen.............................. 187

Abb. 113: Vergleich rekonsturierter Kategorien zwischen den Interviews und Beobachtungen im Bereich interaktionales Wissen .................................... 188

Abb. 114: Schulformspezifische Rekonstruktionsschwerpunkte pädagogischen Wissens .......... 190

Abb. 115: Theoriebasiertes und handlungsorientiertes Verständnis pädagogischen Wissens

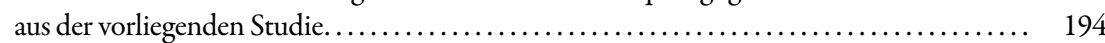

Abb. 116: Rekonstruierter Interaktionsprozess pädagogischen Wissens ..................... 196

Abb. 117: Verhältnis pädagogisches Wissen und didaktisches Dreieck ................... 204

Abb. 118: Auswirkungen von fehlendem pädagogischem Wissen auf das didaktische Dreieck....... 205

Abb. 119: Einflussfaktoren auf pädagogisches Wissen.............................. 207

Abb. 120: Ebenen der Reflexion als Einflussfaktor auf pädagogisches Wissen................ 208

Abb. 121: Felder pädagogischen Wissens von Lehrpersonen........................... 213

\section{Tabellenverzeichnis}

Tab. 1: Beispiele für Konzeptualisierungen pädagogischen Wissen

(in Anlehnung an Voss et al. 2015: 192) .............................. 56

Tab. 2: Thematische Clusterung der Definitionen pädagogischen Wissens $\ldots \ldots \ldots \ldots \ldots \ldots \ldots \ldots$

Tab. 3: Übersicht der Studien zu pädagogischem Wissen

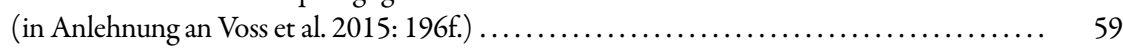

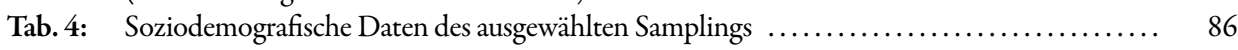

Tab. 5: Soziodemografische Daten - Beobachtungssample .......................... 90

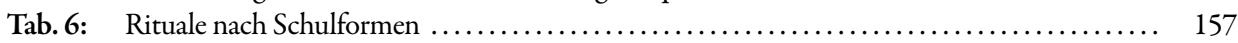

Tab. 7: Sichtbarkeit pädagogischen Wissens im Handeln von Lehrpersonen der

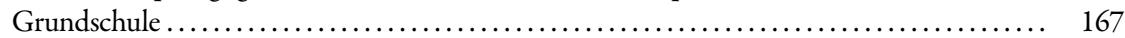

Tab. 8: Sichtbarkeit pädagogischen Wissens im Handeln von Lehrpersonen der Neuen Mittelschule ................................................ 172

Tab. 9: Sichtbarkeit pädagogisches Wissen im Handeln von Gymnasiallehrpersonen ........... 176 
Was verstehen Lehrkräfte aus der Schulpraxis unter dem Begriff pädagogisches Wissen und wie konzeptualisieren sie dieses? Das Wissen von Lehrpersonen im Schulalltag und das damit einhergehende Professionalisierungsverständnis sind zentrale Themen in der Lehrerlnnenbildung. Im vorliegenden Buch wird pädagogisches Wissen von Lehrpersonen unterschiedlicher Schulformen basierend auf einer Interview- und Beobachtungsstudie rekonstruiert und hinsichtlich konzeptueller Erkenntnisse für die Lehrerlnnenbildung aufgearbeitet. Zudem werden Abweichungen und Übereinstimmungen zwischen dem Verständnis pädagogischen Wissens und dem jeweiligen Handeln im Schulalltag sichtbar gemacht. Ausgehend von einer Darstellung unterschiedlicher Wissensformen, wird pädagogisches Wissen und pädagogisches Handeln im Kontext sozialer Zugänge (Mead und Giddens) diskutiert. Es ist das Hauptanliegen dieses Bandes, den Diskurs über das pädagogische Wissen von Lehrpersonen vor dem Hintergrund gesellschaftlicher Transformationsprozesse zu erweitern.

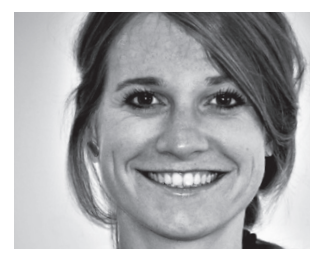

\section{Die Autorin}

Mag. Ann-Kathrin Dittrich, PhD, Jahrgang 1989, wissenschaftliche Mitarbeiterin am Institut für LehrerInnenbildung und Schulforschung der Universität Innsbruck, absolvierte ein Spanisch- sowie Geographie und Wirtschaftskunde-Lehramtsstudium (Sekundarstufe I/II) an der Universität Innsbruck. Ihre Arbeits- und Forschungsschwerpunkte liegen im Bereich der Lehrerlnnenbildung, Professionalisierungsforschung und Schulforschung. 